Supporting Information

\title{
Late-Stage Diversification by Selectivity Switch in meta-C-H Activation: Evidence for Singlet Stabilization
}

Korkit Korvorapun, Rositha Kuniyil, and Lutz Ackermann*

Institut für Organische und Biomolekulare Chemie, Georg-August-Universität Göttingen,

Tammannstraße 2, 37077 Göttingen, Germany

Email: Lutz.Ackermann@ @ chemie.uni-goettingen.de

http://www.ackermann.chemie.uni-goettingen.de/ 


\section{Table of Contents}

$\begin{array}{ll}\text { General Remarks } & \text { S2 }\end{array}$

Optimization Studies $\quad$ S3

Unsuccessful Substrates $\quad$ S6

$\begin{array}{ll}\text { General Procedures } & \text { S7 }\end{array}$

Characterization Data $\quad$ S8

Control Experiments with Complex Ru-I $\quad$ S46

Influence of $\mathrm{PPh}_{3}$ loading in the catalysis $\quad \mathrm{S} 46$

$\begin{array}{ll}\text { Competition Experiments } & \text { S48 }\end{array}$

Reaction with Radical Scavengers $\quad$ S51

Synthesis of Complex Ru-II $\quad$ S53

Synthesis of Complex Ru-III

Synthesis of Complex Ru-IV S56

Catalytic Reaction with Complex trans-Ru-II, Ru-III, and Ru-IV S58

Stoichiometric Reaction of Complex Ru-II $\quad$ S59

Cyclic Voltammetry $\quad$ S60

Racemization Examination $\quad$ S62

$\begin{array}{lr}\text { X-Ray Crystallographic Analysis } & \text { S70 }\end{array}$

$\begin{array}{ll}\text { Fluorescence Spectra } & \text { S112 }\end{array}$

$\begin{array}{ll}\text { Computational Data } & \text { S114 }\end{array}$

References $\quad$ S133

$\begin{array}{ll}\text { NMR Spectra } & \text { S136 }\end{array}$ 


\section{General Remarks}

Catalytic reactions were performed under a $\mathrm{N}_{2}$ atmosphere using pre-dried glassware and standard Schlenk techniques. 1,4-Dioxane was dried over sodium and freshly distilled under $\mathrm{N}_{2}$. The following starting materials were synthesized according to previously described methods: Ru-I, ${ }^{1} \mathbf{R u}-\mathbf{V},{ }^{2}$ 2-arylpyrimidines $\mathbf{1 d}-\mathbf{1 e},{ }^{3}$ purines $\mathbf{1 h}, \mathbf{1 m}-\mathbf{1 o},{ }^{4}$ benzyl chlorides $\mathbf{2} \mathbf{m}-$ 2n, 5n, ${ }^{5}$ and BODIPY 5a. ${ }^{6}$ Other substrates were obtained from commercial sources and were used without further purification. Yields refer to isolated compounds estimated to be $>95 \%$ pure as determined by ${ }^{1} \mathrm{H}-\mathrm{NMR}$ and GC. TLC was performed on Merck TLC Silica Gel $60 \mathrm{~F}_{254}$ with detection under UV light at $254 \mathrm{~nm}$. Chromatographic separations were carried out on Merck Geduran SI-60 (0.040-0.063 mm, 230-400 mesh ASTM). IR spectra were recorded on a Bruker FT-IR alpha-P device. EI-MS was recorded on Jeol AccuTOF at $70 \mathrm{eV}$; ESI-MS was recorded on Bruker Daltonik micrOTOF and maXis and LIFDI with a Linden CMS. Melting points (m.p.) were measured on Stuart ${ }^{\circledR}$ melting point apparatus SMP3, values are uncorrected. Nuclear magnetic resonance (NMR) spectroscopy was performed at 300, 400, 500 or $600 \mathrm{MHz}\left({ }^{1} \mathrm{H}\right.$ NMR), 75, 100 or $125 \mathrm{MHz}\left({ }^{13} \mathrm{C}-\mathrm{NMR}, \mathrm{APT}\right), 282,376$ or $470 \mathrm{MHz}\left({ }^{19} \mathrm{~F}-\mathrm{NMR}\right), 162 \mathrm{MHz}$ $\left({ }^{31} \mathrm{P}-\mathrm{NMR}\right)$ and $121 \mathrm{MHz}\left({ }^{31} \mathrm{P}\left\{{ }^{1} \mathrm{H}\right\}-\mathrm{NMR}\right)$ on Bruker Avance III HD 300, Avance III 300, Avance III 400, Avance III HD 500, Varian Unity-300, Inova 500 and Inova 600 instruments. Chemical shifts $(\delta)$ are provided in ppm and spectra refered to non-deuterated residual solvent signal. Recycling preparative HPLC system from Japan Analytical Industries (LC-92XX II Series, $U V$ and RI Detector) connected to JAIGEL 2HH series column with HPLC grade chloroform. X-ray structures were measured on Bruker APEX-II CCD diffractometer. Fluorescence excitation and emission data in solution were recorded on a Jasco FP-8500 spectrofluorometer. Emission spectra were measured at an excitation wavelength of $502 \mathrm{~nm}$. The bandwidths of excitation and emission were set to 2.5 and $5.0 \mathrm{~nm}$, respectively. The scan speed was adjusted to $500 \mathrm{~nm} / \mathrm{min}$. Analytical HPLC analysis was performed on Agilent 1260/1290 Infinity equipped with Daicel CHIRALPAK IA-3 or IC-3 (4.6 mm x $250 \mathrm{~mm}, 3 \mu \mathrm{m}$ particle size, $1 \mathrm{~mL} / \mathrm{min}$ flow rate). Preparative HPLC from Agilent 1260 Infinity equipped with VP 250/16 Nucleodur 100-10 C18 ec column. 


\section{Optimization Studies}

Table S1. Optimization of bases and temperature. ${ }^{a}$

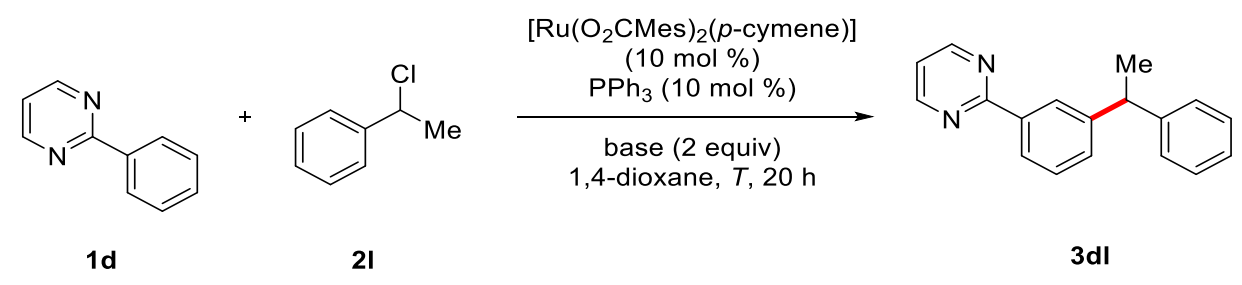

\begin{tabular}{cccc}
\hline Entry & Base & $\boldsymbol{T}\left({ }^{\circ} \mathbf{C}\right)$ & 3dl (\%) \\
\hline $1^{b}$ & $\mathrm{~K}_{2} \mathrm{CO}_{3}$ & 120 & 16 \\
2 & $\mathrm{~K}_{2} \mathrm{CO}_{3}$ & 120 & 39 \\
$\mathbf{3}$ & $\mathrm{K}_{2} \mathrm{CO}_{3}$ & $\mathbf{4 0}$ & $\mathbf{6 2}(\mathbf{7 3})$ \\
4 & $\mathrm{~K}_{2} \mathrm{CO}_{3}$ & 23 & $47(52)$ \\
5 & $\mathrm{KOAc}$ & 23 & $41(46)$ \\
6 & $\mathrm{~K}_{3} \mathrm{PO}_{4}$ & 23 & $51(58)$ \\
7 & $\mathrm{~K}_{3} \mathrm{PO}_{4}$ & $\mathbf{4 0}$ & $\mathbf{6 4}(\mathbf{7 6})$ \\
8 & $\mathrm{~K}_{3} \mathrm{PO}_{4}$ & 60 & $(64)$ \\
9 & $\mathrm{Li}_{3} \mathrm{PO}_{4}$ & 40 & $(5)$ \\
10 & $\mathrm{Na}_{3} \mathrm{PO}_{4}$ & 40 & $59(73)$
\end{tabular}

${ }^{a}$ Reaction conditions: 1d $(0.50 \mathrm{mmol}), 2 \mathrm{l}(1.50 \mathrm{mmol}),\left[\mathrm{Ru}\left(\mathrm{O}_{2} \mathrm{CMes}\right)_{2}(p\right.$-cymene $\left.)\right]$ (10 mol \%), $\mathrm{PPh}_{3}(10 \mathrm{~mol} \%)$, base $(1.00 \mathrm{mmol}), 1,4$-dioxane $(2.0 \mathrm{~mL}), \mathrm{T}, 20 \mathrm{~h}$, under $\mathrm{N}_{2}$; yield of isolated products. The yield in parentheses was determined by ${ }^{1} \mathrm{H}$-NMR using 1,3,5-trimethoxybenzene as internal standard. ${ }^{b}$ without $\mathrm{PPh}_{3}$. 
Table S2. Optimization of ligands. ${ }^{a}$

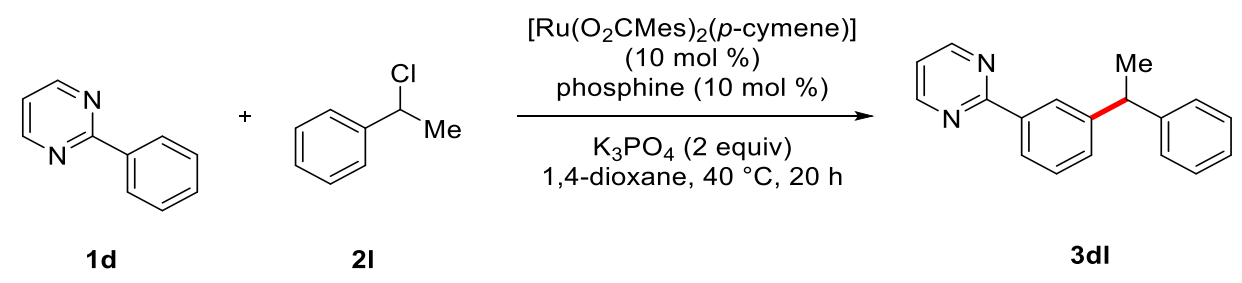

\begin{tabular}{|c|c|c|c|c|c|}
\hline Entry & Phosphine & 3dl (\%) & Entry & Phosphine & 3dl (\%) \\
\hline $\mathbf{1}$ & $\mathbf{P P h}_{3}$ & $64(76)$ & $17^{b}$ & $r a c$-BINAP & (0) \\
\hline 2 & $\mathrm{P}\left(4-\mathrm{FC}_{6} \mathrm{H}_{4}\right)_{3}$ & $65(75)$ & $18^{b}$ & (-)-Cl-MeO-BIPHEP & $(0)$ \\
\hline 3 & $\mathrm{P}\left(4-\mathrm{MeOC}_{6} \mathrm{H}_{4}\right)_{3}$ & (69) & & & \\
\hline 4 & $\mathrm{P}\left(\mathrm{C}_{6} \mathrm{~F}_{5}\right)_{3}$ & (0) & & & \\
\hline 5 & $\mathrm{P}(2 \text {-furyl })_{3}$ & (64) & & & \\
\hline 6 & $\mathrm{P}\left(2,4,6-\left(\mathrm{CH}_{3}\right)_{3} \mathrm{C}_{6} \mathrm{H}_{2}\right)_{3}$ & (0) & & & \\
\hline 7 & $\mathrm{P}\left(2,4,6-\left(\mathrm{OCH}_{3}\right)_{3} \mathrm{C}_{6} \mathrm{H}_{2}\right)_{3}$ & (0) & & & \\
\hline 8 & $\mathrm{P}(1-\mathrm{Ad})_{2} n-\mathrm{Bu}$ & (2) & & & \\
\hline 9 & $\mathrm{P}\left(4-\mathrm{CH}_{3} \mathrm{C}_{6} \mathrm{H}_{4}\right)_{3}$ & (46) & $19^{b}$ & & $(0)$ \\
\hline 10 & $\mathrm{P}\left(2-\mathrm{CH}_{3} \mathrm{C}_{6} \mathrm{H}_{4}\right)_{3}$ & (0) & & $\mathrm{D}_{2} \mathrm{H}$ & \\
\hline 11 & $\mathrm{P}\left(4-\mathrm{CF}_{3} \mathrm{C}_{6} \mathrm{H}_{4}\right)_{3}$ & $65(74)$ & & & \\
\hline 12 & $\mathrm{O}=\mathrm{PPh}_{3}$ & (0) & $20^{b}$ & $n-\mathrm{Bu}_{2} \mathrm{P}(\mathrm{O}) \mathrm{H}$ & $(0)$ \\
\hline 13 & $\mathrm{HPOPh}_{2}$ & (0) & $21^{b}$ & $\mathrm{P}(\mathrm{OPh})_{3}$ & $(21)$ \\
\hline 14 & $\mathrm{P}(n-\mathrm{Bu})_{3}$ & (2) & $22^{b}$ & $\mathrm{P}(\mathrm{OEt})_{3}$ & (15) \\
\hline $15^{b}$ & $\operatorname{IPr}(\mathrm{NHC})$ & (3) & $23^{b}$ & & (30) \\
\hline $16^{c}$ & & (8) & $24^{b}$ & & $(0)$ \\
\hline
\end{tabular}

\footnotetext{
${ }^{a}$ Reaction conditions: 1d $(0.50 \mathrm{mmol}), 2 \mathrm{l}(1.50 \mathrm{mmol}),\left[\mathrm{Ru}\left(\mathrm{O}_{2} \mathrm{CMes}\right)_{2}(p\right.$-cymene $\left.)\right](10 \mathrm{~mol} \%)$, phosphine $(10 \mathrm{~mol} \%), \mathrm{K}_{3} \mathrm{PO}_{4}(1.00 \mathrm{mmol}), 1,4$-dioxane $(2.0 \mathrm{~mL}), 40{ }^{\circ} \mathrm{C}, 20 \mathrm{~h}$, under $\mathrm{N}_{2}$; yield of isolated products. The yield in parentheses was determined by ${ }^{1} \mathrm{H}-\mathrm{NMR}$ using 1,3,5-trimethoxybenzene as internal standard. ${ }^{b} \quad\left[\operatorname{Ru}(\mathrm{OAc})_{2}(p\right.$-cymene $\left.)\right] \quad(10 \mathrm{~mol} \%)$. ${ }^{c}\left[\mathrm{RuCl}_{2}(p \text {-cymene })\right]_{2}(5.0 \mathrm{~mol} \%)$.
} 
Table S3. Optimization of catalyst. ${ }^{a}$

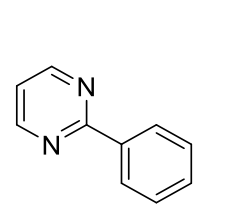

$1 d$

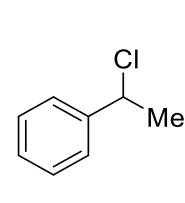

2I
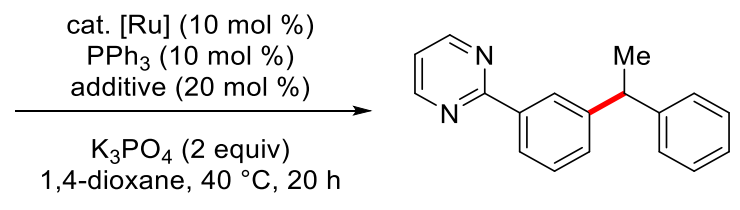

$3 \mathbf{d l}$

\begin{tabular}{|c|c|c|c|}
\hline Entry & cat. $[R u]$ & Additive & 3dl (\%) \\
\hline 1 & - & - & (0) \\
\hline 2 & $\mathrm{RuCl}_{3} \cdot \mathrm{nH}_{2} \mathrm{O}$ & $\mathrm{MesCOOH}$ & $(0)$ \\
\hline $3^{b, c, d}$ & $\mathrm{RuCl}_{3} \cdot \mathrm{nH}_{2} \mathrm{O}$ & KOAc & (6) \\
\hline $4^{e}$ & $\mathrm{RuCl}_{2}\left(\mathrm{PPh}_{3}\right)_{3}$ & $\mathrm{MesCOOH}$ & $(0)$ \\
\hline 5 & $\mathrm{Ru}(\mathrm{OAc})_{2}\left(\mathrm{PPh}_{3}\right)_{2}$ & - & (0) \\
\hline $6^{e}$ & $\mathrm{Ru}(\mathrm{OAc})_{2}\left(\mathrm{PPh}_{3}\right)_{2}$ & - & (0) \\
\hline $7^{e}$ & $\mathrm{Ru}(\mathrm{OAc})_{2}\left(\mathrm{PPh}_{3}\right)_{3}$ & - & $(0)$ \\
\hline $8^{f}$ & {$\left[\mathrm{RuCl}_{2}(p \text {-cymene })\right]_{2}$} & - & $(0)$ \\
\hline 9 & {$\left[\mathrm{Ru}\left(\mathrm{O}_{2} \mathrm{CMes}\right)_{2}(p\right.$-cymene $\left.)\right]$} & - & $64(76)$ \\
\hline 10 & {$\left[\mathrm{Ru}(\mathrm{OAc})_{2}(p\right.$-cymene $\left.)\right]$} & - & $69(76)$ \\
\hline 11 & {$\left[\mathrm{Ru}\left(\mathrm{O}_{2} \mathrm{CAd}\right)_{2}(p\right.$-cymene $\left.)\right]$} & - & $68(76)$ \\
\hline 12 & {$\left[\mathrm{Ru}(\mathrm{OPiv})_{2}(p\right.$-cymene $\left.)\right]$} & - & $68(75)$ \\
\hline $13^{c}$ & {$\left[\mathrm{Ru}(\mathrm{OAc})_{2}(p\right.$-cymene $\left.)\right]$} & - & $68(75)$ \\
\hline $14^{g}$ & {$\left[\mathrm{Ru}(\mathrm{OAc})_{2}(p\right.$-cymene $\left.)\right]$} & - & $(5)$ \\
\hline $15^{h}$ & {$\left[\mathrm{Ru}(\mathrm{OAc})_{2}(p\right.$-cymene $\left.)\right]$} & - & $(42)$ \\
\hline $16^{i}$ & {$\left[\mathrm{Ru}(\mathrm{OAc})_{2}(p\right.$-cymene $\left.)\right]$} & - & (3) \\
\hline $17^{g, h}$ & {$\left[\mathrm{Ru}(\mathrm{OAc})_{2}(p\right.$-cymene $\left.)\right]$} & - & $(56)$ \\
\hline $18^{c, d}$ & {$\left[\mathrm{Ru}(\mathrm{OAc})_{2}(p\right.$-cymene $\left.)\right]$} & - & $68(76)$ \\
\hline $19^{c, d, e}$ & {$\left[\mathrm{Ru}(\mathrm{OAc})_{2}(p\right.$-cymene $\left.)\right]$} & - & $(1)$ \\
\hline $20^{c, d, i}$ & {$\left[\mathrm{Ru}(\mathrm{OAc})_{2}(p\right.$-cymene $\left.)\right]$} & - & $85(85)$ \\
\hline $21^{c, d}$ & $\mathrm{Ru}(\mathrm{OAc})_{2}\left(\mathrm{PPh}_{3}\right)_{2}$ & - & (3) \\
\hline
\end{tabular}

a Reaction conditions: 1d $(0.50 \mathrm{mmol}), 2 \mathrm{l}(1.50 \mathrm{mmol}),[\mathrm{Ru}](10 \mathrm{~mol} \%), \mathrm{PPh}_{3}$ $(10 \mathrm{~mol} \%), \mathrm{K}_{3} \mathrm{PO}_{4}(1.00 \mathrm{mmol}), 1,4$-dioxane $(2.0 \mathrm{~mL}), 40{ }^{\circ} \mathrm{C}, 20 \mathrm{~h}$, under $\mathrm{N}_{2}$; yield of isolated products. The yield in parentheses was determined by ${ }^{1} \mathrm{H}-\mathrm{NMR}$ using 1,3,5-trimethoxybenzene as internal standard. ${ }^{b} \mathrm{NMP} .{ }^{c} \mathrm{~K}_{2} \mathrm{CO}_{3} .{ }^{d} 60{ }^{\circ} \mathrm{C} .{ }^{e}$ without $\mathrm{PPh}_{3}$. ${ }^{f}\left[\mathrm{RuCl}_{2}(p \text {-cymene })\right]_{2}(5.0 \mathrm{~mol} \%) .{ }^{g}[\mathrm{Ru}](5.0 \mathrm{~mol} \%) .{ }^{h} \mathrm{PPh}_{3}(5.0 \mathrm{~mol} \%) .{ }^{i} \mathrm{PPh}_{3}$ (20 mol \%). 
Table S4. Optimization of solvent. ${ }^{a}$

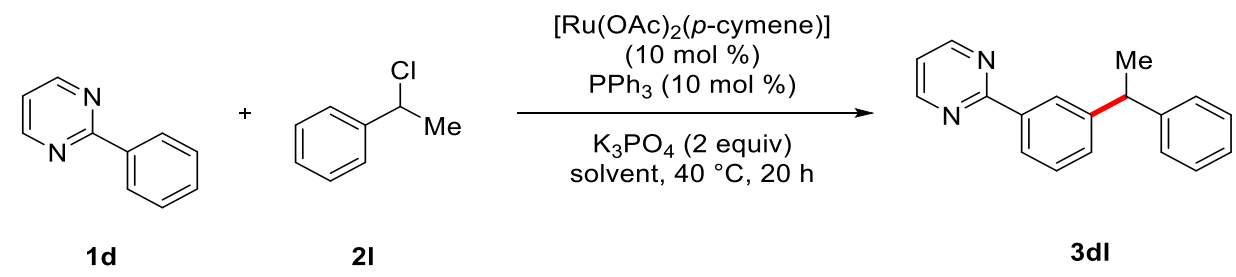

\begin{tabular}{|c|c|c|}
\hline Entry & Solvent & $3 d l(\%)$ \\
\hline 1 & 1,4-dioxane & 69 (76) \\
\hline 2 & 2-MeTHF & $65(71)$ \\
\hline 3 & toluene & 67 (76) \\
\hline 4 & 1,2-DCE & $64(70)$ \\
\hline 5 & $\mathrm{MeCN}$ & $(0)$ \\
\hline \multicolumn{3}{|c|}{$\begin{array}{l}{\left[\mathrm{Ru}(\mathrm{OAc})_{2}(p \text {-cymene })\right](10 \mathrm{~mol} \%), \mathrm{PPh}_{3}(10 \mathrm{~mol} \%), \mathrm{K}_{3} \mathrm{PO}_{4}(1.0 \mathrm{mmol}) \text {, }} \\
\text { solvent }(2.0 \mathrm{~mL}), 40{ }^{\circ} \mathrm{C}, 20 \mathrm{~h} \text {, under } \mathrm{N}_{2} \text {; yield of isolated products. The yield } \\
\text { in parentheses was determined by }{ }^{1} \mathrm{H}-\mathrm{NMR} \text { using } 1,3,5 \text {-trimethoxybenzene as } \\
\text { internal standard. }\end{array}$} \\
\hline
\end{tabular}

\section{Unsuccessful Substrates}

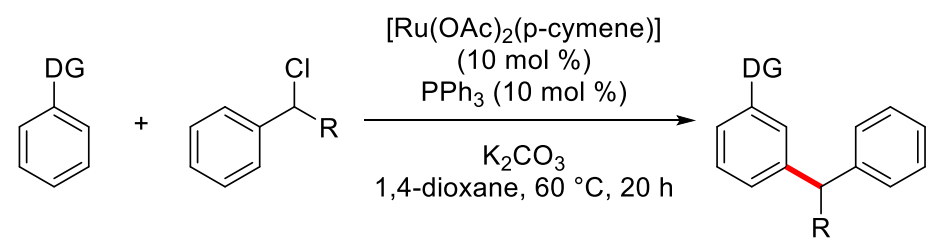<smiles>O=[N+]([O-])C(c1ccccc1)c1cccc(N=Nc2ccccc2)c1</smiles>

$27 \%$<smiles>CON=C(C)c1cccc(Cc2ccc(OC)cc2)c1</smiles>

$0 \%$<smiles>COc1ccc(Cc2cccc(-c3cn4ccccc4n3)c2)cc1</smiles>

$0 \%$<smiles>O=[N+]([O-])C(c1ccccc1)c1cccc(NC2=NCCC=N2)c1</smiles>

$<10 \%^{a}$<smiles>O=[N+]([O-])C(c1ccccc1)c1cccc(Oc2ncccn2)c1</smiles>

$0 \%{ }^{a}$

${ }^{a} \mathrm{~K}_{3} \mathrm{PO}_{4}$ 


\section{General Procedures}

\section{General Procedure A: Ruthenium(II)-catalyzed meta-benzylation of arylheteroarenes 1}

Arylheteroarene 1 (0.50 mmol, 1.00 equiv), [Ru(OAc)2(p-cymene)] (17.7 mg, $50.0 \mu \mathrm{mol}$, $10.0 \mathrm{~mol} \%$ ), $\mathrm{PPh}_{3}\left(13.1 \mathrm{mg}, 50.0 \mu \mathrm{mol}, 10.0 \mathrm{~mol} \%\right.$ ) and $\mathrm{K}_{2} \mathrm{CO}_{3}(138 \mathrm{mg}, 1.00 \mathrm{mmol}$, 2.00 equiv) were placed in a pre-dried $25 \mathrm{~mL}$ Schlenk tube. The tube was evacuated and purged with $\mathrm{N}_{2}$ three times. Benzyl chloride 2 (1.50 mmol, 3.00 equiv) and 1,4-dioxane (2.0 mL) were then added and the mixture was stirred at $60^{\circ} \mathrm{C}$. After $20 \mathrm{~h}$, the resulting mixture was filtered through a pad of silica gel and washed with EtOAc. The filtrate was concentrated in vacuo. Purification of the residue by column chromatography ( $n$-hexane/EtOAc) yielded meta-product 3.

\section{General Procedure B: Ruthenium(II)-catalyzed meta-benzylation or meta-alkylation of} arylpurines 1

Arylpurines 1 (0.25 mmol, 1.00 equiv), [Ru(OAc) $2(p$-cymene $)] \quad(8.9 \mathrm{mg}, 25.0 \mu \mathrm{mol}$, $10.0 \mathrm{~mol} \%), \mathrm{PPh}_{3}(6.6 \mathrm{mg}, 25.0 \mu \mathrm{mol}, 10.0 \mathrm{~mol} \%)$ and $\mathrm{K}_{2} \mathrm{CO}_{3} \quad(69 \mathrm{mg}, 1.00 \mathrm{mmol}$, 2.00 equiv) were placed in a pre-dried $25 \mathrm{~mL}$ Schlenk tube. The tube was evacuated and purged with $\mathrm{N}_{2}$ three times. Alkyl bromide 5 ( $0.50 \mathrm{mmol}, 2.00$ equiv) and 1,4-dioxane $(1.0 \mathrm{~mL})$ were then added and the mixture was stirred at $60{ }^{\circ} \mathrm{C}$. After $20 \mathrm{~h}$, the resulting mixture was filtered through a pad of silica gel and washed with EtOAc. The filtrate was concentrated in vacuo. Purification of the residue by column chromatography ( $n$-hexane/EtOAc) yielded meta-product 6. 


\section{Characterization Data}

\section{2-[3-(4-Methoxybenzyl)phenyl]pyridine (3aa)}

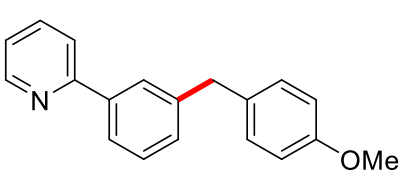

The general procedure A was followed using pyridine 1a $(77.6 \mathrm{mg}$, $0.50 \mathrm{mmol}$ ) and benzyl chloride $2 \mathbf{a}(235 \mathrm{mg}, 1.50 \mathrm{mmol})$. After $20 \mathrm{~h}$, purification by column chromatography ( $n$-hexane/EtOAc 10:1) yielded 3aa $(75.4 \mathrm{mg}, 55 \%)$ as a colorless oil.

${ }^{1} \mathrm{H}-\mathrm{NMR}\left(300 \mathrm{MHz}, \mathrm{CDCl}_{3}\right): \delta=8.69$ (ddd, $\left.J=4.8,1.7,1.0 \mathrm{~Hz}, 1 \mathrm{H}\right), 7.86$ (ddd, $J=1.7,1.5$, $0.6 \mathrm{~Hz}, 1 \mathrm{H}), 7.81$ (ddd, $J=7.8,1.7,1.2 \mathrm{~Hz}, 1 \mathrm{H}), 7.76-7.67$ (m, 2H), 7.39 (ddd, $J=7.8,7.7$, $0.6 \mathrm{~Hz}, 1 \mathrm{H}), 7.25-7.19(\mathrm{~m}, 2 \mathrm{H}), 7.15(\mathrm{~d}, J=8.8 \mathrm{~Hz}, 2 \mathrm{H}), 6.84(\mathrm{~d}, J=8.8 \mathrm{~Hz}, 2 \mathrm{H}), 4.03$ (s, 2H), $3.78(\mathrm{~s}, 3 \mathrm{H})$.

${ }^{13}$ C-NMR (125 MHz, $\left.\mathrm{CDCl}_{3}\right): \delta=157.9\left(\mathrm{C}_{\mathrm{q}}\right), 157.4\left(\mathrm{C}_{\mathrm{q}}\right), 149.5(\mathrm{CH}), 142.0\left(\mathrm{C}_{\mathrm{q}}\right), 139.5\left(\mathrm{C}_{\mathrm{q}}\right)$, $136.5(\mathrm{CH}), 133.0\left(\mathrm{C}_{\mathrm{q}}\right), 129.8(\mathrm{CH}), 129.4(\mathrm{CH}), 128.8(\mathrm{CH}), 127.4(\mathrm{CH}), 124.6(\mathrm{CH}), 121.9$ $(\mathrm{CH}), 120.6(\mathrm{CH}), 113.8(\mathrm{CH}), 55.3\left(\mathrm{CH}_{3}\right), 41.1\left(\mathrm{CH}_{2}\right)$.

IR (ATR): $\tilde{v}=2906,2834,1583,1509,1461,1242,1033,811,774,696 \mathrm{~cm}^{-1}$.

MS (EI) $m / z$ (relative intensity): $275(100)[\mathrm{M}]^{+}, 274(94)[\mathrm{M}-\mathrm{H}]^{+}, 260(45)[\mathrm{M}-\mathrm{Me}]^{+}, 230$ (12), 121 (12), 78 (11), 51 (6).

HR-MS (EI): $m / z$ calcd for $\mathrm{C}_{19} \mathrm{H}_{17} \mathrm{NO}^{+}[\mathrm{M}]^{+} 275.1305$, found 275.1312 .

2-Phenylpyridine (1a) (233 mg, $1.50 \mathrm{mmol}, 3.00$ equiv), [Ru(OAc) 2 (p-cymene)] (17.7 mg, $50.0 \mu \mathrm{mol}, 10.0 \mathrm{~mol} \%)$ and $\mathrm{K}_{2} \mathrm{CO}_{3}(138 \mathrm{mg}, 1.00 \mathrm{mmol}, 2.00$ equiv) were placed in a pre-dried $25 \mathrm{~mL}$ Schlenk tube. The tube was evacuated and purged with $\mathrm{N}_{2}$ three times. Benzyl chloride 2a $(78.3 \mathrm{mg}, 0.50 \mathrm{mmol}, 1.00$ equiv) and 1,4-dioxane $(2.0 \mathrm{~mL})$ were then added and the mixture was stirred at $100{ }^{\circ} \mathrm{C}$. After $20 \mathrm{~h}$, the resulting mixture was filtered through a pad of silica gel and washed with EtOAc. The filtrate was concentrated in vacuo. Purification of the residue by column chromatography ( $n$-hexane/EtOAc $8: 1$ ) yielded monobenzylated product 4aaA (26.4 $\mathrm{mg}, 19 \%)$ as a colorless oil and dibenzylated product $\mathbf{4 a a B}(71.2 \mathrm{mg}, 72 \%)$ as a colorless oil.

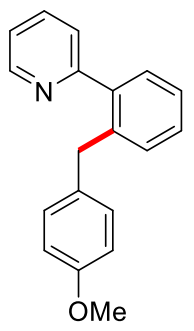

\section{2-[2-(4-Methoxybenzyl)phenyl]pyridine (4aaA)}

${ }^{1} \mathbf{H}$-NMR (300 MHz, $\mathrm{CDCl}_{3}$ ): $\delta=8.67$ (ddd, $\left.J=4.9,1.8,1.0 \mathrm{~Hz}, 1 \mathrm{H}\right), 7.64$ (ddd, $J=7.5,1.8 \mathrm{~Hz}, 1 \mathrm{H}), 7.39-7.18(\mathrm{~m}, 6 \mathrm{H}), 6.88(\mathrm{~d}, J=8.8 \mathrm{~Hz}, 2 \mathrm{H}), 6.71(\mathrm{~d}, J=$ $8.8 \mathrm{~Hz}, 2 \mathrm{H}), 4.05(\mathrm{~s}, 2 \mathrm{H}), 3.73(\mathrm{~s}, 3 \mathrm{H})$. 
${ }^{13}$ C-NMR (125 MHz, $\left.\mathrm{CDCl}_{3}\right): \delta=159.8\left(\mathrm{C}_{\mathrm{q}}\right), 157.5\left(\mathrm{C}_{\mathrm{q}}\right), 149.0(\mathrm{CH}), 140.5\left(\mathrm{C}_{\mathrm{q}}\right), 139.1\left(\mathrm{C}_{\mathrm{q}}\right)$, $136.0(\mathrm{CH}), 133.3\left(\mathrm{C}_{\mathrm{q}}\right), 130.4(\mathrm{CH}), 129.7(\mathrm{CH}), 129.7(\mathrm{CH}), 128.3(\mathrm{CH}), 126.1(\mathrm{CH}), 124.1$ $(\mathrm{CH}), 121.6(\mathrm{CH}), 113.5(\mathrm{CH}), 55.2\left(\mathrm{CH}_{3}\right), 37.9\left(\mathrm{CH}_{2}\right)$.

IR (ATR): $\tilde{v}=3003,2907,2834,1585,1510,1468,1245,1176,1036,753 \mathrm{~cm}^{-1}$.

MS (EI) $m / z$ (relative intensity): $275(75)[\mathrm{M}]^{+}, 274(100)[\mathrm{M}-\mathrm{H}]^{+}, 260(30)[\mathrm{M}-\mathrm{Me}]^{+}, 230$ (18), 167 (45).

HR-MS (ESI): $m / z$ calcd for $\mathrm{C}_{19} \mathrm{H}_{18} \mathrm{NO}^{+}[\mathrm{M}+\mathrm{H}]^{+} 276.1383$, found 276.1384.

The spectral data are in accordance with those reported in the literature. ${ }^{7}$

\section{2-[2,6-Bis(4-methoxybenzyl)phenyl]pyridine (4aaB)}

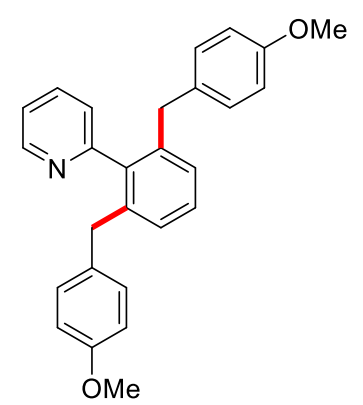

${ }^{1} \mathbf{H}-\mathbf{N M R}\left(600 \mathrm{MHz}, \mathrm{CDCl}_{3}\right): \delta=8.69(\mathrm{ddd}, J=4.9,1.8,1.1 \mathrm{~Hz}, 1 \mathrm{H})$, 7.54 (ddd, $J=7.8,7.7,1.8 \mathrm{~Hz}, 1 \mathrm{H}), 7.25$ (t, $J=7.7 \mathrm{~Hz}, 1 \mathrm{H}), 7.20$ (ddd, $J$ $=7.7,4.9,1.2 \mathrm{~Hz}, 1 \mathrm{H}), 7.09(\mathrm{~d}, J=7.7 \mathrm{~Hz}, 2 \mathrm{H}), 6.92(\mathrm{ddd}, J=7.8,1.2$, $1.1 \mathrm{~Hz}, 1 \mathrm{H}), 6.84(\mathrm{~d}, J=8.7 \mathrm{~Hz}, 4 \mathrm{H}), 6.73(\mathrm{~d}, J=8.7 \mathrm{~Hz}, 4 \mathrm{H}), 3.75(\mathrm{~s}$, $6 \mathrm{H}), 3.72\left(\mathrm{~d}_{\mathrm{AB}}, J=15.6 \mathrm{~Hz}, 2 \mathrm{H}\right), 3.65\left(\mathrm{~d}_{\mathrm{AB}}, J=15.6 \mathrm{~Hz}, 2 \mathrm{H}\right)$.

${ }^{13}$ C-NMR $\left(125 \mathrm{MHz}, \mathrm{CDCl}_{3}\right): \delta=158.8\left(\mathrm{C}_{\mathrm{q}}\right), 157.5\left(\mathrm{C}_{\mathrm{q}}\right), 149.2(\mathrm{CH})$, $140.2\left(\mathrm{C}_{\mathrm{q}}\right), 139.4\left(\mathrm{C}_{\mathrm{q}}\right), 135.5(\mathrm{CH}), 132.9\left(\mathrm{C}_{\mathrm{q}}\right), 129.6(\mathrm{CH}), 128.0(\mathrm{CH}), 127.8(\mathrm{CH}), 125.2$ $(\mathrm{CH}), 121.6(\mathrm{CH}), 113.4(\mathrm{CH}), 55.2\left(\mathrm{CH}_{3}\right), 38.5\left(\mathrm{CH}_{2}\right)$.

IR (ATR): $\tilde{v}=3002,2906,2834,1610,1508,1241,1175,1033,789,750 \mathrm{~cm}^{-1}$.

MS (EI) $m / z$ (relative intensity): $395(94)[\mathrm{M}]^{+}, 394(100)[\mathrm{M}-\mathrm{H}]^{+}, 380(12)[\mathrm{M}-\mathrm{Me}]^{+}, 286$ (29), 272 (25), 242 (9), 121 (9).

HR-MS (ESI): $m / z$ calcd for $\mathrm{C}_{27} \mathrm{H}_{26} \mathrm{NO}_{2}{ }^{+}[\mathrm{M}+\mathrm{H}]^{+}$396.1958, found 396.1962.

\section{2-[4-Methoxy-3-(4-methoxybenzyl)phenyl]pyridine (3ba)}

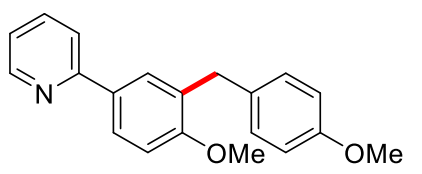

The general procedure A was followed using pyridine $\mathbf{1 b}(92.6 \mathrm{mg}$, $0.50 \mathrm{mmol})$ and benzyl chloride $\mathbf{2 a}(235 \mathrm{mg}, 1.50 \mathrm{mmol})$. After $20 \mathrm{~h}$, purification by column chromatography ( $n$-hexane/EtOAc 6:1) yielded $3 \mathbf{b a}(94.9 \mathrm{mg}, 62 \%)$ as a colorless oil.

${ }^{1} \mathbf{H}-\mathrm{NMR}\left(400 \mathrm{MHz}, \mathrm{CDCl}_{3}\right): \delta=8.64(\mathrm{ddd}, J=4.9,1.9,1.1 \mathrm{~Hz}, 1 \mathrm{H}), 7.86(\mathrm{dd}, J=8.6,2.3 \mathrm{~Hz}$, $1 \mathrm{H}), 7.78(\mathrm{~d}, J=2.3 \mathrm{~Hz}, 1 \mathrm{H}), 7.67(\mathrm{ddd}, J=8.0,7.3,1.9 \mathrm{~Hz}, 1 \mathrm{H}), 7.61$ (ddd, $J=8.0,1.2$, $1.1 \mathrm{~Hz}, 1 \mathrm{H}), 7.18(\mathrm{~d}, J=8.5 \mathrm{~Hz}, 2 \mathrm{H}), 7.14(\mathrm{ddd}, J=7.3,4.9,1.2 \mathrm{~Hz}, 1 \mathrm{H}), 6.96(\mathrm{~d}, J=8.6 \mathrm{~Hz}$, $1 \mathrm{H}), 6.82(\mathrm{~d}, J=8.5 \mathrm{~Hz}, 2 \mathrm{H}), 4.00(\mathrm{~s}, 2 \mathrm{H}), 3.87(\mathrm{~s}, 3 \mathrm{H}), 3.77(\mathrm{~s}, 3 \mathrm{H})$. 
${ }^{13}$ C-NMR (100 MHz, $\left.\mathrm{CDCl}_{3}\right): \delta=158.3\left(\mathrm{C}_{\mathrm{q}}\right), 157.7\left(\mathrm{C}_{\mathrm{q}}\right), 157.3\left(\mathrm{C}_{\mathrm{q}}\right), 149.5(\mathrm{CH}), 136.5(\mathrm{CH})$, $133.0\left(\mathrm{C}_{\mathrm{q}}\right), 131.7\left(\mathrm{C}_{\mathrm{q}}\right), 130.3\left(\mathrm{C}_{\mathrm{q}}\right), 129.7(\mathrm{CH}), 128.9(\mathrm{CH}), 126.1(\mathrm{CH}), 121.3(\mathrm{CH}), 119.8$ $(\mathrm{CH}), 113.6(\mathrm{CH}), 110.6(\mathrm{CH}), 55.5\left(\mathrm{CH}_{3}\right), 55.2\left(\mathrm{CH}_{3}\right), 35.2\left(\mathrm{CH}_{2}\right)$.

IR (ATR): $\tilde{v}=3003,2931,2834,1607,1584,1507,1463,1240,1026,779 \mathrm{~cm}^{-1}$.

MS (ESI) $m / z$ (relative intensity): $306(100)[\mathrm{M}+\mathrm{H}]^{+}$.

HR-MS (ESI): $m / z$ calcd for $\mathrm{C}_{20} \mathrm{H}_{20} \mathrm{NO}_{2}{ }^{+}[\mathrm{M}+\mathrm{H}]^{+} 306.1489$, found 306.1494.

\section{4-Methoxy-2-[3-(4-methoxybenzyl)phenyl]pyridine (3ca)}

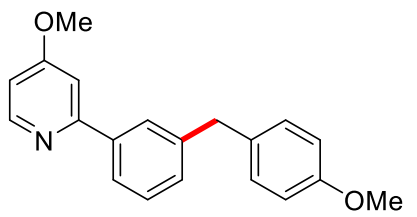

The general procedure A was followed using pyridine 1c $(92.6 \mathrm{mg}$, $0.50 \mathrm{mmol})$ and benzyl chloride $\mathbf{2 a}(235 \mathrm{mg}, 1.50 \mathrm{mmol})$. After $20 \mathrm{~h}$, purification by column chromatography ( $n$-hexane/EtOAc 7:3) yielded 3ca $(99.5 \mathrm{mg}, 65 \%)$ as a colorless oil.

${ }^{1} \mathbf{H}-\mathbf{N M R}\left(300 \mathrm{MHz}, \mathrm{CDCl}_{3}\right): \delta=8.51(\mathrm{dd}, J=5.8,0.5 \mathrm{~Hz}, 1 \mathrm{H}), 7.84-7.82(\mathrm{~m}, 1 \mathrm{H}), 7.77$ (ddt, $J=7.8,1.8,0.9 \mathrm{~Hz}, 1 \mathrm{H}), 7.38(\mathrm{ddd}, J=7.8,7.7,0.6 \mathrm{~Hz}, 1 \mathrm{H}), 7.23-7.19(\mathrm{~m}, 1 \mathrm{H}), 7.20(\mathrm{dd}, J=$ 2.5, $0.5 \mathrm{~Hz}, 1 \mathrm{H}), 7.14(\mathrm{~d}, J=8.8 \mathrm{~Hz}, 2 \mathrm{H}), 6.83(\mathrm{~d}, J=8.8 \mathrm{~Hz}, 2 \mathrm{H}), 6.77(\mathrm{dd}, J=5.8,2.5 \mathrm{~Hz}$, 1H), 4.01 (s, 2H), 3.90 (s, 3H), 3.78 (s, 3H).

${ }^{13}$ C-NMR $\left(125 \mathrm{MHz}, \mathrm{CDCl}_{3}\right): \delta=166.3\left(\mathrm{C}_{\mathrm{q}}\right), 159.1\left(\mathrm{C}_{\mathrm{q}}\right), 157.9\left(\mathrm{C}_{\mathrm{q}}\right), 150.7(\mathrm{CH}), 141.9\left(\mathrm{C}_{\mathrm{q}}\right)$, $139.4\left(\mathrm{C}_{\mathrm{q}}\right), 133.0\left(\mathrm{C}_{\mathrm{q}}\right), 129.8(\mathrm{CH}), 129.5(\mathrm{CH}), 128.7(\mathrm{CH}), 127.5(\mathrm{CH}), 124.6(\mathrm{CH}), 113.8$ $(\mathrm{CH}), 108.0(\mathrm{CH}), 107.0(\mathrm{CH}), 55.3\left(\mathrm{CH}_{3}\right), 55.2\left(\mathrm{CH}_{3}\right), 41.1\left(\mathrm{CH}_{2}\right)$.

IR (ATR): $\tilde{v}=2936,2835,1590,1563,1509,1243,1175,1032,794,699 \mathrm{~cm}^{-1}$.

MS (EI) $m / z$ (relative intensity): 305 (64) $[\mathrm{M}]^{+}, 304(100)[\mathrm{M}-\mathrm{H}]^{+}, 290(25)[\mathrm{M}-\mathrm{Me}]^{+}, 260$ (6) $[\mathrm{M}-\mathrm{Me}-\mathrm{OMe}]^{+}, 121(8), 43(9)$.

HR-MS (EI): $m / z$ calcd for $\mathrm{C}_{20} \mathrm{H}_{19} \mathrm{NO}_{2}{ }^{+}[\mathrm{M}]^{+}$305.1410, found 305.1408.

\section{2-[3-(4-Methoxybenzyl)phenyl]pyrimidine (3da)}

The general procedure A was followed using pyrimidine 1d
$(78.1 \mathrm{mg}, 0.50 \mathrm{mmol})$ and benzyl chloride $2 \mathrm{a}(235 \mathrm{mg}, 1.50 \mathrm{mmol})$. ( $n$-hexane/EtOAc 10:1) yielded 3da (82.2 $\mathrm{mg}, 59 \%)$ as a white soild.

${ }^{1} \mathbf{H}-\mathbf{N M R}\left(600 \mathrm{MHz}, \mathrm{CDCl}_{3}\right): \delta=8.79(\mathrm{~d}, J=4.8 \mathrm{~Hz}, 2 \mathrm{H}), 8.32(\mathrm{ddd}, J=1.8,1.7,0.6 \mathrm{~Hz}, 1 \mathrm{H})$, $8.28(\mathrm{ddd}, J=7.7,1.7,1.5 \mathrm{~Hz}, 1 \mathrm{H}), 7.41$ (dd, $J=7.7,7.6 \mathrm{~Hz}, 1 \mathrm{H}), 7.31-7.28(\mathrm{~m}, 1 \mathrm{H}), 7.17$ (t, $J=4.8 \mathrm{~Hz}, 1 \mathrm{H}), 7.16(\mathrm{~d}, J=8.8 \mathrm{~Hz}, 2 \mathrm{H}), 6.84(\mathrm{~d}, J=8.8 \mathrm{~Hz}, 2 \mathrm{H}), 4.03(\mathrm{~s}, 2 \mathrm{H}), 3.78(\mathrm{~s}, 3 \mathrm{H})$. 
${ }^{13}$ C-NMR $\left(125 \mathrm{MHz}, \mathrm{CDCl}_{3}\right): \delta=164.7\left(\mathrm{C}_{\mathrm{q}}\right), 157.9\left(\mathrm{C}_{\mathrm{q}}\right), 157.0(\mathrm{CH}), 141.9\left(\mathrm{C}_{\mathrm{q}}\right), 137.6\left(\mathrm{C}_{\mathrm{q}}\right)$, $133.1\left(\mathrm{C}_{\mathrm{q}}\right), 131.3(\mathrm{CH}), 129.8(\mathrm{CH}), 128.7(\mathrm{CH}), 128.5(\mathrm{CH}), 125.9(\mathrm{CH}), 118.9(\mathrm{CH}), 113.9$ $(\mathrm{CH}), 55.3\left(\mathrm{CH}_{3}\right), 41.1\left(\mathrm{CH}_{2}\right)$.

IR (ATR): $\tilde{v}=3033,2908,1567,1555,1509,1407,1242,1033,786,696 \mathrm{~cm}^{-1}$.

m.p.: $90-92{ }^{\circ} \mathrm{C}$.

MS (EI) $m / z$ (relative intensity): $276(100)[\mathrm{M}]^{+}, 275$ (53) $[\mathrm{M}-\mathrm{H}]^{+}, 261$ (39) $[\mathrm{M}-\mathrm{Me}]^{+}, 245$ (12)

$[\mathrm{M}-\mathrm{OMe}]^{+}, 231(8), 121(12)$.

HR-MS (EI): $m / z$ calcd for $\mathrm{C}_{18} \mathrm{H}_{16} \mathrm{~N}_{2} \mathrm{O}^{+}[\mathrm{M}]^{+} 276.1257$, found 276.1264 .

\section{2-[4-Fluoro-3-(4-methoxybenzyl)phenyl]pyrimidine (3ea)}

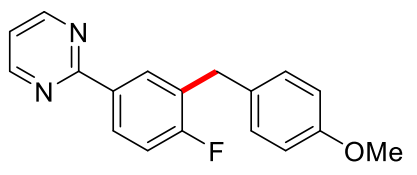

The general procedure $\mathbf{A}$ was followed using pyrimidine $\mathbf{1 e}$ (87.1 $\mathrm{mg}, 0.50 \mathrm{mmol})$ and benzyl chloride $2 \mathbf{a}(235 \mathrm{mg}, 1.50 \mathrm{mmol})$. After $20 \mathrm{~h}$, purification by column chromatography ( $n$-hexane/EtOAc 6:1) yielded 3ea $(74.6 \mathrm{mg}, 51 \%$ ) as a white solid.

${ }^{1} \mathbf{H}-\mathbf{N M R}\left(300 \mathrm{MHz}, \mathrm{CDCl}_{3}\right): \delta=8.75(\mathrm{~d}, J=4.8 \mathrm{~Hz}, 2 \mathrm{H}), 8.36-8.27(\mathrm{~m}, 2 \mathrm{H}), 7.20(\mathrm{~d}, J=$ $8.7 \mathrm{~Hz}, 2 \mathrm{H}), 7.14(\mathrm{dd}, J=9.6,8.5 \mathrm{~Hz}, 1 \mathrm{H}), 7.14(\mathrm{t}, J=4.8 \mathrm{~Hz}, 1 \mathrm{H}), 6.83(\mathrm{~d}, J=8.7 \mathrm{~Hz}, 2 \mathrm{H})$, $4.03(\mathrm{~s}, 2 \mathrm{H}), 3.77(\mathrm{~s}, 3 \mathrm{H})$.

${ }^{13}$ C-NMR $\left(125 \mathrm{MHz}, \mathrm{CDCl}_{3}\right): \delta=163.8\left(\mathrm{C}_{\mathrm{q}}\right), 162.8\left(\mathrm{~d},{ }^{1} J_{\mathrm{C}-\mathrm{F}}=248 \mathrm{~Hz}, \mathrm{C}_{\mathrm{q}}\right), 157.9\left(\mathrm{C}_{\mathrm{q}}\right), 157.0$ $(\mathrm{CH}), 133.6\left(\mathrm{~d},{ }^{4} J_{\mathrm{C}-\mathrm{F}}=3 \mathrm{~Hz}, \mathrm{C}_{\mathrm{q}}\right), 131.8\left(\mathrm{C}_{\mathrm{q}}\right), 131.1\left(\mathrm{~d},{ }^{3} J_{\mathrm{C}-\mathrm{F}}=6 \mathrm{~Hz}, \mathrm{CH}\right), 129.5(\mathrm{CH}), 128.6$ $\left(\mathrm{d},{ }^{2} J_{\mathrm{C}-\mathrm{F}}=16 \mathrm{~Hz}, \mathrm{C}_{\mathrm{q}}\right), 128.1\left(\mathrm{~d},{ }^{3} J_{\mathrm{C}-\mathrm{F}}=9 \mathrm{~Hz}, \mathrm{CH}\right), 118.8(\mathrm{CH}), 115.6\left(\mathrm{~d},{ }^{2} J_{\mathrm{C}-\mathrm{F}}=23 \mathrm{~Hz}, \mathrm{CH}\right)$, $113.9(\mathrm{CH}), 55.2\left(\mathrm{CH}_{3}\right), 34.3\left(\mathrm{~d},{ }^{3} J_{\mathrm{C}-\mathrm{F}}=3 \mathrm{~Hz}, \mathrm{CH}_{2}\right)$.

${ }^{19}$ F-NMR $\left(282 \mathrm{MHz}, \mathrm{CDCl}_{3}\right): \delta=(-114.8)-(-115.0)(\mathrm{m})$.

IR (ATR): $\tilde{v}=3010,2936,1556,1514,1494,1415,1240,1028,796,568 \mathrm{~cm}^{-1}$.

m.p.: $89-90{ }^{\circ} \mathrm{C}$.

MS (EI) $m / z$ (relative intensity): $294(100)[\mathrm{M}]^{+}, 293(55)[\mathrm{M}-\mathrm{H}]^{+}, 279(24)[\mathrm{M}-\mathrm{Me}]^{+}, 263$ (11) $[\mathrm{M}-\mathrm{OMe}]^{+}, 121(13), 91(4)[\mathrm{Bn}]^{+}, 77$ (4), 43 (6).

HR-MS (EI): $\mathrm{m} / z$ calcd for $\mathrm{C}_{18} \mathrm{H}_{15} \mathrm{FN}_{2} \mathrm{O}^{+}[\mathrm{M}]^{+} 294.1163$, found 294.1167.

\section{2-[3-(4-Methoxybenzyl)phenyl]-4,5-dihydrooxazole (3fa)}

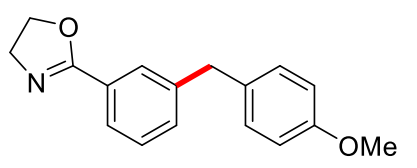

The general procedure A was followed using oxazoline 1f $(73.6 \mathrm{mg}$, $0.50 \mathrm{mmol})$ and benzyl chloride $2 \mathbf{a}(235 \mathrm{mg}, 1.50 \mathrm{mmol})$. After $20 \mathrm{~h}$, purification by column chromatography ( $n$-hexane/EtOAc 7:3) yielded $3 f a(80.7 \mathrm{mg}, 60 \%)$ as a colorless oil. 
${ }^{1} \mathbf{H}-\mathbf{N M R}\left(300 \mathrm{MHz}, \mathrm{CDCl}_{3}\right): \delta=7.83-7.80(\mathrm{~m}, 1 \mathrm{H}), 7.77(\mathrm{ddd}, J=7.2,1.7,1.7 \mathrm{~Hz}, 1 \mathrm{H}), 7.35-$ $7.27(\mathrm{~m}, 2 \mathrm{H}), 7.10(\mathrm{~d}, J=8.8 \mathrm{~Hz}, 2 \mathrm{H}), 6.82(\mathrm{~d}, J=8.8 \mathrm{~Hz}, 2 \mathrm{H}), 4.41(\mathrm{td}, J=9.4,0.8 \mathrm{~Hz}, 2 \mathrm{H})$, $4.04(\mathrm{td}, J=9.4,0.8 \mathrm{~Hz}, 2 \mathrm{H}), 3.94(\mathrm{~s}, 2 \mathrm{H}), 3.77(\mathrm{~s}, 3 \mathrm{H})$.

${ }^{13}$ C-NMR (125 MHz, $\left.\mathrm{CDCl}_{3}\right): \delta=164.6\left(\mathrm{C}_{\mathrm{q}}\right), 157.9\left(\mathrm{C}_{\mathrm{q}}\right), 141.7\left(\mathrm{C}_{\mathrm{q}}\right), 132.7\left(\mathrm{C}_{\mathrm{q}}\right), 131.7(\mathrm{CH})$, $129.7(\mathrm{CH}), 128.5(\mathrm{CH}), 128.4(\mathrm{CH}), 127.8\left(\mathrm{C}_{\mathrm{q}}\right), 125.8(\mathrm{CH}), 113.9(\mathrm{CH}), 67.5\left(\mathrm{CH}_{2}\right), 55.3$ $\left(\mathrm{CH}_{3}\right), 54.9\left(\mathrm{CH}_{2}\right), 40.9\left(\mathrm{CH}_{2}\right)$.

IR (ATR): $\tilde{v}=2904,2835,1647,1509,1242,1176,1033,952,801,707 \mathrm{~cm}^{-1}$.

MS (EI) $m / z$ (relative intensity): $267(100)[\mathrm{M}]^{+}, 266(34)[\mathrm{M}-\mathrm{H}]^{+}, 252(16)[\mathrm{M}-\mathrm{Me}]^{+}, 236(16)$ [M-OMe] $]^{+}, 223$ (10), 208 (10), 165 (15), 152 (14), 121 (25), 105 (17), 89 (6), 77 (7).

HR-MS (EI): $m / z$ calcd for $\mathrm{C}_{17} \mathrm{H}_{17} \mathrm{NO}_{2}{ }^{+}[\mathrm{M}]^{+} 267.1254$, found 267.1262 .

\section{2-[4-Bromo-3-(4-methoxybenzyl)phenyl]-4,5-dihydrooxazole (3ga)}

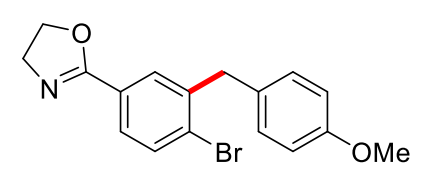

The general procedure A was followed using oxazoline $\mathbf{1 g}(113 \mathrm{mg}$, $0.50 \mathrm{mmol}$ ) and benzyl chloride $\mathbf{2 a}(235 \mathrm{mg}, 1.50 \mathrm{mmol})$. After $20 \mathrm{~h}$, purification by column chromatography ( $n$-hexane/EtOAc 7:3) yielded 3ga (87.2 $\mathrm{mg}, 50 \%)$ as a colorless oil.

${ }^{1} \mathbf{H}-\mathbf{N M R}\left(300 \mathrm{MHz}, \mathrm{CDCl}_{3}\right): \delta=7.78(\mathrm{dd}, J=2.0,0.5 \mathrm{~Hz}, 1 \mathrm{H}), 7.64\left(\mathrm{~d}_{\mathrm{AB}} \mathrm{d}, J=8.3,2.0 \mathrm{~Hz}\right.$, $1 \mathrm{H}), 7.60\left(\mathrm{~d}_{\mathrm{AB}} \mathrm{d}, J=8.3,0.5 \mathrm{~Hz}, 1 \mathrm{H}\right), 7.12(\mathrm{~d}, J=8.8 \mathrm{~Hz}, 2 \mathrm{H}), 6.82(\mathrm{~d}, J=8.8 \mathrm{~Hz}, 2 \mathrm{H}), 4.40$ $(\mathrm{td}, J=9.5,0.8 \mathrm{~Hz}, 2 \mathrm{H}), 4.08(\mathrm{~s}, 2 \mathrm{H}), 4.02(\mathrm{td}, J=9.5,0.8 \mathrm{~Hz}, 2 \mathrm{H}), 3.78(\mathrm{~s}, 3 \mathrm{H})$.

${ }^{13}$ C-NMR (125 MHz, $\left.\mathrm{CDCl}_{3}\right): \delta=163.8\left(\mathrm{C}_{\mathrm{q}}\right), 158.0\left(\mathrm{C}_{\mathrm{q}}\right), 140.9\left(\mathrm{C}_{\mathrm{q}}\right), 132.9(\mathrm{CH}), 131.0\left(\mathrm{C}_{\mathrm{q}}\right)$, $130.5(\mathrm{CH}), 129.7(\mathrm{CH}), 128.1\left(\mathrm{C}_{\mathrm{q}}\right), 127.3(\mathrm{CH}), 127.1\left(\mathrm{C}_{\mathrm{q}}\right), 113.9(\mathrm{CH}), 67.7\left(\mathrm{CH}_{2}\right), 55.2$ $\left(\mathrm{CH}_{3}\right), 55.0\left(\mathrm{CH}_{2}\right), 40.9\left(\mathrm{CH}_{2}\right)$.

IR (ATR): $\tilde{v}=2903,1648,1509,1243,1176,1074,1023,811,722 \mathrm{~cm}^{-1}$.

MS (EI) $m / z$ (relative intensity): $347(98)\left[\mathrm{M}\left({ }^{81} \mathrm{Br}\right)\right]^{+}, 345(100)\left[\mathrm{M}\left({ }^{79} \mathrm{Br}\right)\right]^{+}, 316(16), 266(40)$ $[\mathrm{M}-\mathrm{Br}]^{+}, 223$ (22), 195 (18), 152 (26), 121 (39).

HR-MS (EI): $m / z$ calcd for $\mathrm{C}_{17} \mathrm{H}_{16}{ }^{79} \mathrm{BrNO}_{2}{ }^{+}[\mathrm{M}]^{+} 345.0359$, found 345.0354 .

\section{2-(3-Benzylphenyl)pyrimidine (3db)}

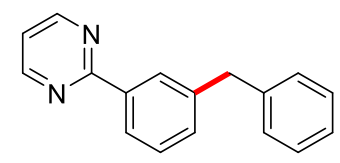

The general procedure A was followed using pyrimidine $1 \mathbf{1 d}(78.1 \mathrm{mg}$, $0.50 \mathrm{mmol}$ ) and benzyl chloride $2 \mathbf{b}(190 \mathrm{mg}, 1.50 \mathrm{mmol})$. After $20 \mathrm{~h}$, purification by column chromatography ( $n$-hexane/EtOAc 10:1) yielded 3db $(63.7 \mathrm{mg}, 52 \%)$ as a white solid. 
${ }^{1} \mathbf{H}-\mathbf{N M R}\left(300 \mathrm{MHz}, \mathrm{CDCl}_{3}\right): \delta=8.79(\mathrm{~d}, J=4.9 \mathrm{~Hz}, 2 \mathrm{H}), 8.36-8.34(\mathrm{~m}, 1 \mathrm{H}), 8.31$ (ddd, $J=$ 7.8, 1.8, 1.3 Hz, 1H), 7.43 (ddd, $J=7.8,7.7,0.6 \mathrm{~Hz}, 1 \mathrm{H}), 7.34-7.20(\mathrm{~m}, 6 \mathrm{H}), 7.16(\mathrm{t}, J=4.9 \mathrm{~Hz}$, $1 \mathrm{H}), 4.10$ (s, 2H).

${ }^{13}$ C-NMR (125 MHz, $\left.\mathrm{CDCl}_{3}\right): \delta=164.6\left(\mathrm{C}_{\mathrm{q}}\right), 157.0(\mathrm{CH}), 141.4\left(\mathrm{C}_{\mathrm{q}}\right), 140.9\left(\mathrm{C}_{\mathrm{q}}\right), 137.7\left(\mathrm{C}_{\mathrm{q}}\right)$, $131.4(\mathrm{CH}), 128.8(\mathrm{CH}), 128.7(\mathrm{CH}), 128.6(\mathrm{CH}), 128.4(\mathrm{CH}), 126.0(\mathrm{CH}), 118.9(\mathrm{CH}), 42.0$ $\left(\mathrm{CH}_{2}\right)$.

IR (ATR): $\tilde{v}=3024,2918,1566,1553,1425,1408,778,759,701,635 \mathrm{~cm}^{-1}$.

m.p.: $76-78^{\circ} \mathrm{C}$.

MS (EI) $m / z$ (relative intensity): 246 (98) [M] $]^{+}, 245$ (100) [M-H $]^{+}, 190$ (8), 165 (24), 152 (5), $122(7), 91(8)[\mathrm{Bn}]^{+}, 43(19)$.

HR-MS (EI): $m / z$ calcd for $\mathrm{C}_{17} \mathrm{H}_{14} \mathrm{~N}_{2}{ }^{+}[\mathrm{M}]^{+} 246.1151$, found 246.1149 .

The spectral data are in accordance with those reported in the literature. ${ }^{8}$

\section{6-(3-Benzylphenyl)-9-iso-propyl-9H-purine (3hb)}<smiles>CC(C)n1cnc2c(-c3cccc(Cc4ccccc4)c3)ncnc21</smiles>
$i-\mathrm{Pr}^{\prime}$

The general procedure A was followed using purine $\mathbf{1 h}(119 \mathrm{mg}, 0.50 \mathrm{mmol})$ and benzyl chloride $\mathbf{2 b}(190 \mathrm{mg}, 1.50 \mathrm{mmol})$. After $20 \mathrm{~h}$, purification by column chromatography ( $n$-hexane/EtOAc 3:1) yielded $\mathbf{3 h b}(116 \mathrm{mg}, 71 \%)$ as a colorless oil.

${ }^{1} \mathbf{H}-\mathbf{N M R}\left(300 \mathrm{MHz}, \mathrm{CDCl}_{3}\right): \delta=9.01(\mathrm{~s}, 1 \mathrm{H}), 8.68$ (ddd, $\left.J=7.7,1.8,1.3 \mathrm{~Hz}, 1 \mathrm{H}\right), 8.62$ (ddd, $J=1.8,1.3,0.6 \mathrm{~Hz}, 1 \mathrm{H}), 8.18(\mathrm{~s}, 1 \mathrm{H}), 7.48(\mathrm{ddd}, J=7.7,7.7,0.6 \mathrm{~Hz}, 1 \mathrm{H}), 7.32$ (dddd, $J=7.7$, 1.3, 1.3, 0.6 Hz, 1H), 7.30-7.15 (m, 5H), 4.98 (hept, $J=6.8 \mathrm{~Hz}, 1 \mathrm{H}), 4.14$ (s, 2H), 1.67 (d, $J=$ $6.8 \mathrm{~Hz}, 6 \mathrm{H})$.

${ }^{13}$ C-NMR (125 MHz, $\left.\mathrm{CDCl}_{3}\right): \delta=154.7\left(\mathrm{C}_{\mathrm{q}}\right), 152.0\left(\mathrm{C}_{\mathrm{q}}\right), 151.9(\mathrm{CH}), 141.8(\mathrm{CH}), 141.4\left(\mathrm{C}_{\mathrm{q}}\right)$, $140.9\left(\mathrm{C}_{\mathrm{q}}\right), 135.9\left(\mathrm{C}_{\mathrm{q}}\right), 131.5\left(\mathrm{C}_{\mathrm{q}}\right.$ and $\left.\mathrm{CH}\right), 129.9(\mathrm{CH}), 128.9(\mathrm{CH}), 128.7(\mathrm{CH}), 128.4(\mathrm{CH})$, $127.9(\mathrm{CH}), 126.0(\mathrm{CH}), 47.3(\mathrm{CH}), 42.1\left(\mathrm{CH}_{2}\right), 22.6\left(\mathrm{CH}_{3}\right)$.

IR (ATR): $\tilde{v}=2977,1567,1494,1324,1217,783,700,646 \mathrm{~cm}^{-1}$.

MS (EI) $m / z$ (relative intensity): 328 (42) $[\mathrm{M}]^{+}, 327(41)[\mathrm{M}-\mathrm{H}]^{+}, 285(100)[\mathrm{M}-i \mathrm{Pr}]^{+}, 165$ (8), $91(6)$.

HR-MS (EI): $m / z$ calcd for $\mathrm{C}_{21} \mathrm{H}_{20} \mathrm{~N}_{4}{ }^{+}[\mathrm{M}]^{+}$328.1682, found 328.1685. 


\section{9-iso-Propyl-6-[3-(4-methoxybenzyl)phenyl]-9H-purine (3ha)}<smiles>COc1ccc(Cc2cccc(-c3ncnc4c3ncn4C(C)C)c2)cc1</smiles>

The general procedure A was followed using purine 1h (119 mg, $0.50 \mathrm{mmol})$ and benzyl chloride $2 \mathrm{a}(235 \mathrm{mg}, 1.50 \mathrm{mmol})$. After $20 \mathrm{~h}$, purification by column chromatography (n-hexane/EtOAc 3:1) yielded 3ha (133 $\mathrm{mg}, 74 \%)$ as a colorless oil.

${ }^{1} \mathbf{H}-\mathrm{NMR}\left(300 \mathrm{MHz}, \mathrm{CDCl}_{3}\right): \delta=9.01(\mathrm{~s}, 1 \mathrm{H}), 8.66$ (ddd, $\left.J=7.8,1.8,1.2 \mathrm{~Hz}, 1 \mathrm{H}\right), 8.59$ (ddd, $J=1.8,1.5,0.6 \mathrm{~Hz}, 1 \mathrm{H}), 8.18(\mathrm{~s}, 1 \mathrm{H}), 7.47$ (ddd, $J=7.8,7.7,0.6 \mathrm{~Hz}, 1 \mathrm{H}), 7.30$ (dddd, $J=7.7$, 1.5, 1.2, $0.6 \mathrm{~Hz}, 1 \mathrm{H}), 7.16(\mathrm{~d}, J=8.8 \mathrm{~Hz}, 2 \mathrm{H}), 6.83(\mathrm{~d}, J=8.8 \mathrm{~Hz}, 2 \mathrm{H}), 4.98$ (hept, $J=6.8 \mathrm{~Hz}$, 1H), 4.07 (s, 2H), 3.77 (s, 3H), 1.67 (d, $J=6.8 \mathrm{~Hz}, 6 \mathrm{H})$.

${ }^{13}$ C-NMR $\left(125 \mathrm{MHz}, \mathrm{CDCl}_{3}\right): \delta=157.9\left(\mathrm{C}_{\mathrm{q}}\right), 154.8\left(\mathrm{C}_{\mathrm{q}}\right), 152.0\left(\mathrm{C}_{\mathrm{q}}\right), 151.9(\mathrm{CH}), 141.9\left(\mathrm{C}_{\mathrm{q}}\right)$, $141.8(\mathrm{CH}), 135.8\left(\mathrm{C}_{\mathrm{q}}\right), 133.1\left(\mathrm{C}_{\mathrm{q}}\right), 131.5\left(\mathrm{C}_{\mathrm{q}}\right), 131.4(\mathrm{CH}), 129.8(\mathrm{CH}), 129.8(\mathrm{CH}), 128.7$ $(\mathrm{CH}), 127.8(\mathrm{CH}), 113.8(\mathrm{CH}), 55.3\left(\mathrm{CH}_{3}\right), 47.3(\mathrm{CH}), 41.2\left(\mathrm{CH}_{2}\right), 22.6\left(\mathrm{CH}_{3}\right)$.

IR (ATR): $\tilde{v}=2977,1567,1509,1324,1243,1217,1033,791,703,646 \mathrm{~cm}^{-1}$.

MS (EI) $m / z$ (relative intensity): $358(84)[\mathrm{M}]^{+}, 357(30)[\mathrm{M}-\mathrm{H}]^{+}, 343(14)[\mathrm{M}-\mathrm{Me}]^{+}, 315$ (100) $[\mathrm{M}-i \mathrm{Pr}]^{+}, 301(43), 121(13), 43(8)$.

HR-MS (EI): $m / z$ calcd for $\mathrm{C}_{22} \mathrm{H}_{22} \mathrm{~N}_{4} \mathrm{O}^{+}[\mathrm{M}]^{+} 358.1788$, found 358.1796 .

\section{9-iso-Propyl-6-[3-(4-methylbenzyl)phenyl]-9H-purine (3hc)}<smiles>Cc1ccc(Cc2cccc(-c3ncnc4c3ncn4C(C)C)c2)cc1</smiles>

The general procedure A was followed using purine $\mathbf{1 h}$ (119 mg, $0.50 \mathrm{mmol})$ and benzyl chloride $2 \mathrm{c}(211 \mathrm{mg}, 1.50 \mathrm{mmol})$. After $20 \mathrm{~h}$, purification by column chromatography ( $n$-hexane/EtOAc $3: 1$ ) yielded 3hc (106 $\mathrm{mg}, 62 \%)$ as a colorless oil.

${ }^{1} \mathbf{H}-\mathbf{N M R}\left(400 \mathrm{MHz}, \mathrm{CDCl}_{3}\right): \delta=9.01(\mathrm{~s}, 1 \mathrm{H}), 8.67$ (ddd, $\left.J=7.8,1.8,1.2 \mathrm{~Hz}, 1 \mathrm{H}\right), 8.61$ (ddd, $J=1.8,1.2,0.6 \mathrm{~Hz}, 1 \mathrm{H}), 8.18(\mathrm{~s}, 1 \mathrm{H}), 7.47$ (ddd, $J=7.8,7.7,0.6 \mathrm{~Hz}, 1 \mathrm{H}), 7.31$ (dddd, $J=7.7$, $1.2,1.2,0.6 \mathrm{~Hz}, 1 \mathrm{H}), 7.14\left(\mathrm{~d}_{\mathrm{AB}}, J=8.0 \mathrm{~Hz}, 2 \mathrm{H}\right), 7.09\left(\mathrm{~d}_{\mathrm{AB}}, J=8.0 \mathrm{~Hz}, 2 \mathrm{H}\right), 4.98$ (hept, $J=$ $6.8 \mathrm{~Hz}, 1 \mathrm{H}), 4.09$ (s, 2H), 2.30 (s, 3H), 1.67 (d, $J=6.8 \mathrm{~Hz}, 6 \mathrm{H})$.

${ }^{13}$ C-NMR (100 MHz, $\left.\mathrm{CDCl}_{3}\right): \delta=154.9\left(\mathrm{C}_{\mathrm{q}}\right), 152.1\left(\mathrm{C}_{\mathrm{q}}\right), 152.0(\mathrm{CH}), 141.9(\mathrm{CH}), 141.8\left(\mathrm{C}_{\mathrm{q}}\right)$, $138.0\left(\mathrm{C}_{\mathrm{q}}\right), 135.9\left(\mathrm{C}_{\mathrm{q}}\right), 135.5\left(\mathrm{C}_{\mathrm{q}}\right), 131.5\left(\mathrm{C}_{\mathrm{q}}\right), 131.5(\mathrm{CH}), 129.9(\mathrm{CH}), 129.1(\mathrm{CH}), 128.8(\mathrm{CH})$, $128.8(\mathrm{CH}), 127.9(\mathrm{CH}), 47.2(\mathrm{CH}), 41.6\left(\mathrm{CH}_{2}\right), 22.6\left(\mathrm{CH}_{3}\right), 21.0\left(\mathrm{CH}_{3}\right)$.

IR (ATR): $\tilde{v}=2977,2919,1567,1495,1445,1324,1217,790,702,646 \mathrm{~cm}^{-1}$.

MS (EI) $m / z$ (relative intensity): 342 (75) $[\mathrm{M}]^{+}, 341(27)[\mathrm{M}-\mathrm{H}]^{+}, 327$ (2) $[\mathrm{M}-\mathrm{Me}]^{+}, 299$ (100) $[\mathrm{M}-i \operatorname{Pr}]^{+}, 285(11), 165(6), 142(7)$.

HR-MS (EI): $m / z$ calcd for $\mathrm{C}_{22} \mathrm{H}_{22} \mathrm{~N}_{4}{ }^{+}[\mathrm{M}]^{+} 342.1839$, found 342.1845. 


\section{9-iso-Propyl-6-\{3-[4-(trifluoromethyl)benzyl]phenyl\}-9H-purine (3hd)}<smiles>CC(C)n1cnc2c(-c3cccc(Cc4ccc(C(F)(F)F)cc4)c3)ncnc21</smiles>

The general procedure A was followed using purine $\mathbf{1 h}$ (119 $\mathrm{mg}$, $0.50 \mathrm{mmol})$ and benzyl chloride $2 \mathrm{~d}(292 \mathrm{mg}, 1.50 \mathrm{mmol})$. After $20 \mathrm{~h}$, purification by column chromatography ( $n$-hexane/EtOAc $3: 1$ ) yielded 3hd (146 $\mathrm{mg}, 73 \%)$ as a colorless oil.

${ }^{1} \mathbf{H}-\mathbf{N M R}\left(500 \mathrm{MHz}, \mathrm{CDCl}_{3}\right): \delta=9.01(\mathrm{~s}, 1 \mathrm{H}), 8.70(\mathrm{ddd}, J=7.8,1.5,1.3 \mathrm{~Hz}, 1 \mathrm{H}), 8.61(\mathrm{dd}, J$ $=1.7,1.5 \mathrm{~Hz}, 1 \mathrm{H}), 8.18(\mathrm{~s}, 1 \mathrm{H}), 7.53(\mathrm{~d}, J=8.0 \mathrm{~Hz}, 2 \mathrm{H}), 7.50(\mathrm{dd}, J=7.8,7.7 \mathrm{~Hz}, 1 \mathrm{H}), 7.36$ $(\mathrm{d}, J=8.0 \mathrm{~Hz}, 2 \mathrm{H}), 7.31$ (ddd, $J=7.7,1.7,1.3 \mathrm{~Hz}, 1 \mathrm{H}$ ), 4.99 (hept, $J=6.9 \mathrm{~Hz}, 1 \mathrm{H}$ ), 4.18 (s, $2 \mathrm{H}), 1.68(\mathrm{~d}, J=6.9 \mathrm{~Hz}, 6 \mathrm{H})$.

${ }^{13} \mathrm{C}-\mathrm{NMR}\left(125 \mathrm{MHz}, \mathrm{CDCl}_{3}\right): \delta=154.6\left(\mathrm{C}_{\mathrm{q}}\right), 152.1\left(\mathrm{C}_{\mathrm{q}}\right), 152.0(\mathrm{CH}), 145.1\left(\mathrm{C}_{\mathrm{q}}\right), 142.0(\mathrm{CH})$, $140.4\left(\mathrm{C}_{\mathrm{q}}\right), 136.2\left(\mathrm{C}_{\mathrm{q}}\right), 131.5\left(\mathrm{C}_{\mathrm{q}}\right), 131.5(\mathrm{CH}), 130.0(\mathrm{CH}), 129.2(\mathrm{CH}), 129.0(\mathrm{CH}), 128.4(\mathrm{q}$, $\left.{ }^{2} J_{\mathrm{C}-\mathrm{F}}=32 \mathrm{~Hz}, \mathrm{C}_{\mathrm{q}}\right), 128.3(\mathrm{CH}), 125.4\left(\mathrm{q},{ }^{3} J_{\mathrm{C}-\mathrm{F}}=4 \mathrm{~Hz}, \mathrm{CH}\right), 124.3\left(\mathrm{q},{ }^{1} J_{\mathrm{C}-\mathrm{F}}=270 \mathrm{~Hz}, \mathrm{C}_{\mathrm{q}}\right), 47.3$ $(\mathrm{CH}), 41.8\left(\mathrm{CH}_{2}\right), 22.6\left(\mathrm{CH}_{3}\right)$.

${ }^{19}$ F-NMR (470 MHz, $\left.\mathrm{CDCl}_{3}\right): \delta=-62.3$ (s).

IR (ATR): $\tilde{v}=2980,1568,1321,1219,1107,1065,1018,788,703,646 \mathrm{~cm}^{-1}$.

MS (EI) $m / z$ (relative intensity): $396(46)[\mathrm{M}]^{+}, 395(58)[\mathrm{M}-\mathrm{H}]^{+}, 353(100)[\mathrm{M}-i \mathrm{Pr}]^{+}, 333$ (17).

HR-MS (EI): $m / z$ calcd for $\mathrm{C}_{22} \mathrm{H}_{19} \mathrm{~F}_{3} \mathrm{~N}_{4}{ }^{+}[\mathrm{M}]^{+} 396.1556$, found 396.1557 .

\section{Ethyl 4-[3-(9-iso-propyl-9H-purin-6-yl)benzyl]benzoate (3he)}<smiles>CCOC(=O)c1ccc(Cc2cccc(-c3ncnc4c3ncn4C(C)C)c2)cc1</smiles>

The general procedure A was followed using purine $\mathbf{1 h}$ (119 $\mathrm{mg}$, $0.50 \mathrm{mmol}$ ) and benzyl chloride $2 \mathrm{e}(298 \mathrm{mg}, 1.50 \mathrm{mmol})$. After $20 \mathrm{~h}$, purification by column chromatography ( $n$-hexane/EtOAc $2: 1$ ) yielded 3he (144 $\mathrm{mg}, 72 \%)$ as a colorless oil.

${ }^{1} \mathbf{H}-\mathbf{N M R}\left(300 \mathrm{MHz}, \mathrm{CDCl}_{3}\right): \delta=9.00(\mathrm{~s}, 1 \mathrm{H}), 8.69$ (ddd, $J=7.8,1.8$, $1.2 \mathrm{~Hz}, 1 \mathrm{H}), 8.60$ (ddd $J=1.8,1.8,0.6 \mathrm{~Hz}, 1 \mathrm{H}), 8.18$ (s, 1H), 7.96 (d, $J=8.6 \mathrm{~Hz}, 2 \mathrm{H}), 7.49$ (ddd, $J=7.8,7.7,0.6 \mathrm{~Hz}, 1 \mathrm{H}), 7.34-7.27(\mathrm{~m}, 3 \mathrm{H}), 4.98$ (hept, $J=6.8 \mathrm{~Hz}, 1 \mathrm{H}), 4.35$ (q, $J=$ $7.1 \mathrm{~Hz}, 2 \mathrm{H}), 4.18(\mathrm{~s}, 2 \mathrm{H}), 1.67(\mathrm{~d}, J=6.8 \mathrm{~Hz}, 6 \mathrm{H}), 1.37(\mathrm{t}, J=7.1 \mathrm{~Hz}, 3 \mathrm{H})$.

${ }^{13}$ C-NMR (125 MHz, $\left.\mathrm{CDCl}_{3}\right): \delta=166.4\left(\mathrm{C}_{\mathrm{q}}\right), 154.5\left(\mathrm{C}_{\mathrm{q}}\right), 152.0\left(\mathrm{C}_{\mathrm{q}}\right), 151.9(\mathrm{CH}), 146.2\left(\mathrm{C}_{\mathrm{q}}\right)$, $141.9(\mathrm{CH}), 140.5\left(\mathrm{C}_{\mathrm{q}}\right), 136.0\left(\mathrm{C}_{\mathrm{q}}\right), 131.5\left(\mathrm{C}_{\mathrm{q}}\right), 131.4(\mathrm{CH}), 130.0(\mathrm{CH}), 129.7(\mathrm{CH}), 128.9$ $(\mathrm{CH}), 128.8(\mathrm{CH}), 128.4\left(\mathrm{C}_{\mathrm{q}}\right), 128.1(\mathrm{CH}), 60.8\left(\mathrm{CH}_{2}\right), 47.3(\mathrm{CH}), 42.0\left(\mathrm{CH}_{2}\right), 22.6\left(\mathrm{CH}_{3}\right), 14.4$ $\left(\mathrm{CH}_{3}\right)$.

IR (ATR): $\tilde{v}=2978,1711,1567,1325,1272,1219,1101,1021,703,646 \mathrm{~cm}^{-1}$. 
MS (EI) $m / z$ (relative intensity): $400(60)[\mathrm{M}]^{+}, 399(100)[\mathrm{M}-\mathrm{H}]^{+}, 371(10)[\mathrm{M}-\mathrm{Et}]^{+}, 357$ (47) $[\mathrm{M}-i \mathrm{Pr}]^{+}, 329$ (21) [M-iPr-Et] $]^{+}, 311$ (48), 285 (19), 283 (26) [M-iPr-CO $\left.{ }_{2} \mathrm{Et}\right]^{+}, 165$ (9), 156 (11), $142(9)$.

HR-MS (EI): $m / z$ calcd for $\mathrm{C}_{24} \mathrm{H}_{24} \mathrm{~N}_{4} \mathrm{O}_{2}{ }^{+}[\mathrm{M}]^{+} 400.1894$, found 400.1883 .

\section{6-[3-(4-Fluorobenzyl)phenyl]-9-iso-propyl-9H-purine (3hf)}<smiles>CC(C)n1cnc2c(-c3cccc(Cc4ccc(F)cc4)c3)ncnc21</smiles>

The general procedure A was followed using purine $\mathbf{1 h}(119 \mathrm{mg}, 0.50 \mathrm{mmol})$ and benzyl chloride $2 \mathbf{f}(217 \mathrm{mg}, 1.50 \mathrm{mmol})$. After $20 \mathrm{~h}$, purification by column chromatography ( $n$-hexane/EtOAc 3:1) yielded 3hf (123 mg, 71\%) as a white solid.

${ }^{1} \mathbf{H}-\mathrm{NMR}\left(600 \mathrm{MHz}, \mathrm{CDCl}_{3}\right): \delta=9.01(\mathrm{~s}, 1 \mathrm{H}), 8.68(\mathrm{ddd}, J=7.8,1.8,1.3 \mathrm{~Hz}, 1 \mathrm{H}), 8.59$ (dd, $J$ $=2.0,1.8 \mathrm{~Hz}, 1 \mathrm{H}), 8.18(\mathrm{~s}, 1 \mathrm{H}), 7.48(\mathrm{dd}, J=7.8,7.7 \mathrm{~Hz}, 1 \mathrm{H}), 7.29(\mathrm{ddd}, J=7.7,2.0,1.3 \mathrm{~Hz}$, 1H), 7.19 (dd, $J=8.8,5.4 \mathrm{~Hz}, 2 \mathrm{H}), 6.96(\mathrm{dd}, J=8.8,8.7 \mathrm{~Hz}, 2 \mathrm{H}), 4.98$ (hept, $J=6.8 \mathrm{~Hz}, 1 \mathrm{H}$ ), $4.09(\mathrm{~s}, 2 \mathrm{H}), 1.67(\mathrm{~d}, J=6.8 \mathrm{~Hz}, 6 \mathrm{H})$.

${ }^{13} \mathrm{C}-\mathrm{NMR}\left(125 \mathrm{MHz}, \mathrm{CDCl}_{3}\right): \delta=161.3\left(\mathrm{~d},{ }^{1} J_{\mathrm{C}-\mathrm{F}}=244 \mathrm{~Hz}, \mathrm{C}_{\mathrm{q}}\right), 154.6\left(\mathrm{C}_{\mathrm{q}}\right), 152.0\left(\mathrm{C}_{\mathrm{q}}\right), 151.9$ $(\mathrm{CH}), 141.8(\mathrm{CH}), 141.2\left(\mathrm{C}_{\mathrm{q}}\right), 136.6\left(\mathrm{~d},{ }^{4} J_{\mathrm{C}-\mathrm{F}}=3 \mathrm{~Hz}, \mathrm{C}_{\mathrm{q}}\right), 136.0\left(\mathrm{C}_{\mathrm{q}}\right), 131.5\left(\mathrm{C}_{\mathrm{q}}\right), 131.3(\mathrm{CH})$, $130.2\left(\mathrm{~d},{ }^{3} J_{\mathrm{C}-\mathrm{F}}=8 \mathrm{~Hz}, \mathrm{CH}\right), 129.8(\mathrm{CH}), 128.8(\mathrm{CH}), 128.0(\mathrm{CH}), 115.1\left(\mathrm{~d},{ }^{2} J_{\mathrm{C}-\mathrm{F}}=21 \mathrm{~Hz}, \mathrm{CH}\right)$, $47.3(\mathrm{CH}), 41.2\left(\mathrm{CH}_{2}\right), 22.6\left(\mathrm{CH}_{3}\right)$.

${ }^{19}$ F-NMR $\left(282 \mathrm{MHz}, \mathrm{CDCl}_{3}\right): \delta=-117.4(\mathrm{tt}, J=8.7,5.4 \mathrm{~Hz})$.

IR (ATR): $\tilde{v}=2980,1567,1506,1444,1326,1221,834,794,704,651 \mathrm{~cm}^{-1}$. m.p.: $95-96^{\circ} \mathrm{C}$.

MS (EI) $m / z$ (relative intensity): $346(41)[\mathrm{M}]^{+}, 345(38)[\mathrm{M}-\mathrm{H}]^{+}, 303(100)[\mathrm{M}-i \mathrm{Pr}]^{+}, 183(5)$, 109 (9), 43 (6).

HR-MS (EI): $m / z$ calcd for $\mathrm{C}_{21} \mathrm{H}_{19} \mathrm{FN}_{4}^{+}[\mathrm{M}]^{+} 346.1588$, found 346.1591 .

\section{6-[3-(4-Chlorobenzyl)phenyl]-9-iso-propyl-9H-purine (3hg)}<smiles>CC(C)n1cnc2c(-c3cccc(Cc4ccc(Cl)cc4)c3)ncnc21</smiles>

The general procedure A was followed using purine $\mathbf{1 h}(119 \mathrm{mg}, 0.50 \mathrm{mmol})$ and benzyl chloride $2 \mathrm{~g}$ ( $242 \mathrm{mg}, 1.50 \mathrm{mmol})$. After $20 \mathrm{~h}$, purification by column chromatography ( $n$-hexane/EtOAc 3:1) yielded 3hg (132 mg, 73\%) as a white solid.

${ }^{1} \mathbf{H}-\mathbf{N M R}\left(300 \mathrm{MHz}, \mathrm{CDCl}_{3}\right): \delta=9.01(\mathrm{~s}, 1 \mathrm{H}), 8.68$ (ddd, $\left.J=7.8,1.8,1.2 \mathrm{~Hz}, 1 \mathrm{H}\right), 8.58$ (ddd, $J=1.8,1.2,0.6 \mathrm{~Hz}, 1 \mathrm{H}), 8.18(\mathrm{~s}, 1 \mathrm{H}), 7.48(\mathrm{ddd}, J=7.8,7.7,0.6 \mathrm{~Hz}, 1 \mathrm{H}), 7.29$ (dddd, $J=7.7$, $1.2,1.2,0.6 \mathrm{~Hz}, 1 \mathrm{H}), 7.25(\mathrm{~d}, J=8.9 \mathrm{~Hz}, 2 \mathrm{H}), 7.17$ (d, $J=8.9 \mathrm{~Hz}, 2 \mathrm{H}), 4.98$ (hept, $J=6.8 \mathrm{~Hz}$, 1H), 4.09 (s, 2H), 1.67 (d, $J=6.8 \mathrm{~Hz}, 6 \mathrm{H})$. 
${ }^{13}$ C-NMR $\left(125 \mathrm{MHz}, \mathrm{CDCl}_{3}\right): \delta=154.6\left(\mathrm{C}_{\mathrm{q}}\right), 152.0\left(\mathrm{C}_{\mathrm{q}}\right), 151.9(\mathrm{CH}), 141.8(\mathrm{CH}), 140.8\left(\mathrm{C}_{\mathrm{q}}\right)$, 139.4 $\left(\mathrm{C}_{\mathrm{q}}\right), 136.0\left(\mathrm{C}_{\mathrm{q}}\right), 131.8\left(\mathrm{C}_{\mathrm{q}}\right), 131.5\left(\mathrm{C}_{\mathrm{q}}\right), 131.3(\mathrm{CH}), 130.2(\mathrm{CH}), 129.9(\mathrm{CH}), 128.8(\mathrm{CH})$, $128.5(\mathrm{CH}), 128.1(\mathrm{CH}), 47.3(\mathrm{CH}), 41.4\left(\mathrm{CH}_{2}\right), 22.6\left(\mathrm{CH}_{3}\right)$.

IR (ATR): $\tilde{v}=2977,1567,1490,1448,1324,1217,1089,781,700,647 \mathrm{~cm}^{-1}$.

m.p.: $104-105^{\circ} \mathrm{C}$.

MS (EI) $m / z$ (relative intensity): $364(16)\left[\mathrm{M}\left({ }^{37} \mathrm{Cl}\right)\right]^{+}, 363(25)\left[\mathrm{M}\left({ }^{37} \mathrm{Cl}\right)-\mathrm{H}\right]^{+}, 362$ (46) $\left[\mathrm{M}\left({ }^{35} \mathrm{Cl}\right)\right]^{+}, 361(51)\left[\mathrm{M}\left({ }^{35} \mathrm{Cl}\right)-\mathrm{H}\right]^{+}, 321(35)\left[\mathrm{M}\left({ }^{37} \mathrm{Cl}\right)-i \mathrm{Pr}\right]^{+}, 319(100)\left[\mathrm{M}\left({ }^{35} \mathrm{Cl}\right)-i \mathrm{Pr}\right]^{+}, 283(16)$ $[\mathrm{M}-i \mathrm{Pr}-\mathrm{Cl}]^{+}, 165$ (7), 142 (10), 125 (9), 43 (7).

HR-MS (EI): $m / z$ calcd for $\mathrm{C}_{21} \mathrm{H}_{19}{ }^{35} \mathrm{ClN}_{4}{ }^{+}[\mathrm{M}]^{+}$362.1293, found 362.1287.

\section{6-[3-(4-Bromobenzyl)phenyl]-9-iso-propyl-9H-purine (3hh)}<smiles>CC(C)n1cnc2c(-c3cccc(Cc4ccc(Br)cc4)c3)ncnc21</smiles>

The general procedure A was followed using purine $\mathbf{1 h}(119 \mathrm{mg}, 0.50 \mathrm{mmol})$ and benzyl chloride $\mathbf{2 h}$ (308 $\mathrm{mg}, 1.50 \mathrm{mmol})$. After $20 \mathrm{~h}$, purification by column chromatography (n-hexane/EtOAc 3:1) yielded 3hh (142 mg, 70\%) as a pale yellow oil.

${ }^{1} \mathbf{H}-\mathrm{NMR}\left(300 \mathrm{MHz}, \mathrm{CDCl}_{3}\right.$ ): $\delta=9.00(\mathrm{~s}, 1 \mathrm{H}), 8.68$ (ddd, $\left.J=7.8,1.8,1.2 \mathrm{~Hz}, 1 \mathrm{H}\right), 8.59$ (ddd, $J=1.8,1.2,0.6 \mathrm{~Hz}, 1 \mathrm{H}), 8.16(\mathrm{~s}, 1 \mathrm{H}), 7.47$ (ddd, $J=7.8,7.7,0.6 \mathrm{~Hz}, 1 \mathrm{H}), 7.38$ (d, $J=8.6 \mathrm{~Hz}$, 2H), 7.28 (dddd, $J=7.7,1.2,1.2,0.6 \mathrm{~Hz}, 1 \mathrm{H}), 7.10(\mathrm{~d}, J=8.6 \mathrm{~Hz}, 2 \mathrm{H}), 4.96$ (hept, $J=6.8 \mathrm{~Hz}$, $1 \mathrm{H}), 4.06(\mathrm{~s}, 2 \mathrm{H}), 1.65(\mathrm{~d}, J=6.8 \mathrm{~Hz}, 6 \mathrm{H})$.

${ }^{13}$ C-NMR (125 MHz, $\left.\mathrm{CDCl}_{3}\right): \delta=154.5\left(\mathrm{C}_{\mathrm{q}}\right), 152.0\left(\mathrm{C}_{\mathrm{q}}\right), 151.8(\mathrm{CH}), 141.8(\mathrm{CH}), 140.7\left(\mathrm{C}_{\mathrm{q}}\right)$, $139.9\left(\mathrm{C}_{\mathrm{q}}\right), 136.0\left(\mathrm{C}_{\mathrm{q}}\right), 131.4\left(\mathrm{C}_{\mathrm{q}}\right), 131.4(\mathrm{CH}), 131.3(\mathrm{CH}), 130.6(\mathrm{CH}), 129.8(\mathrm{CH}), 128.8$ $(\mathrm{CH}), 128.1(\mathrm{CH}), 119.8\left(\mathrm{C}_{\mathrm{q}}\right), 47.2(\mathrm{CH}), 41.4\left(\mathrm{CH}_{2}\right), 22.6\left(\mathrm{CH}_{3}\right)$.

IR (ATR): $\tilde{v}=2977,1567,1486,1325,1218,1070,1011,781,703,646 \mathrm{~cm}^{-1}$.

MS (EI) $m / z$ (relative intensity): 408 (48) $\left[\mathrm{M}\left({ }^{81} \mathrm{Br}\right)\right]^{+}, 407$ (64) $\left[\mathrm{M}\left({ }^{81} \mathrm{Br}\right)-\mathrm{H}\right]^{+}, 406$ (48) $\left[\mathrm{M}\left({ }^{79} \mathrm{Br}\right)\right]^{+}, 405(55)\left[\mathrm{M}\left({ }^{79} \mathrm{Br}\right)-\mathrm{H}\right]^{+}, 365(100)\left[\mathrm{M}\left({ }^{81} \mathrm{Br}\right)-i \mathrm{Pr}\right]^{+}, 363(100)\left[\mathrm{M}\left({ }^{79} \mathrm{Br}\right)-i \mathrm{Pr}\right]^{+}, 283$ (39) $[\mathrm{M}-i \mathrm{Pr}-\mathrm{Br}]^{+}, 165$ (14), 142 (11).

HR-MS (EI): $m / z$ calcd for $\mathrm{C}_{21} \mathrm{H}_{19}{ }^{79} \mathrm{BrN}_{4}{ }^{+}[\mathrm{M}]^{+} 406.0788$, found 406.0778 . 


\section{1-\{4-\{6-[3-(4-Methoxybenzyl)phenyl]-9H-purin-9-yl\}phenyl\}ethan-1-one (3ia)}

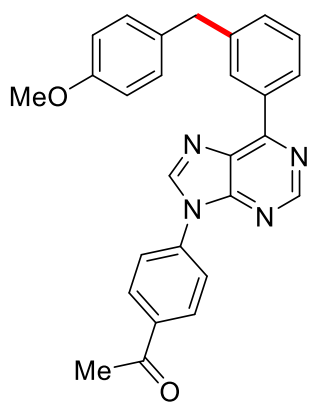

The general procedure A was followed using purine 1i (157 mg, $0.50 \mathrm{mmol})$ and benzyl chloride $2 \mathrm{a}(235 \mathrm{mg}, 1.50 \mathrm{mmol})$. After $20 \mathrm{~h}$, purification by column chromatography ( $n$-hexane/EtOAc 7:3) yielded 3ia $(147 \mathrm{mg}, 68 \%)$ as a white solid.

${ }^{1} \mathbf{H}-\mathrm{NMR}\left(300 \mathrm{MHz}, \mathrm{CDCl}_{3}\right): \delta=9.09$ (s, 1H), 8.70 (ddd, $J=7.8,1.8$, $1.2 \mathrm{~Hz}, 1 \mathrm{H}), 8.64(\mathrm{ddd}, J=1.9,1.8,0.9 \mathrm{~Hz}, 1 \mathrm{H}), 8.46(\mathrm{~s}, 1 \mathrm{H}), 8.21(\mathrm{~d}, J=$ $8.9 \mathrm{~Hz}, 2 \mathrm{H}), 7.97(\mathrm{~d}, J=8.9 \mathrm{~Hz}, 2 \mathrm{H}), 7.50$ (dd, $J=7.8,7.7 \mathrm{~Hz}, 1 \mathrm{H}), 7.35$ (ddd, $J=7.7,1.9$, $1.2 \mathrm{~Hz}, 1 \mathrm{H}), 7.18(\mathrm{~d}, J=8.8 \mathrm{~Hz}, 2 \mathrm{H}), 6.84(\mathrm{~d}, J=8.8 \mathrm{~Hz}, 2 \mathrm{H}), 4.09$ (s, 2H), 3.78 (s, 3H), 2.69 $(\mathrm{s}, 3 \mathrm{H})$.

${ }^{13}$ C-NMR (125 MHz, $\left.\mathrm{CDCl}_{3}\right): \delta=196.4\left(\mathrm{C}_{\mathrm{q}}\right), 157.9\left(\mathrm{C}_{\mathrm{q}}\right), 155.8\left(\mathrm{C}_{\mathrm{q}}\right), 153.1(\mathrm{CH}), 152.0\left(\mathrm{C}_{\mathrm{q}}\right)$, $142.3(\mathrm{CH}), 142.1\left(\mathrm{C}_{\mathrm{q}}\right), 138.2\left(\mathrm{C}_{\mathrm{q}}\right), 136.4\left(\mathrm{C}_{\mathrm{q}}\right), 135.4\left(\mathrm{C}_{\mathrm{q}}\right), 133.0\left(\mathrm{C}_{\mathrm{q}}\right), 131.8(\mathrm{CH}), 131.6\left(\mathrm{C}_{\mathrm{q}}\right)$, $130.1(\mathrm{CH}), 129.9(\mathrm{CH}), 129.8(\mathrm{CH}), 128.8(\mathrm{CH}), 128.0(\mathrm{CH}), 122.9(\mathrm{CH}), 113.9(\mathrm{CH}), 55.3$ $\left(\mathrm{CH}_{3}\right), 41.2\left(\mathrm{CH}_{2}\right), 26.8\left(\mathrm{CH}_{3}\right)$.

IR (ATR): $\tilde{v}=3112,1679,1557,1509,1233,1173,1026,928,835,786 \mathrm{~cm}^{-1}$.

m.p.: $159-160{ }^{\circ} \mathrm{C}$.

MS (EI) $m / z$ (relative intensity): 434 (100) $[\mathrm{M}]^{+}, 433$ (63) $[\mathrm{M}-\mathrm{H}]^{+}, 419$ (53) [M-Me $]^{+}, 210$ (9), $121(9), 43$ (16).

HR-MS (EI): $m / z$ calcd for $\mathrm{C}_{27} \mathrm{H}_{22} \mathrm{~N}_{4} \mathrm{O}_{2}{ }^{+}[\mathrm{M}]^{+} 434.1737$, found 434.1743.

\section{6-[4-Fluoro-3-(4-methylbenzyl)phenyl]-9-iso-propyl-9H-purine (3jc)}

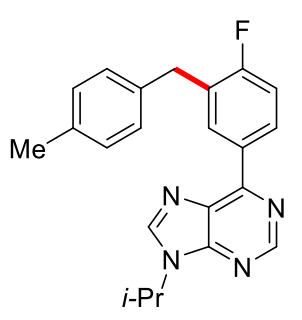

The general procedure $\mathbf{A}$ was followed using purine $\mathbf{1 j}$ (128 $\mathrm{mg}, 0.50 \mathrm{mmol}$ ) and benzyl chloride $2 \mathrm{c}(211 \mathrm{mg}, 1.50 \mathrm{mmol})$. After $20 \mathrm{~h}$, purification by column chromatography ( $n$-hexane/EtOAc $3: 1$ ) and recycling preparative HPLC yielded 3jc $(90.5 \mathrm{mg}, 50 \%)$ as a white soild.

${ }^{1}$ H-NMR $\left(300 \mathrm{MHz}, \mathrm{CDCl}_{3}\right): \delta=8.98(\mathrm{~s}, 1 \mathrm{H}), 8.76(\mathrm{ddd}, J=8.6,5.2$, $2.3 \mathrm{~Hz}, 1 \mathrm{H}), 8.71$ (dd, $J=7.5,2.3 \mathrm{~Hz}, 1 \mathrm{H}), 8.16$ (s, 1H), 7.21 (dd, $J=9.5,8.6 \mathrm{~Hz}, 1 \mathrm{H}), 7.18$ (d, $J=7.9 \mathrm{~Hz}, 2 \mathrm{H}), 7.08(\mathrm{~d}, J=7.9 \mathrm{~Hz}, 2 \mathrm{H}), 4.96$ (hept, $J=6.8 \mathrm{~Hz}, 1 \mathrm{H}), 4.10$ (s, 2H), 2.29 (s, 3H), $1.66(\mathrm{~d}, J=6.8 \mathrm{~Hz}, 6 \mathrm{H})$.

${ }^{13} \mathrm{C}-\mathrm{NMR}\left(125 \mathrm{MHz}, \mathrm{CDCl}_{3}\right): \delta=162.7\left(\mathrm{~d},{ }^{1} J_{\mathrm{C}-\mathrm{F}}=251 \mathrm{~Hz}, \mathrm{C}_{\mathrm{q}}\right), 153.6\left(\mathrm{C}_{\mathrm{q}}\right), 151.9\left(\mathrm{C}_{\mathrm{q}}\right), 151.8$ $(\mathrm{CH}), 141.7(\mathrm{CH}), 136.7\left(\mathrm{C}_{\mathrm{q}}\right), 135.5\left(\mathrm{C}_{\mathrm{q}}\right), 132.6\left(\mathrm{~d},{ }^{3} J_{\mathrm{C}-\mathrm{F}}=6 \mathrm{~Hz}, \mathrm{CH}\right), 131.9\left(\mathrm{~d},{ }^{4} J_{\mathrm{C}-\mathrm{F}}=3 \mathrm{~Hz}\right.$, $\left.\mathrm{C}_{\mathrm{q}}\right), 131.1\left(\mathrm{C}_{\mathrm{q}}\right), 130.2\left(\mathrm{~d},{ }^{3} J_{\mathrm{C}-\mathrm{F}}=9 \mathrm{~Hz}, \mathrm{CH}\right), 129.0(\mathrm{CH}), 128.6\left(\mathrm{~d},{ }^{2} J_{\mathrm{C}-\mathrm{F}}=16 \mathrm{~Hz}, \mathrm{C}_{\mathrm{q}}\right), 128.4$ $(\mathrm{CH}), 115.6\left(\mathrm{~d},{ }^{2} J_{\mathrm{C}-\mathrm{F}}=23 \mathrm{~Hz}, \mathrm{CH}\right), 47.3(\mathrm{CH}), 34.9\left(\mathrm{~d},{ }^{3} J_{\mathrm{C}-\mathrm{F}}=3 \mathrm{~Hz}, \mathrm{CH}_{2}\right), 22.6\left(\mathrm{CH}_{3}\right), 21.0$ $\left(\mathrm{CH}_{3}\right)$. 
${ }^{19}$ F-NMR $\left(282 \mathrm{MHz}, \mathrm{CDCl}_{3}\right): \delta=(-113.6)-(-113.8)(\mathrm{m})$.

IR (ATR): $\tilde{v}=2978,1574,1502,1446,1326,1219,834,806,646 \mathrm{~cm}^{-1}$.

m.p.: $72-74{ }^{\circ} \mathrm{C}$.

MS (EI) $m / z$ (relative intensity): $360(90)[\mathrm{M}]^{+}, 359(26)[\mathrm{M}-\mathrm{H}]^{+}, 345(2)[\mathrm{M}-\mathrm{Me}]^{+}, 317$ (100) $[\mathrm{M}-i \mathrm{Pr}]^{+}, 303(9)$.

HR-MS (EI): $m / z$ calcd for $\mathrm{C}_{22} \mathrm{H}_{21} \mathrm{FN}_{4}{ }^{+}[\mathrm{M}]^{+} 360.1745$, found 360.1748 .

\section{6-[3-(4-Methoxybenzyl)phenyl]-9-(tetrahydro-2H-pyran-2-yl)-9H-purine (3ka)}

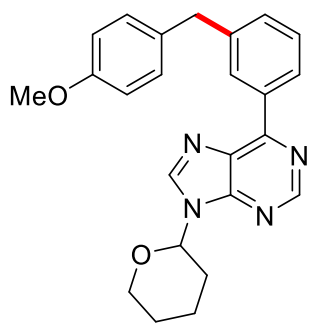

The general procedure A was followed using purine 1k (140 mg, $0.50 \mathrm{mmol})$ and benzyl chloride $2 \mathrm{a}(235 \mathrm{mg}, 1.50 \mathrm{mmol})$. After $20 \mathrm{~h}$, purification by column chromatography ( $n$-hexane/EtOAc $3: 1$ ) yielded 3ka (150 mg, 75\%) as a colorless oil.

${ }^{1} \mathbf{H}-\mathbf{N M R}\left(300 \mathrm{MHz}, \mathrm{CDCl}_{3}\right): \delta=9.01(\mathrm{~s}, 1 \mathrm{H}), 8.68(\mathrm{ddd}, J=7.8,1.8$, $1.2 \mathrm{~Hz}, 1 \mathrm{H}), 8.61$ (ddd, $J=1.8,1.8,0.6 \mathrm{~Hz}, 1 \mathrm{H}), 8.32$ (s, 1H), 7.46 (ddd, $J=7.8,7.7,0.6 \mathrm{~Hz}$, $1 \mathrm{H}), 7.30$ (ddd, $J=7.7,1.8,1.2 \mathrm{~Hz}, 1 \mathrm{H}), 7.15(\mathrm{~d}, J=8.8 \mathrm{~Hz}, 2 \mathrm{H}), 6.81(\mathrm{~d}, J=8.8 \mathrm{~Hz}, 2 \mathrm{H})$, $5.82(\mathrm{dd}, J=9.9,3.0 \mathrm{~Hz}, 1 \mathrm{H}), 4.20-4.13(\mathrm{~m}, 1 \mathrm{H}), 4.06(\mathrm{~s}, 2 \mathrm{H}), 3.82-3.73(\mathrm{~m}, 1 \mathrm{H}), 3.74(\mathrm{~s}, 3 \mathrm{H})$, $2.19-1.98(\mathrm{~m}, 3 \mathrm{H}), 1.86-1.58(\mathrm{~m}, 3 \mathrm{H})$.

${ }^{13}$ C-NMR $\left(125 \mathrm{MHz}, \mathrm{CDCl}_{3}\right): \delta=157.8\left(\mathrm{C}_{\mathrm{q}}\right), 154.8\left(\mathrm{C}_{\mathrm{q}}\right), 152.1(\mathrm{CH}), 151.5\left(\mathrm{C}_{\mathrm{q}}\right), 141.8\left(\mathrm{C}_{\mathrm{q}}\right.$ and $\mathrm{CH}), 135.6\left(\mathrm{C}_{\mathrm{q}}\right), 132.9\left(\mathrm{C}_{\mathrm{q}}\right), 131.4(\mathrm{CH}), 131.0\left(\mathrm{C}_{\mathrm{q}}\right), 129.7(\mathrm{CH}), 128.6(\mathrm{CH}), 127.8(\mathrm{CH})$, 113.7 $(\mathrm{CH}), 81.9(\mathrm{CH}), 68.7\left(\mathrm{CH}_{2}\right), 55.1\left(\mathrm{CH}_{3}\right), 41.1\left(\mathrm{CH}_{2}\right), 31.7\left(\mathrm{CH}_{2}\right), 24.8\left(\mathrm{CH}_{2}\right), 22.8\left(\mathrm{CH}_{2}\right)$. IR (ATR): $\tilde{v}=2946,1567,1509,1323,1243,1083,1042,734,701,643 \mathrm{~cm}^{-1}$.

MS (EI) $m / z$ (relative intensity): 400 (41) $[\mathrm{M}]^{+}, 372(11)[\mathrm{M}-\mathrm{Et}]^{+}, 316(100)[\mathrm{M}-\mathrm{THP}]^{+}, 315$ (76), 301 (52), 121 (22), 85 (33), 67 (11), 41 (12).

HR-MS (EI): $m / z$ calcd for $\mathrm{C}_{24} \mathrm{H}_{24} \mathrm{~N}_{4} \mathrm{O}_{2}{ }^{+}[\mathrm{M}]^{+} 400.1894$, found 400.1903.

$N, N$-Di-iso-propyl-4-\{\{6-[3-(4-methoxybenzyl)phenyl]-9H-purin-9-yl\}methyl\}benzamide (3la)

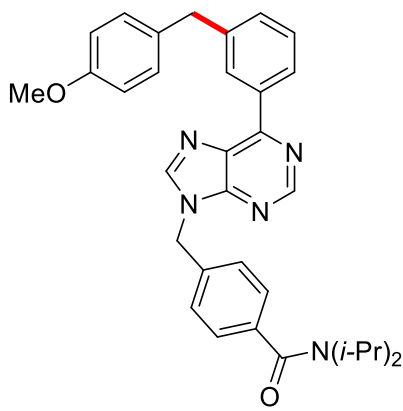

The general procedure A was followed using purine 11 (207 mg, $0.50 \mathrm{mmol})$ and benzyl chloride $2 \mathbf{a}(235 \mathrm{mg}, 1.50 \mathrm{mmol})$. After $20 \mathrm{~h}$, purification by column chromatography ( $n$-hexane/EtOAc 2:3) yielded 3la (167 mg, 63\%) as a white soild.

${ }^{1}$ H-NMR $\left(400 \mathrm{MHz}, \mathrm{CDCl}_{3}\right.$ ): $\delta=9.04$ (s, 1H), 8.68 (ddd, $J=7.8$, $1.8,1.2 \mathrm{~Hz}, 1 \mathrm{H}), 8.62(\mathrm{ddd}, J=1.8,1.8,0.6 \mathrm{~Hz}, 1 \mathrm{H}), 8.10(\mathrm{~s}, 1 \mathrm{H})$, 
$7.47(\mathrm{dd}, J=7.8,7.7 \mathrm{~Hz}, 1 \mathrm{H}), 7.33-7.28(\mathrm{~m}, 5 \mathrm{H}), 7.15(\mathrm{~d}, J=8.8 \mathrm{~Hz}, 2 \mathrm{H}), 6.82(\mathrm{~d}, J=8.8 \mathrm{~Hz}$, 2H), 5.47 (s, 2H), 4.06 (s, 2H), 3.75 (s, 3H), 3.87-3.39 (br, 2H), 1.78-0.88 (br, 12H).

${ }^{13} \mathrm{C}-\mathrm{NMR}\left(100 \mathrm{MHz}, \mathrm{CDCl}_{3}\right): \delta=170.1\left(\mathrm{C}_{\mathrm{q}}\right), 157.9\left(\mathrm{C}_{\mathrm{q}}\right), 155.0\left(\mathrm{C}_{\mathrm{q}}\right), 152.6(\mathrm{CH}), 152.4\left(\mathrm{C}_{\mathrm{q}}\right)$, 144.0 (CH), $142.0\left(\mathrm{C}_{\mathrm{q}}\right), 139.2\left(\mathrm{C}_{\mathrm{q}}\right), 135.7\left(\mathrm{C}_{\mathrm{q}}\right), 133.1\left(\mathrm{C}_{\mathrm{q}}\right), 131.6(\mathrm{CH}), 130.9\left(\mathrm{C}_{\mathrm{q}}\right), 129.9(\mathrm{CH})$, $129.8(\mathrm{CH}), 128.8(\mathrm{CH}), 127.9(\mathrm{CH}), 127.8(\mathrm{CH}), 126.4(\mathrm{CH}), 113.8(\mathrm{CH}), 55.2\left(\mathrm{CH}_{3}\right), 51.6-$ 50.0 (br, $\mathrm{CH}), 46.8\left(\mathrm{CH}_{2}\right), 46.6-45.2(\mathrm{br}, \mathrm{CH}), 41.1\left(\mathrm{CH}_{2}\right), 20.6\left(\mathrm{CH}_{3}\right)$.

IR (ATR): $\tilde{v}=2967,1623,1568,1509,1439,1323,1244,1035,794,703 \mathrm{~cm}^{-1}$.

m.p.: $68-70{ }^{\circ} \mathrm{C}$.

MS (EI) $m / z$ (relative intensity): $533(56)[\mathrm{M}]^{+}, 532(10)[\mathrm{M}-\mathrm{H}]^{+}, 518(5)[\mathrm{M}-\mathrm{Me}]^{+}, 490$ (21) $[\mathrm{M}-i \mathrm{Pr}]^{+}, 433(100)\left[\mathrm{M}-\mathrm{N}(i \mathrm{Pr})_{2}\right]^{+}, 405$ (18) $\left[\mathrm{M}-\mathrm{C}(\mathrm{O}) \mathrm{N}(i \mathrm{Pr})_{2}\right]^{+}, 315$ (25), 217 (14), 118 (19), $91(23)[\mathrm{Bn}]^{+}, 58(14), 43(52)$.

HR-MS (EI): $m / z$ calcd for $\mathrm{C}_{33} \mathrm{H}_{35} \mathrm{~N}_{5} \mathrm{O}_{2}{ }^{+}[\mathrm{M}]^{+}$533.2785, found 533.2807.

\section{6-[3-(3,5-Dimethoxybenzyl)phenyl]-9-iso-propyl-9H-purine (3hi)}

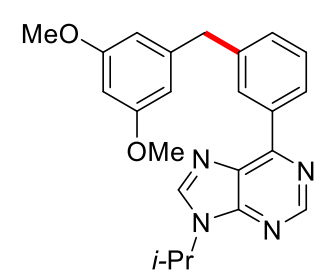

The general procedure A was followed using purine $\mathbf{1 h}$ (119 mg, $0.50 \mathrm{mmol})$ and benzyl chloride $2 \mathbf{i}(280 \mathrm{mg}, 1.50 \mathrm{mmol})$. After $20 \mathrm{~h}$, purification by column chromatography ( $n$-hexane/EtOAc $2: 1$ ) yielded 3hi $(130 \mathrm{mg}, 67 \%)$ as a colorless oil.

${ }^{1} \mathbf{H}-\mathbf{N M R}\left(300 \mathrm{MHz}, \mathrm{CDCl}_{3}\right.$ ): $\delta=8.99(\mathrm{~s}, 1 \mathrm{H}), 8.67$ (ddd, $J=7.8,1.8,1.2 \mathrm{~Hz}, 1 \mathrm{H}$ ), 8.62 (ddd, $J=1.8,1.5,0.6 \mathrm{~Hz}, 1 \mathrm{H}), 8.14(\mathrm{~s}, 1 \mathrm{H}), 7.46(\mathrm{ddd}, J=7.8,7.7,0.6 \mathrm{~Hz}, 1 \mathrm{H}), 7.32$ (dddd, $J=7.7$, $1.5,1.2,0.6 \mathrm{~Hz}, 1 \mathrm{H}), 6.40(\mathrm{~d}, J=2.3 \mathrm{~Hz}, 2 \mathrm{H}), 6.29(\mathrm{t}, J=2.3 \mathrm{~Hz}, 1 \mathrm{H}), 4.94$ (hept, $J=6.8 \mathrm{~Hz}$, 1H), 4.05 (s, 2H), $3.72(\mathrm{~s}, 6 \mathrm{H}), 1.63(\mathrm{~d}, J=6.8 \mathrm{~Hz}, 6 \mathrm{H})$.

${ }^{13}$ C-NMR $\left(125 \mathrm{MHz}, \mathrm{CDCl}_{3}\right): \delta=160.6\left(\mathrm{C}_{\mathrm{q}}\right), 154.5\left(\mathrm{C}_{\mathrm{q}}\right), 151.9\left(\mathrm{C}_{\mathrm{q}}\right), 151.8(\mathrm{CH}), 143.1\left(\mathrm{C}_{\mathrm{q}}\right)$, $141.7(\mathrm{CH}), 140.9\left(\mathrm{C}_{\mathrm{q}}\right), 135.8\left(\mathrm{C}_{\mathrm{q}}\right), 131.3\left(\mathrm{C}_{\mathrm{q}}\right), 131.3(\mathrm{CH}), 129.8(\mathrm{CH}), 128.6(\mathrm{CH}), 127.9$ $(\mathrm{CH}), 107.0(\mathrm{CH}), 98.0(\mathrm{CH}), 55.1\left(\mathrm{CH}_{3}\right), 47.2(\mathrm{CH}), 42.2\left(\mathrm{CH}_{2}\right), 22.5\left(\mathrm{CH}_{3}\right)$.

IR (ATR): $\tilde{v}=2936,1567,1457,1324,1204,1145,1063,791,703,646 \mathrm{~cm}^{-1}$.

MS (EI) $m / z$ (relative intensity): $388(97)[\mathrm{M}]^{+}, 387(58)[\mathrm{M}-\mathrm{H}]^{+}, 373(4)[\mathrm{M}-\mathrm{Me}]^{+}, 345$ (100) $[\mathrm{M}-i \mathrm{Pr}]^{+}, 331$ (10) [M-iPr-Me] $]^{+}, 313$ (20), 299 (8), 173 (8), 151 (6), 43 (14).

HR-MS (EI): $m / z$ calcd for $\mathrm{C}_{23} \mathrm{H}_{24} \mathrm{~N}_{4} \mathrm{O}_{2}{ }^{+}[\mathrm{M}]^{+} 388.1894$, found 388.1894. 


\section{9-iso-Propyl-6-[3-(naphthalen-1-ylmethyl)phenyl]-9H-purine (3hj)}

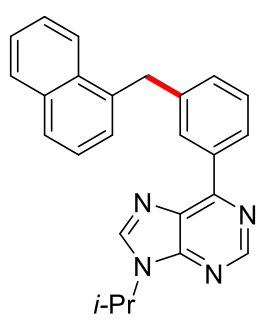

The general procedure A was followed using purine $\mathbf{1 h}(119 \mathrm{mg}, 0.50 \mathrm{mmol})$ and benzyl chloride $2 \mathbf{j}$ ( $265 \mathrm{mg}, 1.50 \mathrm{mmol}$ ) at $80{ }^{\circ} \mathrm{C}$. After $20 \mathrm{~h}$, purification by column chromatography ( $n$-hexane/EtOAc 3:1) yielded $3 \mathbf{h j}(127 \mathrm{mg}, 67 \%$ ) as a white solid.

${ }^{1} \mathbf{H}-\mathrm{NMR}\left(300 \mathrm{MHz}, \mathrm{CDCl}_{3}\right): \delta=9.02(\mathrm{~s}, 1 \mathrm{H}), 8.73(\mathrm{ddd}, J=1.8,1.5,0.6 \mathrm{~Hz}$, 1H), 8.67 (ddd, $J=7.8,1.8,1.2 \mathrm{~Hz}, 1 \mathrm{H}), 8.17$ (s, 1H), 8.09-8.03 (m, 1H), 7.89-7.83 (m, 1H), 7.76 (br d, $J=8.1 \mathrm{~Hz}, 1 \mathrm{H}$ ), 7.48-7.40 (m, 4H), 7.35 (ddt, $J=7.0,1.4,0.8 \mathrm{~Hz}, 1 \mathrm{H}$ ), 7.26 (dddd, $J=7.6,1.5,1.2,0.6 \mathrm{~Hz}, 1 \mathrm{H}), 4.98$ (hept, $J=6.8 \mathrm{~Hz}, 1 \mathrm{H}), 4.61(\mathrm{~s}, 2 \mathrm{H}), 1.67$ (d, $J=6.8 \mathrm{~Hz}, 6 \mathrm{H})$. ${ }^{13}$ C-NMR (125 MHz, $\left.\mathrm{CDCl}_{3}\right): \delta=154.8\left(\mathrm{C}_{\mathrm{q}}\right), 152.0\left(\mathrm{C}_{\mathrm{q}}\right), 151.9(\mathrm{CH}), 141.8(\mathrm{CH}), 141.0\left(\mathrm{C}_{\mathrm{q}}\right)$, $136.5\left(\mathrm{C}_{\mathrm{q}}\right), 135.9\left(\mathrm{C}_{\mathrm{q}}\right), 133.8\left(\mathrm{C}_{\mathrm{q}}\right), 132.0\left(\mathrm{C}_{\mathrm{q}}\right), 131.5\left(\mathrm{C}_{\mathrm{q}}\right), 131.2(\mathrm{CH}), 129.9(\mathrm{CH}), 128.7(\mathrm{CH})$, $128.5(\mathrm{CH}), 127.9(\mathrm{CH}), 127.3(\mathrm{CH}), 127.1(\mathrm{CH}), 125.9(\mathrm{CH}), 125.5(\mathrm{CH}), 125.4(\mathrm{CH}), 124.2$ $(\mathrm{CH}), 47.3(\mathrm{CH}), 39.2\left(\mathrm{CH}_{2}\right), 22.6\left(\mathrm{CH}_{3}\right)$.

IR (ATR): $\tilde{v}=2978,1569,1444,1324,1225,791,705,647,571 \mathrm{~cm}^{-1}$.

m.p.: $114-116^{\circ} \mathrm{C}$.

MS (ESI) $m / z$ (relative intensity): $757(6)[2 \mathrm{M}+\mathrm{H}]^{+}, 401$ (2) $[\mathrm{M}+\mathrm{Na}]^{+}, 379(100)[\mathrm{M}+\mathrm{H}]^{+}$.

HR-MS (ESI): $m / z$ calcd for $\mathrm{C}_{25} \mathrm{H}_{23} \mathrm{~N}_{4}^{+}[\mathrm{M}+\mathrm{H}]^{+} 379.1917$, found 379.1920 .

\section{1,4-Bis[3-(9-iso-propyl-9H-purin-6-yl)benzyl]benzene (3hk)}

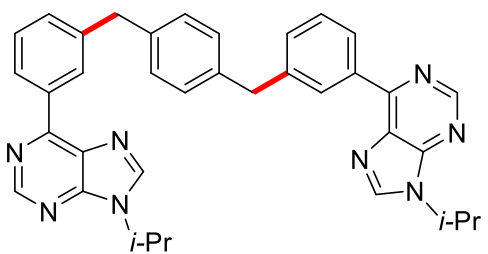

Arylpurine $\mathbf{1 h}$ (297 mg, $1.25 \mathrm{mmol}, 2.50$ equiv), benzyl chloride $2 \mathbf{k}$ (87.6 mg, $0.50 \mathrm{mmol}, 1.00$ equiv), $\left[\mathrm{Ru}(\mathrm{OAc})_{2}(p-\right.$ cymene)] (17.7 mg, $50.0 \mu \mathrm{mol}, 10.0 \mathrm{~mol} \%), \mathrm{PPh}_{3}(13.1 \mathrm{mg}$, $50.0 \mu \mathrm{mol}, 10.0 \mathrm{~mol} \%)$ and $\mathrm{K}_{2} \mathrm{CO}_{3}(276 \mathrm{mg}, 2.00 \mathrm{mmol}$,

4.00 equiv) were placed in a pre-dried $25 \mathrm{~mL}$ Schlenk tube. The tube was evacuated and purged with $\mathrm{N}_{2}$ three times. 1,4-Dioxane $(2.0 \mathrm{~mL})$ was then added and the mixture was stirred at 60 ${ }^{\circ} \mathrm{C}$. After $20 \mathrm{~h}$, the resulting mixture was filtered through a pad of silica gel and washed with EtOAc. The filtrate was concentrated in vacuo. Purification of the residue by column chromatography ( $n$-hexane/EtOAc 1:2) yielded 3hk (139 mg, 48\%) as a white solid.

${ }^{1}$ H-NMR (300 MHz, $\mathrm{CDCl}_{3}$ ): $\delta=8.99$ (s, 2H), 8.65 (ddd, $J=7.8,1.8,1.2 \mathrm{~Hz}, 2 \mathrm{H}$ ), 8.61 (ddd, $J=1.8,1.8,0.6 \mathrm{~Hz}, 2 \mathrm{H}), 8.15(\mathrm{~s}, 2 \mathrm{H}), 7.45$ (ddd, $J=7.8,7.7,0.6 \mathrm{~Hz}, 2 \mathrm{H}), 7.30$ (ddd, $J=7.7$, 1.8, $1.2 \mathrm{~Hz}, 2 \mathrm{H}), 7.16(\mathrm{~s}, 4 \mathrm{H}), 4.96$ (hept, $J=6.8 \mathrm{~Hz}, 2 \mathrm{H}), 4.08(\mathrm{~s}, 4 \mathrm{H}), 1.65$ (d, $J=6.8 \mathrm{~Hz}$, $12 \mathrm{H})$. 
${ }^{13} \mathrm{C}-\mathrm{NMR}\left(125 \mathrm{MHz}, \mathrm{CDCl}_{3}\right): \delta=154.7\left(\mathrm{C}_{\mathrm{q}}\right), 151.9\left(\mathrm{C}_{\mathrm{q}}\right), 151.8(\mathrm{CH}), 141.7(\mathrm{CH}), 141.5\left(\mathrm{C}_{\mathrm{q}}\right)$, 138.6 $\left(\mathrm{C}_{\mathrm{q}}\right), 135.8\left(\mathrm{C}_{\mathrm{q}}\right), 131.4\left(\mathrm{C}_{\mathrm{q}}\right.$ and $\left.\mathrm{CH}\right), 129.9(\mathrm{CH}), 128.9(\mathrm{CH}), 128.6(\mathrm{CH}), 127.8(\mathrm{CH})$, $47.2(\mathrm{CH}), 41.6\left(\mathrm{CH}_{2}\right), 22.6\left(\mathrm{CH}_{3}\right)$.

IR (ATR): $\tilde{v}=2975,1570,1441,1326,1218,790,699,646,582 \mathrm{~cm}^{-1}$.

m.p.: $156-158{ }^{\circ} \mathrm{C}$.

MS (EI) $m / z$ (relative intensity): $578(100)[\mathrm{M}]^{+}, 536$ (17) $[\mathrm{M}-i \mathrm{Pr}]^{+}, 493$ (12) $[\mathrm{M}-i \mathrm{Pr}-i \mathrm{Pr}]^{+}$, 285 (21), 246 (12), 209 (17), 43 (6).

HR-MS (EI): $m / z$ calcd for $\mathrm{C}_{36} \mathrm{H}_{34} \mathrm{~N}_{8}{ }^{+}[\mathrm{M}]^{+}$578.2901, found 578.2900.

\section{2-[3-(1-Phenylethyl)phenyl]pyrimidine (3dl)}<smiles>CC(c1ccccc1)c1cccc(-c2ncccn2)c1</smiles>

The general procedure A was followed using pyrimidine 1d $(78.1 \mathrm{mg}$, $0.50 \mathrm{mmol})$ and benzyl chloride $2 \mathrm{l}(211 \mathrm{mg}, 1.50 \mathrm{mmol})$. After $20 \mathrm{~h}$, purification by column chromatography (n-hexane/EtOAc 10:1) and recycling preparative HPLC yielded $\mathbf{3 d l}(88.6 \mathrm{mg}, 68 \%)$ as a white solid.

${ }^{1} \mathbf{H}-\mathbf{N M R}\left(300 \mathrm{MHz}, \mathrm{CDCl}_{3}\right): \delta=8.76(\mathrm{~d}, J=4.9 \mathrm{~Hz}, 2 \mathrm{H}), 8.38(\mathrm{dd}, J=1.8,1.8 \mathrm{~Hz}, 1 \mathrm{H}), 8.28$ (ddd, $J=7.6,1.8,1.3 \mathrm{~Hz}, 1 \mathrm{H}), 7.40$ (dd, $J=7.6,7.6 \mathrm{~Hz}, 1 \mathrm{H}), 7.33-7.29$ (m, 1H), 7.29-7.24 $(\mathrm{m}, 4 \mathrm{H}), 7.20-7.14(\mathrm{~m}, 1 \mathrm{H}), 7.11(\mathrm{t}, J=4.9 \mathrm{~Hz}, 1 \mathrm{H}), 4.27(\mathrm{q}, J=7.2 \mathrm{~Hz}, 1 \mathrm{H}), 1.71(\mathrm{~d}, J=$ $7.2 \mathrm{~Hz}, 3 \mathrm{H})$.

${ }^{13}$ C-NMR (125 MHz, $\left.\mathrm{CDCl}_{3}\right): \delta=164.7\left(\mathrm{C}_{\mathrm{q}}\right), 157.0(\mathrm{CH}), 146.6\left(\mathrm{C}_{\mathrm{q}}\right), 146.1\left(\mathrm{C}_{\mathrm{q}}\right), 137.5\left(\mathrm{C}_{\mathrm{q}}\right)$, $130.1(\mathrm{CH}), 128.6(\mathrm{CH}), 128.3(\mathrm{CH}), 127.5(\mathrm{CH}), 127.3(\mathrm{CH}), 126.0(\mathrm{CH}), 125.9(\mathrm{CH}), 118.9$ $(\mathrm{CH}), 44.9(\mathrm{CH}), 21.9\left(\mathrm{CH}_{3}\right)$.

IR (ATR): $\tilde{v}=3027,2966,1567,1553,1422,1407,794,765,698 \mathrm{~cm}^{-1}$.

m.p.: $78-79^{\circ} \mathrm{C}$.

MS (EI) $m / z$ (relative intensity): 260 (42) $[\mathrm{M}]^{+}, 259$ (14) $[\mathrm{M}-\mathrm{H}]^{+}, 245$ (100) $[\mathrm{M}-\mathrm{Me}]^{+}, 190$ (6), 165 (18), 122 (7).

HR-MS (EI): $m / z$ calcd for $\mathrm{C}_{18} \mathrm{H}_{16} \mathrm{~N}_{2}{ }^{+}[\mathrm{M}]^{+} 260.1308$, found 260.1321 .

\section{2-\{3-[1-(4-Fluorophenyl)ethyl]phenyl\}pyrimidine (3dm)}<smiles>CC(c1ccc(F)cc1)c1cccc(-c2ncccn2)c1</smiles>

The general procedure A was followed using pyrimidine 1d $(78.1 \mathrm{mg}$, $0.50 \mathrm{mmol}$ ) and benzyl chloride $2 \mathrm{~m}$ (238 mg, $1.50 \mathrm{mmol})$. After $20 \mathrm{~h}$, purification by column chromatography (n-hexane/EtOAc 10:1) yielded 3dm (113 mg, 81\%) as a white solid.

${ }^{1} \mathbf{H}-\mathbf{N M R}\left(300 \mathrm{MHz}, \mathrm{CDCl}_{3}\right): \delta=8.80(\mathrm{~d}, J=4.9 \mathrm{~Hz}, 2 \mathrm{H}), 8.34(\mathrm{ddt}, J=1.9,1.5,0.6 \mathrm{~Hz}, 1 \mathrm{H})$, 8.28 (dddd, $J=7.7,1.5,1.2,0.3 \mathrm{~Hz}, 1 \mathrm{H}), 7.42(\mathrm{dd}, J=7.7,7.7 \mathrm{~Hz}, 1 \mathrm{H}), 7.30(\mathrm{dddd}, J=7.7$, 
1.9, 1.2, $0.6 \mathrm{~Hz}, 1 \mathrm{H}), 7.25-7.20(\mathrm{~m}, 2 \mathrm{H}), 7.18(\mathrm{t}, J=4.9 \mathrm{~Hz}, 1 \mathrm{H}), 6.97(\mathrm{dd}, J=8.7,8.7 \mathrm{~Hz}$, $2 \mathrm{H}), 4.26(\mathrm{q}, J=7.3 \mathrm{~Hz}, 1 \mathrm{H}), 1.70(\mathrm{~d}, J=7.3 \mathrm{~Hz}, 3 \mathrm{H})$.

${ }^{13} \mathrm{C}-\mathrm{NMR}\left(125 \mathrm{MHz}, \mathrm{CDCl}_{3}\right): \delta=164.7\left(\mathrm{C}_{\mathrm{q}}\right), 161.2\left(\mathrm{~d},{ }^{1} J_{\mathrm{C}-\mathrm{F}}=244 \mathrm{~Hz}, \mathrm{C}_{\mathrm{q}}\right), 157.1(\mathrm{CH}), 146.5$ $\left(\mathrm{C}_{\mathrm{q}}\right), 141.8\left(\mathrm{~d},{ }^{4} J_{\mathrm{C}-\mathrm{F}}=3 \mathrm{~Hz}, \mathrm{C}_{\mathrm{q}}\right), 137.7\left(\mathrm{C}_{\mathrm{q}}\right), 130.0(\mathrm{CH}), 128.9\left(\mathrm{~d},{ }^{3} J_{\mathrm{C}-\mathrm{F}}=8 \mathrm{~Hz}, \mathrm{CH}\right), 128.7$ $(\mathrm{CH}), 127.2(\mathrm{CH}), 126.1(\mathrm{CH}), 119.0(\mathrm{CH}), 115.0\left(\mathrm{~d},{ }^{2} J_{\mathrm{C}-\mathrm{F}}=21 \mathrm{~Hz}, \mathrm{CH}\right), 44.2(\mathrm{CH}), 22.1$ $\left(\mathrm{CH}_{3}\right)$.

${ }^{19}$ F-NMR $\left(282 \mathrm{MHz}, \mathrm{CDCl}_{3}\right): \delta=-117.5(\mathrm{tt}, J=8.7,5.4 \mathrm{~Hz})$.

IR (ATR): $\tilde{v}=2961,1567,1554,1505,1406,1219,1158,835,782,690 \mathrm{~cm}^{-1}$.

m.p.: $88-90{ }^{\circ} \mathrm{C}$.

MS (EI) $m / z$ (relative intensity): 278 (38) [M] $]^{+}, 263$ (100) [M-Me] $]^{+}, 243$ (11), 208 (7), 183 (17).

HR-MS (EI): $m / z$ calcd for $\mathrm{C}_{18} \mathrm{H}_{15} \mathrm{FN}_{2}{ }^{+}[\mathrm{M}]^{+}$278.1214, found 278.1211 .

\section{2-[3-(1-Phenylethyl)phenyl]pyridine (3al)}<smiles>CC(c1ccccc1)c1cccc(-c2ccccn2)c1</smiles>

The general procedure A was followed using pyridine 1a $(77.6 \mathrm{mg}$, $0.50 \mathrm{mmol}$ ) and benzyl chloride 21 (211 $\mathrm{mg}, 1.50 \mathrm{mmol})$. After $20 \mathrm{~h}$, purification by column chromatography ( $n$-hexane/EtOAc 10:1) and recycling preparative HPLC yielded 3al $(96.2 \mathrm{mg}, 74 \%)$ as a colorless oil.

${ }^{1} \mathbf{H}-\mathbf{N M R}\left(300 \mathrm{MHz}, \mathrm{CDCl}_{3}\right): \delta=8.67(\mathrm{ddd}, J=4.8,1.8,1.0 \mathrm{~Hz}, 1 \mathrm{H}), 7.89(\mathrm{dd}, J=1.9,1.9 \mathrm{~Hz}$, $1 \mathrm{H}), 7.78(\mathrm{ddd}, J=7.7,1.9,1.2 \mathrm{~Hz}, 1 \mathrm{H}), 7.75-7.65(\mathrm{~m}, 2 \mathrm{H}), 7.38(\mathrm{dd}, J=7.7,7.7 \mathrm{~Hz}, 1 \mathrm{H})$, $7.32-7.13(\mathrm{~m}, 7 \mathrm{H}), 4.25(\mathrm{q}, J=7.2 \mathrm{~Hz}, 1 \mathrm{H}), 1.70(\mathrm{~d}, J=7.2 \mathrm{~Hz}, 3 \mathrm{H})$.

${ }^{13} \mathrm{C}-\mathrm{NMR}\left(125 \mathrm{MHz}, \mathrm{CDCl}_{3}\right): \delta=157.5\left(\mathrm{C}_{\mathrm{q}}\right), 149.5(\mathrm{CH}), 146.8\left(\mathrm{C}_{\mathrm{q}}\right), 146.1\left(\mathrm{C}_{\mathrm{q}}\right), 139.4\left(\mathrm{C}_{\mathrm{q}}\right)$, $136.5(\mathrm{CH}), 128.7(\mathrm{CH}), 128.3(\mathrm{CH}), 128.2(\mathrm{CH}), 127.6(\mathrm{CH}), 126.3(\mathrm{CH}), 126.0(\mathrm{CH}), 124.7$ $(\mathrm{CH}), 121.9(\mathrm{CH}), 120.6(\mathrm{CH}), 44.9(\mathrm{CH}), 22.0\left(\mathrm{CH}_{3}\right)$.

IR (ATR): $\tilde{v}=2966,1583,1565,1433,1414,761,696,614 \mathrm{~cm}^{-1}$.

MS (EI) $m / z$ (relative intensity): $259(63)[\mathrm{M}]^{+}, 258(100)[\mathrm{M}-\mathrm{H}]^{+}, 244(90)[\mathrm{M}-\mathrm{Me}]^{+}, 165$ (20), 78 (12), 51 (9).

HR-MS (ESI): $m / z$ calcd for $\mathrm{C}_{19} \mathrm{H}_{18} \mathrm{~N}^{+}[\mathrm{M}+\mathrm{H}]^{+}$260.1434, found 260.1435.

The spectral data are in accordance with those reported in the literature. ${ }^{8-9}$

\section{2-\{3-[1-(2-Chlorophenyl)ethyl]phenyl $\}$ pyrimidine (3dn)}

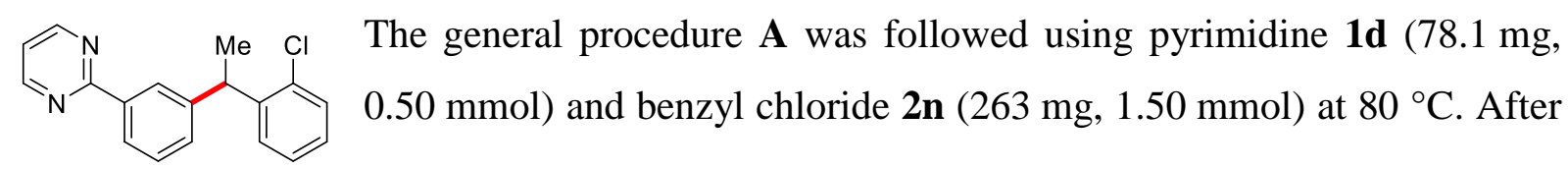


$20 \mathrm{~h}$, purification by column chromatography ( $n$-hexane/EtOAc 10:1) yielded 3dn (76.3 mg, $52 \%)$ as a colorless oil.

${ }^{1} \mathbf{H}-\mathrm{NMR}\left(300 \mathrm{MHz}, \mathrm{CDCl}_{3}\right): \delta=8.80(\mathrm{~d}, J=4.8 \mathrm{~Hz}, 2 \mathrm{H}), 8.39(\mathrm{ddd}, J=1.9,1.7,0.8 \mathrm{~Hz}, 1 \mathrm{H})$, 8.30 (ddd, $J=7.7,1.7,1.4 \mathrm{~Hz}, 1 \mathrm{H}), 7.42(\mathrm{ddd}, J=7.7,7.7,0.8 \mathrm{~Hz}, 1 \mathrm{H}), 7.36$ (dd, $J=7.6$, $1.5 \mathrm{~Hz}, 1 \mathrm{H}), 7.33$ (dddd, $J=7.7,1.9,1.4,0.6 \mathrm{~Hz}, 1 \mathrm{H}), 7.27$ (dd, $J=7.6,2.0 \mathrm{~Hz}, 1 \mathrm{H}), 7.21$ (ddd, $J=7.6,7.5,1.5 \mathrm{~Hz}, 1 \mathrm{H}), 7.17(\mathrm{t}, J=4.8 \mathrm{~Hz}, 1 \mathrm{H}), 7.13(\mathrm{ddd}, J=7.6,7.5,2.0 \mathrm{~Hz}, 1 \mathrm{H}), 4.77(\mathrm{q}$, $J=7.2 \mathrm{~Hz}, 1 \mathrm{H}), 1.70(\mathrm{~d}, J=7.2 \mathrm{~Hz}, 3 \mathrm{H})$.

${ }^{13}$ C-NMR $\left(125 \mathrm{MHz}, \mathrm{CDCl}_{3}\right): \delta=164.7\left(\mathrm{C}_{\mathrm{q}}\right), 157.1(\mathrm{CH}), 145.2\left(\mathrm{C}_{\mathrm{q}}\right), 143.5\left(\mathrm{C}_{\mathrm{q}}\right), 137.6\left(\mathrm{C}_{\mathrm{q}}\right)$, $133.8\left(\mathrm{C}_{\mathrm{q}}\right), 130.3(\mathrm{CH}), 129.5(\mathrm{CH}), 128.6(\mathrm{CH}), 128.6(\mathrm{CH}), 127.4(\mathrm{CH}), 127.3(\mathrm{CH}), 126.9$ $(\mathrm{CH}), 126.1(\mathrm{CH}), 118.9(\mathrm{CH}), 41.2(\mathrm{CH}), 21.2\left(\mathrm{CH}_{3}\right)$.

IR (ATR): $\tilde{v}=2968,1567,1553,1422,1407,1034,793,757,699,635 \mathrm{~cm}^{-1}$.

MS (EI) $m / z$ (relative intensity): $296(31)\left[\mathrm{M}\left({ }^{37} \mathrm{Cl}\right)\right]^{+}, 294(89)\left[\mathrm{M}\left({ }^{35} \mathrm{Cl}\right)\right]^{+}, 281(35)\left[\mathrm{M}\left({ }^{37} \mathrm{Cl}\right)-\right.$ $\mathrm{Me}]^{+}, 279(100)\left[\mathrm{M}\left({ }^{35} \mathrm{Cl}\right)-\mathrm{Me}\right]^{+}, 259(24)[\mathrm{M}-\mathrm{Cl}]^{+}, 243$ (60) $[\mathrm{M}-\mathrm{Me}-\mathrm{Cl}]^{+}, 190$ (26), 165 (12), 129 (10), 122 (10), 103 (14), 77 (13).

HR-MS (ESI): $m / z$ calcd for $\mathrm{C}_{18} \mathrm{H}_{16}{ }^{35} \mathrm{ClN}_{2}{ }^{+}[\mathrm{M}+\mathrm{H}]^{+}$295.0997, found 295.0999.

\section{2-[3-(1-Phenylethyl)phenyl]-4,5-dihydrooxazole (3fl)}

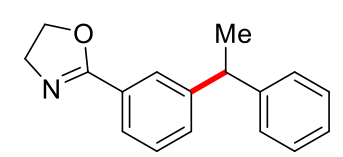

The general procedure A was followed using oxazoline $\mathbf{1 f}(73.6 \mathrm{mg}$, $0.50 \mathrm{mmol})$ and benzyl chloride $21(211 \mathrm{mg}, 1.50 \mathrm{mmol})$. After $20 \mathrm{~h}$, purification by column chromatography ( $n$-hexane/EtOAc $3: 1)$ and recycling preparative HPLC yielded $\mathbf{3 f l}(65.6 \mathrm{mg}, 52 \%)$ as a colorless oil.

${ }^{1} \mathbf{H}-\mathbf{N M R}\left(400 \mathrm{MHz}, \mathrm{CDCl}_{3}\right): \delta=7.89-7.87(\mathrm{~m}, 1 \mathrm{H}), 7.79-7.74(\mathrm{~m}, 1 \mathrm{H}), 7.35-7.26(\mathrm{~m}, 4 \mathrm{H})$, 7.24-7.15 (m, 3H), $4.42(\mathrm{td}, J=9.5,0.6 \mathrm{~Hz}, 2 \mathrm{H}), 4.19(\mathrm{q}, J=7.3 \mathrm{~Hz}, 1 \mathrm{H}), 4.05(\mathrm{t}, J=9.5 \mathrm{~Hz}$, $2 \mathrm{H}), 1.66(\mathrm{~d}, J=7.3 \mathrm{~Hz}, 3 \mathrm{H})$.

${ }^{13} \mathrm{C}-\mathrm{NMR}\left(100 \mathrm{MHz}, \mathrm{CDCl}_{3}\right): \delta=164.8\left(\mathrm{C}_{\mathrm{q}}\right), 146.6\left(\mathrm{C}_{\mathrm{q}}\right), 145.9\left(\mathrm{C}_{\mathrm{q}}\right), 130.7(\mathrm{CH}), 128.4(\mathrm{CH})$, $127.8\left(\mathrm{C}_{\mathrm{q}}\right), 127.6(\mathrm{CH}), 127.2(\mathrm{CH}), 126.1(\mathrm{CH}), 126.0(\mathrm{CH}), 67.5\left(\mathrm{CH}_{2}\right), 54.9\left(\mathrm{CH}_{2}\right), 44.7$ $(\mathrm{CH}), 21.7\left(\mathrm{CH}_{3}\right)$.

IR (ATR): $\tilde{v}=2967,2875,1647,1450,1356,1264,1178,1066,947,700 \mathrm{~cm}^{-1}$.

MS (EI) $m / z$ (relative intensity): 251 (69) [M] $]^{+}, 236$ (100) [M-Me] $]^{+}, 221$ (12), 193 (40), 192 (40), 178 (15), 165 (68), 103 (19), 89 (9), 77 (16).

HR-MS (EI): $m / z$ calcd for $\mathrm{C}_{17} \mathrm{H}_{17} \mathrm{NO}^{+}[\mathrm{M}]^{+} 251.1305$, found 251.1316. 


\section{9-iso-Propyl-6-[3-(1-phenylethyl)phenyl]-9H-purine (3hl)}

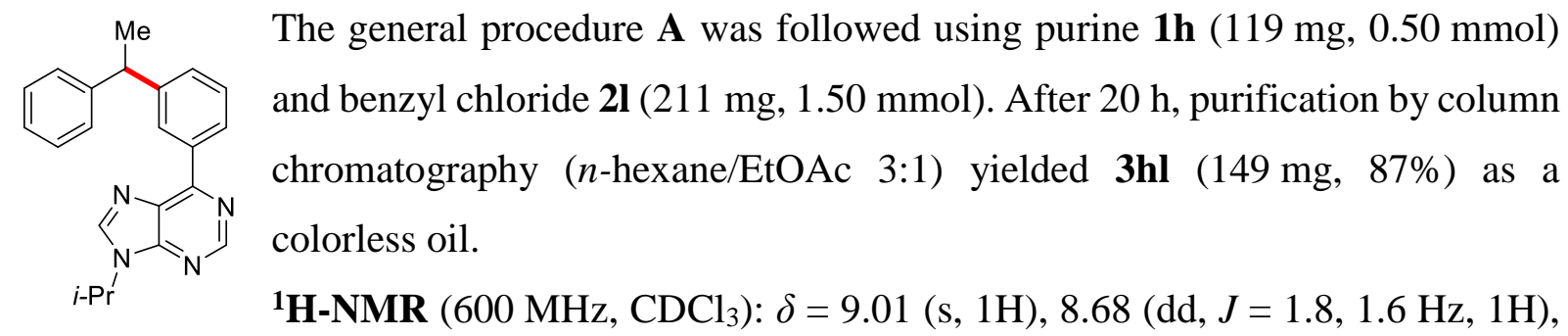

$8.66(\mathrm{ddd}, J=7.7,1.6,1.4 \mathrm{~Hz}, 1 \mathrm{H}), 8.18(\mathrm{~s}, 1 \mathrm{H}), 7.47$ (dd, $J=7.7,7.7 \mathrm{~Hz}, 1 \mathrm{H}), 7.35-7.33$ (m, 1H), 7.31-7.27 (m, 4H), 7.19-7.16 (m, 1H), 4.98 (hept, $J=6.8 \mathrm{~Hz}, 1 \mathrm{H}), 4.32$ (q, $J=7.2 \mathrm{~Hz}$, $1 \mathrm{H}), 1.74(\mathrm{~d}, J=7.2 \mathrm{~Hz}, 3 \mathrm{H}), 1.67(\mathrm{~d}, J=6.8 \mathrm{~Hz}, 6 \mathrm{H})$.

${ }^{13}$ C-NMR $\left(125 \mathrm{MHz}, \mathrm{CDCl}_{3}\right): \delta=154.9\left(\mathrm{C}_{\mathrm{q}}\right), 152.0\left(\mathrm{C}_{\mathrm{q}}\right), 151.9(\mathrm{CH}), 146.7\left(\mathrm{C}_{\mathrm{q}}\right), 146.1\left(\mathrm{C}_{\mathrm{q}}\right)$, $141.8(\mathrm{CH}), 135.8\left(\mathrm{C}_{\mathrm{q}}\right), 131.5\left(\mathrm{C}_{\mathrm{q}}\right), 130.2(\mathrm{CH}), 128.6(\mathrm{CH}), 128.6(\mathrm{CH}), 128.3(\mathrm{CH}), 127.9$ $(\mathrm{CH}), 127.6(\mathrm{CH}), 125.9(\mathrm{CH}), 47.3(\mathrm{CH}), 45.0(\mathrm{CH}), 22.7\left(\mathrm{CH}_{3}\right), 22.0\left(\mathrm{CH}_{3}\right)$.

IR (ATR): $\tilde{v}=2973,1566,1494,1446,1324,1218,799,700,646,573 \mathrm{~cm}^{-1}$.

MS (EI) $m / z$ (relative intensity): $342(81)[\mathrm{M}]^{+}, 341(63)[\mathrm{M}-\mathrm{H}]^{+}, 327$ (34) [M-Me] $]^{+}, 299$ (95) $[\mathrm{M}-i \mathrm{Pr}]^{+}, 285$ (100) $[\mathrm{M}-i \mathrm{Pr}-\mathrm{Me}]^{+}, 165$ (14), 142 (8).

HR-MS (EI): $m / z$ calcd for $\mathrm{C}_{22} \mathrm{H}_{22} \mathrm{~N}_{4}{ }^{+}[\mathrm{M}]^{+} 342.1839$, found 342.1832.

\section{5,5-Difluoro-10-\{4-[3-(9-iso-propyl-9H-purin-6-yl)benzyl]phenyl\}-1,3,7,9-tetramethyl-} $5 H-4 \lambda^{4}, 5 \lambda^{4}$-dipyrrolo[1,2-c:2',1'-f][1,3,2]diazaborinine (6a)

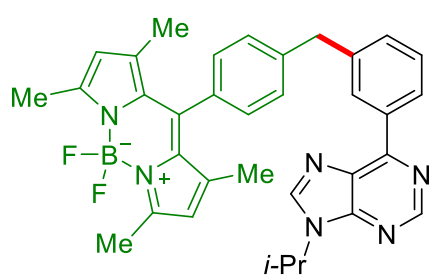

The general procedure $\mathbf{B}$ was followed using purine $\mathbf{1 h}(23.9 \mathrm{mg}$, $0.10 \mathrm{mmol}$ ) and benzyl chloride 5a $(75 \mathrm{mg}, 0.20 \mathrm{mmol})$. After $20 \mathrm{~h}$, purification by column chromatography ( $n$-hexane/EtOAc $7: 3)$ yielded $\mathbf{6 a}(26.1 \mathrm{mg}, 45 \%)$ as an orange solid.

${ }^{1} \mathbf{H}-\mathbf{N M R}\left(600 \mathrm{MHz}, \mathrm{CDCl}_{3}\right): \delta=9.00(\mathrm{~s}, 1 \mathrm{H}), 8.71(\mathrm{ddd}, J=7.8,1.7,1.3 \mathrm{~Hz}, 1 \mathrm{H}), 8.57$ (dd, $J$ $=2.0,1.7 \mathrm{~Hz}, 1 \mathrm{H}), 8.18(\mathrm{~s}, 1 \mathrm{H}), 7.50(\mathrm{dd}, J=7.8,7.7 \mathrm{~Hz}, 1 \mathrm{H}), 7.37(\mathrm{~d}, J=8.1 \mathrm{~Hz}, 2 \mathrm{H}), 7.31$ (ddd, $J=7.7,2.0,1.3 \mathrm{~Hz}, 1 \mathrm{H}), 7.20$ (d, $J=8.1 \mathrm{~Hz}, 2 \mathrm{H}$ ), 5.96 (s, 2H), 4.99 (hept, $J=6.8 \mathrm{~Hz}$, 1H), 4.22 (s, 2H), 2.54 (s, 6H), $1.68(\mathrm{~d}, J=6.8 \mathrm{~Hz}, 6 \mathrm{H}), 1.39$ (s, 6H).

${ }^{13}$ C-NMR (125 MHz, $\left.\mathrm{CDCl}_{3}\right): \delta=155.1\left(\mathrm{C}_{\mathrm{q}}\right), 154.6\left(\mathrm{C}_{\mathrm{q}}\right), 152.0\left(\mathrm{C}_{\mathrm{q}}\right), 151.9(\mathrm{CH}), 143.1\left(\mathrm{C}_{\mathrm{q}}\right)$, $141.8\left(\mathrm{C}_{\mathrm{q}}\right.$ and $\left.\mathrm{CH}\right), 141.8\left(\mathrm{C}_{\mathrm{q}}\right), 141.0\left(\mathrm{C}_{\mathrm{q}}\right), 136.0\left(\mathrm{C}_{\mathrm{q}}\right), 132.7\left(\mathrm{C}_{\mathrm{q}}\right), 131.4\left(\mathrm{C}_{\mathrm{q}}\right), 131.2(\mathrm{CH}), 129.8$ $(\mathrm{CH}), 129.7(\mathrm{CH}), 128.8(\mathrm{CH}), 128.1(\mathrm{CH}), 128.0(\mathrm{CH}), 121.0(\mathrm{CH}), 47.3(\mathrm{CH}), 41.7\left(\mathrm{CH}_{2}\right)$, $22.7\left(\mathrm{CH}_{3}\right), 14.6\left(\mathrm{CH}_{3}\right), 14.5\left(\mathrm{CH}_{3}\right)$.

${ }^{11}$ B-NMR $\left(128 \mathrm{MHz}, \mathrm{CDCl}_{3}\right): \delta=0.8\left(\mathrm{t},{ }^{1} J_{\mathrm{B}-\mathrm{F}}=33.1 \mathrm{~Hz}\right)$.

${ }^{19}$ F-NMR $\left(376 \mathrm{MHz}, \mathrm{CDCl}_{3}\right): \delta=-146.3\left(\mathrm{q},{ }^{1} J_{\mathrm{F}-\mathrm{B}}=33.1 \mathrm{~Hz}\right)$.

IR (ATR): $\tilde{v}=2925,1542,1508,1306,1191,1155,976,731,703,647 \mathrm{~cm}^{-1}$. 
m.p.: $170-172{ }^{\circ} \mathrm{C}$ (decomp.).

MS (ESI) $m / z$ (relative intensity): $1150(5)[2 \mathrm{M}+\mathrm{H}]^{+}, 597(4)[\mathrm{M}+\mathrm{Na}]^{+}, 575(100)[\mathrm{M}+\mathrm{H}]^{+}, 177$ (6), 117 (19).

HR-MS (ESI): $m / z$ calcd for $\mathrm{C}_{34} \mathrm{H}_{34} \mathrm{BF}_{2} \mathrm{~N}_{6}{ }^{+}[\mathrm{M}+\mathrm{H}]^{+}$575.2906, found 575.2903.

UV-Vis: $\lambda_{\max }\left(1.0 \mathrm{mg} / \mathrm{L}\right.$ in $\left.\mathrm{CHCl}_{3}\right)=502 \mathrm{~nm}$.

Em: $\lambda_{\max }\left(1.0 \mathrm{mg} / \mathrm{L}\right.$ in $\left.\mathrm{CHCl}_{3}\right)=514 \mathrm{~nm}$.

$\left\{(3 \mathrm{a} R, 4 R, 6 R, 6 \mathrm{a} R)-6-\left\{6-\left\{3-\left[4-\left(5,5-D i f l u o r o-1,3,7,9-t e t r a m e t h y l-5 H-4 \lambda^{4}, 5 \lambda^{4}\right.\right.\right.\right.\right.$-dipyrrolo[1,2$\left.c: 2 ', 1^{\prime}-f\right][1,3,2]$ diazaborinin-10-yl)benzyl]phenyl\}-9H-purin-9-yl\}-2,2-

dimethyltetrahydrofuro[3,4- $d][1,3]$ dioxol-4-yl\}methyl diethyl phosphate (6b)

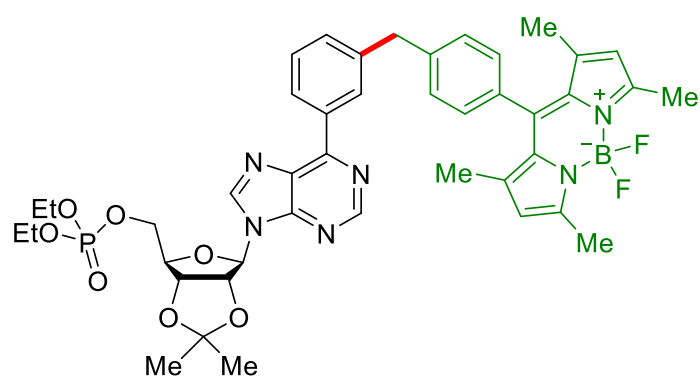

The general procedure B was followed using purine $1 \mathrm{~m}(50.5 \mathrm{mg}, 0.10 \mathrm{mmol})$ and benzyl chloride 5a (75 mg, $0.20 \mathrm{mmol})$. After $20 \mathrm{~h}$, purification by column chromatography ( $n$-hexane/EtOAc $3: 7)$ yielded $6 \mathbf{b}(12.9 \mathrm{mg}, 15 \%)$ as an orange solid.

${ }^{1} \mathbf{H}-\mathbf{N M R}\left(300 \mathrm{MHz}, \mathrm{CDCl}_{3}\right): \delta=9.01(\mathrm{~s}, 1 \mathrm{H}), 8.71(\mathrm{~d}, J=7.8 \mathrm{~Hz}, 1 \mathrm{H}), 8.57$ (s, 1H), 8.29 (s, 1H), $7.50(\mathrm{dd}, J=7.8,7.8 \mathrm{~Hz}, 1 \mathrm{H}), 7.37$ (d, $J=8.0 \mathrm{~Hz}, 2 \mathrm{H}), 7.32(\mathrm{~d}, J=7.8 \mathrm{~Hz}, 1 \mathrm{H}), 7.21(\mathrm{~d}$, $J=8.0 \mathrm{~Hz}, 2 \mathrm{H}), 6.27(\mathrm{~d}, J=2.6 \mathrm{~Hz}, 1 \mathrm{H}), 5.97(\mathrm{~s}, 2 \mathrm{H}), 5.45(\mathrm{dd}, J=6.3,2.6 \mathrm{~Hz}, 1 \mathrm{H}), 5.13(\mathrm{dd}$, $J=6.3,3.0 \mathrm{~Hz}, 1 \mathrm{H}), 4.58-4.51(\mathrm{~m}, 1 \mathrm{H}), 4.30(\mathrm{ddd}, J=10.8,6.3,4.4 \mathrm{~Hz}, 1 \mathrm{H}), 4.26-4.17(\mathrm{~m}$, $1 \mathrm{H}), 4.22(\mathrm{~s}, 2 \mathrm{H}), 4.13-3.98(\mathrm{~m}, 4 \mathrm{H}), 2.54(\mathrm{~s}, 6 \mathrm{H}), 1.66(\mathrm{~s}, 3 \mathrm{H}), 1.42(\mathrm{~s}, 3 \mathrm{H}), 1.40(\mathrm{~s}, 6 \mathrm{H}), 1.31-$ $1.22(\mathrm{~m}, 6 \mathrm{H})$.

${ }^{13} \mathrm{C}-\mathrm{NMR}\left(125 \mathrm{MHz}, \mathrm{CDCl}_{3}\right): \delta=155.3\left(\mathrm{C}_{\mathrm{q}}\right), 155.1\left(\mathrm{C}_{\mathrm{q}}\right), 152.5(\mathrm{CH}), 151.7\left(\mathrm{C}_{\mathrm{q}}\right), 143.2(\mathrm{CH})$, $143.1\left(\mathrm{C}_{\mathrm{q}}\right), 141.8\left(\mathrm{C}_{\mathrm{q}}\right), 141.8\left(\mathrm{C}_{\mathrm{q}}\right), 141.2\left(\mathrm{C}_{\mathrm{q}}\right), 135.7\left(\mathrm{C}_{\mathrm{q}}\right), 132.8\left(\mathrm{C}_{\mathrm{q}}\right), 131.7\left(\mathrm{C}_{\mathrm{q}}\right), 131.6(\mathrm{CH})$, $131.5\left(\mathrm{C}_{\mathrm{q}}\right), 129.9(\mathrm{CH}), 129.8(\mathrm{CH}), 128.9(\mathrm{CH}), 128.3(\mathrm{CH}), 128.1(\mathrm{CH}), 121.1(\mathrm{CH}), 114.8$ $\left(\mathrm{C}_{\mathrm{q}}\right), 91.1(\mathrm{CH}), 85.2\left(\mathrm{~d},{ }^{3} J_{\mathrm{C}-\mathrm{P}}=8 \mathrm{~Hz}, \mathrm{CH}\right), 84.2(\mathrm{CH}), 81.3(\mathrm{CH}), 66.6\left(\mathrm{~d},{ }^{2} J_{\mathrm{C}-\mathrm{P}}=6 \mathrm{~Hz}, \mathrm{CH}_{2}\right)$, $64.1\left(\mathrm{~d},{ }^{2} J_{\mathrm{C}-\mathrm{P}}=6 \mathrm{~Hz}, \mathrm{CH}_{2}\right), 64.1\left(\mathrm{~d},{ }^{2} J_{\mathrm{C}-\mathrm{P}}=6 \mathrm{~Hz}, \mathrm{CH}_{2}\right), 41.6\left(\mathrm{CH}_{2}\right), 27.2\left(\mathrm{CH}_{3}\right), 25.3\left(\mathrm{CH}_{3}\right)$, $16.1\left(\mathrm{~d},{ }^{3} J_{\mathrm{C}-\mathrm{P}}=7 \mathrm{~Hz}, \mathrm{CH}_{3}\right), 16.1\left(\mathrm{~d},{ }^{3} J_{\mathrm{C}-\mathrm{P}}=7 \mathrm{~Hz}, \mathrm{CH}_{3}\right), 14.6\left(\mathrm{t},{ }^{4} J_{\mathrm{C}-\mathrm{F}}=2 \mathrm{~Hz}, \mathrm{CH}_{3}\right), 14.4\left(\mathrm{CH}_{3}\right)$. ${ }^{11} \mathbf{B}-\mathrm{NMR}\left(128 \mathrm{MHz}, \mathrm{CDCl}_{3}\right): \delta=0.8\left(\mathrm{t},{ }^{1} \mathrm{~J}_{\mathrm{B}-\mathrm{F}}=33.0 \mathrm{~Hz}\right)$.

${ }^{19}$ F-NMR $\left(376 \mathrm{MHz}, \mathrm{CDCl}_{3}\right): \delta=-146.3\left(\mathrm{q},{ }^{1} J_{\mathrm{F}-\mathrm{B}}=33.0 \mathrm{~Hz}\right)$.

${ }^{31} \mathbf{P}-\mathbf{N M R}\left(162 \mathrm{MHz}, \mathrm{CDCl}_{3}\right): \delta=(-0.8)-(-1.2)(\mathrm{m})$.

IR (ATR): $\tilde{v}=2927,1543,1509,1306,1192,1155,1020,972,752,643 \mathrm{~cm}^{-1}$.

MS (ESI) $m / z$ (relative intensity): $863(100)[\mathrm{M}+\mathrm{Na}]^{+}, 841(13)[\mathrm{M}+\mathrm{H}]^{+}, 821(16)[\mathrm{M}-\mathrm{F}]^{+}$.

HR-MS (ESI): $m / z$ calcd for $\mathrm{C}_{43} \mathrm{H}_{48} \mathrm{BF}_{2} \mathrm{~N}_{6} \mathrm{O}_{7} \mathrm{PNa}^{+}[\mathrm{M}+\mathrm{Na}]^{+}$863.3283, found 863.3273. 
UV-Vis: $\lambda_{\max }\left(1.0 \mathrm{mg} / \mathrm{L}\right.$ in $\left.\mathrm{CHCl}_{3}\right)=502 \mathrm{~nm}$.

$\mathbf{E}_{\mathbf{m}}: \boldsymbol{\lambda}_{\max }\left(1.0 \mathrm{mg} / \mathrm{L}\right.$ in $\left.\mathrm{CHCl}_{3}\right)=514 \mathrm{~nm}$.

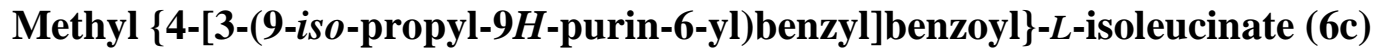

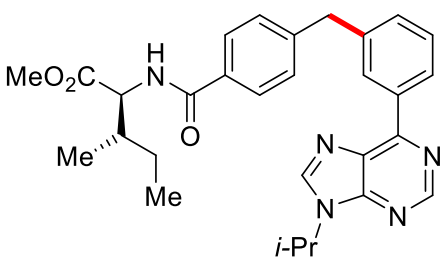

The general procedure B was followed using purine 1h $(59.6 \mathrm{mg}$, $0.25 \mathrm{mmol}$ ) and benzyl chloride $5 \mathbf{b}(149 \mathrm{mg}, 0.50 \mathrm{mmol})$. After $20 \mathrm{~h}$, purification by column chromatography ( $n$-hexane/EtOAc 1:1) yielded $\mathbf{6 c}(92.4 \mathrm{mg}, 74 \%)$ as a viscous colorless oil.

${ }^{1} \mathbf{H}-\mathrm{NMR}\left(600 \mathrm{MHz}, \mathrm{CDCl}_{3}\right): \delta=9.00$ (s, 1H), 8.68 (ddd, $\left.J=7.8,1.6,1.4 \mathrm{~Hz}, 1 \mathrm{H}\right), 8.60$ (dd, $J$ $=1.8,1.6 \mathrm{~Hz}, 1 \mathrm{H}), 8.18(\mathrm{~s}, 1 \mathrm{H}), 7.72(\mathrm{~d}, J=8.2 \mathrm{~Hz}, 2 \mathrm{H}), 7.48(\mathrm{dd}, J=7.8,7.7 \mathrm{~Hz}, 1 \mathrm{H}), 7.32$ $(\mathrm{d}, J=8.2 \mathrm{~Hz}, 2 \mathrm{H}), 7.31-7.27(\mathrm{~m}, 1 \mathrm{H}), 6.61(\mathrm{~d}, J=8.5 \mathrm{~Hz}, 1 \mathrm{H}), 4.97$ (hept, $J=6.8 \mathrm{~Hz}, 1 \mathrm{H})$, $4.79(\mathrm{dd}, J=8.5,4.9 \mathrm{~Hz}, 1 \mathrm{H}), 4.16(\mathrm{~s}, 2 \mathrm{H}), 3.74(\mathrm{~s}, 3 \mathrm{H}), 2.06-1.85(\mathrm{~m}, 1 \mathrm{H}), 1.66$ (d, $J=6.8 \mathrm{~Hz}$, $6 \mathrm{H}), 1.58-1.42(\mathrm{~m}, 1 \mathrm{H}), 1.31-1.13(\mathrm{~m}, 1 \mathrm{H}), 0.94(\mathrm{~d}, J=6.5 \mathrm{~Hz}, 3 \mathrm{H}), 0.94(\mathrm{t}, J=7.3 \mathrm{~Hz}, 3 \mathrm{H})$. ${ }^{13} \mathrm{C}-\mathrm{NMR}\left(125 \mathrm{MHz}, \mathrm{CDCl}_{3}\right): \delta=172.4\left(\mathrm{C}_{\mathrm{q}}\right), 166.8\left(\mathrm{C}_{\mathrm{q}}\right), 154.5\left(\mathrm{C}_{\mathrm{q}}\right), 152.0\left(\mathrm{C}_{\mathrm{q}}\right), 151.9(\mathrm{CH})$, 145.0 $\left(\mathrm{C}_{\mathrm{q}}\right), 141.9(\mathrm{CH}), 140.5\left(\mathrm{C}_{\mathrm{q}}\right), 136.0\left(\mathrm{C}_{\mathrm{q}}\right), 131.9\left(\mathrm{C}_{\mathrm{q}}\right), 131.4\left(\mathrm{C}_{\mathrm{q}}\right), 131.4(\mathrm{CH}), 129.9(\mathrm{CH})$, $129.1(\mathrm{CH}), 128.8(\mathrm{CH}), 128.1(\mathrm{CH}), 127.2(\mathrm{CH}), 56.7(\mathrm{CH}), 52.1\left(\mathrm{CH}_{3}\right), 47.3(\mathrm{CH}), 41.9$ $\left(\mathrm{CH}_{2}\right), 38.3(\mathrm{CH}), 25.4\left(\mathrm{CH}_{2}\right), 22.6\left(\mathrm{CH}_{3}\right), 15.5\left(\mathrm{CH}_{3}\right), 11.7\left(\mathrm{CH}_{3}\right)$.

IR (ATR): $\tilde{v}=3311,2966,1737,1646,1569,1496,1326,1218,733,647 \mathrm{~cm}^{-1}$.

MS (EI) $\mathrm{m} / z$ (relative intensity): 499 (9) $[\mathrm{M}]^{+}, 440$ (53) $\left[\mathrm{M}-\mathrm{CO}_{2} \mathrm{Me}^{+}, 355\right.$ (100) [M-(H-Leu$\mathrm{OMe})]^{+}, 328$ (19), 285 (32), 156 (12).

HR-MS (EI): $m / z$ calcd for $\mathrm{C}_{29} \mathrm{H}_{33} \mathrm{~N}_{5} \mathrm{O}_{3}{ }^{+}[\mathrm{M}]^{+} 499.2578$, found 499.2586.

\section{Methyl}

$\{4-\{3-\{9-\{(3 \mathrm{a} R, 4 R, 6 R, 6 \mathrm{a} R)-6-\{[($ diethoxyphosphoryl $)$ oxy $]$ methyl $\}-2,2-$ dimethyltetrahydrofuro[3,4- $d][1,3]$ dioxol-4-yl\}-9H-purin-6-yl $\}$ benzyl $\}$ benzoyl $\}-L$ valinate (6d)

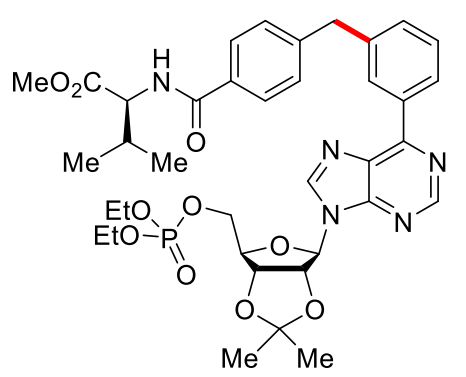

The general procedure $\mathbf{B}$ was followed using purine 1m (126 mg, $0.25 \mathrm{mmol})$ and benzyl chloride $S-5 \mathrm{c}(142 \mathrm{mg}, 0.50 \mathrm{mmol})$. After $20 \mathrm{~h}$, purification by column chromatography ( $n$-hexane/EtOAc 1:3) yielded $\mathbf{6 d}(110 \mathrm{mg}, 59 \%)$ as a viscous colorless oil.

${ }^{1} \mathbf{H}-\mathbf{N M R}\left(600 \mathrm{MHz}, \mathrm{CDCl}_{3}\right): \delta=9.02(\mathrm{~s}, 1 \mathrm{H}), 8.68$ (ddd, $J=7.8$, $1.6,1.4 \mathrm{~Hz}, 1 \mathrm{H}), 8.60(\mathrm{dd}, J=1.8,1.6 \mathrm{~Hz}, 1 \mathrm{H}), 8.29$ (s, 1H), 7.74 (d, $J=8.3 \mathrm{~Hz}, 2 \mathrm{H}), 7.49$ (dd, $J=7.8,7.7 \mathrm{~Hz}, 1 \mathrm{H}), 7.33$ (d, $J=8.3 \mathrm{~Hz}, 2 \mathrm{H}), 7.31$ (ddd, $J=7.7$, 1.8, 1.4 Hz, 1H), $6.58(\mathrm{~d}, J=8.7 \mathrm{~Hz}, 1 \mathrm{H}), 6.26(\mathrm{~d}, J=2.5 \mathrm{~Hz}, 1 \mathrm{H}), 5.45(\mathrm{dd}, J=6.3,2.5 \mathrm{~Hz}$, 1H), $5.13(\mathrm{dd}, J=6.3,3.1 \mathrm{~Hz}, 1 \mathrm{H}), 4.76(\mathrm{dd}, J=8.7,4.9 \mathrm{~Hz}, 1 \mathrm{H}), 4.53(\mathrm{dddd}, J=4.9,4.5,3.1$, 
$1.2 \mathrm{~Hz}, 1 \mathrm{H}), 4.29$ (ddd, $J=11.0,6.2,4.5 \mathrm{~Hz}, 1 \mathrm{H}), 4.21(\mathrm{ddd}, J=11.0,6.9,4.9 \mathrm{~Hz}, 1 \mathrm{H}), 4.17$ (s, 2H), 4.10-4.00 (m, 4H), $3.76(\mathrm{~s}, 3 \mathrm{H}), 2.25(\mathrm{pd}, J=6.9,4.9 \mathrm{~Hz}, 1 \mathrm{H}), 1.65(\mathrm{~s}, 3 \mathrm{H}), 1.41$ (s, $3 \mathrm{H}), 1.27(\mathrm{td}, J=7.1,1.0 \mathrm{~Hz}, 3 \mathrm{H}), 1.24(\mathrm{td}, J=7.1,1.0 \mathrm{~Hz}, 3 \mathrm{H}), 0.99(\mathrm{~d}, J=6.9 \mathrm{~Hz}, 3 \mathrm{H}), 0.96$ $(\mathrm{d}, J=6.9 \mathrm{~Hz}, 3 \mathrm{H})$.

${ }^{13}$ C-NMR $\left(125 \mathrm{MHz}, \mathrm{CDCl}_{3}\right): \delta=172.5\left(\mathrm{C}_{\mathrm{q}}\right), 166.9\left(\mathrm{C}_{\mathrm{q}}\right), 155.0\left(\mathrm{C}_{\mathrm{q}}\right), 152.4(\mathrm{CH}), 151.6\left(\mathrm{C}_{\mathrm{q}}\right)$, $144.9\left(\mathrm{C}_{\mathrm{q}}\right), 143.1(\mathrm{CH}), 140.7\left(\mathrm{C}_{\mathrm{q}}\right), 135.7\left(\mathrm{C}_{\mathrm{q}}\right), 132.0\left(\mathrm{C}_{\mathrm{q}}\right), 131.6\left(\mathrm{C}_{\mathrm{q}}\right.$ and $\left.\mathrm{CH}\right), 130.0(\mathrm{CH})$, $129.1(\mathrm{CH}), 128.9(\mathrm{CH}), 128.2(\mathrm{CH}), 127.3(\mathrm{CH}), 114.7\left(\mathrm{C}_{\mathrm{q}}\right), 91.1(\mathrm{CH}), 85.3\left(\mathrm{~d},{ }^{3} J_{\mathrm{C}-\mathrm{P}}=8 \mathrm{~Hz}\right.$, $\mathrm{CH}), 84.2(\mathrm{CH}), 81.4(\mathrm{CH}), 66.6\left(\mathrm{~d},{ }^{2} J_{\mathrm{C}-\mathrm{P}}=6 \mathrm{~Hz}, \mathrm{CH}_{2}\right), 64.0\left(\mathrm{~d},{ }^{2} J_{\mathrm{C}-\mathrm{P}}=6 \mathrm{~Hz}, \mathrm{CH}_{2}\right), 63.9(\mathrm{~d}$, $\left.{ }^{2} J_{\mathrm{C}-\mathrm{P}}=6 \mathrm{~Hz}, \mathrm{CH}_{2}\right), 57.4(\mathrm{CH}), 52.2\left(\mathrm{CH}_{3}\right), 41.9\left(\mathrm{CH}_{2}\right), 31.7(\mathrm{CH}), 27.3\left(\mathrm{CH}_{3}\right), 25.4\left(\mathrm{CH}_{3}\right), 19.1$ $\left(\mathrm{CH}_{3}\right), 18.0\left(\mathrm{CH}_{3}\right), 16.1\left(\mathrm{~d},{ }^{3} \mathrm{~J}_{\mathrm{C}-\mathrm{P}}=7 \mathrm{~Hz}, \mathrm{CH}_{3}\right)$.

${ }^{31} \mathbf{P}\left\{{ }^{1} \mathbf{H}\right\}$-NMR $\left(121 \mathrm{MHz}, \mathrm{CDCl}_{3}\right): \delta=-1.1$ (s).

IR (ATR): $\tilde{v}=3327,2981,1739,1648,1569,1498,1260,1209,1020,733 \mathrm{~cm}^{-1}$.

MS (ESI) $m / z$ (relative intensity): $774(33)[\mathrm{M}+\mathrm{Na}]^{+}, 752(100)[\mathrm{M}+\mathrm{H}]^{+}$.

HR-MS (ESI): $m / z$ calcd for $\mathrm{C}_{37} \mathrm{H}_{47} \mathrm{~N}_{5} \mathrm{O}_{10} \mathrm{P}^{+}[\mathrm{M}+\mathrm{H}]^{+} 752.3055$, found 752.3055 .

\section{Methyl \{4-[3-(9-iso-propyl-9H-purin-6-yl)benzyl]benzoyl $\}-L$-valinate $(S$-6e)}

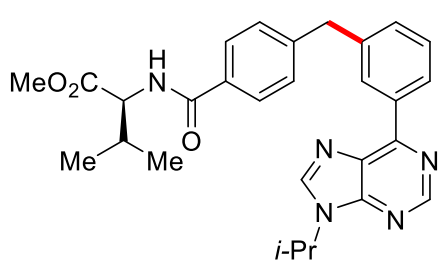

The general procedure B was followed using purine $1 \mathbf{h}(59.6 \mathrm{mg}$, $0.25 \mathrm{mmol}$ ) and benzyl chloride $S-5 \mathrm{c}(142 \mathrm{mg}, 0.50 \mathrm{mmol})$. After $20 \mathrm{~h}$, purification by column chromatography ( $n$-hexane/EtOAc $1: 1)$ yielded $s-6 e(85.3 \mathrm{mg}, 70 \%)$ as a white solid.

${ }^{1} \mathbf{H}-\mathbf{N M R}\left(600 \mathrm{MHz}, \mathrm{CDCl}_{3}\right): \delta=9.00(\mathrm{~s}, 1 \mathrm{H}), 8.68(\mathrm{ddd}, J=7.8,1.6,1.2 \mathrm{~Hz}, 1 \mathrm{H}), 8.60(\mathrm{dd}, J$ $=1.8,1.6 \mathrm{~Hz}, 1 \mathrm{H}), 8.18(\mathrm{~s}, 1 \mathrm{H}), 7.73(\mathrm{~d}, J=8.4 \mathrm{~Hz}, 2 \mathrm{H}), 7.49(\mathrm{dd}, J=7.8,7.7 \mathrm{~Hz}, 1 \mathrm{H}), 7.33$ $(\mathrm{d}, J=8.4 \mathrm{~Hz}, 2 \mathrm{H}), 7.30(\mathrm{ddd}, J=7.7,1.8,1.2 \mathrm{~Hz}, 1 \mathrm{H}), 6.57$ (d, $J=8.7 \mathrm{~Hz}, 1 \mathrm{H}), 4.98$ (hept, $J$ $=6.8 \mathrm{~Hz}, 1 \mathrm{H}), 4.76(\mathrm{dd}, J=8.7,4.9 \mathrm{~Hz}, 1 \mathrm{H}), 4.17(\mathrm{~s}, 2 \mathrm{H}), 3.75(\mathrm{~s}, 3 \mathrm{H}), 2.25$ (heptd, $J=6.9$, $4.9 \mathrm{~Hz}, 1 \mathrm{H}), 1.67(\mathrm{~d}, J=6.8 \mathrm{~Hz}, 6 \mathrm{H}), 0.99(\mathrm{~d}, J=6.9 \mathrm{~Hz}, 3 \mathrm{H}), 0.96(\mathrm{~d}, J=6.9 \mathrm{~Hz}, 3 \mathrm{H})$. ${ }^{13}$ C-NMR $\left(125 \mathrm{MHz}, \mathrm{CDCl}_{3}\right): \delta=172.5\left(\mathrm{C}_{\mathrm{q}}\right), 167.0\left(\mathrm{C}_{\mathrm{q}}\right), 154.5\left(\mathrm{C}_{\mathrm{q}}\right), 152.0\left(\mathrm{C}_{\mathrm{q}}\right), 151.9(\mathrm{CH})$, 145.0 $\left(\mathrm{C}_{\mathrm{q}}\right), 141.9(\mathrm{CH}), 140.6\left(\mathrm{C}_{\mathrm{q}}\right), 136.1\left(\mathrm{C}_{\mathrm{q}}\right), 132.0\left(\mathrm{C}_{\mathrm{q}}\right), 131.4\left(\mathrm{C}_{\mathrm{q}}\right), 131.4(\mathrm{CH}), 129.9(\mathrm{CH})$, $129.1(\mathrm{CH}), 128.9(\mathrm{CH}), 128.1(\mathrm{CH}), 127.2(\mathrm{CH}), 57.4(\mathrm{CH}), 52.2\left(\mathrm{CH}_{3}\right), 47.3(\mathrm{CH}), 41.9$ $\left(\mathrm{CH}_{2}\right), 31.7(\mathrm{CH}), 22.6\left(\mathrm{CH}_{3}\right), 19.1\left(\mathrm{CH}_{3}\right), 18.0\left(\mathrm{CH}_{3}\right)$.

IR (ATR): $\tilde{v}=3321,2965,1737,1644,1568,1495,1325,1214,783,646 \mathrm{~cm}^{-1}$. m.p.: $62-64{ }^{\circ} \mathrm{C}$.

MS (EI) $m / z$ (relative intensity): 485 (14) $[\mathrm{M}]^{+}, 453$ (8) $[\mathrm{M}-\mathrm{OMe}]^{+}, 426$ (49) $\left[\mathrm{M}-\mathrm{CO}_{2} \mathrm{Me}\right]^{+}$, 355 (100) [M-(H-Val-OMe)] , 328 (23), 285 (34), 165 (7), 156 (13), 142 (8), 43 (16).

HR-MS (EI): $m / z$ calcd for $\mathrm{C}_{28} \mathrm{H}_{31} \mathrm{~N}_{5} \mathrm{O}_{3}{ }^{+}[\mathrm{M}]^{+}$485.2421, found 485.2409. 


\section{Methyl \{4-[3-(9-iso-propyl-9H-purin-6-yl)benzyl]benzoyl $\}-D$-valinate $(R$-6e)}

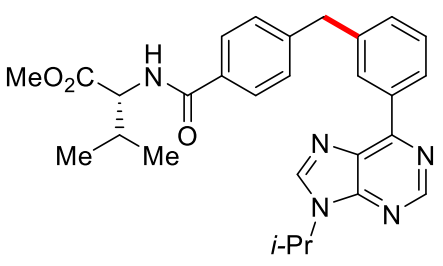

The general procedure B was followed using purine $\mathbf{1 h}(59.6 \mathrm{mg}$, $0.25 \mathrm{mmol})$ and benzyl chloride $R-5 \mathrm{c}(142 \mathrm{mg}, 0.50 \mathrm{mmol})$. After $20 \mathrm{~h}$, purification by column chromatography ( $n$-hexane/EtOAc $1: 1)$ yielded $R-6 \mathbf{e}(92.9 \mathrm{mg}, 77 \%)$ as a viscous colorless oil.

${ }^{1} \mathbf{H}-\mathbf{N M R}\left(600 \mathrm{MHz}, \mathrm{CDCl}_{3}\right): \delta=9.01(\mathrm{~s}, 1 \mathrm{H}), 8.68(\mathrm{ddd}, J=7.8,1.5,1.3 \mathrm{~Hz}, 1 \mathrm{H}), 8.61(\mathrm{dd}, J$ $=1.7,1.5 \mathrm{~Hz}, 1 \mathrm{H}), 8.18(\mathrm{~s}, 1 \mathrm{H}), 7.73(\mathrm{~d}, J=8.5 \mathrm{~Hz}, 2 \mathrm{H}), 7.49(\mathrm{dd}, J=7.8,7.7 \mathrm{~Hz}, 1 \mathrm{H}), 7.33$ $(\mathrm{d}, J=8.5 \mathrm{~Hz}, 2 \mathrm{H}), 7.30(\mathrm{ddd}, J=7.7,1.7,1.3 \mathrm{~Hz}, 1 \mathrm{H}), 6.57$ (d, $J=8.7 \mathrm{~Hz}, 1 \mathrm{H}), 4.98$ (hept, $J$ $=6.8 \mathrm{~Hz}, 1 \mathrm{H}), 4.76(\mathrm{dd}, J=8.7,4.9 \mathrm{~Hz}, 1 \mathrm{H}), 4.18(\mathrm{~s}, 2 \mathrm{H}), 3.76(\mathrm{~s}, 3 \mathrm{H}), 2.25(\mathrm{pd}, J=6.9$, $4.9 \mathrm{~Hz}, 1 \mathrm{H}), 1.67(\mathrm{~d}, J=6.8 \mathrm{~Hz}, 6 \mathrm{H}), 0.99(\mathrm{~d}, J=6.9 \mathrm{~Hz}, 3 \mathrm{H}), 0.96(\mathrm{~d}, J=6.9 \mathrm{~Hz}, 3 \mathrm{H})$.

${ }^{13}$ C-NMR (125 MHz, $\left.\mathrm{CDCl}_{3}\right): \delta=172.5\left(\mathrm{C}_{\mathrm{q}}\right), 167.0\left(\mathrm{C}_{\mathrm{q}}\right), 154.5\left(\mathrm{C}_{\mathrm{q}}\right), 152.0\left(\mathrm{C}_{\mathrm{q}}\right), 151.9(\mathrm{CH})$, $145.0\left(\mathrm{C}_{\mathrm{q}}\right), 141.9(\mathrm{CH}), 140.6\left(\mathrm{C}_{\mathrm{q}}\right), 136.1\left(\mathrm{C}_{\mathrm{q}}\right), 132.0\left(\mathrm{C}_{\mathrm{q}}\right), 131.5\left(\mathrm{C}_{\mathrm{q}}\right), 131.4(\mathrm{CH}), 129.9(\mathrm{CH})$, $129.2(\mathrm{CH}), 128.9(\mathrm{CH}), 128.1(\mathrm{CH}), 127.2(\mathrm{CH}), 57.4(\mathrm{CH}), 52.2\left(\mathrm{CH}_{3}\right), 47.3(\mathrm{CH}), 41.9$ $\left(\mathrm{CH}_{2}\right), 31.7(\mathrm{CH}), 22.7\left(\mathrm{CH}_{3}\right), 19.1\left(\mathrm{CH}_{3}\right), 18.0\left(\mathrm{CH}_{3}\right)$.

IR (ATR): $\tilde{v}=3319,2966,1736,1644,1568,1495,1325,1213,703,646 \mathrm{~cm}^{-1}$.

MS (EI) $m / z$ (relative intensity): 485 (10) $[\mathrm{M}]^{+}, 426$ (46) $\left[\mathrm{M}-\mathrm{CO}_{2} \mathrm{Me}\right]^{+}, 355$ (100) [M-(H-ValOMe) $]^{+}, 328$ (15), 285 (31), 156 (14).

HR-MS (ESI): $m / z$ calcd for $\mathrm{C}_{28} \mathrm{H}_{32} \mathrm{~N}_{5} \mathrm{O}_{3}{ }^{+}[\mathrm{M}+\mathrm{H}]^{+} 486.2500$, found 486.2497.

\section{Methyl \{4-[3-(9-iso-propyl-9H-purin-6-yl)benzyl]benzoyl $\}-L$-serinate ( $S$-6f)}

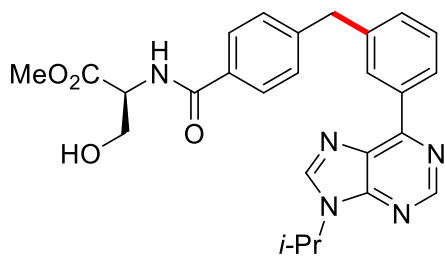

The general procedure B was followed using purine $1 \mathbf{h}(59.6 \mathrm{mg}$, $0.25 \mathrm{mmol})$ and benzyl chloride $S-5 \mathbf{d}(136 \mathrm{mg}, 0.50 \mathrm{mmol})$. After $20 \mathrm{~h}$, purification by column chromatography ( $n$-hexane/EtOAc 1:9) yielded $s$-6f $(59.1 \mathrm{mg}, 50 \%)$ as a colorless oil.

${ }^{1} \mathbf{H}-\mathbf{N M R}\left(500 \mathrm{MHz}, \mathrm{CDCl}_{3}\right): \delta=8.99(\mathrm{~s}, 1 \mathrm{H}), 8.64(\mathrm{ddd}, J=7.8,1.6,1.4 \mathrm{~Hz}, 1 \mathrm{H}), 8.55(\mathrm{dd}, J$ $=1.7,1.6 \mathrm{~Hz}, 1 \mathrm{H}), 8.19(\mathrm{~s}, 1 \mathrm{H}), 7.71(\mathrm{~d}, J=8.3 \mathrm{~Hz}, 2 \mathrm{H}), 7.48$ (dd, $J=7.8,7.7 \mathrm{~Hz}, 1 \mathrm{H}), 7.30$ $7.26(\mathrm{~m}, 1 \mathrm{H}), 7.28(\mathrm{~d}, J=8.3 \mathrm{~Hz}, 2 \mathrm{H}), 7.12(\mathrm{~d}, J=7.3 \mathrm{~Hz}, 1 \mathrm{H}), 4.97$ (hept, $J=6.8 \mathrm{~Hz}, 1 \mathrm{H})$, 4.81 (ddd, $J=7.3,3.7,3.5 \mathrm{~Hz}, 1 \mathrm{H}), 4.14$ (s, 2H), 4.02 (dd, $J=11.2,3.7 \mathrm{~Hz}, 1 \mathrm{H}), 3.97$ (dd, $J=$ 11.2, $3.5 \mathrm{~Hz}, 1 \mathrm{H}), 3.77$ (s, 3H), 3.18 (br s, 1H), 1.66 (d, $J=6.8 \mathrm{~Hz}, 6 \mathrm{H})$.

${ }^{13}$ C-NMR (125 MHz, $\left.\mathrm{CDCl}_{3}\right): \delta=171.0\left(\mathrm{C}_{\mathrm{q}}\right), 167.5\left(\mathrm{C}_{\mathrm{q}}\right), 154.6\left(\mathrm{C}_{\mathrm{q}}\right), 152.1\left(\mathrm{C}_{\mathrm{q}}\right), 152.0(\mathrm{CH})$, $145.3\left(\mathrm{C}_{\mathrm{q}}\right), 142.1(\mathrm{CH}), 140.6\left(\mathrm{C}_{\mathrm{q}}\right), 136.0\left(\mathrm{C}_{\mathrm{q}}\right), 131.5(\mathrm{CH}), 131.4\left(\mathrm{C}_{\mathrm{q}}\right), 131.3\left(\mathrm{C}_{\mathrm{q}}\right), 130.0(\mathrm{CH})$, $129.2(\mathrm{CH}), 129.0(\mathrm{CH}), 128.2(\mathrm{CH}), 127.4(\mathrm{CH}), 63.4\left(\mathrm{CH}_{2}\right), 55.1(\mathrm{CH}), 52.8\left(\mathrm{CH}_{3}\right), 47.3$ $(\mathrm{CH}), 41.8\left(\mathrm{CH}_{2}\right), 22.5\left(\mathrm{CH}_{3}\right)$.

IR (ATR): $\tilde{v}=3355,2980,1741,1644,1569,1497,1326,1219,703,647 \mathrm{~cm}^{-1}$. 
MS (EI) $m / z$ (relative intensity): $473(7)[\mathrm{M}]^{+}, 472(5)[\mathrm{M}-\mathrm{H}]^{+}, 455(5)\left[\mathrm{M}-\mathrm{H}_{2} \mathrm{O}\right]^{+}, 443$ (32) $[\mathrm{M}-\mathrm{CHOH}]^{+}, 414$ (8) $\left[\mathrm{M}-\mathrm{CO}_{2} \mathrm{Me}\right]^{+}, 400$ (13), 372 (12), 355 (100) [M-(H-Ser-OMe) $]^{+}, 328$ (23), 285 (41), 165 (10), 156 (20), 142 (13).

HR-MS (EI): $m / z$ calcd for $\mathrm{C}_{26} \mathrm{H}_{27} \mathrm{~N}_{5} \mathrm{O}_{4}{ }^{+}[\mathrm{M}]^{+} 473.2058$, found 473.2079.

\section{Methyl \{4-[3-(9-iso-propyl-9H-purin-6-yl)benzyl]benzoyl $\}-D / L$-serinate ( $\mathrm{rac}-6 \mathrm{f})$}<smiles>COC(=O)C(CO)NC(=O)c1ccc(Cc2cccc(-c3ncnc4c3ncn4C(C)C)c2)cc1</smiles>

The general procedure B was followed using purine 1h $(59.6 \mathrm{mg}$, $0.25 \mathrm{mmol}$ ) and benzyl chloride $\mathrm{rac}-\mathbf{5 d}(136 \mathrm{mg}, 0.50 \mathrm{mmol})$. After $20 \mathrm{~h}$, purification by column chromatography ( $n$-hexane/EtOAc 1:9) yielded rac-6f $(65.5 \mathrm{mg}, 55 \%)$ as a white

solid.

${ }^{1} \mathbf{H}-\mathrm{NMR}\left(600 \mathrm{MHz}, \mathrm{CDCl}_{3}\right): \delta=8.99$ (s, 1H), 8.65 (ddd, $\left.J=7.8,1.6,1.5 \mathrm{~Hz}, 1 \mathrm{H}\right), 8.57$ (dd, $J$ $=1.6,1.6 \mathrm{~Hz}, 1 \mathrm{H}), 8.18(\mathrm{~s}, 1 \mathrm{H}), 7.72(\mathrm{~d}, J=8.3 \mathrm{~Hz}, 2 \mathrm{H}), 7.48(\mathrm{dd}, J=7.8,7.7 \mathrm{~Hz}, 1 \mathrm{H}), 7.31-$ $7.28(\mathrm{~m}, 1 \mathrm{H}), 7.29(\mathrm{~d}, J=8.3 \mathrm{~Hz}, 2 \mathrm{H}), 7.09(\mathrm{~d}, J=7.2 \mathrm{~Hz}, 1 \mathrm{H}), 4.97$ (hept, $J=6.8 \mathrm{~Hz}, 1 \mathrm{H}$ ), 4.83 (ddd, $J=7.2,3.7,3.5 \mathrm{~Hz}, 1 \mathrm{H}), 4.15$ (s, 2H), 4.03 (dd, $J=11.3,3.7 \mathrm{~Hz}, 1 \mathrm{H}), 3.99$ (dd, $J=$ 11.3, 3.5 Hz, 1H), 3.78 (s, 3H), 3.01 (br s, 1H), 1.66 (d, $J=6.8 \mathrm{~Hz}, 6 \mathrm{H})$.

${ }^{13}$ C-NMR (125 MHz, $\left.\mathrm{CDCl}_{3}\right): \delta=170.9\left(\mathrm{C}_{\mathrm{q}}\right), 167.3\left(\mathrm{C}_{\mathrm{q}}\right), 154.5\left(\mathrm{C}_{\mathrm{q}}\right), 152.0\left(\mathrm{C}_{\mathrm{q}}\right), 151.9(\mathrm{CH})$, $145.3\left(\mathrm{C}_{\mathrm{q}}\right), 142.0(\mathrm{CH}), 140.5\left(\mathrm{C}_{\mathrm{q}}\right), 136.0\left(\mathrm{C}_{\mathrm{q}}\right), 131.4\left(\mathrm{C}_{\mathrm{q}}\right.$ and $\left.\mathrm{CH}\right), 131.3\left(\mathrm{C}_{\mathrm{q}}\right), 129.9(\mathrm{CH})$, $129.1(\mathrm{CH}), 128.9(\mathrm{CH}), 128.1(\mathrm{CH}), 127.3(\mathrm{CH}), 63.5\left(\mathrm{CH}_{2}\right), 55.2(\mathrm{CH}), 52.8\left(\mathrm{CH}_{3}\right), 47.3$ $(\mathrm{CH}), 41.9\left(\mathrm{CH}_{2}\right), 22.6\left(\mathrm{CH}_{3}\right)$.

IR (ATR): $\tilde{v}=3333,2976,1739,1643,1569,1496,1326,1218,703,646 \mathrm{~cm}^{-1}$.

m.p.: $73-75^{\circ} \mathrm{C}$.

MS (EI) $m / z$ (relative intensity): 473 (4) $[\mathrm{M}]^{+}, 472(3)[\mathrm{M}-\mathrm{H}]^{+}, 455$ (11) $\left[\mathrm{M}-\mathrm{H}_{2} \mathrm{O}\right]^{+}, 443$ (29) $[\mathrm{M}-\mathrm{CHOH}]^{+}, 414(5)\left[\mathrm{M}-\mathrm{CO}_{2} \mathrm{Me}\right]^{+}, 400$ (9), 372 (7), 355 (100) [M-(H-Ser-OMe) $]^{+}, 328(16)$, 311 (14), 285 (38), 165 (9), 156 (20), 142 (13).

HR-MS (EI): $m / z$ calcd for $\mathrm{C}_{26} \mathrm{H}_{27} \mathrm{~N}_{5} \mathrm{O}_{4}{ }^{+}[\mathrm{M}]^{+}$473.2058, found 473.2055.

Methyl $\quad N$-\{4-[3-(9-iso-propyl-9H-purin-6-yl)benzyl]benzoyl\}-S-(4-methylbenzyl)- $L$ cysteinate $(6 \mathrm{~g})$

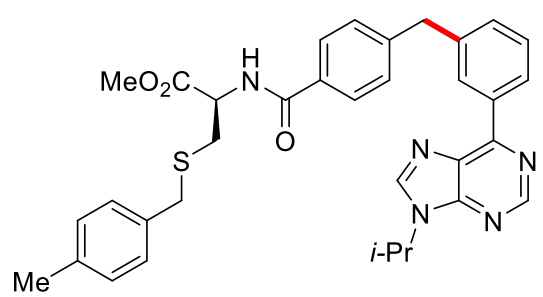

The general procedure $\mathbf{B}$ was followed using purine $\mathbf{1 h}$ (59.6 mg, $0.25 \mathrm{mmol}$ ) and benzyl chloride 5e (196 mg, $0.50 \mathrm{mmol})$. After $20 \mathrm{~h}$, purification by column chromatography ( $n$-hexane/EtOAc 1:1) yielded 6g (117 mg, $79 \%$ ) as a viscous colorless oil. 
${ }^{1} \mathbf{H}-\mathbf{N M R}\left(600 \mathrm{MHz}, \mathrm{CDCl}_{3}\right): \delta=9.01(\mathrm{~s}, 1 \mathrm{H}), 8.69(\mathrm{ddd}, J=7.8,1.6,1.3 \mathrm{~Hz}, 1 \mathrm{H}), 8.62(\mathrm{dd}, J$ $=1.9,1.6 \mathrm{~Hz}, 1 \mathrm{H}), 8.18(\mathrm{~s}, 1 \mathrm{H}), 7.72(\mathrm{~d}, J=8.3 \mathrm{~Hz}, 2 \mathrm{H}), 7.49(\mathrm{dd}, J=7.8,7.7 \mathrm{~Hz}, 1 \mathrm{H}), 7.33$ $(\mathrm{d}, J=8.3 \mathrm{~Hz}, 2 \mathrm{H}), 7.30(\mathrm{ddd}, J=7.7,1.9,1.3 \mathrm{~Hz}, 1 \mathrm{H}), 7.14(\mathrm{~d}, J=7.8 \mathrm{~Hz}, 2 \mathrm{H}), 7.06(\mathrm{~d}, J=$ $7.8 \mathrm{~Hz}, 2 \mathrm{H}), 6.86(\mathrm{~d}, J=7.5 \mathrm{~Hz}, 1 \mathrm{H}), 4.98$ (hept, $J=6.9 \mathrm{~Hz}, 1 \mathrm{H}), 4.98$ (ddd, $J=7.5,5.3,5.1 \mathrm{~Hz}$, $1 \mathrm{H}), 4.17(\mathrm{~s}, 2 \mathrm{H}), 3.76(\mathrm{~s}, 3 \mathrm{H}), 3.67\left(\mathrm{~d}_{\mathrm{AB}}, J=13.5 \mathrm{~Hz}, 1 \mathrm{H}\right), 3.66\left(\mathrm{~d}_{\mathrm{AB}}, J=13.5 \mathrm{~Hz}, 1 \mathrm{H}\right), 3.01$ $\left(\mathrm{d}_{\mathrm{AB}} \mathrm{d}, J=13.9,5.1 \mathrm{~Hz}, 1 \mathrm{H}\right), 2.96\left(\mathrm{~d}_{\mathrm{AB}} \mathrm{d}, J=13.9,5.3 \mathrm{~Hz}, 1 \mathrm{H}\right), 2.27(\mathrm{~s}, 3 \mathrm{H}), 1.67(\mathrm{~d}, J=6.9 \mathrm{~Hz}$, $6 \mathrm{H})$.

${ }^{13}$ C-NMR $\left(125 \mathrm{MHz}, \mathrm{CDCl}_{3}\right): \delta=171.2\left(\mathrm{C}_{\mathrm{q}}\right), 166.7\left(\mathrm{C}_{\mathrm{q}}\right), 154.5\left(\mathrm{C}_{\mathrm{q}}\right), 152.0\left(\mathrm{C}_{\mathrm{q}}\right), 151.9(\mathrm{CH})$, 145.2 $\left(\mathrm{C}_{\mathrm{q}}\right), 141.9(\mathrm{CH}), 140.5\left(\mathrm{C}_{\mathrm{q}}\right), 136.8\left(\mathrm{C}_{\mathrm{q}}\right), 136.1\left(\mathrm{C}_{\mathrm{q}}\right), 134.4\left(\mathrm{C}_{\mathrm{q}}\right), 131.4\left(\mathrm{C}_{\mathrm{q}}\right), 131.4(\mathrm{CH})$, $129.9(\mathrm{CH}), 129.2(\mathrm{CH}), 129.1(\mathrm{CH}), 128.8(\mathrm{CH}), 128.6(\mathrm{CH}), 128.1(\mathrm{CH}), 127.3(\mathrm{CH}), 52.7$ $\left(\mathrm{CH}_{3}\right), 52.0(\mathrm{CH}), 47.3(\mathrm{CH}), 41.9\left(\mathrm{CH}_{2}\right), 36.5\left(\mathrm{CH}_{2}\right), 33.5\left(\mathrm{CH}_{2}\right), 22.6\left(\mathrm{CH}_{3}\right), 21.1\left(\mathrm{CH}_{3}\right)$.

IR (ATR): $\tilde{v}=3329,2979,1741,1650,1569,1494,1325,1216,731,647 \mathrm{~cm}^{-1}$.

MS (ESI) $m / z$ (relative intensity): 1209 (16) $[2 \mathrm{M}+\mathrm{Na}]^{+}, 1187(9)[2 \mathrm{M}+\mathrm{H}]^{+}, 616(100)[\mathrm{M}+\mathrm{Na}]^{+}$, $594(47)[\mathrm{M}+\mathrm{H}]^{+}$.

HR-MS (ESI): $m / z$ calcd for $\mathrm{C}_{34} \mathrm{H}_{35} \mathrm{~N}_{5} \mathrm{O}_{3} \mathrm{SNa}^{+}[\mathrm{M}+\mathrm{Na}]^{+}$616.2353, found 616.2372.

\section{Methyl \{4-[3-(9-iso-propyl-9H-purin-6-yl)benzyl]benzoyl\}-L-tryptophanate (6h)}

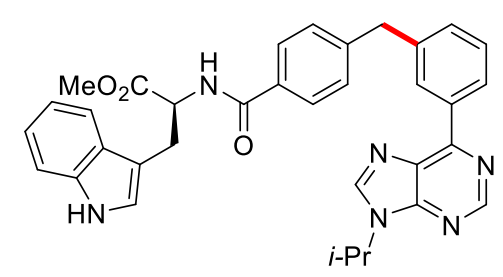

The general procedure $\mathbf{B}$ was followed using purine $\mathbf{1 h}$ (59.6 mg, $0.25 \mathrm{mmol})$ and benzyl chloride $\mathbf{5 f}(186 \mathrm{mg}$, $0.50 \mathrm{mmol})$. After $20 \mathrm{~h}$, purification by column chromatography ( $n$-hexane/EtOAc 1:2) yielded $\mathbf{6 h}(96.5 \mathrm{mg}, 67 \%)$ as a viscous

colorless oil.

${ }^{1} \mathbf{H}-\mathbf{N M R}\left(600 \mathrm{MHz}, \mathrm{CDCl}_{3}\right): \delta=9.01(\mathrm{~s}, 1 \mathrm{H}), 8.67$ (ddd, $\left.J=7.8,1.6,1.4 \mathrm{~Hz}, 1 \mathrm{H}\right), 8.58(\mathrm{dd}, J$ $=1.8,1.6 \mathrm{~Hz}, 1 \mathrm{H}), 8.23$ (br s, 1H), $8.18(\mathrm{~s}, 1 \mathrm{H}), 7.60(\mathrm{~d}, J=8.4 \mathrm{~Hz}, 2 \mathrm{H}), 7.53$ (dd, $J=7.9$, $1.1 \mathrm{~Hz}, 1 \mathrm{H}), 7.48(\mathrm{dd}, J=7.8,7.7 \mathrm{~Hz}, 1 \mathrm{H}), 7.32(\mathrm{~d}, J=8.2,1.0 \mathrm{~Hz}, 1 \mathrm{H}), 7.30-7.26(\mathrm{~m}, 1 \mathrm{H})$, $7.25(\mathrm{~d}, J=8.4 \mathrm{~Hz}, 2 \mathrm{H}), 7.16(\mathrm{ddd}, J=8.2,7.0,1.1 \mathrm{~Hz}, 1 \mathrm{H}), 7.06(\mathrm{ddd}, J=7.9,7.0,1.0 \mathrm{~Hz}$, $1 \mathrm{H}), 6.97(\mathrm{~d}, J=2.4 \mathrm{~Hz}, 1 \mathrm{H}), 6.63(\mathrm{~d}, J=7.7 \mathrm{~Hz}, 1 \mathrm{H}), 5.12(\mathrm{dt}, J=7.7,5.3 \mathrm{~Hz}, 1 \mathrm{H}), 4.98$ (hept, $J=6.8 \mathrm{~Hz}, 1 \mathrm{H}), 4.13(\mathrm{~s}, 2 \mathrm{H}), 3.69(\mathrm{~s}, 3 \mathrm{H}), 3.42(\mathrm{~d}, J=5.3 \mathrm{~Hz}, 2 \mathrm{H}), 1.67(\mathrm{~d}, J=6.8 \mathrm{~Hz}, 6 \mathrm{H})$.

${ }^{13} \mathrm{C}-\mathrm{NMR}\left(125 \mathrm{MHz}, \mathrm{CDCl}_{3}\right): \delta=172.2\left(\mathrm{C}_{\mathrm{q}}\right), 166.6\left(\mathrm{C}_{\mathrm{q}}\right), 154.5\left(\mathrm{C}_{\mathrm{q}}\right), 152.0\left(\mathrm{C}_{\mathrm{q}}\right), 151.8(\mathrm{CH})$, $144.9\left(\mathrm{C}_{\mathrm{q}}\right), 142.0(\mathrm{CH}), 140.6\left(\mathrm{C}_{\mathrm{q}}\right), 136.0\left(\mathrm{C}_{\mathrm{q}}\right), 135.9\left(\mathrm{C}_{\mathrm{q}}\right), 131.7\left(\mathrm{C}_{\mathrm{q}}\right), 131.4(\mathrm{CH}), 131.4\left(\mathrm{C}_{\mathrm{q}}\right)$, $129.9(\mathrm{CH}), 129.1(\mathrm{CH}), 128.9(\mathrm{CH}), 128.1(\mathrm{CH}), 127.5\left(\mathrm{C}_{\mathrm{q}}\right), 127.2(\mathrm{CH}), 122.7(\mathrm{CH}), 122.2$ $(\mathrm{CH}), 119.6(\mathrm{CH}), 118.6(\mathrm{CH}), 111.2(\mathrm{CH}), 110.0\left(\mathrm{C}_{\mathrm{q}}\right), 53.4(\mathrm{CH}), 52.4\left(\mathrm{CH}_{3}\right), 47.3(\mathrm{CH}), 41.8$ $\left(\mathrm{CH}_{2}\right), 27.7\left(\mathrm{CH}_{2}\right), 22.6\left(\mathrm{CH}_{3}\right)$.

IR (ATR): $\tilde{v}=3300,2980,1737,1644,1570,1495,1327,1218,736,647 \mathrm{~cm}^{-1}$. 
MS (ESI) $m / z$ (relative intensity): $1168(5)[2 \mathrm{M}+\mathrm{Na}]^{+}, 1145(19)[2 \mathrm{M}+\mathrm{H}]^{+}, 595(10)[\mathrm{M}+\mathrm{Na}]^{+}$, $573(100)[\mathrm{M}+\mathrm{H}]^{+}$.

HR-MS (ESI): $m / z$ calcd for $\mathrm{C}_{34} \mathrm{H}_{33} \mathrm{~N}_{6} \mathrm{O}_{3}{ }^{+}[\mathrm{M}+\mathrm{H}]^{+}$573.2609, found 573.2608.

\section{Methyl \{4-[3-(9-iso-propyl-9H-purin-6-yl)benzyl]benzoyl\}-L-tyrosinate (6i)}<smiles>CC(=O)C(Cc1ccc(O)cc1)NC(=O)c1ccc(Cc2cccc(-c3ncnc4c3ncn4C(F)(F)F)c2)cc1</smiles>

The general procedure $\mathbf{B}$ was followed using purine $\mathbf{1 h}$ (59.6 $\mathrm{mg}, 0.25 \mathrm{mmol})$ and benzyl chloride $\mathbf{5 g}$ (174 $\mathrm{mg}$, $0.50 \mathrm{mmol})$. After $20 \mathrm{~h}$, purification by column chromatography ( $n$-hexane/EtOAc 1:2) yielded 6i (72.2 mg,

$53 \%)$ as a viscous colorless oil.

${ }^{1} \mathbf{H}-\mathbf{N M R}\left(600 \mathrm{MHz}, \mathrm{CDCl}_{3}\right): \delta=9.01(\mathrm{~s}, 1 \mathrm{H}), 8.62(\mathrm{ddd}, J=7.8,1.6,1.4 \mathrm{~Hz}, 1 \mathrm{H}), 8.53(\mathrm{dd}, J$ $=1.8,1.6 \mathrm{~Hz}, 1 \mathrm{H}), 8.20(\mathrm{~s}, 1 \mathrm{H}), 7.61(\mathrm{~d}, J=8.3 \mathrm{~Hz}, 2 \mathrm{H}), 7.45(\mathrm{dd}, J=7.8,7.7 \mathrm{~Hz}, 1 \mathrm{H}), 7.28-$ $7.25(\mathrm{~m}, 1 \mathrm{H}), 7.23(\mathrm{~d}, J=8.3 \mathrm{~Hz}, 2 \mathrm{H}), 6.91(\mathrm{~d}, J=8.5 \mathrm{~Hz}, 2 \mathrm{H}), 6.86$ (br s, 1H), 6.69 (d, $J=$ $8.5 \mathrm{~Hz}, 2 \mathrm{H}), 6.59$ (d, $J=7.7 \mathrm{~Hz}, 1 \mathrm{H}), 5.00$ (dt, $J=7.7,5.6 \mathrm{~Hz}, 1 \mathrm{H}), 4.98$ (hept, $J=6.8 \mathrm{~Hz}, 1 \mathrm{H}$ ), $4.10(\mathrm{~s}, 2 \mathrm{H}), 3.72$ (s, 3H), 3.16 (dd, $J=14.0,5.6 \mathrm{~Hz}, 1 \mathrm{H}), 3.09$ (dd, $J=14.0,5.6 \mathrm{~Hz}, 1 \mathrm{H}), 1.66$ $(\mathrm{d}, J=6.8 \mathrm{~Hz}, 6 \mathrm{H})$.

${ }^{13}$ C-NMR (125 MHz, $\left.\mathrm{CDCl}_{3}\right): \delta=172.0\left(\mathrm{C}_{\mathrm{q}}\right), 166.8\left(\mathrm{C}_{\mathrm{q}}\right), 155.3\left(\mathrm{C}_{\mathrm{q}}\right), 154.7\left(\mathrm{C}_{\mathrm{q}}\right), 152.0\left(\mathrm{C}_{\mathrm{q}}\right)$, $151.9(\mathrm{CH}), 145.1\left(\mathrm{C}_{\mathrm{q}}\right), 142.1(\mathrm{CH}), 140.6\left(\mathrm{C}_{\mathrm{q}}\right), 135.8\left(\mathrm{C}_{\mathrm{q}}\right), 131.6\left(\mathrm{C}_{\mathrm{q}}\right), 131.5(\mathrm{CH}), 131.3\left(\mathrm{C}_{\mathrm{q}}\right)$, $130.3(\mathrm{CH}), 130.0(\mathrm{CH}), 129.1(\mathrm{CH}), 128.9(\mathrm{CH}), 128.1(\mathrm{CH}), 127.2(\mathrm{CH}), 127.1\left(\mathrm{C}_{\mathrm{q}}\right), 115.6$ $(\mathrm{CH}), 53.7(\mathrm{CH}), 52.4\left(\mathrm{CH}_{3}\right), 47.4(\mathrm{CH}), 41.8\left(\mathrm{CH}_{2}\right), 37.1\left(\mathrm{CH}_{2}\right), 22.6\left(\mathrm{CH}_{3}\right)$.

IR (ATR): $\tilde{v}=3269,2978,1736,1639,1569,1326,1217,784,703,646 \mathrm{~cm}^{-1}$.

MS (EI) $\mathrm{m} / \mathrm{z}$ (relative intensity): 549 (3) $[\mathrm{M}]^{+}, 490$ (5) $\left[\mathrm{M}-\mathrm{CO}_{2} \mathrm{Me}\right]^{+}, 443$ (100) [M-(4OHBn $)]^{+}, 400$ (40) [M-(4-OHBn)-iPr] $]^{+}, 372$ (24), 355 (58) [M-(H-Tyr-OMe) $]^{+}, 328$ (37), 313 (18), 285 (45), 178 (8), 147 (7), 107 (19), 43 (8).

HR-MS (EI): $m / z$ calcd for $\mathrm{C}_{32} \mathrm{H}_{31} \mathrm{~N}_{5} \mathrm{O}_{4}{ }^{+}[\mathrm{M}]^{+}$549.2371, found 549.2387.

\section{Methyl \{4-[3-(9-iso-propyl-9H-purin-6-yl)benzyl]benzoyl $\}-L$-prolyl- $L$-leucinate $(6 j)$}

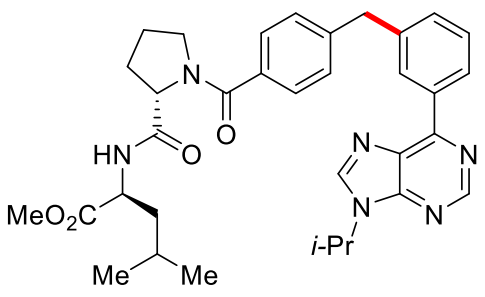

${ }^{1}$ H-NMR $\left(600 \mathrm{MHz}, \mathrm{CDCl}_{3}\right): \delta=8.99(\mathrm{~s}, 1 \mathrm{H}), 8.68(\mathrm{~d}, J=7.8 \mathrm{~Hz}, 1 \mathrm{H}), 8.58(\mathrm{~s}, 1 \mathrm{H}), 8.18(\mathrm{~s}$, 1H), $7.48(\mathrm{dd}, J=7.8,7.8 \mathrm{~Hz}, 1 \mathrm{H}), 7.41$ (d, $J=8.0 \mathrm{~Hz}, 2 \mathrm{H}), 7.34$ (d, $J=7.8 \mathrm{~Hz}, 1 \mathrm{H}), 7.30$ (d,
The general procedure $\mathbf{B}$ was followed using purine $\mathbf{1 h}$ (59.6 $\mathrm{mg}, \quad 0.25 \mathrm{mmol})$ and benzyl chloride $\mathbf{5 h}$ (198 $\mathrm{mg}$, $0.50 \mathrm{mmol}$ ). After $20 \mathrm{~h}$, purification by column chromatography ( $n$-hexane/EtOAc 1:4) yielded $\mathbf{6 j}(107 \mathrm{mg}, 72 \%$ ) as a viscous colorless oil. 
$J=7.8 \mathrm{~Hz}, 1 \mathrm{H}), 7.28(\mathrm{~d}, J=8.0 \mathrm{~Hz}, 2 \mathrm{H}), 4.98$ (hept, $J=6.8 \mathrm{~Hz}, 1 \mathrm{H}), 4.78(\mathrm{dd}, J=7.9,4.7 \mathrm{~Hz}$, $1 \mathrm{H}), 4.54(\mathrm{ddd}, J=8.0,7.8,4.5 \mathrm{~Hz}, 1 \mathrm{H}), 4.14(\mathrm{~s}, 2 \mathrm{H}), 3.71$ (s, 3H), 3.54-3.48 (m, 1H), 3.48$3.42(\mathrm{~m}, 1 \mathrm{H}), 2.48-2.38(\mathrm{~m}, 1 \mathrm{H}), 2.06-1.97(\mathrm{~m}, 2 \mathrm{H}), 1.83-1.75(\mathrm{~m}, 1 \mathrm{H}), 1.66(\mathrm{~d}, J=6.8 \mathrm{~Hz}$, $6 \mathrm{H}), 1.67-1.53(\mathrm{~m}, 3 \mathrm{H}), 0.88(\mathrm{~d}, J=6.1 \mathrm{~Hz}, 3 \mathrm{H}), 0.87$ (d, $J=6.4 \mathrm{~Hz}, 3 \mathrm{H})$.

${ }^{13}$ C-NMR (125 MHz, $\left.\mathrm{CDCl}_{3}\right): \delta=173.0\left(\mathrm{C}_{\mathrm{q}}\right), 170.9\left(\mathrm{C}_{\mathrm{q}}\right), 170.7\left(\mathrm{C}_{\mathrm{q}}\right), 154.5\left(\mathrm{C}_{\mathrm{q}}\right), 152.0\left(\mathrm{C}_{\mathrm{q}}\right)$, $151.9(\mathrm{CH}), 143.4\left(\mathrm{C}_{\mathrm{q}}\right), 141.9(\mathrm{CH}), 140.6\left(\mathrm{C}_{\mathrm{q}}\right), 136.0\left(\mathrm{C}_{\mathrm{q}}\right), 133.9\left(\mathrm{C}_{\mathrm{q}}\right), 131.4\left(\mathrm{C}_{\mathrm{q}}\right.$ and $\left.\mathrm{CH}\right)$, $129.9(\mathrm{CH}), 128.8(\mathrm{CH}), 128.8(\mathrm{CH}), 128.1(\mathrm{CH}), 127.2(\mathrm{CH}), 59.6(\mathrm{CH}), 52.2\left(\mathrm{CH}_{3}\right), 51.1$ $(\mathrm{CH}), 50.4\left(\mathrm{CH}_{2}\right), 47.3(\mathrm{CH}), 41.9\left(\mathrm{CH}_{2}\right), 41.2\left(\mathrm{CH}_{2}\right), 27.0\left(\mathrm{CH}_{2}\right), 25.5\left(\mathrm{CH}_{2}\right), 25.0(\mathrm{CH}), 22.8$ $\left(\mathrm{CH}_{3}\right), 22.6\left(\mathrm{CH}_{3}\right), 21.9\left(\mathrm{CH}_{3}\right)$.

IR (ATR): $\tilde{v}=3282,2957,1743,1681,1614,1570,1428,1327,1221,648 \mathrm{~cm}^{-1}$.

MS (EI) $m / z$ (relative intensity): $596(5)[\mathrm{M}]^{+}, 424$ (30) $[\mathrm{M}-(\mathrm{C}(\mathrm{O})-\mathrm{Leu}-\mathrm{OMe})]^{+}, 355$ (100) [M(H-Pro-Leu-OMe)] $]^{+}, 328$ (11), 285 (18), 156 (9), 43 (6).

HR-MS (EI): $m / z$ calcd for $\mathrm{C}_{34} \mathrm{H}_{40} \mathrm{~N}_{6} \mathrm{O}_{4}{ }^{+}[\mathrm{M}]^{+}$596.3106, found 596.3124.

\section{Methyl \{4-[3-(9-iso-propyl-9H-purin-6-yl)benzyl]benzoyl\}- $L$-leucyl- $L$-methioninate (6k)}

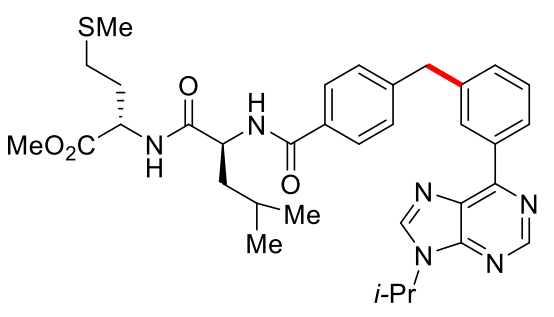

The general procedure $\mathbf{B}$ was followed using purine $\mathbf{1 h}$ (59.6 mg, $0.25 \mathrm{mmol})$ and benzyl chloride $\mathbf{5 i}$ (215 $\mathrm{mg}$, $0.50 \mathrm{mmol})$. After $20 \mathrm{~h}$, purification by column chromatography ( $n$-hexane/EtOAc 1:2) yielded 6k (117 mg, $74 \%)$ as a viscous colorless oil.

${ }^{1} \mathbf{H}-\mathbf{N M R}\left(600 \mathrm{MHz}, \mathrm{CDCl}_{3}\right): \delta=9.00(\mathrm{~s}, 1 \mathrm{H}), 8.68(\mathrm{ddd}, J=7.8,1.6,1.3 \mathrm{~Hz}, 1 \mathrm{H}), 8.59(\mathrm{dd}, J$ $=1.9,1.6 \mathrm{~Hz}, 1 \mathrm{H}), 8.19(\mathrm{~s}, 1 \mathrm{H}), 7.70(\mathrm{~d}, J=8.3 \mathrm{~Hz}, 2 \mathrm{H}), 7.48(\mathrm{dd}, J=7.8,7.7 \mathrm{~Hz}, 1 \mathrm{H}), 7.30$ $(\mathrm{d}, J=8.3 \mathrm{~Hz}, 2 \mathrm{H}), 7.28(\mathrm{ddd}, J=7.7,1.9,1.3 \mathrm{~Hz}, 1 \mathrm{H}), 6.92(\mathrm{~d}, J=8.0 \mathrm{~Hz}, 1 \mathrm{H}), 6.61(\mathrm{~d}, J=$ $8.2 \mathrm{~Hz}, 1 \mathrm{H}), 4.98$ (hept, $J=6.8 \mathrm{~Hz}, 1 \mathrm{H}), 4.72-4.66(\mathrm{~m}, 2 \mathrm{H}), 4.15(\mathrm{~s}, 2 \mathrm{H}), 3.74(\mathrm{~s}, 3 \mathrm{H}), 2.45$ (t, $J=7.4 \mathrm{~Hz}, 2 \mathrm{H}), 2.17-2.09(\mathrm{~m}, 1 \mathrm{H}), 2.02-1.93(\mathrm{~m}, 1 \mathrm{H}), 1.99$ (s, 3H), 1.79-1.61 (m, 3H), 1.67 $(\mathrm{d}, J=6.8 \mathrm{~Hz}, 6 \mathrm{H}), 0.95(\mathrm{~d}, J=6.4 \mathrm{~Hz}, 3 \mathrm{H}), 0.95(\mathrm{~d}, J=6.3 \mathrm{~Hz}, 3 \mathrm{H})$.

${ }^{13}$ C-NMR $\left(125 \mathrm{MHz}, \mathrm{CDCl}_{3}\right): \delta=171.9\left(\mathrm{C}_{\mathrm{q}}\right), 171.7\left(\mathrm{C}_{\mathrm{q}}\right), 167.1\left(\mathrm{C}_{\mathrm{q}}\right), 154.4\left(\mathrm{C}_{\mathrm{q}}\right), 152.0\left(\mathrm{C}_{\mathrm{q}}\right)$, $151.8(\mathrm{CH}), 145.2\left(\mathrm{C}_{\mathrm{q}}\right), 142.0(\mathrm{CH}), 140.5\left(\mathrm{C}_{\mathrm{q}}\right), 135.9\left(\mathrm{C}_{\mathrm{q}}\right), 131.5\left(\mathrm{C}_{\mathrm{q}}\right), 131.4\left(\mathrm{C}_{\mathrm{q}}\right.$ and $\left.\mathrm{CH}\right)$, $129.9(\mathrm{CH}), 129.2(\mathrm{CH}), 128.9(\mathrm{CH}), 128.1(\mathrm{CH}), 127.2(\mathrm{CH}), 52.5\left(\mathrm{CH}_{3}\right), 52.0(\mathrm{CH}), 51.6$ $(\mathrm{CH}), 47.3(\mathrm{CH}), 41.9\left(\mathrm{CH}_{2}\right), 41.1\left(\mathrm{CH}_{2}\right), 31.5\left(\mathrm{CH}_{2}\right), 30.0\left(\mathrm{CH}_{2}\right), 24.9(\mathrm{CH}), 22.9\left(\mathrm{CH}_{3}\right), 22.6$ $\left(\mathrm{CH}_{3}\right), 22.3\left(\mathrm{CH}_{3}\right), 15.5\left(\mathrm{CH}_{3}\right)$.

IR (ATR): $\tilde{v}=3272,2954,1742,1632,1568,1325,1218,784,703,646 \mathrm{~cm}^{-1}$.

MS (EI) $m / z$ (relative intensity): 630 (1) $[\mathrm{M}]^{+}, 615$ (1) $[\mathrm{M}-\mathrm{Me}]^{+}, 569$ (6) $\left[\mathrm{M}-\mathrm{SMe}_{2}\right]^{+}, 556$ (33) $[\mathrm{M}-\mathrm{EtSMe}]^{+}, 500$ (32) $[\mathrm{M}-\mathrm{EtSMe}-\mathrm{Bu}]^{+}, 468$ (11) [M-EtSMe-Bu-OMe] $]^{+}, 440$ (20) [M- 
EtSMe-Bu-CO $\left.{ }_{2} \mathrm{Me}\right]^{+}, 355$ (100) [M-(H-Leu-Met-OMe)] $]^{+}, 328$ (17), 313 (7), 285 (30), 156 (10), 43 (8).

HR-MS (EI): $m / z$ calcd for $\mathrm{C}_{34} \mathrm{H}_{42} \mathrm{~N}_{6} \mathrm{O}_{4} \mathrm{~S}^{+}[\mathrm{M}]^{+}$630.2983, found 630.2984.

(R)-2,5,7,8-Tetramethyl-2-[(4R,8R)-4,8,12-trimethyltridecyl]chroman-6-yl 4-[3-(9-isopropyl-9H-purin-6-yl)benzyl]benzoate (6l)

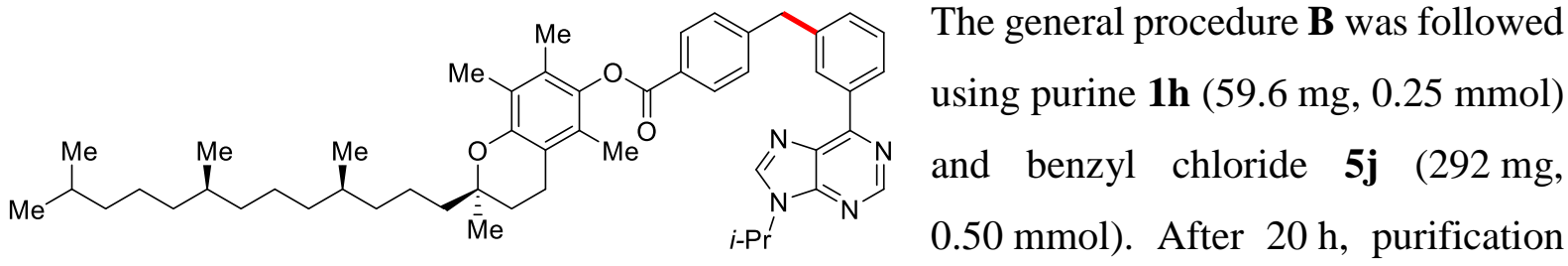
by column chromatography ( $n$-hexane/EtOAc 3:1) yielded $\mathbf{6 l}(127 \mathrm{mg}, 65 \%)$ as a viscous pale yellow oil.

${ }^{1} \mathbf{H}-\mathrm{NMR}\left(600 \mathrm{MHz}, \mathrm{CDCl}_{3}\right): \delta=9.03(\mathrm{~s}, 1 \mathrm{H}), 8.73$ (ddd, $\left.J=7.8,1.6,1.4 \mathrm{~Hz}, 1 \mathrm{H}\right), 8.68$ (dd, $J$ $=1.7,1.6 \mathrm{~Hz}, 1 \mathrm{H}), 8.19(\mathrm{~s}, 1 \mathrm{H}), 8.18(\mathrm{~d}, J=8.3 \mathrm{~Hz}, 2 \mathrm{H}), 7.52(\mathrm{dd}, J=7.8,7.7 \mathrm{~Hz}, 1 \mathrm{H}), 7.41$ (d, $J=8.3 \mathrm{~Hz}, 2 \mathrm{H}), 7.36$ (ddd, $J=7.7,1.7,1.4 \mathrm{~Hz}, 1 \mathrm{H}$ ), 4.99 (hept, $J=6.8 \mathrm{~Hz}, 1 \mathrm{H}$ ), 4.23 (s, 2H), $2.61(\mathrm{dd}, J=7.0,6.8 \mathrm{~Hz}, 2 \mathrm{H}), 2.12$ (s, 3H), 2.05 (s, 3H), 2.01 (s, 3H), 1.83 (dt, $J=13.9$, $7.0 \mathrm{~Hz}, 1 \mathrm{H}), 1.80-1.73(\mathrm{~m}, 1 \mathrm{H}), 1.68$ (d, $J=6.8 \mathrm{~Hz}, 6 \mathrm{H}), 1.62-1.02(\mathrm{~m}, 24 \mathrm{H}), 0.90-0.83$ (m, $12 \mathrm{H})$.

${ }^{13} \mathrm{C}-\mathrm{NMR}\left(125 \mathrm{MHz}, \mathrm{CDCl}_{3}, 1: 1\right.$ Conformer A and B): $\delta=164.9\left(\mathrm{C}_{\mathrm{q}}\right), 154.4\left(\mathrm{C}_{\mathrm{q}}\right), 152.0\left(\mathrm{C}_{\mathrm{q}}\right)$, $151.9(\mathrm{CH}), 149.3\left(\mathrm{C}_{\mathrm{q}}\right), 146.9\left(\mathrm{C}_{\mathrm{q}}\right), 141.9(\mathrm{CH}), 140.5\left(\mathrm{C}_{\mathrm{q}}\right), 140.3\left(\mathrm{C}_{\mathrm{q}}\right), 136.1\left(\mathrm{C}_{\mathrm{q}}\right), 131.5\left(\mathrm{C}_{\mathrm{q}}\right)$, $131.4(\mathrm{CH}), 130.3(\mathrm{CH}), 130.0(\mathrm{CH}), 129.0(\mathrm{CH}), 128.9(\mathrm{CH}), 128.2(\mathrm{CH}), 127.5\left(\mathrm{C}_{\mathrm{q}}\right), 126.8$ $\left(\mathrm{C}_{\mathrm{q}}\right), 125.0\left(\mathrm{C}_{\mathrm{q}}\right), 122.9\left(\mathrm{C}_{\mathrm{q}}\right), 117.3\left(\mathrm{C}_{\mathrm{q}}\right), 75.0\left(\mathrm{C}_{\mathrm{q}}\right), 47.3(\mathrm{CH}), 42.1\left(\mathrm{CH}_{2}\right), 40.4$ and $39.7\left(\mathrm{CH}_{2}\right)$, $39.4\left(\mathrm{CH}_{2}\right), 37.5\left(\mathrm{CH}_{2}\right), 37.5\left(\mathrm{CH}_{2}\right), 37.3\left(\mathrm{CH}_{2}\right), 32.8(\mathrm{CH}), 32.8$ and $32.7(\mathrm{CH}), 31.3$ and 31.1 $\left(\mathrm{CH}_{2}\right), 28.0(\mathrm{CH}), 24.8\left(\mathrm{CH}_{2}\right), 24.5\left(\mathrm{CH}_{2}\right), 24.2$ and $23.8\left(\mathrm{CH}_{3}\right), 22.8\left(\mathrm{CH}_{3}\right), 22.7\left(\mathrm{CH}_{3}\right), 22.6$ $\left(\mathrm{CH}_{3}\right), 21.1\left(\mathrm{CH}_{2}\right), 20.7\left(\mathrm{CH}_{2}\right), 19.8\left(\mathrm{CH}_{3}\right), 19.7\left(\mathrm{CH}_{3}\right), 13.1\left(\mathrm{CH}_{3}\right), 12.3\left(\mathrm{CH}_{3}\right), 11.9\left(\mathrm{CH}_{3}\right)$. Conformer $\mathrm{A}$ and $\mathrm{B}$ originate from the hindered rotation around the $\mathrm{C}-\mathrm{C}$ bond to the ester group.

IR (ATR): $\tilde{v}=2925,1732,1571,1459,1327,1273,1237,1092,704,648 \mathrm{~cm}^{-1}$.

MS (ESI) $m / z$ (relative intensity): $808(14)[\mathrm{M}+\mathrm{Na}]^{+}, 786(100)[\mathrm{M}+\mathrm{H}]^{+}$.

HR-MS (ESI): $m / z$ calcd for $\mathrm{C}_{51} \mathrm{H}_{69} \mathrm{~N}_{4} \mathrm{O}_{3}{ }^{+}[\mathrm{M}+\mathrm{H}]^{+}$785.5364, found 785.5349. 


\section{didodecanoate $(R-6 \mathrm{~m})$}

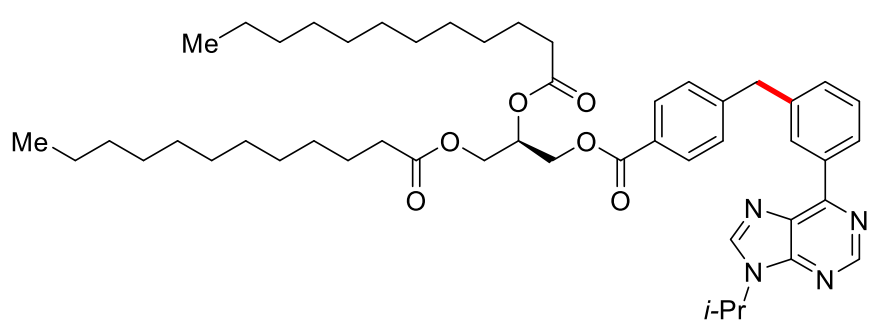

The general procedure $\mathbf{B}$ was followed using purine $\mathbf{1 h}(59.6 \mathrm{mg}, 0.25 \mathrm{mmol})$ and benzyl chloride $R$-5 $\mathbf{k}$ (305 $\mathrm{mg}$, $0.50 \mathrm{mmol}$ ). After $20 \mathrm{~h}$, purification by column

( $n$-hexane/EtOAc 4:1) yielded $R$-6m (153 mg, 75\%) as a yellow oil.

${ }^{1}$ H-NMR (400 MHz, $\left.\mathrm{CDCl}_{3}\right): \delta=9.01(\mathrm{~s}, 1 \mathrm{H}), 8.70(\mathrm{ddd}, J=7.8,1.6,1.4 \mathrm{~Hz}, 1 \mathrm{H}), 8.62(\mathrm{dd}, J$ $=1.8,1.6 \mathrm{~Hz}, 1 \mathrm{H}), 8.18(\mathrm{~s}, 1 \mathrm{H}), 7.93(\mathrm{~d}, J=8.3 \mathrm{~Hz}, 2 \mathrm{H}), 7.49(\mathrm{dd}, J=7.8,7.7 \mathrm{~Hz}, 1 \mathrm{H}), 7.32$ (d, $J=8.3 \mathrm{~Hz}, 2 \mathrm{H}), 7.31-7.27(\mathrm{~m}, 1 \mathrm{H}), 5.40$ (dddd, $J=6.0,5.9,4.4,4.3 \mathrm{~Hz}, 1 \mathrm{H}), 4.98$ (hept, $J$ $=6.8 \mathrm{~Hz}, 1 \mathrm{H}), 4.49\left(\mathrm{~d}_{\mathrm{AB}} \mathrm{d}, J=11.9,4.3 \mathrm{~Hz}, 1 \mathrm{H}\right), 4.39\left(\mathrm{~d}_{\mathrm{AB}} \mathrm{d}, J=11.9,6.0 \mathrm{~Hz}, 1 \mathrm{H}\right), 4.37(\mathrm{dd}, J$ $=11.9,4.4 \mathrm{~Hz}, 1 \mathrm{H}), 4.22(\mathrm{dd}, J=11.9,5.9 \mathrm{~Hz}, 1 \mathrm{H}), 4.18(\mathrm{~s}, 2 \mathrm{H}), 2.31(\mathrm{t}, J=7.5 \mathrm{~Hz}, 2 \mathrm{H}), 2.31$ (t, $J=7.6 \mathrm{~Hz}, 2 \mathrm{H}), 1.68(\mathrm{~d}, J=6.8 \mathrm{~Hz}, 6 \mathrm{H}), 1.64-1.55(\mathrm{~m}, 4 \mathrm{H}), 1.33-1.17(\mathrm{~m}, 32 \mathrm{H}), 0.87$ (t, $J$ $=7.0 \mathrm{~Hz}, 3 \mathrm{H}), 0.87(\mathrm{t}, J=7.1 \mathrm{~Hz}, 3 \mathrm{H})$.

${ }^{13}$ C-NMR $\left(100 \mathrm{MHz}, \mathrm{CDCl}_{3}\right): \delta=173.3\left(\mathrm{C}_{\mathrm{q}}\right), 172.9\left(\mathrm{C}_{\mathrm{q}}\right), 165.9\left(\mathrm{C}_{\mathrm{q}}\right), 154.6\left(\mathrm{C}_{\mathrm{q}}\right), 152.1\left(\mathrm{C}_{\mathrm{q}}\right)$, 152.0 $(\mathrm{CH}), 146.9\left(\mathrm{C}_{\mathrm{q}}\right), 142.0(\mathrm{CH}), 140.4\left(\mathrm{C}_{\mathrm{q}}\right), 136.2\left(\mathrm{C}_{\mathrm{q}}\right), 131.5\left(\mathrm{C}_{\mathrm{q}}\right), 131.5(\mathrm{CH}), 130.0(\mathrm{CH})$, $130.0(\mathrm{CH}), 129.1(\mathrm{CH}), 129.0(\mathrm{CH}), 128.3(\mathrm{CH}), 127.5\left(\mathrm{C}_{\mathrm{q}}\right), 68.9(\mathrm{CH}), 62.7\left(\mathrm{CH}_{2}\right), 62.2$ $\left(\mathrm{CH}_{2}\right), 47.2(\mathrm{CH}), 42.0\left(\mathrm{CH}_{2}\right), 34.2\left(\mathrm{CH}_{2}\right), 34.0\left(\mathrm{CH}_{2}\right), 31.9\left(\mathrm{CH}_{2}\right), 29.6\left(\mathrm{CH}_{2}\right), 29.4\left(\mathrm{CH}_{2}\right)$, $29.4\left(\mathrm{CH}_{2}\right), 29.3\left(\mathrm{CH}_{2}\right), 29.2\left(\mathrm{CH}_{2}\right), 29.1\left(\mathrm{CH}_{2}\right), 29.0\left(\mathrm{CH}_{2}\right), 24.9\left(\mathrm{CH}_{2}\right), 24.8\left(\mathrm{CH}_{2}\right), 22.7$ $\left(\mathrm{CH}_{2}\right), 22.6\left(\mathrm{CH}_{3}\right), 14.1\left(\mathrm{CH}_{3}\right)$.

IR (ATR): $\tilde{v}=2923,2853,1726,1569,1326,1269,1102,783,703,647 \mathrm{~cm}^{-1}$.

MS (ESI) $m / z$ (relative intensity): $1622(33)[2 \mathrm{M}+\mathrm{H}]^{+}, 834(32)[\mathrm{M}+\mathrm{Na}]^{+}, 812(100)[\mathrm{M}+\mathrm{H}]^{+}$.

HR-MS (ESI): $m / z$ calcd for $\mathrm{C}_{49} \mathrm{H}_{71} \mathrm{~N}_{4} \mathrm{O}_{6}{ }^{+}[\mathrm{M}+\mathrm{H}]^{+} 811.5368$, found 811.5372 .

\section{3-\{\{4-[3-(9-iso-Propyl-9H-purin-6-yl)benzyl]benzoyl\}oxy\}propane-1,2-diyl}

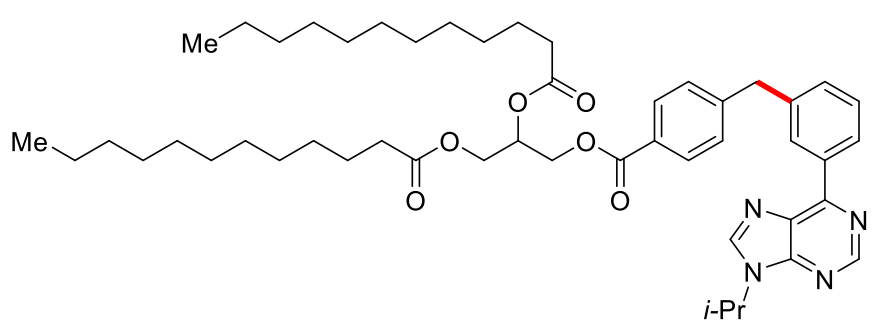

\section{didodecanoate (rac-6m)}

The general procedure $\mathbf{B}$ was followed using purine $\mathbf{1 h}(59.6 \mathrm{mg}, 0.25 \mathrm{mmol})$ and benzyl chloride rac-5k $(305 \mathrm{mg}$, $0.50 \mathrm{mmol})$. After $20 \mathrm{~h}$, purification by

column chromatography ( $n$-hexane/EtOAc 4:1) yielded rac-6m $(153 \mathrm{mg}, 75 \%$ ) as a yellow oil. ${ }^{1} \mathbf{H}-\mathbf{N M R}\left(600 \mathrm{MHz}, \mathrm{CDCl}_{3}\right): \delta=9.00(\mathrm{~s}, 1 \mathrm{H}), 8.70$ (ddd, $\left.J=7.8,1.7,1.5 \mathrm{~Hz}, 1 \mathrm{H}\right), 8.62$ (dd, $J$ $=1.8,1.7 \mathrm{~Hz}, 1 \mathrm{H}), 8.18(\mathrm{~s}, 1 \mathrm{H}), 7.93(\mathrm{~d}, J=8.3 \mathrm{~Hz}, 2 \mathrm{H}), 7.49(\mathrm{dd}, J=7.8,7.7 \mathrm{~Hz}, 1 \mathrm{H}), 7.32$ 
(d, $J=8.3 \mathrm{~Hz}, 2 \mathrm{H}), 7.31-7.27(\mathrm{~m}, 1 \mathrm{H}), 5.40$ (dddd, $J=5.9,5.8,4.3,4.2 \mathrm{~Hz}, 1 \mathrm{H}), 4.98$ (hept, $J$ $=6.8 \mathrm{~Hz}, 1 \mathrm{H}), 4.48\left(\mathrm{~d}_{\mathrm{AB}} \mathrm{d}, J=11.9,4.3 \mathrm{~Hz}, 1 \mathrm{H}\right), 4.39\left(\mathrm{~d}_{\mathrm{AB}} \mathrm{d}, J=11.9,5.8 \mathrm{~Hz}, 1 \mathrm{H}\right), 4.37(\mathrm{dd}, J$ $=11.9,4.2 \mathrm{~Hz}, 1 \mathrm{H}), 4.22(\mathrm{dd}, J=11.9,5.9 \mathrm{~Hz}, 1 \mathrm{H}), 4.17(\mathrm{~s}, 2 \mathrm{H}), 2.31(\mathrm{t}, J=7.5 \mathrm{~Hz}, 2 \mathrm{H}), 2.30$ $(\mathrm{t}, J=7.5 \mathrm{~Hz}, 2 \mathrm{H}), 1.67(\mathrm{~d}, J=6.8 \mathrm{~Hz}, 6 \mathrm{H}), 1.65-1.53(\mathrm{~m}, 4 \mathrm{H}), 1.34-1.15(\mathrm{~m}, 32 \mathrm{H}), 0.86(\mathrm{t}, J$ $=6.6 \mathrm{~Hz}, 3 \mathrm{H}), 0.86(\mathrm{t}, J=6.6 \mathrm{~Hz}, 3 \mathrm{H})$.

${ }^{13}$ C-NMR $\left(125 \mathrm{MHz}, \mathrm{CDCl}_{3}\right): \delta=173.1\left(\mathrm{C}_{\mathrm{q}}\right), 172.7\left(\mathrm{C}_{\mathrm{q}}\right), 165.8\left(\mathrm{C}_{\mathrm{q}}\right), 154.4\left(\mathrm{C}_{\mathrm{q}}\right), 152.0\left(\mathrm{C}_{\mathrm{q}}\right)$, $151.9(\mathrm{CH}), 146.7\left(\mathrm{C}_{\mathrm{q}}\right), 141.9(\mathrm{CH}), 140.3\left(\mathrm{C}_{\mathrm{q}}\right), 136.1\left(\mathrm{C}_{\mathrm{q}}\right), 131.4\left(\mathrm{C}_{\mathrm{q}}\right), 131.3(\mathrm{CH}), 129.9(\mathrm{CH})$, $129.9(\mathrm{CH}), 129.0(\mathrm{CH}), 128.9(\mathrm{CH}), 128.2(\mathrm{CH}), 127.4\left(\mathrm{C}_{\mathrm{q}}\right), 68.9(\mathrm{CH}), 62.7\left(\mathrm{CH}_{2}\right), 62.2$ $\left(\mathrm{CH}_{2}\right), 47.3(\mathrm{CH}), 42.0\left(\mathrm{CH}_{2}\right), 34.3\left(\mathrm{CH}_{2}\right), 34.1\left(\mathrm{CH}_{2}\right), 31.9\left(\mathrm{CH}_{2}\right), 29.6\left(\mathrm{CH}_{2}\right), 29.5\left(\mathrm{CH}_{2}\right)$, $29.5\left(\mathrm{CH}_{2}\right), 29.4\left(\mathrm{CH}_{2}\right), 29.3\left(\mathrm{CH}_{2}\right), 29.1\left(\mathrm{CH}_{2}\right), 29.1\left(\mathrm{CH}_{2}\right), 25.0\left(\mathrm{CH}_{2}\right), 24.9\left(\mathrm{CH}_{2}\right), 22.7$ $\left(\mathrm{CH}_{2}\right), 22.6\left(\mathrm{CH}_{3}\right), 14.2\left(\mathrm{CH}_{3}\right)$.

IR (ATR): $\tilde{v}=2922,2853,1725,1569,1326,1269,1102,783,704,647 \mathrm{~cm}^{-1}$.

MS (ESI) $m / z$ (relative intensity): $834(1)[\mathrm{M}+\mathrm{Na}]^{+}, 812(100)[\mathrm{M}+\mathrm{H}]^{+}$.

HR-MS (ESI): $m / z$ calcd for $\mathrm{C}_{49} \mathrm{H}_{71} \mathrm{~N}_{4} \mathrm{O}_{6}{ }^{+}[\mathrm{M}+\mathrm{H}]^{+} 811.5368$, found 811.5352 .

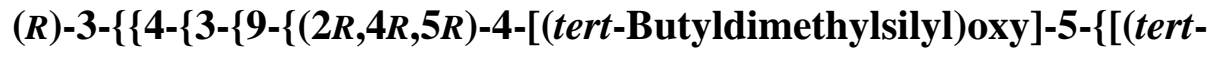

\section{butyldimethylsilyl)oxy]methyl\}tetrahydrofuran-2-yl\}-9H-purin-6-}

yl\}benzyl\}benzoyl\}oxy\}propane-1,2-diyl didodecanoate (6n)

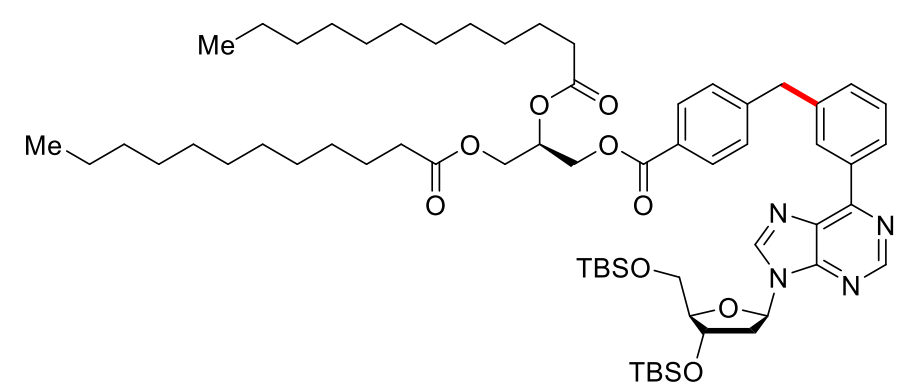

The general procedure $\mathbf{B}$ was followed using purine 1n (135 mg, $0.25 \mathrm{mmol})$ and benzyl chloride $R-\mathbf{5 k}$ (305 mg, $0.50 \mathrm{mmol})$. After $20 \mathrm{~h}$, purification by column chromatography (n-hexane/EtOAc 6:1) yielded $\mathbf{6 n}$

(195 $\mathrm{mg}, 70 \%)$ as a yellow oil.

${ }^{1} \mathbf{H}-\mathbf{N M R}\left(400 \mathrm{MHz}, \mathrm{CDCl}_{3}\right): \delta=8.99(\mathrm{~s}, 1 \mathrm{H}), 8.70$ (ddd, $\left.J=7.8,1.6,1.4 \mathrm{~Hz}, 1 \mathrm{H}\right), 8.61$ (dd, $J$ $=1.8,1.6 \mathrm{~Hz}, 1 \mathrm{H}), 8.42(\mathrm{~s}, 1 \mathrm{H}), 7.93(\mathrm{~d}, J=8.3 \mathrm{~Hz}, 2 \mathrm{H}), 7.49(\mathrm{dd}, J=7.8,7.7 \mathrm{~Hz}, 1 \mathrm{H}), 7.32$ $(\mathrm{d}, J=8.3 \mathrm{~Hz}, 2 \mathrm{H}), 7.31-7.27(\mathrm{~m}, 1 \mathrm{H}), 6.57(\mathrm{dd}, J=6.7,6.3 \mathrm{~Hz}, 1 \mathrm{H}), 5.40$ (dddd, $J=6.0,5.9$, $4.4,4.3 \mathrm{~Hz}, 1 \mathrm{H}), 4.65(\mathrm{ddd}, J=5.9,3.6,3.3 \mathrm{~Hz}, 1 \mathrm{H}), 4.49\left(\mathrm{~d}_{\mathrm{AB}}, J=11.9,4.3 \mathrm{~Hz}, 1 \mathrm{H}\right), 4.40$ $\left(\mathrm{d}_{\mathrm{ABd}}, J=11.9,6.0 \mathrm{~Hz}, 1 \mathrm{H}\right), 4.37(\mathrm{dd}, J=11.9,4.4 \mathrm{~Hz}, 1 \mathrm{H}), 4.22(\mathrm{dd}, J=11.9,5.9 \mathrm{~Hz}, 1 \mathrm{H})$, $4.17(\mathrm{~s}, 2 \mathrm{H}), 4.06(\mathrm{ddd}, J=4.3,3.3,3.3 \mathrm{~Hz}, 1 \mathrm{H}), 3.88\left(\mathrm{~d}_{\mathrm{ABd}} \mathrm{d}, J=11.2,4.3 \mathrm{~Hz}, 1 \mathrm{H}\right), 3.80\left(\mathrm{~d}_{\mathrm{AB}}\right.$, $J=11.2,3.3 \mathrm{~Hz}, 1 \mathrm{H}), 2.71(\mathrm{ddd}, J=13.0,6.7,5.9 \mathrm{~Hz}, 1 \mathrm{H}), 2.49(\mathrm{ddd}, J=13.0,6.3,3.6 \mathrm{~Hz}$, $1 \mathrm{H}), 2.31(\mathrm{t}, J=7.5 \mathrm{~Hz}, 2 \mathrm{H}), 2.31(\mathrm{t}, J=7.6 \mathrm{~Hz}, 2 \mathrm{H}), 1.67-1.54(\mathrm{~m}, 4 \mathrm{H}), 1.33-1.17(\mathrm{~m}, 32 \mathrm{H})$, 
$0.93(\mathrm{~s}, 9 \mathrm{H}), 0.91(\mathrm{~s}, 9 \mathrm{H}), 0.87(\mathrm{t}, J=6.9 \mathrm{~Hz}, 3 \mathrm{H}), 0.87(\mathrm{t}, J=6.9 \mathrm{~Hz}, 3 \mathrm{H}), 0.12(\mathrm{~s}, 6 \mathrm{H}), 0.09$ (s, 3H), $0.09(\mathrm{~s}, 3 \mathrm{H})$.

${ }^{13}$ C-NMR $\left(100 \mathrm{MHz}, \mathrm{CDCl}_{3}\right): \delta=173.3\left(\mathrm{C}_{\mathrm{q}}\right), 172.9\left(\mathrm{C}_{\mathrm{q}}\right), 165.9\left(\mathrm{C}_{\mathrm{q}}\right), 154.7\left(\mathrm{C}_{\mathrm{q}}\right), 152.2(\mathrm{CH})$, $152.0\left(\mathrm{C}_{\mathrm{q}}\right), 146.9\left(\mathrm{C}_{\mathrm{q}}\right), 142.8(\mathrm{CH}), 140.4\left(\mathrm{C}_{\mathrm{q}}\right), 136.0\left(\mathrm{C}_{\mathrm{q}}\right), 131.7\left(\mathrm{C}_{\mathrm{q}}\right), 131.5(\mathrm{CH}), 130.0(\mathrm{CH})$, $129.1(\mathrm{CH}), 129.0(\mathrm{CH}), 128.4(\mathrm{CH}), 127.5\left(\mathrm{C}_{\mathrm{q}}\right), 88.0(\mathrm{CH}), 84.5(\mathrm{CH}), 72.0(\mathrm{CH}), 68.9(\mathrm{CH})$, $62.8\left(\mathrm{CH}_{2}\right), 62.7\left(\mathrm{CH}_{2}\right), 62.2\left(\mathrm{CH}_{2}\right), 42.0\left(\mathrm{CH}_{2}\right), 41.1\left(\mathrm{CH}_{2}\right), 34.2\left(\mathrm{CH}_{2}\right), 34.0\left(\mathrm{CH}_{2}\right), 31.9$ $\left(\mathrm{CH}_{2}\right), 29.6\left(\mathrm{CH}_{2}\right), 29.4\left(\mathrm{CH}_{2}\right), 29.4\left(\mathrm{CH}_{2}\right), 29.3\left(\mathrm{CH}_{2}\right), 29.2\left(\mathrm{CH}_{2}\right), 29.1\left(\mathrm{CH}_{2}\right), 29.0\left(\mathrm{CH}_{2}\right)$, $25.9\left(\mathrm{CH}_{3}\right), 25.8\left(\mathrm{CH}_{3}\right), 24.9\left(\mathrm{CH}_{2}\right), 24.8\left(\mathrm{CH}_{2}\right), 22.7\left(\mathrm{CH}_{2}\right), 18.4\left(\mathrm{C}_{\mathrm{q}}\right), 18.0\left(\mathrm{C}_{\mathrm{q}}\right), 14.1\left(\mathrm{CH}_{3}\right)$, $-4.7\left(\mathrm{CH}_{3}\right),-4.8\left(\mathrm{CH}_{3}\right),-5.4\left(\mathrm{CH}_{3}\right),-5.5\left(\mathrm{CH}_{3}\right)$.

IR (ATR): $\tilde{v}=2925,2854,1727,1570,1256,1107,835,778,703,646 \mathrm{~cm}^{-1}$.

MS (ESI) $m / z$ (relative intensity): $2227(38)[2 \mathrm{M}+\mathrm{H}]^{+}, 1136(39)[\mathrm{M}+\mathrm{Na}]^{+}, 1114(100)[\mathrm{M}+\mathrm{H}]^{+}$.

HR-MS (ESI): $m / z$ calcd for $\mathrm{C}_{63} \mathrm{H}_{101} \mathrm{~N}_{4} \mathrm{O}_{9} \mathrm{Si}_{2}{ }^{+}[\mathrm{M}+\mathrm{H}]^{+} 1113.7102$, found 1113.7102 .

\section{3-\{\{4-[3-(9-iso-Propyl-9H-purin-6-yl)benzyl]benzoyl\}oxy\}propane-1,2-diyl dioleate (6o)}

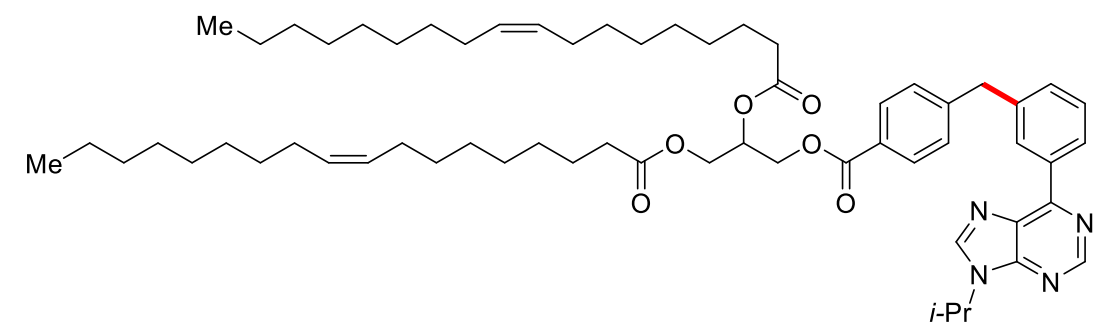

The general procedure $\mathbf{B}$ was followed using purine 1h $(59.6 \mathrm{mg}, \quad 0.25 \mathrm{mmol})$ and benzyl chloride $\mathbf{5 l}$ (387 mg, $0.50 \mathrm{mmol})$. After $20 \mathrm{~h}$, purification by column chromatography ( $n$-hexane/EtOAc 4:1) yielded 6o (174 mg, 71\%) as a pale yellow oil.

${ }^{1} \mathbf{H}$-NMR $\left(600 \mathrm{MHz}, \mathrm{CDCl}_{3}\right): \delta=9.00(\mathrm{~s}, 1 \mathrm{H}), 8.70(\mathrm{ddd}, J=7.8,1.7,1.5 \mathrm{~Hz}, 1 \mathrm{H}), 8.62(\mathrm{dd}, J$ $=1.8,1.7 \mathrm{~Hz}, 1 \mathrm{H}), 8.18(\mathrm{~s}, 1 \mathrm{H}), 7.93(\mathrm{~d}, J=8.3 \mathrm{~Hz}, 2 \mathrm{H}), 7.49(\mathrm{dd}, J=7.8,7.7 \mathrm{~Hz}, 1 \mathrm{H}), 7.32$ (d, $J=8.3 \mathrm{~Hz}, 2 \mathrm{H}), 7.31-7.27(\mathrm{~m}, 1 \mathrm{H}), 5.44-5.25$ (m, 5H), 4.98 (hept, $J=6.8 \mathrm{~Hz}, 1 \mathrm{H}), 4.48$ $\left(\mathrm{d}_{\mathrm{AB}} \mathrm{d}, J=11.9,4.3 \mathrm{~Hz}, 1 \mathrm{H}\right), 4.39\left(\mathrm{~d}_{\mathrm{AB}} \mathrm{d}, J=11.9,5.6 \mathrm{~Hz}, 1 \mathrm{H}\right), 4.37(\mathrm{dd}, J=11.9,4.1 \mathrm{~Hz}, 1 \mathrm{H})$, $4.22(\mathrm{dd}, J=11.9,5.9 \mathrm{~Hz}, 1 \mathrm{H}), 4.18(\mathrm{~s}, 2 \mathrm{H}), 2.31(\mathrm{t}, J=7.5 \mathrm{~Hz}, 2 \mathrm{H}), 2.30(\mathrm{t}, J=7.5 \mathrm{~Hz}, 2 \mathrm{H})$, 2.08-1.91 (m, 8H), $1.67(\mathrm{~d}, J=6.8 \mathrm{~Hz}, 6 \mathrm{H}), 1.64-1.52(\mathrm{~m}, 4 \mathrm{H}), 1.37-1.18$ (m, 40H), 0.87 (t, $J$ $=6.6 \mathrm{~Hz}, 3 \mathrm{H}), 0.87(\mathrm{t}, J=6.6 \mathrm{~Hz}, 3 \mathrm{H})$.

${ }^{13}$ C-NMR (125 MHz, $\left.\mathrm{CDCl}_{3}\right): \delta=173.1\left(\mathrm{C}_{\mathrm{q}}\right), 172.7\left(\mathrm{C}_{\mathrm{q}}\right), 165.8\left(\mathrm{C}_{\mathrm{q}}\right), 154.4\left(\mathrm{C}_{\mathrm{q}}\right), 152.0\left(\mathrm{C}_{\mathrm{q}}\right)$, $151.9(\mathrm{CH}), 146.7\left(\mathrm{C}_{\mathrm{q}}\right), 141.8(\mathrm{CH}), 140.3\left(\mathrm{C}_{\mathrm{q}}\right), 136.1\left(\mathrm{C}_{\mathrm{q}}\right), 131.4\left(\mathrm{C}_{\mathrm{q}}\right), 131.3(\mathrm{CH}), 129.9(\mathrm{CH})$, $129.9(\mathrm{CH}), 129.6(\mathrm{CH}), 129.6(\mathrm{CH}), 129.0(\mathrm{CH}), 128.8(\mathrm{CH}), 128.2(\mathrm{CH}), 127.4\left(\mathrm{C}_{\mathrm{q}}\right), 68.9$ $(\mathrm{CH}), 62.7\left(\mathrm{CH}_{2}\right), 62.2\left(\mathrm{CH}_{2}\right), 47.3(\mathrm{CH}), 42.0\left(\mathrm{CH}_{2}\right), 34.2\left(\mathrm{CH}_{2}\right), 34.1\left(\mathrm{CH}_{2}\right), 31.9\left(\mathrm{CH}_{2}\right), 29.8$ $\left(\mathrm{CH}_{2}\right), 29.7\left(\mathrm{CH}_{2}\right), 29.6\left(\mathrm{CH}_{2}\right), 29.3\left(\mathrm{CH}_{2}\right), 29.2\left(\mathrm{CH}_{2}\right), 29.1\left(\mathrm{CH}_{2}\right), 29.1\left(\mathrm{CH}_{2}\right), 29.1\left(\mathrm{CH}_{2}\right)$, $27.3\left(\mathrm{CH}_{2}\right), 27.2\left(\mathrm{CH}_{2}\right), 24.9\left(\mathrm{CH}_{2}\right), 24.9\left(\mathrm{CH}_{2}\right), 22.7\left(\mathrm{CH}_{2}\right), 22.6\left(\mathrm{CH}_{3}\right), 14.2\left(\mathrm{CH}_{3}\right)$. 
IR (ATR): $\tilde{v}=2923,2853,1726,1570,1458,1269,1175,1096,703,647 \mathrm{~cm}^{-1}$.

MS (ESI) $m / z$ (relative intensity): $1951(14)[2 \mathrm{M}+\mathrm{H}]^{+}, 976(100)[\mathrm{M}+\mathrm{H}]^{+}$.

HR-MS (ESI): $m / z$ calcd for $\mathrm{C}_{61} \mathrm{H}_{91} \mathrm{~N}_{4} \mathrm{O}_{6}{ }^{+}[\mathrm{M}+\mathrm{H}]^{+}$975.6933, found 975.6929.

$3-\{\{4-\{3-\{9-\{(3 \mathrm{a} R, 4 R, 6 R, 6 \mathrm{a} R)-6-\{[($ Diethoxyphosphoryl)oxy $]$ methyl $\}-2,2-$

dimethyltetrahydrofuro[3,4- $d][1,3]$ dioxol-4-yl\}-9H-purin-6-

yl\}benzyl\}benzoyl\}oxy\}propane-1,2-diyl dioleate (6p)

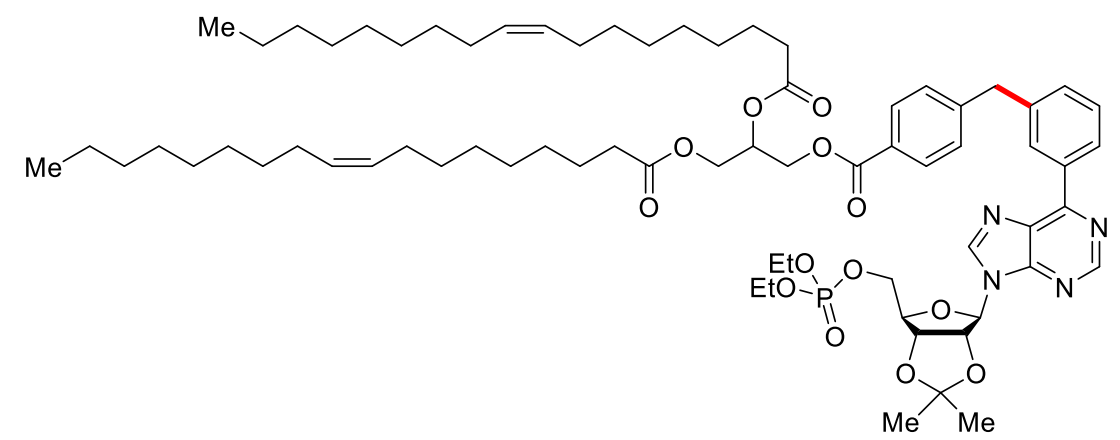

The general procedure $\mathbf{B}$ was followed using purine $1 \mathbf{m}(126 \mathrm{mg}, 0.25 \mathrm{mmol})$ and benzyl chloride $\mathbf{5 l}$ (387 mg, $\quad 0.50 \mathrm{mmol}$ ). After $20 \mathrm{~h}$, purification by column chromatography

( $n$-hexane/EtOAc 3:2) yielded 6p (172 $\mathrm{mg}, 55 \%)$ as a greenish yellow oil.

${ }^{1} \mathbf{H}-\mathbf{N M R}\left(600 \mathrm{MHz}, \mathrm{CDCl}_{3}\right): \delta=9.01(\mathrm{~s}, 1 \mathrm{H}), 8.69$ (ddd, $\left.J=7.8,1.6,1.4 \mathrm{~Hz}, 1 \mathrm{H}\right), 8.61(\mathrm{dd}, J$ $=1.9,1.6 \mathrm{~Hz}, 1 \mathrm{H}), 8.28(\mathrm{~s}, 1 \mathrm{H}), 7.93(\mathrm{~d}, J=8.4 \mathrm{~Hz}, 2 \mathrm{H}), 7.48(\mathrm{dd}, J=7.8,7.7 \mathrm{~Hz}, 1 \mathrm{H}), 7.31$ $(\mathrm{d}, J=8.4 \mathrm{~Hz}, 2 \mathrm{H}), 7.31-7.28(\mathrm{~m}, 1 \mathrm{H}), 6.26(\mathrm{~d}, J=2.6 \mathrm{~Hz}, 1 \mathrm{H}), 5.45(\mathrm{dd}, J=6.3,2.6 \mathrm{~Hz}, 1 \mathrm{H})$, 5.39 (dddd, $J=6.0,5.9,4.3,4.3 \mathrm{~Hz}, 1 \mathrm{H}), 5.36-5.27(\mathrm{~m}, 4 \mathrm{H}), 5.13(\mathrm{dd}, J=6.3,3.1 \mathrm{~Hz}, 1 \mathrm{H})$, 4.53 (dddd, $J=4.5,4.5,3.1,1.1 \mathrm{~Hz}, 1 \mathrm{H}), 4.48\left(\mathrm{~d}_{\mathrm{ABd}}, J=11.9,4.3 \mathrm{~Hz}, 1 \mathrm{H}\right), 4.38\left(\mathrm{~d}_{\mathrm{ABd}}, J=\right.$ $11.9,6.0 \mathrm{~Hz}, 1 \mathrm{H}), 4.36(\mathrm{dd}, J=11.9,4.3 \mathrm{~Hz}, 1 \mathrm{H}), 4.29(\mathrm{ddd}, J=10.9,6.3,4.5 \mathrm{~Hz}, 1 \mathrm{H}), 4.21$ (dd, $J=11.9,5.9 \mathrm{~Hz}, 1 \mathrm{H}), 4.20$ (ddd, $J=10.9,6.8,4.5 \mathrm{~Hz}, 1 \mathrm{H}), 4.17$ (s, 2H), 4.09-4.00 (m, $4 \mathrm{H}), 2.30(\mathrm{t}, J=7.5 \mathrm{~Hz}, 2 \mathrm{H}), 2.30(\mathrm{t}, J=7.6 \mathrm{~Hz}, 2 \mathrm{H}), 2.06-1.95(\mathrm{~m}, 8 \mathrm{H}), 1.64(\mathrm{~s}, 3 \mathrm{H}), 1.63-$ $1.55(\mathrm{~m}, 4 \mathrm{H}), 1.41$ (s, 3H), 1.36-1.19 (m, 46H), 0.86 (t, $J=7.2 \mathrm{~Hz}, 3 \mathrm{H}), 0.86$ (t, $J=7.1 \mathrm{~Hz}$, $3 \mathrm{H})$.

${ }^{13} \mathrm{C}-\mathrm{NMR}\left(125 \mathrm{MHz}, \mathrm{CDCl}_{3}\right): \delta=173.1\left(\mathrm{C}_{\mathrm{q}}\right), 172.7\left(\mathrm{C}_{\mathrm{q}}\right), 165.7\left(\mathrm{C}_{\mathrm{q}}\right), 154.9\left(\mathrm{C}_{\mathrm{q}}\right), 152.3(\mathrm{CH})$, 151.6 $\left(\mathrm{C}_{\mathrm{q}}\right), 146.6\left(\mathrm{C}_{\mathrm{q}}\right), 143.1(\mathrm{CH}), 140.4\left(\mathrm{C}_{\mathrm{q}}\right), 135.7\left(\mathrm{C}_{\mathrm{q}}\right), 131.6\left(\mathrm{C}_{\mathrm{q}}\right.$ and $\left.\mathrm{CH}\right), 129.9(\mathrm{CH})$, $129.9(\mathrm{CH}), 129.8(\mathrm{CH}), 129.6(\mathrm{CH}), 129.6(\mathrm{CH}), 129.0(\mathrm{CH}), 128.9(\mathrm{CH}), 128.3(\mathrm{CH}), 127.4$ $\left(\mathrm{C}_{\mathrm{q}}\right), 114.6\left(\mathrm{C}_{\mathrm{q}}\right), 91.0(\mathrm{CH}), 85.3\left(\mathrm{~d},{ }^{3} J_{\mathrm{C}-\mathrm{P}}=8 \mathrm{~Hz}, \mathrm{CH}\right), 84.2(\mathrm{CH}), 81.3(\mathrm{CH}), 68.9(\mathrm{CH}), 66.6$ $\left(\mathrm{d},{ }^{2} J_{\mathrm{C}-\mathrm{P}}=6 \mathrm{~Hz}, \mathrm{CH}_{2}\right), 64.1\left(\mathrm{~d},{ }^{2} J_{\mathrm{C}-\mathrm{P}}=5 \mathrm{~Hz}, \mathrm{CH}_{2}\right), 64.1\left(\mathrm{~d},{ }^{2} J_{\mathrm{C}-\mathrm{P}}=5 \mathrm{~Hz}, \mathrm{CH}_{2}\right), 62.7\left(\mathrm{CH}_{2}\right)$, $62.2\left(\mathrm{CH}_{2}\right), 42.0\left(\mathrm{CH}_{2}\right), 34.2\left(\mathrm{CH}_{2}\right), 34.1\left(\mathrm{CH}_{2}\right), 31.9\left(\mathrm{CH}_{2}\right), 29.8\left(\mathrm{CH}_{2}\right), 29.7\left(\mathrm{CH}_{2}\right), 29.6$ $\left(\mathrm{CH}_{2}\right), 29.3\left(\mathrm{CH}_{2}\right), 29.2\left(\mathrm{CH}_{2}\right), 29.1\left(\mathrm{CH}_{2}\right), 29.1\left(\mathrm{CH}_{2}\right), 29.0\left(\mathrm{CH}_{2}\right), 27.3\left(\mathrm{CH}_{2}\right), 27.2\left(\mathrm{CH}_{3}\right)$, $27.2\left(\mathrm{CH}_{2}\right), 25.4\left(\mathrm{CH}_{3}\right), 24.9\left(\mathrm{CH}_{2}\right), 24.9\left(\mathrm{CH}_{2}\right), 22.7\left(\mathrm{CH}_{2}\right), 16.11\left(\mathrm{~d},{ }^{3} J_{\mathrm{C}-\mathrm{P}}=7 \mathrm{~Hz}, \mathrm{CH}_{3}\right), 14.2$ $\left(\mathrm{CH}_{3}\right)$. 
${ }^{31} \mathbf{P}-\mathbf{N M R}\left(162 \mathrm{MHz}, \mathrm{CDCl}_{3}\right): \delta=(-0.8)-(-1.2)(\mathrm{m})$.

IR (ATR): $\tilde{v}=2923,2853,1741,1569,1268,1097,1021,703,645 \mathrm{~cm}^{-1}$.

MS (ESI) $m / z$ (relative intensity): $2484(15)[2 \mathrm{M}+\mathrm{H}]^{+}, 1264(32)[\mathrm{M}+\mathrm{Na}]^{+}, 1242(100)[\mathrm{M}+\mathrm{H}]^{+}$, $632(4)[\mathrm{M}+\mathrm{H}+\mathrm{Na}]^{2+}$.

HR-MS (ESI): $m / z$ calcd for $\mathrm{C}_{70} \mathrm{H}_{106} \mathrm{~N}_{4} \mathrm{O}_{13} \mathrm{P}^{+}[\mathrm{M}+\mathrm{H}]^{+}$1241.7489, found 1241.7480 .

$(2 R, 3 R, 4 S, 5 R, 6 S)$-2-(Acetoxymethyl)-6-\{4-[3-(9-iso-propyl-9H-purin-6yl)benzyl]phenoxy\}tetrahydro-2H-pyran-3,4,5-triyl triacetate (6q)

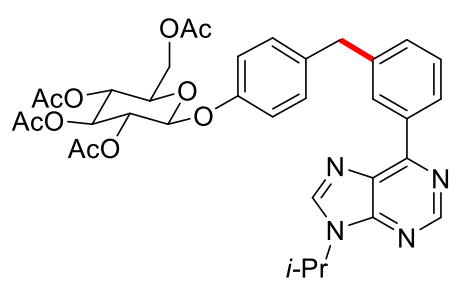

The general procedure $\mathbf{B}$ was followed using purine $\mathbf{1 h}(119 \mathrm{mg}$, $0.50 \mathrm{mmol})$ and benzyl chloride $\mathbf{5 m}(473 \mathrm{mg}, 1.00 \mathrm{mmol})$. After $20 \mathrm{~h}$, purification by column chromatography ( $n$-hexane/EtOAc 1:1) yielded 6q (240 $\mathrm{mg}, 71 \%)$ as a white solid.

${ }^{1} \mathbf{H}-\mathbf{N M R}\left(300 \mathrm{MHz}, \mathrm{CDCl}_{3}\right): \delta=9.00(\mathrm{~s}, 1 \mathrm{H}), 8.67$ (ddd, $\left.J=7.8,1.8,1.2 \mathrm{~Hz}, 1 \mathrm{H}\right), 8.58$ (ddd, $J=1.8,1.8,0.6 \mathrm{~Hz}, 1 \mathrm{H}), 8.18(\mathrm{~s}, 1 \mathrm{H}), 7.47(\mathrm{ddd}, J=7.8,7.7,0.5 \mathrm{~Hz}, 1 \mathrm{H}), 7.29(\mathrm{ddd}, J=7.7$, $1.8,1.2 \mathrm{~Hz}, 1 \mathrm{H}), 7.16(\mathrm{~d}, J=8.7 \mathrm{~Hz}, 2 \mathrm{H}), 6.91(\mathrm{~d}, J=8.7 \mathrm{~Hz}, 2 \mathrm{H}), 5.29-5.22(\mathrm{~m}, 2 \mathrm{H}), 5.14$ $(\mathrm{dd}, J=9.9,9.7 \mathrm{~Hz}, 1 \mathrm{H}), 5.03(\mathrm{~d}, J=7.8 \mathrm{~Hz}, 1 \mathrm{H}), 4.98$ (hept, $J=6.8 \mathrm{~Hz}, 1 \mathrm{H}), 4.27$ (dd, $J=$ 12.3, $5.3 \mathrm{~Hz}, 1 \mathrm{H}), 4.14(\mathrm{dd}, J=12.3,2.5 \mathrm{~Hz}, 1 \mathrm{H}), 4.08$ (s, 2H), 3.82 (ddd, $J=9.9,5.3,2.5 \mathrm{~Hz}$, 1H), 2.04 (s, 3H), 2.04 (s, 3H), 2.03 (s, 3H), 2.02 (s, 3H), 1.68 (d, J=6.8 Hz, 6H).

${ }^{13} \mathrm{C}-\mathrm{NMR}\left(125 \mathrm{MHz}, \mathrm{CDCl}_{3}\right): \delta=170.4\left(\mathrm{C}_{\mathrm{q}}\right), 170.0\left(\mathrm{C}_{\mathrm{q}}\right), 169.2\left(\mathrm{C}_{\mathrm{q}}\right), 169.1\left(\mathrm{C}_{\mathrm{q}}\right), 155.2\left(\mathrm{C}_{\mathrm{q}}\right)$, 154.6 $\left(\mathrm{C}_{\mathrm{q}}\right), 152.0\left(\mathrm{C}_{\mathrm{q}}\right), 151.9(\mathrm{CH}), 141.8(\mathrm{CH}), 141.4\left(\mathrm{C}_{\mathrm{q}}\right), 136.1\left(\mathrm{C}_{\mathrm{q}}\right), 135.9\left(\mathrm{C}_{\mathrm{q}}\right), 131.5\left(\mathrm{C}_{\mathrm{q}}\right)$, $131.3(\mathrm{CH}), 129.9(\mathrm{CH}), 129.8(\mathrm{CH}), 128.7(\mathrm{CH}), 128.0(\mathrm{CH}), 117.1(\mathrm{CH}), 99.3(\mathrm{CH}), 72.8$ $(\mathrm{CH}), 72.0(\mathrm{CH}), 71.2(\mathrm{CH}), 68.4(\mathrm{CH}), 62.0\left(\mathrm{CH}_{2}\right), 47.3(\mathrm{CH}), 41.6\left(\mathrm{CH}_{2}\right), 22.6\left(\mathrm{CH}_{3}\right), 20.7$ $\left(\mathrm{CH}_{3}\right), 20.7\left(\mathrm{CH}_{3}\right), 20.7\left(\mathrm{CH}_{3}\right), 20.6\left(\mathrm{CH}_{3}\right)$.

IR (ATR): $\tilde{v}=2977,1746,1570,1508,1367,1213,1035,704,647,598 \mathrm{~cm}^{-1}$.

m.p.: $182-184{ }^{\circ} \mathrm{C}$.

MS (ESI) $m / z$ (relative intensity): $697(8)[\mathrm{M}+\mathrm{Na}]^{+}, 675(100)[\mathrm{M}+\mathrm{H}]^{+}$.

HR-MS (ESI): $m / z$ calcd for $\mathrm{C}_{35} \mathrm{H}_{39} \mathrm{~N}_{4} \mathrm{O}_{10}{ }^{+}[\mathrm{M}+\mathrm{H}]^{+}$675.2661, found 675.2656. 
$(2 R, 3 R, 4 S, 5 R, 6 S)-2-($ Acetoxymethyl)-6- $\{4-\{3-\{9-[(2 R, 3 R, 4 R, 5 R)-3,4-d i a c e t o x y-5-$ (acetoxymethyl)tetrahydrofuran-2-yl]-9H-purin-6-yl\}benzyl\}phenoxy\}tetrahydro-2Hpyran-3,4,5-triyl triacetate (6r)

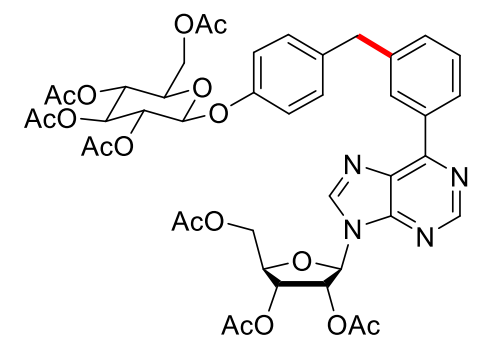

The general procedure B was followed using purine 10 (182 $\mathrm{mg}$, $0.40 \mathrm{mmol})$ and benzyl chloride $\mathbf{5 m}(379 \mathrm{mg}, 0.80 \mathrm{mmol})$. After $20 \mathrm{~h}$, purification by column chromatography ( $n$-hexane/EtOAc $2: 3)$ yielded $6 \mathbf{r}(231 \mathrm{mg}, 67 \%)$ as a viscous pale yellow oil.

${ }^{1} \mathbf{H}-\mathbf{N M R}\left(600 \mathrm{MHz}, \mathrm{CDCl}_{3}\right): \delta=9.01(\mathrm{~s}, 1 \mathrm{H}), 8.64$ (ddd, $J=7.8$, 1.8, $1.2 \mathrm{~Hz}, 1 \mathrm{H}), 8.56(\mathrm{dd}, J=1.8,1.7 \mathrm{~Hz}, 1 \mathrm{H}), 8.27(\mathrm{~s}, 1 \mathrm{H}), 7.47(\mathrm{dd}, J=7.8,7.6 \mathrm{~Hz}, 1 \mathrm{H})$, 7.30 (ddd, $J=7.6,1.7,1.2 \mathrm{~Hz}, 1 \mathrm{H}), 7.16(\mathrm{~d}, J=8.7 \mathrm{~Hz}, 2 \mathrm{H}), 6.91$ (d, $J=8.7 \mathrm{~Hz}, 2 \mathrm{H}), 6.29$ (d, $J=5.4 \mathrm{~Hz}, 1 \mathrm{H}), 6.00(\mathrm{dd}, J=5.5,5.4 \mathrm{~Hz}, 1 \mathrm{H}), 5.70(\mathrm{dd}, J=5.5,4.5 \mathrm{~Hz}, 1 \mathrm{H}), 5.28-5.22(\mathrm{~m}$, 2H), $5.14(\mathrm{dd}, J=10.0,9.1 \mathrm{~Hz}, 1 \mathrm{H}), 5.02(\mathrm{~d}, J=7.6 \mathrm{~Hz}, 1 \mathrm{H}), 4.49-4.45$ (m, 2H), 4.42-4.37 (m, 1H), $4.27(\mathrm{dd}, J=12.3,5.3 \mathrm{~Hz}, 1 \mathrm{H}), 4.13(\mathrm{dd}, J=12.3,2.4 \mathrm{~Hz}, 1 \mathrm{H}), 4.07$ (s, 2H), 3.82 (ddd, $J=10.1,5.3,2.4 \mathrm{~Hz}, 1 \mathrm{H}), 2.16$ (s, 3H), 2.13 (s, 3H), 2.08 (s, 3H), 2.04 (s, 3H), 2.03 (s, $3 \mathrm{H}), 2.03(\mathrm{~s}, 3 \mathrm{H}), 2.02(\mathrm{~s}, 3 \mathrm{H})$.

${ }^{13}$ C-NMR (125 MHz, $\left.\mathrm{CDCl}_{3}\right): \delta=170.4\left(\mathrm{C}_{\mathrm{q}}\right), 170.1\left(\mathrm{C}_{\mathrm{q}}\right), 170.0\left(\mathrm{C}_{\mathrm{q}}\right), 169.4\left(\mathrm{C}_{\mathrm{q}}\right), 169.2\left(\mathrm{C}_{\mathrm{q}}\right)$, $169.1\left(\mathrm{C}_{\mathrm{q}}\right), 155.3\left(\mathrm{C}_{\mathrm{q}}\right), 155.2\left(\mathrm{C}_{\mathrm{q}}\right), 152.5(\mathrm{CH}), 151.9\left(\mathrm{C}_{\mathrm{q}}\right), 142.4(\mathrm{CH}), 141.5\left(\mathrm{C}_{\mathrm{q}}\right), 135.9\left(\mathrm{C}_{\mathrm{q}}\right)$, $135.5\left(\mathrm{C}_{\mathrm{q}}\right), 131.6(\mathrm{CH}), 131.6\left(\mathrm{C}_{\mathrm{q}}\right), 129.9(\mathrm{CH}), 129.8(\mathrm{CH}), 128.8(\mathrm{CH}), 128.0(\mathrm{CH}), 117.1$ $(\mathrm{CH}), 99.3(\mathrm{CH}), 86.4(\mathrm{CH}), 80.4(\mathrm{CH}), 73.1(\mathrm{CH}), 72.8(\mathrm{CH}), 72.0(\mathrm{CH}), 71.2(\mathrm{CH}), 70.7$ $(\mathrm{CH}), 68.3(\mathrm{CH}), 63.1\left(\mathrm{CH}_{2}\right), 62.0\left(\mathrm{CH}_{2}\right), 41.2\left(\mathrm{CH}_{2}\right), 20.8\left(\mathrm{CH}_{3}\right), 20.7\left(\mathrm{CH}_{3}\right), 20.7\left(\mathrm{CH}_{3}\right), 20.6$ $\left(\mathrm{CH}_{3}\right), 20.6\left(\mathrm{CH}_{3}\right), 20.6\left(\mathrm{CH}_{3}\right), 20.4\left(\mathrm{CH}_{3}\right)$.

IR (ATR): $\tilde{v}=1745,1569,1508,1368,1216,1038,907,703,642,600 \mathrm{~cm}^{-1}$.

MS (ESI) $m / z$ (relative intensity): $913(37)[\mathrm{M}+\mathrm{Na}]^{+}, 891(100)[\mathrm{M}+\mathrm{H}]^{+}, 465$ (38).

HR-MS (ESI): $m / z$ calcd for $\mathrm{C}_{43} \mathrm{H}_{47} \mathrm{~N}_{4} \mathrm{O}_{17}{ }^{+}[\mathrm{M}+\mathrm{H}]^{+}$891.2931, found 891.2923.

$(2 R, 3 R, 4 S, 5 R, 6 S)-2-($ Acetoxymethyl)-6- $\{4-\{3-\{9-\{(3 \mathrm{a} R, 4 R, 6 R, 6 \mathrm{a} R)-6-$

$\{[($ diethoxyphosphoryl)oxy]methyl $\}-2,2-d i m e t h y l t e t r a h y d r o f u r o[3,4-d][1,3]$ dioxol-4-yl $\}$ -

$9 H$-purin-6-yl\}benzyl\}phenoxy\}tetrahydro-2H-pyran-3,4,5-triyl triacetate $(6 \mathrm{~s})$

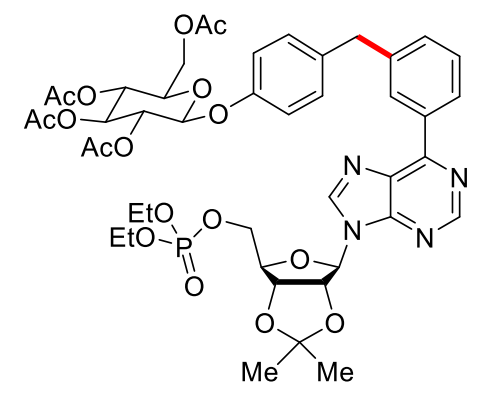

The general procedure B was followed using purine 1m $(99.0 \mathrm{mg}$, $0.20 \mathrm{mmol})$ and benzyl chloride $\mathbf{5 m}(189 \mathrm{mg}, 0.40 \mathrm{mmol})$. After $20 \mathrm{~h}$, purification by column chromatography ( $n$-hexane/EtOAc $1: 4)$ yielded $6 \mathbf{s}(130 \mathrm{mg}, 69 \%)$ as a viscous pale yellow oil.

${ }^{1} \mathbf{H}$-NMR (600 MHz, $\left.\mathrm{CDCl}_{3}\right): \delta=8.99(\mathrm{~s}, 1 \mathrm{H}), 8.64$ (ddd, $J=7.8$, 1.6, 1.4 Hz, 1H), 8.55 (dd, $J=1.6,1.6 \mathrm{~Hz}, 1 \mathrm{H}), 8.26$ (s, 1H), 7.45 
$(\mathrm{dd}, J=7.8,7.8 \mathrm{~Hz}, 1 \mathrm{H}), 7.28(\mathrm{ddd}, J=7.8,1.6,1.4 \mathrm{~Hz}, 1 \mathrm{H}), 7.14(\mathrm{~d}, J=8.7 \mathrm{~Hz}, 2 \mathrm{H}), 6.89$ (d, $J=8.7 \mathrm{~Hz}, 2 \mathrm{H}), 6.24(\mathrm{~d}, J=2.5 \mathrm{~Hz}, 1 \mathrm{H}), 5.43(\mathrm{dd}, J=6.3,2.5 \mathrm{~Hz}, 1 \mathrm{H}), 5.27-5.20(\mathrm{~m}, 2 \mathrm{H})$, $5.12(\mathrm{dd}, J=10.1,9.1 \mathrm{~Hz}, 1 \mathrm{H}), 5.10(\mathrm{dd}, J=6.3,3.0 \mathrm{~Hz}, 1 \mathrm{H}), 5.01(\mathrm{~d}, J=7.7 \mathrm{~Hz}, 1 \mathrm{H}), 4.51$ (dddd, $J=5.1,4.5,3.0,1.2 \mathrm{~Hz}, 1 \mathrm{H}), 4.27$ (ddd, $J=11.0,6.4,4.5 \mathrm{~Hz}, 1 \mathrm{H}), 4.25(\mathrm{dd}, J=12.2$, $5.3 \mathrm{~Hz}, 1 \mathrm{H}), 4.19$ (ddd, $J=11.0,6.9,5.1 \mathrm{~Hz}, 1 \mathrm{H}), 4.11(\mathrm{dd}, J=12.2,2.4 \mathrm{~Hz}, 1 \mathrm{H}), 4.07-3.99$ (m, 6H), $3.80(\mathrm{ddd}, J=10.1,5.3,2.4 \mathrm{~Hz}, 1 \mathrm{H}), 2.02(\mathrm{~s}, 3 \mathrm{H}), 2.01(\mathrm{~s}, 3 \mathrm{H}), 2.01(\mathrm{~s}, 3 \mathrm{H}), 2.00$ (s, $3 \mathrm{H}), 1.62(\mathrm{~s}, 3 \mathrm{H}), 1.39(\mathrm{~s}, 3 \mathrm{H}), 1.25(\mathrm{td}, J=7.2,1.1 \mathrm{~Hz}, 3 \mathrm{H}), 1.22(\mathrm{td}, J=7.1,1.1 \mathrm{~Hz}, 3 \mathrm{H})$.

${ }^{13}$ C-NMR (125 MHz, $\left.\mathrm{CDCl}_{3}\right): \delta=170.4\left(\mathrm{C}_{\mathrm{q}}\right), 170.0\left(\mathrm{C}_{\mathrm{q}}\right), 169.2\left(\mathrm{C}_{\mathrm{q}}\right), 169.1\left(\mathrm{C}_{\mathrm{q}}\right), 155.2\left(\mathrm{C}_{\mathrm{q}}\right)$, $155.1\left(\mathrm{C}_{\mathrm{q}}\right), 152.4(\mathrm{CH}), 151.6\left(\mathrm{C}_{\mathrm{q}}\right), 143.1(\mathrm{CH}), 141.5\left(\mathrm{C}_{\mathrm{q}}\right), 135.9\left(\mathrm{C}_{\mathrm{q}}\right), 135.6\left(\mathrm{C}_{\mathrm{q}}\right), 131.6\left(\mathrm{C}_{\mathrm{q}}\right)$, $131.6(\mathrm{CH}), 129.9(\mathrm{CH}), 129.8(\mathrm{CH}), 128.8(\mathrm{CH}), 128.1(\mathrm{CH}), 117.1(\mathrm{CH}), 114.7\left(\mathrm{C}_{\mathrm{q}}\right), 99.3$ $(\mathrm{CH}), 91.0(\mathrm{CH}), 85.3\left(\mathrm{~d},{ }^{3} J_{\mathrm{C}-\mathrm{P}}=8 \mathrm{~Hz}, \mathrm{CH}\right), 84.2(\mathrm{CH}), 81.4(\mathrm{CH}), 72.8(\mathrm{CH}), 72.0(\mathrm{CH}), 71.2$ $(\mathrm{CH}), 68.3(\mathrm{CH}), 66.6\left(\mathrm{~d},{ }^{2} J_{\mathrm{C}-\mathrm{P}}=5 \mathrm{~Hz}, \mathrm{CH}_{2}\right), 64.1\left(\mathrm{~d},{ }^{2} J_{\mathrm{C}-\mathrm{P}}=6 \mathrm{~Hz}, \mathrm{CH}_{2}\right), 64.1\left(\mathrm{~d},{ }^{2} J_{\mathrm{C}-\mathrm{P}}=6 \mathrm{~Hz}\right.$, $\left.\mathrm{CH}_{2}\right), 62.0\left(\mathrm{CH}_{2}\right), 41.2\left(\mathrm{CH}_{2}\right), 27.2\left(\mathrm{CH}_{3}\right), 25.4\left(\mathrm{CH}_{3}\right), 20.7\left(\mathrm{CH}_{3}\right), 20.7\left(\mathrm{CH}_{3}\right), 20.7\left(\mathrm{CH}_{3}\right)$, $20.6\left(\mathrm{CH}_{3}\right), 16.1\left(\mathrm{~d},{ }^{3} J_{\mathrm{C}-\mathrm{P}}=7 \mathrm{~Hz}, \mathrm{CH}_{3}\right)$.

${ }^{31} \mathbf{P}\left\{{ }^{1} \mathbf{H}\right\}-N M R\left(121 \mathrm{MHz}, \mathrm{CDCl}_{3}\right): \delta=-1.1(\mathrm{~s})$.

IR (ATR): $\tilde{v}=2985,1754,1569,1508,1373,1213,1029,733,702,645 \mathrm{~cm}^{-1}$.

MS (ESI) $m / z$ (relative intensity): $1904(8)[2 \mathrm{M}+\mathrm{Na}]^{+}, 1882(13)[2 \mathrm{M}+\mathrm{H}]^{+}, 963(66)[\mathrm{M}+\mathrm{Na}]^{+}$, $941(100)[\mathrm{M}+\mathrm{H}]^{+}, 655(3), 482$ (12).

HR-MS (ESI): $m / z$ calcd for $\mathrm{C}_{44} \mathrm{H}_{54} \mathrm{~N}_{4} \mathrm{O}_{17} \mathrm{P}^{+}[\mathrm{M}+\mathrm{H}]^{+} 941.3216$, found 941.3219 .

\section{$(2 R, 3 S, 4 S, 5 R, 6 S)-2-(H y d r o x y m e t h y l)-6-\{2-[3-(9-i s o-p r o p y l-9 H-p u r i n-6-$}

\section{yl)benzyl]phenoxy\}tetrahydro-2H-pyran-3,4,5-triol (6t)}

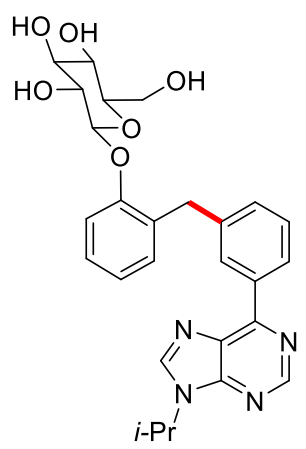

The general procedure B was followed using purine 1 h $(119 \mathrm{mg}$, $0.50 \mathrm{mmol})$ and benzyl chloride $5 \mathbf{n}(305 \mathrm{mg}, 1.00 \mathrm{mmol})$ at $80^{\circ} \mathrm{C}$. After $20 \mathrm{~h}$, purification by column chromatography $\left(\mathrm{CH}_{2} \mathrm{Cl}_{2}\right.$ /acetone $\left.1: 1\right)$ and reverse phase $\mathrm{HPLC}\left(\mathrm{H}_{2} \mathrm{O} / \mathrm{MeCN} 60: 40\right)$ yielded $6 \mathbf{t}(139 \mathrm{mg}, 55 \%)$ as a viscous colorless oil.

${ }^{1} \mathbf{H}-\mathbf{N M R}\left(600 \mathrm{MHz}\right.$, acetone- $\left.d_{6}\right): \delta=8.96(\mathrm{~s}, 1 \mathrm{H}), 8.90(\mathrm{dd}, J=1.7,1.7 \mathrm{~Hz}$, $1 \mathrm{H}), 8.70$ (ddd, $J=7.6,1.7,1.6 \mathrm{~Hz}, 1 \mathrm{H}), 8.63$ (s, $1 \mathrm{H}), 7.49$ (ddd, $J=7.6$, $1.7,1.6 \mathrm{~Hz}, 1 \mathrm{H}), 7.46(\mathrm{dd}, J=7.6,7.6 \mathrm{~Hz}, 1 \mathrm{H}), 7.31(\mathrm{dd}, J=7.4,1.7 \mathrm{~Hz}, 1 \mathrm{H}), 7.19(\mathrm{dd}, J=$ 8.3, $1.5 \mathrm{~Hz}, 1 \mathrm{H}), 7.16(\mathrm{ddd}, J=8.3,7.2,1.7 \mathrm{~Hz}, 1 \mathrm{H}), 6.97(\mathrm{ddd}, J=7.4,7.2,1.5 \mathrm{~Hz}, 1 \mathrm{H}), 5.26$ (br s, 1H), 5.02 (hept, $J=6.8 \mathrm{~Hz}, 1 \mathrm{H}), 5.02$ (d, $J=7.6 \mathrm{~Hz}, 1 \mathrm{H}), 4.48$ (br s, 1H), 4.30 (br s, 1H), $4.19\left(\mathrm{~d}_{\mathrm{AB}}, J=14.2 \mathrm{~Hz}, 1 \mathrm{H}\right), 4.13\left(\mathrm{~d}_{\mathrm{AB}}, J=14.2 \mathrm{~Hz}, 1 \mathrm{H}\right), 3.90-3.84(\mathrm{~m}, 1 \mathrm{H}), 3.75-3.66(\mathrm{~m}$, 
$3 \mathrm{H}), 3.55(\mathrm{t}, J=8.9 \mathrm{~Hz}, 1 \mathrm{H}), 3.53-3.49(\mathrm{~m}, 1 \mathrm{H}), 3.46(\mathrm{t}, J=9.2 \mathrm{~Hz}, 1 \mathrm{H}), 1.69(\mathrm{~d}, J=6.8 \mathrm{~Hz}$, $6 \mathrm{H})$.

${ }^{13}$ C-NMR (125 MHz, acetone- $\left.d_{6}\right): \delta=156.4\left(\mathrm{C}_{\mathrm{q}}\right), 154.4\left(\mathrm{C}_{\mathrm{q}}\right), 153.1\left(\mathrm{C}_{\mathrm{q}}\right), 152.4(\mathrm{CH}), 144.6$ $(\mathrm{CH}), 142.3\left(\mathrm{C}_{\mathrm{q}}\right), 136.5\left(\mathrm{C}_{\mathrm{q}}\right), 132.2(\mathrm{CH}), 131.9\left(\mathrm{C}_{\mathrm{q}}\right), 131.6(\mathrm{CH}), 131.4\left(\mathrm{C}_{\mathrm{q}}\right), 131.0(\mathrm{CH}), 129.0$ $(\mathrm{CH}), 128.3(\mathrm{CH}), 128.1(\mathrm{CH}), 122.7(\mathrm{CH}), 116.1(\mathrm{CH}), 102.1(\mathrm{CH}), 78.2(\mathrm{CH}), 77.7(\mathrm{CH})$, $74.5(\mathrm{CH}), 71.3(\mathrm{CH}), 62.7\left(\mathrm{CH}_{2}\right), 48.4(\mathrm{CH}), 37.2\left(\mathrm{CH}_{2}\right), 22.4\left(\mathrm{CH}_{3}\right), 22.4\left(\mathrm{CH}_{3}\right)$.

IR (ATR): $\tilde{v}=3340,2919,1572,1491,1454,1328,1223,1072,1043,648 \mathrm{~cm}^{-1}$.

MS (ESI) $m / z$ (relative intensity): $529(42)[\mathrm{M}+\mathrm{Na}]^{+}, 507(100)[\mathrm{M}+\mathrm{H}]^{+}$.

HR-MS (ESI): $m / z$ calcd for $\mathrm{C}_{27} \mathrm{H}_{31} \mathrm{~N}_{4} \mathrm{O}_{6}{ }^{+}[\mathrm{M}+\mathrm{H}]^{+}$507.2238, found 507.2249.

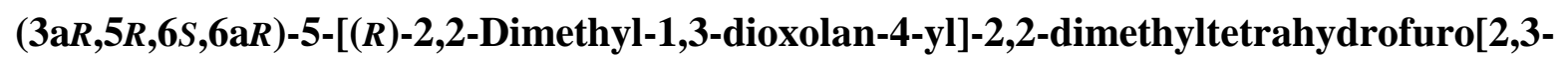
$d][1,3]$ dioxol-6-yl 2-[3-(9-iso-propyl-9H-purin-6-yl)phenyl]acetate (6u)

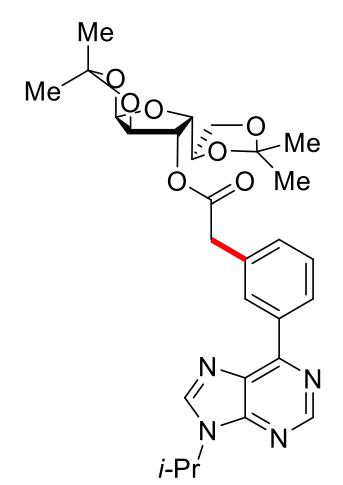

The general procedure B was followed using purine $\mathbf{1 h}(119 \mathrm{mg}$, $0.50 \mathrm{mmol})$ and alkyl bromide 5 o $(381 \mathrm{mg}, 1.00 \mathrm{mmol})$. After $20 \mathrm{~h}$, purification by column chromatography ( $n$-hexane/EtOAc 3:2) yielded $\mathbf{6 u}$ (145 mg, 54\%) as a colorless oil.

${ }^{1} \mathbf{H}-\mathbf{N M R}\left(600 \mathrm{MHz}, \mathrm{CDCl}_{3}\right): \delta=9.00(\mathrm{~s}, 1 \mathrm{H}), 8.76(\mathrm{ddd}, J=7.8,1.7$, $1.5 \mathrm{~Hz}, 1 \mathrm{H}), 8.68(\mathrm{dd}, J=1.7,1.6 \mathrm{~Hz}, 1 \mathrm{H}), 8.18(\mathrm{~s}, 1 \mathrm{H}), 7.52(\mathrm{dd}, J=7.8$, $7.7 \mathrm{~Hz}, 1 \mathrm{H}), 7.45$ (ddd, $J=7.7,1.6,1.5 \mathrm{~Hz}, 1 \mathrm{H}), 5.85$ (d, $J=3.6 \mathrm{~Hz}, 1 \mathrm{H})$, $5.30(\mathrm{~d}, J=2.9 \mathrm{~Hz}, 1 \mathrm{H}), 4.98$ (hept, $J=6.8 \mathrm{~Hz}, 1 \mathrm{H}), 4.47$ (d, $J=3.6 \mathrm{~Hz}, 1 \mathrm{H}), 4.18\left(\mathrm{~d}_{\mathrm{AB}} \mathrm{d}, J=\right.$ 8.0, $2.9 \mathrm{~Hz}, 1 \mathrm{H}), 4.16\left(\mathrm{~d}_{\mathrm{AB}} \mathrm{dd}, J=8.0,5.7,4.8 \mathrm{~Hz}, 1 \mathrm{H}\right), 3.98\left(\mathrm{~d}_{\mathrm{AB}} \mathrm{d}, J=8.6,5.7 \mathrm{~Hz}, 1 \mathrm{H}\right), 3.96$ $\left(\mathrm{d}_{\mathrm{AB}} \mathrm{d}, J=8.6,4.8 \mathrm{~Hz}, 1 \mathrm{H}\right), 3.82\left(\mathrm{~d}_{\mathrm{AB}}, J=15.3 \mathrm{~Hz}, 1 \mathrm{H}\right), 3.80\left(\mathrm{~d}_{\mathrm{AB}}, J=15.3 \mathrm{~Hz}, 1 \mathrm{H}\right), 1.67(\mathrm{~d}, J$ $=6.8 \mathrm{~Hz}, 6 \mathrm{H}), 1.49(\mathrm{~s}, 3 \mathrm{H}), 1.36(\mathrm{~s}, 3 \mathrm{H}), 1.26(\mathrm{~s}, 3 \mathrm{H}), 1.20(\mathrm{~s}, 3 \mathrm{H})$.

${ }^{13} \mathrm{C}-\mathrm{NMR}\left(125 \mathrm{MHz}, \mathrm{CDCl}_{3}\right): \delta=169.8\left(\mathrm{C}_{\mathrm{q}}\right), 154.0\left(\mathrm{C}_{\mathrm{q}}\right), 152.1\left(\mathrm{C}_{\mathrm{q}}\right), 151.9(\mathrm{CH}), 141.9(\mathrm{CH})$, $136.1\left(\mathrm{C}_{\mathrm{q}}\right), 133.7\left(\mathrm{C}_{\mathrm{q}}\right), 131.6(\mathrm{CH}), 131.4\left(\mathrm{C}_{\mathrm{q}}\right), 130.2(\mathrm{CH}), 128.9(\mathrm{CH}), 128.8(\mathrm{CH}), 112.2$ $\left(\mathrm{C}_{\mathrm{q}}\right), 109.2\left(\mathrm{C}_{\mathrm{q}}\right), 105.0(\mathrm{CH}), 83.2(\mathrm{CH}), 79.9(\mathrm{CH}), 76.4(\mathrm{CH}), 72.3(\mathrm{CH}), 67.2\left(\mathrm{CH}_{2}\right), 47.3$ $(\mathrm{CH}), 41.4\left(\mathrm{CH}_{2}\right), 26.9\left(\mathrm{CH}_{3}\right), 26.8\left(\mathrm{CH}_{3}\right), 26.3\left(\mathrm{CH}_{3}\right), 25.1\left(\mathrm{CH}_{3}\right), 22.6\left(\mathrm{CH}_{3}\right)$.

IR (ATR): $\tilde{v}=2984,1742,1570,1372,1215,1072,1018,843,703,647 \mathrm{~cm}^{-1}$.

MS (ESI) $m / z$ (relative intensity): $1100(12)[2 \mathrm{M}+\mathrm{Na}]^{+}, 561(43)[\mathrm{M}+\mathrm{Na}]^{+}, 539(100)[\mathrm{M}+\mathrm{H}]^{+}$. HR-MS (ESI): $m / z$ calcd for $\mathrm{C}_{28} \mathrm{H}_{35} \mathrm{~N}_{4} \mathrm{O}_{7}{ }^{+}[\mathrm{M}+\mathrm{H}]^{+}$539.2500, found 539.2490. 


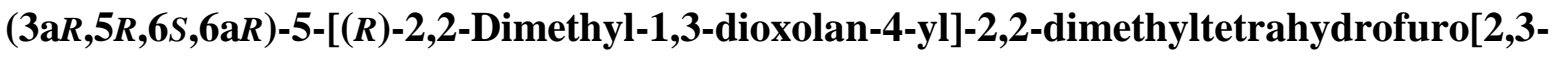
d][1,3]dioxol-6-yl 2-[3-(9-iso-propyl-9H-purin-6-yl)phenyl]-2-methylpropanoate (6v)

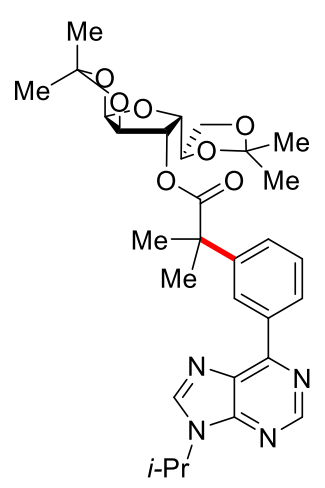

The general procedure B was followed using purine $\mathbf{1 h}(119 \mathrm{mg}$, $0.50 \mathrm{mmol}$ ) and alkyl bromide $\mathbf{5 p}(409 \mathrm{mg}, 1.00 \mathrm{mmol})$. After $20 \mathrm{~h}$, purification by column chromatography ( $n$-hexane/EtOAc $2: 1$ ) yielded $\mathbf{6 v}$ (241 mg, 76\%) as a colorless oil.

${ }^{1} \mathbf{H}-\mathbf{N M R}\left(600 \mathrm{MHz}, \mathrm{CDCl}_{3}\right): \delta=9.00(\mathrm{~s}, 1 \mathrm{H}), 8.79(\mathrm{dd}, J=1.8,1.7 \mathrm{~Hz}$, $1 \mathrm{H}), 8.74$ (ddd, $J=7.5,1.7,1.7 \mathrm{~Hz}, 1 \mathrm{H}), 8.18$ (s, 1H), 7.52 (dd, $J=7.7$, $7.5 \mathrm{~Hz}, 1 \mathrm{H}), 7.49$ (ddd, $J=7.7,1.8,1.7 \mathrm{~Hz}, 1 \mathrm{H}), 5.66(\mathrm{~d}, J=3.6 \mathrm{~Hz}, 1 \mathrm{H})$, $5.31(\mathrm{~d}, J=3.1 \mathrm{~Hz}, 1 \mathrm{H}), 4.98$ (hept, $J=6.8 \mathrm{~Hz}, 1 \mathrm{H}), 4.48(\mathrm{~d}, J=3.6 \mathrm{~Hz}, 1 \mathrm{H}), 4.16$ (dd, $J=8.0$, $3.1 \mathrm{~Hz}, 1 \mathrm{H}), 4.01(\mathrm{ddd}, J=8.0,6.1,5.3 \mathrm{~Hz}, 1 \mathrm{H}), 3.88\left(\mathrm{~d}_{\mathrm{AB}} \mathrm{d}, J=8.6,6.1 \mathrm{~Hz}, 1 \mathrm{H}\right), 3.85\left(\mathrm{~d}_{\mathrm{ABd}}\right.$, $J=8.6,5.3 \mathrm{~Hz}, 1 \mathrm{H}), 1.71(\mathrm{~s}, 3 \mathrm{H}), 1.68-1.66(\mathrm{~m}, 9 \mathrm{H}), 1.48(\mathrm{~s}, 3 \mathrm{H}), 1.36(\mathrm{~s}, 3 \mathrm{H}), 1.23$ (s, 3H), $1.19(\mathrm{~s}, 3 \mathrm{H})$.

${ }^{13}$ C-NMR $\left(125 \mathrm{MHz}, \mathrm{CDCl}_{3}\right): \delta=175.1\left(\mathrm{C}_{\mathrm{q}}\right), 154.3\left(\mathrm{C}_{\mathrm{q}}\right), 152.0\left(\mathrm{C}_{\mathrm{q}}\right), 151.9(\mathrm{CH}), 144.5\left(\mathrm{C}_{\mathrm{q}}\right)$, $141.9(\mathrm{CH}), 135.9\left(\mathrm{C}_{\mathrm{q}}\right), 131.4\left(\mathrm{C}_{\mathrm{q}}\right), 128.6(\mathrm{CH}), 128.5(\mathrm{CH}), 128.0(\mathrm{CH}), 126.9(\mathrm{CH}), 112.1$ $\left(\mathrm{C}_{\mathrm{q}}\right), 109.1\left(\mathrm{C}_{\mathrm{q}}\right), 105.1(\mathrm{CH}), 83.0(\mathrm{CH}), 80.2(\mathrm{CH}), 76.3(\mathrm{CH}), 72.3(\mathrm{CH}), 67.3\left(\mathrm{CH}_{2}\right), 47.3$ $(\mathrm{CH}), 47.1\left(\mathrm{C}_{\mathrm{q}}\right), 26.9\left(\mathrm{CH}_{3}\right), 26.8\left(\mathrm{CH}_{3}\right), 26.8\left(\mathrm{CH}_{3}\right), 26.3\left(\mathrm{CH}_{3}\right), 26.1\left(\mathrm{CH}_{3}\right), 25.1\left(\mathrm{CH}_{3}\right), 22.7$ $\left(\mathrm{CH}_{3}\right)$.

IR (ATR): $\tilde{v}=2983,1737,1569,1372,1217,1140,1073,1020,845,647 \mathrm{~cm}^{-1}$.

MS (ESI) $m / z$ (relative intensity): $1156(6)[2 \mathrm{M}+\mathrm{Na}]^{+}, 589(14)[\mathrm{M}+\mathrm{Na}]^{+}, 567(100)[\mathrm{M}+\mathrm{H}]^{+}$.

HR-MS (ESI): $m / z$ calcd for $\mathrm{C}_{30} \mathrm{H}_{39} \mathrm{~N}_{4} \mathrm{O}_{7}{ }^{+}[\mathrm{M}+\mathrm{H}]^{+}$567.2813, found 567.2812.

$[(3 \mathrm{a} R, 5 R, 6 S, 6 \mathrm{a} R)-6-H y d r o x y-2,2-d i m e t h y l t e t r a h y d r o f u r o[2,3-d][1,3]$ dioxol-5-yl]methyl 2[3-(9-iso-propyl-9H-purin-6-yl)phenyl]-2-methylpropanoate (6w)

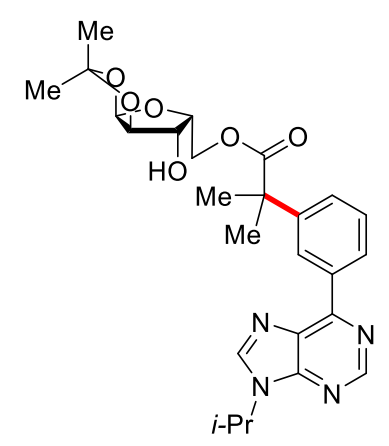

The general procedure B was followed using purine $\mathbf{1 h}(119 \mathrm{mg}$, $0.50 \mathrm{mmol})$ and alkyl bromide $\mathbf{5 q}(339 \mathrm{mg}, 1.00 \mathrm{mmol})$. After $20 \mathrm{~h}$, purification by column chromatography (n-hexane/EtOAc 1:1) and recycling preparative HPLC yielded $6 \mathbf{w}(169 \mathrm{mg}, 68 \%)$ as a viscous colorless oil.

${ }^{1} \mathbf{H}-\mathbf{N M R}\left(600 \mathrm{MHz}, \mathrm{CDCl}_{3}\right): \delta=8.97(\mathrm{~s}, 1 \mathrm{H}), 8.58(\mathrm{ddd}, J=6.7,2.3$, $2.1 \mathrm{~Hz}, 1 \mathrm{H}), 8.28(\mathrm{dd}, J=2.3,1.4 \mathrm{~Hz}, 1 \mathrm{H}), 8.22(\mathrm{~s}, 1 \mathrm{H}), 7.58-7.53(\mathrm{~m}$, 2H), $5.92(\mathrm{~d}, J=3.5 \mathrm{~Hz}, 1 \mathrm{H}), 5.78(\mathrm{~d}, J=4.5 \mathrm{~Hz}, 1 \mathrm{H}), 4.99$ (hept, $J=6.8 \mathrm{~Hz}, 1 \mathrm{H}), 4.74$ (dd, $J$ $=10.3,10.3 \mathrm{~Hz}, 1 \mathrm{H}), 4.40(\mathrm{~d}, J=3.5 \mathrm{~Hz}, 1 \mathrm{H}), 4.28(\mathrm{ddd}, J=10.3,4.6,3.2 \mathrm{~Hz}, 1 \mathrm{H}), 4.13(\mathrm{dd}$, 
$J=10.3,4.6 \mathrm{~Hz}, 1 \mathrm{H}), 3.85(\mathrm{dd}, J=4.5,3.2 \mathrm{~Hz}, 1 \mathrm{H}), 1.70(\mathrm{~s}, 3 \mathrm{H}), 1.68(\mathrm{~d}, J=6.8 \mathrm{~Hz}, 3 \mathrm{H}), 1.68$ $(\mathrm{d}, J=6.8 \mathrm{~Hz}, 3 \mathrm{H}), 1.57(\mathrm{~s}, 3 \mathrm{H}), 1.40(\mathrm{~s}, 3 \mathrm{H}), 1.24(\mathrm{~s}, 3 \mathrm{H})$.

${ }^{13}$ C-NMR (125 MHz, $\left.\mathrm{CDCl}_{3}\right): \delta=176.1\left(\mathrm{C}_{\mathrm{q}}\right), 155.0\left(\mathrm{C}_{\mathrm{q}}\right), 152.1\left(\mathrm{C}_{\mathrm{q}}\right), 151.7(\mathrm{CH}), 145.4\left(\mathrm{C}_{\mathrm{q}}\right)$, $142.6(\mathrm{CH}), 135.1\left(\mathrm{C}_{\mathrm{q}}\right), 131.4\left(\mathrm{C}_{\mathrm{q}}\right), 128.9(\mathrm{CH}), 128.8(\mathrm{CH}), 127.2(\mathrm{CH}), 111.6\left(\mathrm{C}_{\mathrm{q}}\right), 105.0$ $(\mathrm{CH}), 85.6(\mathrm{CH}), 77.6(\mathrm{CH}), 72.7(\mathrm{CH}), 60.7\left(\mathrm{CH}_{2}\right), 47.6(\mathrm{CH}), 47.1\left(\mathrm{C}_{\mathrm{q}}\right), 28.0\left(\mathrm{CH}_{3}\right), 26.9$ $\left(\mathrm{CH}_{3}\right), 26.2\left(\mathrm{CH}_{3}\right), 25.8\left(\mathrm{CH}_{3}\right), 22.7\left(\mathrm{CH}_{3}\right), 22.6\left(\mathrm{CH}_{3}\right)$.

IR (ATR): $\tilde{v}=2978,1729,1569,1326,1217,1147,1070,1012,702,647 \mathrm{~cm}^{-1}$.

MS (ESI) $m / z$ (relative intensity): $1016(65)[2 \mathrm{M}+\mathrm{Na}]^{+}, 994(6)[2 \mathrm{M}+\mathrm{H}]^{+}, 519(66)[\mathrm{M}+\mathrm{Na}]^{+}$, $497(100)[\mathrm{M}+\mathrm{H}]^{+}$.

HR-MS (ESI): $m / z$ calcd for $\mathrm{C}_{26} \mathrm{H}_{33} \mathrm{~N}_{4} \mathrm{O}_{6}{ }^{+}[\mathrm{M}+\mathrm{H}]^{+}$497.2395, found 497.2384.

\section{\{(3aR,4R,6R,6a $R)-6-[2,4-D i o x o-3,4-d i h y d r o p y r i m i d i n-1(2 H)-y l]-2,2-$}

dimethyltetrahydrofuro[3,4-d][1,3]dioxol-4-yl]methyl 4-[3-(9-iso-propyl-9H-purin-6yl)benzyl]benzoate $(6 x)$

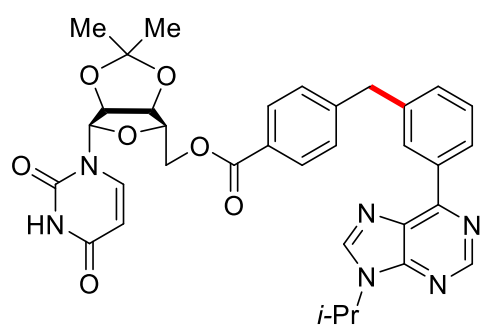

The general procedure $\mathbf{B}$ was followed using purine $\mathbf{1 h}$ (59.6 $\mathrm{mg}, \quad 0.25 \mathrm{mmol})$ and benzyl chloride $\mathbf{5 r}$ (219 $\mathrm{mg}$, $0.50 \mathrm{mmol})$. After $20 \mathrm{~h}$, purification by column chromatography ( $n$-hexane/EtOAc $1: 3)$ yielded $\mathbf{6 x}(87.3 \mathrm{mg}, 55 \%)$ as a white solid.

${ }^{1}$ H-NMR $\left(600 \mathrm{MHz}, \mathrm{CDCl}_{3}\right): \delta=9.62(\mathrm{~d}, J=2.1 \mathrm{~Hz}, 1 \mathrm{H}), 8.99(\mathrm{~s}, 1 \mathrm{H}), 8.66(\mathrm{ddd}, J=7.8,1.6$, $1.4 \mathrm{~Hz}, 1 \mathrm{H}), 8.54$ (dd, $J=1.8,1.6 \mathrm{~Hz}, 1 \mathrm{H}), 8.20$ (s, 1H), 7.88 (d, $J=8.3 \mathrm{~Hz}, 2 \mathrm{H}), 7.50$ (dd, $J=$ 7.8, 7.7 Hz, 1H), 7.33-7.30 (m, 1H), $7.30(\mathrm{~d}, J=8.3 \mathrm{~Hz}, 2 \mathrm{H}), 7.24(\mathrm{~d}, J=8.1 \mathrm{~Hz}, 1 \mathrm{H}), 5.64(\mathrm{~d}$, $J=2.1 \mathrm{~Hz}, 1 \mathrm{H}), 5.52(\mathrm{dd}, J=8.1,2.1 \mathrm{~Hz}, 1 \mathrm{H}), 5.03(\mathrm{dd}, J=6.3,2.1 \mathrm{~Hz}, 1 \mathrm{H}), 4.97$ (hept, $J=$ $6.8 \mathrm{~Hz}, 1 \mathrm{H}), 4.90(\mathrm{dd}, J=6.3,3.4 \mathrm{~Hz}, 1 \mathrm{H}), 4.61(\mathrm{dd}, J=12.1,3.4 \mathrm{~Hz}, 1 \mathrm{H}), 4.55(\mathrm{ddd}, J=4.9$, $3.4,3.4 \mathrm{~Hz}, 1 \mathrm{H}), 4.44(\mathrm{dd}, J=12.1,4.9 \mathrm{~Hz}, 1 \mathrm{H}), 4.17\left(\mathrm{~d}_{\mathrm{AB}}, J=15.5 \mathrm{~Hz}, 1 \mathrm{H}\right), 4.15\left(\mathrm{~d}_{\mathrm{AB}}, J=\right.$ $15.5 \mathrm{~Hz}, 1 \mathrm{H}), 1.66(\mathrm{~d}, J=6.8 \mathrm{~Hz}, 6 \mathrm{H}), 1.56(\mathrm{~s}, 3 \mathrm{H}), 1.35$ (s, 3H).

${ }^{13}$ C-NMR $\left(125 \mathrm{MHz}, \mathrm{CDCl}_{3}\right): \delta=165.8\left(\mathrm{C}_{\mathrm{q}}\right), 162.8\left(\mathrm{C}_{\mathrm{q}}\right), 154.6\left(\mathrm{C}_{\mathrm{q}}\right), 152.0\left(\mathrm{C}_{\mathrm{q}}\right), 151.9(\mathrm{CH})$, $149.9\left(\mathrm{C}_{\mathrm{q}}\right), 147.1\left(\mathrm{C}_{\mathrm{q}}\right), 142.1(\mathrm{CH}), 140.8(\mathrm{CH}), 140.1\left(\mathrm{C}_{\mathrm{q}}\right), 136.1\left(\mathrm{C}_{\mathrm{q}}\right), 131.5(\mathrm{CH}), 131.3\left(\mathrm{C}_{\mathrm{q}}\right)$, $130.0(\mathrm{CH}), 129.6(\mathrm{CH}), 129.0(\mathrm{CH}), 128.9(\mathrm{CH}), 128.3(\mathrm{CH}), 127.3\left(\mathrm{C}_{\mathrm{q}}\right), 114.3\left(\mathrm{C}_{\mathrm{q}}\right), 102.3$ $(\mathrm{CH}), 95.0(\mathrm{CH}), 85.4(\mathrm{CH}), 85.1(\mathrm{CH}), 81.2(\mathrm{CH}), 64.4\left(\mathrm{CH}_{2}\right), 47.3(\mathrm{CH}), 42.0\left(\mathrm{CH}_{2}\right), 27.2$ $\left(\mathrm{CH}_{3}\right), 25.3\left(\mathrm{CH}_{3}\right), 22.6\left(\mathrm{CH}_{3}\right)$.

IR (ATR): $\tilde{v}=2983,1688,1570,1456,1378,1270,1216,1087,750,647 \mathrm{~cm}^{-1}$.

m.p.: $87-90{ }^{\circ} \mathrm{C}$.

MS (ESI) $m / z$ (relative intensity): $661(3)[\mathrm{M}+\mathrm{Na}]^{+}, 639(100)[\mathrm{M}+\mathrm{H}]^{+}$. 
HR-MS (ESI): $m / z$ calcd for $\mathrm{C}_{34} \mathrm{H}_{35} \mathrm{~N}_{6} \mathrm{O}_{7}{ }^{+}[\mathrm{M}+\mathrm{H}]^{+}$639.2562, found 639.2562.

$\{(3 \mathrm{a} R, 4 R, 6 R, 6 \mathrm{a} R)-6-[2,4-D i o x 0-3,4-d i h y d r o p y r i m i d i n-1(2 H)-y l]-2,2-$ dimethyltetrahydrofuro[3,4-d][1,3]dioxol-4-yl\}methyl $\quad 4-\{3-\{9-\{(3 \mathrm{a} R, 4 R, 6 R, 6 \mathrm{a} R)-6-$ $\{[($ diethoxyphosphoryl)oxy]methyl $\}-2,2-d i m e t h y l t e t r a h y d r o f u r o[3,4-d][1,3]$ dioxol-4-yl $\}$ 9H-purin-6-yl\}benzyl\}benzoate (6y)

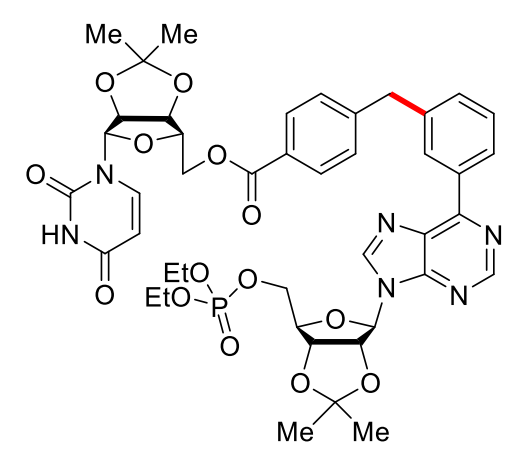
The general procedure $\mathbf{B}$ was followed using purine $\mathbf{1 m}$ (126 mg, $0.25 \mathrm{mmol})$ and benzyl chloride $\mathbf{5 r}$ (219 $\mathrm{mg}$, $0.50 \mathrm{mmol})$. After $20 \mathrm{~h}$, purification by column chromatography $\left(\mathrm{CH}_{2} \mathrm{Cl}_{2} /\right.$ acetone $\left.3: 1\right)$ yielded $\mathbf{6 y}(83.5 \mathrm{mg}$, $37 \%)$ as a viscous colorless oil.

${ }^{1} \mathbf{H}-\mathbf{N M R}\left(600 \mathrm{MHz}, \mathrm{CDCl}_{3}\right): \delta=9.24(\mathrm{~d}, J=2.2 \mathrm{~Hz}, 1 \mathrm{H}), 9.01$ $(\mathrm{s}, 1 \mathrm{H}), 8.66(\mathrm{ddd}, J=7.8,1.6,1.4 \mathrm{~Hz}, 1 \mathrm{H}), 8.55(\mathrm{dd}, J=1.6$, $1.6 \mathrm{~Hz}, 1 \mathrm{H}), 8.31(\mathrm{~s}, 1 \mathrm{H}), 7.88(\mathrm{~d}, J=8.3 \mathrm{~Hz}, 2 \mathrm{H}), 7.50(\mathrm{dd}, J=7.8,7.7 \mathrm{~Hz}, 1 \mathrm{H}), 7.34$ (ddd, $J$ $=7.7,1.6,1.4 \mathrm{~Hz}, 1 \mathrm{H}), 7.32(\mathrm{~d}, J=8.3 \mathrm{~Hz}, 2 \mathrm{H}), 7.24(\mathrm{~d}, J=8.1 \mathrm{~Hz}, 1 \mathrm{H}), 6.26(\mathrm{~d}, J=2.5 \mathrm{~Hz}$, $1 \mathrm{H}), 5.66(\mathrm{~d}, J=2.1 \mathrm{~Hz}, 1 \mathrm{H}), 5.51(\mathrm{dd}, J=8.1,2.2 \mathrm{~Hz}, 1 \mathrm{H}), 5.45(\mathrm{dd}, J=6.3,2.5 \mathrm{~Hz}, 1 \mathrm{H})$, $5.13(\mathrm{dd}, J=6.3,3.1 \mathrm{~Hz}, 1 \mathrm{H}), 5.02(\mathrm{dd}, J=6.3,2.1 \mathrm{~Hz}, 1 \mathrm{H}), 4.90(\mathrm{dd}, J=6.3,3.3 \mathrm{~Hz}, 1 \mathrm{H})$, $4.62(\mathrm{dd}, J=12.1,3.3 \mathrm{~Hz}, 1 \mathrm{H}), 4.56-4.51(\mathrm{~m}, 2 \mathrm{H}), 4.45$ (dd, $J=12.1,4.8 \mathrm{~Hz}, 1 \mathrm{H}), 4.29$ (ddd, $J=11.1,6.3,4.6 \mathrm{~Hz}, 1 \mathrm{H}), 4.20(\mathrm{ddd}, J=11.1,6.9,5.3 \mathrm{~Hz}, 1 \mathrm{H}), 4.18\left(\mathrm{~d}_{\mathrm{AB}}, J=15.5 \mathrm{~Hz}, 1 \mathrm{H}\right)$, $4.15\left(\mathrm{~d}_{\mathrm{AB}}, J=15.5 \mathrm{~Hz}, 1 \mathrm{H}\right), 4.11-4.01(\mathrm{~m}, 4 \mathrm{H}), 1.64(\mathrm{~s}, 3 \mathrm{H}), 1.57(\mathrm{~s}, 3 \mathrm{H}), 1.41(\mathrm{~s}, 3 \mathrm{H}), 1.35$ (s, $3 \mathrm{H}), 1.27(\mathrm{td}, J=7.1,0.9 \mathrm{~Hz}, 3 \mathrm{H}), 1.24(\mathrm{td}, J=7.1,1.0 \mathrm{~Hz}, 3 \mathrm{H})$.

${ }^{13}$ C-NMR $\left(125 \mathrm{MHz}, \mathrm{CDCl}_{3}\right): \delta=165.8\left(\mathrm{C}_{\mathrm{q}}\right), 162.5\left(\mathrm{C}_{\mathrm{q}}\right), 155.0\left(\mathrm{C}_{\mathrm{q}}\right), 152.3(\mathrm{CH}), 151.6\left(\mathrm{C}_{\mathrm{q}}\right)$, $149.9\left(\mathrm{C}_{\mathrm{q}}\right), 147.0\left(\mathrm{C}_{\mathrm{q}}\right), 143.4(\mathrm{CH}), 140.7(\mathrm{CH}), 140.3\left(\mathrm{C}_{\mathrm{q}}\right), 135.7\left(\mathrm{C}_{\mathrm{q}}\right), 131.8(\mathrm{CH}), 131.6\left(\mathrm{C}_{\mathrm{q}}\right)$, $130.0(\mathrm{CH}), 129.6(\mathrm{CH}), 129.1(\mathrm{CH}), 129.0(\mathrm{CH}), 128.4(\mathrm{CH}), 127.4\left(\mathrm{C}_{\mathrm{q}}\right), 114.7\left(\mathrm{C}_{\mathrm{q}}\right), 114.3$ $\left(\mathrm{C}_{\mathrm{q}}\right), 102.4(\mathrm{CH}), 94.9(\mathrm{CH}), 91.0(\mathrm{CH}), 85.4(\mathrm{CH}), 85.3\left(\mathrm{~d},{ }^{3} J_{\mathrm{C}-\mathrm{P}}=8 \mathrm{~Hz}, \mathrm{CH}\right), 85.1(\mathrm{CH}), 84.2$ $(\mathrm{CH}), 81.4(\mathrm{CH}), 81.2(\mathrm{CH}), 66.6\left(\mathrm{~d},{ }^{2} J_{\mathrm{C}-\mathrm{P}}=5 \mathrm{~Hz}, \mathrm{CH}_{2}\right), 64.4\left(\mathrm{CH}_{2}\right), 64.1\left(\mathrm{~d},{ }^{2} J_{\mathrm{C}-\mathrm{P}}=5 \mathrm{~Hz}\right.$, $\left.\mathrm{CH}_{2}\right), 64.1\left(\mathrm{~d},{ }^{2} \mathrm{~J}_{\mathrm{C}-\mathrm{P}}=6 \mathrm{~Hz}, \mathrm{CH}_{2}\right), 42.0\left(\mathrm{CH}_{2}\right), 27.2\left(\mathrm{CH}_{3}\right), 27.2\left(\mathrm{CH}_{3}\right), 25.4\left(\mathrm{CH}_{3}\right), 25.3\left(\mathrm{CH}_{3}\right)$, $16.1\left(\mathrm{~d},{ }^{3} J_{\mathrm{C}-\mathrm{P}}=7 \mathrm{~Hz}, \mathrm{CH}_{3}\right)$.

${ }^{31} \mathbf{P}\left\{{ }^{1} \mathbf{H}\right\}$-NMR $\left(121 \mathrm{MHz}, \mathrm{CDCl}_{3}\right): \delta=-1.1(\mathrm{~s})$.

IR (ATR): $\tilde{v}=3493,2988,1694,1571,1382,1270,1213,1088,1029,866 \mathrm{~cm}^{-1}$.

MS (ESI) $m / z$ (relative intensity): $1832(2)[2 \mathrm{M}+\mathrm{Na}]^{+}, 1811(4)[2 \mathrm{M}+\mathrm{H}]^{+}, 928(12)[\mathrm{M}+\mathrm{Na}]^{+}$, $906(100)[\mathrm{M}+\mathrm{H}]^{+}$.

HR-MS (ESI): $m / z$ calcd for $\mathrm{C}_{43} \mathrm{H}_{50} \mathrm{~N}_{6} \mathrm{O}_{14} \mathrm{P}^{+}[\mathrm{M}+\mathrm{H}]^{+}$905.3117, found 905.3120. 


\section{Control Experiments with Complex Ru-I}

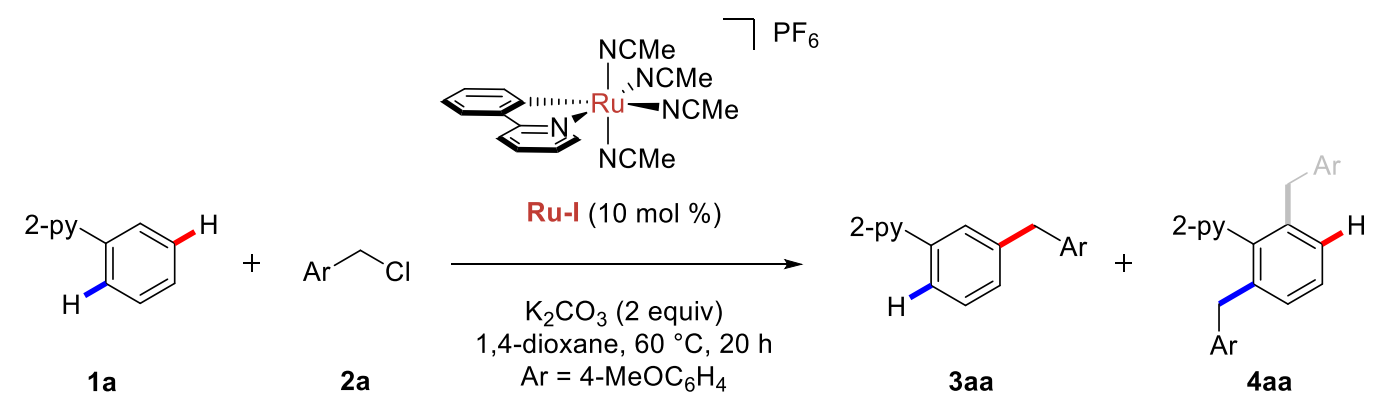

\begin{tabular}{|c|c|c|c|c|}
\hline Entry & $\begin{array}{c}\mathrm{PPh}_{3} \\
(10 \mathrm{~mol} \%)\end{array}$ & $\begin{array}{c}\text { KOAc } \\
(20 \mathrm{~mol} \%)\end{array}$ & Yield of 3aa & Yield of $\mathbf{4 a a}$ \\
\hline 1 & X & $M$ & $9 \%$ & $\begin{array}{c}14 \% \\
\text { (only mono) }\end{array}$ \\
\hline 2 & & & $5 \%$ & $\begin{array}{c}2 \% \\
\text { (only mono) } \\
\end{array}$ \\
\hline 3 & & & - & $\begin{array}{c}76 \%, \text { mono: }: \mathrm{di}=1.0: 7.4 \\
(66 \%, \text { mono: } \mathrm{di}=1.0: 7.3)\end{array}$ \\
\hline 4 & & & $80 \%(72 \%)$ & $15 \%$, mono: $\mathrm{di}=1.0: 2.8$ \\
\hline
\end{tabular}

Yields were determined by ${ }^{1} \mathrm{H}-\mathrm{NMR}$ using 1,3,5-trimethoxybenzene as the internal standard. Isolated yields are given in parentheses.

\section{Influence of $\mathrm{PPh}_{3}$ loading in the catalysis}
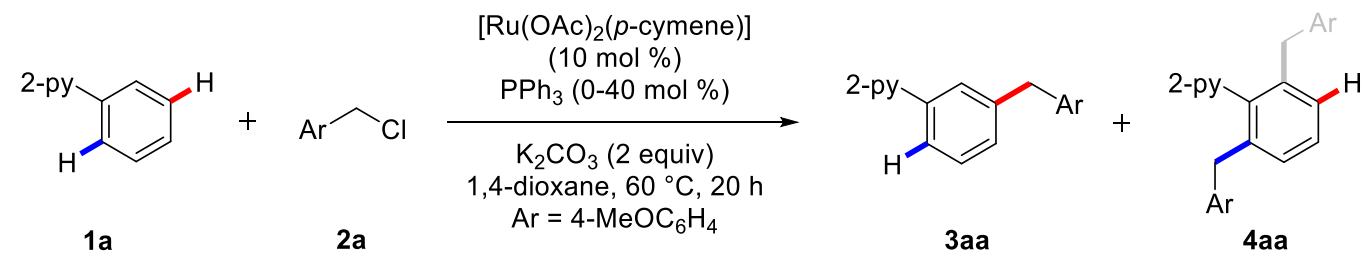

2-Phenylpyridine (1a) $(77.6 \mathrm{mg}, 0.50 \mathrm{mmol}, 1.00$ equiv), [Ru(OAc) 2 (p-cymene)] (17.7 mg, $50.0 \mu \mathrm{mol}, 10.0 \mathrm{~mol} \%), \mathrm{PPh}_{3}(0-40 \mathrm{~mol} \%)$ and $\mathrm{K}_{2} \mathrm{CO}_{3}(138 \mathrm{mg}, 1.00 \mathrm{mmol}, 2.00$ equiv) were placed in a pre-dried $25 \mathrm{~mL}$ Schlenk tube. The tube was evacuated and purged with $\mathrm{N}_{2}$ three times. Benzyl chloride 2a (235 mg, $1.50 \mathrm{mmol}, 3.00$ equiv) and 1,4-dioxane (2.0 mL) were then added and the mixture was stirred at $60{ }^{\circ} \mathrm{C}$. After $20 \mathrm{~h}$, the resulting mixture was filtered through a pad of silica gel and washed with EtOAc. The filtrate was concentrated in vacuo. The yields were determined by ${ }^{1} \mathrm{H}-\mathrm{NMR}$ using 1,3,5-trimethoxybenzene as the internal standard. 
Table S5. Conversion with different amount of $\mathrm{PPh}_{3}$.

\begin{tabular}{cccc}
\hline Entry & PPh3 $($ x mol \%) & 3aa $(\%)$ & 4aa $(\%)^{\mathrm{a}}$ \\
\hline 1 & 0 & 22 & $18(1.0: 2.6)$ \\
3 & 5 & 30 & $56(1.0: 1.3)$ \\
4 & 10 & 52 & $25(1.0: 7.3)$ \\
5 & 15 & 76 & $6(1.0: 1.0)$ \\
6 & 20 & 65 & 0 \\
7 & 25 & 27 & 0 \\
8 & 30 & 27 & 0 \\
9 & 35 & 16 & 0 \\
9 & 40 & 15 & 0 \\
\hline $\begin{array}{l}a \\
\text { combined yield of mono- and dibenzylated } \\
\text { products and the ratio of mono- to dibenzylated }\end{array}$ \\
\hline
\end{tabular}

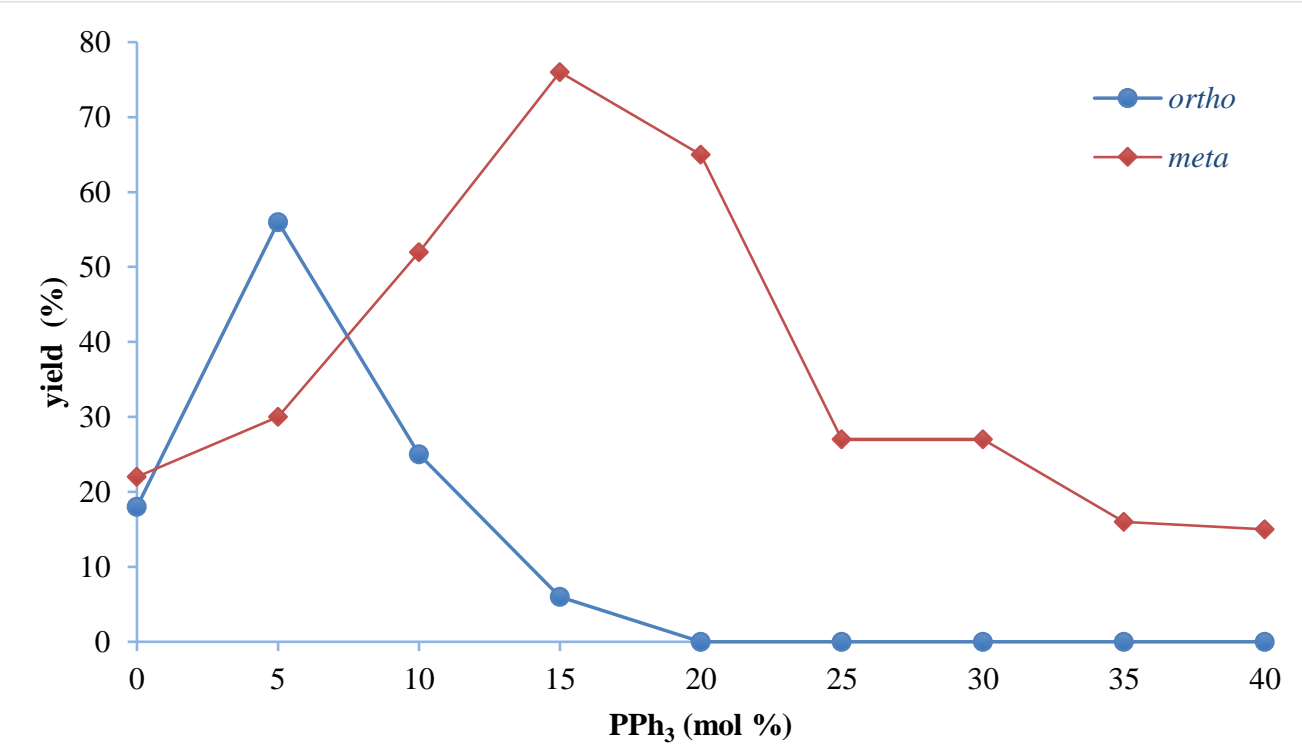

Figure S1. Influence of $\mathrm{PPh}_{3}$ loading in ruthenium(II)-catalyzed benzylation. 


\section{Competition Experiments}

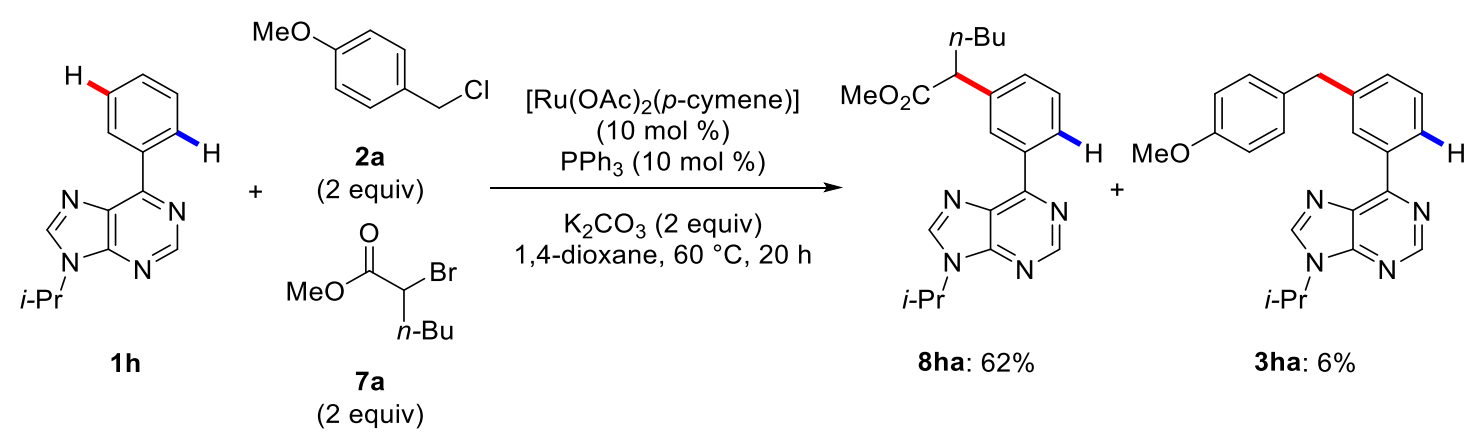

Purine (1h) (119 mg, $0.50 \mathrm{mmol}, 1.00$ equiv), [Ru(OAc) 2 (p-cymene)] (17.7 mg, $50.0 \mu \mathrm{mol}$, $10.0 \mathrm{~mol} \%$ ), $\mathrm{PPh}_{3}(13.1 \mathrm{mg}, 50.0 \mu \mathrm{mol}, 10.0 \mathrm{~mol} \%)$ and $\mathrm{K}_{2} \mathrm{CO}_{3}(138 \mathrm{mg}, 1.00 \mathrm{mmol}$, 2.00 equiv) were placed in a pre-dried $25 \mathrm{~mL}$ Schlenk tube. The tube was evacuated and purged with $\mathrm{N}_{2}$ three times. Benzyl chloride $\mathbf{2 a}$ (157 mg, $1.00 \mathrm{mmol}, 2.00$ equiv), alkyl bromide 7a (209 $\mathrm{mg}, 1.00 \mathrm{mmol}, 2.00$ equiv) and 1,4-dioxane $(2.0 \mathrm{~mL}$ ) were then added and the mixture was stirred at $60^{\circ} \mathrm{C}$. After $20 \mathrm{~h}$, the resulting mixture was filtered through a pad of silica gel and washed with EtOAc. The filtrate was concentrated in vacuo. Purification of the residue by column chromatography ( $n$-hexane/EtOAc 3:1) yielded $\mathbf{8 h a}{ }^{10}(114 \mathrm{mg}, 62 \%)$ as a colorless oil and 3ha $(11.3 \mathrm{mg}, 6 \%)$ as a colorless oil.

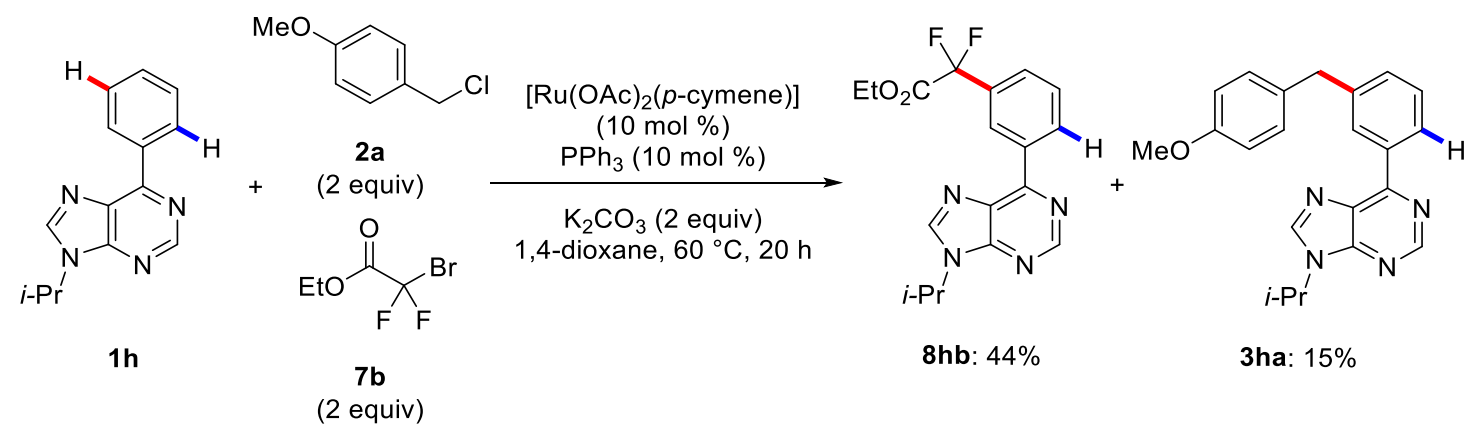

Purine (1h) (119 mg, $0.50 \mathrm{mmol}, 1.00$ equiv), [Ru(OAc) 2 (p-cymene)] (17.7 mg, $50.0 \mu \mathrm{mol}$, $10.0 \mathrm{~mol} \%$ ), $\mathrm{PPh}_{3}(13.1 \mathrm{mg}, 50.0 \mu \mathrm{mol}, 10.0 \mathrm{~mol} \%)$ and $\mathrm{K}_{2} \mathrm{CO}_{3}(138 \mathrm{mg}, 1.00 \mathrm{mmol}$, 2.00 equiv) were placed in a pre-dried $25 \mathrm{~mL}$ Schlenk tube. The tube was evacuated and purged with $\mathrm{N}_{2}$ three times. Benzyl chloride 2a (157 mg, $1.00 \mathrm{mmol}, 2.00$ equiv), alkyl bromide $\mathbf{7 b}$ (203 mg, $1.00 \mathrm{mmol}, 2.00$ equiv) and 1,4-dioxane (2.0 mL) were then added and the mixture was stirred at $60^{\circ} \mathrm{C}$. After $20 \mathrm{~h}$, the resulting mixture was filtered through a pad of silica gel and washed with EtOAc. The filtrate was concentrated in vacuo. Purification of the residue by column chromatography ( $n$-hexane/EtOAc 3:1) yielded $\mathbf{8 h b ^ { 1 1 }}(79.3 \mathrm{mg}, 44 \%)$ as a colorless oil and $3 \mathbf{h a}(27.7 \mathrm{mg}, 15 \%)$ as a colorless oil. 

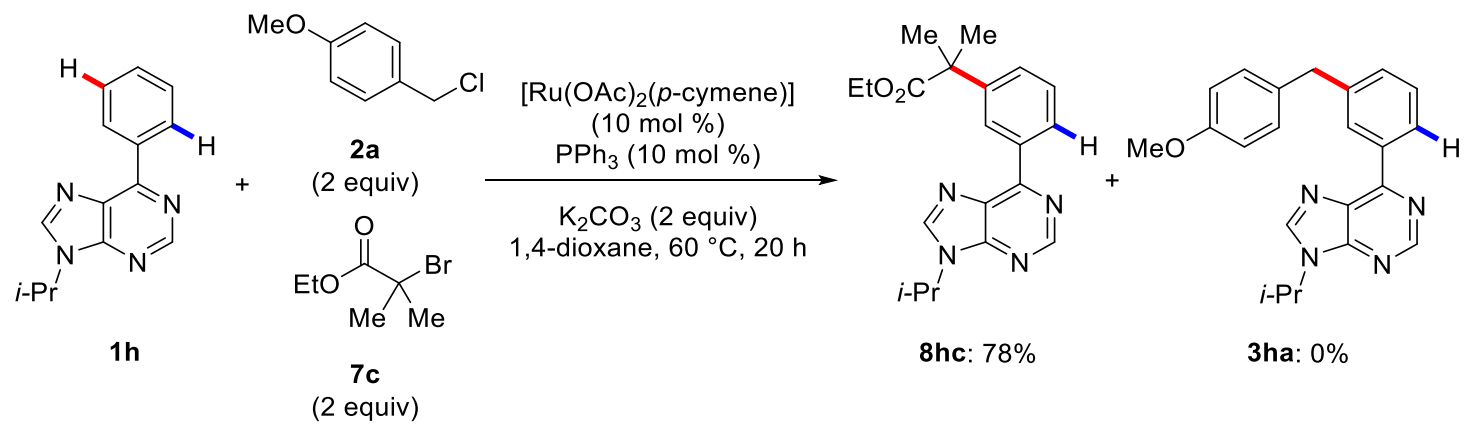

8hc: $78 \%$

3ha: $0 \%$

Purine (1h) (119 mg, $0.50 \mathrm{mmol}, 1.00$ equiv), [Ru(OAc) 2 (p-cymene)] (17.7 mg, $50.0 \mu \mathrm{mol}$, $10.0 \mathrm{~mol} \%$ ), $\mathrm{PPh}_{3}(13.1 \mathrm{mg}, 50.0 \mu \mathrm{mol}, 10.0 \mathrm{~mol} \%)$ and $\mathrm{K}_{2} \mathrm{CO}_{3}(138 \mathrm{mg}, 1.00 \mathrm{mmol}$, 2.00 equiv) were placed in a pre-dried $25 \mathrm{~mL}$ Schlenk tube. The tube was evacuated and purged with $\mathrm{N}_{2}$ three times. Benzyl chloride $2 \mathbf{a}$ (157 mg, $1.00 \mathrm{mmol}, 2.00$ equiv), alkyl bromide 7c (195 mg, $1.00 \mathrm{mmol}, 2.00$ equiv) and 1,4-dioxane $(2.0 \mathrm{~mL})$ were then added and the mixture was stirred at $60{ }^{\circ} \mathrm{C}$. After $20 \mathrm{~h}$, the resulting mixture was filtered through a pad of silica gel and washed with EtOAc. The filtrate was concentrated in vacuo. Purification of the residue by column chromatography ( $n$-hexane/EtOAc 3:1) yielded $\mathbf{8 h c}(137 \mathrm{mg}, 78 \%)$ as a colorless oil.

\section{Ethyl 2-[3-(9-isopropyl-9H-purin-6-yl)phenyl]-2-methylpropanoate (8hc)}

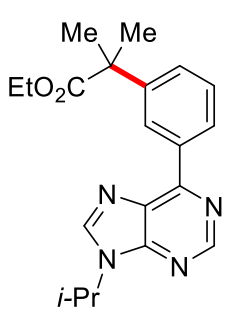

${ }^{1} \mathbf{H}-\mathrm{NMR}\left(600 \mathrm{MHz}, \mathrm{CDCl}_{3}\right): \delta=9.01(\mathrm{~s}, 1 \mathrm{H}), 8.81(\mathrm{dd}, J=1.8,1.7 \mathrm{~Hz}, 1 \mathrm{H})$, $8.71(\mathrm{ddd}, J=7.5,1.7,1.6 \mathrm{~Hz}, 1 \mathrm{H}), 8.18(\mathrm{~s}, 1 \mathrm{H}), 7.51(\mathrm{dd}, J=7.8,7.5 \mathrm{~Hz}, 1 \mathrm{H})$, 7.48 (ddd, $J=7.8,1.8,1.6 \mathrm{~Hz}, 1 \mathrm{H}), 4.98$ (hept, $J=6.8 \mathrm{~Hz}, 1 \mathrm{H}), 4.14$ (q, $J=$ $7.1 \mathrm{~Hz}, 2 \mathrm{H}), 1.68(\mathrm{~s}, 6 \mathrm{H}), 1.67(\mathrm{~d}, J=6.8 \mathrm{~Hz}, 6 \mathrm{H}), 1.18(\mathrm{t}, J=7.1 \mathrm{~Hz}, 3 \mathrm{H})$.

${ }^{13}$ C-NMR $\left(125 \mathrm{MHz}, \mathrm{CDCl}_{3}\right): \delta=176.5\left(\mathrm{C}_{\mathrm{q}}\right), 154.6\left(\mathrm{C}_{\mathrm{q}}\right), 152.0\left(\mathrm{C}_{\mathrm{q}}\right), 151.9$ $(\mathrm{CH}), 145.2\left(\mathrm{C}_{\mathrm{q}}\right), 141.8(\mathrm{CH}), 135.8\left(\mathrm{C}_{\mathrm{q}}\right), 131.5\left(\mathrm{C}_{\mathrm{q}}\right), 128.4(\mathrm{CH}), 128.4(\mathrm{CH}), 128.3(\mathrm{CH})$, $126.8(\mathrm{CH}), 60.8\left(\mathrm{CH}_{2}\right), 47.2(\mathrm{CH}), 46.8\left(\mathrm{C}_{\mathrm{q}}\right), 26.7\left(\mathrm{CH}_{3}\right), 22.7\left(\mathrm{CH}_{3}\right), 14.1\left(\mathrm{CH}_{3}\right)$.

IR (ATR): $\tilde{v}=2977,1722,1567,1324,1218,1142,1025,798,702,646 \mathrm{~cm}^{-1}$.

MS (EI) $m / z$ (relative intensity): $352(18)[\mathrm{M}]^{+}, 323$ (4) $[\mathrm{M}-\mathrm{Et}]^{+}, 279$ (89) $\left[\mathrm{M}-\mathrm{CO}_{2} \mathrm{Et}\right]^{+}, 237$ (100) $\left[\mathrm{M}-\mathrm{C}(\mathrm{Me})_{2} \mathrm{CO}_{2} \mathrm{Et}\right]^{+}, 221(12)$.

HR-MS (EI): $m / z$ calcd for $\mathrm{C}_{20} \mathrm{H}_{24} \mathrm{~N}_{4} \mathrm{O}_{2}{ }^{+}[\mathrm{M}]^{+} 352.1894$, found 352.1897. 
<smiles>CC(C)n1cnc2c(-c3ccccc3)ncnc21</smiles>

$1 \mathrm{~h}$<smiles>CCc1ccc(OC)cc1</smiles>

$2 a$

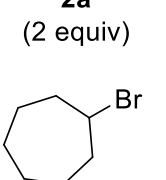

$7 d$
$\left[\mathrm{Ru}(\mathrm{OAc})_{2}(p\right.$-cymene $\left.)\right]$ $(10 \mathrm{~mol} \%)$

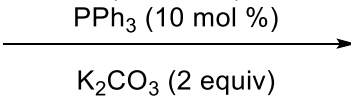

1,4-dioxane, $60^{\circ} \mathrm{C}, 20 \mathrm{~h}$

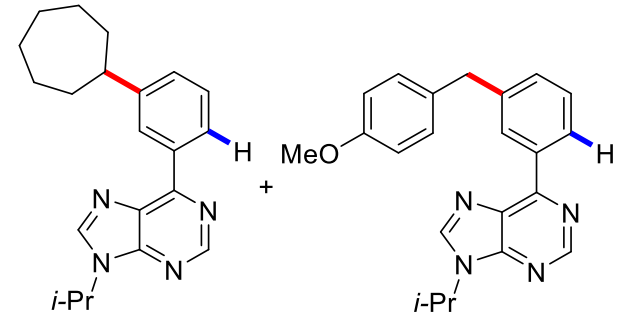

8hd: $0 \%$

3ha: $68 \%$

Purine (1h) (119 mg, $0.50 \mathrm{mmol}, 1.00$ equiv), [Ru(OAc) 2 (p-cymene)] (17.7 mg, $50.0 \mu \mathrm{mol}$, $10.0 \mathrm{~mol} \%), \mathrm{PPh}_{3}(13.1 \mathrm{mg}, 50.0 \mu \mathrm{mol}, 10.0 \mathrm{~mol} \%)$ and $\mathrm{K}_{2} \mathrm{CO}_{3}(138 \mathrm{mg}, 1.00 \mathrm{mmol}$, 2.00 equiv) were placed in a pre-dried $25 \mathrm{~mL}$ Schlenk tube. The tube was evacuated and purged with $\mathrm{N}_{2}$ three times. Benzyl chloride $2 \mathbf{a}(157 \mathrm{mg}, 1.00 \mathrm{mmol}, 2.00$ equiv), alkyl bromide 7d (177 mg, $1.00 \mathrm{mmol}, 2.00$ equiv) and 1,4-dioxane $(2.0 \mathrm{~mL})$ were then added and the mixture was stirred at $60{ }^{\circ} \mathrm{C}$. After $20 \mathrm{~h}$, the resulting mixture was filtered through a pad of silica gel and washed with EtOAc. The filtrate was concentrated in vacuo. Purification of the residue by column chromatography ( $n$-hexane/EtOAc 3:1) yielded 3ha (122 mg, 68\%) as a colorless oil. 


\section{Reaction with Radical Scavengers}

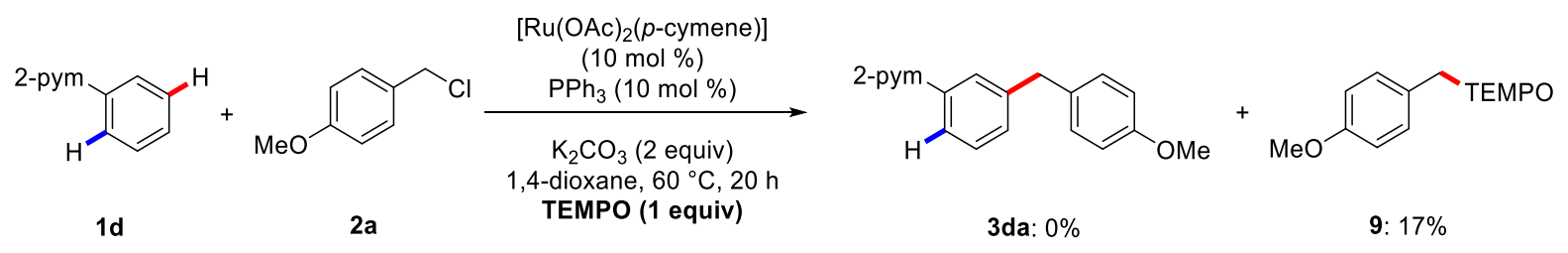

2-Phenylpyrimidine (1d) $(78.1 \mathrm{mg}, 0.50 \mathrm{mmol}, 1.00$ equiv), [Ru(OAc) 2 (p-cymene)] (17.7 mg, $50.0 \mu \mathrm{mol}, 10.0 \mathrm{~mol} \%$ ), $\mathrm{PPh}_{3}(13.1 \mathrm{mg}, 50.0 \mu \mathrm{mol}, 10.0 \mathrm{~mol} \%), \mathrm{K}_{2} \mathrm{CO}_{3}(138 \mathrm{mg}, 1.00 \mathrm{mmol}$, 2.00 equiv) and TEMPO (78.2 mg, $0.50 \mathrm{mmol}, 1.00$ equiv) were placed in a pre-dried $25 \mathrm{~mL}$ Schlenk tube. The tube was evacuated and purged with $\mathrm{N}_{2}$ three times. Benzyl chloride $\mathbf{2 a}$ (235 mg, $1.50 \mathrm{mmol}, 3.00$ equiv) and 1,4-dioxane $(2.0 \mathrm{~mL})$ were then added and the mixture was stirred at $60{ }^{\circ} \mathrm{C}$. After $20 \mathrm{~h}$, the resulting mixture was filtered through a pad of silica gel and washed with EtOAc. The filtrate was concentrated in vacuo. Purification of the residue by column chromatography ( $n$-hexane/EtOAc 20:1) followed by recycling preparative HPLC yielded TEMPO-adduct $9(23.0 \mathrm{mg}, 17 \%)$ as a colorless oil and recovered 1d $(61.0 \mathrm{mg}, 78 \%)$ as a colorless oil.

\section{1-[(4-Methoxybenzyl)oxy]-2,2,6,6-tetramethylpiperidine (9)}

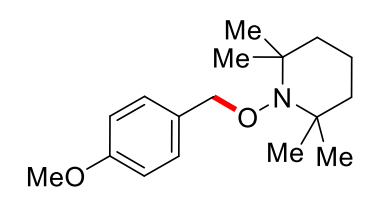

${ }^{1} \mathbf{H}$-NMR $\left(300 \mathrm{MHz}, \mathrm{CDCl}_{3}\right): \delta=7.29(\mathrm{~d}, J=8.7 \mathrm{~Hz}, 2 \mathrm{H}), 6.88(\mathrm{~d}, J=$ $8.7 \mathrm{~Hz}, 2 \mathrm{H}), 4.74(\mathrm{~s}, 2 \mathrm{H}), 3.81(\mathrm{~s}, 3 \mathrm{H}), 1.62-1.31(\mathrm{~m}, 6 \mathrm{H}), 1.26(\mathrm{~s}, 6 \mathrm{H})$,

${ }^{13} \mathrm{C}-\mathrm{NMR}\left(75 \mathrm{MHz}, \mathrm{CDCl}_{3}\right): \delta=159.0\left(\mathrm{C}_{\mathrm{q}}\right), 130.4\left(\mathrm{C}_{\mathrm{q}}\right), 129.2(\mathrm{CH}), 113.6(\mathrm{CH}), 78.4\left(\mathrm{CH}_{2}\right)$, $60.0\left(\mathrm{C}_{\mathrm{q}}\right), 55.3\left(\mathrm{CH}_{3}\right), 39.7\left(\mathrm{CH}_{2}\right), 33.2\left(\mathrm{CH}_{3}\right), 20.3\left(\mathrm{CH}_{3}\right), 17.1\left(\mathrm{CH}_{2}\right)$.

IR (ATR): $\tilde{v}=2930,1613,1513,1359,1247,1173,1036,821,695,603 \mathrm{~cm}^{-1}$.

MS (ESI) $m / z$ (relative intensity): 278 (100) $[\mathrm{M}+\mathrm{H}]^{+}, 243$ (3), 137 (16).

HR-MS (ESI): $m / z$ calcd for $\mathrm{C}_{17} \mathrm{H}_{28} \mathrm{NO}_{2}{ }^{+}[\mathrm{M}+\mathrm{H}]^{+}$278.2115, found 278.2120.

The spectral data are in accordance with those reported in the literature. ${ }^{12}$ 


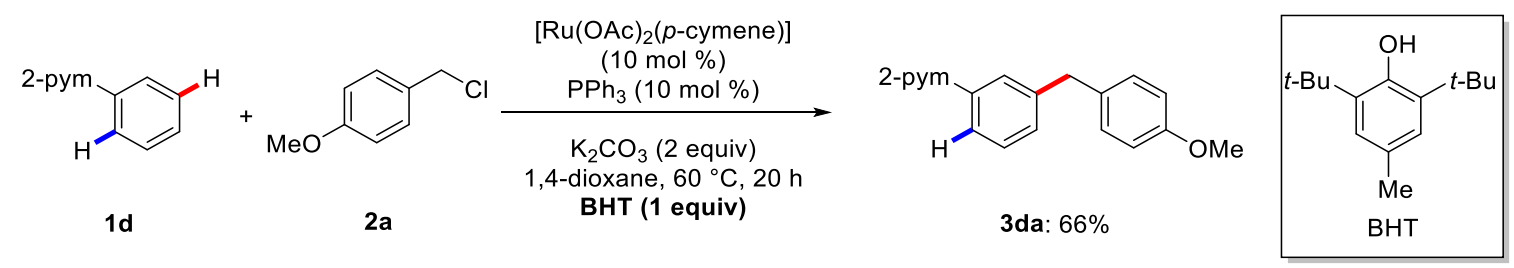

2-Phenylpyrimidine (1d) $(78.1 \mathrm{mg}, 0.50 \mathrm{mmol}, 1.00 \text { equiv), [Ru(OAc) })_{2}(p$-cymene)] (17.7 mg, $50.0 \mu \mathrm{mol}, 10.0 \mathrm{~mol} \%), \mathrm{PPh}_{3}(13.1 \mathrm{mg}, 50.0 \mu \mathrm{mol}, 10.0 \mathrm{~mol} \%), \mathrm{K}_{2} \mathrm{CO}_{3}(138 \mathrm{mg}, 1.00 \mathrm{mmol}$, 2.00 equiv) and BHT (110 mg, $0.50 \mathrm{mmol}, 1.00$ equiv) were placed in a pre-dried $25 \mathrm{~mL}$ Schlenk tube. The tube was evacuated and purged with $\mathrm{N}_{2}$ three times. Benzyl chloride $2 \mathbf{a}$ (235 mg, $1.50 \mathrm{mmol}, 3.00$ equiv) and 1,4-dioxane $(2.0 \mathrm{~mL})$ were then added and the mixture was stirred at $60{ }^{\circ} \mathrm{C}$. After $20 \mathrm{~h}$, the resulting mixture was filtered through a pad of silica gel and washed with EtOAc. The filtrate was concentrated in vacuo. Purification of the residue by column chromatography ( $n$-hexane/EtOAc 10:1) yielded 3da (91.4 mg, $66 \%$ ) as a colorless oil.

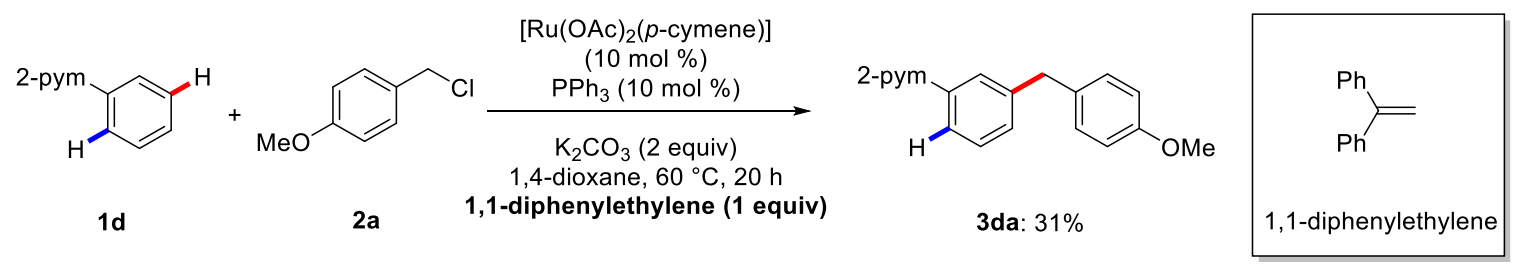

2-Phenylpyrimidine (1d) $(78.1 \mathrm{mg}, 0.50 \mathrm{mmol}, 1.00$ equiv), [Ru(OAc) 2 (p-cymene)] (17.7 mg, $50.0 \mu \mathrm{mol}, 10.0 \mathrm{~mol} \%), \mathrm{PPh}_{3}(13.1 \mathrm{mg}, 50.0 \mu \mathrm{mol}, 10.0 \mathrm{~mol} \%), \mathrm{K}_{2} \mathrm{CO}_{3}(138 \mathrm{mg}, 1.00 \mathrm{mmol}$, 2.00 equiv) and 1,1-diphenylethylene ( $90.1 \mathrm{mg}, 0.50 \mathrm{mmol}, 1.00$ equiv) were placed in a predried $25 \mathrm{~mL}$ Schlenk tube. The tube was evacuated and purged with $\mathrm{N}_{2}$ three times. Benzyl chloride $2 \mathbf{a}$ (235 mg, $1.50 \mathrm{mmol}, 3.00$ equiv) and 1,4-dioxane $(2.0 \mathrm{~mL})$ were then added and the mixture was stirred at $60{ }^{\circ} \mathrm{C}$. After $20 \mathrm{~h}$, the resulting mixture was filtered throough a pad of silica gel and washed with EtOAc. The filtrate was concentrated in vacuo. Purification of the residue by column chromatography ( $n$-hexane/EtOAc 10:1) yielded 3da (42.3 $\mathrm{mg}, 31 \%$ ) as a colorless oil and recovered $\mathbf{1 d}(39.2 \mathrm{mg}, 50 \%)$ as a colorless oil. 


\section{Synthesis of Complex Ru-II}

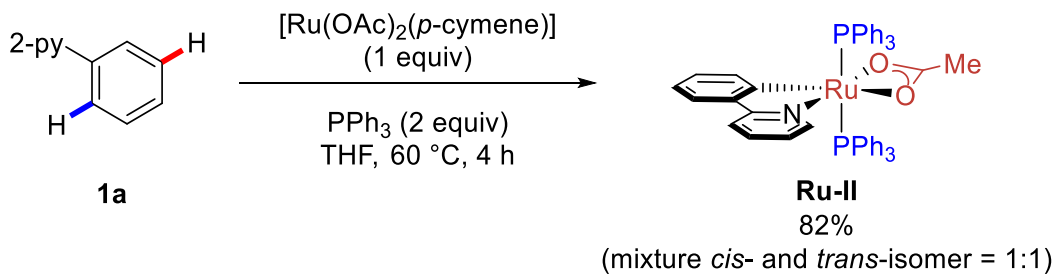

2-Phenylpyridine (1a) (155 mg, $1.00 \mathrm{mmol}, 1.00$ equiv), [Ru(OAc) 2 (p-cymene)] (353 mg, 1.00 mmol, 1.00 equiv), and $\mathrm{PPh}_{3}(525 \mathrm{mg}, 2.00 \mathrm{mmol}, 2.00$ equiv) were placed in a pre-dried $25 \mathrm{~mL}$ Schlenk tube. The tube was evacuated and purged with $\mathrm{N}_{2}$ three times. THF $(2 \mathrm{~mL})$ was added and the mixture was stirred at $60{ }^{\circ} \mathrm{C}$ for $4 \mathrm{~h}$. An orange solid formed during this time and the reaction was cooled to ambient temperature. The orange solid was collected and washed with THF $(5.0 \mathrm{~mL})$ and dry $n$-hexane $(2 \times 10 \mathrm{~mL})$. The orange solid was dried under vacuum, providing complex Ru-II (690 mg, 82\%).

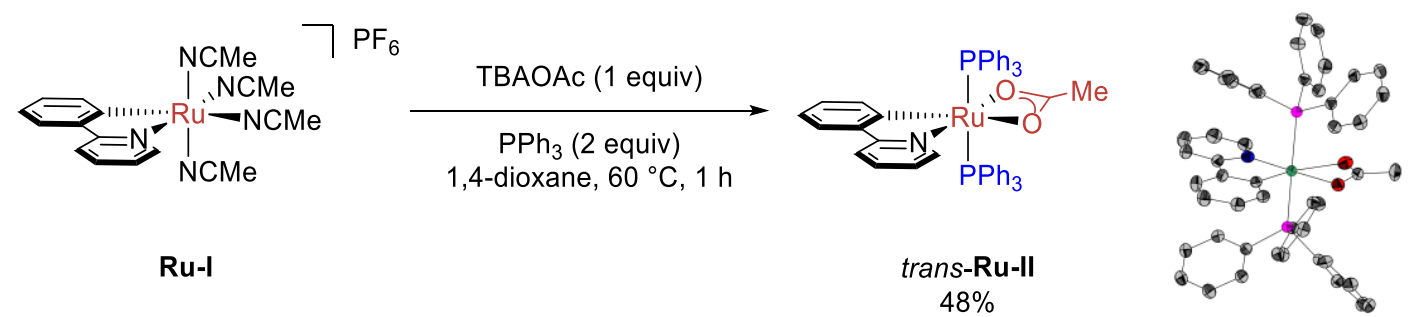

Ru-I (282 mg, $0.50 \mathrm{mmol}, 1.00$ equiv), TBAOAc (151 mg, $0.50 \mathrm{mmol}, 1.00$ equiv), and $\mathrm{PPh}_{3}$ (262 mg, $1.00 \mathrm{mmol}, 2.00$ equiv) were placed in a pre-dried $25 \mathrm{~mL}$ Schlenk tube. The tube was evacuated and purged with $\mathrm{N}_{2}$ three times. 1,4-Dioxane $(1.6 \mathrm{~mL})$ was added and the mixture was stirred at $60{ }^{\circ} \mathrm{C}$ for $1 \mathrm{~h}$. Then, the reaction was cooled to ambient temperature. The orange solid was collected and washed with THF $(5.0 \mathrm{~mL})$ and dry $n$-hexane $(2 \times 10 \mathrm{~mL})$. The orange solid was dried under vacuum, providing complex trans-Ru-II (203 mg, 48\%). Suitable crystals of trans-Ru-II for X-ray crystallography were grown by slow crystallization from $\mathrm{THF} / n$-hexane (see X-Ray crystallographic analysis section).

\section{Characteristic Data for trans-Ru-II}

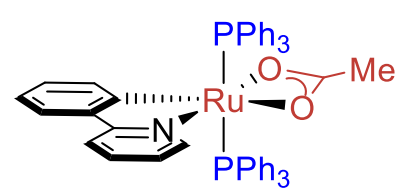

${ }^{1}$ H-NMR $\left(300 \mathrm{MHz}, \mathrm{CD}_{2} \mathrm{Cl}_{2}\right): \delta=8.79(\mathrm{~d}, J=5.7 \mathrm{~Hz}, 1 \mathrm{H}), 7.90(\mathrm{~d}$, $J=7.4 \mathrm{~Hz}, 1 \mathrm{H}), 7.30-7.06(\mathrm{~m}, 30 \mathrm{H}), 6.93-6.84(\mathrm{~m}, 1 \mathrm{H}), 6.74$ (dd, $J$ $=7.4,1.7 \mathrm{~Hz}, 1 \mathrm{H}), 6.64(\mathrm{~d}, J=8.2 \mathrm{~Hz}, 1 \mathrm{H}), 6.53(\mathrm{ddd}, J=7.4,7.1$,

$1.7 \mathrm{~Hz}, 1 \mathrm{H}), 6.46(\mathrm{dd}, J=7.4,7.1 \mathrm{~Hz}, 1 \mathrm{H}), 6.34(\mathrm{ddd}, J=7.2,5.7,1.5 \mathrm{~Hz}, 1 \mathrm{H}), 0.77$ (s, 3H). 
${ }^{13}$ C-NMR (75 MHz, $\left.\mathrm{CD}_{2} \mathrm{Cl}_{2}\right): \delta=184.5\left(\mathrm{dd}, J=23,11 \mathrm{~Hz}, \mathrm{C}_{\mathrm{q}}\right), 180.9\left(\mathrm{C}_{\mathrm{q}}\right), 169.8\left(\mathrm{C}_{\mathrm{q}}\right), 153.2$ $(\mathrm{CH}), 148.8\left(\mathrm{C}_{\mathrm{q}}\right), 140.7(\mathrm{CH}), 134.7\left(\mathrm{t},{ }^{2} J_{\mathrm{C}-\mathrm{P}}+{ }^{4} J_{\mathrm{C}-\mathrm{P}}=11 \mathrm{~Hz}, \mathrm{CH}\right), 133.0\left(\mathrm{t},{ }^{1} J_{\mathrm{C}-\mathrm{P}}+{ }^{3} J_{\mathrm{C}-\mathrm{P}}=\right.$ $\left.35 \mathrm{~Hz}, \mathrm{C}_{\mathrm{q}}\right), 131.9(\mathrm{CH}), 129.1(\mathrm{CH}), 127.7\left(\mathrm{t},{ }^{3} J_{\mathrm{C}-\mathrm{P}}+{ }^{5} J_{\mathrm{C}-\mathrm{P}}=9 \mathrm{~Hz}, \mathrm{CH}\right), 125.3(\mathrm{CH}), 123.5$ $(\mathrm{CH}), 118.8(\mathrm{CH}), 118.0(\mathrm{CH}), 117.9(\mathrm{CH}), 22.8\left(\mathrm{CH}_{3}\right)$.

${ }^{31} \mathbf{P}\left\{{ }^{1} \mathbf{H}\right\}$-NMR $\left(121 \mathrm{MHz}, \mathrm{CD}_{2} \mathrm{Cl}_{2}\right): \delta=41.2(\mathrm{~s})$.

IR (ATR): $\tilde{v}=3047,1536,1431,1090,1067,745,693,672,510,493 \mathrm{~cm}^{-1}$.

m.p.: $100-103{ }^{\circ} \mathrm{C}$ (decomp.).

MS (LIFDI) $m / z$ (relative intensity): $839.2(100)[\mathrm{M}]^{+}$. 


\section{Synthesis of Complex Ru-III}

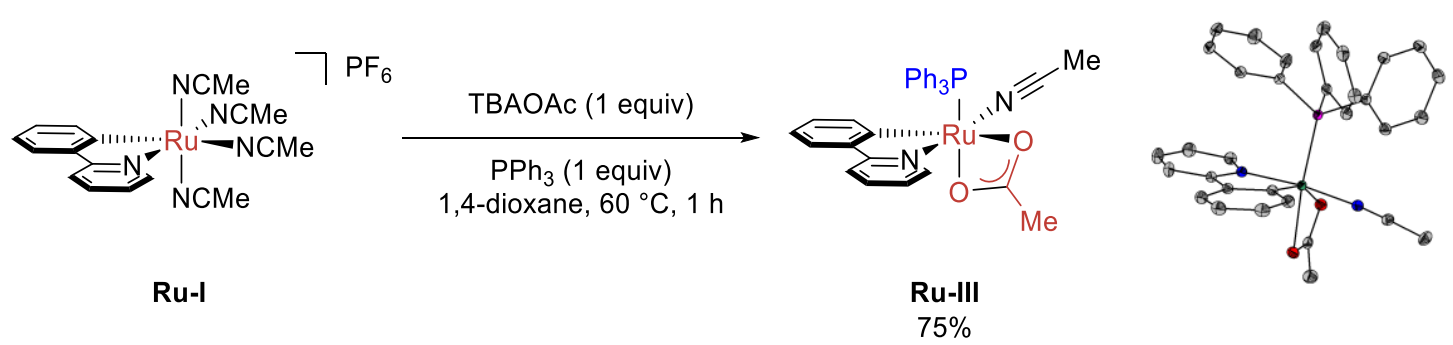

Ru-I (282 mg, 0.50 mmol, 1.00 equiv), TBAOAc (151 mg, $0.50 \mathrm{mmol}, 1.00$ equiv), and $\mathrm{PPh}_{3}$ (131 mg, $0.50 \mathrm{mmol}, 1.00$ equiv) were placed in a pre-dried $25 \mathrm{~mL}$ Schlenk tube. The tube was evacuated and purged with $\mathrm{N}_{2}$ three times. 1,4-Dioxane $(1.6 \mathrm{~mL})$ was added and the mixture was stirred at $60{ }^{\circ} \mathrm{C}$ for $1 \mathrm{~h}$. Then, the reaction was cooled to ambient temperature. The orange solid was collected and washed with THF $(5.0 \mathrm{~mL})$ and dry $n$-hexane $(2 \times 10 \mathrm{~mL})$. The orange solid was dried under vacuum, providing complex Ru-III (234 mg, 75\%). Suitable crystals of $\mathbf{R u}$-III for X-ray crystallography were grown by slow crystallization from $\mathrm{CH}_{2} \mathrm{Cl}_{2} / n$-hexane (see X-Ray crystallographic analysis section).

${ }^{1} \mathbf{H}-\mathbf{N M R}\left(300 \mathrm{MHz}, \mathrm{CD}_{2} \mathrm{Cl}_{2}\right): \delta=8.44(\mathrm{~d}, J=5.9 \mathrm{~Hz}, 1 \mathrm{H}), 7.61(\mathrm{~d}, J=7.6 \mathrm{~Hz}, 1 \mathrm{H}), 7.47-7.34$ (m, 3H), 7.30-7.19 (m, 3H), 7.19-7.08 (m, 12H), 6.83 (ddd, $J=7.5,7.3,1.5 \mathrm{~Hz}, 1 \mathrm{H}), 6.78-$ $6.67(\mathrm{~m}, 2 \mathrm{H}), 2.12(\mathrm{~s}, 3 \mathrm{H}), 1.89$ (s, 3H).

${ }^{13}$ C-NMR $\left(100 \mathrm{MHz}, \mathrm{CD}_{2} \mathrm{Cl}_{2}\right): \delta=187.3\left(\mathrm{~d},{ }^{2} J_{\mathrm{C}-\mathrm{P}}=16 \mathrm{~Hz}, \mathrm{C}_{\mathrm{q}}\right), 185.3\left(\mathrm{C}_{\mathrm{q}}\right), 167.4\left(\mathrm{C}_{\mathrm{q}}\right), 151.8$ $(\mathrm{CH}), 146.6\left(\mathrm{C}_{\mathrm{q}}\right), 140.0(\mathrm{CH}), 135.7\left(\mathrm{~d},{ }^{1} J_{\mathrm{C}-\mathrm{P}}=43 \mathrm{~Hz}, \mathrm{C}_{\mathrm{q}}\right), 134.7(\mathrm{CH}), 133.6\left(\mathrm{~d},{ }^{2} J_{\mathrm{C}-\mathrm{P}}=10 \mathrm{~Hz}\right.$, $\mathrm{CH}), 129.1\left(\mathrm{~d},{ }^{4} J_{\mathrm{C}-\mathrm{P}}=2 \mathrm{~Hz}, \mathrm{CH}\right), 127.9\left(\mathrm{~d},{ }^{3} J_{\mathrm{C}-\mathrm{P}}=9 \mathrm{~Hz}, \mathrm{CH}\right), 126.8(\mathrm{CH}), 123.9(\mathrm{CH}), 122.1$ $\left(\mathrm{C}_{\mathrm{q}}\right), 120.6(\mathrm{CH}), 119.1(\mathrm{CH}), 118.0(\mathrm{CH}), 24.7\left(\mathrm{CH}_{3}\right), 4.9\left(\mathrm{CH}_{3}\right)$.

${ }^{31} \mathbf{P}\left\{{ }^{1} \mathbf{H}\right\}$-NMR $\left(162 \mathrm{MHz}, \mathrm{CD}_{2} \mathrm{Cl}_{2}\right): \delta=65.7$ (s).

IR (ATR): $\tilde{v}=3052,2241,1538,1474,1434,1092,751,696,664,518 \mathrm{~cm}^{-1}$.

m.p.: $147-149{ }^{\circ} \mathrm{C}$ (decomp.).

MS (LIFDI) $m / z$ (relative intensity): $618.2(100)[\mathrm{M}]^{+}$. 


\section{Synthesis of Complex Ru-IV}
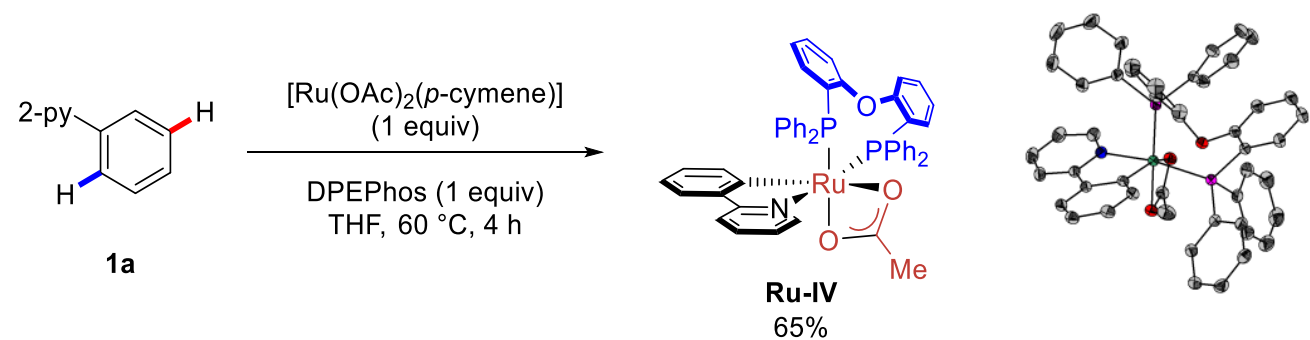

2-Phenylpyridine (1a) (77.6 mg, $0.50 \mathrm{mmol}, 1.00$ equiv), [Ru(OAc) 2 (p-cymene) $]$ (177 mg, 0.50 mmol, 1.00 equiv), and bis[2-(diphenylphosphino)phenyl]ether (DPEPhos, $269 \mathrm{mg}$, $0.50 \mathrm{mmol}, 1.00$ equiv) were placed in a pre-dried $25 \mathrm{~mL}$ Schlenk tube. The tube was evacuated and purged with $\mathrm{N}_{2}$ three times. THF $(2.0 \mathrm{~mL})$ was added and the mixture was stirred at $60{ }^{\circ} \mathrm{C}$ for $4 \mathrm{~h}$. A yellow solid formed during this time and the reaction was cooled to ambient temperature. The yellow solid was collected and washed with dry $n$-hexane $(2 \times 10 \mathrm{~mL})$. The yellow solid was dried under vacuum, providing complex Ru-IV (276 mg, 65\%). Suitable crystals of Ru-IV for X-ray crystallography were grown by slow crystallization from $\mathrm{THF} / n$-hexane (see X-Ray crystallographic analysis section).

${ }^{1} \mathbf{H}-\mathbf{N M R}\left(400 \mathrm{MHz}, \mathrm{CD}_{2} \mathrm{Cl}_{2}\right): \delta=7.94-7.89(\mathrm{~m}, 1 \mathrm{H}), 7.73(\mathrm{~d}, J=8.2 \mathrm{~Hz}, 1 \mathrm{H}), 7.58(\mathrm{~d}, J=$ $7.8 \mathrm{~Hz}, 1 \mathrm{H}), 7.54-7.06(\mathrm{~m}, 20 \mathrm{H}), 6.91(\mathrm{dd}, J=7.4,7.4 \mathrm{~Hz}, 1 \mathrm{H}), 6.87-6.79$ (m, 2H), 6.74-6.62 $(\mathrm{m}, 5 \mathrm{H}), 6.57(\mathrm{dd}, J=8.3,8.3 \mathrm{~Hz}, 1 \mathrm{H}), 6.43(\mathrm{dd}, J=6.5,6.5 \mathrm{~Hz}, 1 \mathrm{H}), 6.23(\mathrm{dd}, J=7.5,7.5 \mathrm{~Hz}$, $1 \mathrm{H}), 6.08(\mathrm{dd}, J=8.7,8.7 \mathrm{~Hz}, 2 \mathrm{H}), 0.87(\mathrm{~d}, J=1.7 \mathrm{~Hz}, 3 \mathrm{H})$.

${ }^{13}$ C-NMR $\left(100 \mathrm{MHz}, \mathrm{CD}_{2} \mathrm{Cl}_{2}\right): \delta=184.5\left(\mathrm{~d},{ }^{3} J_{\mathrm{C}-\mathrm{P}}=2 \mathrm{~Hz}, \mathrm{C}_{\mathrm{q}}\right), 182.0\left(\mathrm{dd},{ }^{3} J_{\mathrm{C}-\mathrm{P}}=17,8 \mathrm{~Hz}, \mathrm{C}_{\mathrm{q}}\right)$, $164.5\left(\mathrm{C}_{\mathrm{q}}\right), 160.7\left(\mathrm{~d},{ }^{2} J_{\mathrm{C}-\mathrm{P}}=8 \mathrm{~Hz}, \mathrm{C}_{\mathrm{q}}\right), 158.9\left(\mathrm{~d},{ }^{2} J_{\mathrm{C}-\mathrm{P}}=8 \mathrm{~Hz}, \mathrm{C}_{\mathrm{q}}\right), 151.1(\mathrm{CH}), 146.6\left(\mathrm{~d},{ }^{2} J_{\mathrm{C}-\mathrm{P}}\right.$ $\left.=1 \mathrm{~Hz}, \mathrm{C}_{\mathrm{q}}\right), 145.3\left(\mathrm{~d},{ }^{4} J_{\mathrm{C}-\mathrm{P}}=4 \mathrm{~Hz}, \mathrm{CH}\right), 136.8\left(\mathrm{~d},{ }^{1} J_{\mathrm{C}-\mathrm{P}}=42 \mathrm{~Hz}, \mathrm{C}_{\mathrm{q}}\right), 136.3\left(\mathrm{~d},{ }^{1} J_{\mathrm{C}-\mathrm{P}}=43 \mathrm{~Hz}\right.$, $\left.\mathrm{C}_{\mathrm{q}}\right), 136.1-135.5(\mathrm{~m}, \mathrm{CH}), 135.4(\mathrm{CH}), 135.2\left(\mathrm{~d},{ }^{1} J_{\mathrm{C}-\mathrm{P}}=39 \mathrm{~Hz}, \mathrm{C}_{\mathrm{q}}\right), 135.2-134.6(\mathrm{br}, \mathrm{CH})$, $134.0\left(\mathrm{~d},{ }^{1} J_{\mathrm{C}-\mathrm{P}}=37 \mathrm{~Hz}, \mathrm{C}_{\mathrm{q}}\right), 134.1-133.6(\mathrm{~m}, \mathrm{CH}), 131.6\left(\mathrm{~d},{ }^{2} J_{\mathrm{C}-\mathrm{P}}=9 \mathrm{~Hz}, \mathrm{CH}\right), 131.3(\mathrm{CH})$, $131.1(\mathrm{CH}), 129.3(\mathrm{CH}), 128.6\left(\mathrm{~d},{ }^{4} J_{\mathrm{C}-\mathrm{P}}=2 \mathrm{~Hz}, \mathrm{CH}\right), 127.9\left(\mathrm{~d},{ }^{4} J_{\mathrm{C}-\mathrm{P}}=2 \mathrm{~Hz}, \mathrm{CH}\right), 127.8\left(\mathrm{~d},{ }^{3} J_{\mathrm{C}-}\right.$ $\mathrm{P}=7 \mathrm{~Hz}, \mathrm{CH}), 127.7\left(\mathrm{~d},{ }^{3} J_{\mathrm{C}-\mathrm{P}}=7 \mathrm{~Hz}, \mathrm{CH}\right), 127.4\left(\mathrm{~d},{ }^{1} J_{\mathrm{C}-\mathrm{P}}=39 \mathrm{~Hz}, \mathrm{C}_{\mathrm{q}}\right), 127.2\left(\mathrm{~d},{ }^{3} J_{\mathrm{C}-\mathrm{P}}=9 \mathrm{~Hz}\right.$, $\mathrm{CH}), 126.9\left(\mathrm{~d},{ }^{3} J_{\mathrm{C}-\mathrm{P}}=8 \mathrm{~Hz}, \mathrm{CH}\right), 125.7(\mathrm{CH}), 125.4\left(\mathrm{~d},{ }^{1} J_{\mathrm{C}-\mathrm{P}}=38 \mathrm{~Hz}, \mathrm{C}_{\mathrm{q}}\right), 124.7\left(\mathrm{~d},{ }^{3} J_{\mathrm{C}-\mathrm{P}}=\right.$ $6 \mathrm{~Hz}, \mathrm{CH}), 124.4\left(\mathrm{~d},{ }^{3} J_{\mathrm{C}-\mathrm{P}}=5 \mathrm{~Hz}, \mathrm{CH}\right), 123.3\left(\mathrm{~d},{ }^{3} J_{\mathrm{C}-\mathrm{P}}=6 \mathrm{~Hz}, \mathrm{CH}\right), 122.9(\mathrm{CH}), 120.1\left(\mathrm{~d},{ }^{4} J_{\mathrm{C}-}\right.$ $\mathrm{P}=3 \mathrm{~Hz}, \mathrm{CH}), 119.7(\mathrm{CH}), 118.6(\mathrm{br}, \mathrm{CH}), 117.6\left(\mathrm{~d},{ }^{4} J_{\mathrm{C}-\mathrm{P}}=2 \mathrm{~Hz}, \mathrm{CH}\right), 23.6\left(\mathrm{~d},{ }^{4} J_{\mathrm{C}-\mathrm{P}}=2 \mathrm{~Hz}\right.$, $\left.\mathrm{CH}_{3}\right)$.

${ }^{31} \mathbf{P}\left\{{ }^{1} \mathbf{H}\right\}$-NMR $\left(162 \mathrm{MHz}, \mathrm{CD}_{2} \mathrm{Cl}_{2}\right): \delta=59.5(\mathrm{~d}, J=37.7 \mathrm{~Hz}), 46.7(\mathrm{~d}, J=37.7 \mathrm{~Hz})$.

IR (ATR): $\tilde{v}=3050,1578,1454,1433,1232,1091,744,695,673,518 \mathrm{~cm}^{-1}$.

m.p.: $215-217^{\circ} \mathrm{C}$ (decomp.). 
MS (LIFDI) $m / z$ (relative intensity): 853.1 (100) [M] ${ }^{+}$. 


\section{Catalytic Reaction with Complex trans-Ru-II, Ru-III, and Ru-IV}

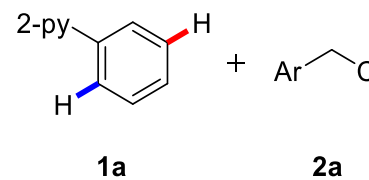

$2 a$

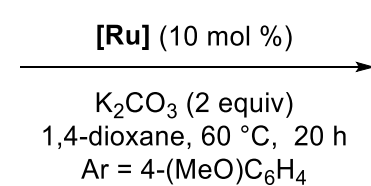

without KOAc

with $\mathrm{KOAc}(10 \mathrm{~mol} \%)$

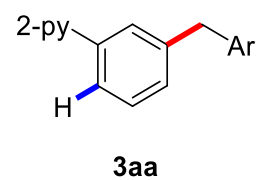

trans-Ru-II: $\quad 39 \%$

Ru-III: $\quad 50 \%$

Ru-IV: $\quad 0 \%$

trans-Ru-II: $\quad 54 \%$

Ru-III: $59 \%$

Ru-IV: $\quad 0 \%$

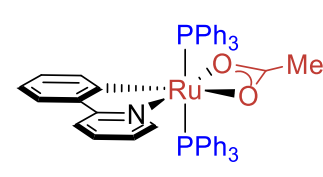

Ru-II

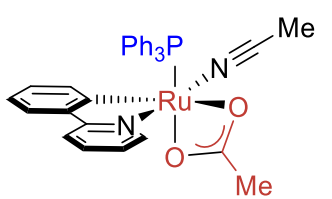

Ru-III

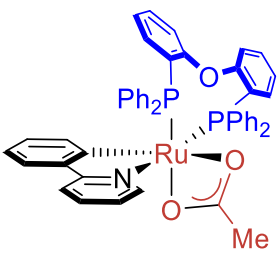

Ru-IV

\section{Reaction with Complex Ru-II and Ru-IV}

2-Phenylpyridine (1a) (38.8 mg, $0.25 \mathrm{mmol}, 1.00$ equiv), Ru-II (21.0 mg, $25.0 \mu \mathrm{mol}$, $10.0 \mathrm{~mol} \%)$ or Ru-IV $(21.3 \mathrm{mg}, 25.0 \mu \mathrm{mol}, 10.0 \mathrm{~mol} \%)$ and $\mathrm{K}_{2} \mathrm{CO}_{3}(69 \mathrm{mg}, 0.50 \mathrm{mmol}$, 2.00 equiv) were placed in a pre-dried $25 \mathrm{~mL}$ Schlenk tube. The tube was evacuated and purged with $\mathrm{N}_{2}$ three times. Benzyl chloride $2 \mathrm{a}(118 \mathrm{mg}, 0.75 \mathrm{mmol}, 3.00$ equiv) and 1,4-dioxane $(1.0 \mathrm{~mL})$ were then added and the mixture was stirred at $60^{\circ} \mathrm{C}$. After $20 \mathrm{~h}$, the resulting mixture was filtered through a pad of silica gel and washed with EtOAc. The filtrate was concentrated in vacuo. Purification of the residue by column chromatography ( $n$-hexane/EtOAc 10:1) yielded meta-product 3aa.

\section{Reaction with Complex Ru-III}

2-Phenylpyridine (1a) (77.6 mg, 0.5 mmol, 1.00 equiv), Ru-III (30.9 mg, $50.0 \mu \mathrm{mol}$, $10.0 \mathrm{~mol} \%$ ) and $\mathrm{K}_{2} \mathrm{CO}_{3}(139 \mathrm{mg}, 1.00 \mathrm{mmol}, 2.00$ equiv) were placed in a pre-dried $25 \mathrm{~mL}$ Schlenk tube. The tube was evacuated and purged with $\mathrm{N}_{2}$ three times. Benzyl chloride 2a (235 mg, $1.5 \mathrm{mmol}, 3.00$ equiv) and 1,4-dioxane $(2.0 \mathrm{~mL})$ were then added and the mixture was stirred at $60{ }^{\circ} \mathrm{C}$. After $20 \mathrm{~h}$, the resulting mixture was filtered through a pad of silica gel and washed with EtOAc. The filtrate was concentrated in vacuo. Purification of the residue by column chromatography ( $n$-hexane/EtOAc 10:1) yielded meta-adduct 3aa. 


\section{Stoichiometric Reaction of Complex Ru-II}

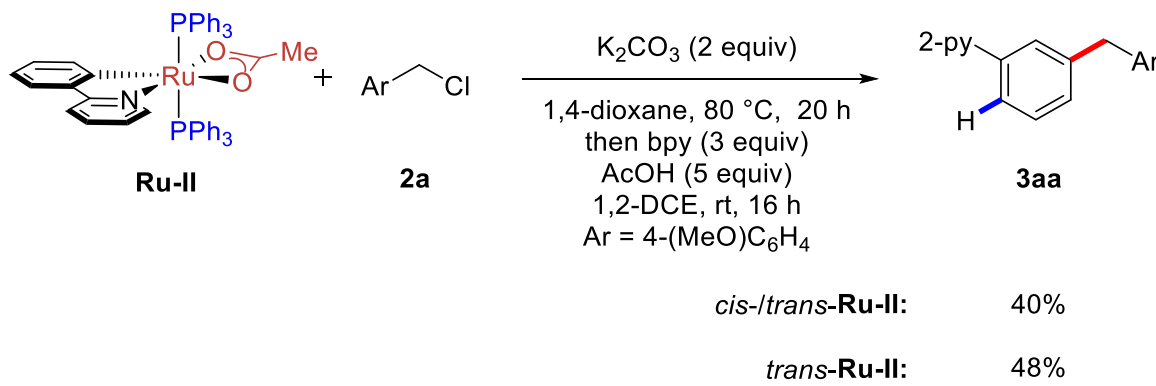

Complex Ru-II (84.0 mg, $0.10 \mathrm{mmol}, 1.00$ equiv) and $\mathrm{K}_{2} \mathrm{CO}_{3} \quad(28.0 \mathrm{mg}, 0.20 \mathrm{mmol}$, 2.00 equiv) were placed in a pre-dried $25 \mathrm{~mL}$ Schlenk tube. The tube was evacuated and purged with $\mathrm{N}_{2}$ three times. Benzyl chloride 2a $(23.5 \mathrm{mg}, 0.15 \mathrm{mmol}, 1.50$ equiv) and 1,4-dioxane $(1.0 \mathrm{~mL})$ were then added and the mixture was stirred at $80{ }^{\circ} \mathrm{C}$ for $20 \mathrm{~h}$. At ambient temperature, 2,2'-bipyridine ( $46.9 \mathrm{mg}, 0.30 \mathrm{mmol}, 3.00$ equiv), $\mathrm{AcOH}$ (30.0 mg, $0.50 \mathrm{mmol}, 5.00$ equiv) and 1,2-DCE $(1 \mathrm{~mL})$ were added, and the resulting mixture was stirred at ambient temperature for $16 \mathrm{~h}$. Then, the reaction mixture was quenched with sat. aq. $\mathrm{NaHCO}_{3}$ solution $(10 \mathrm{~mL})$ and the aqueous layer was extracted with $\mathrm{CH}_{2} \mathrm{Cl}_{2}(3 \times 10 \mathrm{~mL})$. The combined organic layers were dried over $\mathrm{Na}_{2} \mathrm{SO}_{4}$ and concentrated in vacuo. Purification of the residue by column chromatography ( -hexane/EtOAc 10:1) yielded meta-product 3aa (in case of cis-/trans-RuII: $11.0 \mathrm{mg}, 40 \%$, in case of trans-Ru-II: $13.2 \mathrm{mg}, 48 \%$ ) as a colorless oil. 


\section{Cyclic Voltammetry}

Cyclic voltammetric analysis was carried out with a Metrohm Autolab PGSTAT204 workstation and analysis was performed with Nova 2.1.4 software. A glassy-carbon electrode ( $3 \mathrm{~mm}$-diameter, disc-electrode) was used as the working electrode, a Pt wire was used as the auxiliary electrode and a $\mathrm{Ag} / \mathrm{AgCl}$ electrode was used as the reference. Measurements were carried out at a scan rate of $100 \mathrm{mV} \mathrm{s}^{-1}$.

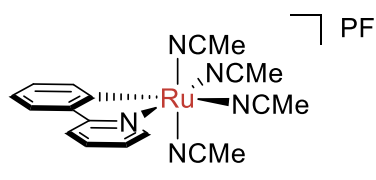

Ru-I

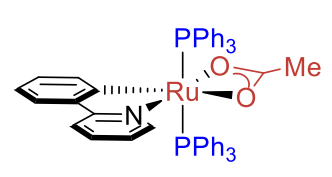

Ru-II

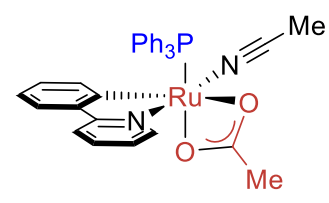

Ru-III

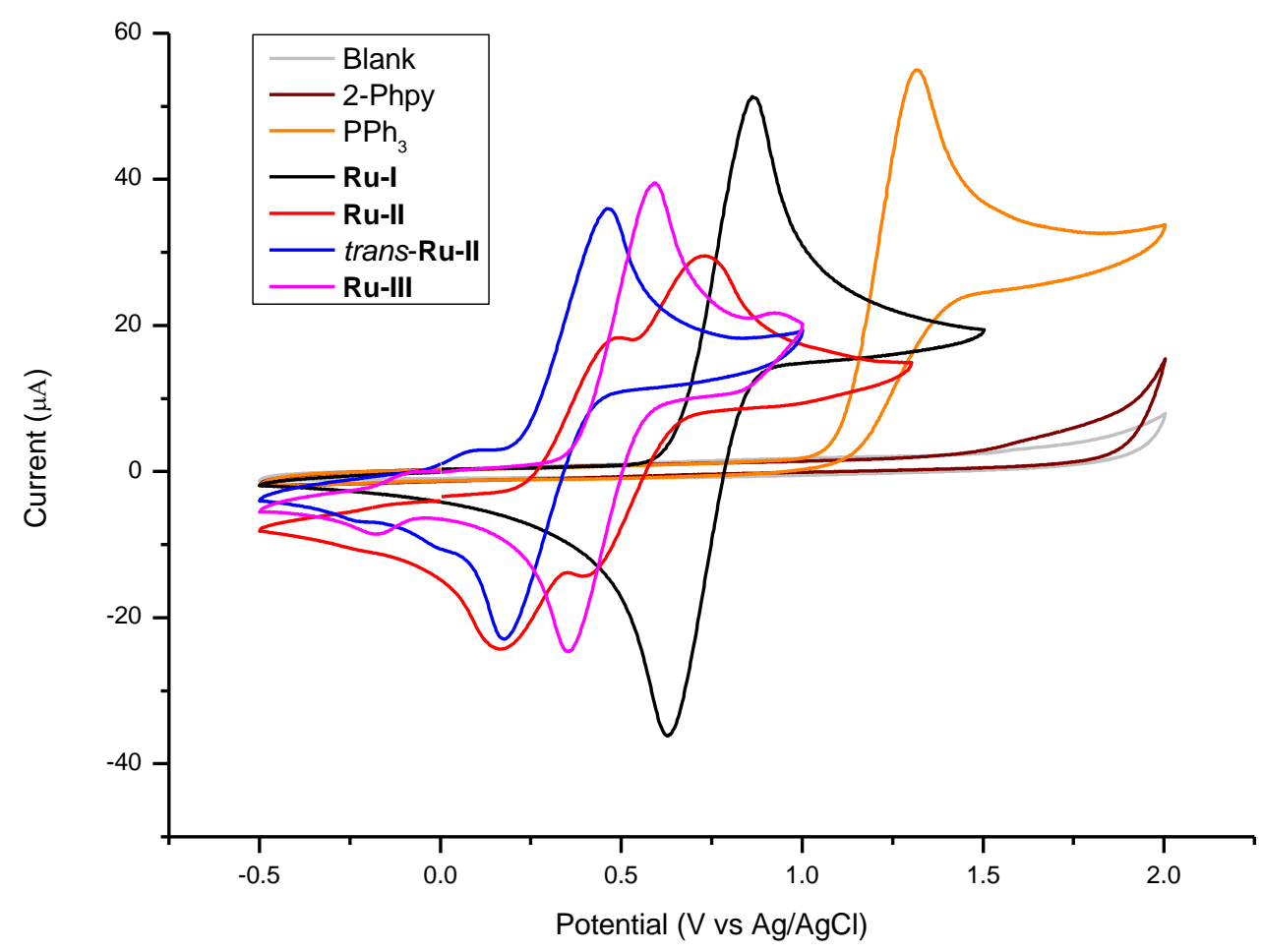

Figure S2. Cyclic Voltammogram at $100 \mathrm{mV} \mathrm{s}^{-1}$

Cyclic voltammograms at $100 \mathrm{mV} \mathrm{s}^{-1}$ in 1 ,2-DCE. $n$-Bu $\mathrm{NPF}_{6}$ (0.1 $\mathrm{M}$ in $\left.1,2-\mathrm{DCE}\right)$, concentration of substrates $4 \mathrm{mM}$. $E_{1 / 2}$ of $\mathbf{R u}-\mathbf{I}=0.75 \mathrm{~V}, E_{1 / 2}$ of cis-/trans-Ru-II $=0.33$ and $0.56 \mathrm{~V}, E_{1 / 2}$ of trans-Ru-II $=0.32 \mathrm{~V}, E_{1 / 2}$ of $\mathbf{R u}-\mathbf{I I I}=0.47 \mathrm{~V}$. 


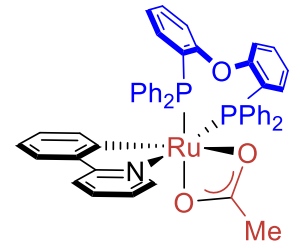

Ru-IV

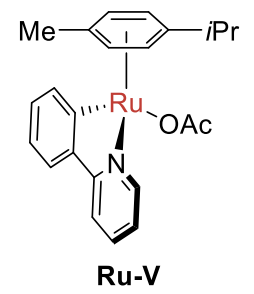

Ru-V

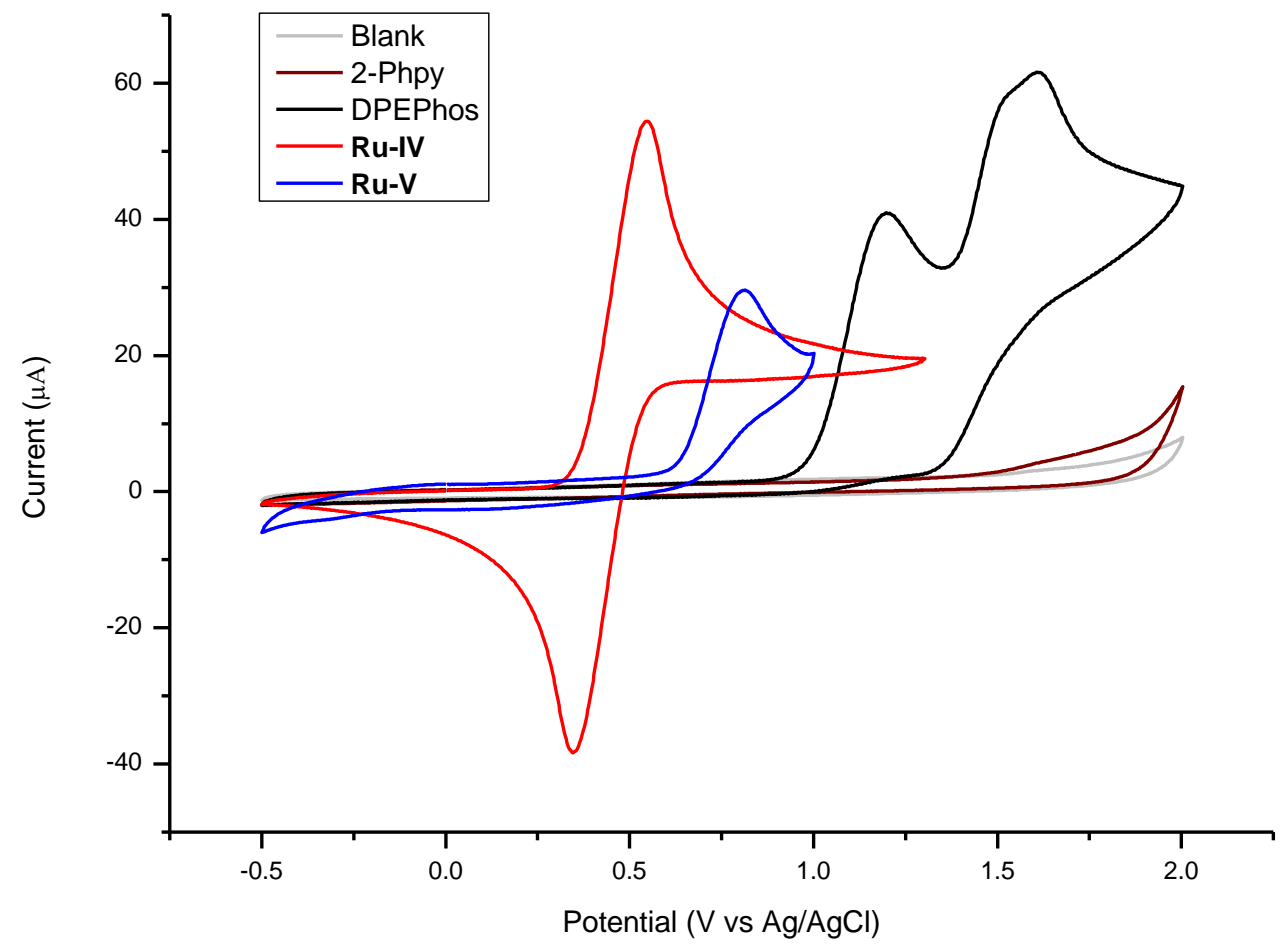

Figure S3. Cyclic Voltammogram at $100 \mathrm{mV} \mathrm{s}^{-1}$

Cyclic voltammograms at $100 \mathrm{mV} \mathrm{s}^{-1}$ in 1,2 -DCE. $n-\mathrm{Bu}_{4} \mathrm{NPF}_{6}(0.1 \mathrm{M}$ in $1,2-\mathrm{DCE})$, concentration of substrates $4 \mathrm{mM}$. $E_{1 / 2}$ of $\mathbf{R u}-\mathbf{I V}=0.44 \mathrm{~V}, E_{\mathrm{ox}}$ of $\mathbf{R u}-\mathbf{V}=0.81 \mathrm{~V}$. 


\section{Racemization Examination}

Benzyl chlorides $S-\mathbf{5 c}$ and $R-\mathbf{5 c}$ were examined by HPLC with a Daicel CHIRALPAK IA-3 (4.6 $\mathrm{mm}$ x $250 \mathrm{~mm}, 3 \mu \mathrm{m}$ particle size) $n$-hexane $/ i$-PrOH 80:20, $1 \mathrm{~mL} / \mathrm{min}$ flow rate, detection at $250 \mathrm{~nm}$. $S-\mathbf{5 c}: t_{r}=13.6 \mathrm{~min} . \quad R-\mathbf{5 c}: t_{r}=9.9 \mathrm{~min}$.<smiles>CC(=O)C(NC(=O)c1ccc(CCl)cc1)C(C)C</smiles>
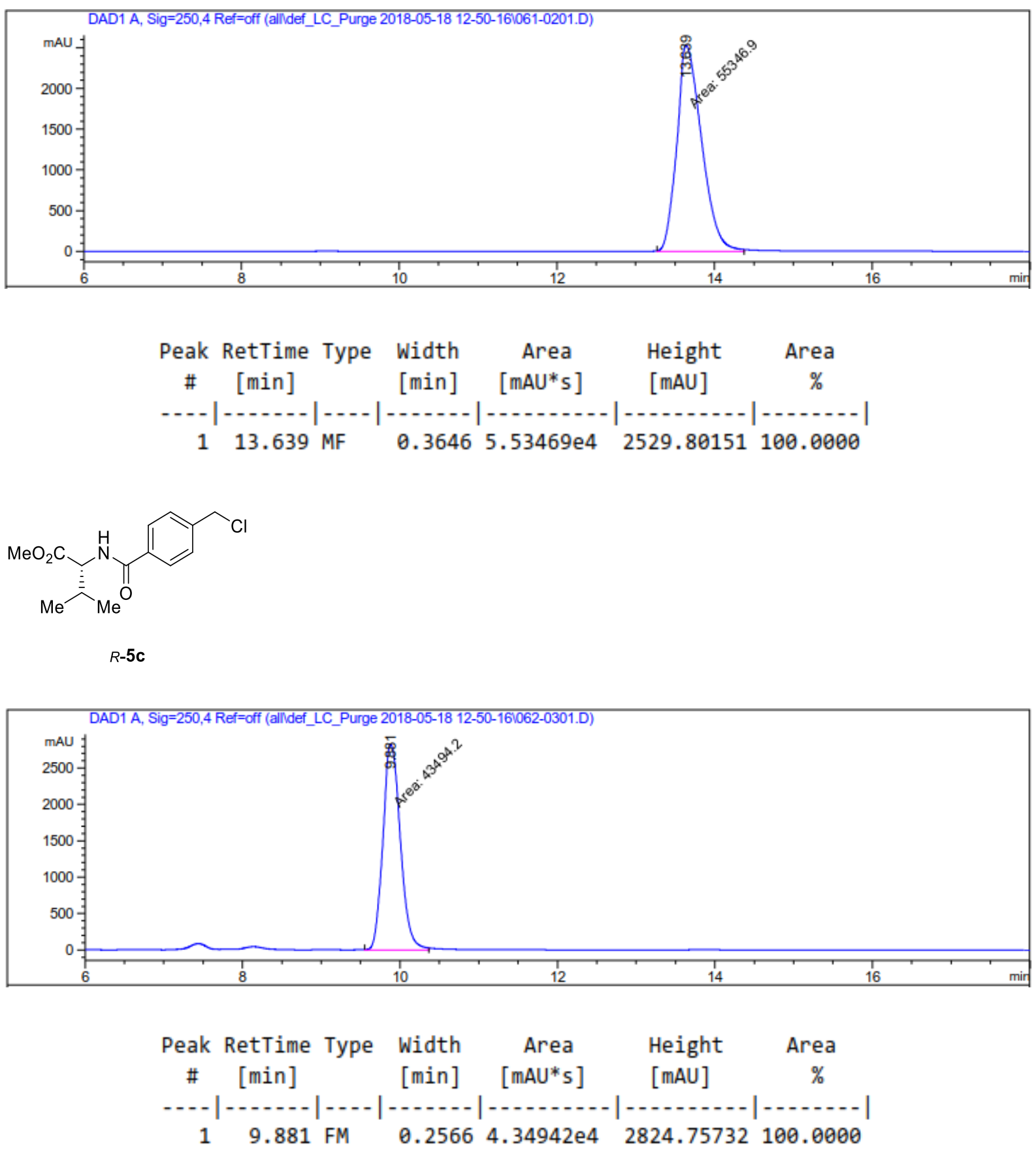
Mixture of $S-\mathbf{5 c}$ and $R-\mathbf{5 c}$
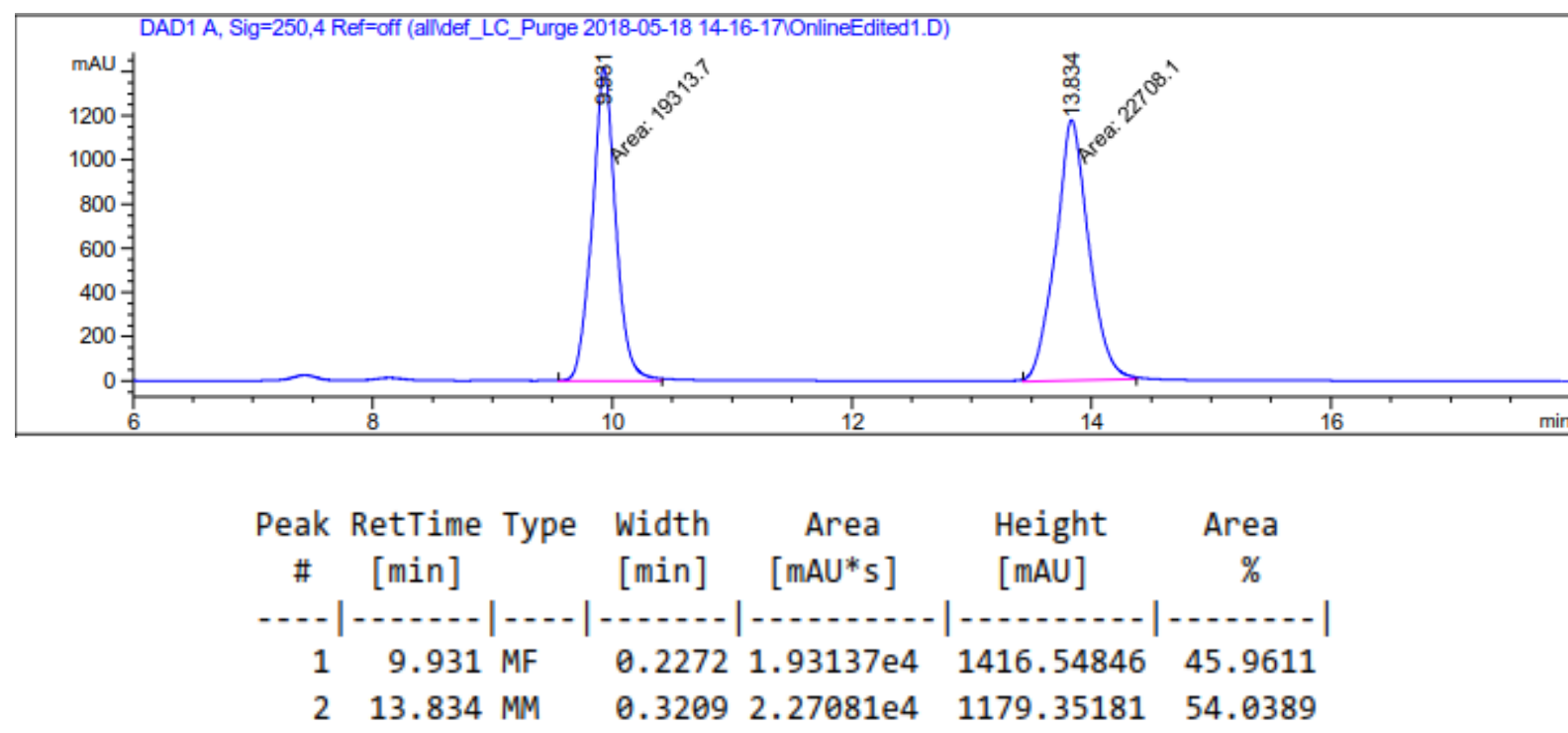

Benzyl chlorides rac-5d and $S-\mathbf{5 d}$ were examined by HPLC with a Daicel CHIRALPAK IA-3 (4.6 $\mathrm{mm}$ x $250 \mathrm{~mm}, 3 \mu \mathrm{m}$ particle size) $n$-hexane $/ i$-PrOH 70:30, $1 \mathrm{~mL} / \mathrm{min}$ flow rate, detection at $250 \mathrm{~nm} . S-\mathbf{5 d}: t_{r}=11.8 \mathrm{~min} . R-\mathbf{5 d}: t_{r}=9.1 \mathrm{~min}$.<smiles>CC(=O)C(CO)NC(=O)c1ccc(CCl)cc1</smiles>
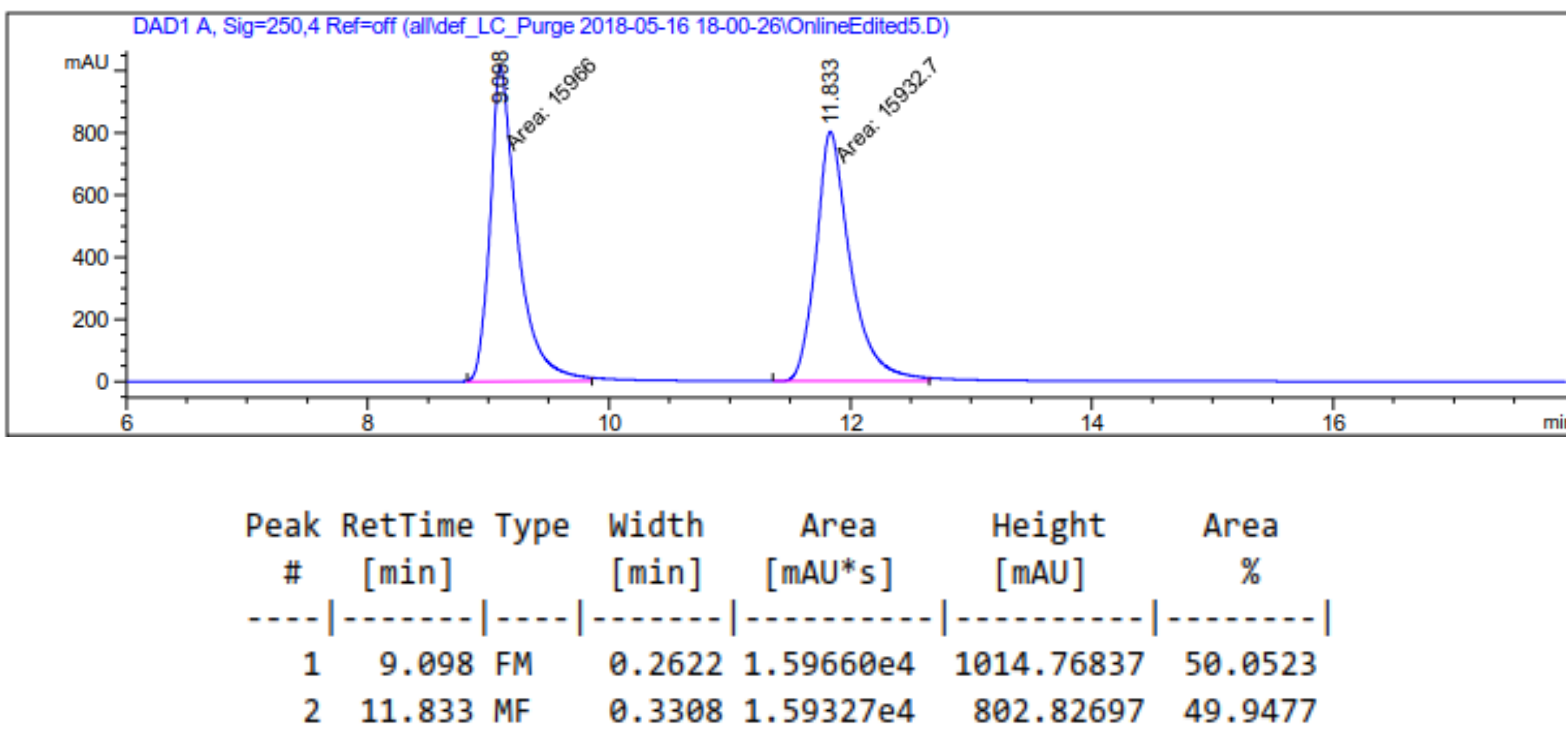

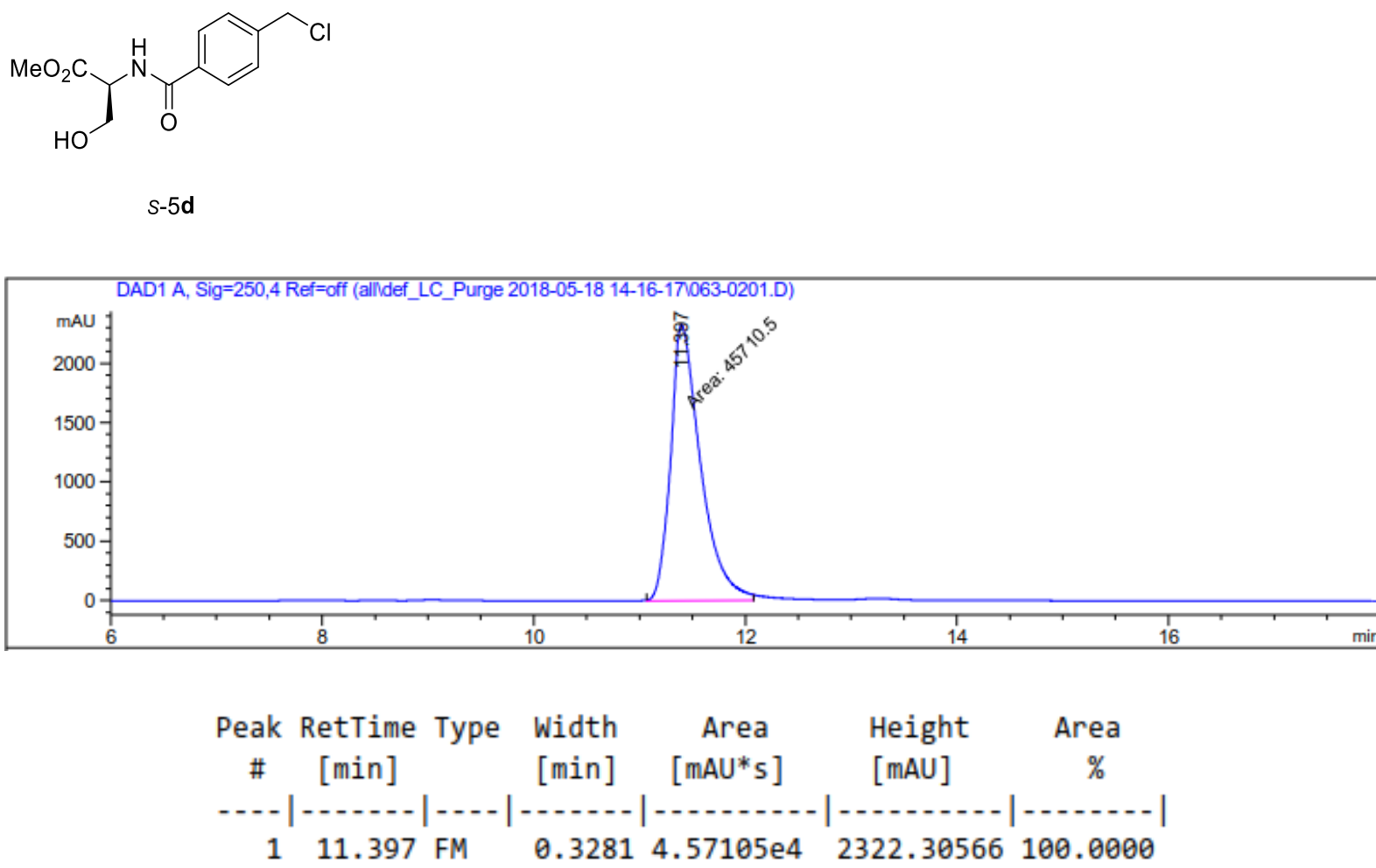

Benzyl chlorides $r a c-5 \mathbf{k}$ and $R-\mathbf{5} \mathbf{k}$ were examined by HPLC with a Daicel CHIRALPAK IC-3 (4.6 mm x $250 \mathrm{~mm}, 3 \mu \mathrm{m}$ particle size) $n$-hexane/EtOAc 95:5, $1 \mathrm{~mL} / \mathrm{min}$ flow rate, detection at $273 \mathrm{~nm} . R-\mathbf{5 k}: t_{r}=15.0 \mathrm{~min} . S-5 \mathbf{k}: t_{r}=19.3 \mathrm{~min}$.<smiles>CCCCCCCCCCCC(=O)OCC(COC(=O)c1ccc(CCl)cc1)OC(=O)CCCCCCC</smiles>
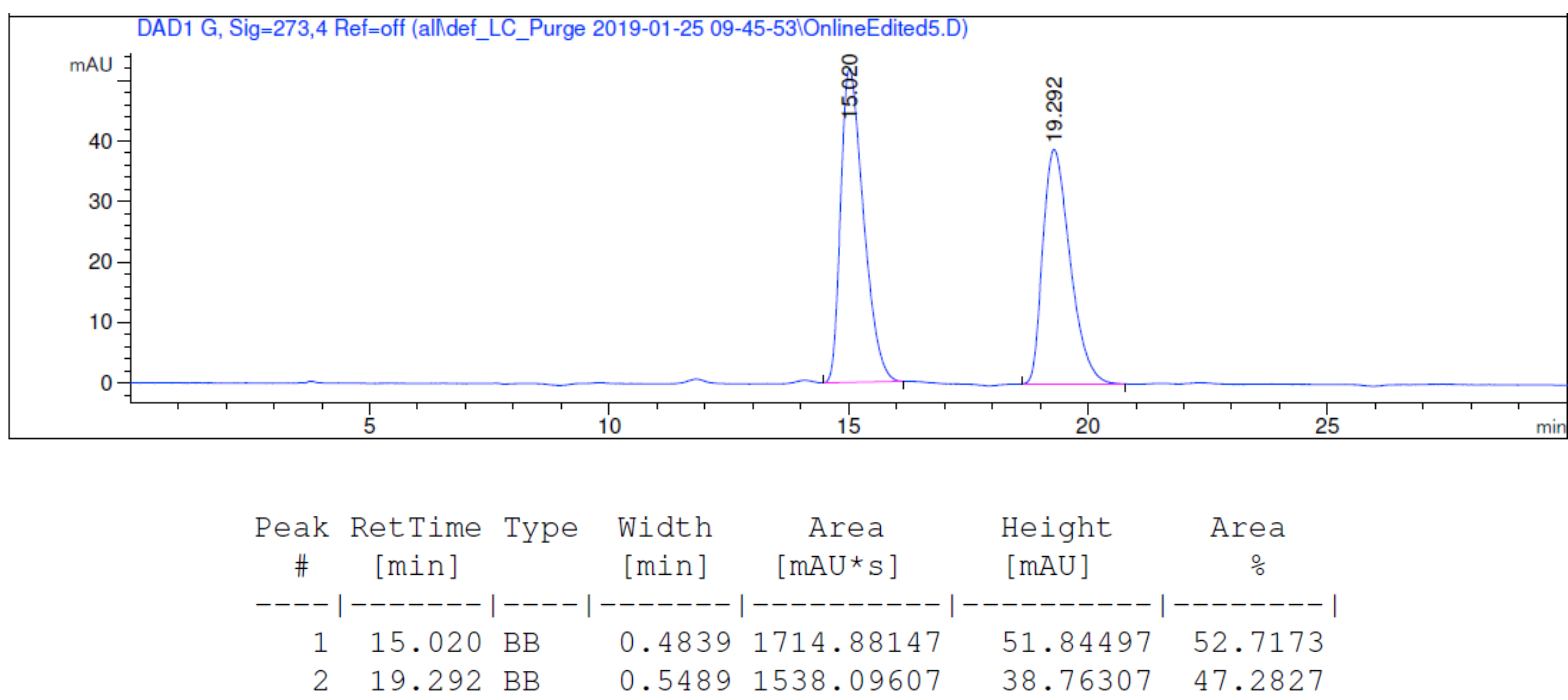
<smiles>CCCCCCCCCCCC(=O)OCC(COC(=O)c1ccc(CCl)cc1)OC(=O)CCCCCCCCCCC</smiles>

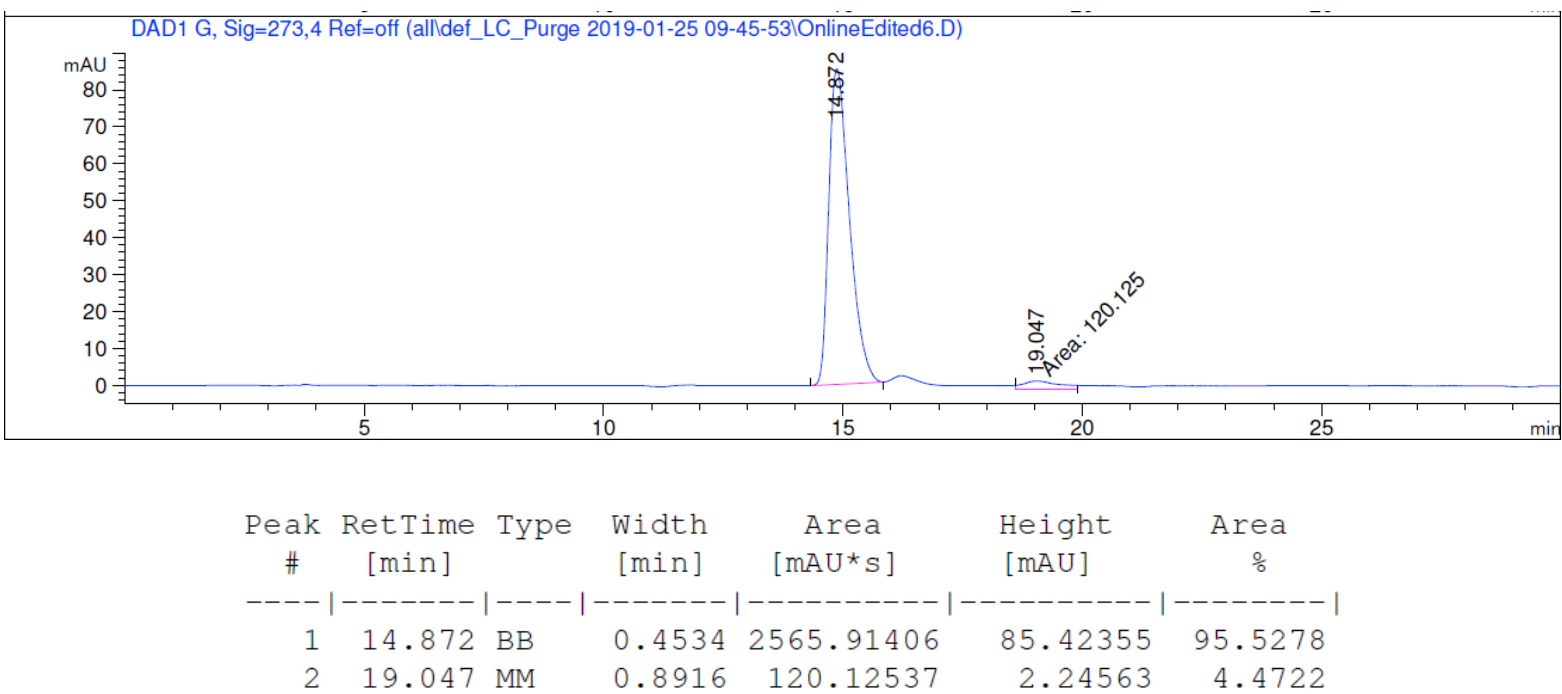

Compound $S$-6e and $R$-6e were examined by HPLC with a Daicel CHIRALPAK IA-3 $(4.6 \mathrm{~mm}$ x $250 \mathrm{~mm}, 3 \mu \mathrm{m}$ particle size) $n$-hexane $/ i-\mathrm{PrOH} 50: 50,1 \mathrm{~mL} / \mathrm{min}$ flow rate, detection at 250 nm. $S$-6e: $t_{r}=10.1$ min. $R$-6e: $t_{r}=7.8 \mathrm{~min}$.<smiles>CC(=O)C(NC(=O)c1ccc(Cc2cccc(-c3ncnc4c3ncn4C(C)C)c2)cc1)C(C)C</smiles>

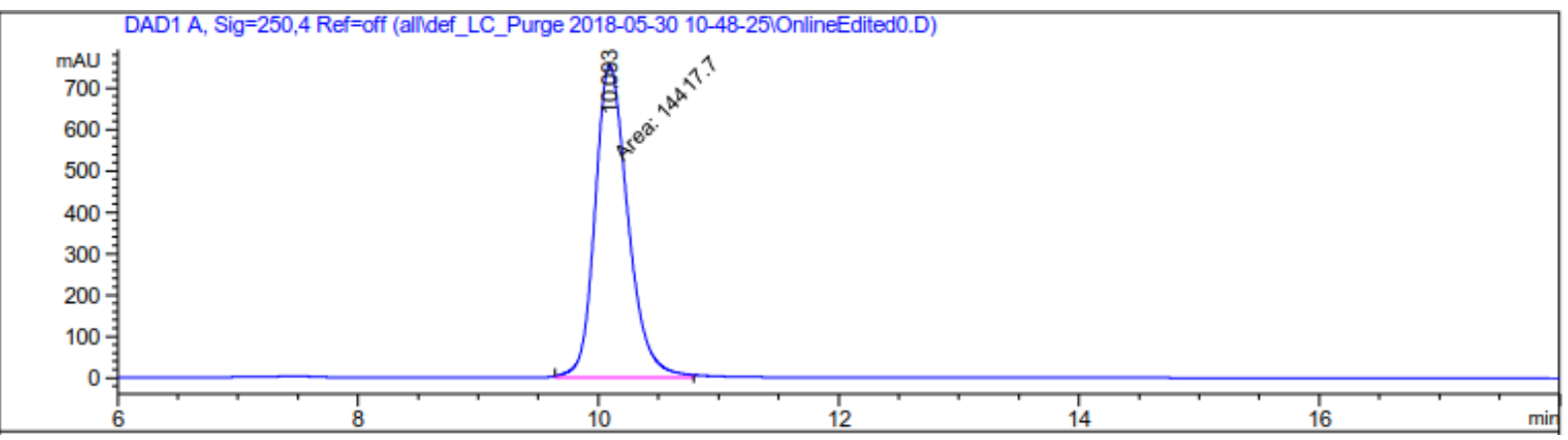




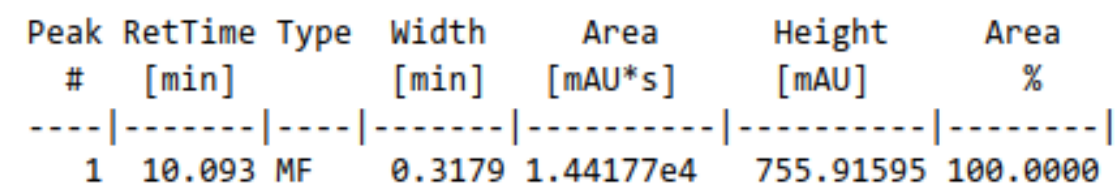<smiles>CC(=O)C(NC(=O)c1ccc(Cc2cccc(-c3ncnc4c3ncn4C(C)C)c2)cc1)C(C)C</smiles>

$R-6 e$
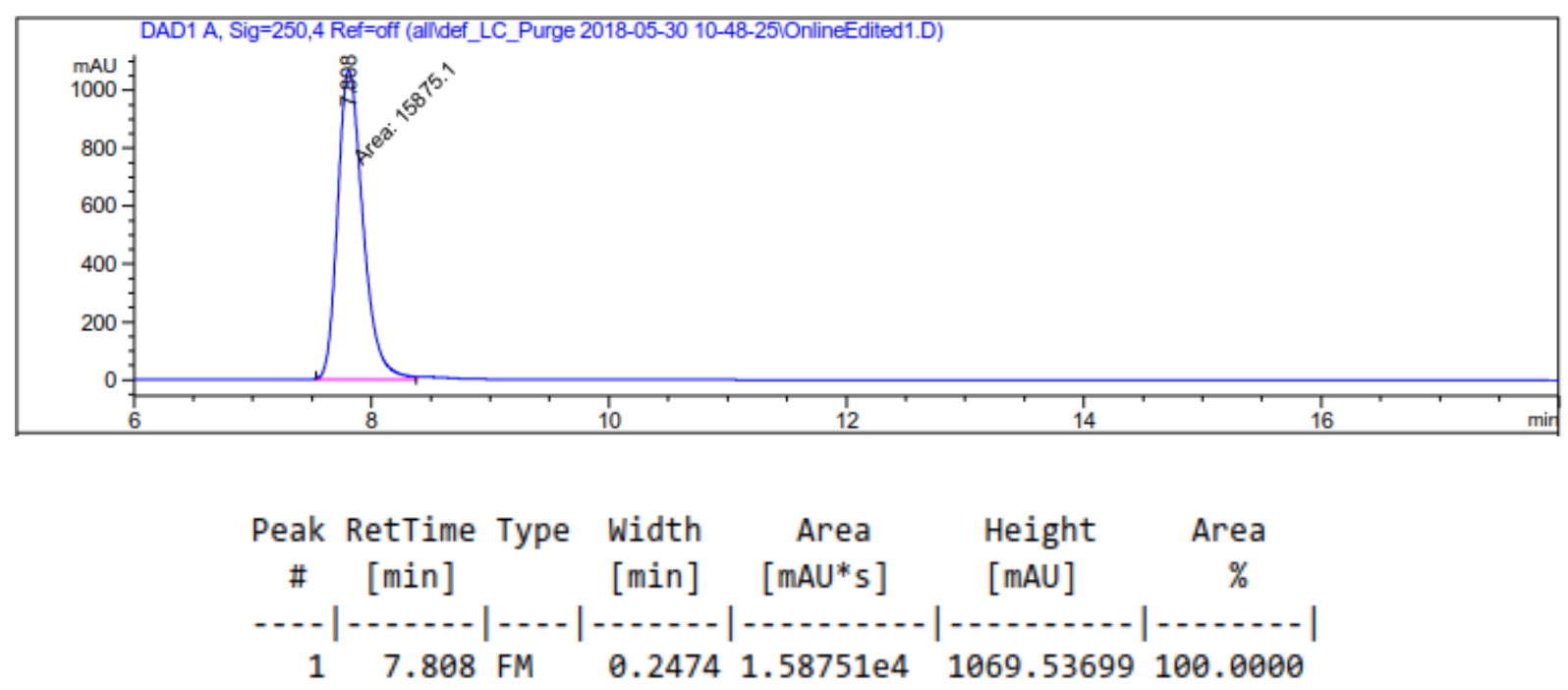

Mixture of $S-6 \mathbf{e}$ and $R-6 \mathbf{e}$
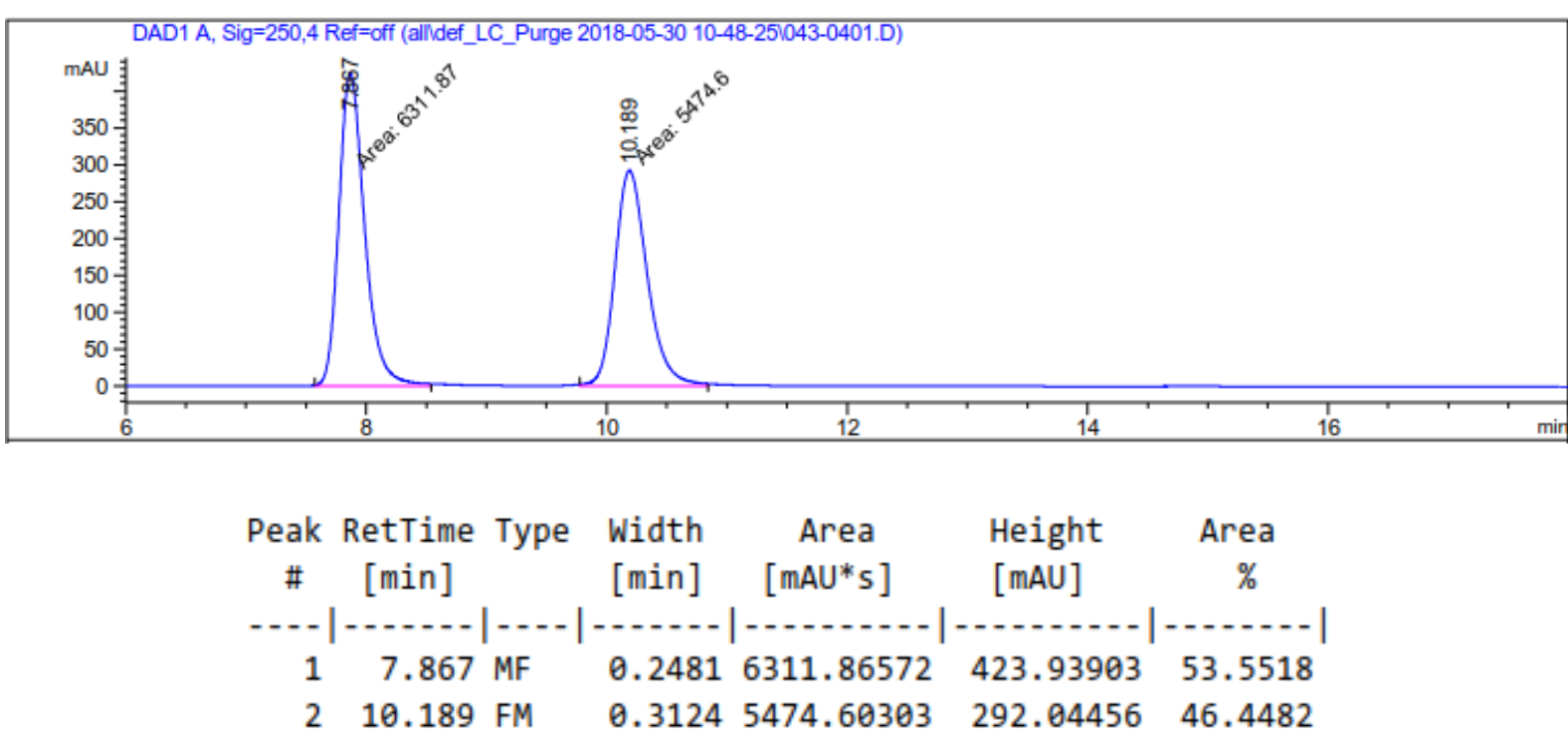
Compound rac-6f and $S$-6f were examined by HPLC with a Daicel CHIRALPAK IA-3 (4.6 mm x $250 \mathrm{~mm}, 3 \mu \mathrm{m}$ particle size) $n$-hexane $/ i-\mathrm{PrOH} 50: 50,1 \mathrm{~mL} / \mathrm{min}$ flow rate, detection at 250 nm. $S$-6f: $t_{r}=10.1$ min. $R$-6f: $t_{r}=8.0 \mathrm{~min}$.<smiles>CC(=O)C(CO)NC(=O)c1ccc(Cc2cccc(-c3ncnc4c3ncn4C(F)(F)F)c2)cc1</smiles>
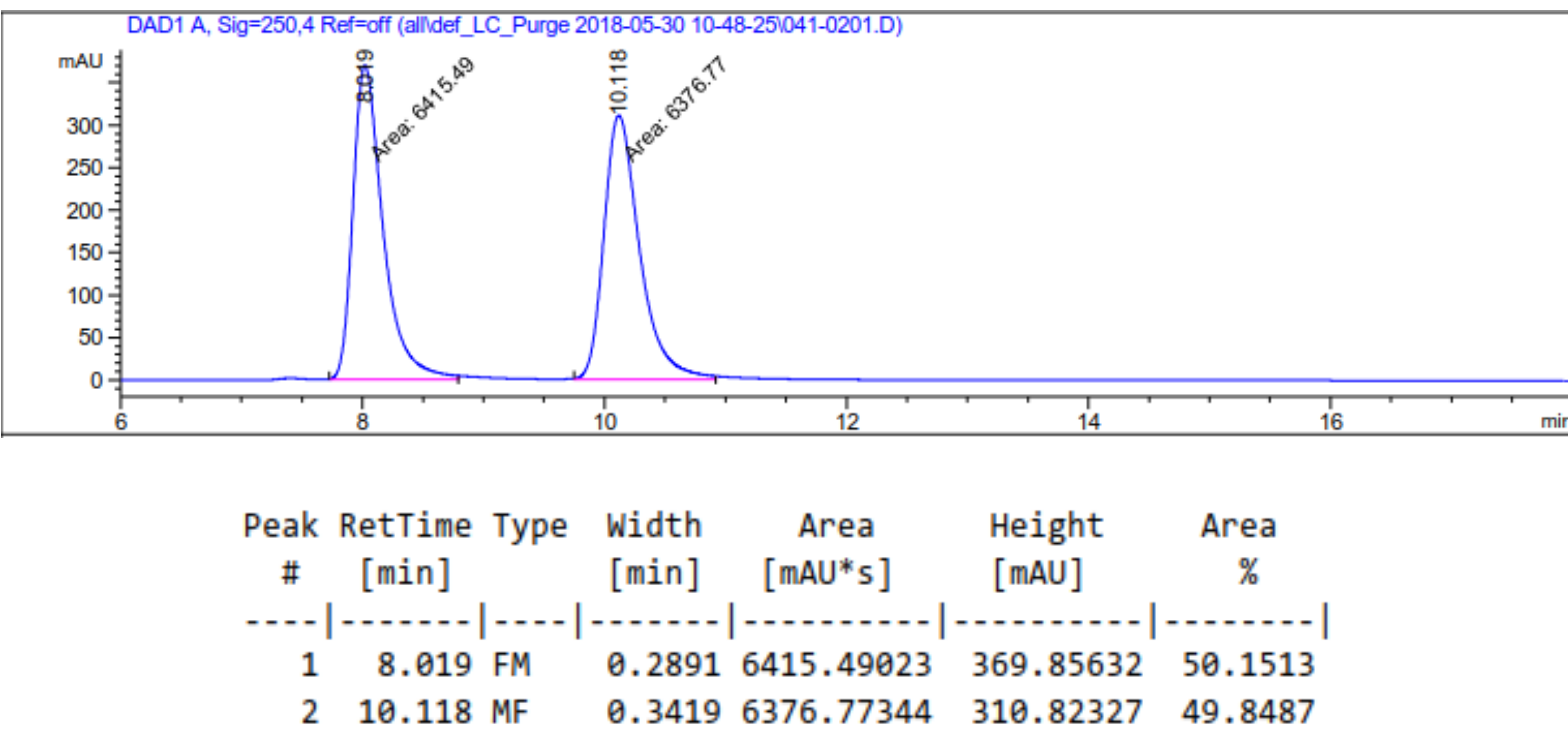<smiles>CC(=O)C(CO)NC(=O)c1ccc(Cc2cccc(-c3ncnc4c3ncn4C(F)(F)F)c2)cc1</smiles>

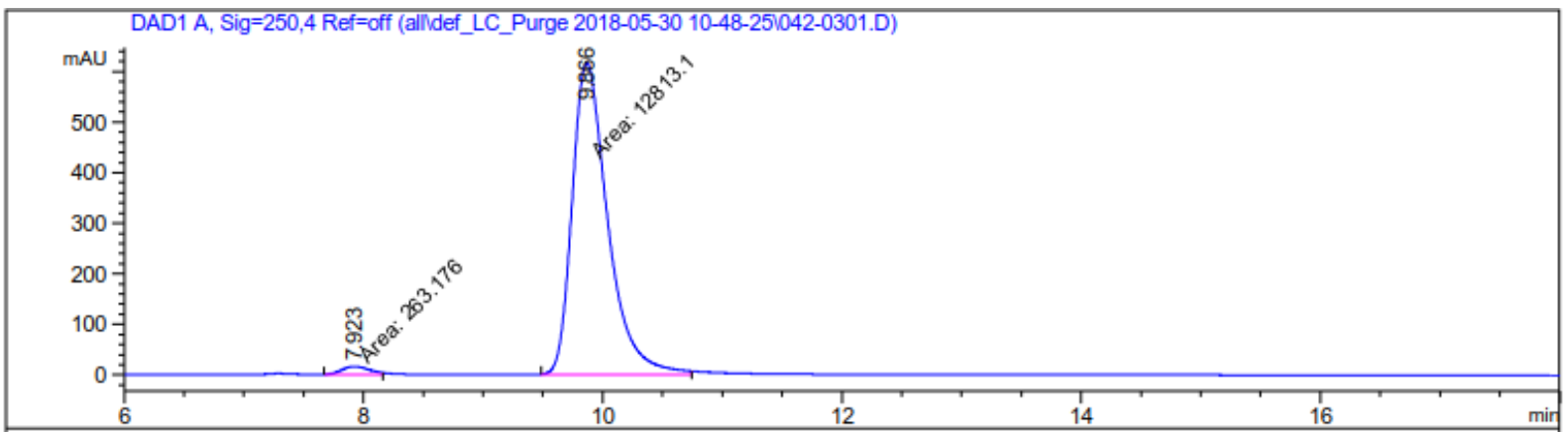




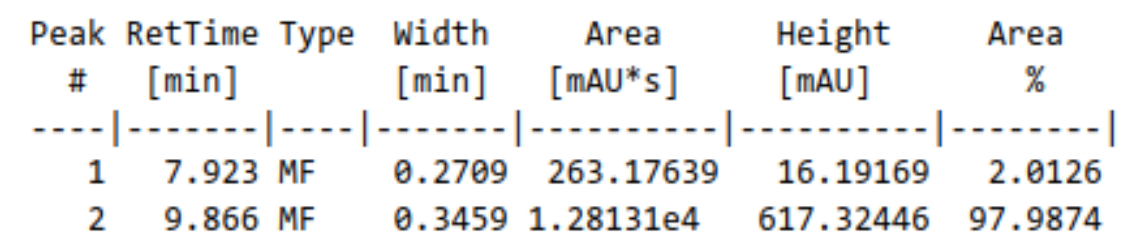

Compound rac-6m and $R-6 \mathbf{m}$ were examined by HPLC with a Daicel CHIRALPAK IC-3 (4.6 $\mathrm{mm}$ x $250 \mathrm{~mm}, 3 \mu \mathrm{m}$ particle size) $n$-hexane/EtOAc $85: 15,1 \mathrm{~mL} / \mathrm{min}$ flow rate, detection at $274 \mathrm{~nm} . R-\mathbf{6 m}: t_{r}=13.6 \mathrm{~min} . S-6 \mathrm{~m}: t_{r}=14.8 \mathrm{~min}$.<smiles>CCCCCCCCCCCCC(=O)OC(COC(=O)CCCCCCCCCCC)COC(=O)c1ccc(Cc2cccc(-c3ncnc4c3ncn4C(F)F)c2)cc1</smiles>

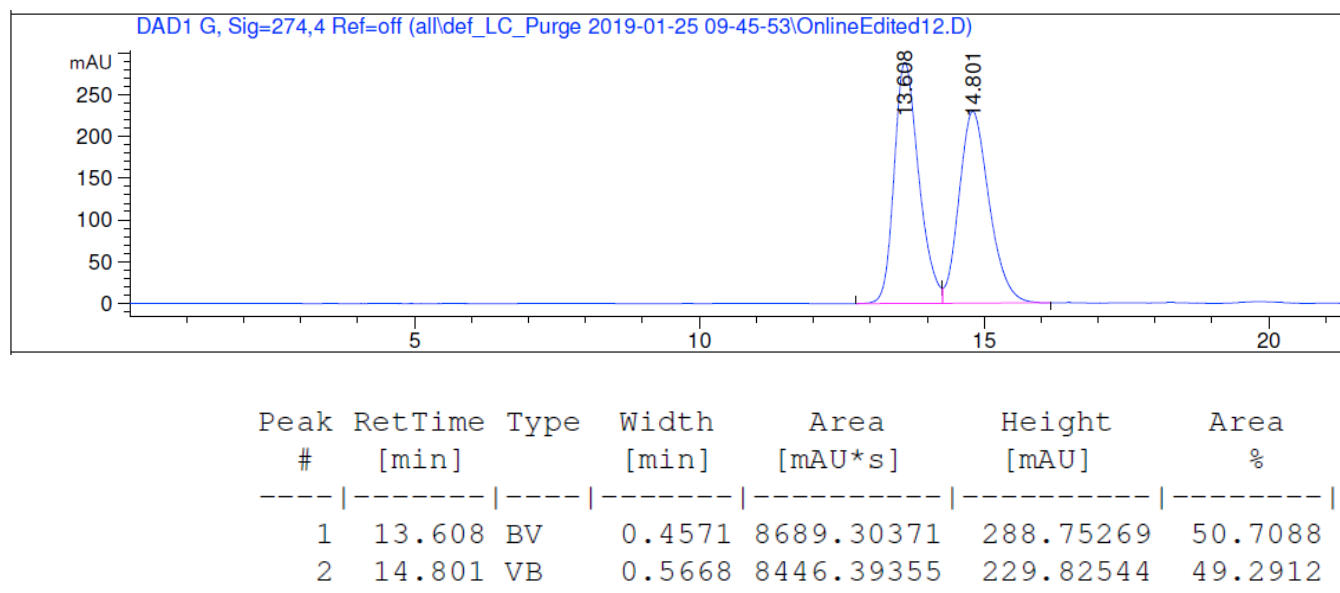<smiles>CCCCCCCCCCCCC(=O)OCC(COC(=O)CCCCCCCCCCC)OC(=O)c1ccc(Cc2cccc(-c3ncnc4c3ncn4C(C)C)c2)cc1</smiles>

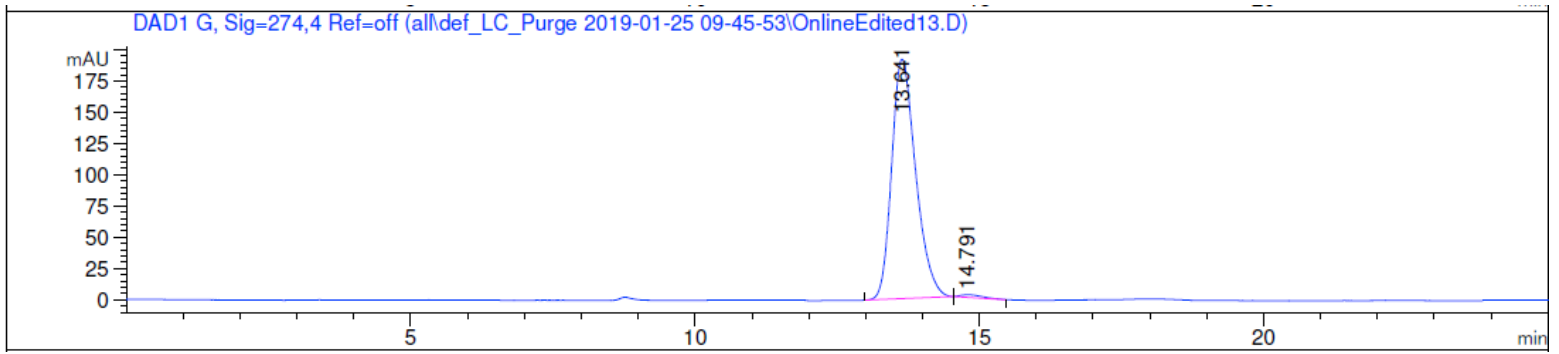




\begin{tabular}{|c|c|c|c|c|c|c|}
\hline $\begin{array}{c}\text { Peak } \\
\#\end{array}$ & $\begin{array}{c}\text { RetTime } \\
\text { [min] }\end{array}$ & Type & $\begin{array}{c}\text { Width } \\
{[\text { min }]}\end{array}$ & $\begin{array}{c}\text { Area } \\
{\left[\mathrm{mAU}{ }^{*} \mathrm{~S}\right]}\end{array}$ & $\begin{array}{l}\text { Height } \\
{[\mathrm{mAU}]}\end{array}$ & $\begin{array}{c}\text { Area } \\
\frac{\circ}{6}\end{array}$ \\
\hline 1 & 13.641 & BB & 0.4463 & 5623.72949 & 191.69371 & 99.0091 \\
\hline 2 & 14.791 & BB & 0.3248 & 56.28119 & 2.05320 & 0.9909 \\
\hline
\end{tabular}




\section{X-Ray Crystallographic Analysis}

The crystal was kept at $100.01 \mathrm{~K}$ during data collection. Using Olex $2,{ }^{13}$ the structure was solved with the $\mathrm{XT}^{14}$ structure solution program using Intrinsic Phasing and refined with the $\mathrm{XL}^{15}$ refinement package using Least Squares minimisation.

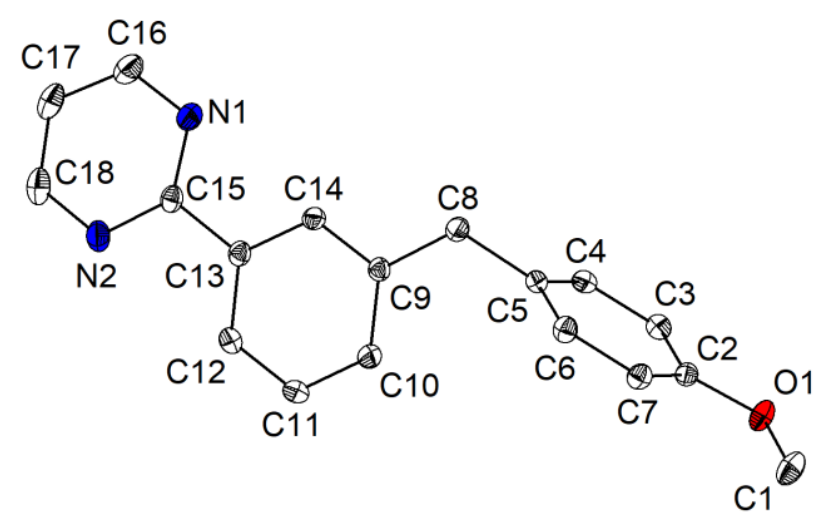

Figure S4. Molecular structure of 3da with thermal ellipoids at 50\% probability level. The hydrogen atoms are omitted for clarity.

Crystal Data for $\mathrm{C}_{18} \mathrm{H}_{16} \mathrm{~N}_{2} \mathrm{O}(M=276.33 \mathrm{~g} / \mathrm{mol})$ : orthorhombic, space group Pbca (no. 61), $a=$ 18.7085(6) $\AA, b=6.2302(2) \AA, c=24.4878(10) \AA, V=2854.24(17) \AA^{3}, Z=8, T=100.0 \mathrm{~K}$, $\mu(\mathrm{MoK} \alpha)=0.081 \mathrm{~mm}^{-1}$, Dcalc $=1.286 \mathrm{~g} / \mathrm{cm}^{3}, 37416$ reflections measured $\left(4.354^{\circ} \leq 2 \Theta \leq\right.$ $\left.59.152^{\circ}\right), 3996$ unique $\left(R_{\text {int }}=0.0318, R_{\text {sigma }}=0.0169\right)$ which were used in all calculations. The final $R_{1}$ was $0.0436(\mathrm{I}>2 \sigma(\mathrm{I}))$ and $w R_{2}$ was 0.1102 (all data).

Table S6. Crystal data and structure refinement for 3da

\begin{tabular}{|l|l|}
\hline Compound & 3da \\
\hline CCDC number & 1915683 \\
\hline Identification code & Pbca \\
\hline Empirical formula & $\mathrm{C}_{18} \mathrm{H}_{16} \mathrm{~N}_{2} \mathrm{O}$ \\
\hline Formula weight & 276.33 \\
\hline Temperature/K & 100.0 \\
\hline Crystal system & orthorhombic \\
\hline Space group & Pbca \\
\hline
\end{tabular}




\begin{tabular}{|c|c|}
\hline $\mathrm{a} / \AA ̊$ & $18.7085(6)$ \\
\hline $\mathrm{b} / \AA ̊$ & $6.2302(2)$ \\
\hline $\mathrm{c} / \AA ̊ \AA$ & $24.4878(10)$ \\
\hline$\alpha /^{\circ}$ & 90 \\
\hline$\beta /{ }^{\circ}$ & 90 \\
\hline$\gamma /{ }^{\circ}$ & 90 \\
\hline Volume $/ \AA^{3}$ & 2854.24(17) \\
\hline $\mathrm{Z}$ & 8 \\
\hline$\rho_{\text {calc } g / \mathrm{cm}^{3}}$ & 1.286 \\
\hline$\mu / \mathrm{mm}^{-1}$ & 0.081 \\
\hline $\mathrm{F}(000)$ & 1168.0 \\
\hline Crystal size $/ \mathrm{mm}^{3}$ & $0.251 \times 0.204 \times 0.076$ \\
\hline Radiation & $\operatorname{MoK} \alpha(\lambda=0.71073)$ \\
\hline \multicolumn{2}{|c|}{$2 \Theta$ range for data collection $/{ }^{\circ} 4.354$ to 59.152} \\
\hline Index ranges & $-21 \leq \mathrm{h} \leq 25,-8 \leq \mathrm{k} \leq 8,-33 \leq 1 \leq 33$ \\
\hline Reflections collected & 37416 \\
\hline Independent reflections & $3996\left[R_{\text {int }}=0.0318, R_{\text {sigma }}=0.0169\right]$ \\
\hline Data/restraints/parameters & $3996 / 0 / 191$ \\
\hline Goodness-of-fit on $\mathrm{F}^{2}$ & 1.068 \\
\hline Final $R$ indexes $[\mathrm{I}>=2 \sigma(\mathrm{I})]$ & $\mathrm{R}_{1}=0.0436, \mathrm{wR}_{2}=0.1080$ \\
\hline Final $\mathrm{R}$ indexes [all data] & $\mathrm{R}_{1}=0.0469, \mathrm{wR}_{2}=0.1102$ \\
\hline Largest diff. peak/hole / e $\AA^{-3}$ & $30.37 /-0.23$ \\
\hline
\end{tabular}

Table S7. Bond lengths [^] for 3da

Atom Atom Length/Å

O1 C1 1.4292(13)

O1 C2 1.3748(12)

N1 C15 1.3420(13)

N1 C16 1.3384(14)

$\mathrm{N} 2 \quad \mathrm{C} 15 \quad 1.3436(13)$

$\mathrm{N} 2 \quad \mathrm{C} 18 \quad 1.3394(15)$

\section{Atom Atom Length/Å}

C6 C7 1.3970(14)

C8 C9 1.5199(13)

C9 C10 1.3965(14)

C9 C14 1.3961(13)

C10 C11 1.3915(14)

C11 C12 1.3880(14) 


\section{Atom Atom Length/ ̊}

C2 C3 1.3954(13)

C2 C7 1.3883(14)

C3 C4 1.3854(14)

C4 C5 1.4001(13)

C5 C6 1.3907(13)

C5 C8 1.5123(13)

\section{Atom Atom Length/Å}

C12 C13 1.3999(14)

C13 C14 1.3976(13)

C13 C15 1.4850(14)

C16 C17 1.3807(17)

C17 C18 1.3810(18)

Table S8. Bond angles $\left[{ }^{\circ}\right]$ for $\mathbf{3 d a}$

\section{Atom Atom Atom Angle $/^{\circ}$}

C2 $\quad$ O1 $\quad$ C1 $117.38(8)$

C16 N1 C15 116.26(10)

C18 N2 C15 116.37(10)

O1 C2 C3 115.48(9)

O1 $\quad$ C2 $\quad$ C7 $124.56(9)$

$\begin{array}{llll}\text { C7 } & \text { C2 } & \text { C3 } & 119.95(9)\end{array}$

C4 C3 C2 119.97(9)

C3 C4 C5 121.13(9)

C4 C5 C8 120.37(9)

C6 $\quad$ C5 $\quad$ C4 117.99(9)

C6 $\quad$ C5 $\quad$ C8 $121.62(9)$

C5 $\quad$ C6 $\quad$ C7 $121.62(9)$

C2 C7 C6 119.33(9)

C5 $\quad$ C8 $\quad$ C9 $115.13(8)$

C10 C9 C8 121.71(9)

\section{Atom Atom Atom Angle ${ }^{\circ}$}

C14 C9 C8 119.58(9)

C14 C9 C10 118.70(9)

C11 C10 C9 120.51(9)

C12 C11 C10 120.57(9)

C11 C12 C13 119.69(9)

C12 C13 C15 119.76(9)

C14 C13 C12 119.40(9)

C14 C13 C15 120.79(9)

C9 $\mathrm{C} 14 \quad \mathrm{C} 13 \quad 121.12(9)$

$\mathrm{N} 1 \quad \mathrm{C} 15 \quad \mathrm{~N} 2 \quad 125.58(10)$

N1 C15 C13 $117.25(9)$

N2 $\quad$ C15 $\quad$ C13 $117.17(9)$

N1 $\quad \mathrm{C} 16 \quad \mathrm{C} 17 \quad 122.81(11)$

C16 C17 C18 116.37(10)

N2 $\quad \mathrm{C} 18 \quad \mathrm{C} 17 \quad 122.60(11)$ 


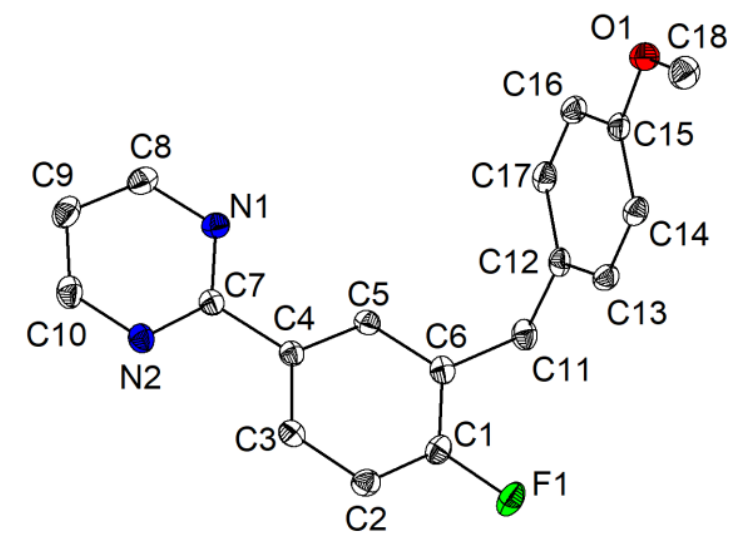

Figure S5. Molecular structure of 3ea with thermal ellipoids at 50\% probability level. The hydrogen atoms are omitted for clarity.

Crystal Data for $\mathrm{C}_{18} \mathrm{H}_{15} \mathrm{FN}_{2} \mathrm{O}(M=294.32 \mathrm{~g} / \mathrm{mol})$ : monoclinic, space group P2 $1 / \mathrm{c}$ (no. 14), $a=$ 8.1191(4) $\AA, b=5.6386(3) \AA, c=31.0628(17) \AA, \beta=93.276(2)^{\circ}, V=1419.74(13) \AA^{3}, Z=4$, $T=99.98 \mathrm{~K}, \mu(\mathrm{MoK} \alpha)=0.096 \mathrm{~mm}^{-1}$, Dcalc $=1.377 \mathrm{~g} / \mathrm{cm}^{3}, 20105$ reflections measured $\left(5.026^{\circ}\right.$ $\left.\leq 2 \Theta \leq 59.122^{\circ}\right), 3964$ unique $\left(R_{\text {int }}=0.0168, \mathrm{R}_{\text {sigma }}=0.0125\right)$ which were used in all calculations. The final $R_{1}$ was $0.0370(\mathrm{I}>2 \sigma(\mathrm{I}))$ and $w R_{2}$ was 0.1001 (all data).

Table S9. Crystal data and structure refinement for 3ea

\begin{tabular}{|l|l|}
\hline Compound & 3ea \\
\hline CCDC number & 1915686 \\
\hline Identification code & mo_0182_CG_0m \\
\hline Empirical formula & $\mathrm{C}_{18} \mathrm{H}_{15} \mathrm{FN}_{2} \mathrm{O}$ \\
\hline Formula weight & 294.32 \\
\hline Temperature/K & 99.98 \\
\hline Crystal system & monoclinic \\
\hline Space group & $\mathrm{P} 2{ }_{1} / \mathrm{c}$ \\
\hline $\mathrm{a} / \AA$ & $8.1191(4)$ \\
\hline $\mathrm{b} / \AA$ & $5.6386(3)$ \\
\hline $\mathrm{c} / \AA$ & $31.0628(17)$ \\
\hline$\alpha /{ }^{\circ}$ & 90 \\
\hline$\beta /{ }^{\circ}$ & $93.276(2)$ \\
\hline
\end{tabular}




\begin{tabular}{|c|c|}
\hline$\gamma /{ }^{\circ}$ & 90 \\
\hline Volume $/ \AA^{3}$ & $1419.74(13)$ \\
\hline $\mathrm{Z}$ & 4 \\
\hline$\rho_{\text {calc }} \mathrm{g} / \mathrm{cm}^{3}$ & 1.377 \\
\hline$\mu / \mathrm{mm}^{-1}$ & 0.096 \\
\hline $\mathrm{F}(000)$ & 616.0 \\
\hline Crystal size $/ \mathrm{mm}^{3}$ & $0.426 \times 0.412 \times 0.326$ \\
\hline Radiation & $\operatorname{MoK} \alpha(\lambda=0.71073)$ \\
\hline \multicolumn{2}{|c|}{$2 \Theta$ range for data collection $/{ }^{\circ} 5.026$ to 59.122} \\
\hline Index ranges & $-11 \leq \mathrm{h} \leq 11,-7 \leq \mathrm{k} \leq 7,-43 \leq 1 \leq 39$ \\
\hline Reflections collected & 20105 \\
\hline Independent reflections & $3964\left[R_{\text {int }}=0.0168, R_{\text {sigma }}=0.0125\right]$ \\
\hline Data/restraints/parameters & $3964 / 0 / 200$ \\
\hline Goodness-of-fit on $\mathrm{F}^{2}$ & 1.039 \\
\hline Final $\mathrm{R}$ indexes $[\mathrm{I}>=2 \sigma(\mathrm{I})]$ & $\mathrm{R}_{1}=0.0370, \mathrm{wR}_{2}=0.0984$ \\
\hline Final $\mathrm{R}$ indexes [all data] & $\mathrm{R}_{1}=0.0389, \mathrm{wR}_{2}=0.1001$ \\
\hline Largest diff. peak/hole / e $\AA^{-}$ & $0.43 /-0.19$ \\
\hline
\end{tabular}

Table S10. Bond lengths $[\AA]$ for 3ea

\begin{tabular}{|c|c|c|c|c|c|}
\hline \multicolumn{3}{|c|}{ Atom Atom Length/Å } & \multicolumn{3}{|c|}{ Atom Atom Length/Å } \\
\hline $\mathrm{F} 1$ & $\mathrm{C} 1$ & $1.3658(10)$ & $\mathrm{C} 4$ & $\mathrm{C} 7$ & $1.4836(12)$ \\
\hline $\mathrm{O} 1$ & $\mathrm{C} 15$ & $1.3692(11)$ & $\mathrm{C} 5$ & C6 & $1.3964(12)$ \\
\hline $\mathrm{O} 1$ & $\mathrm{C} 18$ & $1.4255(12)$ & C6 & $\mathrm{C} 11$ & $1.5157(12)$ \\
\hline N1 & $\mathrm{C} 7$ & $1.3444(12)$ & $\mathrm{C} 8$ & C9 & $1.3867(14)$ \\
\hline N1 & $\mathrm{C} 8$ & $1.3381(12)$ & C9 & $\mathrm{C} 10$ & $1.3840(15)$ \\
\hline $\mathrm{N} 2$ & $\mathrm{C} 7$ & $1.3431(11)$ & $\mathrm{C} 11$ & $\mathrm{C} 12$ & $1.5145(12)$ \\
\hline N2 & $\mathrm{C} 10$ & $1.3342(12)$ & $\mathrm{C} 12$ & $\mathrm{C} 13$ & $1.3920(12)$ \\
\hline $\mathrm{C} 1$ & $\mathrm{C} 2$ & $1.3830(13)$ & $\mathrm{C} 12$ & $\mathrm{C} 17$ & $1.3964(13)$ \\
\hline $\mathrm{C} 1$ & C6 & $1.3860(13)$ & $\mathrm{C} 13$ & $\mathrm{C} 14$ & $1.3952(13)$ \\
\hline $\mathrm{C} 2$ & $\mathrm{C} 3$ & $1.3880(12)$ & $\mathrm{C} 14$ & $\mathrm{C} 15$ & $1.3929(12)$ \\
\hline $\mathrm{C} 3$ & $\mathrm{C} 4$ & $1.3963(12)$ & $\mathrm{C} 15$ & $\mathrm{C} 16$ & $1.3945(13)$ \\
\hline
\end{tabular}




\section{Atom Atom Length/̊̊}

C4 C5 1.3949(12)

\section{Atom Atom Length/Å}

C16 C17 1.3873(13)

Table S11. Bond angles $\left[^{\circ}\right]$ for 3ea

\begin{tabular}{|c|c|c|c|c|c|c|c|}
\hline \multicolumn{4}{|c|}{ Atom Atom Atom Angle ${ }^{\circ}$} & \multicolumn{4}{|c|}{ Atom Atom Atom Angle $/^{\circ}$} \\
\hline $\mathrm{C} 15$ & $\mathrm{O} 1$ & $\mathrm{C} 18$ & $116.57(8)$ & $\mathrm{N} 2$ & $\mathrm{C} 7$ & N1 & $125.87(8)$ \\
\hline $\mathrm{C} 8$ & N1 & $\mathrm{C} 7$ & $116.46(8)$ & $\mathrm{N} 2$ & $\mathrm{C} 7$ & $\mathrm{C} 4$ & $116.77(8)$ \\
\hline $\mathrm{C} 10$ & $\mathrm{~N} 2$ & $\mathrm{C} 7$ & $116.10(8)$ & $\mathrm{N} 1$ & $\mathrm{C} 8$ & C9 & $122.20(9)$ \\
\hline $\mathrm{F} 1$ & $\mathrm{C} 1$ & $\mathrm{C} 2$ & $117.78(8)$ & $\mathrm{C} 10$ & C9 & $\mathrm{C} 8$ & $116.53(9)$ \\
\hline $\mathrm{F} 1$ & $\mathrm{C} 1$ & C6 & $118.51(8)$ & $\mathrm{N} 2$ & $\mathrm{C} 10$ & C9 & $122.85(9)$ \\
\hline $\mathrm{C} 2$ & $\mathrm{C} 1$ & C6 & $123.71(8)$ & $\mathrm{C} 12$ & $\mathrm{C} 11$ & C6 & $111.51(7)$ \\
\hline $\mathrm{C} 1$ & $\mathrm{C} 2$ & $\mathrm{C} 3$ & $118.22(8)$ & $\mathrm{C} 13$ & $\mathrm{C} 12$ & $\mathrm{C} 11$ & $120.96(8)$ \\
\hline $\mathrm{C} 2$ & $\mathrm{C} 3$ & $\mathrm{C} 4$ & $120.48(8)$ & C13 & $\mathrm{C} 12$ & C17 & $117.98(8)$ \\
\hline $\mathrm{C} 3$ & $\mathrm{C} 4$ & $\mathrm{C} 7$ & $119.94(8)$ & C17 & $\mathrm{C} 12$ & C11 & $121.03(8)$ \\
\hline $\mathrm{C} 5$ & $\mathrm{C} 4$ & $\mathrm{C} 3$ & $119.27(8)$ & $\mathrm{C} 12$ & $\mathrm{C} 13$ & C14 & $121.67(8)$ \\
\hline $\mathrm{C} 5$ & $\mathrm{C} 4$ & $\mathrm{C} 7$ & $120.79(8)$ & $\mathrm{C} 15$ & $\mathrm{C} 14$ & C13 & $119.26(8)$ \\
\hline $\mathrm{C} 4$ & C5 & C6 & $121.60(8)$ & O1 & $\mathrm{C} 15$ & C14 & $124.28(8)$ \\
\hline $\mathrm{C} 1$ & C6 & $\mathrm{C} 5$ & $116.69(8)$ & $\mathrm{O} 1$ & C15 & C16 & $115.79(8)$ \\
\hline $\mathrm{C} 1$ & C6 & $\mathrm{C} 11$ & $122.40(8)$ & C14 & $\mathrm{C} 15$ & C16 & $119.92(8)$ \\
\hline $\mathrm{C} 5$ & C6 & $\mathrm{C} 11$ & $120.90(8)$ & C17 & $\mathrm{C} 16$ & C15 & $119.86(8)$ \\
\hline N1 & $\mathrm{C} 7$ & $\mathrm{C} 4$ & $117.36(8)$ & $\mathrm{C} 16$ & $\mathrm{C} 17$ & C12 & $121.29(8)$ \\
\hline
\end{tabular}

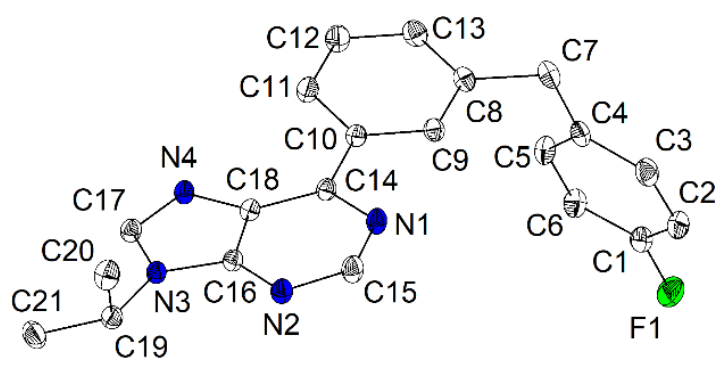

Figure S6. Molecular structure of $\mathbf{3 h f}$ with thermal ellipoids at 50\% probability level. The hydrogen atoms are omitted for clarity. 
Crystal Data for $\mathrm{C}_{21} \mathrm{H}_{19} \mathrm{FN}_{4}(M=346.40 \mathrm{~g} / \mathrm{mol})$ : monoclinic, space group $\mathrm{P} 2{ }_{1} / \mathrm{c}$ (no. 14), $a=$ 11.2425(7) $\AA, b=9.3295(6) \AA, c=16.3348(11) \AA, \beta=94.764(2)^{\circ}, V=1707.39(19) \AA^{3}, Z=4$, $T=99.98 \mathrm{~K}, \mu(\mathrm{MoK} \alpha)=0.090 \mathrm{~mm}^{-1}$, Dcalc $=1.348 \mathrm{~g} / \mathrm{cm}^{3}, 5632$ reflections measured $\left(5.004^{\circ}\right.$ $\left.\leq 2 \Theta \leq 63.084^{\circ}\right), 5632$ unique $\left(R_{\text {int }}=0.0, \mathrm{R}_{\text {sigma }}=0.0206\right)$ which were used in all calculations . The final $R_{1}$ was $0.0392(\mathrm{I}>2 \sigma(\mathrm{I}))$ and $w R_{2}$ was 0.1096 (all data).

Table S12. Crystal data and structure refinement for $3 \mathbf{h f}$

\begin{tabular}{|c|c|}
\hline Compound & $3 \mathrm{hf}$ \\
\hline CCDC number & 1915675 \\
\hline Identification code & mo_0272_CG_0m_4 \\
\hline Empirical formula & $\mathrm{C}_{21} \mathrm{H}_{19} \mathrm{FN}_{4}$ \\
\hline Formula weight & 346.40 \\
\hline Temperature/K & 99.98 \\
\hline Crystal system & monoclinic \\
\hline Space group & $\mathrm{P} 2_{1} / \mathrm{c}$ \\
\hline $\mathrm{a} / \AA$ & $11.2425(7)$ \\
\hline $\mathrm{b} / \AA ̊ \AA$ & $9.3295(6)$ \\
\hline $\mathrm{c} / \AA$ & $16.3348(11)$ \\
\hline$\alpha /^{\circ}$ & 90 \\
\hline$\beta /^{\circ}$ & $94.764(2)$ \\
\hline$\gamma /{ }^{\circ}$ & 90 \\
\hline Volume $/ \AA^{3}$ & $1707.39(19)$ \\
\hline $\mathrm{Z}$ & 4 \\
\hline$\rho_{\text {calc }} \mathrm{g} / \mathrm{cm}^{3}$ & 1.348 \\
\hline$\mu / \mathrm{mm}^{-1}$ & 0.090 \\
\hline $\mathrm{F}(000)$ & 728.0 \\
\hline Crystal size $/ \mathrm{mm}^{3}$ & $0.424 \times 0.247 \times 0.21$ \\
\hline Radiation & $\operatorname{MoK} \alpha(\lambda=0.71073)$ \\
\hline $2 \Theta$ range for data collection $/{ }^{\circ}$ & 5.004 to 63.084 \\
\hline Index ranges & $-16 \leq \mathrm{h} \leq 16,13 \leq \mathrm{k} \leq 0,24 \leq 1 \leq 0$ \\
\hline Reflections collected & 5632 \\
\hline
\end{tabular}




\begin{tabular}{|l|l|}
\hline Independent reflections & $5632\left[\mathrm{R}_{\text {int }}=0.0, \mathrm{R}_{\text {sigma }}=0.0206\right]$ \\
\hline Data/restraints/parameters & $5632 / 0 / 237$ \\
\hline Goodness-of-fit on $\mathrm{F}^{2}$ & 1.041 \\
\hline Final $\mathrm{R}$ indexes $[\mathrm{I}>=2 \sigma(\mathrm{I})]$ & $\mathrm{R}_{1}=0.0392, \mathrm{wR}_{2}=0.1076$ \\
\hline Final $\mathrm{R}$ indexes [all data $]$ & $\mathrm{R}_{1}=0.0428, \mathrm{wR}_{2}=0.1096$ \\
\hline Largest diff. peak/hole / e $\AA^{-3}$ & $0.44 /-0.27$ \\
\hline
\end{tabular}

Table S13. Bond lengths [̊̊] for $\mathbf{3 h f}$

\begin{tabular}{|c|c|c|c|c|c|}
\hline \multicolumn{3}{|c|}{ Atom Atom Length/Å } & \multicolumn{3}{|c|}{ Atom Atom Length/Å } \\
\hline $\mathrm{F} 1$ & $\mathrm{C} 1$ & $1.3554(11)$ & $\mathrm{C} 4$ & $\mathrm{C} 7$ & $1.5150(12)$ \\
\hline N1 & $\mathrm{C} 14$ & $1.3502(10)$ & $\mathrm{C} 5$ & C6 & $1.3911(13)$ \\
\hline N1 & $\mathrm{C} 15$ & $1.3397(11)$ & $\mathrm{C} 7$ & $\mathrm{C} 8$ & $1.5132(12)$ \\
\hline $\mathrm{N} 2$ & $\mathrm{C} 15$ & $1.3367(11)$ & $\mathrm{C} 8$ & C9 & $1.3925(11)$ \\
\hline $\mathrm{N} 2$ & $\mathrm{C} 16$ & $1.3337(10)$ & $\mathrm{C} 8$ & $\mathrm{C} 13$ & $1.3960(12)$ \\
\hline N3 & $\mathrm{C} 16$ & $1.3730(10)$ & C9 & $\mathrm{C} 10$ & $1.4001(11)$ \\
\hline N3 & $\mathrm{C} 17$ & $1.3691(10)$ & $\mathrm{C} 10$ & $\mathrm{C} 11$ & $1.4004(11)$ \\
\hline N3 & C19 & $1.4762(10)$ & $\mathrm{C} 10$ & $\mathrm{C} 14$ & $1.4770(11)$ \\
\hline N4 & $\mathrm{C} 17$ & $1.3179(11)$ & $\mathrm{C} 11$ & $\mathrm{C} 12$ & $1.3882(12)$ \\
\hline N4 & $\mathrm{C} 18$ & $1.3913(10)$ & $\mathrm{C} 12$ & $\mathrm{C} 13$ & $1.3908(12)$ \\
\hline $\mathrm{C} 1$ & $\mathrm{C} 2$ & $1.3774(13)$ & $\mathrm{C} 14$ & $\mathrm{C} 18$ & $1.4032(11)$ \\
\hline $\mathrm{C} 1$ & C6 & $1.3841(12)$ & $\mathrm{C} 16$ & $\mathrm{C} 18$ & $1.4076(11)$ \\
\hline $\mathrm{C} 2$ & $\mathrm{C} 3$ & $1.3891(14)$ & C19 & $\mathrm{C} 20$ & $1.5227(13)$ \\
\hline $\mathrm{C} 3$ & $\mathrm{C} 4$ & $1.3948(12)$ & C19 & $\mathrm{C} 21$ & $1.5200(12)$ \\
\hline $\mathrm{C} 4$ & $\mathrm{C} 5$ & $1.3969(11)$ & & & \\
\hline
\end{tabular}

Table S14. Bond angles $\left[^{\circ}\right]$ for $\mathbf{3 h f}$

\section{Atom Atom Atom Angle ${ }^{\circ}$}

C15 N1 C14 119.49(7)

C16 N2 C15 111.22(7)

C16 N3 C19 125.59(7)

C17 N3 C16 105.56(7)

C17 N3 C19 128.70(7)

\section{Atom Atom Atom Angle ${ }^{\circ}$}

C9 $\quad$ C10 $\quad$ C11 $119.40(7)$

C9 $\quad \mathrm{C} 10 \quad \mathrm{C} 14 \quad 119.41(7)$

C11 C10 C14 121.19(7)

C12 C11 C10 119.80(8)

C11 C12 C13 120.20(8) 


\begin{tabular}{|c|c|c|c|c|c|c|c|}
\hline \multicolumn{4}{|c|}{ Atom Atom Atom Angle ${ }^{\circ}$} & \multicolumn{4}{|c|}{ Atom Atom Atom Angle ${ }^{\circ}$} \\
\hline $\mathrm{C} 17$ & N4 & $\mathrm{C} 18$ & $104.01(7)$ & $\mathrm{C} 12$ & $\mathrm{C} 13$ & $\mathrm{C} 8$ & $120.91(8)$ \\
\hline $\mathrm{F} 1$ & $\mathrm{C} 1$ & $\mathrm{C} 2$ & $118.70(8)$ & $\mathrm{N} 1$ & $\mathrm{C} 14$ & $\mathrm{C} 10$ & $117.00(7)$ \\
\hline $\mathrm{F} 1$ & $\mathrm{C} 1$ & C6 & $118.57(8)$ & $\mathrm{N} 1$ & $\mathrm{C} 14$ & $\mathrm{C} 18$ & $117.75(7)$ \\
\hline $\mathrm{C} 2$ & $\mathrm{C} 1$ & C6 & $122.73(9)$ & $\mathrm{C} 18$ & $\mathrm{C} 14$ & $\mathrm{C} 10$ & $125.25(7)$ \\
\hline $\mathrm{C} 1$ & $\mathrm{C} 2$ & $\mathrm{C} 3$ & $117.97(8)$ & $\mathrm{N} 2$ & $\mathrm{C} 15$ & $\mathrm{~N} 1$ & $128.32(8)$ \\
\hline $\mathrm{C} 2$ & $\mathrm{C} 3$ & $\mathrm{C} 4$ & $121.76(8)$ & $\mathrm{N} 2$ & $\mathrm{C} 16$ & $\mathrm{~N} 3$ & $126.86(7)$ \\
\hline C3 & $\mathrm{C} 4$ & $\mathrm{C} 5$ & $118.12(8)$ & $\mathrm{N} 2$ & $\mathrm{C} 16$ & $\mathrm{C} 18$ & $126.84(7)$ \\
\hline $\mathrm{C} 3$ & $\mathrm{C} 4$ & $\mathrm{C} 7$ & $120.23(8)$ & $\mathrm{N} 3$ & $\mathrm{C} 16$ & $\mathrm{C} 18$ & $106.29(7)$ \\
\hline $\mathrm{C} 5$ & $\mathrm{C} 4$ & $\mathrm{C} 7$ & $121.63(8)$ & N4 & $\mathrm{C} 17$ & N3 & $114.51(7)$ \\
\hline C6 & $\mathrm{C} 5$ & $\mathrm{C} 4$ & $121.33(8)$ & N4 & C18 & C14 & $134.05(7)$ \\
\hline $\mathrm{C} 1$ & C6 & $\mathrm{C} 5$ & $118.10(8)$ & N4 & C18 & C16 & $109.63(7)$ \\
\hline $\mathrm{C} 8$ & $\mathrm{C} 7$ & $\mathrm{C} 4$ & $114.04(7)$ & C14 & $\mathrm{C} 18$ & $\mathrm{C} 16$ & $116.32(7)$ \\
\hline C9 & $\mathrm{C} 8$ & $\mathrm{C} 7$ & $120.19(8)$ & N3 & C19 & C20 & $109.47(7)$ \\
\hline C9 & $\mathrm{C} 8$ & $\mathrm{C} 13$ & $118.62(8)$ & $\mathrm{N} 3$ & C19 & $\mathrm{C} 21$ & $110.16(7)$ \\
\hline C13 & $\mathrm{C} 8$ & $\mathrm{C} 7$ & $121.19(8)$ & $\mathrm{C} 21$ & C19 & C20 & $112.01(7)$ \\
\hline C8 & $\mathrm{C} 9$ & $\mathrm{C} 10$ & $121.07(7)$ & & & & \\
\hline
\end{tabular}

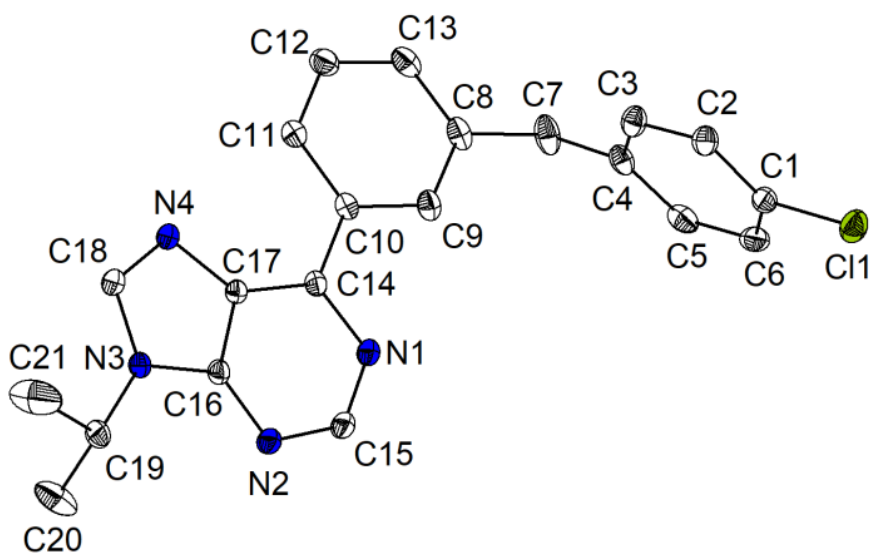

Figure S7. Molecular structure of $\mathbf{3 h g}$ with thermal ellipoids at $50 \%$ probability level. The hydrogen atoms are omitted for clarity.

Crystal Data for $\mathrm{C}_{21} \mathrm{H}_{19} \mathrm{ClN}_{4}(M=362.85 \mathrm{~g} / \mathrm{mol})$ : triclinic, space group P-1 (no. 2), $a=$ 8.9649(8) $\AA, b=9.9520(7) \AA, c=11.7814(11) \AA, \alpha=100.554(3)^{\circ}, \beta=105.986(3)^{\circ}, \gamma=$ 
$111.878(2)^{\circ}, \quad V=888.28(13) \AA^{3}, Z=2, T=100.0 \mathrm{~K}, \mu(\mathrm{MoK} \alpha)=0.227 \mathrm{~mm}^{-1}$, Dcalc $=$ $1.357 \mathrm{~g} / \mathrm{cm}^{3}, 12425$ reflections measured $\left(4.656^{\circ} \leq 2 \Theta \leq 63.036^{\circ}\right), 5883$ unique $\left(R_{\text {int }}=0.0176\right.$, $\left.\mathrm{R}_{\text {sigma }}=0.0255\right)$ which were used in all calculations. The final $R_{1}$ was $0.0376(\mathrm{I}>2 \sigma(\mathrm{I}))$ and $w R_{2}$ was 0.1059 (all data).

Table S15. Crystal data and structure refinement for 3 hg

\begin{tabular}{|c|c|}
\hline Compound & 3hg \\
\hline CCDC number & 1915610 \\
\hline Identification code & mo_0273_CG_0m \\
\hline Empirical formula & $\mathrm{C}_{21} \mathrm{H}_{19} \mathrm{ClN}_{4}$ \\
\hline Formula weight & 362.85 \\
\hline Temperature/K & 100.0 \\
\hline Crystal system & triclinic \\
\hline Space group & $\mathrm{P}-1$ \\
\hline $\mathrm{a} / \AA$ & $8.9649(8)$ \\
\hline $\mathrm{b} / \AA$ & $9.9520(7)$ \\
\hline $\mathrm{c} / \AA$ & $11.7814(11)$ \\
\hline$\alpha /^{\circ}$ & $100.554(3)$ \\
\hline$\beta /{ }^{\circ}$ & $105.986(3)$ \\
\hline$\gamma /{ }^{\circ}$ & $111.878(2)$ \\
\hline Volume $/ \AA^{3}$ & $888.28(13)$ \\
\hline $\mathrm{Z}$ & 2 \\
\hline$\rho_{\text {calc }} \mathrm{g} / \mathrm{cm}^{3}$ & 1.357 \\
\hline$\mu / \mathrm{mm}^{-1}$ & 0.227 \\
\hline $\mathrm{F}(000)$ & 380.0 \\
\hline Crystal size $/ \mathrm{mm}^{3}$ & $0.287 \times 0.198 \times 0.162$ \\
\hline Radiation & $\operatorname{MoK} \alpha(\lambda=0.71073)$ \\
\hline \multicolumn{2}{|c|}{$2 \Theta$ range for data collection $/{ }^{\circ} 4.656$ to 63.036} \\
\hline Index ranges & $-13 \leq \mathrm{h} \leq 9,-14 \leq \mathrm{k} \leq 14,-17 \leq 1 \leq 17$ \\
\hline Reflections collected & 12425 \\
\hline Independent reflections & $5883\left[\mathrm{R}_{\text {int }}=0.0176, \mathrm{R}_{\text {sigma }}=0.0255\right]$ \\
\hline
\end{tabular}




\begin{tabular}{|l|l|}
\hline Data/restraints/parameters & $5883 / 0 / 237$ \\
\hline Goodness-of-fit on $\mathrm{F}^{2}$ & 1.078 \\
\hline Final $\mathrm{R}$ indexes $[\mathrm{I}>=2 \sigma(\mathrm{I})]$ & $\mathrm{R}_{1}=0.0376, \mathrm{wR}_{2}=0.1031$ \\
\hline Final $\mathrm{R}$ indexes [all data] & $\mathrm{R}_{1}=0.0407, \mathrm{wR}_{2}=0.1059$ \\
\hline Largest diff. peak/hole / e $\AA^{-3}$ & $0.49 /-0.52$ \\
\hline
\end{tabular}

Table S16. Bond lengths [Å] for $\mathbf{3 h g}$

Atom Atom Length/Å

Cl1 C1 1.7425(10)

$\mathrm{N} 1 \quad \mathrm{C} 14 \quad 1.3485(12)$

N1 C15 1.3365(12)

$\mathrm{N} 2 \quad \mathrm{C} 15 \quad 1.3376(12)$

N2 C16 1.3310(12)

N3 C16 1.3715(11)

N3 C18 $1.3729(12)$

N3 C19 1.4755(12)

N4 C17 $1.3905(12)$

N4 C18 1.3172(12)

C1 C2 1.3885(13)

C1 C6 1.3821(14)

C2 C3 1.3915(14)

C3 C4 1.3956(14)

C4 C5 1.3920(15)

\section{Atom Atom Length/Å}

C4 C7 1.5099(15)

C5 C6 1.3913(15)

C7 C8 1.5123(14)

C8 C9 1.3949(13)

C8 C13 1.3933(15)

C9 C10 1.4013(13)

C10 C11 1.3992(13)

C10 C14 1.4782(13)

C11 C12 1.3905(13)

C12 C13 1.3885(15)

C14 C17 1.4048(12)

C16 C17 1.4063(12)

C19 C20 1.5140(16)

C19 C21 1.5035(16)

Table S17. Bond angles $\left[^{\circ}\right]$ for $3 \mathrm{hg}$

Atom Atom Atom Angle ${ }^{\circ}$

C15 N1 C14 119.56(8)

C16 N2 C15 111.07(8)

C16 N3 C18 105.30(8)

C16 N3 C19 124.64(8)

C18 N3 C19 130.03(8)

$\begin{array}{llll}\text { C18 N4 C17 104.07(8) } & \text { N }\end{array}$
Atom Atom Atom Angle $/^{\circ}$

C9 $\quad \mathrm{C} 10 \quad \mathrm{C} 14 \quad 119.50(8)$

C11 C10 C9 119.16(9)

C11 C10 $\mathrm{C} 14 \quad$ 121.32(8)

C12 C11 C10 119.88(9)

C13 C12 C11 120.36(10)

C12 C13 C8 120.72(9) 


\begin{tabular}{|c|c|c|c|c|c|c|c|}
\hline \multicolumn{4}{|c|}{ Atom Atom Atom Angle ${ }^{\circ}$} & \multicolumn{4}{|c|}{ Atom Atom Atom Angle ${ }^{\circ}$} \\
\hline $\mathrm{C} 2$ & $\mathrm{C} 1$ & $\mathrm{Cl1}$ & $119.39(8)$ & N1 & $\mathrm{C} 14$ & $\mathrm{C} 10$ & $117.03(8)$ \\
\hline C6 & $\mathrm{C} 1$ & $\mathrm{Cl1}$ & $118.85(8)$ & N1 & $\mathrm{C} 14$ & $\mathrm{C} 17$ & $117.62(8)$ \\
\hline C6 & $\mathrm{C} 1$ & $\mathrm{C} 2$ & $121.76(9)$ & $\mathrm{C} 17$ & $\mathrm{C} 14$ & $\mathrm{C} 10$ & $125.36(8)$ \\
\hline $\mathrm{C} 1$ & $\mathrm{C} 2$ & $\mathrm{C} 3$ & $118.39(9)$ & N1 & $\mathrm{C} 15$ & $\mathrm{~N} 2$ & $128.45(9)$ \\
\hline $\mathrm{C} 2$ & $\mathrm{C} 3$ & $\mathrm{C} 4$ & 121.37(9) & $\mathrm{N} 2$ & $\mathrm{C} 16$ & N3 & $126.45(8)$ \\
\hline $\mathrm{C} 3$ & $\mathrm{C} 4$ & $\mathrm{C} 7$ & 121.12(9) & $\mathrm{N} 2$ & $\mathrm{C} 16$ & $\mathrm{C} 17$ & $126.96(8)$ \\
\hline $\mathrm{C} 5$ & $\mathrm{C} 4$ & $\mathrm{C} 3$ & $118.47(9)$ & $\mathrm{N} 3$ & $\mathrm{C} 16$ & $\mathrm{C} 17$ & $106.58(8)$ \\
\hline $\mathrm{C} 5$ & $\mathrm{C} 4$ & $\mathrm{C} 7$ & $120.31(9)$ & N4 & $\mathrm{C} 17$ & $\mathrm{C} 14$ & $134.12(8)$ \\
\hline C6 & C5 & $\mathrm{C} 4$ & $121.19(9)$ & N4 & $\mathrm{C} 17$ & $\mathrm{C} 16$ & $109.54(8)$ \\
\hline $\mathrm{C} 1$ & C6 & $\mathrm{C} 5$ & 118.82(9) & $\mathrm{C} 14$ & $\mathrm{C} 17$ & $\mathrm{C} 16$ & $116.33(8)$ \\
\hline $\mathrm{C} 4$ & $\mathrm{C} 7$ & $\mathrm{C} 8$ & $115.92(8)$ & N4 & C18 & N3 & $114.51(8)$ \\
\hline C9 & $\mathrm{C} 8$ & $\mathrm{C} 7$ & $120.82(10)$ & $\mathrm{N} 3$ & C19 & $\mathrm{C} 20$ & $110.46(8)$ \\
\hline C13 & $\mathrm{C} 8$ & $\mathrm{C} 7$ & $120.33(9)$ & N3 & C19 & $\mathrm{C} 21$ & $110.84(9)$ \\
\hline C13 & $\mathrm{C} 8$ & C9 & $118.79(9)$ & $\mathrm{C} 21$ & C19 & $\mathrm{C} 20$ & $112.70(12)$ \\
\hline $\mathrm{C} 8$ & $\mathrm{C} 9$ & C10 & 121.08(9) & & & & \\
\hline
\end{tabular}

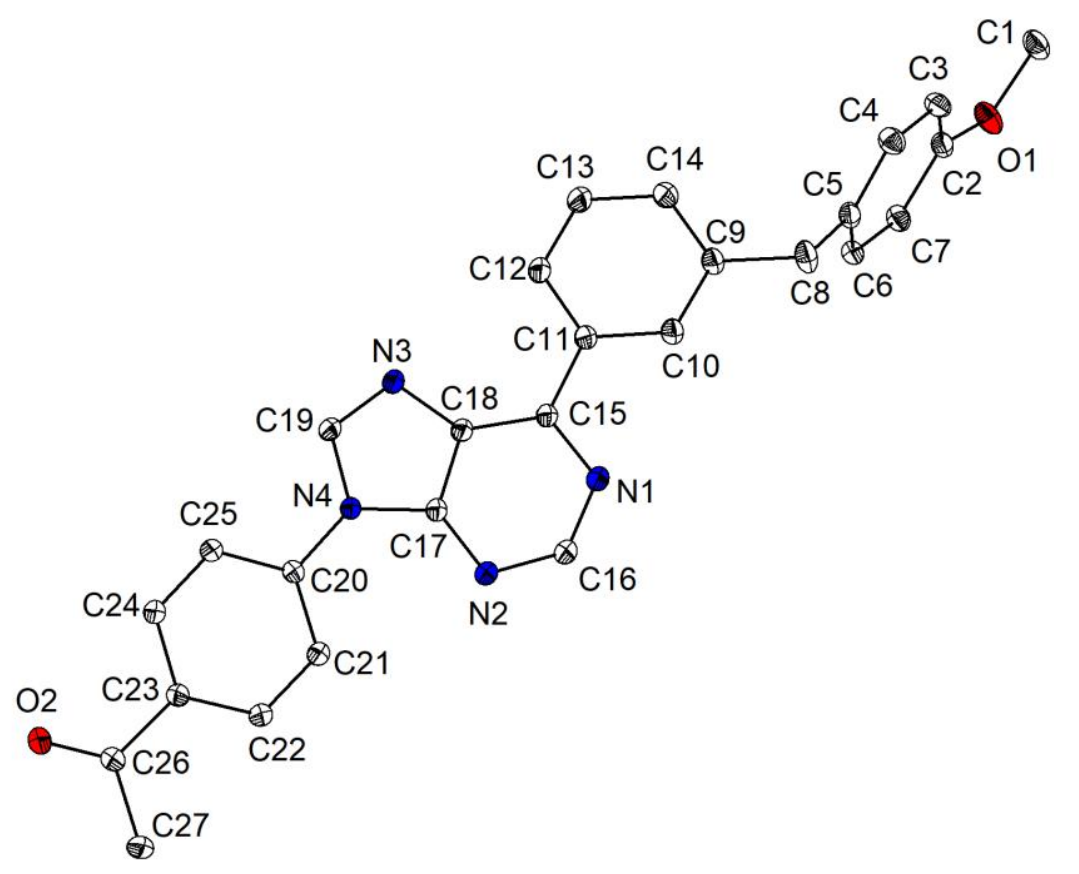

Figure S8. Molecular structure of 3ia with thermal ellipoids at 50\% probability level. The hydrogen atoms are omitted for clarity. 
Crystal Data for $\mathrm{C}_{27} \mathrm{H}_{22} \mathrm{~N}_{4} \mathrm{O}_{2}(M=434.48 \mathrm{~g} / \mathrm{mol})$ : triclinic, space group P-1 (no. 2), $a=$ 6.3344(7) $\AA$, $b=12.4251(18) \AA, c=13.9524(19) \AA, \alpha=106.848(5)^{\circ}, \beta=91.291(5)^{\circ}, \gamma=$ 93.640(5) $)^{\circ}, V=1047.9(2) \AA^{3}, Z=2, T=100.01 \mathrm{~K}, \mu(\mathrm{MoK} \alpha)=0.089 \mathrm{~mm}^{-1}$, Dcalc $=$ $1.377 \mathrm{~g} / \mathrm{cm}^{3}, 38890$ reflections measured $\left(5.22^{\circ} \leq 2 \Theta \leq 63.094^{\circ}\right), 7003$ unique $\left(R_{\text {int }}=0.0243\right.$, $\left.\mathrm{R}_{\text {sigma }}=0.0175\right)$ which were used in all calculations. The final $R_{1}$ was $0.0393(\mathrm{I}>2 \sigma(\mathrm{I}))$ and $w R_{2}$ was 0.1124 (all data).

Table S18. Crystal data and structure refinement for 3ia

\begin{tabular}{|c|c|}
\hline Compound & 3ia \\
\hline CCDC number & 1915681 \\
\hline Identification code & mo_0250_CG_0m \\
\hline Empirical formula & $\mathrm{C}_{27} \mathrm{H}_{22} \mathrm{~N}_{4} \mathrm{O}_{2}$ \\
\hline Formula weight & 434.48 \\
\hline Temperature/K & 100.01 \\
\hline Crystal system & triclinic \\
\hline Space group & $\mathrm{P}-1$ \\
\hline $\mathrm{a} / \AA$ & $6.3344(7)$ \\
\hline $\mathrm{b} / \AA ̊$ & $12.4251(18)$ \\
\hline $\mathrm{c} / \AA$ & $13.9524(19)$ \\
\hline$\alpha /^{\circ}$ & $106.848(5)$ \\
\hline$\beta /{ }^{\circ}$ & $91.291(5)$ \\
\hline$\gamma /{ }^{\circ}$ & $93.640(5)$ \\
\hline Volume $/ \AA^{3}$ & 1047.9(2) \\
\hline $\mathrm{Z}$ & 2 \\
\hline$\rho_{\text {calc }} \mathrm{g} / \mathrm{cm}^{3}$ & 1.377 \\
\hline$\mu / \mathrm{mm}^{-1}$ & 0.089 \\
\hline $\mathrm{F}(000)$ & 456.0 \\
\hline Crystal size $/ \mathrm{mm}^{3}$ & $0.567 \times 0.17 \times 0.062$ \\
\hline Radiation & $\operatorname{MoK} \alpha(\lambda=0.71073)$ \\
\hline \multicolumn{2}{|c|}{$2 \Theta$ range for data collection $/{ }^{\circ} 5.22$ to 63.094} \\
\hline Index ranges & $-9 \leq \mathrm{h} \leq 9,-18 \leq \mathrm{k} \leq 18,-20 \leq 1 \leq 20$ \\
\hline
\end{tabular}




\begin{tabular}{|l|l|}
\hline Reflections collected & 38890 \\
\hline Independent reflections & $7003\left[\mathrm{R}_{\text {int }}=0.0243, \mathrm{R}_{\text {sigma }}=0.0175\right]$ \\
\hline Data/restraints/parameters & $7003 / 0 / 300$ \\
\hline Goodness-of-fit on $\mathrm{F}^{2}$ & 1.045 \\
\hline Final R indexes [I $>=2 \sigma(\mathrm{I})]$ & $\mathrm{R}_{1}=0.0393, \mathrm{wR}_{2}=0.1089$ \\
\hline Final $\mathrm{R}$ indexes [all data] & $\mathrm{R}_{1}=0.0433, \mathrm{wR}_{2}=0.1124$ \\
\hline Largest diff. peak/hole / e $\AA^{-3}$ & $0.47 /-0.23$ \\
\hline
\end{tabular}

Table S19. Bond lengths $[\AA ̊]$ for 3ia

Atom Atom Length/Å

O1 C1 1.4302(11)

O1 C2 1.3665(10)

$\mathrm{O} 2 \quad \mathrm{C} 26 \quad 1.2206(11)$

N1 C15 1.3497(11)

$\mathrm{N} 1 \quad \mathrm{C} 16 \quad 1.3356(11)$

$\mathrm{N} 2 \quad \mathrm{C} 16 \quad 1.3425(11)$

$\mathrm{N} 2 \quad \mathrm{C} 17 \quad 1.3333(10)$

N3 C18 1.3907(11)

N3 C19 1.3055(11)

N4 C17 1.3881(10)

N4 C19 1.3875(11)

N4 C20 1.4193(11)

C2 C3 1.3901(12)

C2 C7 1.3984(12)

C3 C4 1.3973(12)

C4 C5 1.3902(12)

C5 C6 1.3994(12)

C5 C8 1.5085(12)

C6 C7 1.3844(12)

\section{Atom Atom Length/Å}

C8 C9 1.5137(12)

C9 C10 1.3900(12)

C9 C14 1.3993(13)

C10 C11 1.4028(11)

C11 C12 1.4009(12)

C11 C15 1.4799(12)

C12 C13 1.3930(13)

C13 C14 1.3911(13)

C15 C18 1.4012(11)

C17 C18 1.4052(11)

C20 C21 1.3907(12)

C20 C25 1.3985(11)

C21 C22 1.3892(12)

C22 C23 1.3957(12)

C23 C24 1.3966(12)

C23 C26 1.4906(12)

C24 C25 1.3853(12)

C26 C27 1.5076(13) 
Table S20. Bond angles $\left[{ }^{\circ}\right]$ for 3 ia

Atom Atom Atom Angle $/^{\circ}$

C2 $\quad$ O1 C1 116.58(7)

C16 N1 C15 119.31(7)

C17 N2 C16 111.49(7)

C19 N3 C18 104.44(7)

C17 N4 C20 129.35(7)

C19 N4 C17 105.13(7)

C19 N4 C20 125.52(7)

O1 C2 C3 124.61(8)

O1 C2 C7 115.72(8)

C3 $\quad$ C2 $\quad$ C7 $119.66(8)$

C2 C3 C4 119.19(8)

C5 $\quad \mathrm{C} 4 \quad \mathrm{C} 3 \quad 121.92(8)$

C4 C5 C6 117.86(8)

C4 C5 C8 121.14(8)

C6 $\quad$ C5 C8 120.92(8)

C7 C6 C5 121.06(8)

C6 $\quad$ C7 $\quad$ C2 $120.24(8)$

C5 $\quad$ C8 $89 \quad 116.24(7)$

C10 C9 C8 119.52(8)

C10 C9 C14 118.68(8)

C14 C9 C8 121.75(8)

C9 $\mathrm{C} 10 \quad \mathrm{C} 11 \quad 121.58(8)$

C10 C11 C15 118.78(7)

C12 C11 C10 119.01(8)

C12 C11 C15 122.14(8)

C13 C12 C11 119.62(8)

\section{Atom Atom Atom Angle ${ }^{\circ}$}

C14 C13 C12 120.74(9)

C13 C14 C9 120.36(8)

$\mathrm{N} 1 \quad \mathrm{C} 15 \quad \mathrm{C} 11 \quad 116.88(7)$

N1 C15 C18 $117.56(8)$

C18 C15 C11 125.50(8)

$\mathrm{N} 1 \quad \mathrm{C} 16 \quad \mathrm{~N} 2 \quad 128.45(8)$

$\mathrm{N} 2 \quad \mathrm{C} 17 \quad \mathrm{~N} 4 \quad 128.29(8)$

N2 $\quad$ C17 $\quad$ C18 $125.97(8)$

N4 $\quad$ C17 $\quad$ C18 $105.73(7)$

N3 $\quad$ C18 C15 $132.49(8)$

N3 $\quad$ C18 C17 $110.28(7)$

C15 C18 C17 117.21(7)

N3 $\quad$ C19 N4 114.39(7)

C21 C20 N4 120.61(7)

$\begin{array}{llll}\mathrm{C} 21 & \mathrm{C} 20 & \mathrm{C} 25 & 120.25(8)\end{array}$

C25 C20 N4 119.14(7)

C22 C21 C20 119.57(8)

C21 C22 C23 120.87(8)

C22 C23 C24 118.79(8)

C22 C23 C26 122.55(8)

C24 C23 C26 118.65(7)

C25 C24 C23 120.92(8)

C24 C25 C20 119.50(8)

O2 $\quad$ C26 $\quad$ C23 $120.55(8)$

O2 $\quad \begin{array}{lll}\mathrm{C} 26 & \mathrm{C} 27 & 121.26(8)\end{array}$

C23 C26 C27 118.16(7) 


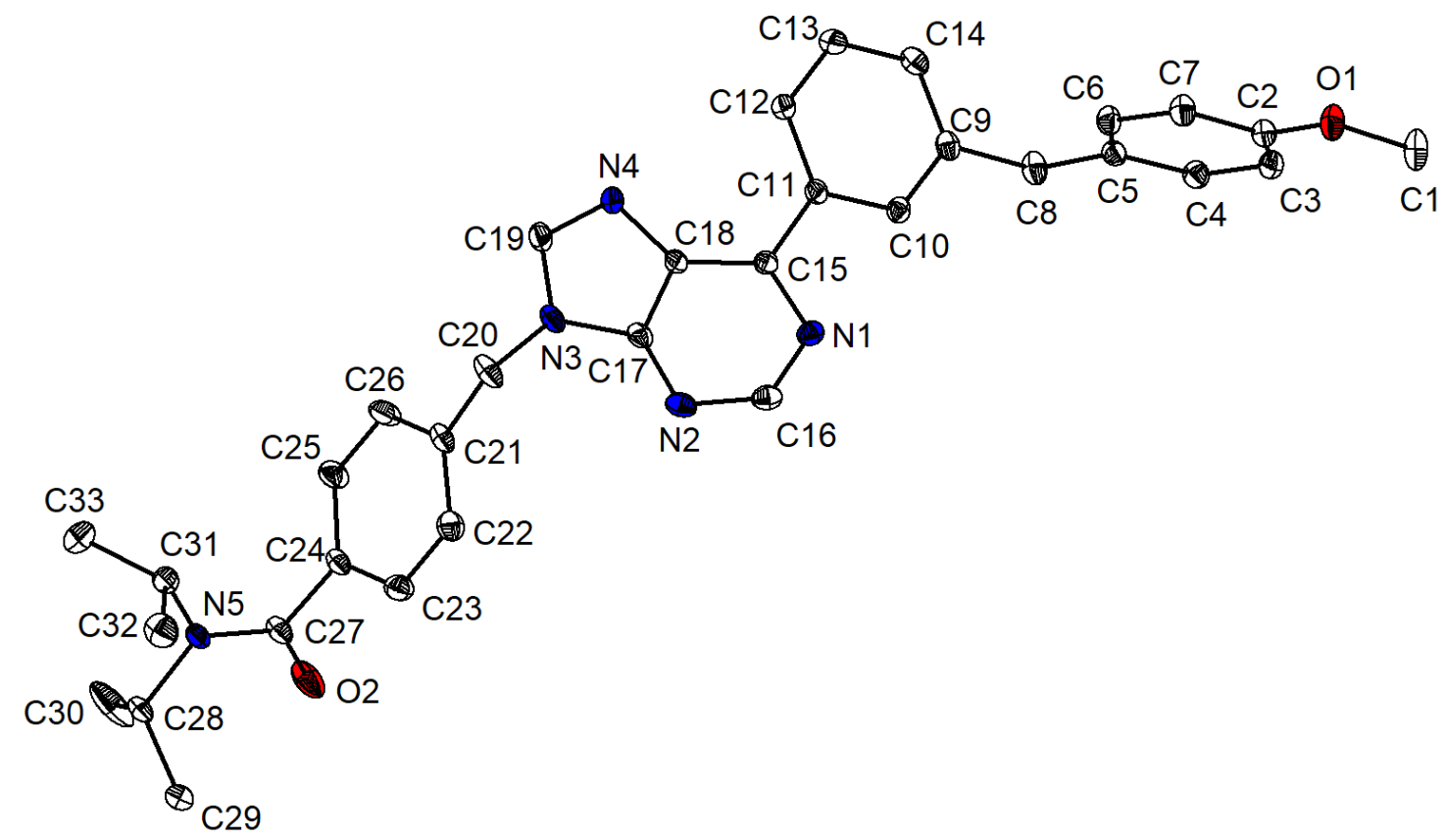

Figure S9. Molecular structure of 3la with thermal ellipoids at 50\% probability level. The hydrogen atoms are omitted for clarity.

Crystal Data for $\mathrm{C}_{33} \mathrm{H}_{35} \mathrm{~N}_{5} \mathrm{O}_{2}(M=533.66 \mathrm{~g} / \mathrm{mol})$ : monoclinic, space group P2 $1 / \mathrm{n}$ (no. 14), $a=$ 10.1416(6) $\mathrm{A}, b=27.4937(13) \AA, c=10.2461(6) \AA, \beta=101.977(2)^{\circ}, V=2794.7(3) \AA^{3}, Z=4$, $T=100.0 \mathrm{~K}, \mu(\mathrm{MoK} \alpha)=0.081 \mathrm{~mm}^{-1}$, Dcalc $=1.268 \mathrm{~g} / \mathrm{cm}^{3}, 44140$ reflections measured $\left(5.03^{\circ}\right.$ $\left.\leq 2 \Theta \leq 62.986^{\circ}\right), 9275$ unique $\left(R_{\text {int }}=0.0219, R_{\text {sigma }}=0.0176\right)$ which were used in all calculations. The final $R_{1}$ was $0.0409(\mathrm{I}>2 \sigma(\mathrm{I}))$ and $w R_{2}$ was 0.1117 (all data).

Table S21. Crystal data and structure refinement for 3la

\begin{tabular}{|l|l|}
\hline Compound & 3la \\
\hline CCDC number & 1915682 \\
\hline Identification code & mo_0241_CG_Om \\
\hline Empirical formula & $\mathrm{C}_{33} \mathrm{H}_{35} \mathrm{~N}_{5} \mathrm{O}_{2}$ \\
\hline Formula weight & 533.66 \\
\hline Temperature/K & 100.0 \\
\hline Crystal system & monoclinic \\
\hline Space group & $\mathrm{P} 2{ }_{1} / \mathrm{n}$ \\
\hline a/A & $10.1416(6)$ \\
\hline
\end{tabular}




\begin{tabular}{|c|c|}
\hline $\mathrm{b} / \AA$ & $27.4937(13)$ \\
\hline $\mathrm{c} / \AA ̊$ & 10.2461(6) \\
\hline$\alpha /^{\circ}$ & 90 \\
\hline$\beta /^{\circ}$ & 101.977(2) \\
\hline$\gamma /{ }^{\circ}$ & 90 \\
\hline Volume $/ \AA^{3}$ & 2794.7(3) \\
\hline $\mathrm{Z}$ & 4 \\
\hline$\rho_{\text {calc }} \mathrm{g} / \mathrm{cm}^{3}$ & 1.268 \\
\hline$\mu / \mathrm{mm}^{-1}$ & 0.081 \\
\hline $\mathrm{F}(000)$ & 1136.0 \\
\hline Crystal size $/ \mathrm{mm}^{3}$ & $0.438 \times 0.434 \times 0.37$ \\
\hline Radiation & $\operatorname{MoK} \alpha(\lambda=0.71073)$ \\
\hline $2 \Theta$ range for data collection/ & 5.03 to 62.986 \\
\hline Index ranges & $-14 \leq \mathrm{h} \leq 14,-40 \leq \mathrm{k} \leq 40,-15 \leq 1 \leq 14$ \\
\hline Reflections collected & 44140 \\
\hline Independent reflections & $9275\left[R_{\text {int }}=0.0219, R_{\text {sigma }}=0.0176\right]$ \\
\hline Data/restraints/parameters & $9275 / 0 / 366$ \\
\hline Goodness-of-fit on $\mathrm{F}^{2}$ & 1.039 \\
\hline Final $\mathrm{R}$ indexes $[\mathrm{I}>=2 \sigma(\mathrm{I})]$ & $\mathrm{R}_{1}=0.0409, \mathrm{wR}_{2}=0.1081$ \\
\hline Final $\mathrm{R}$ indexes [all data] & $\mathrm{R}_{1}=0.0455, \mathrm{w} \mathrm{R}_{2}=0.1117$ \\
\hline Largest diff. peak/hole / e $\AA^{-}$ & $0.46 /-0.24$ \\
\hline
\end{tabular}

Table S22. Bond lengths $[\AA ̊]$ for 3la

Atom Atom Length/Å

O1 C1 1.4265(12)

O1 C2 1.3681(10)

O2 C27 1.2366(11)

N1 C15 1.3529(10)

N1 C16 1.3418(11)

$\mathrm{N} 2 \quad \mathrm{C} 16 \quad 1.3392(12)$

$\mathrm{N} 2 \quad \mathrm{C} 17 \quad 1.3324(11)$
Atom Atom Length/ ̊

C8 C9 1.5110(12)

C9 C10 1.3938(12)

C9 C14 1.3978(12)

C10 C11 1.3999(11)

C11 C12 1.4006(11)

C11 C15 1.4787(11)

C12 C13 1.3941(12) 


\section{Atom Atom Length/Å}

N3 C17 $1.3718(11)$

N3 C19 1.3727(12)

N3 C20 1.4611(11)

N4 C18 1.3921(10)

N4 C19 1.3156(11)

N5 C27 1.3496(11)

N5 C28 $1.4869(10)$

N5 C31 1.4843(11)

C2 C3 1.3954(12)

C2 C7 1.3937(12)

C3 C4 1.3942(12)

C4 C5 1.3964(11)

C5 C6 1.3940(11)

C5 C8 1.5155(12)

C6 C7 1.3892(12)

\section{Atom Atom Length/}

C13 C14 1.3936(12)

C15 C18 1.3996(11)

C17 C18 1.4062(11)

C20 C21 1.5135(12)

C21 C22 1.3898(12)

C21 C26 1.3963(12)

C22 C23 1.3948(12)

C23 C24 1.3904(12)

C24 C25 1.3940(12)

C24 C27 1.5105(11)

C25 C26 1.3939(12)

C28 C29 1.5193(13)

C28 C30 1.5176(14)

C31 C32 1.5266(14)

C31 C33 1.5277(13)

Table S23. Bond angles $\left[{ }^{\circ}\right]$ for 3la

\section{Atom Atom Atom Angle ${ }^{\circ}$}

C2 $\quad$ O1 C1 116.90(7)

C16 N1 C15 119.01(7)

C17 N2 C16 111.16(7)

C17 N3 C19 106.04(7)

C17 N3 C20 125.94(8)

C19 N3 C20 127.37(8)

C19 N4 C18 104.00(7)

C27 N5 C28 120.29(7)

C27 N5 C31 122.97(7)

C31 N5 C28 116.05(7)

O1 C2 C3 124.48(8)

O1 C2 C7 115.57(8)

$\begin{array}{llll}\text { C7 } & \text { C2 } & \text { C3 } & 119.95(8)\end{array}$

\section{Atom Atom Atom Angle $/^{\circ}$}

N1 C15 C11 117.52(7)

N1 C15 C18 118.02(7)

C18 C15 C11 124.46(7)

N2 C16 N1 128.49(8)

$\begin{array}{llll}\mathrm{N} 2 & \mathrm{C} 17 & \mathrm{~N} 3 & 127.32(8)\end{array}$

N2 $\quad$ C17 $\quad$ C18 $126.84(8)$

N3 $\quad$ C17 $\quad$ C18 $105.80(7)$

N4 $\quad$ C18 C15 $133.51(7)$

N4 $\quad$ C18 $\quad$ C17 $110.02(7)$

C15 C18 C17 116.45(7)

N4 $\quad$ C19 N3 114.12(8)

N3 $\quad$ C20 C21 $115.36(7)$

C22 C21 C20 119.83(8) 


\begin{tabular}{lllllllll}
\multicolumn{1}{c}{ Atom Atom Atom Angle/ } & & \multicolumn{3}{c}{ Atom Atom Atom Angle ${ }^{\circ}$} \\
$\mathrm{C} 4$ & $\mathrm{C} 3$ & $\mathrm{C} 2$ & $119.17(8)$ & & $\mathrm{C} 22$ & $\mathrm{C} 21$ & $\mathrm{C} 26$ & $118.82(8)$ \\
$\mathrm{C} 3$ & $\mathrm{C} 4$ & $\mathrm{C} 5$ & $121.69(8)$ & $\mathrm{C} 26$ & $\mathrm{C} 21$ & $\mathrm{C} 20$ & $121.09(8)$ \\
$\mathrm{C} 4$ & $\mathrm{C} 5$ & $\mathrm{C} 8$ & $119.26(7)$ & $\mathrm{C} 21$ & $\mathrm{C} 22$ & $\mathrm{C} 23$ & $120.83(8)$ \\
$\mathrm{C} 6$ & $\mathrm{C} 5$ & $\mathrm{C} 4$ & $117.99(8)$ & $\mathrm{C} 24$ & $\mathrm{C} 23$ & $\mathrm{C} 22$ & $120.03(8)$ \\
$\mathrm{C} 6$ & $\mathrm{C} 5$ & $\mathrm{C} 8$ & $122.74(7)$ & $\mathrm{C} 23$ & $\mathrm{C} 24$ & $\mathrm{C} 25$ & $119.44(7)$ \\
$\mathrm{C} 7$ & $\mathrm{C} 6$ & $\mathrm{C} 5$ & $121.27(8)$ & $\mathrm{C} 23$ & $\mathrm{C} 24$ & $\mathrm{C} 27$ & $118.20(8)$ \\
$\mathrm{C} 6$ & $\mathrm{C} 7$ & $\mathrm{C} 2$ & $119.92(8)$ & $\mathrm{C} 25$ & $\mathrm{C} 24$ & $\mathrm{C} 27$ & $122.36(8)$ \\
$\mathrm{C} 9$ & $\mathrm{C} 8$ & $\mathrm{C} 5$ & $115.97(7)$ & $\mathrm{C} 26$ & $\mathrm{C} 25$ & $\mathrm{C} 24$ & $120.14(8)$ \\
$\mathrm{C} 10$ & $\mathrm{C} 9$ & $\mathrm{C} 8$ & $120.18(8)$ & $\mathrm{C} 25$ & $\mathrm{C} 26$ & $\mathrm{C} 21$ & $120.51(8)$ \\
$\mathrm{C} 10$ & $\mathrm{C} 9$ & $\mathrm{C} 14$ & $118.59(8)$ & $\mathrm{O} 2$ & $\mathrm{C} 27$ & $\mathrm{~N} 5$ & $123.26(8)$ \\
$\mathrm{C} 14$ & $\mathrm{C} 9$ & $\mathrm{C} 8$ & $121.21(8)$ & $\mathrm{O} 2$ & $\mathrm{C} 27$ & $\mathrm{C} 24$ & $119.35(8)$ \\
$\mathrm{C} 9$ & $\mathrm{C} 10$ & $\mathrm{C} 11$ & $121.30(7)$ & $\mathrm{N} 5$ & $\mathrm{C} 27$ & $\mathrm{C} 24$ & $117.35(7)$ \\
$\mathrm{C} 10$ & $\mathrm{C} 11$ & $\mathrm{C} 12$ & $119.37(7)$ & $\mathrm{N} 5$ & $\mathrm{C} 28$ & $\mathrm{C} 29$ & $112.50(7)$ \\
$\mathrm{C} 10$ & $\mathrm{C} 11$ & $\mathrm{C} 15$ & $119.10(7)$ & $\mathrm{N} 5$ & $\mathrm{C} 28$ & $\mathrm{C} 30$ & $112.69(7)$ \\
$\mathrm{C} 12$ & $\mathrm{C} 11$ & $\mathrm{C} 15$ & $121.53(7)$ & $\mathrm{C} 30$ & $\mathrm{C} 28$ & $\mathrm{C} 29$ & $112.42(9)$ \\
$\mathrm{C} 13$ & $\mathrm{C} 12$ & $\mathrm{C} 11$ & $119.70(8)$ & $\mathrm{N} 5$ & $\mathrm{C} 31$ & $\mathrm{C} 32$ & $111.04(7)$ \\
$\mathrm{C} 14$ & $\mathrm{C} 13$ & $\mathrm{C} 12$ & $120.27(8)$ & $\mathrm{C} 31$ & $\mathrm{C} 33$ & $111.36(8)$ \\
$\mathrm{C} 13$ & $\mathrm{C} 14$ & $\mathrm{C} 9$ & $120.76(8)$ & $\mathrm{C} 31$ & $\mathrm{C} 33$ & $112.64(8)$
\end{tabular}

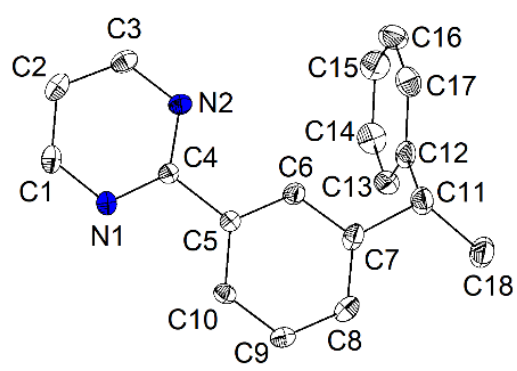

Figure S10. Molecular structure of 3dl with thermal ellipoids at 50\% probability level. The hydrogen atoms are omitted for clarity.

Crystal Data for $\mathrm{C}_{18} \mathrm{H}_{16} \mathrm{~N}_{2}(M=260.33 \mathrm{~g} / \mathrm{mol})$ : orthorhombic, space group Pca2 1 (no. 29), $a=$ 12.2928(3) $\AA, b=6.01020(10) \AA, c=36.9034(9) \AA, V=2726.50(10) \AA^{3}, Z=8, T=99.97 \mathrm{~K}$, $\mu(\mathrm{MoK} \alpha)=0.075 \mathrm{~mm}^{-1}$, Dcalc $=1.268 \mathrm{~g} / \mathrm{cm}^{3}, 28332$ reflections measured $\left(6.624^{\circ} \leq 2 \Theta \leq\right.$ 
$\left.59.142^{\circ}\right), 7604$ unique $\left(R_{\text {int }}=0.0264, \mathrm{R}_{\text {sigma }}=0.0245\right)$ which were used in all calculations. The final $R_{1}$ was 0.0431 (I $\left.>2 \sigma(\mathrm{I})\right)$ and $w R_{2}$ was 0.1162 (all data).

Table S24. Crystal data and structure refinement for $\mathbf{3 d l}$

\begin{tabular}{|c|c|}
\hline Compound & 3dl \\
\hline CCDC number & 1915687 \\
\hline Identification code & Pca21 \\
\hline Empirical formula & $\mathrm{C}_{18} \mathrm{H}_{16} \mathrm{~N}_{2}$ \\
\hline Formula weight & 260.33 \\
\hline Temperature/K & 99.97 \\
\hline Crystal system & orthorhombic \\
\hline Space group & Pca2 1 \\
\hline $\mathrm{a} / \AA ̊$ & $12.2928(3)$ \\
\hline $\mathrm{b} / \AA$ & $6.01020(10)$ \\
\hline $\mathrm{c} / \AA$ & 36.9034(9) \\
\hline$\alpha /^{\circ}$ & 90 \\
\hline$\beta /{ }^{\circ}$ & 90 \\
\hline$\gamma /{ }^{\circ}$ & 90 \\
\hline Volume $/ \AA^{3}$ & $2726.50(10)$ \\
\hline $\mathrm{Z}$ & 8 \\
\hline$\rho_{\text {calc }} \mathrm{g} / \mathrm{cm}^{3}$ & 1.268 \\
\hline$\mu / \mathrm{mm}^{-1}$ & 0.075 \\
\hline $\mathrm{F}(000)$ & 1104.0 \\
\hline Crystal size $/ \mathrm{mm}^{3}$ & $0.309 \times 0.268 \times 0.244$ \\
\hline Radiation & $\operatorname{MoK} \alpha(\lambda=0.71073)$ \\
\hline \multicolumn{2}{|c|}{$2 \Theta$ range for data collection $/{ }^{\circ} 6.624$ to 59.142} \\
\hline Index ranges & $-17 \leq \mathrm{h} \leq 17,-8 \leq \mathrm{k} \leq 8,-51 \leq 1 \leq 51$ \\
\hline Reflections collected & 28332 \\
\hline Independent reflections & $7604\left[R_{\text {int }}=0.0264, R_{\text {sigma }}=0.0245\right]$ \\
\hline Data/restraints/parameters & $7604 / 151 / 452$ \\
\hline Goodness-of-fit on $\mathrm{F}^{2}$ & 1.050 \\
\hline
\end{tabular}




\begin{tabular}{|l|l|}
\hline Final $\mathrm{R}$ indexes $[\mathrm{I}>=2 \sigma(\mathrm{I})]$ & $\mathrm{R}_{1}=0.0431, \mathrm{wR}_{2}=0.1153$ \\
\hline Final R indexes [all data] & $\mathrm{R}_{1}=0.0443, \mathrm{wR}_{2}=0.1162$ \\
\hline Largest diff. peak/hole $/ \mathrm{e} \AA^{-3}$ & $0.52 /-0.25$ \\
\hline Flack parameter & $-0.2(5)$ \\
\hline
\end{tabular}

Table S25. Bond lengths $[\AA]$ for 3dl

\begin{tabular}{lllllll}
\multicolumn{2}{c}{ Atom Atom Length/A } & \multicolumn{3}{c}{ Atom Atom Length/Å } \\
N1 & C1 & $1.342(3)$ & C7 & C11 & $1.541(3)$ \\
N1 & C4 & $1.337(2)$ & C8 & C9 & $1.403(3)$ \\
N2 & C3 & $1.334(3)$ & C9 & C10 & $1.388(3)$ \\
N2 & C4 & $1.342(3)$ & $\mathrm{C} 11$ & C12 & $1.524(3)$ \\
C1 & C2 & $1.378(3)$ & $\mathrm{C} 11$ & $\mathrm{C} 18$ & $1.522(3)$ \\
C2 & C3 & $1.391(3)$ & $\mathrm{C} 12$ & $\mathrm{C} 13$ & $1.393(3)$ \\
$\mathrm{C} 4$ & $\mathrm{C} 5$ & $1.486(3)$ & $\mathrm{C} 12$ & $\mathrm{C} 17$ & $1.384(4)$ \\
$\mathrm{C} 5$ & $\mathrm{C} 6$ & $1.394(3)$ & $\mathrm{C} 13$ & $\mathrm{C} 14$ & $1.397(3)$ \\
$\mathrm{C} 5$ & $\mathrm{C} 10$ & $1.396(3)$ & $\mathrm{C} 14$ & $\mathrm{C} 15$ & $1.388(4)$ \\
$\mathrm{C} 6$ & $\mathrm{C} 7$ & $1.399(3)$ & $\mathrm{C} 15$ & $\mathrm{C} 16$ & $1.379(4)$ \\
$\mathrm{C} 7$ & $\mathrm{C} 8$ & $1.391(3)$ & $\mathrm{C} 16$ & $\mathrm{C} 17$ & $1.385(4)$
\end{tabular}

Table S26. Bond angles $\left[{ }^{\circ}\right]$ for $\mathbf{3 d l}$

Atom Atom Atom Angle $^{\circ}$
$\begin{array}{llll}\text { C4 } & \text { N1 } & \text { C1 } & 116.03(18) \\ \text { C3 } & \text { N2 } & \text { C4 } & 116.4(2) \\ \text { N1 } & \text { C1 } & \text { C2 } & 122.9(2) \\ \text { C1 } & \text { C2 } & \text { C3 } & 116.23(19) \\ \text { N2 } & \text { C3 } & \text { C2 } & 122.4(2) \\ \text { N1 } & \text { C4 } & \text { N2 } & 126.02(18) \\ \text { N1 } & \text { C4 } & \text { C5 } & 117.10(17) \\ \text { N2 } & \text { C4 } & \text { C5 } & 116.87(17) \\ \text { C6 } & \text { C5 } & \text { C4 } & 119.36(17) \\ \text { C6 } & \text { C5 } & \text { C10 } & 119.46(18) \\ \text { C10 } & \text { C5 } & \text { C4 } & 121.18(17)\end{array}$

Atom Atom Atom Angle $/^{\circ}$

C7 C8 C9 $120.75(19)$

C10 C9 C8 120.37(19)

C9 $\quad \mathrm{C} 10 \quad \mathrm{C} 5 \quad 119.62(19)$

C12 C11 C7 109.22(17)

C18 C11 C7 113.9(2)

C18 C11 C12 111.3(2)

C13 C12 C11 122.1(2)

C17 C12 C11 119.1(2)

C17 C12 C13 118.8(2)

C12 C13 C14 120.3(2)

C15 C14 C13 119.8(2) 


\begin{tabular}{lllllllll}
\multicolumn{3}{c}{ Atom Atom Atom Angle $/^{\circ}$} & \multicolumn{3}{c}{ Atom Atom Atom Angle $/^{\circ}$} \\
C5 & C6 & C7 & $121.70(19)$ & C16 & C15 & C14 & $119.9(2)$ \\
C6 & C7 & C11 & $117.46(19)$ & C15 & C16 & C17 & $120.1(2)$ \\
C8 & C7 & C6 & $118.1(2)$ & C12 & C17 & C16 & $121.1(2)$ \\
C8 & C7 & C11 & $124.44(19)$ & & & &
\end{tabular}

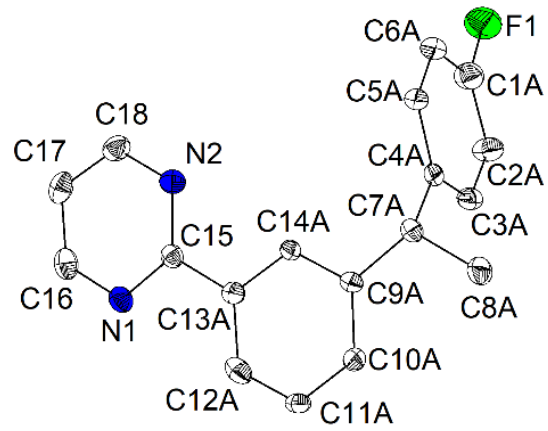

Figure S11. Molecular structure of $\mathbf{3 d m}$ with thermal ellipoids at 50\% probability level. The hydrogen atoms are omitted for clarity.

Crystal Data for $\mathrm{C}_{18} \mathrm{H}_{15} \mathrm{FN}_{2}(M=278.32 \mathrm{~g} / \mathrm{mol})$ : monoclinic, space group P2 $1 / \mathrm{c}$ (no. 14), $a=$ 19.3115(8) $\AA, b=5.8472(3) \AA, c=12.7090(5) \AA, \beta=106.294(2)^{\circ}, V=1377.44(11) \AA^{3}, Z=4$, $T=100.01 \mathrm{~K}, \mu(\mathrm{MoK} \alpha)=0.089 \mathrm{~mm}^{-1}$, Dcalc $=1.342 \mathrm{~g} / \mathrm{cm}^{3}, 39008$ reflections measured $\left(4.394^{\circ} \leq 2 \Theta \leq 59.996^{\circ}\right), 4019$ unique $\left(R_{\text {int }}=0.0356, \mathrm{R}_{\text {sigma }}=0.0218\right)$ which were used in all calculations. The final $R_{1}$ was 0.0533 (I $\left.>2 \sigma(\mathrm{I})\right)$ and $w R_{2}$ was 0.1230 (all data).

Table S27. Crystal data and structure refinement for $\mathbf{3 d m}$

\begin{tabular}{|l|l|}
\hline Compound & $\mathbf{3 d m}$ \\
\hline CCDC number & 1915684 \\
\hline Identification code & mo_0065_CG_0m \\
\hline Empirical formula & $\mathrm{C}_{18} \mathrm{H}_{15} \mathrm{FN}_{2}$ \\
\hline Formula weight & 278.32 \\
\hline Temperature/K & 100.01 \\
\hline Crystal system & monoclinic \\
\hline Space group & $\mathrm{P} 2{ }_{1} / \mathrm{c}$ \\
\hline a/ & $19.3115(8)$ \\
\hline
\end{tabular}




\begin{tabular}{|c|c|}
\hline $\mathrm{b} / \AA ̊ \AA$ & $5.8472(3)$ \\
\hline $\mathrm{c} / \AA ̊$ & $12.7090(5)$ \\
\hline$\alpha /^{\circ}$ & 90 \\
\hline$\beta /^{\circ}$ & 106.294(2) \\
\hline$\gamma /{ }^{\circ}$ & 90 \\
\hline Volume $/ \AA^{3}$ & 1377.44(11) \\
\hline $\mathrm{Z}$ & 4 \\
\hline$\rho_{\text {calc }} \mathrm{g} / \mathrm{cm}^{3}$ & 1.342 \\
\hline$\mu / \mathrm{mm}^{-1}$ & 0.089 \\
\hline $\mathrm{F}(000)$ & 584.0 \\
\hline Crystal size $/ \mathrm{mm}^{3}$ & $0.29 \times 0.17 \times 0.065$ \\
\hline Radiation & $\operatorname{MoK} \alpha(\lambda=0.71073)$ \\
\hline \multicolumn{2}{|c|}{$2 \Theta$ range for data collection $/{ }^{\circ} 4.394$ to 59.996} \\
\hline Index ranges & $-26 \leq \mathrm{h} \leq 27,-8 \leq \mathrm{k} \leq 8,-17 \leq 1 \leq 17$ \\
\hline Reflections collected & 39008 \\
\hline Independent reflections & $4019\left[R_{\text {int }}=0.0356, R_{\text {sigma }}=0.0218\right]$ \\
\hline Data/restraints/parameters & $4019 / 36 / 295$ \\
\hline Goodness-of-fit on $\mathrm{F}^{2}$ & 1.183 \\
\hline Final $R$ indexes $[I>=2 \sigma(I)]$ & $\mathrm{R}_{1}=0.0533, \mathrm{wR}_{2}=0.1197$ \\
\hline Final $\mathrm{R}$ indexes [all data] & $\mathrm{R}_{1}=0.0588, \mathrm{wR}_{2}=0.1230$ \\
\hline Largest diff. peak/hole / e $\AA^{-3}$ & $\sqrt[3]{0.40 /-0.33}$ \\
\hline
\end{tabular}

Table S28. Bond lengths $[\AA ̊]$ for $\mathbf{3 d m}$
Atom Atom Length/Å
Atom Atom Length/Å
F1 C1A 1.374(3)
C7A C8A 1.528(3)
N1 C15 1.3446(17)
C7A C9A 1.528(3)
N1 C16 1.3374(19)
C9A C10A1.391(3)
N2 C15 1.3429(17)
C9A C14A1.395(3)
N2 C18 1.3355(18)
C10AC11A 1.388(4)
C1A C2A 1.373(4)
C11AC12A 1.375(4)
C1A C6A 1.382(4)
C12AC13A 1.399(4) 
Atom Atom Length/Å

C2A C3A 1.382(4)

C3A C4A 1.396(3)

C4A C5A 1.392(3)

C4A C7A 1.526(3)

C5A C6A 1.395(4)

\section{Atom Atom Length/Å}

C13A C14A 1.395(3)

C13AC15 1.478(3)

C16 C17 1.379(2)

C17 C18 1.380(2)

Table S29. Bond angles $\left[{ }^{\circ}\right]$ for $\mathbf{3 d m}$

\begin{tabular}{|c|c|c|c|c|c|c|c|}
\hline \multicolumn{4}{|c|}{ Atom Atom Atom Angle $/^{\circ}$} & \multicolumn{4}{|c|}{ Atom Atom Atom Angle ${ }^{\circ}$} \\
\hline $\mathrm{C} 16$ & N1 & $\mathrm{C} 15$ & $116.14(12)$ & \multicolumn{4}{|c|}{ C10AC9A C7A 123.7(2) } \\
\hline $\mathrm{C} 18$ & $\mathrm{~N} 2$ & $\mathrm{C} 15$ & $116.38(12)$ & \multicolumn{4}{|c|}{ C10AC9A C14A 117.9(2) } \\
\hline $\mathrm{F} 1$ & $\mathrm{C} 1 \mathrm{~A}$ & C6A & $114.0(4)$ & \multicolumn{4}{|c|}{ C14AC9A C7A 118.4(2) } \\
\hline $\mathrm{C} 2 \mathrm{~A}$ & $\mathrm{C} 1 \mathrm{~A}$ & $\mathrm{~F} 1$ & $122.3(4)$ & \multicolumn{4}{|c|}{ C11AC10AC9A 120.9(2) } \\
\hline $\mathrm{C} 2 \mathrm{~A}$ & $\mathrm{C} 1 \mathrm{~A}$ & C6A & $123.7(3)$ & \multicolumn{4}{|c|}{ C12AC11AC10A 120.2(3) } \\
\hline $\mathrm{C} 1 \mathrm{~A}$ & $\mathrm{C} 2 \mathrm{~A}$ & $\mathrm{C} 3 \mathrm{~A}$ & $117.9(3)$ & \multicolumn{4}{|c|}{ C11AC12AC13A 120.8(3) } \\
\hline $\mathrm{C} 2 \mathrm{~A}$ & $\mathrm{C} 3 \mathrm{~A}$ & $\mathrm{C} 4 \mathrm{~A}$ & $121.3(2)$ & \multicolumn{4}{|c|}{ C12AC13AC15 122.2(3) } \\
\hline $\mathrm{C} 3 \mathrm{~A}$ & $\mathrm{C} 4 \mathrm{~A}$ & C7A & $120.0(2)$ & \multicolumn{4}{|c|}{ C14AC13AC12A 118.0(2) } \\
\hline C5A & $\mathrm{C} 4 \mathrm{~A}$ & $\mathrm{C} 3 \mathrm{~A}$ & $118.6(2)$ & \multicolumn{4}{|c|}{ C14AC13AC15 119.7(3) } \\
\hline C5A & $\mathrm{C} 4 \mathrm{~A}$ & C7A & $121.4(3)$ & \multicolumn{4}{|c|}{ C13AC14AC9A 122.2(2) } \\
\hline $\mathrm{C} 4 \mathrm{~A}$ & C5A & C6A & $121.4(2)$ & N1 & $\mathrm{C} 15$ & C13A & $118.11(19)$ \\
\hline $\mathrm{C} 1 \mathrm{~A}$ & C6A & $\mathrm{C} 5 \mathrm{~A}$ & $117.1(3)$ & $\mathrm{N} 2$ & $\mathrm{C} 15$ & $\mathrm{~N} 1$ & $125.54(13)$ \\
\hline $\mathrm{C} 4 \mathrm{~A}$ & C7A & $\mathrm{C} 8 \mathrm{~A}$ & $111.63(18)$ & $\mathrm{N} 2$ & $\mathrm{C} 15$ & C13A & $116.36(19)$ \\
\hline $\mathrm{C} 4 \mathrm{~A}$ & C7A & C9A & $109.42(19)$ & N1 & $\mathrm{C} 16$ & C17 & $122.83(13)$ \\
\hline \multirow[t]{2}{*}{ C8A } & C7A & C9A & $114.46(18)$ & C16 & $\mathrm{C} 17$ & C18 & $116.39(13)$ \\
\hline & & & & $\mathrm{N} 2$ & C18 & C17 & $122.71(14)$ \\
\hline
\end{tabular}




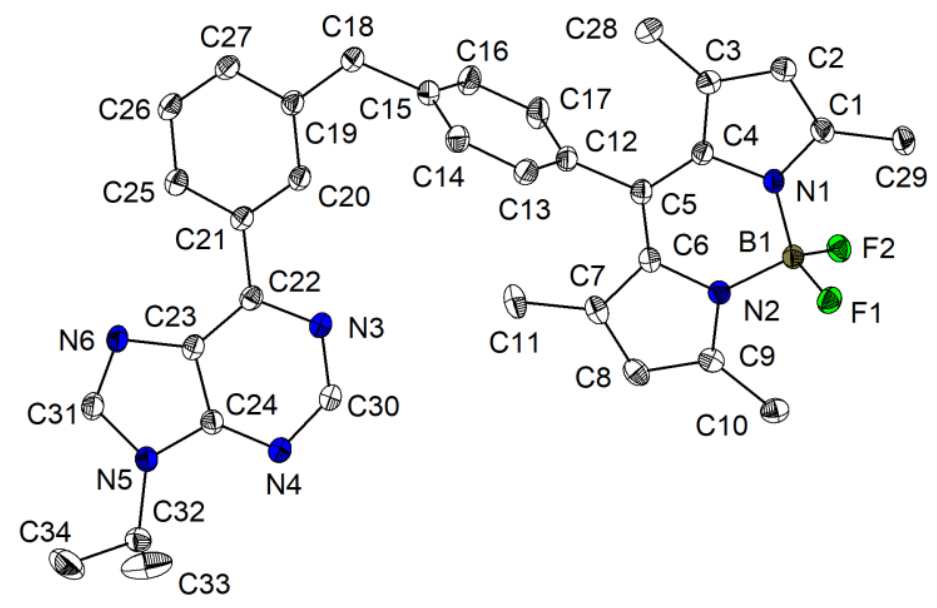

Figure S12. Molecular structure of $6 \mathbf{a}$ with thermal ellipoids at 50\% probability level. The hydrogen atoms are omitted for clarity.

Crystal Data for $\mathrm{C}_{34} \mathrm{H}_{33} \mathrm{BF}_{2} \mathrm{~N}_{6}(M=574.47 \mathrm{~g} / \mathrm{mol})$ : triclinic, space group P-1 (no. 2), $a=$ 10.4593(10) $\mathrm{A}, b=16.8136(17) \AA, c=16.9094(11) \AA, \alpha=94.418(2)^{\circ}, \beta=94.020(3)^{\circ}, \gamma=$ 90.953(3) $)^{\circ}, \quad V=2956.8(5) \AA^{3}, Z=4, T=100.0 \mathrm{~K}, \mu(\mathrm{MoK} \alpha)=0.086 \mathrm{~mm}^{-1}$, Dcalc $=$ $1.290 \mathrm{~g} / \mathrm{cm}^{3}, 108206$ reflections measured $\left(4.646^{\circ} \leq 2 \Theta \leq 61.34^{\circ}\right), 18151$ unique $\left(R_{\text {int }}=0.0389\right.$, $\left.\mathrm{R}_{\text {sigma }}=0.0271\right)$ which were used in all calculations. The final $R_{1}$ was $0.0464(\mathrm{I}>2 \sigma(\mathrm{I}))$ and $w R_{2}$ was 0.1302 (all data).

Table S30. Crystal data and structure refinement for $\mathbf{6 a}$

\begin{tabular}{|l|l|}
\hline Compound & $\mathbf{6 a}$ \\
\hline CCDC number & 1915685 \\
\hline Identification code & $0408 \_C G_{-} 0 \mathrm{~m}$ \\
\hline Empirical formula & $\mathrm{C}_{34} \mathrm{H}_{33} \mathrm{BF}_{2} \mathrm{~N}_{6}$ \\
\hline Formula weight & 574.47 \\
\hline Temperature/K & 100.0 \\
\hline Crystal system & triclinic \\
\hline Space group & $\mathrm{P}-1$ \\
\hline $\mathrm{a} / \AA$ & $10.4593(10)$ \\
\hline $\mathrm{b} / \AA$ & $16.8136(17)$ \\
\hline $\mathrm{c} / \AA$ & $16.9094(11)$ \\
\hline$\alpha /{ }^{\circ}$ & $94.418(2)$ \\
\hline & \multicolumn{1}{|c}{ s94 }
\end{tabular}




\begin{tabular}{|l|l|}
\hline$\beta /{ }^{\circ}$ & $94.020(3)$ \\
\hline$\gamma /{ }^{\circ}$ & $90.953(3)$ \\
\hline Volume/ $\AA^{3}$ & $2956.8(5)$ \\
\hline$Z$ & 4 \\
\hline$\rho_{\text {calcg }} / \mathrm{cm}^{3}$ & 1.290 \\
\hline$\mu / \mathrm{mm}^{-1}$ & 0.086 \\
\hline $\mathrm{F}(000)$ & 1208.0 \\
\hline Crystal size/mm & \\
\hline Radiation & $0.607 \times 0.465 \times 0.242$ \\
\hline $2 \Theta$ range for data collection/ & 4.646 to 61.34 \\
\hline Index ranges & $-14 \leq \mathrm{h} \leq 15,-24 \leq \mathrm{k} \leq 24,-24 \leq 1 \leq 24$ \\
\hline Reflections collected & 108206 \\
\hline Independent reflections & $18151\left[\mathrm{R}_{\text {int }}=0.0389, \mathrm{R}_{\text {sigma }}=0.0271\right]$ \\
\hline Data/restraints/parameters & $18151 / 0 / 787$ \\
\hline Goodness-of-fit on $\mathrm{F}^{2}$ & 1.031 \\
\hline Final R indexes $[\mathrm{I}>=2 \sigma(\mathrm{I})]$ & $\mathrm{R}_{1}=0.0464, \mathrm{wR}_{2}=0.1217$ \\
\hline Final $\mathrm{R}$ indexes $[$ all data $]$ & $\mathrm{R}_{1}=0.0569, \mathrm{wR}_{2}=0.1302$ \\
\hline Largest diff. peak/hole / e $\AA^{-3}$ & $0.41 /-0.39$ \\
\hline
\end{tabular}

Table S31. Bond lengths $[\AA]$ for $6 \mathbf{a}$

\section{Atom Atom Length/Å}

F1 B1 1.3996(14)

F2 B1 1.3927(14)

N1 C1 1.3475(14)

$\mathrm{N} 1 \quad \mathrm{C} 4 \quad 1.4020(14)$

$\mathrm{N} 1 \quad \mathrm{~B} 1 \quad 1.5470(15)$

N2 C6 1.4010(14)

$\mathrm{N} 2 \quad \mathrm{C} 9 \quad 1.3491(14)$

$\mathrm{N} 2$ B1 1.5453(15)

N3 C22 1.3502(14)

N3 $\quad$ C30 $1.3373(15)$

\section{Atom Atom Length/Å}

C6 C7 1.4289(15)

C7 C8 1.3877(17)

C7 C11 1.4966(16)

C8 C9 1.4069(16)

C9 C10 1.4859(16)

C12 C13 1.3906(16)

C12 C17 1.3926(16)

C13 C14 1.3928(16)

C14 C15 1.3921(17)

C15 C16 1.3950(17) 


\begin{tabular}{|c|c|c|c|c|c|}
\hline \multicolumn{3}{|c|}{ Atom Atom Length/Å } & \multicolumn{3}{|c|}{ Atom Atom Length/Å } \\
\hline N4 & $\mathrm{C} 24$ & $1.3329(14)$ & $\mathrm{C} 15$ & $\mathrm{C} 18$ & $1.5119(16)$ \\
\hline $\mathrm{N} 4$ & $\mathrm{C} 30$ & $1.3398(15)$ & $\mathrm{C} 16$ & $\mathrm{C} 17$ & $1.3896(16)$ \\
\hline N5 & $\mathrm{C} 24$ & $1.3710(14)$ & $\mathrm{C} 18$ & C19 & $1.5179(16)$ \\
\hline N5 & $\mathrm{C} 31$ & $1.3715(15)$ & C19 & $\mathrm{C} 20$ & $1.3929(16)$ \\
\hline N5 & $\mathrm{C} 32$ & $1.4766(15)$ & C19 & $\mathrm{C} 27$ & $1.3986(15)$ \\
\hline N6 & $\mathrm{C} 23$ & $1.3863(14)$ & $\mathrm{C} 20$ & $\mathrm{C} 21$ & $1.3993(16)$ \\
\hline N6 & $\mathrm{C} 31$ & $1.3127(16)$ & $\mathrm{C} 21$ & $\mathrm{C} 22$ & $1.4786(15)$ \\
\hline $\mathrm{C} 1$ & $\mathrm{C} 2$ & $1.4041(16)$ & $\mathrm{C} 21$ & $\mathrm{C} 25$ & $1.4015(15)$ \\
\hline $\mathrm{C} 1$ & $\mathrm{C} 29$ & $1.4879(16)$ & $\mathrm{C} 22$ & $\mathrm{C} 23$ & $1.3978(16)$ \\
\hline $\mathrm{C} 2$ & $\mathrm{C} 3$ & $1.3863(16)$ & $\mathrm{C} 23$ & $\mathrm{C} 24$ & $1.4073(16)$ \\
\hline $\mathrm{C} 3$ & $\mathrm{C} 4$ & $1.4261(16)$ & $\mathrm{C} 25$ & $\mathrm{C} 26$ & $1.3905(16)$ \\
\hline $\mathrm{C} 3$ & $\mathrm{C} 28$ & $1.4987(16)$ & $\mathrm{C} 26$ & $\mathrm{C} 27$ & $1.3877(17)$ \\
\hline $\mathrm{C} 4$ & $\mathrm{C} 5$ & $1.3954(15)$ & $\mathrm{C} 32$ & C33 & $1.500(2)$ \\
\hline $\mathrm{C} 5$ & C6 & $1.3939(16)$ & $\mathrm{C} 32$ & C34 & $1.5037(19)$ \\
\hline $\mathrm{C} 5$ & C12 & $1.4910(15)$ & & & \\
\hline
\end{tabular}

Table S32. Bond angles $\left[^{\circ}\right]$ for $\mathbf{6 a}$

\begin{tabular}{|c|c|c|c|c|c|c|c|}
\hline \multicolumn{4}{|c|}{ Atom Atom Atom Angle $/^{\circ}$} & \multicolumn{4}{|c|}{ Atom Atom Atom Angle $/^{\circ}$} \\
\hline $\mathrm{C} 1$ & N1 & $\mathrm{C} 4$ & $108.40(9)$ & $\mathrm{C} 12$ & $\mathrm{C} 13$ & $\mathrm{C} 14$ & $120.02(11)$ \\
\hline $\mathrm{C} 1$ & N1 & B1 & $125.97(9)$ & $\mathrm{C} 15$ & $\mathrm{C} 14$ & $\mathrm{C} 13$ & $121.08(11)$ \\
\hline $\mathrm{C} 4$ & N1 & B1 & $125.63(9)$ & $\mathrm{C} 14$ & $\mathrm{C} 15$ & $\mathrm{C} 16$ & $118.40(10)$ \\
\hline C6 & $\mathrm{N} 2$ & B1 & $125.75(9)$ & $\mathrm{C} 14$ & $\mathrm{C} 15$ & $\mathrm{C} 18$ & $121.26(11)$ \\
\hline C9 & $\mathrm{N} 2$ & C6 & $108.57(9)$ & $\mathrm{C} 16$ & $\mathrm{C} 15$ & $\mathrm{C} 18$ & $120.29(11)$ \\
\hline C9 & $\mathrm{N} 2$ & B1 & $125.66(9)$ & $\mathrm{C} 17$ & $\mathrm{C} 16$ & $\mathrm{C} 15$ & $120.88(11)$ \\
\hline $\mathrm{C} 30$ & N3 & $\mathrm{C} 22$ & $118.63(10)$ & $\mathrm{C} 16$ & $\mathrm{C} 17$ & $\mathrm{C} 12$ & $120.25(11)$ \\
\hline $\mathrm{C} 24$ & N4 & $\mathrm{C} 30$ & $111.08(10)$ & $\mathrm{C} 15$ & $\mathrm{C} 18$ & $\mathrm{C} 19$ & $113.56(10)$ \\
\hline $\mathrm{C} 24$ & N5 & $\mathrm{C} 31$ & $105.50(10)$ & $\mathrm{C} 20$ & C19 & $\mathrm{C} 18$ & $121.67(10)$ \\
\hline $\mathrm{C} 24$ & N5 & C32 & $125.65(10)$ & $\mathrm{C} 20$ & C19 & $\mathrm{C} 27$ & $118.66(11)$ \\
\hline C31 & N5 & C32 & $128.73(10)$ & $\mathrm{C} 27$ & C19 & $\mathrm{C} 18$ & $119.64(10)$ \\
\hline C31 & N6 & $\mathrm{C} 23$ & $103.92(10)$ & C19 & $\mathrm{C} 20$ & $\mathrm{C} 21$ & $121.09(10)$ \\
\hline N1 & $\mathrm{C} 1$ & $\mathrm{C} 2$ & 109.01(10) & $\mathrm{C} 20$ & $\mathrm{C} 21$ & $\mathrm{C} 22$ & $119.32(10)$ \\
\hline
\end{tabular}




\section{Atom Atom Atom Angle/ ${ }^{\circ}$}

N1 C1 C29 123.13(11)

$\begin{array}{llll}\mathrm{C} 2 & \mathrm{C} 1 & \mathrm{C} 29 & 127.85(11)\end{array}$

C3 C2 C1 108.62(10)

C2 C3 C4 106.09(10)

C2 $\quad$ C3 $\quad$ C28 124.72(11)

C4 C3 C28 129.18(11)

N1 C4 C3 107.86(9)

C5 C4 N1 120.38(10)

C5 C4 C3 131.61(10)

C4 C5 C12 118.72(10)

C6 C5 C4 121.09(10)

C6 C5 C12 120.19(10)

N2 C6 C7 107.79(10)

C5 C6 N2 120.33(10)

C5 C6 C7 131.71(10)

C6 C7 C11 128.87(11)

C8 C7 C6 106.15(10)

C8 $\quad$ C7 C11 124.97(11)

C7 C8 C9 108.55(10)

N2 C9 C8 108.93(10)

N2 C9 C10 122.61(10)

C8 C9 C10 128.47(11)

C13 C12 C5 120.36(10)

C13 C12 C17 119.37(10)

C17 C12 C5 120.24(10)

\section{Atom Atom Atom Angle ${ }^{\circ}$}

C20 C21 C25 119.37(10)

C25 C21 C22 121.30(10)

N3 C22 C21 117.52(10)

N3 C22 C23 118.04(10)

C23 C22 C21 124.44(10)

N6 C23 C22 133.07(11)

N6 C23 C24 109.96(10)

C22 C23 C24 116.93(10)

N4 C24 N5 127.74(11)

N4 C24 C23 126.21(10)

N5 $\quad$ C24 C23 106.02(10)

C26 C25 C21 119.71(11)

C27 C26 C25 120.35(10)

C26 C27 C19 120.78(11)

N3 $\quad$ C30 N4 129.10(11)

N6 C31 N5 114.60(10)

N5 C32 C33 111.27(10)

N5 $\quad$ C32 C34 110.42(11)

C33 C32 C34 113.29(14)

F1 B1 N1 110.21(9)

F1 B1 N2 110.45(9)

F2 B1 F1 108.57(9)

F2 B1 N1 110.56(9)

F2 B1 N2 110.59(9)

N2 B1 N1 106.46(9) 


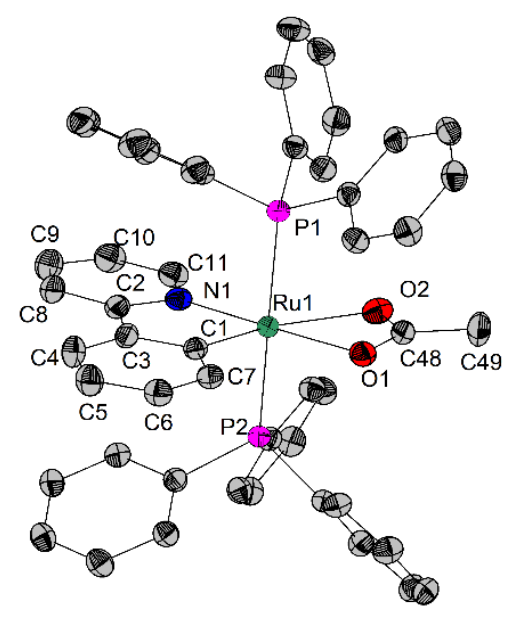

Figure S13. Molecular structure of trans-Ru-II with thermal ellipoids at 50\% probability level. The hydrogen atoms and THF are omitted for clarity.

Crystal Data for $\mathrm{C}_{55} \mathrm{H}_{53} \mathrm{NO}_{3.5} \mathrm{P}_{2} \mathrm{Ru}(M=946.99 \mathrm{~g} / \mathrm{mol})$ : monoclinic, space group $\mathrm{P} 2{ }_{1} / \mathrm{n}$ (no. 14), $a=11.9019(7) \AA, b=15.2039(9) \AA, c=24.7562(15) \AA, \beta=93.907(3)^{\circ}, V=4469.4(5) \AA^{3}$, $Z=4, T=99.99 \mathrm{~K}, \mu(\mathrm{MoK} \alpha)=0.471 \mathrm{~mm}^{-1}$, Dcalc $=1.407 \mathrm{~g} / \mathrm{cm}^{3}, 13699$ reflections measured $\left(4.57^{\circ} \leq 2 \Theta \leq 61.146^{\circ}\right), 13699$ unique $\left(R_{\text {int }}=0, \mathrm{R}_{\text {sigma }}=0.0303\right)$ which were used in all calculations. The final $R_{1}$ was $0.0435(\mathrm{I}>2 \sigma(\mathrm{I}))$ and $w R_{2}$ was 0.1183 (all data).

Table S33. Crystal data and structure refinement for trans-Ru-II

\begin{tabular}{|l|l|}
\hline Compound & trans-Ru-II \\
\hline CCDC number & 1915676 \\
\hline Identification code & $0473 \_C G \_0 m_{-} 4$ \\
\hline Empirical formula & $\mathrm{C}_{55} \mathrm{H}_{53} \mathrm{NO}_{3.5} \mathrm{P}_{2} \mathrm{Ru}$ \\
\hline Formula weight & 946.99 \\
\hline Temperature/K & 99.99 \\
\hline Crystal system & monoclinic \\
\hline Space group & $\mathrm{P} 2{ }_{1} / \mathrm{n}$ \\
\hline $\mathrm{a} / \AA$ & $11.9019(7)$ \\
\hline $\mathrm{b} / \AA$ & $15.2039(9)$ \\
\hline $\mathrm{c} / \AA$ & $24.7562(15)$ \\
\hline$\alpha /{ }^{\circ}$ & 90 \\
\hline$\beta /{ }^{\circ}$ & $93.907(3)$ \\
\hline
\end{tabular}




\begin{tabular}{|l|l|}
\hline$\gamma /{ }^{\circ}$ & 90 \\
\hline Volume/ $\AA^{3}$ & $4469.4(5)$ \\
\hline$Z$ & 4 \\
\hline$\rho_{\text {calcg/cm }}^{3}$ & 1.407 \\
\hline$\mu / \mathrm{mm}^{-1}$ & 0.471 \\
\hline $\mathrm{F}(000)$ & 1968.0 \\
\hline Crystal size/mm & \\
\hline Radiation & $0.317 \times 0.086 \times 0.063$ \\
\hline $2 \Theta$ range for data collection/ & 4.57 to 61.146 \\
\hline Index ranges & $-16 \leq \mathrm{h} \leq 16,0 \leq \mathrm{k} \leq 21,0 \leq 1 \leq 35$ \\
\hline Reflections collected & 13699 \\
\hline Independent reflections & $13699\left[\mathrm{R}_{\text {int }}=0, \mathrm{R}_{\text {sigma }}=0.0303\right]$ \\
\hline Data/restraints/parameters & $13699 / 30 / 587$ \\
\hline Goodness-of-fit on $\mathrm{F}^{2}$ & 1.048 \\
\hline Final R indexes [I $>=2 \sigma(\mathrm{I})]$ & $\mathrm{R}_{1}=0.0435, \mathrm{wR}_{2}=0.1132$ \\
\hline Final R indexes [all data] & $\mathrm{R}_{1}=0.0516, \mathrm{wR}_{2}=0.1183$ \\
\hline Largest diff. peak/hole / e $\AA^{-3}$ & $1.34 /-0.94$ \\
\hline
\end{tabular}

Table S34. Selected bond lengths $[\AA]$ for trans-Ru-II

\begin{tabular}{|c|c|c|c|c|c|}
\hline \multicolumn{2}{|c|}{ Atom Atom } & \multirow{2}{*}{$\begin{array}{l}\text { Length/Å } \\
2.3421(5)\end{array}$} & \multicolumn{2}{|c|}{ Atom Atom } & \multirow{2}{*}{$\begin{array}{r}\text { Length/Å } \\
1.392(3)\end{array}$} \\
\hline Ru1 & $\mathrm{P} 1$ & & C16 & $\mathrm{C} 17$ & \\
\hline Ru1 & P2 & $2.3355(5)$ & $\mathrm{C} 18$ & C19 & $1.395(3)$ \\
\hline Ru1 & $\mathrm{O} 1$ & $2.1592(16)$ & $\mathrm{C} 18$ & $\mathrm{C} 23$ & $1.395(3)$ \\
\hline Ru1 & $\mathrm{O} 2$ & $2.3317(17)$ & C19 & $\mathrm{C} 20$ & $1.387(3)$ \\
\hline Ru1 & N1 & $2.0554(18)$ & $\mathrm{C} 20$ & $\mathrm{C} 21$ & $1.380(4)$ \\
\hline Ru1 & $\mathrm{C} 1$ & $2.0031(17)$ & $\mathrm{C} 21$ & $\mathrm{C} 22$ & $1.380(4)$ \\
\hline P1 & $\mathrm{C} 12$ & $1.8378(19)$ & $\mathrm{C} 22$ & $\mathrm{C} 23$ & $1.386(3)$ \\
\hline P1 & $\mathrm{C} 18$ & $1.828(2)$ & $\mathrm{C} 24$ & $\mathrm{C} 25$ & $1.397(3)$ \\
\hline P1 & $\mathrm{C} 24$ & $1.8314(19)$ & $\mathrm{C} 24$ & $\mathrm{C} 29$ & $1.392(3)$ \\
\hline $\mathrm{P} 2$ & $\mathrm{C} 30$ & $1.832(2)$ & $\mathrm{C} 25$ & $\mathrm{C} 26$ & $1.389(3)$ \\
\hline P2 & C36 & $1.8312(19)$ & $\mathrm{C} 26$ & $\mathrm{C} 27$ & $1.392(3)$ \\
\hline
\end{tabular}




\begin{tabular}{|c|c|c|c|c|c|}
\hline \multicolumn{2}{|c|}{ Atom Atom } & \multirow{2}{*}{$\begin{array}{r}\text { Length/Å } \\
1.837(2)\end{array}$} & \multicolumn{2}{|c|}{ Atom Atom } & \multirow{2}{*}{$\begin{array}{r}\text { Length/Å } \\
1.383(3)\end{array}$} \\
\hline $\mathrm{P} 2$ & $\mathrm{C} 42$ & & $\mathrm{C} 27$ & $\mathrm{C} 28$ & \\
\hline $\mathrm{O} 1$ & $\mathrm{C} 48$ & $1.264(2)$ & $\mathrm{C} 28$ & $\mathrm{C} 29$ & $1.393(3)$ \\
\hline $\mathrm{O} 2$ & $\mathrm{C} 48$ & $1.261(2)$ & $\mathrm{C} 30$ & $\mathrm{C} 31$ & $1.390(3)$ \\
\hline N1 & $\mathrm{C} 2$ & $1.387(3)$ & $\mathrm{C} 30$ & $\mathrm{C} 35$ & $1.398(3)$ \\
\hline N1 & $\mathrm{C} 11$ & $1.379(3)$ & C31 & $\mathrm{C} 32$ & $1.398(3)$ \\
\hline $\mathrm{C} 1$ & $\mathrm{C} 3$ & $1.382(3)$ & $\mathrm{C} 32$ & C33 & $1.382(3)$ \\
\hline $\mathrm{C} 1$ & $\mathrm{C} 7$ & $1.386(2)$ & $\mathrm{C} 33$ & $\mathrm{C} 34$ & $1.390(3)$ \\
\hline $\mathrm{C} 2$ & $\mathrm{C} 3$ & $1.451(3)$ & C34 & C35 & $1.386(3)$ \\
\hline $\mathrm{C} 2$ & $\mathrm{C} 8$ & $1.397(3)$ & $\mathrm{C} 36$ & $\mathrm{C} 37$ & $1.393(3)$ \\
\hline $\mathrm{C} 3$ & $\mathrm{C} 4$ & $1.397(3)$ & $\mathrm{C} 36$ & $\mathrm{C} 41$ & $1.398(3)$ \\
\hline $\mathrm{C} 4$ & $\mathrm{C} 5$ & $1.385(3)$ & $\mathrm{C} 37$ & C38 & $1.392(3)$ \\
\hline $\mathrm{C} 5$ & C6 & $1.387(3)$ & C38 & C39 & $1.384(3)$ \\
\hline C6 & $\mathrm{C} 7$ & $1.383(3)$ & C39 & $\mathrm{C} 40$ & $1.381(3)$ \\
\hline $\mathrm{C} 8$ & C9 & $1.381(3)$ & $\mathrm{C} 40$ & $\mathrm{C} 41$ & $1.386(3)$ \\
\hline $\mathrm{C} 9$ & $\mathrm{C} 10$ & $1.390(4)$ & $\mathrm{C} 42$ & $\mathrm{C} 43$ & $1.392(3)$ \\
\hline $\mathrm{C} 10$ & $\mathrm{C} 11$ & $1.385(3)$ & $\mathrm{C} 42$ & $\mathrm{C} 47$ & 1.397(3) \\
\hline $\mathrm{C} 12$ & $\mathrm{C} 13$ & $1.394(3)$ & $\mathrm{C} 43$ & $\mathrm{C} 44$ & $1.386(3)$ \\
\hline $\mathrm{C} 12$ & $\mathrm{C} 17$ & $1.388(3)$ & $\mathrm{C} 44$ & $\mathrm{C} 45$ & $1.387(3)$ \\
\hline $\mathrm{C} 13$ & $\mathrm{C} 14$ & $1.394(3)$ & $\mathrm{C} 45$ & $\mathrm{C} 46$ & $1.384(3)$ \\
\hline $\mathrm{C} 14$ & $\mathrm{C} 15$ & $1.383(3)$ & $\mathrm{C} 46$ & $\mathrm{C} 47$ & $1.395(3)$ \\
\hline $\mathrm{C} 15$ & $\mathrm{C} 16$ & $1.386(3)$ & $\mathrm{C} 48$ & C49 & $1.489(3)$ \\
\hline
\end{tabular}

Table S35. Selected bond angles $\left[{ }^{\circ}\right]$ for trans-Ru-II

\begin{tabular}{|c|c|c|c|c|c|c|c|}
\hline \multicolumn{3}{|c|}{ Atom Atom Atom } & \multirow{2}{*}{$\begin{array}{l}\text { Angle }^{\circ}{ }^{2} \\
172.681(17)\end{array}$} & \multicolumn{3}{|c|}{ Atom Atom Atom } & \multirow{2}{*}{$\begin{array}{l}\text { Angle }^{\circ} \\
\quad 119.96(15)\end{array}$} \\
\hline $\mathrm{P} 2$ & Ru1 & $\mathrm{P} 1$ & & $\mathrm{C} 17$ & $\mathrm{C} 12$ & $\mathrm{P} 1$ & \\
\hline $\mathrm{O} 1$ & Ru1 & $\mathrm{P} 1$ & $91.03(4)$ & $\mathrm{C} 17$ & $\mathrm{C} 12$ & $\mathrm{C} 13$ & $118.84(18)$ \\
\hline $\mathrm{O} 1$ & Ru1 & $\mathrm{P} 2$ & $92.19(4)$ & $\mathrm{C} 14$ & $\mathrm{C} 13$ & $\mathrm{C} 12$ & $120.6(2)$ \\
\hline $\mathrm{O} 1$ & Ru1 & $\mathrm{O} 2$ & $57.94(6)$ & $\mathrm{C} 15$ & $\mathrm{C} 14$ & $\mathrm{C} 13$ & $120.0(2)$ \\
\hline $\mathrm{O} 2$ & Ru1 & $\mathrm{P} 1$ & $85.36(4)$ & $\mathrm{C} 14$ & $\mathrm{C} 15$ & $\mathrm{C} 16$ & 119.67(19) \\
\hline $\mathrm{O} 2$ & Ru1 & $\mathrm{P} 2$ & $90.78(4)$ & $\mathrm{C} 15$ & $\mathrm{C} 16$ & $\mathrm{C} 17$ & $120.4(2)$ \\
\hline $\mathrm{N} 1$ & Ru1 & $\mathrm{P} 1$ & $88.04(5)$ & $\mathrm{C} 12$ & $\mathrm{C} 17$ & $\mathrm{C} 16$ & $120.5(2)$ \\
\hline
\end{tabular}




\section{Atom Atom Atom}

N1 Ru1 P2

N1 Ru1 O1

N1 Ru1 O2

C1 Ru1 P1

C1 Ru1 P2

C1 Ru1 O1

C1 Ru1 O2

C1 Ru1 N1

C12 P1 Ru1

C18 P1 Ru1

C18 P1 C12

C18 P1 C24

C24 P1 Ru1

C24 P1 C12

C30 P2 Ru1

C30 P2 C42

C36 P2 Ru1

C36 P2 C30

C36 P2 C42

C42 P2 Ru1

C48 O1 Ru1

C48 O2 Ru1

C2 N1 Ru1

C11 N1 Ru1

C11 N1 C2

C3 C1 Ru1

C3 $\quad$ C1 1

C7 $\mathrm{C} 1 \quad \mathrm{Ru} 1$

N1 $\mathrm{C} 2 \quad \mathrm{C} 3$

N1 $\quad$ C2 $\quad$ C8
Angle $/^{\circ}$

88.70(5)

179.02(6)

121.66(6)

93.47(5)

92.29(5)

101.95(6)

159.78(6)

78.41(7)

115.92(7)

115.73(6)

101.27(9)

103.56(9)

115.02(6)

103.44(9)

115.18(6)

104.39(9)

115.02(6)

102.04(9)

102.48(9)

115.91(6)

95.11(13)

87.27(13)

115.92(13)

126.77(16)

117.31(18)

118.20(13)

118.03(17)

123.77(14)

113.97(17)

121.79(19)
Atom Atom Atom

C19 C18 P1

C19 C18 C23

C23 C18 P1

C20 $\quad 19 \quad$ C18

C21 C20 C19

C22 C21 C20

$\begin{array}{lll}\mathrm{C} 21 & \mathrm{C} 22 & \mathrm{C} 23\end{array}$

C22 C23 C18

C25 C24 P1

C29 C24 P1

C29 $\mathrm{C} 24 \quad \mathrm{C} 25$

C26 C25 C24

$\begin{array}{lll}\mathrm{C} 25 & \mathrm{C} 26 & \mathrm{C} 27\end{array}$

C28 C27 C26

C27 C28 C29

C24 C29 $\quad 28$

C31 C30 P2

$\begin{array}{lll}\text { C31 } & \text { C30 } & \text { C35 }\end{array}$

C35 C30 P2

C30 C31 C32

C33 C32 C31

C32 $\quad$ C33 $\quad$ C34

C35 C34 C33

C34 C35 C30

C37 C36 P2

C37 $\quad$ C36 $\quad$ C41

C41 C36 P2

C38 C37 C36

C39 $\quad 338 \quad$ C37

$\begin{array}{lll}\text { C40 } & \text { C39 } & \text { C38 }\end{array}$
Angle/ ${ }^{\circ}$

122.35(16)

118.57(19)

119.01(16)

120.7(2)

119.8(2)

120.3(2)

120.1(2)

$120.5(2)$

117.64(15)

123.60(15)

118.73(18)

120.78(19)

$119.8(2)$

119.8(2)

120.3(2)

120.51(19)

122.18(15)

118.87(18)

118.83(15)

120.29(19)

120.39(19)

119.54(19)

120.31(19)

120.59(18)

122.21(14)

118.53(17)

119.20(15)

120.64(19)

120.21(19)

119.51(18) 


\begin{tabular}{lllrrllr}
\multicolumn{2}{c}{ Atom Atom Atom } & \multicolumn{1}{c}{ Angle ${ }^{\circ}$} & & Atom Atom Atom & \multicolumn{1}{c}{ Angle ${ }^{\circ}$} \\
C8 & C2 & C3 & $124.23(19)$ & C39 & C40 & C41 & $120.67(19)$ \\
C1 & C3 & C2 & $113.47(17)$ & C40 & C41 & C36 & $120.42(19)$ \\
C1 & C3 & C4 & $120.81(18)$ & C43 & C42 & P2 & $118.07(15)$ \\
C4 & C3 & C2 & $125.70(19)$ & C43 & C42 & C47 & $118.63(18)$ \\
C5 & C4 & C3 & $120.1(2)$ & C47 & C42 & P2 & $123.20(15)$ \\
C4 & C5 & C6 & $119.6(2)$ & C44 & C43 & C42 & $120.85(19)$ \\
C7 & C6 & C5 & $119.4(2)$ & C43 & C44 & C45 & $120.17(19)$ \\
C6 & C7 & C1 & $122.04(19)$ & C46 & C45 & C44 & $119.8(2)$ \\
C9 & C8 & C2 & $119.7(2)$ & C45 & C46 & C47 & $120.1(2)$ \\
C8 & C9 & C10 & $119.2(2)$ & C46 & C47 & C42 & $120.46(18)$ \\
C11 & C10 & C9 & $120.0(2)$ & O1 & C48 & C49 & $119.0(2)$ \\
N1 & C11 & C10 & $122.0(2)$ & O2 & C48 & O1 & $119.45(19)$ \\
C13 & C12 & P1 & $121.20(15)$ & O2 & C48 & C49 & $121.5(2)$
\end{tabular}

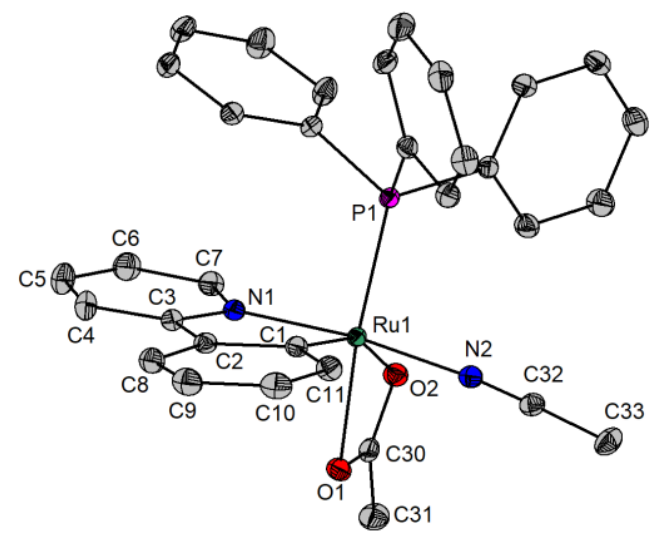

Figure S14. Molecular structure of Ru-III with thermal ellipoids at 50\% probability level.

The hydrogen atoms are omitted for clarity.

Crystal Data for $\mathrm{C}_{33} \mathrm{H}_{29} \mathrm{~N}_{2} \mathrm{O}_{2} \mathrm{PRu}(M=617.62 \mathrm{~g} / \mathrm{mol})$ : orthorhombic, space group Pna2 1 (no. 33), $a=16.2264(8) \AA, b=9.3716(4) \AA, c=17.7053(10) \AA, V=2692.4(2) \AA^{3}, Z=4, T=$ $100.1 \mathrm{~K}, \mu(\mathrm{MoK} \alpha)=0.676 \mathrm{~mm}^{-1}$, Dcalc $=1.524 \mathrm{~g} / \mathrm{cm}^{3}, 57514$ reflections measured $\left(4.602^{\circ} \leq\right.$ $\left.2 \Theta \leq 63.044^{\circ}\right), 8977$ unique $\left(R_{\text {int }}=0.0242, \mathrm{R}_{\text {sigma }}=0.0160\right)$ which were used in all calculations . The final $R_{1}$ was $0.0155(\mathrm{I}>2 \sigma(\mathrm{I}))$ and $w R_{2}$ was 0.0412 (all data). 
Table S36. Crystal data and structure refinement for Ru-III

\begin{tabular}{|c|c|}
\hline Compound & Ru-III \\
\hline CCDC number & 1915689 \\
\hline Identification code & Pna21 \\
\hline Empirical formula & $\mathrm{C}_{33} \mathrm{H}_{29} \mathrm{~N}_{2} \mathrm{O}_{2} \mathrm{PRu}$ \\
\hline Formula weight & 617.62 \\
\hline Temperature/K & 100.1 \\
\hline Crystal system & orthorhombic \\
\hline Space group & Pna2 1 \\
\hline $\mathrm{a} / \AA$ & $16.2264(8)$ \\
\hline $\mathrm{b} / \AA$ & $9.3716(4)$ \\
\hline $\mathrm{c} / \AA$ & $17.7053(10)$ \\
\hline$\alpha / /^{\circ}$ & 90 \\
\hline$\beta /{ }^{\circ}$ & 90 \\
\hline$\gamma /{ }^{\circ}$ & 90 \\
\hline Volume $/ \AA^{3}$ & $2692.4(2)$ \\
\hline $\mathrm{Z}$ & 4 \\
\hline$\rho_{\text {calc }} \mathrm{g} / \mathrm{cm}^{3}$ & 1.524 \\
\hline$\mu / \mathrm{mm}^{-1}$ & 0.676 \\
\hline $\mathrm{F}(000)$ & 1264.0 \\
\hline Crystal size $/ \mathrm{mm}^{3}$ & $0.305 \times 0.139 \times 0.09$ \\
\hline Radiation & $\operatorname{MoK} \alpha(\lambda=0.71073)$ \\
\hline $2 \Theta$ range for data collection $/{ }^{\circ}$ & 4.602 to 63.044 \\
\hline Index ranges & $-23 \leq \mathrm{h} \leq 23,-13 \leq \mathrm{k} \leq 13,-26 \leq 1 \leq 26$ \\
\hline Reflections collected & 57514 \\
\hline Independent reflections & $8977\left[\mathrm{R}_{\text {int }}=0.0242, \mathrm{R}_{\text {sigma }}=0.0160\right]$ \\
\hline Data/restraints/parameters & $8977 / 1 / 354$ \\
\hline Goodness-of-fit on $\mathrm{F}^{2}$ & 1.034 \\
\hline Final $\mathrm{R}$ indexes $[\mathrm{I}>=2 \sigma(\mathrm{I})]$ & $\mathrm{R}_{1}=0.0155, \mathrm{wR} 2=0.0408$ \\
\hline Final $\mathrm{R}$ indexes [all data] & $\mathrm{R}_{1}=0.0160, \mathrm{wR}_{2}=0.0412$ \\
\hline Largest diff. peak/hole / e $\AA^{-3}$ & $0.37 /-0.43$ \\
\hline
\end{tabular}


Table S37. Bond lengths [̊] for Ru-III

\begin{tabular}{|c|c|c|c|c|c|}
\hline \multicolumn{2}{|c|}{ Atom Atom } & \multirow{2}{*}{$\begin{array}{l}\text { Length/̊̊ } \\
2.2451(5)\end{array}$} & \multicolumn{2}{|c|}{ Atom Atom } & \multirow{2}{*}{$\begin{array}{r}\text { Length/Å } \\
1.393(3)\end{array}$} \\
\hline Ru1 & $\mathrm{P} 1$ & & $\mathrm{C} 9$ & $\mathrm{C} 10$ & \\
\hline Ru1 & $\mathrm{O} 1$ & $2.2216(12)$ & $\mathrm{C} 10$ & C11 & $1.389(2)$ \\
\hline Ru1 & $\mathrm{O} 2$ & $2.2956(11)$ & $\mathrm{C} 12$ & $\mathrm{C} 13$ & $1.397(2)$ \\
\hline Ru1 & N1 & $2.0531(13)$ & $\mathrm{C} 12$ & $\mathrm{C} 17$ & $1.394(2)$ \\
\hline Ru1 & $\mathrm{N} 2$ & $2.0148(14)$ & $\mathrm{C} 13$ & $\mathrm{C} 14$ & $1.395(2)$ \\
\hline Ru1 & $\mathrm{C} 1$ & $2.0085(15)$ & C14 & $\mathrm{C} 15$ & $1.394(3)$ \\
\hline P1 & $\mathrm{C} 12$ & $1.8372(16)$ & C15 & $\mathrm{C} 16$ & $1.388(3)$ \\
\hline P1 & $\mathrm{C} 18$ & $1.8283(16)$ & $\mathrm{C} 16$ & $\mathrm{C} 17$ & $1.391(2)$ \\
\hline $\mathrm{P} 1$ & $\mathrm{C} 24$ & $1.8394(16)$ & $\mathrm{C} 18$ & C19 & $1.396(2)$ \\
\hline $\mathrm{O} 1$ & $\mathrm{C} 30$ & $1.267(2)$ & $\mathrm{C} 18$ & $\mathrm{C} 23$ & $1.400(2)$ \\
\hline $\mathrm{O} 2$ & $\mathrm{C} 30$ & $1.2635(19)$ & C19 & $\mathrm{C} 20$ & $1.395(2)$ \\
\hline N1 & $\mathrm{C} 3$ & $1.3643(19)$ & $\mathrm{C} 20$ & $\mathrm{C} 21$ & $1.381(2)$ \\
\hline N1 & $\mathrm{C} 7$ & $1.345(2)$ & $\mathrm{C} 21$ & $\mathrm{C} 22$ & $1.393(3)$ \\
\hline $\mathrm{N} 2$ & $\mathrm{C} 32$ & $1.147(2)$ & $\mathrm{C} 22$ & $\mathrm{C} 23$ & $1.390(2)$ \\
\hline $\mathrm{C} 1$ & $\mathrm{C} 2$ & $1.424(2)$ & $\mathrm{C} 24$ & $\mathrm{C} 25$ & $1.396(2)$ \\
\hline $\mathrm{C} 1$ & $\mathrm{C} 11$ & $1.412(2)$ & $\mathrm{C} 24$ & $\mathrm{C} 29$ & $1.398(2)$ \\
\hline $\mathrm{C} 2$ & $\mathrm{C} 3$ & $1.456(2)$ & $\mathrm{C} 25$ & $\mathrm{C} 26$ & $1.395(2)$ \\
\hline $\mathrm{C} 2$ & $\mathrm{C} 8$ & $1.403(2)$ & $\mathrm{C} 26$ & $\mathrm{C} 27$ & $1.383(3)$ \\
\hline $\mathrm{C} 3$ & $\mathrm{C} 4$ & $1.396(2)$ & $\mathrm{C} 27$ & $\mathrm{C} 28$ & $1.390(3)$ \\
\hline $\mathrm{C} 4$ & $\mathrm{C} 5$ & $1.380(3)$ & $\mathrm{C} 28$ & $\mathrm{C} 29$ & $1.390(2)$ \\
\hline $\mathrm{C} 5$ & C6 & 1.394(3) & $\mathrm{C} 30$ & $\mathrm{C} 31$ & $1.507(2)$ \\
\hline C6 & $\mathrm{C} 7$ & $1.383(2)$ & $\mathrm{C} 32$ & $\mathrm{C} 33$ & $1.460(2)$ \\
\hline $\mathrm{C} 8$ & C9 & $1.382(3)$ & & & \\
\hline
\end{tabular}


Table S38. Bond angles $\left[{ }^{\circ}\right]$ for Ru-III

\begin{tabular}{|c|c|c|c|c|c|c|c|}
\hline \multicolumn{3}{|c|}{ Atom Atom Atom } & \multirow{2}{*}{$\begin{array}{l}\text { Angle }^{\circ} \\
\qquad 105.56(3)\end{array}$} & \multicolumn{3}{|c|}{ Atom Atom Atom } & \multirow{2}{*}{$\begin{array}{l}\text { Angle }^{\circ} \\
120.03(16)\end{array}$} \\
\hline $\mathrm{P} 1$ & Ru1 & $\mathrm{O} 2$ & & $\mathrm{C} 5$ & $\mathrm{C} 4$ & $\mathrm{C} 3$ & \\
\hline $\mathrm{O} 1$ & Ru1 & $\mathrm{P} 1$ & $163.66(3)$ & $\mathrm{C} 4$ & $\mathrm{C} 5$ & C6 & $118.98(16)$ \\
\hline $\mathrm{O} 1$ & Ru1 & $\mathrm{O} 2$ & $58.10(4)$ & $\mathrm{C} 7$ & C6 & $\mathrm{C} 5$ & $118.58(16)$ \\
\hline $\mathrm{N} 1$ & Ru1 & $\mathrm{P} 1$ & $92.21(4)$ & $\mathrm{N} 1$ & $\mathrm{C} 7$ & C6 & $122.93(16)$ \\
\hline $\mathrm{N} 1$ & Ru1 & $\mathrm{O} 1$ & $88.19(5)$ & C9 & $\mathrm{C} 8$ & $\mathrm{C} 2$ & $120.10(15)$ \\
\hline $\mathrm{N} 1$ & Ru1 & $\mathrm{O} 2$ & $91.65(5)$ & $\mathrm{C} 8$ & C9 & $\mathrm{C} 10$ & $119.48(16)$ \\
\hline $\mathrm{N} 2$ & $\mathrm{Ru} 1$ & $\mathrm{P} 1$ & $93.88(4)$ & $\mathrm{C} 11$ & $\mathrm{C} 10$ & C9 & $120.47(16)$ \\
\hline $\mathrm{N} 2$ & Ru1 & $\mathrm{O} 1$ & $87.33(5)$ & $\mathrm{C} 10$ & $\mathrm{C} 11$ & $\mathrm{C} 1$ & $122.38(16)$ \\
\hline $\mathrm{N} 2$ & Ru1 & $\mathrm{O} 2$ & $91.42(5)$ & $\mathrm{C} 13$ & $\mathrm{C} 12$ & $\mathrm{P} 1$ & $123.40(12)$ \\
\hline $\mathrm{N} 2$ & Ru1 & $\mathrm{N} 1$ & $172.18(6)$ & $\mathrm{C} 17$ & $\mathrm{C} 12$ & $\mathrm{P} 1$ & $118.07(12)$ \\
\hline $\mathrm{C} 1$ & $\mathrm{Ru} 1$ & $\mathrm{P} 1$ & $95.99(4)$ & $\mathrm{C} 17$ & $\mathrm{C} 12$ & $\mathrm{C} 13$ & $118.48(14)$ \\
\hline $\mathrm{C} 1$ & Ru1 & $\mathrm{O} 1$ & $100.17(5)$ & $\mathrm{C} 14$ & $\mathrm{C} 13$ & $\mathrm{C} 12$ & $120.72(16)$ \\
\hline $\mathrm{C} 1$ & Ru1 & $\mathrm{O} 2$ & $157.26(5)$ & $\mathrm{C} 15$ & $\mathrm{C} 14$ & $\mathrm{C} 13$ & $119.83(16)$ \\
\hline $\mathrm{C} 1$ & Ru1 & $\mathrm{N} 1$ & $80.16(6)$ & $\mathrm{C} 16$ & $\mathrm{C} 15$ & $\mathrm{C} 14$ & $119.97(16)$ \\
\hline $\mathrm{C} 1$ & Ru1 & N2 & $94.33(6)$ & $\mathrm{C} 15$ & $\mathrm{C} 16$ & $\mathrm{C} 17$ & $119.78(16)$ \\
\hline $\mathrm{C} 12$ & $\mathrm{P} 1$ & Ru1 & $121.81(5)$ & $\mathrm{C} 16$ & $\mathrm{C} 17$ & $\mathrm{C} 12$ & $121.19(15)$ \\
\hline $\mathrm{C} 12$ & $\mathrm{P} 1$ & $\mathrm{C} 24$ & $101.16(7)$ & C19 & $\mathrm{C} 18$ & $\mathrm{P} 1$ & $121.80(12)$ \\
\hline C18 & $\mathrm{P} 1$ & Ru1 & $113.59(5)$ & C19 & $\mathrm{C} 18$ & $\mathrm{C} 23$ & $118.57(14)$ \\
\hline C18 & $\mathrm{P} 1$ & $\mathrm{C} 12$ & $102.78(7)$ & $\mathrm{C} 23$ & $\mathrm{C} 18$ & $\mathrm{P} 1$ & $119.34(12)$ \\
\hline $\mathrm{C} 18$ & $\mathrm{P} 1$ & $\mathrm{C} 24$ & $101.24(7)$ & $\mathrm{C} 20$ & C19 & $\mathrm{C} 18$ & $120.50(15)$ \\
\hline $\mathrm{C} 24$ & $\mathrm{P} 1$ & Ru1 & $113.57(5)$ & $\mathrm{C} 21$ & $\mathrm{C} 20$ & C19 & $120.46(16)$ \\
\hline C30 & $\mathrm{O} 1$ & Ru1 & $92.44(9)$ & C20 & $\mathrm{C} 21$ & $\mathrm{C} 22$ & $119.60(18)$ \\
\hline C30 & $\mathrm{O} 2$ & Ru1 & $89.14(10)$ & $\mathrm{C} 23$ & $\mathrm{C} 22$ & $\mathrm{C} 21$ & $120.18(17)$ \\
\hline $\mathrm{C} 3$ & N1 & Ru1 & $115.95(10)$ & $\mathrm{C} 22$ & $\mathrm{C} 23$ & $\mathrm{C} 18$ & $120.66(15)$ \\
\hline $\mathrm{C} 7$ & $\mathrm{~N} 1$ & Ru1 & $125.24(11)$ & $\mathrm{C} 25$ & $\mathrm{C} 24$ & $\mathrm{P} 1$ & $119.44(12)$ \\
\hline $\mathrm{C} 7$ & N1 & $\mathrm{C} 3$ & $118.82(13)$ & $\mathrm{C} 25$ & $\mathrm{C} 24$ & $\mathrm{C} 29$ & $119.11(14)$ \\
\hline C32 & $\mathrm{N} 2$ & Ru1 & 174.61(14) & C29 & $\mathrm{C} 24$ & $\mathrm{P} 1$ & $121.43(12)$ \\
\hline $\mathrm{C} 2$ & $\mathrm{C} 1$ & Ru1 & 113.99(11) & C26 & $\mathrm{C} 25$ & $\mathrm{C} 24$ & $120.21(15)$ \\
\hline $\mathrm{C} 11$ & $\mathrm{C} 1$ & Ru1 & $129.80(12)$ & $\mathrm{C} 27$ & $\mathrm{C} 26$ & $\mathrm{C} 25$ & $120.23(16)$ \\
\hline $\mathrm{C} 11$ & $\mathrm{C} 1$ & $\mathrm{C} 2$ & $115.52(14)$ & $\mathrm{C} 26$ & $\mathrm{C} 27$ & $\mathrm{C} 28$ & $119.99(16)$ \\
\hline
\end{tabular}




\begin{tabular}{llllllll}
\multicolumn{2}{c}{ Atom Atom Atom } & \multicolumn{1}{l}{ Angle $^{\circ}$} & \multicolumn{2}{c}{ Atom Atom Atom } & \multicolumn{1}{c}{ Angle/ } \\
C1 & C2 & C3 & $115.34(13)$ & C27 & C28 & C29 & $120.04(16)$ \\
C8 & C2 & C1 & $122.04(14)$ & C28 & C29 & C24 & $120.42(15)$ \\
C8 & C2 & C3 & $122.62(15)$ & O1 & C30 & C31 & $120.13(15)$ \\
N1 & C3 & C2 & $113.71(13)$ & O2 & C30 & O1 & $120.31(15)$ \\
N1 & C3 & C4 & $120.63(15)$ & O2 & C30 & C31 & $119.57(15)$ \\
C4 & C3 & C2 & $125.62(15)$ & N2 & C32 & C33 & $177.99(19)$
\end{tabular}

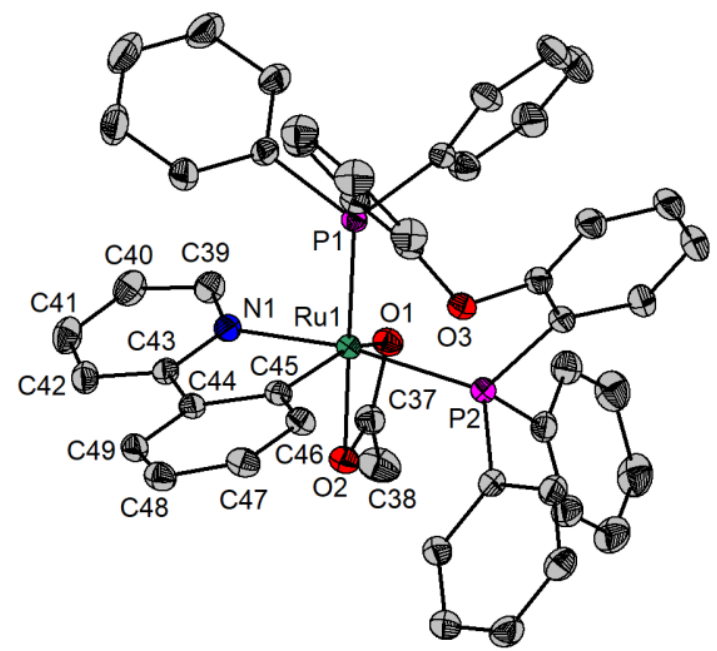

Figure S15. Molecular structure of Ru-IV with thermal ellipoids at 50\% probability level.

The hydrogen atoms are omitted for clarity.

Crystal Data for $\mathrm{C}_{49} \mathrm{H}_{39} \mathrm{NO}_{3} \mathrm{P}_{2} \mathrm{Ru}(M=852.82 \mathrm{~g} / \mathrm{mol})$ : monoclinic, space group C2/c (no. 15), $a=19.1587(12) \AA, b=20.6734(11) \AA, c=20.6880(9) \AA, \beta=107.466(2)^{\circ}, V=7816.2(7) \AA^{3}$, $Z=8, T=149.96 \mathrm{~K}, \mu(\mathrm{MoK} \alpha)=0.529 \mathrm{~mm}^{-1}$, Dcalc $=1.449 \mathrm{~g} / \mathrm{cm}^{3}, 182744$ reflections measured $\left(5.086^{\circ} \leq 2 \Theta \leq 63.122^{\circ}\right), 13014$ unique $\left(R_{\text {int }}=0.0258, R_{\text {sigma }}=0.0112\right)$ which were used in all calculations. The final $R_{1}$ was 0.0217 (I $\left.>2 \sigma(\mathrm{I})\right)$ and $w R_{2}$ was 0.0600 (all data).

Table S39. Crystal data and structure refinement for Ru-IV

\begin{tabular}{|l|l|}
\hline Compound & Ru-IV \\
\hline CCDC number & 1915688 \\
\hline Identification code & 0527_CG_0m \\
\hline & S106
\end{tabular}




\begin{tabular}{|c|c|}
\hline Empirical formula & $\mathrm{C}_{49} \mathrm{H}_{39} \mathrm{NO}_{3} \mathrm{P}_{2} \mathrm{Ru}$ \\
\hline Formula weight & 852.82 \\
\hline Temperature/K & 149.96 \\
\hline Crystal system & monoclinic \\
\hline Space group & $\mathrm{C} 2 / \mathrm{c}$ \\
\hline $\mathrm{a} / \AA$ & $19.1587(12)$ \\
\hline $\mathrm{b} / \AA$ & $20.6734(11)$ \\
\hline $\mathrm{c} / \AA$ & 20.6880(9) \\
\hline$\alpha /^{\circ}$ & 90 \\
\hline$\beta /{ }^{\circ}$ & $107.466(2)$ \\
\hline$\gamma /{ }^{\circ}$ & 90 \\
\hline Volume $/ \AA^{3}$ & $7816.2(7)$ \\
\hline $\mathrm{Z}$ & 8 \\
\hline$\rho_{\text {calc }} \mathrm{g} / \mathrm{cm}^{3}$ & 1.449 \\
\hline$\mu / \mathrm{mm}^{-1}$ & 0.529 \\
\hline $\mathrm{F}(000)$ & 3504.0 \\
\hline Crystal size $/ \mathrm{mm}^{3}$ & $0.529 \times 0.31 \times 0.154$ \\
\hline Radiation & $\operatorname{MoK} \alpha(\lambda=0.71073)$ \\
\hline $2 \Theta$ range for data collection $/{ }^{\circ}$ & 5.086 to 63.122 \\
\hline Index ranges & $-28 \leq \mathrm{h} \leq 28,-29 \leq \mathrm{k} \leq 29,-28 \leq 1 \leq 30$ \\
\hline Reflections collected & 182744 \\
\hline Independent reflections & $13014\left[\mathrm{R}_{\text {int }}=0.0258, \mathrm{R}_{\mathrm{sigma}}=0.0112\right]$ \\
\hline Data/restraints/parameters & 13014/0/506 \\
\hline Goodness-of-fit on $\mathrm{F}^{2}$ & 1.052 \\
\hline Final $R$ indexes $[\mathrm{I}>=2 \sigma(\mathrm{I})]$ & $\mathrm{R}_{1}=0.0217, \mathrm{w} \mathrm{R}_{2}=0.0583$ \\
\hline Final $\mathrm{R}$ indexes [all data] & $\mathrm{R}_{1}=0.0240, \mathrm{wR}_{2}=0.0600$ \\
\hline Largest diff. peak/hole / e $\AA^{-3}$ & $0.44 /-0.54$ \\
\hline
\end{tabular}


Table S40. Selected bond lengths $[\AA ̊]$ for Ru-IV

\begin{tabular}{|c|c|c|c|c|c|}
\hline \multicolumn{2}{|c|}{ Atom Atom } & \multirow{2}{*}{$\begin{array}{l}\text { Length/Å } \\
2.2377(3)\end{array}$} & \multicolumn{2}{|c|}{ Atom Atom } & \multirow{2}{*}{$\begin{array}{r}\text { Length/Å } \\
1.3885(17)\end{array}$} \\
\hline $\mathrm{Ru} 1$ & $\mathrm{P} 1$ & & $\mathrm{C} 15$ & $\mathrm{C} 16$ & \\
\hline Ru1 & $\mathrm{P} 2$ & $2.3263(3)$ & $\mathrm{C} 16$ & $\mathrm{C} 17$ & $1.3830(18)$ \\
\hline $\mathrm{Ru} 1$ & $\mathrm{O} 1$ & $2.2928(8)$ & $\mathrm{C} 17$ & $\mathrm{C} 18$ & $1.3903(16)$ \\
\hline Ru1 & $\mathrm{O} 2$ & $2.1987(8)$ & C19 & $\mathrm{C} 20$ & $1.3870(14)$ \\
\hline Ru 1 & N1 & $2.0836(9)$ & C19 & $\mathrm{C} 24$ & $1.3922(15)$ \\
\hline Ru1 & C37 & $2.5928(11)$ & $\mathrm{C} 20$ & $\mathrm{C} 21$ & $1.3865(17)$ \\
\hline Ru1 & $\mathrm{C} 45$ & $2.0302(10)$ & $\mathrm{C} 21$ & $\mathrm{C} 22$ & $1.3808(19)$ \\
\hline $\mathrm{P} 1$ & $\mathrm{C} 1$ & $1.8379(11)$ & $\mathrm{C} 22$ & $\mathrm{C} 23$ & $1.3910(16)$ \\
\hline $\mathrm{P} 1$ & $\mathrm{C} 7$ & $1.8263(10)$ & $\mathrm{C} 23$ & $\mathrm{C} 24$ & $1.3990(15)$ \\
\hline $\mathrm{P} 1$ & $\mathrm{C} 13$ & $1.8506(10)$ & $\mathrm{C} 25$ & $\mathrm{C} 26$ & $1.3963(15)$ \\
\hline $\mathrm{P} 2$ & $\mathrm{C} 24$ & $1.8388(10)$ & $\mathrm{C} 25$ & $\mathrm{C} 30$ & $1.3989(16)$ \\
\hline $\mathrm{P} 2$ & $\mathrm{C} 25$ & $1.8503(11)$ & $\mathrm{C} 26$ & $\mathrm{C} 27$ & $1.3934(17)$ \\
\hline $\mathrm{P} 2$ & C31 & $1.8325(11)$ & $\mathrm{C} 27$ & $\mathrm{C} 28$ & $1.381(2)$ \\
\hline $\mathrm{O} 1$ & C37 & $1.2627(13)$ & $\mathrm{C} 28$ & $\mathrm{C} 29$ & $1.389(2)$ \\
\hline $\mathrm{O} 2$ & $\mathrm{C} 37$ & $1.2700(13)$ & $\mathrm{C} 29$ & $\mathrm{C} 30$ & $1.3893(17)$ \\
\hline $\mathrm{O} 3$ & $\mathrm{C} 14$ & $1.3899(13)$ & $\mathrm{C} 31$ & $\mathrm{C} 32$ & $1.3923(15)$ \\
\hline $\mathrm{O} 3$ & $\mathrm{C} 19$ & $1.3839(13)$ & C31 & $\mathrm{C} 36$ & $1.3966(15)$ \\
\hline N1 & C39 & $1.3452(14)$ & C32 & C33 & $1.3908(15)$ \\
\hline N1 & $\mathrm{C} 43$ & $1.3561(13)$ & C33 & $\mathrm{C} 34$ & $1.3822(18)$ \\
\hline $\mathrm{C} 1$ & $\mathrm{C} 2$ & $1.3936(15)$ & $\mathrm{C} 34$ & $\mathrm{C} 35$ & $1.3881(17)$ \\
\hline $\mathrm{C} 1$ & C6 & $1.3966(15)$ & $\mathrm{C} 35$ & $\mathrm{C} 36$ & $1.3897(16)$ \\
\hline $\mathrm{C} 2$ & $\mathrm{C} 3$ & $1.3926(16)$ & $\mathrm{C} 37$ & C38 & $1.5027(16)$ \\
\hline $\mathrm{C} 3$ & $\mathrm{C} 4$ & $1.377(2)$ & C39 & $\mathrm{C} 40$ & $1.3827(16)$ \\
\hline $\mathrm{C} 4$ & $\mathrm{C} 5$ & $1.380(2)$ & $\mathrm{C} 40$ & $\mathrm{C} 41$ & $1.3874(18)$ \\
\hline $\mathrm{C} 5$ & C6 & $1.3904(18)$ & C41 & $\mathrm{C} 42$ & $1.3819(16)$ \\
\hline $\mathrm{C} 7$ & $\mathrm{C} 8$ & $1.3952(15)$ & $\mathrm{C} 42$ & $\mathrm{C} 43$ & $1.3977(14)$ \\
\hline $\mathrm{C} 7$ & $\mathrm{C} 12$ & $1.3906(14)$ & $\mathrm{C} 43$ & $\mathrm{C} 44$ & $1.4616(14)$ \\
\hline $\mathrm{C} 8$ & C9 & $1.3844(16)$ & $\mathrm{C} 44$ & $\mathrm{C} 45$ & $1.4264(14)$ \\
\hline C9 & $\mathrm{C} 10$ & $1.385(2)$ & $\mathrm{C} 44$ & C49 & $1.4016(14)$ \\
\hline C10 & $\mathrm{C} 11$ & $1.379(2)$ & $\mathrm{C} 45$ & $\mathrm{C} 46$ & $1.4080(14)$ \\
\hline
\end{tabular}




\section{Atom Atom Length/Å}

C11 C12 1.3948(16)

C13 C14 1.3961(15)

C13 C18 1.4026(15)

C14 C15 1.3863(15)
Atom Atom Length/Å

C46 C47 1.3919(15)

C47 C48 1.3908(16)

C48 C49 1.3826(16)

Table S41. Selected bond angles $\left[^{\circ}\right]$ for Ru-IV

\begin{tabular}{|c|c|c|c|c|c|c|c|}
\hline \multicolumn{3}{|c|}{ Atom Atom Atom } & \multirow{2}{*}{$\begin{array}{l}\text { Angle }^{\circ}{ }^{\circ} \\
\quad 96.827(9)\end{array}$} & \multicolumn{3}{|c|}{ Atom Atom Atom } & \multirow{2}{*}{$\begin{array}{l}\text { Angle }^{\circ} \\
\quad 122.74(8)\end{array}$} \\
\hline P1 & Ru1 & $\mathrm{P} 2$ & & $\mathrm{C} 18$ & $\mathrm{C} 13$ & $\mathrm{P} 1$ & \\
\hline $\mathrm{P} 1$ & Ru1 & O1 & $111.67(2)$ & $\mathrm{O} 3$ & $\mathrm{C} 14$ & $\mathrm{C} 13$ & $119.50(9)$ \\
\hline P1 & Ru1 & $\mathrm{C} 37$ & $140.68(3)$ & $\mathrm{C} 15$ & $\mathrm{C} 14$ & $\mathrm{O} 3$ & $117.78(10)$ \\
\hline $\mathrm{P} 2$ & Ru1 & $\mathrm{C} 37$ & $88.90(3)$ & $\mathrm{C} 15$ & $\mathrm{C} 14$ & $\mathrm{C} 13$ & $122.62(10)$ \\
\hline $\mathrm{O} 1$ & $\mathrm{Ru} 1$ & $\mathrm{P} 2$ & $90.52(2)$ & $\mathrm{C} 14$ & $\mathrm{C} 15$ & $\mathrm{C} 16$ & $119.60(11)$ \\
\hline $\mathrm{O} 1$ & Ru1 & $\mathrm{C} 37$ & $29.14(3)$ & $\mathrm{C} 17$ & $\mathrm{C} 16$ & $\mathrm{C} 15$ & $119.49(11)$ \\
\hline $\mathrm{O} 2$ & Ru1 & $\mathrm{P} 1$ & $169.67(2)$ & $\mathrm{C} 16$ & $\mathrm{C} 17$ & $\mathrm{C} 18$ & $120.21(11)$ \\
\hline $\mathrm{O} 2$ & Ru1 & $\mathrm{P} 2$ & $86.58(2)$ & $\mathrm{C} 17$ & $\mathrm{C} 18$ & $\mathrm{C} 13$ & $121.78(11)$ \\
\hline $\mathrm{O} 2$ & $\mathrm{Ru} 1$ & $\mathrm{O} 1$ & $58.41(3)$ & $\mathrm{O} 3$ & C19 & $\mathrm{C} 20$ & $122.38(10)$ \\
\hline $\mathrm{O} 2$ & Ru1 & $\mathrm{C} 37$ & $29.29(3)$ & $\mathrm{O} 3$ & C19 & $\mathrm{C} 24$ & $115.08(9)$ \\
\hline N1 & Ru1 & $\mathrm{P} 1$ & $93.69(3)$ & $\mathrm{C} 20$ & C19 & $\mathrm{C} 24$ & $122.43(10)$ \\
\hline N1 & Ru1 & $\mathrm{P} 2$ & $169.30(3)$ & $\mathrm{C} 21$ & $\mathrm{C} 20$ & C19 & $118.66(11)$ \\
\hline N1 & Ru1 & O1 & $83.73(3)$ & $\mathrm{C} 22$ & $\mathrm{C} 21$ & $\mathrm{C} 20$ & $120.59(11)$ \\
\hline N1 & Ru1 & $\mathrm{O} 2$ & $82.72(3)$ & $\mathrm{C} 21$ & $\mathrm{C} 22$ & $\mathrm{C} 23$ & $119.98(11)$ \\
\hline N1 & Ru1 & C37 & $81.68(3)$ & $\mathrm{C} 22$ & $\mathrm{C} 23$ & $\mathrm{C} 24$ & $120.86(11)$ \\
\hline $\mathrm{C} 45$ & Ru1 & $\mathrm{P} 1$ & $91.38(3)$ & C19 & $\mathrm{C} 24$ & $\mathrm{P} 2$ & $116.57(8)$ \\
\hline $\mathrm{C} 45$ & Ru1 & $\mathrm{P} 2$ & $101.60(3)$ & C19 & $\mathrm{C} 24$ & $\mathrm{C} 23$ & $117.42(10)$ \\
\hline $\mathrm{C} 45$ & Ru1 & O1 & $152.61(3)$ & $\mathrm{C} 23$ & $\mathrm{C} 24$ & $\mathrm{P} 2$ & $126.01(9)$ \\
\hline $\mathrm{C} 45$ & Ru1 & $\mathrm{O} 2$ & $97.50(3)$ & $\mathrm{C} 26$ & $\mathrm{C} 25$ & $\mathrm{P} 2$ & $123.27(9)$ \\
\hline $\mathrm{C} 45$ & Ru1 & N1 & $80.07(4)$ & $\mathrm{C} 26$ & $\mathrm{C} 25$ & $\mathrm{C} 30$ & $118.14(10)$ \\
\hline $\mathrm{C} 45$ & Ru1 & $\mathrm{C} 37$ & $125.59(4)$ & $\mathrm{C} 30$ & $\mathrm{C} 25$ & $\mathrm{P} 2$ & $118.58(8)$ \\
\hline $\mathrm{C} 1$ & $\mathrm{P} 1$ & $\mathrm{Ru} 1$ & $112.82(3)$ & $\mathrm{C} 27$ & $\mathrm{C} 26$ & $\mathrm{C} 25$ & $120.49(12)$ \\
\hline $\mathrm{C} 1$ & $\mathrm{P} 1$ & $\mathrm{C} 13$ & $99.47(5)$ & $\mathrm{C} 28$ & $\mathrm{C} 27$ & $\mathrm{C} 26$ & $120.65(12)$ \\
\hline
\end{tabular}




\begin{tabular}{|c|c|c|c|c|c|c|c|}
\hline \multicolumn{3}{|c|}{ Atom Atom Atom } & \multirow{2}{*}{$\begin{array}{l}\text { Angle }^{\circ} \\
\qquad 113.34(3)\end{array}$} & \multicolumn{3}{|c|}{ Atom Atom Atom } & \multirow{2}{*}{$\begin{array}{l}\text { Angle }^{\circ} \\
119.63(12)\end{array}$} \\
\hline $\mathrm{C} 7$ & $\mathrm{P} 1$ & Ru1 & & $\mathrm{C} 27$ & $\mathrm{C} 28$ & $\mathrm{C} 29$ & \\
\hline $\mathrm{C} 7$ & $\mathrm{P} 1$ & $\mathrm{C} 1$ & $100.59(5)$ & $\mathrm{C} 28$ & $\mathrm{C} 29$ & $\mathrm{C} 30$ & $119.83(13)$ \\
\hline $\mathrm{C} 7$ & $\mathrm{P} 1$ & $\mathrm{C} 13$ & $102.72(5)$ & $\mathrm{C} 29$ & $\mathrm{C} 30$ & $\mathrm{C} 25$ & $121.24(12)$ \\
\hline $\mathrm{C} 13$ & $\mathrm{P} 1$ & Ru1 & $124.57(3)$ & $\mathrm{C} 32$ & C31 & $\mathrm{P} 2$ & $122.92(8)$ \\
\hline $\mathrm{C} 24$ & $\mathrm{P} 2$ & Ru1 & $123.17(3)$ & $\mathrm{C} 32$ & $\mathrm{C} 31$ & $\mathrm{C} 36$ & $118.78(10)$ \\
\hline $\mathrm{C} 24$ & $\mathrm{P} 2$ & $\mathrm{C} 25$ & $99.76(5)$ & C36 & $\mathrm{C} 31$ & $\mathrm{P} 2$ & $118.23(8)$ \\
\hline $\mathrm{C} 25$ & $\mathrm{P} 2$ & Ru1 & $111.22(3)$ & $\mathrm{C} 33$ & $\mathrm{C} 32$ & $\mathrm{C} 31$ & $120.69(10)$ \\
\hline C31 & $\mathrm{P} 2$ & Ru1 & $117.34(3)$ & $\mathrm{C} 34$ & $\mathrm{C} 33$ & $\mathrm{C} 32$ & $120.16(11)$ \\
\hline $\mathrm{C} 31$ & $\mathrm{P} 2$ & $\mathrm{C} 24$ & $101.20(5)$ & $\mathrm{C} 33$ & $\mathrm{C} 34$ & $\mathrm{C} 35$ & $119.70(11)$ \\
\hline C31 & $\mathrm{P} 2$ & $\mathrm{C} 25$ & $100.65(5)$ & $\mathrm{C} 34$ & $\mathrm{C} 35$ & $\mathrm{C} 36$ & $120.33(11)$ \\
\hline $\mathrm{C} 37$ & $\mathrm{O} 1$ & Ru1 & $88.72(6)$ & $\mathrm{C} 35$ & C36 & C31 & $120.32(11)$ \\
\hline C37 & $\mathrm{O} 2$ & Ru1 & $92.83(6)$ & $\mathrm{O} 1$ & C37 & Ru1 & $62.14(5)$ \\
\hline C19 & $\mathrm{O} 3$ & $\mathrm{C} 14$ & $118.07(8)$ & $\mathrm{O} 1$ & $\mathrm{C} 37$ & $\mathrm{O} 2$ & $119.99(10)$ \\
\hline C39 & N1 & Ru1 & $125.21(7)$ & $\mathrm{O} 1$ & $\mathrm{C} 37$ & C38 & $120.32(10)$ \\
\hline C39 & N1 & $\mathrm{C} 43$ & $119.79(9)$ & $\mathrm{O} 2$ & $\mathrm{C} 37$ & Ru1 & $57.88(5)$ \\
\hline C43 & N1 & Ru1 & $114.43(7)$ & $\mathrm{O} 2$ & $\mathrm{C} 37$ & $\mathrm{C} 38$ & $119.68(10)$ \\
\hline $\mathrm{C} 2$ & $\mathrm{C} 1$ & $\mathrm{P} 1$ & $122.72(8)$ & C 38 & C37 & Ru1 & $177.39(9)$ \\
\hline $\mathrm{C} 2$ & $\mathrm{C} 1$ & C6 & $118.59(10)$ & N1 & C39 & $\mathrm{C} 40$ & $122.52(11)$ \\
\hline C6 & $\mathrm{C} 1$ & $\mathrm{P} 1$ & 118.67(8) & C39 & $\mathrm{C} 40$ & C41 & $118.15(11)$ \\
\hline $\mathrm{C} 3$ & $\mathrm{C} 2$ & $\mathrm{C} 1$ & $120.69(12)$ & $\mathrm{C} 42$ & C41 & $\mathrm{C} 40$ & $119.64(10)$ \\
\hline $\mathrm{C} 4$ & $\mathrm{C} 3$ & $\mathrm{C} 2$ & $120.18(13)$ & C41 & $\mathrm{C} 42$ & $\mathrm{C} 43$ & $119.79(10)$ \\
\hline $\mathrm{C} 3$ & $\mathrm{C} 4$ & $\mathrm{C} 5$ & $119.69(12)$ & $\mathrm{N} 1$ & $\mathrm{C} 43$ & $\mathrm{C} 42$ & $119.94(10)$ \\
\hline $\mathrm{C} 4$ & C5 & C6 & $120.74(12)$ & N1 & $\mathrm{C} 43$ & $\mathrm{C} 44$ & $113.83(9)$ \\
\hline $\mathrm{C} 5$ & C6 & $\mathrm{C} 1$ & $120.10(12)$ & $\mathrm{C} 42$ & $\mathrm{C} 43$ & $\mathrm{C} 44$ & $126.20(9)$ \\
\hline $\mathrm{C} 8$ & $\mathrm{C} 7$ & $\mathrm{P} 1$ & $115.36(8)$ & $\mathrm{C} 45$ & $\mathrm{C} 44$ & $\mathrm{C} 43$ & $116.48(9)$ \\
\hline $\mathrm{C} 12$ & $\mathrm{C} 7$ & $\mathrm{P} 1$ & $125.30(8)$ & C49 & $\mathrm{C} 44$ & $\mathrm{C} 43$ & 121.34(9) \\
\hline $\mathrm{C} 12$ & $\mathrm{C} 7$ & $\mathrm{C} 8$ & 119.31(10) & C49 & $\mathrm{C} 44$ & $\mathrm{C} 45$ & $122.16(9)$ \\
\hline C9 & $\mathrm{C} 8$ & $\mathrm{C} 7$ & $120.16(11)$ & $\mathrm{C} 44$ & $\mathrm{C} 45$ & Ru1 & $112.30(7)$ \\
\hline $\mathrm{C} 10$ & C9 & $\mathrm{C} 8$ & $120.18(12)$ & C46 & $\mathrm{C} 45$ & $\mathrm{Ru} 1$ & $132.00(8)$ \\
\hline $\mathrm{C} 11$ & $\mathrm{C} 10$ & C9 & $120.14(11)$ & $\mathrm{C} 46$ & $\mathrm{C} 45$ & C44 & $115.37(9)$ \\
\hline
\end{tabular}




\begin{tabular}{lllrllll} 
Atom Atom Atom & \multicolumn{1}{c}{ Angle $^{\circ}$} & \multicolumn{3}{c}{ Atom Atom Atom } & \multicolumn{1}{c}{ Angle ${ }^{\circ}$} \\
C10 & C11 & C12 & $120.01(12)$ & C47 & C46 & C45 & $122.18(10)$ \\
C7 & C12 & C11 & $120.09(11)$ & C48 & C47 & C46 & $120.97(10)$ \\
C14 & C13 & P1 & $120.96(8)$ & C49 & C48 & C47 & $119.01(10)$ \\
C14 & C13 & C18 & $116.28(9)$ & C48 & C49 & C44 & $120.24(10)$
\end{tabular}




\section{Fluorescence Spectra}

Concentration of sample: $1 \mathrm{mg} / \mathrm{L}$ in $\mathrm{CHCl}_{3}$

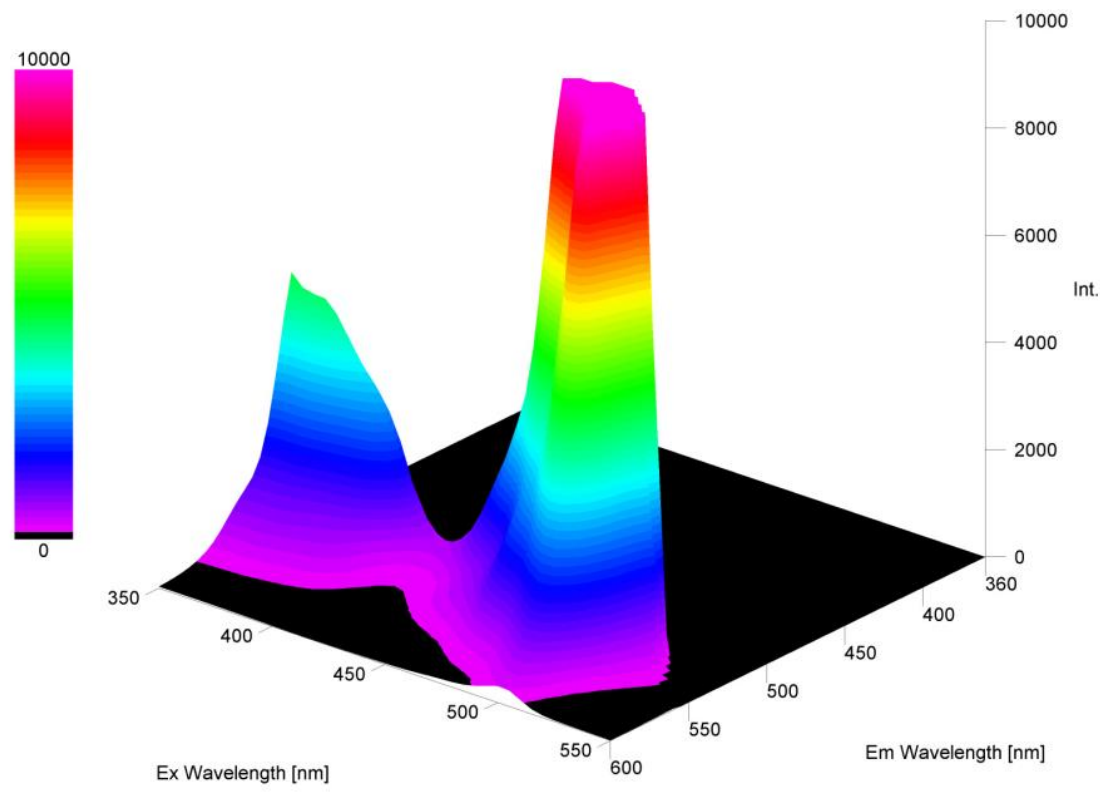

Figure S16. Excitation/emission fluorescence spectra of $\mathbf{6 a}$.

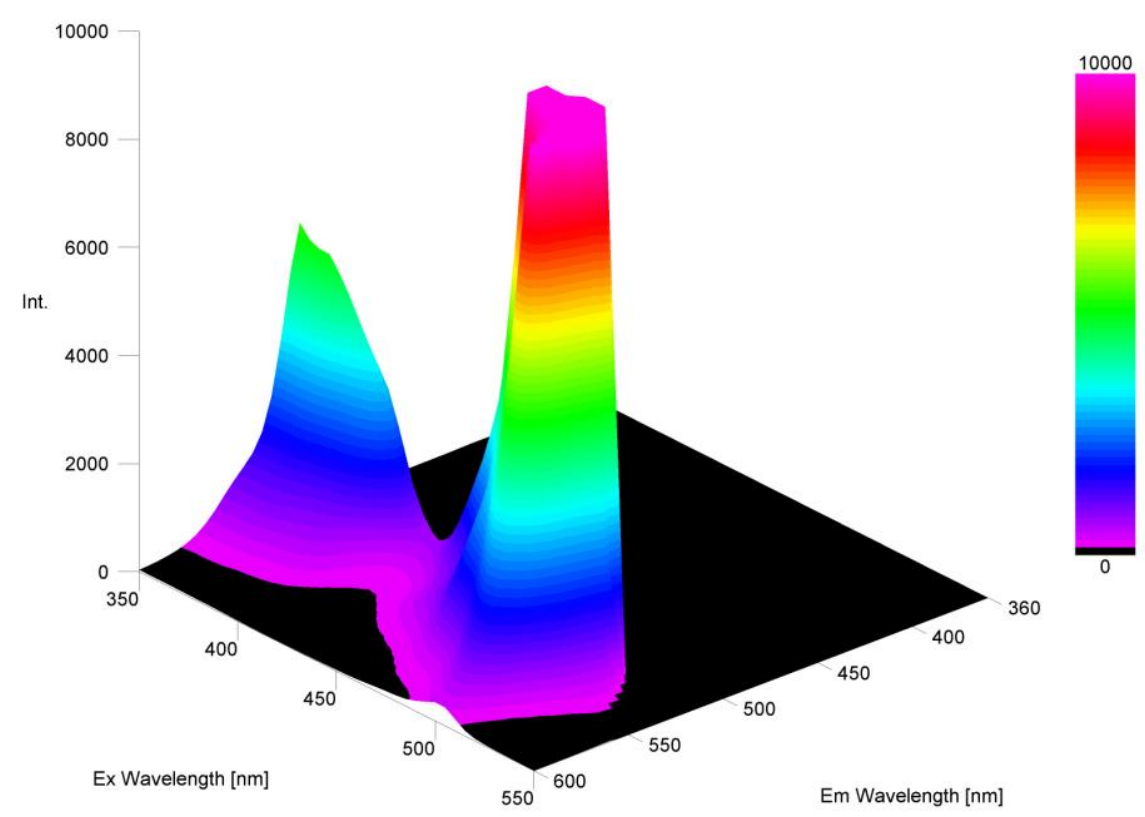

Figure S17. Excitation/emission fluorescence spectra of $\mathbf{6 b}$. 


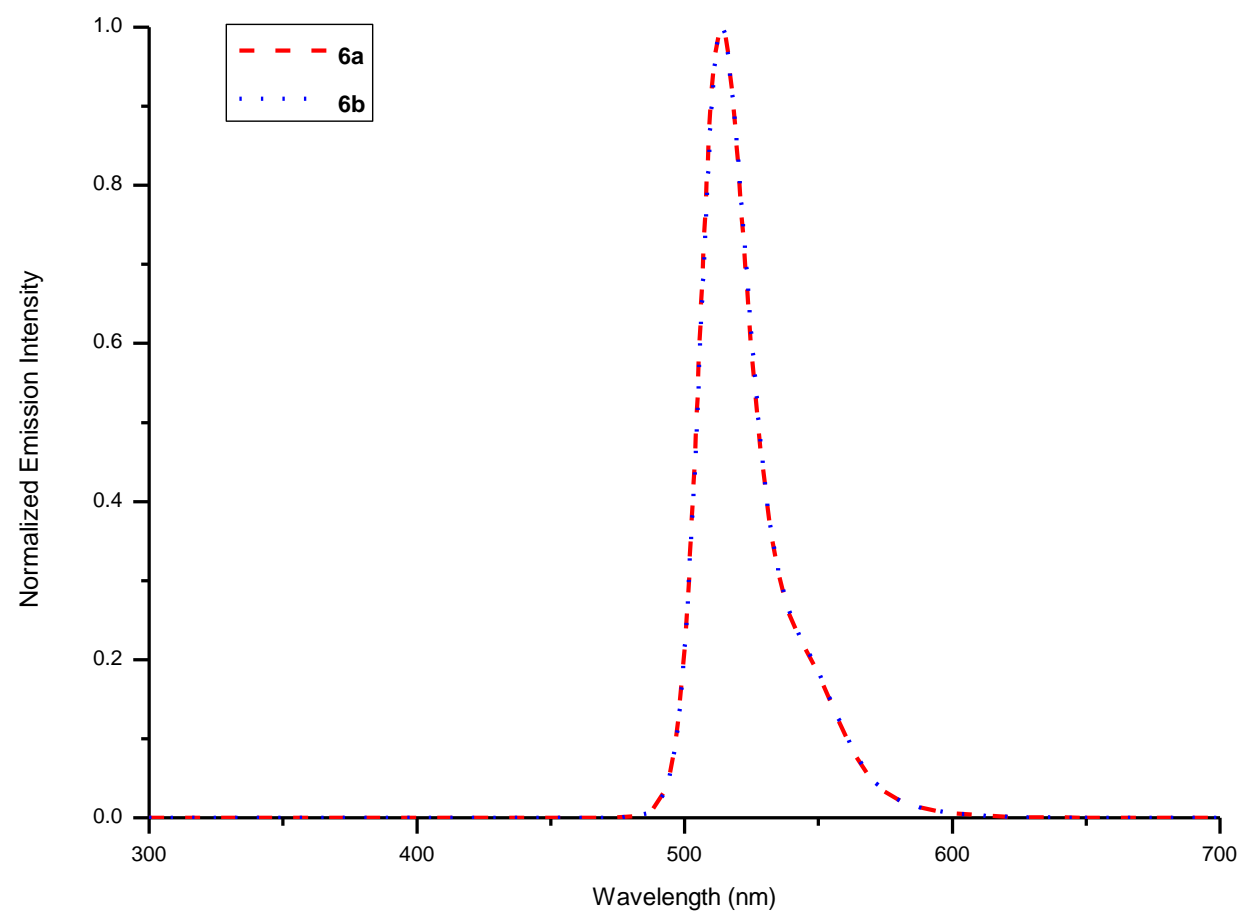

Figure S18. Emission fluorescence spectra of $6 \mathbf{a}$ and $\mathbf{6 b}$ (excitation at $502 \mathrm{~nm}$ ). 


\section{Computational Data}

All the calculations were carried out by using DFT with the Gaussian 16, Revision A.03 package. ${ }^{16}$ Geometry optimization of all the stationary points were carried out at the TPSS ${ }^{17}$ level of theory in combination with D3 dispersion corrections with Becke-Johnson damping scheme (D3BJ). ${ }^{18}$ Ruthenium was described with def2-TZVP ${ }^{19}$ basis set in combination with Stuttgart-Dresden effective core potentials. ${ }^{20}$ For all other atoms, def2-TZVP basis set was used. Analytical frequency calculations were carried out at the same level of theory in order to identify all the intermediates (no imaginary frequencies) and to provide thermal and nonthermal corrections to the free energy in gas-phase at $298.15 \mathrm{~K}$ and $1 \mathrm{~atm}$.

The electronic energy was then refined through $\mathrm{PBE}^{21}$ single-point calculations on the optimized structures including dispersion corrections (D3BJ) using the same basis set as for the geometry optimization. Solvent effects were taken into consideration in the single-point calculations. These were implicitly included through the use of the $\mathrm{SMD}^{22}$ model with a dielectric constant of $\varepsilon=2.2099$, which corresponds to 1,4-dioxane. The same method was also used to calculate Wiberg bond indices (WBI). These were performed by using NBO 6.0 software. ${ }^{23}$ Plots of orbitals were constructed by using Gauss View 5 software. ${ }^{24}$

Unless and otherwise stated, all the reported energies are Gibbs free energies in $\mathrm{kcal} \mathrm{mol}^{-1}$, which were calculated by adding the gas-phase thermal and non-thermal corrections at 298.15 K to the single-point energies.

Table S42. Calculated energy values of intermediates $\mathbf{C}$ and $\mathbf{B}$ at the X/def2-TZVP+SMD(1,4dioxane)//TPSS-D3(BJ)/def2-TZVP level of theory (in $\mathrm{kcal} \mathrm{mol}^{-1}$ ).

\begin{tabular}{ccc}
\hline & & \\
\hline PBE0-D3(BJ) & 0.0 & 20.0 \\
\hline B3LYP-D3(BJ) & 0.0 & 21.4 \\
\hline \hline PW6B95-D3(BJ) & 0.0 & 22.6 \\
\hline wB97XD & 0.0 & 19.4 \\
\hline
\end{tabular}


Table S43. Bond length and Wiberg Bond Indices (WBI) of $\mathrm{Ru}-\mathrm{C}$ bond in intermediates $\mathbf{C}$ and B at the PBE0-D3(BJ)//def2-TZVP+SMD(1,4-dioxane)//TPSS-D3(BJ)/def2-TZVP level of theory.

\begin{tabular}{lcc}
\hline Structure & Ru-C Bond Length $(\stackrel{\AA}{\mathbf{A}})$ & WBI (Ru-C) \\
\hline & & \\
\end{tabular}

Table S44. Calculated energy values for the isomers of intermediate $\mathbf{C}$ (singlet states) at the PBE0-D3(BJ)// def2-TZVP+SMD(1,4-dioxane)//TPSS-D3(BJ)/def2-TZVP level of theory (in kcal mol ${ }^{-1}$ ).

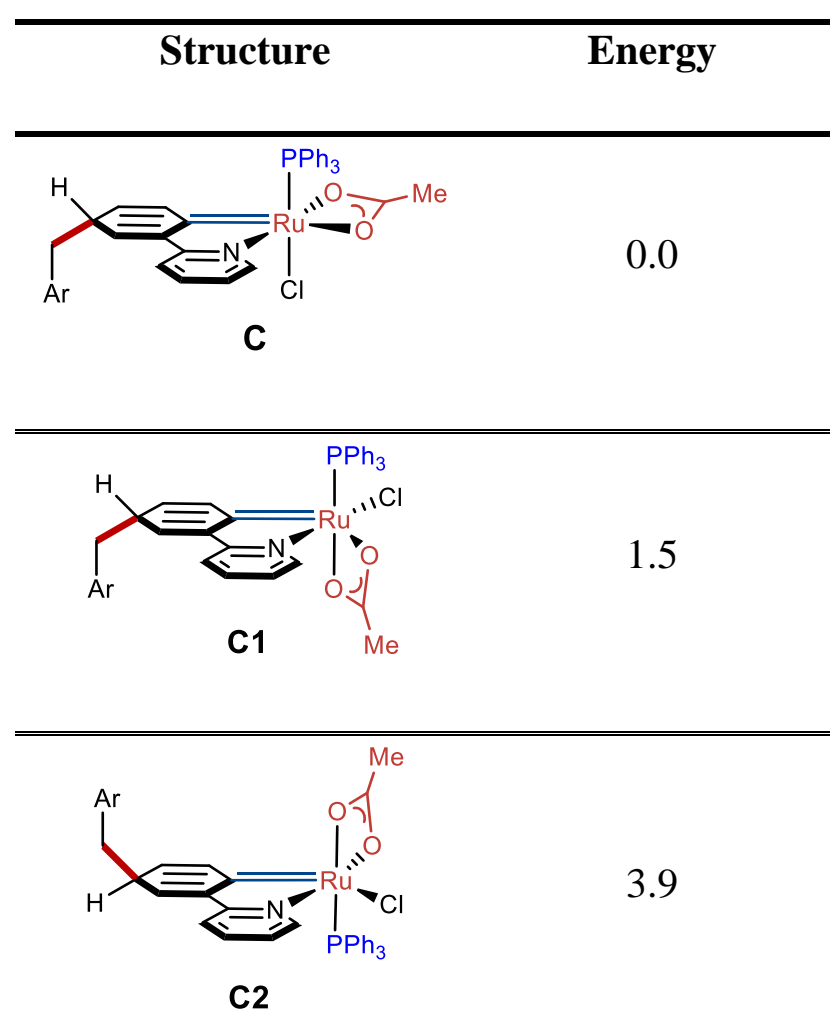


Table S45. Calculated energy values of intermediates $\mathbf{C}, \mathbf{C 3}$ and $\mathbf{C 4}$ (singlet states) which are characterized by benzylations at meta, para and ortho-positions, respectively, with respect to the directing group at the PBE0-D3(BJ)//def2-TZVP+SMD(1,4-dioxane)//TPSSD3(BJ)/def2-TZVP level of theory (in $\mathrm{kcal} \mathrm{mol}^{-1}$ ).

Structure

Table S46. Calculated electronic energies at the PBE0-D3(BJ)/def2-TZVP+SMD(1,4-dioxane)//TPSS-D3(BJ)/def2-TZVP level of theory and total Gibbs free energies with dispersion corrections for all reported structures (in Hartree).

\begin{tabular}{ccc}
\hline Structure & Electronic Energy & Total Gibbs Free Energy \\
\hline B & -2682.631672 & -2682.084970 \\
\hline C & -2682.668692 & -2682.116877 \\
\hline C1 & -2682.667857 & -2682.114454 \\
\hline \hline C2 3 & -2682.664403 & -2682.110587 \\
\hline \hline C4 & -2682.633155 & -2682.082197 \\
\hline
\end{tabular}



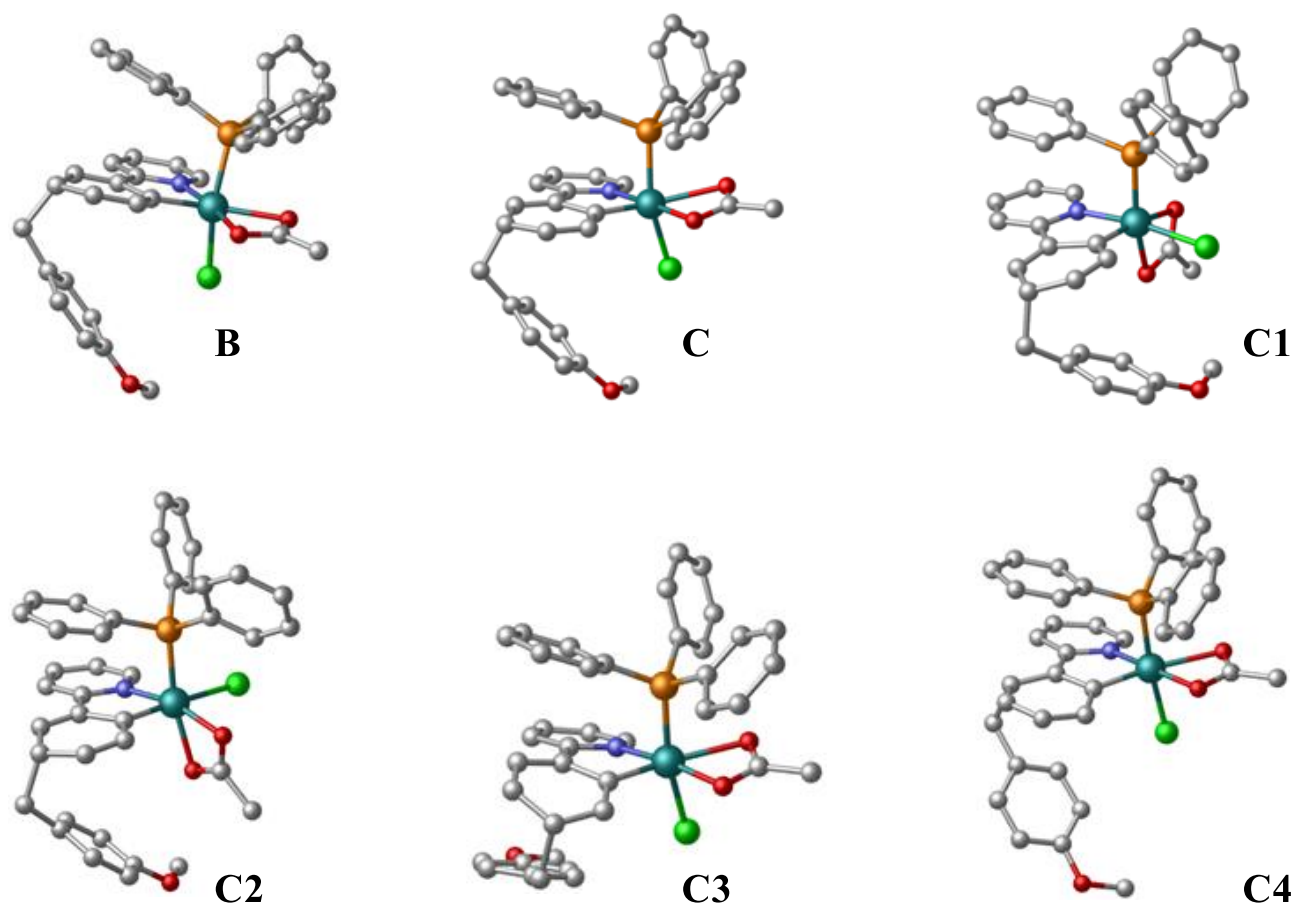

Figure S19. Geometries of all the structures obtained at the PBE0-D3(BJ)/def2-TZVP+SMD (1,4-dioxane)//TPSS-D3(BJ)/def2-TZVP level of theory. Non-participating $\mathrm{H}$ atoms were omitted for clarity.

\section{Cartesian Coordinates of the Optimized Geometries}

\section{B}

Lowest frequency $=7.850 \mathrm{~cm}^{-1}$

Charge $=0$, Multiplicity $=3$
$\mathrm{Ru} \quad 0.017432 \quad-0.722250 \quad-0.712677$
$\begin{array}{llll}\text { O } & -0.153374 & -2.527170 & 0.350912\end{array}$
C $\quad 0.669418-3.185444 \quad-0.379796$
C $\quad 0.992726-4.613673-0.053433$
P $\quad 1.973466 \quad-0.059841 \quad 0.294921$
C $\quad 3.341796 \quad 0.239859 \quad-0.883321$
C $\quad 3.363877 \quad-0.448688 \quad-2.103451$
C $\quad 4.410102-0.248030 \quad-3.003954$
C $\quad 5.442749 \quad 0.637453 \quad-2.695219$
C $\quad 5.433895 \quad 1.316565-1.475314$ 

$\begin{array}{llll}\text { C } & 4.391492 & 1.117882 & -0.572140\end{array}$
$\begin{array}{llll}\text { C } & 1.839992 & 1.533620 & 1.185053\end{array}$
$\begin{array}{llll}\text { C } & 1.543293 & 1.585207 & 2.552571\end{array}$
$\begin{array}{llll}\text { C } & 1.286840 & 2.806349 & 3.172386\end{array}$
C $\quad 1.327211 \quad 3.991256 \quad 2.437643$
$\begin{array}{llll}\text { C } & 1.627392 & 3.949159 & 1.076207\end{array}$
C $\quad 1.877464 \quad 2.729836 \quad 0.451702$
$\begin{array}{llll}\text { C } & 2.695815 & -1.233957 & 1.499142\end{array}$
C $4.028997-1.658411 \quad 1.420661$
$\begin{array}{llll}\text { C } & 4.525141 & -2.591151 & 2.330896\end{array}$
$\begin{array}{llll}\text { C } & 3.698739 & -3.108597 & 3.328617\end{array}$
C $\quad 2.367526 \quad-2.696841 \quad 3.408168$
$\begin{array}{llll}\text { C } & 1.865362 & -1.773977 & 2.493508\end{array}$
O $1.221366 \quad-2.576337 \quad-1.348900$
$\begin{array}{llll}\text { C } & -1.032917 & 0.324607 & 0.608463\end{array}$
$\begin{array}{llll}\text { C } & -1.301637 & 1.685458 & 0.274896\end{array}$
$\begin{array}{llll}\text { C } & -1.976959 & 2.527117 & 1.135327\end{array}$
$\begin{array}{llll}\text { C } & -2.609986 & 2.025818 & 2.391843\end{array}$
$\begin{array}{llll}\text { C } & -2.114685 & 0.659405 & 2.761493\end{array}$
$\begin{array}{llll}\text { C } & -1.435200 & -0.140567 & 1.891365\end{array}$
C $\quad-0.756900 \quad 2.097996 \quad-1.007697$
N $\quad-0.035180 \quad 1.121730 \quad-1.638127$
$\begin{array}{llll}\mathrm{H} & -2.117408 & 3.576699 & 0.886823\end{array}$
$\begin{array}{llll}\mathrm{H} & -1.210364 & -1.166499 & 2.170218\end{array}$
$\begin{array}{llll}\text { H } & -2.417229 & 0.265611 & 3.730204\end{array}$
H $4.388895 \quad 1.647692 \quad 0.375428$
H $\quad 4.417711 \quad-0.788610 \quad-3.946343$
$\begin{array}{llll}\mathrm{H} & 6.239338 & 2.001776 & -1.225789\end{array}$
H $\quad 6.254874 \quad 0.795386 \quad-3.399529$
H $\quad 4.677404 \quad-1.267547 \quad 0.643636$
$\mathrm{H} \quad 0.820965 \quad-1.487086 \quad 2.533097$
H $\quad 5.559685 \quad-2.914874 \quad 2.257122$
H $\quad 1.713278 \quad-3.104942 \quad 4.173449$
H $4.087442 \quad-3.836382 \quad 4.035454$ 


$$
\begin{array}{cccc}
\mathrm{H} & 1.507421 & 0.671897 & 3.136026 \\
\mathrm{H} & 2.100741 & 2.707560 & -0.610606 \\
\mathrm{H} & 1.052116 & 2.829182 & 4.232809 \\
\mathrm{H} & 1.658861 & 4.865520 & 0.493288 \\
\mathrm{H} & 1.125156 & 4.941811 & 2.923252 \\
\mathrm{H} & 2.573358 & -1.156693 & -2.329046 \\
\mathrm{H} & 0.125475 & -5.105191 & 0.392386 \\
\mathrm{H} & 1.316571 & -5.142925 & -0.951302 \\
\mathrm{H} & 1.810578 & -4.625070 & 0.677121 \\
\mathrm{C} & -0.903142 & 3.352779 & -1.609720 \\
\mathrm{C} & -0.309436 & 3.610437 & -2.836697 \\
\mathrm{H} & -1.486235 & 4.113458 & -1.102906 \\
\mathrm{H} & -0.417998 & 4.583986 & -3.305435 \\
\mathrm{C} & 0.418053 & 2.596732 & -3.466299 \\
\mathrm{C} & 0.523189 & 1.364910 & -2.839477 \\
\mathrm{H} & 0.888094 & 2.747223 & -4.431776 \\
\mathrm{H} & 1.057339 & 0.536162 & -3.287769 \\
\mathrm{Cl} & -1.611334 & -1.384172 & -2.284269 \\
\mathrm{H} & -2.419289 & 2.737707 & 3.212462 \\
\mathrm{C} & -4.198854 & 2.000762 & 2.248150 \\
\mathrm{H} & -4.509661 & 2.990357 & 1.891330 \\
\mathrm{H} & -4.621470 & 1.858810 & 3.249982 \\
\mathrm{C} & -4.666002 & 0.907305 & 1.328480 \\
\mathrm{C} & -4.568997 & 1.027067 & -0.060122 \\
\mathrm{C} & -5.102246 & -0.321076 & 1.846321 \\
\mathrm{C} & -4.861365 & -0.037904 & -0.911644 \\
\mathrm{H} & -4.230781 & 1.964368 & -0.494026 \\
\mathrm{C} & -5.411800 & -1.390197 & 1.017251 \\
\mathrm{H} & -5.187738 & -0.444209 & 2.923947 \\
\mathrm{C} & -5.275287 & -1.259408 & -0.370527 \\
\mathrm{H} & -4.733637 & -5.082936 & -1.980530 \\
& -5.335182 & -2.294463 & -2.520866
\end{array}
$$



H $\quad-4.286475 \quad-2.051545 \quad-2.725282$
H $\quad-5.573779 \quad-3.285647 \quad-2.909069$
H $\quad-5.992605 \quad-1.547995 \quad-2.986268$
C
Lowest frequency $=8.720 \mathrm{~cm}^{-1}$
Charge $=0$, Multiplicity $=1$
$\begin{array}{llll}\mathrm{Ru} & -0.002594 & -0.819027 & -0.609115\end{array}$
$\begin{array}{llll}\text { O } & 0.164848 & -2.631222 & 0.455677\end{array}$
$\begin{array}{llll}\text { C } & 1.063433 & -3.173026 & -0.282197\end{array}$
$\begin{array}{llll}\mathrm{P} & 1.966176 & 0.009026 & 0.264851\end{array}$
$\begin{array}{llll}\text { C } & 3.274276 & 0.375610 & -0.961991\end{array}$
C $3.249183 \quad-0.233207 \quad-2.222562$
C $\quad 4.243482 \quad 0.052711 \quad-3.158579$
$\begin{array}{llll}\text { C } & 5.270573 & 0.942540 & -2.845600\end{array}$
C $\quad 5.308052 \quad 1.544883 \quad-1.586232$
$\begin{array}{llll}\text { C } & 4.316084 & 1.264026 & -0.649477\end{array}$
$\begin{array}{llll}\text { C } & 1.764839 & 1.628601 & 1.100071\end{array}$
C $\quad 1.474325 \quad 1.714425 \quad 2.467845$
C $\quad 1.163521 \quad 2.943026 \quad 3.047393$
$\begin{array}{llll}\text { C } & 1.141299 & 4.103050 & 2.271487\end{array}$
C $\quad 1.436585 \quad 4.027225 \quad 0.910454$
$\begin{array}{llll}\text { C } & 1.742109 & 2.799372 & 0.327058\end{array}$
$\begin{array}{llll}\text { C } & 2.793708 & -1.054606 & 1.502850\end{array}$
$\begin{array}{llll}\text { C } & 4.151709 & -1.388422 & 1.424167\end{array}$
$\begin{array}{llll}\text { C } & 4.723537 & -2.234191 & 2.374265\end{array}$
$\begin{array}{llll}\text { C } & 3.947230 & -2.754387 & 3.410196\end{array}$
C $\quad 2.590159 \quad-2.437316 \quad 3.486561$
$\begin{array}{llll}\text { C } & 2.013154 & -1.602535 & 2.532379\end{array}$
O $1.546218 \quad-2.522174 \quad-1.258263$
$\begin{array}{llll}\text { C } & -1.009995 & 0.204227 & 0.599126\end{array}$
$\begin{array}{llll}\text { C } & -1.275179 & 1.603699 & 0.254669\end{array}$
C $\quad-1.912883 \quad 2.441135 \quad 1.103938$ 

$\begin{array}{llll}\text { C } & -2.509107 & 1.971753 & 2.383359\end{array}$
$\begin{array}{llll}\text { C } & -2.118492 & 0.575128 & 2.741489\end{array}$
$\begin{array}{llll}\text { C } & -1.475526 & -0.250190 & 1.886188\end{array}$
$\begin{array}{llll}\text { C } & -0.838649 & 1.937561 & -1.099587\end{array}$
N $\quad-0.225196 \quad 0.882179 \quad-1.719578$
$\begin{array}{llll}\mathrm{H} & -2.095797 & 3.480737 & 0.834448\end{array}$
$\mathrm{H} \quad-1.286870 \quad-1.286958 \quad 2.153034$
$\mathrm{H} \quad-2.456140 \quad 0.205257 \quad 3.708319$
$\mathrm{H} \quad 4.345471 \quad 1.739646 \quad 0.326427$
H $\quad 4.215417 \quad-0.426879 \quad-4.133103$
$\mathrm{H} \quad 6.109028 \quad 2.234150 \quad-1.333284$
H $\quad 6.042497 \quad 1.164584 \quad-3.577427$
H $\quad 4.759617 \quad-0.998410 \quad 0.614635$
H $\quad 0.948630 \quad-1.398341 \quad 2.560151$
H $\quad 5.777017 \quad-2.489858 \quad 2.300646$
H $\quad 1.974550 \quad-2.855848 \quad 4.277855$
H $\quad 4.394633 \quad-3.415429 \quad 4.147330$
H $\quad 1.491459 \quad 0.821069 \quad 3.082529$
H $\quad 1.963990 \quad 2.749582 \quad-0.734310$
$\mathrm{H} \quad 0.940971 \quad 2.993190 \quad 4.109808$
$\mathrm{H} \quad 1.424585 \quad 4.923936 \quad 0.296816$
H $\quad 0.901389 \quad 5.060055 \quad 2.726544$
H $2.467797 \quad-0.949597 \quad-2.447338$
C $\quad-1.041306 \quad 3.142082-1.770910$
C $\quad-0.624762 \quad 3.276872 \quad-3.090851$
H $\quad-1.525718 \quad 3.964637 \quad-1.254633$
$\mathrm{H} \quad-0.770510 \quad 4.212283 \quad-3.622351$
C $\quad-0.035101 \quad 2.180753 \quad-3.723921$
C $\quad 0.138217 \quad 1.002166 \quad-3.012622$
$\mathrm{H} \quad 0.284127 \quad 2.230050 \quad-4.759480$
$\mathrm{H} \quad 0.571522 \quad 0.114573 \quad-3.456795$
Cl $\quad-1.526504 \quad-1.873916 \quad-2.062105$
$\mathrm{H} \quad-2.240452 \quad 2.667493 \quad 3.193721$
$\begin{array}{llll}\text { C } & -4.097353 & 2.049301 & 2.265151\end{array}$ 

H $\quad-4.355487 \quad 3.051785 \quad 1.903911$
$\begin{array}{llll}\mathrm{H} & -4.508187 & 1.943220 & 3.275002\end{array}$
$\begin{array}{llll}\text { C } & -4.634885 & 0.967516 & 1.366876\end{array}$
$\begin{array}{llll}\text { C } & -4.510544 & 1.040213 & -0.022831\end{array}$
$\begin{array}{llll}\text { C } & -5.151925 & -0.216119 & 1.913561\end{array}$
C $\quad-4.837999 \quad-0.036238-0.846373$
$\mathrm{H} \quad-4.122249 \quad 1.945074 \quad-0.482637$
C $\quad-5.501771 \quad-1.292877 \quad 1.111605$
H $\quad-5.265071 \quad-0.300557 \quad 2.992521$
C $\quad-5.321269-1.218987 \quad-0.276268$
H $\quad-4.684030 \quad 0.043440 \quad-1.915708$
$\mathrm{H} \quad-5.890154-2.211952 \quad 1.539462$
$\begin{array}{llll}\mathrm{O} & -5.644770 & -2.339832 & -0.984066\end{array}$
C $\quad-5.328014 \quad-2.338727 \quad-2.384052$
H $\quad-4.261016 \quad-2.143883 \quad-2.536531$
H $\quad-5.582036 \quad-3.338930 \quad-2.736782$
Н $\quad-5.932749-1.594369-2.918757$
$\begin{array}{llll}\text { C } & 1.564562 & -4.550965 & 0.058572\end{array}$
H $\quad 1.830019 \quad-5.090823 \quad-0.852756$
H $2.466463 \quad-4.448439 \quad 0.674047$
H $\quad 0.813699 \quad-5.102038 \quad 0.627818$

\section{C1}

Lowest frequency $=17.420 \mathrm{~cm}^{-1}$

Charge $=0$, Multiplicity $=1$
$\begin{array}{llll}\mathrm{Ru} & -0.128288 & 0.697765 & 0.539910\end{array}$
$\begin{array}{llll}\text { O } & -1.578847 & 1.598526 & 1.837637\end{array}$
$\begin{array}{llll}\text { C } & -0.768025 & 2.482988 & 2.297192\end{array}$
P $\quad 1.822678 \quad 0.143172 \quad-0.526976$
C $3.168460 \quad 1.334656-0.199536$
$\begin{array}{llll}\text { C } & 2.824578 & 2.674032 & 0.021525\end{array}$
$\begin{array}{llll}\text { C } & 3.827865 & 3.628109 & 0.192761\end{array}$
$\begin{array}{llll}\text { C } & 5.170327 & 3.254301 & 0.145512\end{array}$ 

$\begin{array}{llll}\text { C } & 5.515809 & 1.920132 & -0.085111\end{array}$
$\begin{array}{llll}\text { C } & 4.519970 & 0.963721 & -0.264227\end{array}$
$\begin{array}{llll}\text { C } & 2.423290 & -1.466344 & 0.101268\end{array}$
$\begin{array}{llll}\text { C } & 1.943418 & -2.651485 & -0.477068\end{array}$
$\begin{array}{llll}\text { C } & 2.248314 & -3.888252 & 0.086631\end{array}$
$\begin{array}{llll}\text { C } & 3.024579 & -3.959219 & 1.243939\end{array}$
$\begin{array}{llll}\text { C } & 3.492733 & -2.785163 & 1.834741\end{array}$
$\begin{array}{llll}\text { C } & 3.193644 & -1.546843 & 1.269411\end{array}$
$\begin{array}{llll}\text { C } & 1.968188 & -0.057815 & -2.347228\end{array}$
$\begin{array}{llll}\text { C } & 2.934567 & -0.911731 & -2.900970\end{array}$
C $\quad 3.093043 \quad-0.993564 \quad-4.282682$
$\begin{array}{llll}\text { C } & 2.296408 & -0.217902 & -5.126819\end{array}$
$\begin{array}{llll}\text { C } & 1.346821 & 0.644801 & -4.580321\end{array}$
$\begin{array}{llll}\text { C } & 1.182239 & 0.729478 & -3.197855\end{array}$
$\begin{array}{llll}\text { O } & 0.449977 & 2.443349 & 1.952605\end{array}$
$\begin{array}{llll}\text { C } & -0.914943 & -0.795415 & -0.325234\end{array}$
$\begin{array}{llll}\text { C } & -1.041055 & -2.003118 & 0.511144\end{array}$
$\begin{array}{llll}\text { C } & -1.779252 & -3.066955 & 0.113732\end{array}$
$\begin{array}{llll}\text { C } & -2.502400 & -3.098495 & -1.182709\end{array}$
$\begin{array}{llll}\text { C } & -2.098344 & -1.994825 & -2.100757\end{array}$
$\begin{array}{llll}\text { C } & -1.403897 & -0.914481 & -1.680832\end{array}$
$\begin{array}{llll}\text { C } & -0.374299 & -1.897188 & 1.809998\end{array}$
$\begin{array}{llll}\mathrm{N} & 0.167236 & -0.659695 & 2.022130\end{array}$
H $\quad-1.930674 \quad-3.919002 \quad 0.776051$
H $\quad-1.228289 \quad-0.081724 \quad-2.351589$
H $\quad-2.482393 \quad-2.032746 \quad-3.118437$
H $\quad 4.792724 \quad-0.070154 \quad-0.455404$
$\mathrm{H} \quad 3.554952 \quad 4.665214 \quad 0.365818$
H $\quad 6.561094 \quad 1.626582 \quad-0.131285$
$\begin{array}{llll}\mathrm{H} & 5.949109 & 3.999587 & 0.283429\end{array}$
H $\quad 3.561724 \quad-1.517872 \quad-2.254810$

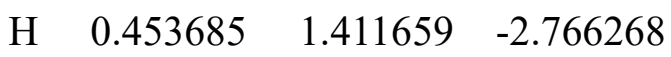
H $\quad 3.841579 \quad-1.662317 \quad-4.699225$
$\begin{array}{llll}\mathrm{H} & 0.729158 & 1.259563 & -5.229419\end{array}$ 

H $\quad 2.421136 \quad-0.283108 \quad-6.204372$
H $\quad 1.326217 \quad-2.601768 \quad-1.369222$
$\begin{array}{llll}\mathrm{H} & 3.554591 & -0.636707 & 1.738709\end{array}$
H $\quad 1.872732 \quad-4.796931 \quad-0.376031$
H $\quad 4.089034 \quad-2.831374 \quad 2.741786$
H $\quad 3.260723 \quad-4.923542 \quad 1.685137$
$\begin{array}{llll}\mathrm{H} & 1.778035 & 2.960248 & 0.057177\end{array}$
$\begin{array}{llll}\text { C } & -0.262199 & -2.898219 & 2.773329\end{array}$
$\begin{array}{llll}\text { C } & 0.384506 & -2.633753 & 3.975155\end{array}$
$\begin{array}{llll}\mathrm{H} & -0.677867 & -3.880541 & 2.574368\end{array}$
H $\quad 0.479129 \quad-3.407254 \quad 4.730855$
C $\quad 0.910251 \quad-1.358215 \quad 4.188506$
C $\quad 0.786957 \quad-0.400836 \quad 3.193260$
H $\quad 1.420842 \quad-1.104705 \quad 5.111659$
$\begin{array}{llll}\mathrm{H} & 1.182413 & 0.604200 & 3.291430\end{array}$
$\begin{array}{lllll}\mathrm{Cl} & -0.792362 & 2.397937 & -1.018148\end{array}$
$\mathrm{H} \quad-2.345792 \quad-4.078145 \quad-1.659811$
C $\quad-4.083199 \quad-3.011178 \quad-0.918965$
H $\quad-4.309321 \quad-3.646627 \quad-0.055291$
$\mathrm{H} \quad-4.584230 \quad-3.440728 \quad-1.792330$

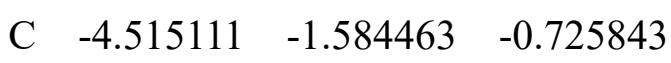
$\begin{array}{llll}\text { C } & -4.168072 & -0.859045 & 0.417392\end{array}$
$\begin{array}{llll}\text { C } & -5.107896 & -0.886429 & -1.788329\end{array}$
$\begin{array}{llll}\text { C } & -4.331288 & 0.523627 & 0.482521\end{array}$
$\begin{array}{llll}\mathrm{H} & -3.715875 & -1.367528 & 1.264217\end{array}$
$\begin{array}{llll}\text { C } & -5.304780 & 0.486004 & -1.733419\end{array}$
$\begin{array}{llll}\mathrm{H} & -5.393194 & -1.427436 & -2.688606\end{array}$
$\begin{array}{llll}\text { C } & -4.878544 & 1.206334 & -0.609457\end{array}$
$\begin{array}{llll}\mathrm{H} & -3.978793 & 1.058268 & 1.355397\end{array}$
$\begin{array}{llll}\mathrm{H} & -5.747395 & 1.026085 & -2.564742\end{array}$
$\begin{array}{llll}\text { O } & -5.034718 & 2.560249 & -0.667248\end{array}$
$\begin{array}{llll}\text { C } & -4.363921 & 3.328689 & 0.345083\end{array}$
$\begin{array}{llll}\mathrm{H} & -3.298240 & 3.079567 & 0.366451\end{array}$
$\begin{array}{llll}\mathrm{H} & -4.506318 & 4.370432 & 0.054614\end{array}$ 


$\begin{array}{lrll}\mathrm{H} & -4.815928 & 3.154909 & 1.330420 \\ \mathrm{C} & -1.295436 & 3.563110 & 3.204044 \\ \mathrm{H} & -0.512688 & 3.903471 & 3.885407 \\ \mathrm{H} & -1.602403 & 4.412653 & 2.582854 \\ \mathrm{H} & -2.166643 & 3.210199 & 3.760637\end{array}$

\section{C2}

Lowest frequency $=17.370 \mathrm{~cm}^{-1}$

Charge $=0$, Multiplicity $=1$
$\mathrm{Ru} \quad-0.090413 \quad-0.885980 \quad-0.381288$
$\begin{array}{llll}\text { O } & -0.623532 & -2.388321 & 1.050034\end{array}$
C $\quad-1.686860 \quad-2.657920 \quad 0.392703$
$\begin{array}{llll}\mathrm{P} & 2.006099 & -0.155561 & 0.272355\end{array}$
$\begin{array}{llll}\text { C } & 3.421687 & -0.028045 & -0.898800\end{array}$
C $\quad 3.389420 \quad-0.638416 \quad-2.155423$
C $4.483538-0.530556-3.016451$
$\begin{array}{llll}\text { C } & 5.617512 & 0.180613 & -2.629936\end{array}$
$\begin{array}{llll}\text { C } & 5.660976 & 0.782706 & -1.369624\end{array}$
$\begin{array}{lllll}\text { C } & 4.571734 & 0.678707 & -0.509908\end{array}$
C $\quad 1.948982 \quad 1.561399 \quad 0.907539$
$\begin{array}{llll}\text { C } & 1.547002 & 1.832557 & 2.223060\end{array}$
$\begin{array}{llll}\text { C } & 1.344718 \quad 3.145140 & 2.643335\end{array}$
C $\quad 1.529287 \quad 4.205154 \quad 1.753753$
$\begin{array}{llll}\text { C } & 1.923743 & 3.944268 & 0.441285\end{array}$
$\begin{array}{llll}\text { C } & 2.134273 & 2.632561 & 0.020859\end{array}$
$\begin{array}{llll}\text { C } & 2.715499 & -1.158565 & 1.624269\end{array}$
$\begin{array}{llll}\text { C } & 3.577989 & -0.632933 & 2.596992\end{array}$
$\begin{array}{llll}\text { C } & 4.135193 & -1.468174 & 3.562927\end{array}$
$\begin{array}{llll}\text { C } & 3.844204 & -2.833939 & 3.558388\end{array}$
$\begin{array}{llll}\text { C } & 2.994302 & -3.361329 & 2.585999\end{array}$
$\begin{array}{llll}\text { C } & 2.426211 & -2.530014 & 1.621714\end{array}$
O $\quad-1.944428 \quad-1.946220 \quad-0.639642$
$\begin{array}{llll}\text { C } & -0.887277 & 0.513998 & 0.633733\end{array}$ 

$\begin{array}{llll}\text { C } & -1.048580 & 1.796398 & -0.059280\end{array}$
$\begin{array}{llll}\text { C } & -1.654479 & 2.855889 & 0.527729\end{array}$
$\begin{array}{llll}\text { C } & -2.312963 & 2.754865 & 1.855882\end{array}$
$\begin{array}{llll}\text { C } & -1.973523 & 1.503979 & 2.590379\end{array}$
$\begin{array}{llll}\text { C } & -1.348575 & 0.461152 & 2.001134\end{array}$
$\begin{array}{llll}\text { C } & -0.575276 & 1.772560 & -1.445869\end{array}$
$\begin{array}{llll}\mathrm{N} & -0.110795 & 0.539966 & -1.830075\end{array}$
$\begin{array}{llll}\mathrm{H} & -1.780232 & 3.797847 & -0.004957\end{array}$
H $\quad-1.171342 \quad-0.457698 \quad 2.553324$
$\begin{array}{llll}\mathrm{H} & -2.320198 & 1.428550 & 3.620183\end{array}$
$\mathrm{H} \quad 4.612276 \quad 1.157081 \quad 0.464013$
H $\quad 4.447083 \quad-1.014088 \quad-3.988983$
$\begin{array}{llll}\mathrm{H} & 6.544463 & 1.332412 & -1.056274\end{array}$
$\begin{array}{llll}\mathrm{H} & 6.468422 & 0.261894 & -3.301019\end{array}$
$\mathrm{H} \quad 3.810162 \quad 0.427883 \quad 2.605572$
H $\quad 1.760932 \quad-2.933988 \quad 0.864163$
H $\quad 4.798422 \quad-1.053303 \quad 4.317278$
H $\quad 2.766432 \quad-4.423467 \quad 2.577517$
H $\quad 4.280137 \quad-3.483891 \quad 4.312382$
$\mathrm{H} \quad 1.381450 \quad 1.013216 \quad 2.914644$
H $\quad 2.442402 \quad 2.437301 \quad-1.001532$
H $\quad 1.036393 \quad 3.339394 \quad 3.667019$
$\mathrm{H} \quad 2.068266 \quad 4.762458 \quad-0.259050$
$\mathrm{H} \quad 1.370216 \quad 5.228190 \quad 2.083563$
$\mathrm{H} \quad 2.524238 \quad-1.232830 \quad-2.431107$
$\begin{array}{llll}\text { C } & -0.614105 & 2.831638 & -2.351187\end{array}$
$\begin{array}{llll}\text { C } & -0.197852 & 2.640454 & -3.664064\end{array}$
$\begin{array}{llll}\mathrm{H} & -0.970082 & 3.801713 & -2.019530\end{array}$
$\begin{array}{llll}\mathrm{H} & -0.218518 & 3.461390 & -4.374179\end{array}$
$\begin{array}{lllll}\text { C } & 0.232547 & 1.369606 & -4.051439\end{array}$
$\begin{array}{llll}\text { C } & 0.259763 & 0.347094 & -3.115327\end{array}$
$\begin{array}{llll}\mathrm{H} & 0.550098 & 1.166131 & -5.068736\end{array}$
H $\quad 0.574779 \quad-0.664907 \quad-3.347755$
$\begin{array}{llll}\mathrm{Cl} & 0.826182 & -2.734445 & -1.748230\end{array}$ 

H $\quad-2.065319 \quad 3.636749 \quad 2.466298$
$\begin{array}{llll}\text { C } & -3.895412 & 2.823113 & 1.644662\end{array}$
H $\quad-4.111542 \quad 3.730029 \quad 1.068546$
$\mathrm{H} \quad-4.351825 \quad 2.937886 \quad 2.633626$
$\begin{array}{llll}\text { C } & -4.417144 & 1.588903 & 0.961369\end{array}$
C $\quad-4.286874 \quad 1.410041 \quad-0.418253$
$\begin{array}{llll}\text { C } & -4.932960 & 0.522175 & 1.711028\end{array}$
$\begin{array}{llll}\text { C } & -4.608994 & 0.202132 & -1.034989\end{array}$
H $\quad-3.899769 \quad 2.219816 \quad-1.031437$
$\begin{array}{llll}\text { C } & -5.274546 & -0.683812 & 1.115225\end{array}$
$\begin{array}{llll}\mathrm{H} & -5.050676 & 0.632991 & 2.786844\end{array}$
C $\quad-5.086751 \quad-0.860056 \quad-0.261457$
H $\quad-4.457926 \quad 0.089693 \quad-2.101990$
$\mathrm{H} \quad-5.662273 \quad-1.511219 \quad 1.701657$
$\begin{array}{llll}\text { O } & -5.391943 & -2.097394 & -0.756408\end{array}$
C $\quad \begin{array}{llll}-5.014421 & -2.362464 & -2.116019\end{array}$
H $\quad-3.939333 \quad-2.200790 \quad-2.247136$
H $\quad-5.261356-3.411381 \quad-2.284200$
$\mathrm{H} \quad-5.588370 \quad-1.734684 \quad-2.809393$
$\begin{array}{llll}\text { C } & -2.589436 & -3.785091 & 0.796601\end{array}$
$\mathrm{H} \quad-3.629982 \quad-3.530584 \quad 0.582672$
H $\quad-2.321643 \quad-4.669992 \quad 0.207577$
H $\quad-2.454743 \quad-4.016717 \quad 1.855090$

\section{C3}

Lowest frequency $=17.030 \mathrm{~cm}^{-1}$

Charge $=0$, Multiplicity $=1$
$\mathrm{Ru} \quad 0.240286 \quad 0.146729 \quad-1.180812$
$\begin{array}{llll}\text { O } & 1.279801 & -1.142767 & -2.512692\end{array}$
$\begin{array}{llll}\text { C } & 2.022625 & -0.223461 & -3.002989\end{array}$
$\begin{array}{llll}\mathrm{P} & 1.889266 & 0.084534 & 0.368493\end{array}$
$\begin{array}{llll}C & 2.615451 & 1.723306 & 0.763655\end{array}$
$\begin{array}{llll}\text { C } & 2.385499 & 2.801814 & -0.098681\end{array}$ 

$\begin{array}{llll}\text { C } & 2.915261 & 4.059613 & 0.191189\end{array}$
$\begin{array}{llll}\text { C } & 3.680186 & 4.251202 & 1.341431\end{array}$
$\begin{array}{llll}\text { C } & 3.921097 & 3.178279 & 2.202789\end{array}$
$\begin{array}{llll}\text { C } & 3.391489 & 1.921953 & 1.916582\end{array}$
$\begin{array}{llll}\text { C } & 1.470327 & -0.513530 & 2.055065\end{array}$
$\begin{array}{llll}\text { C } & 1.683087 & -1.837433 & 2.457455\end{array}$
$\begin{array}{llll}\text { C } & 1.261082 & -2.273215 & 3.712549\end{array}$
C $\quad 0.618562 \quad-1.393580 \quad 4.584746$
$\begin{array}{llll}\text { C } & 0.399514 & -0.072749 & 4.192796\end{array}$
$\begin{array}{llll}\text { C } & 0.816973 & 0.362171 & 2.936306\end{array}$
$\begin{array}{llll}\text { C } & 3.337036 & -0.938685 & -0.099023\end{array}$
$\begin{array}{llll}\text { C } & 4.646841 & -0.444564 & -0.116345\end{array}$
$\begin{array}{llll}\text { C } & 5.702466 & -1.260015 & -0.525717\end{array}$
$\begin{array}{llll}\text { C } & 5.461650 & -2.576119 & -0.919949\end{array}$
$\begin{array}{llll}\text { C } & 4.156478 & -3.071961 & -0.919468\end{array}$
$\begin{array}{llll}\text { C } & 3.099423 & -2.255481 & -0.524722\end{array}$
$\begin{array}{llll}\text { O } & 1.921936 & 0.953727 & -2.531922\end{array}$
$\begin{array}{llll}\text { C } & -0.787574 & -1.200330 & -0.155742\end{array}$
$\begin{array}{llll}\text { C } & -1.471942 & -0.685876 & 1.014981\end{array}$
$\begin{array}{llll}\text { C } & -1.947832 & -1.549647 & 2.046432\end{array}$
$\begin{array}{llll}\text { C } & -2.056592 & -2.878923 & 1.796864\end{array}$
$\begin{array}{llll}\text { C } & -1.800769 & -3.389720 & 0.434401\end{array}$
$\begin{array}{llll}\text { C } & -0.937372 & -2.539435 & -0.410389\end{array}$
$\begin{array}{llll}\text { C } & -1.614586 & 0.731813 & 0.999192\end{array}$
$\begin{array}{llll}\mathrm{N} & -0.941400 & 1.327542 & -0.059124\end{array}$
H $\quad-2.230894 \quad-1.140101 \quad 3.011846$

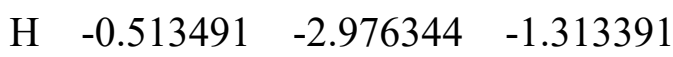
$\mathrm{H} \quad-1.481960 \quad-4.437900 \quad 0.429828$
$\begin{array}{llll}\mathrm{H} & 3.574126 & 1.091945 & 2.593089\end{array}$
H $\quad 2.731297 \quad 4.889209 \quad-0.485985$
H $\quad 4.519506 \quad 3.320935 \quad 3.098612$
$\begin{array}{llll}\mathrm{H} & 4.089975 & 5.231981 & 1.567371\end{array}$
$\begin{array}{llll}\mathrm{H} & 4.841621 & 0.581237 & 0.178700\end{array}$
$\begin{array}{llll}\mathrm{H} & 2.079097 & -2.620993 & -0.577117\end{array}$ 


\begin{tabular}{|c|c|c|c|}
\hline & 6.713897 & -0.862970 & -0.539230 \\
\hline - & 3.958629 & -4.089088 & -1.246452 \\
\hline I & 6.285019 & -3.208978 & -1.239672 \\
\hline & 2.193944 & -2.528294 & 1.794996 \\
\hline & 0.646823 & 1.392945 & 2.640965 \\
\hline I & 1.441358 & -3.302178 & 4.011824 \\
\hline I & -0.098487 & 0.622306 & 4.863715 \\
\hline $\mathrm{I}$ & 0.294034 & -1.734479 & 5.564009 \\
\hline $\mathrm{I}$ & 1.811049 & 2.639276 & -1.003704 \\
\hline C & -2.444410 & 1.492324 & 1.841452 \\
\hline C & -2.645023 & 2.835077 & 1.581531 \\
\hline $\mathrm{I}$ & -2.942989 & 1.000333 & 2.669477 \\
\hline $\mathrm{H}$ & -3.288229 & 3.432694 & 2.219304 \\
\hline C & -2.028396 & 3.401967 & 0.454034 \\
\hline $\mathrm{C}$ & -1.207355 & 2.622757 & -0.343802 \\
\hline $\mathrm{H}$ & -2.175044 & 4.446789 & 0.200530 \\
\hline $\mathrm{H}$ & -0.709536 & 3.012691 & -1.223673 \\
\hline $\mathrm{C}$ & -1.213589 & 0.651923 & -3.025890 \\
\hline $\mathrm{H}$ & -2.458997 & -3.560642 & 2.541008 \\
\hline $\mathrm{C}$ & -3.276647 & -3.445049 & -0.322226 \\
\hline $\mathrm{H}$ & -3.856830 & -4.188742 & 0.231311 \\
\hline $\mathrm{H}$ & -3.070092 & -3.830570 & -1.323534 \\
\hline $\mathrm{C}$ & -3.965581 & -2.123901 & -0.359474 \\
\hline $\mathrm{C}$ & -4.876111 & -1.753632 & 0.645246 \\
\hline $\mathrm{C}$ & -3.657641 & -1.177903 & -1.343461 \\
\hline $\mathrm{C}$ & -5.452855 & -0.492720 & 0.667266 \\
\hline $\mathrm{H}$ & -5.128327 & -2.466085 & 1.427678 \\
\hline $\mathrm{C}$ & -4.222369 & 0.094753 & -1.338129 \\
\hline $\mathrm{H}$ & -2.929496 & -1.402070 & -2.117534 \\
\hline C & -5.116959 & 0.444182 & -0.323249 \\
\hline $\mathrm{H}$ & -6.156819 & -0.207054 & 1.443066 \\
\hline $\mathrm{H}$ & -3.908535 & 0.796944 & -2.100588 \\
\hline $\mathrm{O}$ & -5.701356 & 1.675640 & -0.20656 \\
\hline $\mathrm{C}$ & -5.395367 & 2.631292 & -1.23087 \\
\hline
\end{tabular}




$$
\begin{array}{llll}
\text { H } & -5.725665 & 2.268578 & -2.211505 \\
\text { H } & -5.947564 & 3.532308 & -0.960859 \\
\text { H } & -4.320927 & 2.842355 & -1.261121 \\
\text { C } & 3.024633 & -0.555474 & -4.072776 \\
\text { H } & 3.279422 & 0.338535 & -4.644529 \\
\text { H } & 3.932375 & -0.937251 & -3.590085 \\
\text { H } & 2.632080 & -1.334300 & -4.730267
\end{array}
$$

\section{C4}

Lowest frequency $=16.180 \mathrm{~cm}^{-1}$

Charge $=0$, Multiplicity $=1$

$$
\begin{array}{cccc}
\mathrm{Ru} & -0.142449 & 0.847410 & -0.768005 \\
\mathrm{O} & -0.388826 & 2.780267 & 0.054414 \\
\mathrm{C} & -1.143347 & 3.214094 & -0.889614 \\
\mathrm{P} & -1.983699 & 0.075624 & 0.325767 \\
\mathrm{C} & -3.424431 & -0.378896 & -0.718331 \\
\mathrm{C} & -3.493180 & 0.085030 & -2.037367 \\
\mathrm{C} & -4.574101 & -0.262100 & -2.849500 \\
\mathrm{C} & -5.595132 & -1.071285 & -2.353084 \\
\mathrm{C} & -5.538123 & -1.531879 & -1.035412 \\
\mathrm{C} & -4.459691 & -1.189270 & -0.223340 \\
\mathrm{C} & -1.737602 & -1.493899 & 1.255687 \\
\mathrm{C} & -1.321776 & -1.492890 & 2.594109 \\
\mathrm{C} & -1.026531 & -2.687947 & 3.248541 \\
\mathrm{C} & -1.133984 & -3.904209 & 2.572881 \\
\mathrm{C} & -1.535977 & -3.915536 & 1.236525 \\
\mathrm{C} & -1.832398 & -2.720803 & 0.582503 \\
\mathrm{C} & -2.688692 & 1.212861 & 1.579334 \\
\mathrm{C} & -4.054950 & 1.503196 & 1.674919 \\
\mathrm{C} & -4.518878 & 2.399134 & 2.638885 \\
\mathrm{C} & -3.625362 & 3.011079 & 3.517641 \\
\mathrm{C} & -2.259645 & 2.738812 & 3.418383 \\
\mathrm{C} & -1.793106 & 1.856991 & 2.447193
\end{array}
$$



$\begin{array}{llll}\mathrm{O} & -1.484855 & 2.418545 & -1.816167\end{array}$
$\begin{array}{llll}\text { C } & 1.027204 & -0.128896 & 0.485423\end{array}$
$\begin{array}{llll}\text { C } & 1.192154 & -1.518021 & 0.246982\end{array}$
$\begin{array}{llll}\text { C } & 1.987426 & -2.383451 & 1.147831\end{array}$
$\begin{array}{llll}\text { C } & 2.287523 & -1.787161 & 2.464800\end{array}$
$\begin{array}{llll}\text { C } & 2.186784 & -0.439879 & 2.650429\end{array}$
$\begin{array}{llll}\text { C } & 1.653656 & 0.391809 & 1.628614\end{array}$
C $\quad 0.672947-1.945376 \quad-1.004654$
N $\quad 0.092194 \quad-0.899256 \quad-1.716190$
H $\quad 1.551710 \quad-3.385608 \quad 1.246045$
H $1.655872 \quad 1.470454 \quad 1.773838$
$\mathrm{H} \quad 2.527441 \quad 0.012490 \quad 3.578131$
H $\quad-4.414570 \quad-1.558496 \quad 0.797190$
H $\quad-4.616672 \quad 0.104589 \quad-3.871463$
H $\quad-6.332983 \quad-2.159508 \quad-0.641412$
Н $\quad-6.434768 \quad-1.342299 \quad-2.987473$
$\begin{array}{llll}\mathrm{H} & -4.757407 & 1.039391 & 0.990301\end{array}$
$\mathrm{H} \quad-0.727907 \quad 1.688074 \quad 2.328791$
$\mathrm{H} \quad-5.581120 \quad 2.620226 \quad 2.699291$
$\mathrm{H} \quad-1.555032 \quad 3.230100 \quad 4.083735$
H $\quad-3.989059 \quad 3.709038 \quad 4.266776$
H $\quad-1.237322 \quad-0.553964 \quad 3.131477$
H $\quad-2.140455 \quad-2.741596 \quad-0.458195$
$\mathrm{H} \quad-0.714124 \quad-2.666395 \quad 4.288876$
$\begin{array}{llll}\mathrm{H} & -1.620934 & -4.856252 & 0.698837\end{array}$
H $\quad-0.909058-4.836056 \quad 3.084549$
H $\quad-2.710690 \quad 0.737781 \quad-2.408322$
C $\quad 0.757282 \quad-3.229732 \quad-1.574109$
C $\quad 0.355909 \quad-3.433024 \quad-2.882282$
H $\quad 1.126426 \quad-4.051895 \quad-0.969001$
H $\quad 0.412584 \quad-4.419290 \quad-3.332026$
C $\quad-0.112948-2.335875 \quad-3.623431$
C $\quad-0.224672 \quad-1.096937-3.014497$
H $\quad-0.409647 \quad-2.445004 \quad-4.661079$ 

H $\quad-0.599496 \quad-0.222607 \quad-3.534661$
Cl $1.632635 \quad 1.752266 \quad-2.107052$
H $\quad 2.722484 \quad-2.427911 \quad 3.227006$
$\begin{array}{llll}\text { C } & 3.440646 & -2.651353 & 0.445251\end{array}$
H $3.198377 \quad-3.083775 \quad-0.530027$
H $3.936012 \quad-3.412513 \quad 1.055165$
$\begin{array}{llll}\text { C } & 4.294626 & -1.431249 & 0.315966\end{array}$
$\begin{array}{llll}\text { C } & 4.043871 & -0.468858 & -0.666757\end{array}$
$\begin{array}{llll}\text { C } & 5.344040 & -1.192621 & 1.217744\end{array}$
$\begin{array}{llll}\text { C } & 4.797491 & 0.699411 & -0.754588\end{array}$
H $3.226375 \quad-0.594239-1.369926$
$\begin{array}{llll}\text { C } & 6.113670 & -0.041305 & 1.140990\end{array}$
H $\quad 5.557697 \quad-1.923502 \quad 1.995289$
$\begin{array}{llll}\text { C } & 5.834927 & 0.918671 & 0.155392\end{array}$
$\mathrm{H} \quad 4.528280 \quad 1.429737 \quad-1.507749$
$\begin{array}{llll}\mathrm{H} & 6.926270 & 0.142174 & 1.837231\end{array}$
$\begin{array}{llll}\text { O } & 6.621211 & 2.038012 & 0.169721\end{array}$
$\begin{array}{llll}\text { C } & 6.332444 & 3.042639 & -0.811330\end{array}$
H $\quad 5.308072 \quad 3.416814 \quad-0.699876$
H $\quad 7.047153 \quad 3.844633 \quad-0.623754$
$\mathrm{H} \quad 6.469551 \quad 2.648697 \quad-1.825697$
C $\quad-1.640136 \quad 4.633553 \quad-0.846372$
$\mathrm{H} \quad-1.853225 \quad 4.989719 \quad-1.855934$
$\mathrm{H} \quad-2.567830 \quad 4.661507 \quad-0.262017$
$\mathrm{H} \quad-0.906805 \quad 5.277794 \quad-0.356880$ 


\section{References}

(1) (a) Simonetti, M.; Cannas, D. M.; Just-Baringo, X.; Vitorica-Yrezabal, I. J.; Larrosa, I. Cyclometallated ruthenium catalyst enables late-stage directed arylation of pharmaceuticals. Nat. Chem. 2018, 10, 724-731. (b) Flegeau, E. F.; Bruneau, C.; Dixneuf, P. H.; Jutand, A. Autocatalysis for C-H Bond Activation by Ruthenium(II) Complexes in Catalytic Arylation of Functional Arenes. J. Am. Chem. Soc. 2011, 133, 10161-10170.

(2) Li, Z.-Y.; Li, L.; Li, Q.-L.; Jing, K.; Xu, H.; Wang, G.-W. Ruthenium-Catalyzed metaSelective $\mathrm{C}-\mathrm{H}$ Mono- and Difluoromethylation of Arenes through ortho-Metalation Strategy. Chem. Eur. J. 2017, 23, 3285-3290.

(3) Bégouin, J.-M.; Gosmini, C. Cobalt-Catalyzed Cross-Coupling Between In Situ Prepared Arylzinc Halides and 2-Chloropyrimidine or 2-Chloropyrazine. J. Org. Chem. 2009, 74, 3221-3224.

(4) (a) Ali, M. A.; Yao, X.; Sun, H.; Lu, H. [RhCp* $\left.\mathrm{Cl}_{2}\right]_{2}$-Catalyzed Directed $N$-Boc Amidation of Arenes “on Water”. Org. Lett. 2015, 17, 1513-1516. (b) Martín-Ortíz, M.; Gómez-Gallego, M.; Ramírez de Arellano, C.; Sierra, M. A. The Selective Synthesis of Metallanucleosides and Metallanucleotides: A New Tool for the Functionalization of Nucleic Acids. Chem. Eur. J. 2012, 18, 12603-12608. (c) Oza, V. B.; Corcoran, R. C. A Mild Preparation of Protected Phosphate Esters From Alcohols. J. Org. Chem. 1995, 60, 3680-3684.

(5) Sun, L.; Peng, G.; Niu, H.; Wang, Q.; Li, C. A Highly Chemoselective and Rapid Chlorination of Benzyl Alcohols under Neutral Conditions. Synthesis 2008, 24, 39193924.

(6) Li, Z.; Chen, Q.-Y.; Wang, P.-D.; Wu, Y. Multifunctional BODIPY derivatives to image cancer cells and sense copper(II) ions in living cells. RSC Adv. 2013, 3, 5524-5528.

(7) Ackermann, L.; Novák, P. Regioselective Ruthenium-Catalyzed Direct Benzylations of Arenes through C-H Bond Cleavages. Org. Lett. 2009, 11, 4966-4969.

(8) Li, B.; Fang, S.-L.; Huang, D.-Y.; Shi, B.-F. Ru-Catalyzed Meta-C-H Benzylation of Arenes with Toluene Derivatives. Org. Lett. 2017, 19, 3950-3953.

(9) Li, G.; Li, D.; Zhang, J.; Shi, D.-Q.; Zhao,Y. Ligand-Enabled Regioselectivity in the Oxidative Cross-coupling of Arenes with Toluenes and Cycloalkanes Using Ruthenium Catalysts: Tuning the Site-Selectivity from the ortho to meta Positions. ACS Catal. 2017, 7, 4138-4143. 
(10) Korvorapun, K.; Kaplaneris, N.; Rogge, T.; Warratz, S.; Stückl, A. C.; Ackermann, L. Sequential meta-/ortho-C-H Functionalizations by One-Pot Ruthenium(II/III) Catalysis. ACS Catal. 2018, 8, 886-892.

(11) Fumagalli, F.; Warratz, S.; Zhang, S.-K.; Rogge, T.; Zhu, C.; Stückl, A. C.; Ackermann, L. Arene-Ligand-Free Ruthenium(II/III) Manifold for meta-C-H Alkylation: Remote Purine Diversification. Chem. Eur. J. 2018, 24, 3984-3988.

(12) Huo, H.; Harms, K.; Meggers, E. Catalytic, Enantioselective Addition of Alkyl Radicals to Alkenes via Visible-Light-Activated Photoredox Catalysis with a Chiral Rhodium Complex. J. Am. Chem. Soc. 2016, 138, 6936-6939.

(13) Dolomanov, O. V.; Bourhis, L. J.; Gildea, R. J.; Howard, J. A. K.; Puschmann, H. OLEX2: a complete structure solution, refinement and analysis program. J. Appl. Cryst. 2009, 42, 339-341.

(14) Sheldrick, G. M. SHELXT - Integrated space-group and crystal-structure determination. Acta Cryst. A 2015, 71, 3-8.

(15) Sheldrick, G. M. A short history of SHELX. Acta Cryst. A 2008, 64, 112-122.

(16) Frisch, M. J.; Trucks, G. W.; Schlegel, H. B.; Scuseria, G. E.; Robb, M. A.; Cheeseman, J. R.; Scalmani, G.; Barone, V.; Petersson, G. A.; Nakatsuji, H.; Li, X.; Caricato, M.; Marenich, A. V.; Bloino, J.; Janesko, B. G.; Gomperts, R.; Mennucci, B.; Hratchian, H. P.; Ortiz, J. V.; Izmaylov, A. F.; Sonnenberg, J. L.; Williams-Young, D.; Ding, F.; Lipparini, F.; Egidi, F.; Goings, J.; Peng, B.; Petrone, A.; Henderson, T.; Ranasinghe, D.; Zakrzewski, V. G.; Gao, J.; Rega, N.; Zheng, G.; Liang, W.; Hada, M.; Ehara, M.; Toyota, K.; Fukuda, R.; Hasegawa, J.; Ishida, M.; Nakajima, T.; Honda, Y.; Kitao, O.; Nakai, H.; Vreven, T.; Throssell, K.; Montgomery, Jr.; J. A.; Peralta, J. E.; Ogliaro, F.; Bearpark, M. J.; Heyd, J. J.; Brothers, E. N.; Kudin, K. N.; Staroverov, V. N.; Keith, T. A.; Kobayashi, R.; Normand, J.; Raghavachari, K.; Rendell, A. P.; Burant, J. C.; Iyengar, S. S.; Tomasi, J.; Cossi, M.; Millam, J. M.; Klene, M.; Adamo, C.; Cammi, R.; Ochterski, J. W.; Martin, R. L.; Morokuma, K.; Farkas, O.; Foresman, J. B.; Fox, D. J. Gaussian 16, revision A.03; Gaussian, Inc.: Wallingford, CT, 2016.

(17) Tao, J.; Perdew, J. P.; Staroverov, V. N.; Scuseria, G. E. Climbing the Density Functional Ladder: Nonempirical Meta-Generalized Gradient Approximation Designed for Molecules and Solids. Phys. Rev. Lett. 2003, 91, 146401.

(18) (a) Grimme, S.; Ehrlich, S.; Goerigk, L. Effect of the damping function in dispersion corrected density functional theory. J. Comput. Chem. 2011, 32, 1456-1465. (b) Grimme, S.; Antony, J.; Ehrlich, S.; Krieg, H. A consistent and accurate ab initio parametrization 
of density functional dispersion correction (DFT-D) for the 94 elements H-Pu. J. Chem. Phys. 2010, 132, 154104-154119.

(19) Weigend, F.; Ahlrichs, R. Balanced basis sets of split valence, triple zeta valence and quadruple zeta valence quality for $\mathrm{H}$ to Rn: Design and assessment of accuracy. Phys. Chem. Chem. Phys. 2005, 7, 3297-3305.

(20) Andrae, D.; Häußermann, U.; Dolg, M.; Stoll, H.; Preuß, H. Energy-adjusted ab initio pseudopotentials for the second and third row transition elements. Theor. Chim. Acta. 1990, 77, 123-141.

(21) Adamo, C.; Barone, V. Toward reliable density functional methods without adjustable parameters: The PBE0 model. J. Chem. Phys. 1999, 110, 6158-6159.

(22) Marenich, A. V.; Cramer, C. J.; Truhlar, D. G. Universal Solvation Model Based on Solute Electron Density and on a Continuum Model of the Solvent Defined by the Bulk Dielectric Constant and Atomic Surface Tensions. J. Phys. Chem. B. 2009, 113, 63786396.

(23) Glendening, E. D.; Landis, C. R.; Weinhold, F. NBO 6.0: Natural bond orbital analysis program. J. Comput. Chem. 2013, 34, 1429-1437.

(24) Dennington, R.; Keith, T. A.; Millam, J. M. GaussView, Version 5; Semichem, Inc.: Shawnee Mission, KS, 2009. 


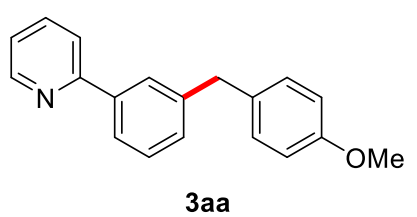

(300 MHz, $\mathrm{CDCl}_{3}$ )

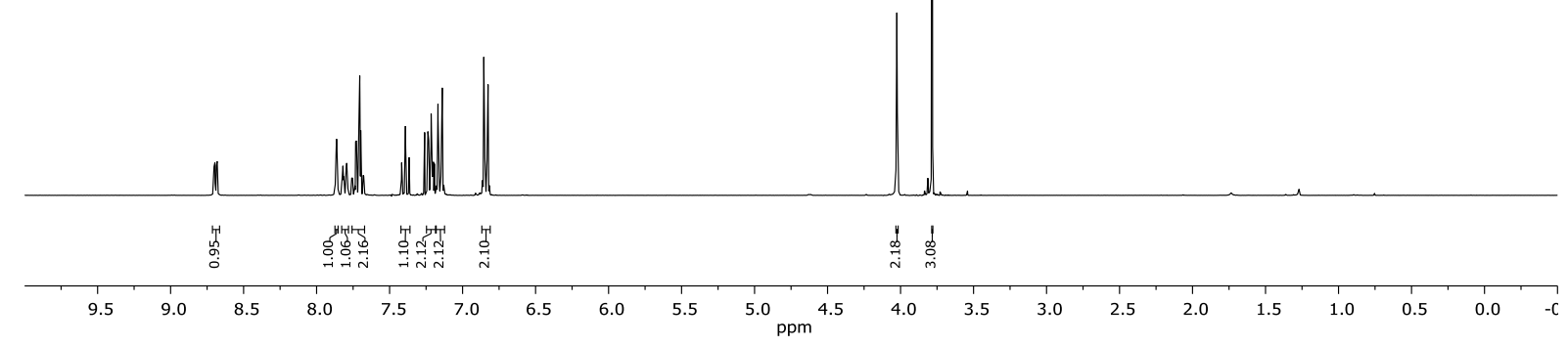

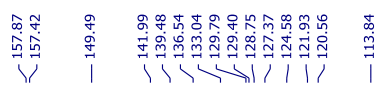

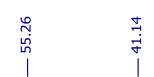<smiles>COc1ccc(Cc2cccc(-c3ccccn3)c2)cc1</smiles>

(125 MHz, $\mathrm{CDCl}_{3}$ )

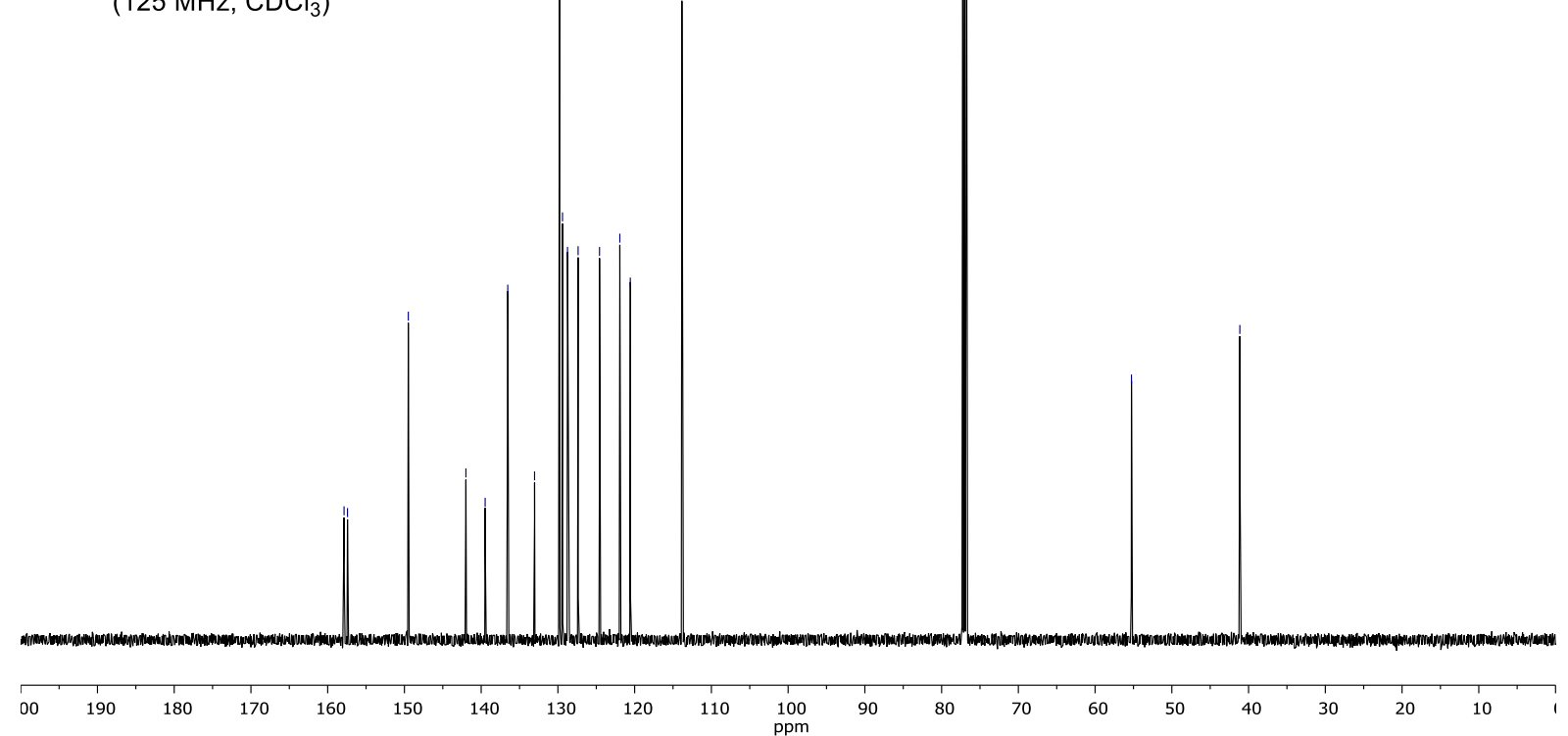




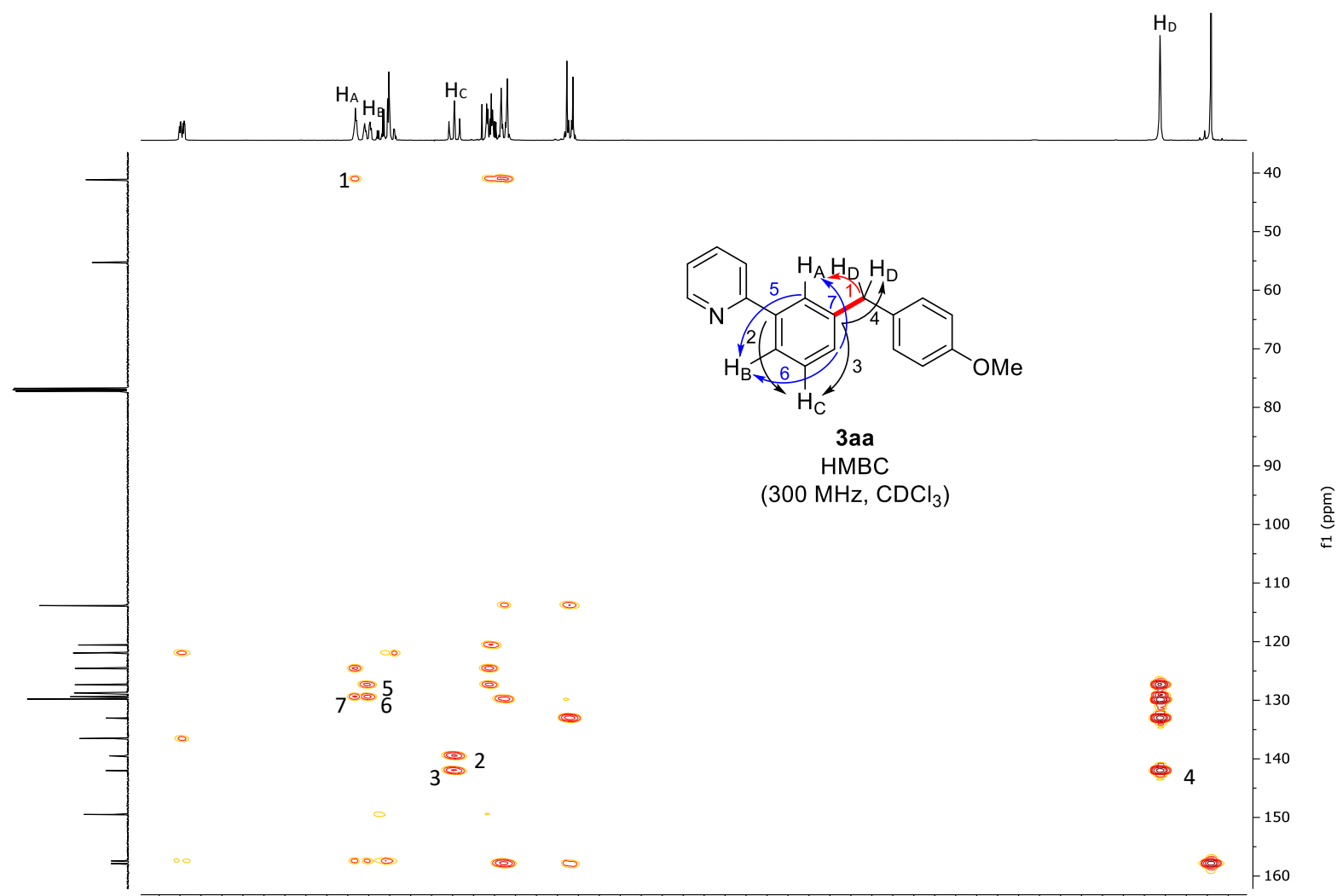

$\begin{array}{lllllllllllllllllllllllllllllllll}8.8 & 8.6 & 8.4 & 8.2 & 8.0 & 7.8 & 7.6 & 7.4 & 7.2 & 7.0 & 6.8 & 6.6 & 6.4 & 6.2 & 6.0 & 5.8 & 5.6 & 5.4 & 5.2 & 5.0 & 4.8 & 4.6 & 4.4 & 4.2 & 4.0 & 3.8\end{array}$

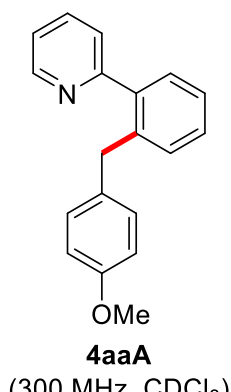

$\left(300 \mathrm{MHz} \mathrm{CDCl}_{3}\right)$

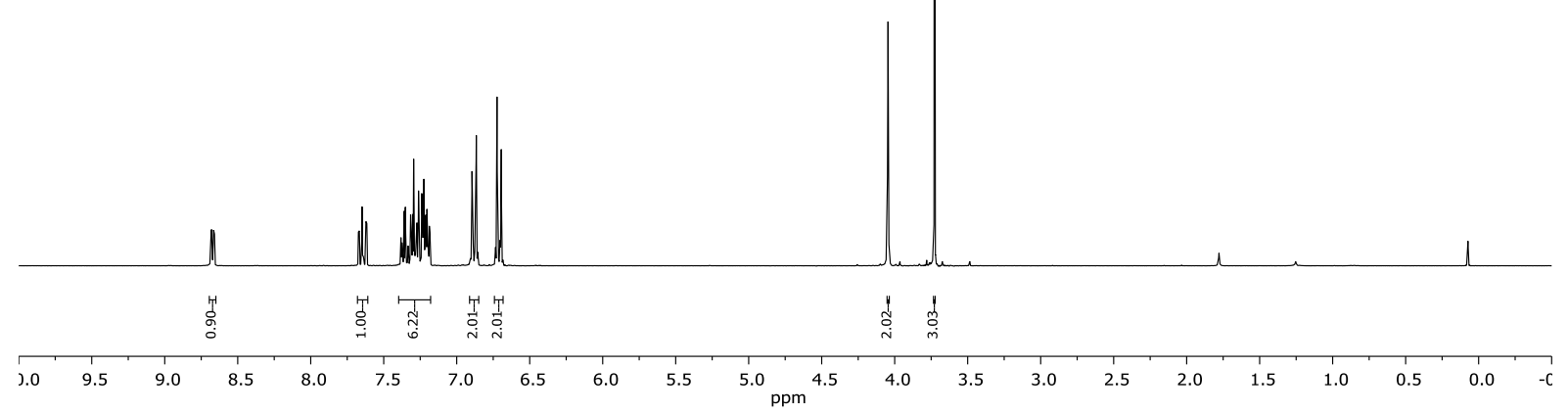



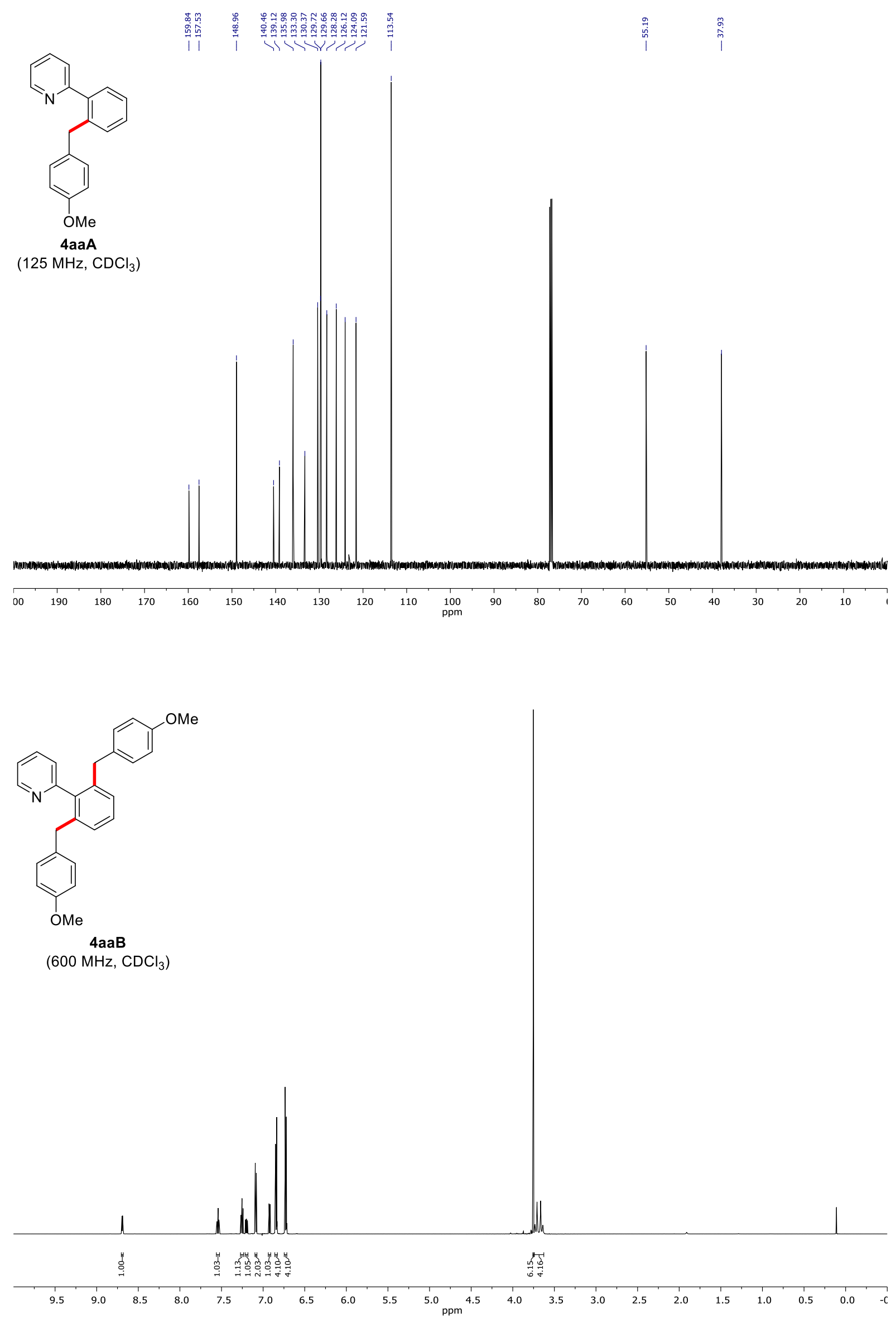

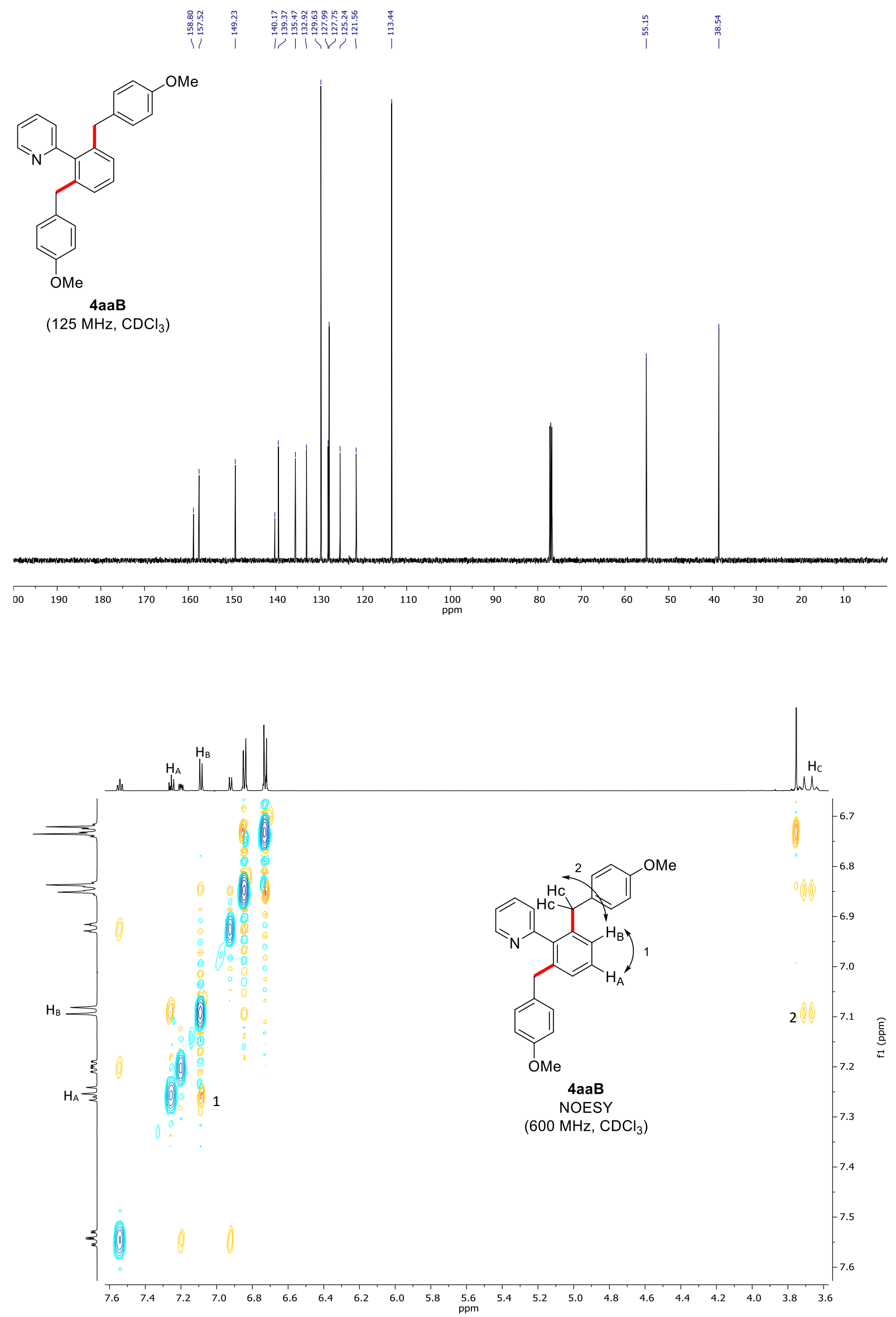

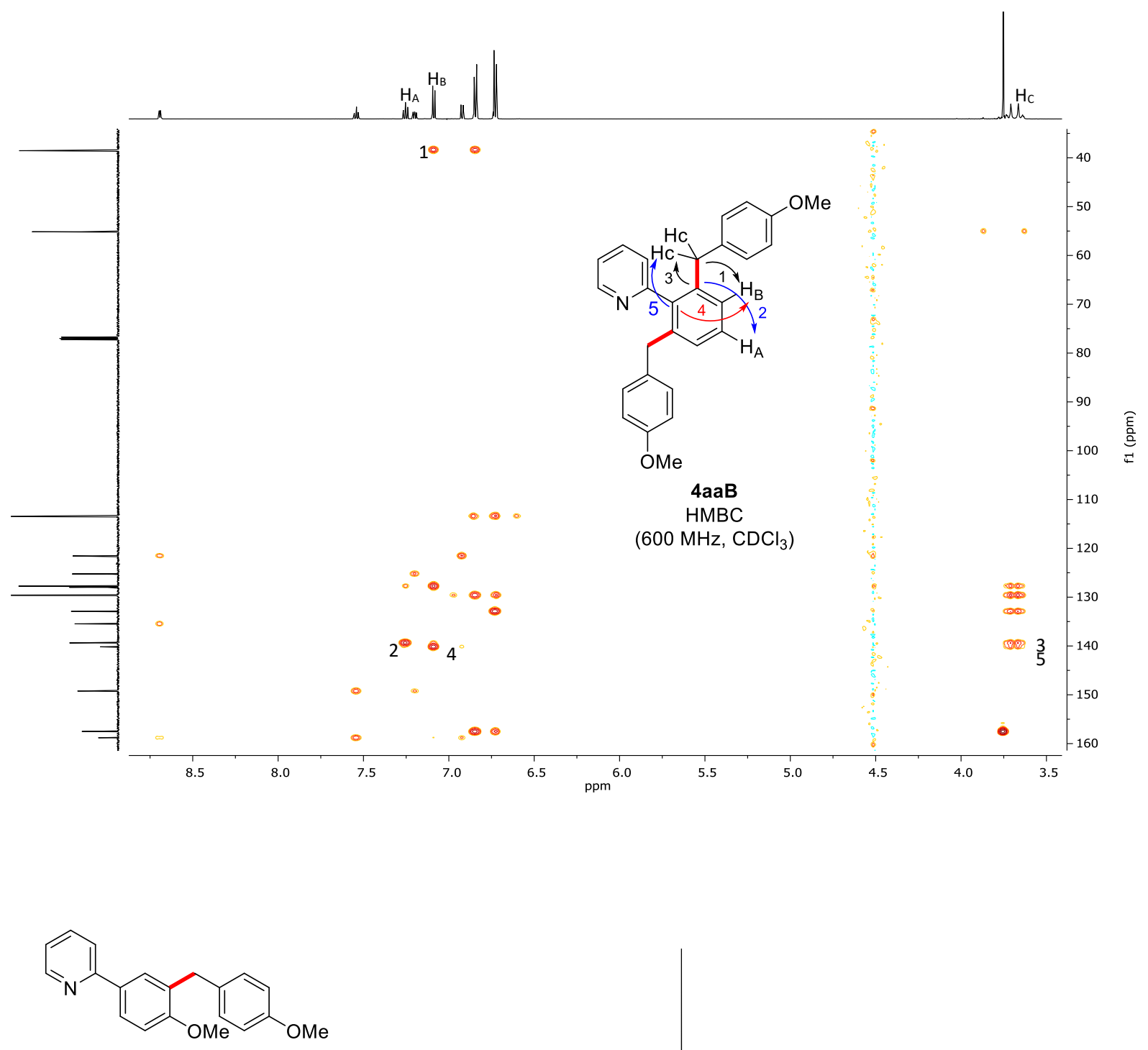

$3 \mathrm{ba}$

(400 MHz, $\mathrm{CDCl}_{3}$ )

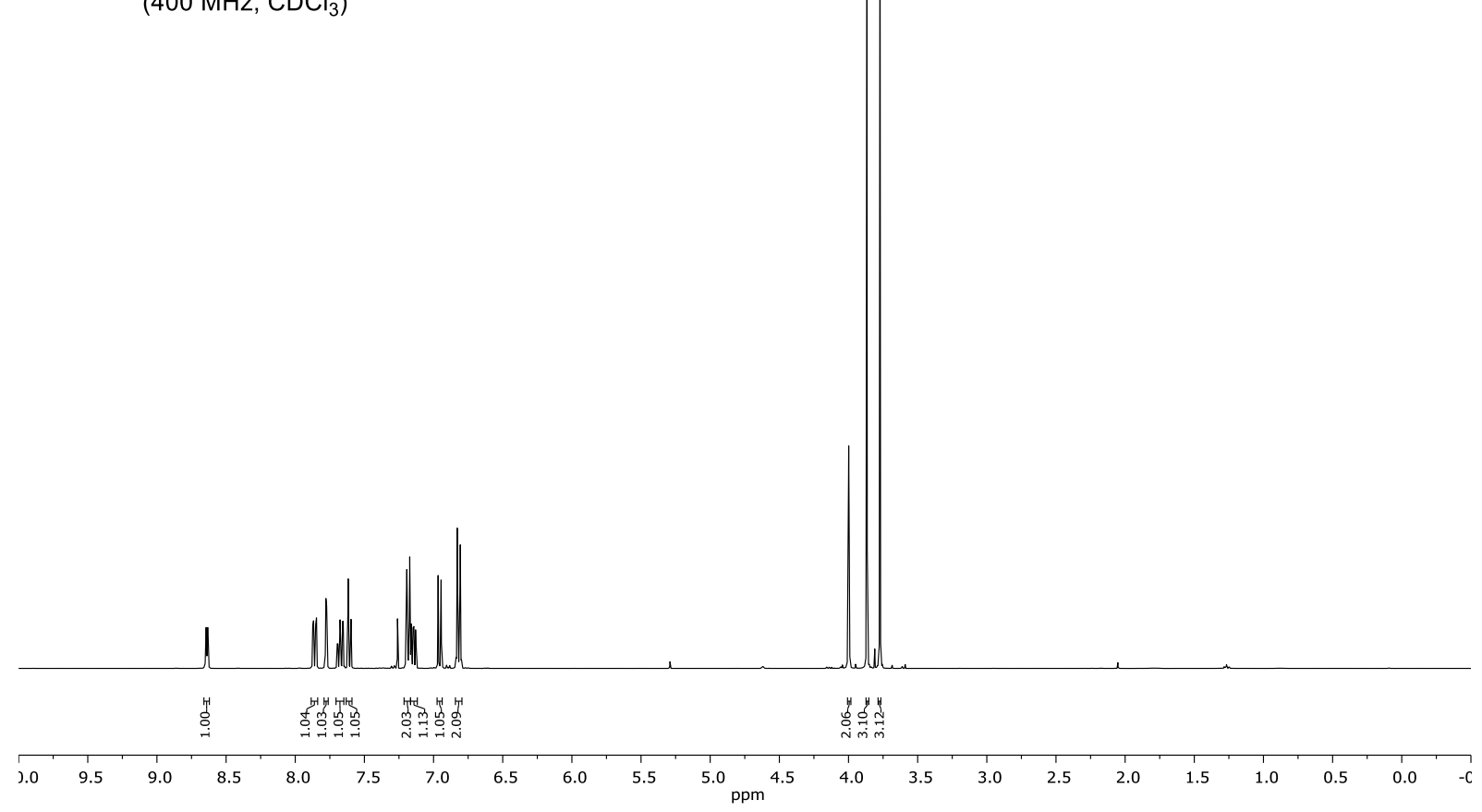




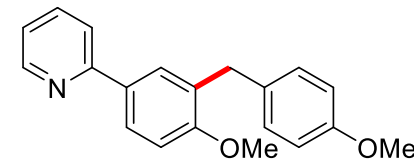

3 ba

(100 MHz, $\mathrm{CDCl}_{3}$ )

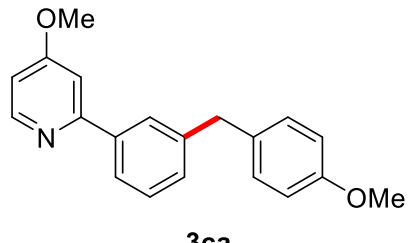

(300 MHz, $\mathrm{CDCl}_{3}$ )

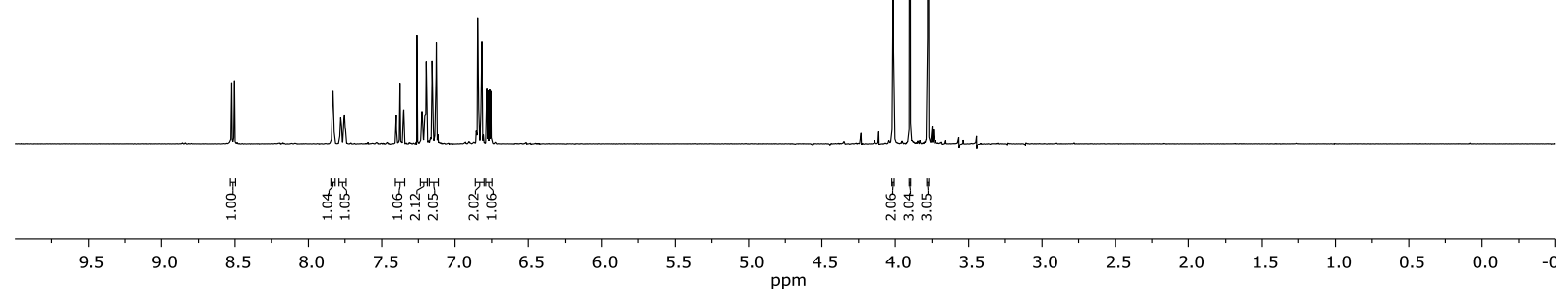




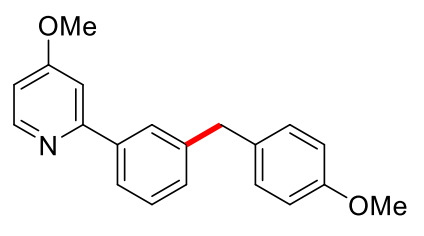

$3 \mathrm{ca}$

$\left(125 \mathrm{MHz}, \mathrm{CDCl}_{3}\right)$

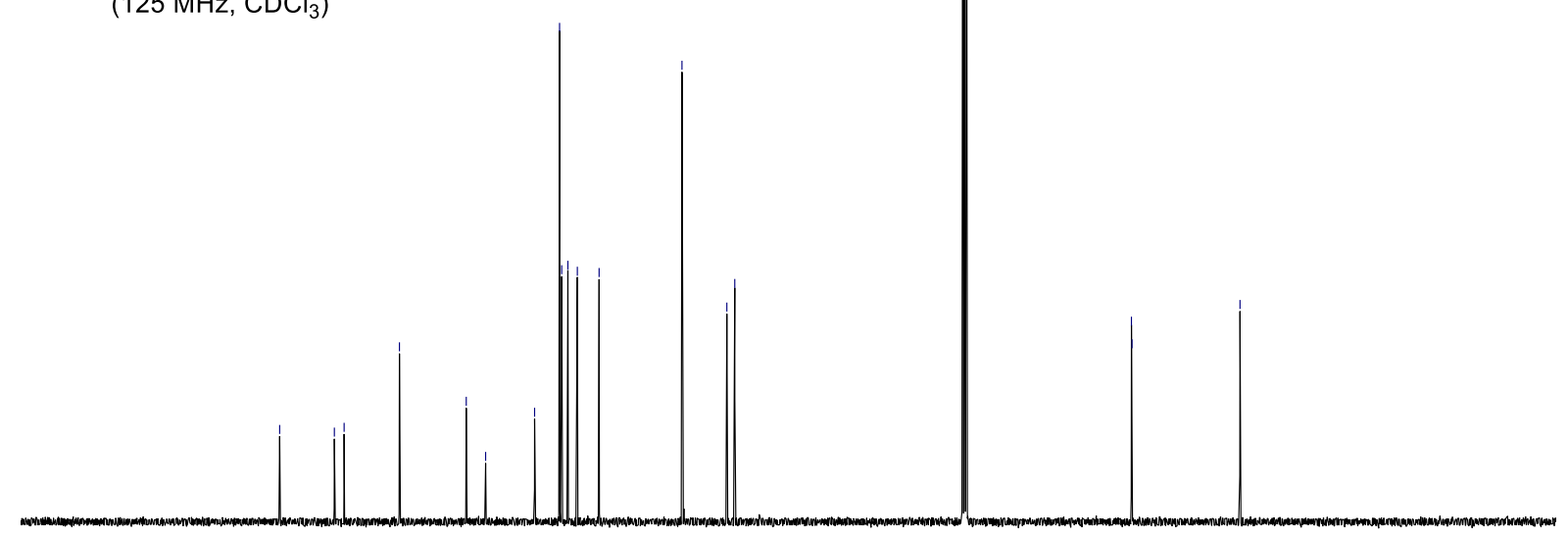

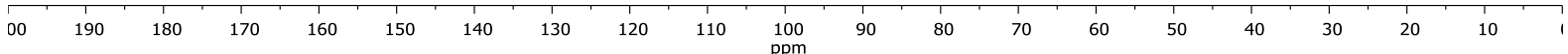

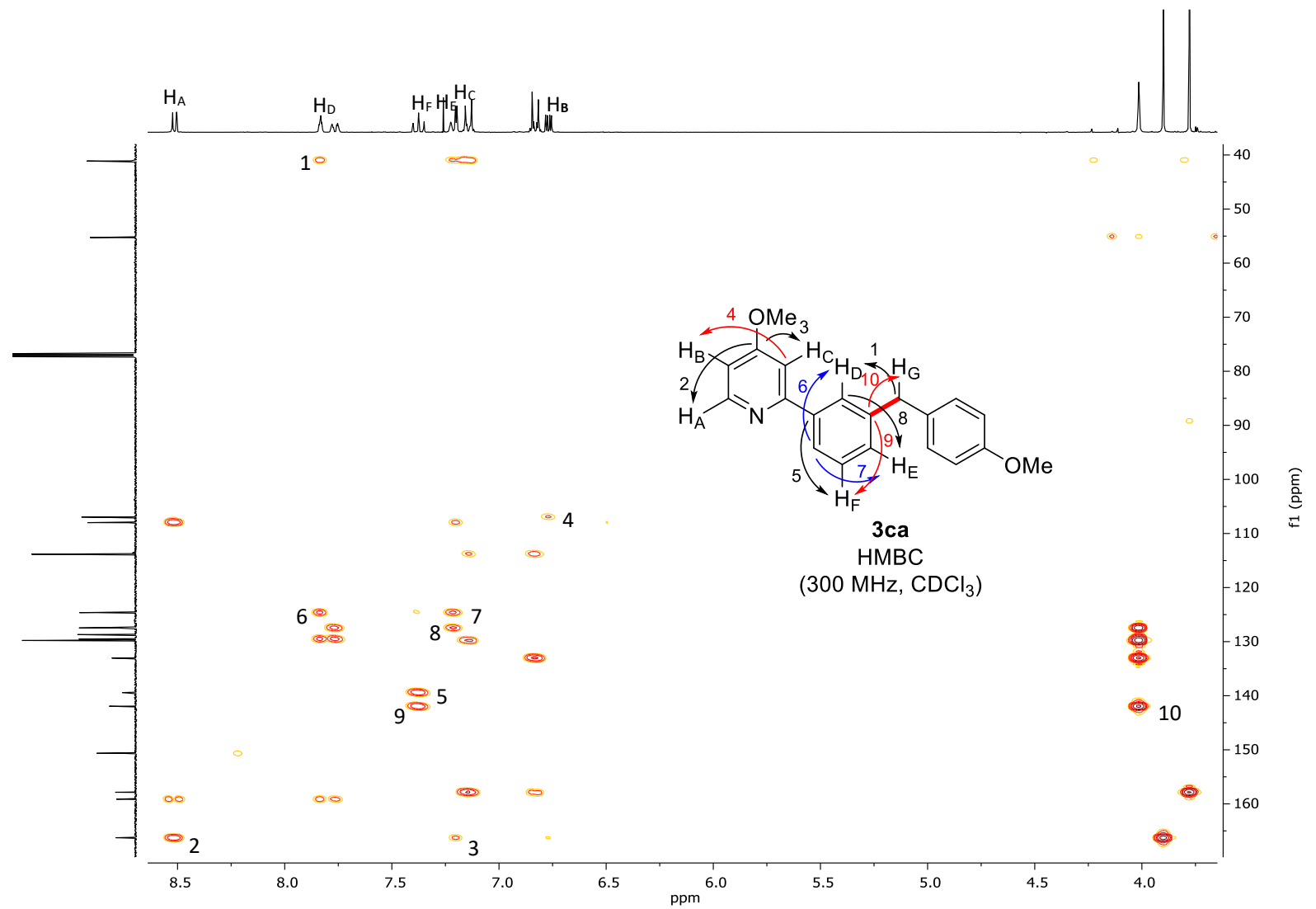



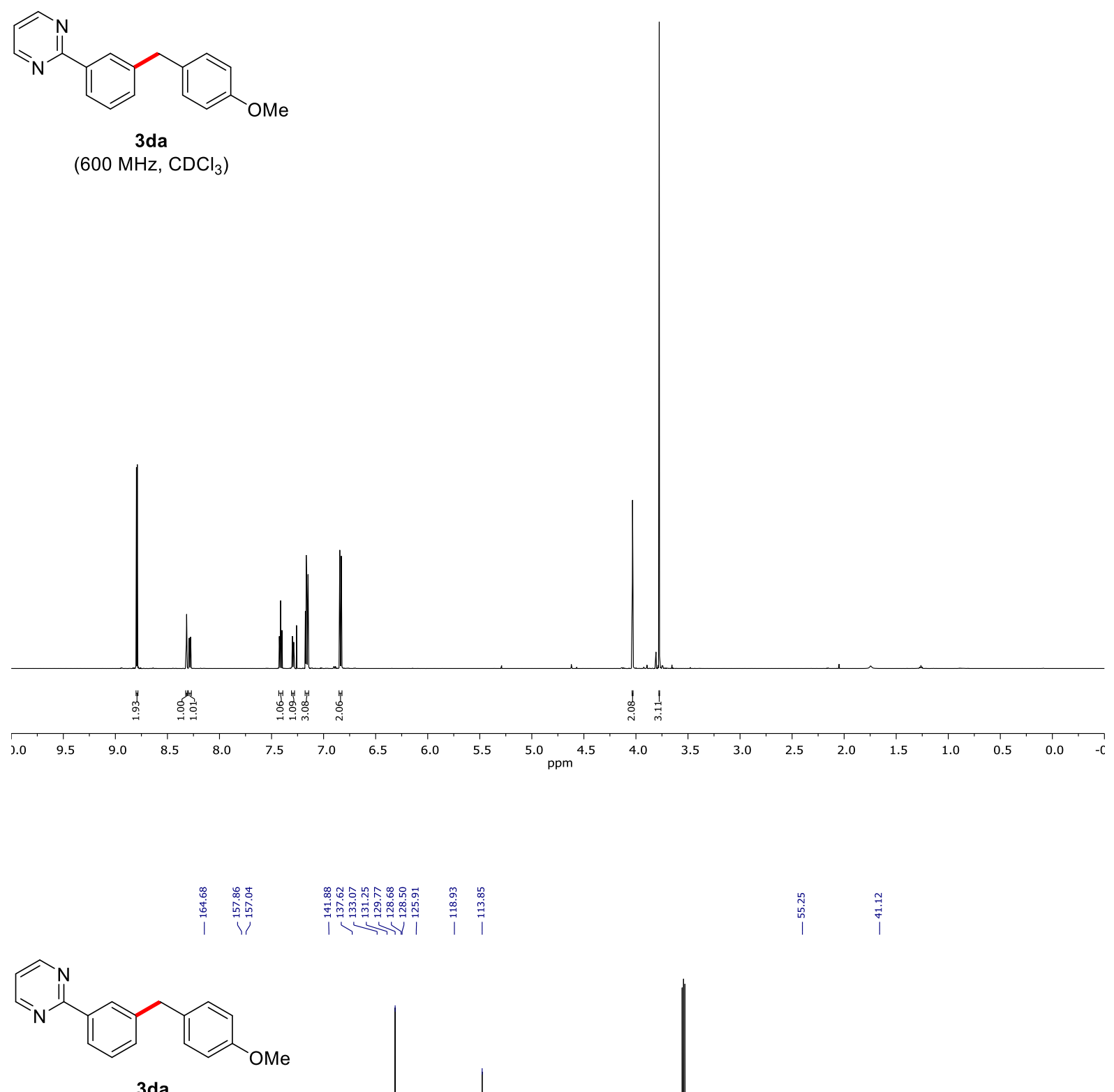

(125 $\mathrm{MHz}, \mathrm{CDCl}_{3}$ )

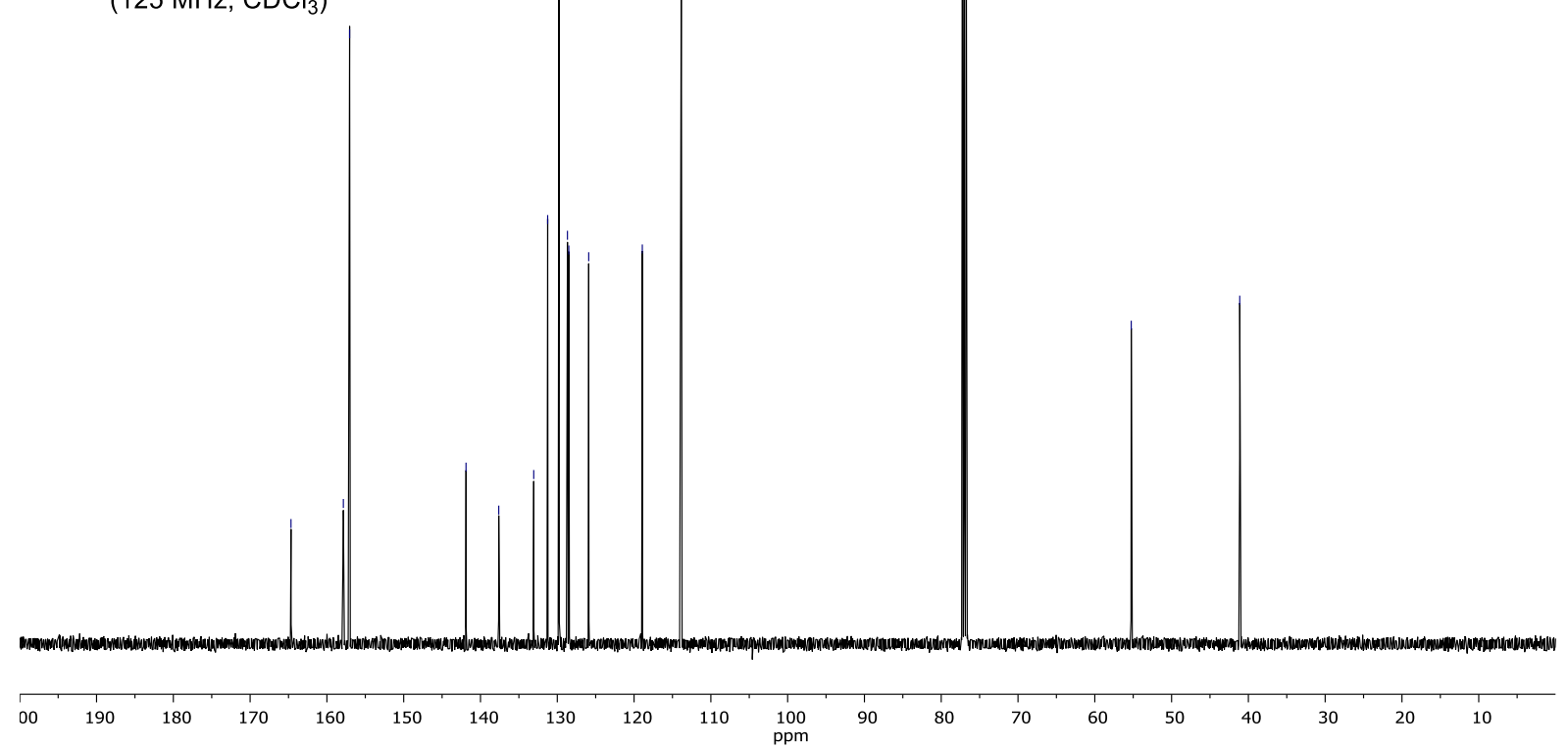



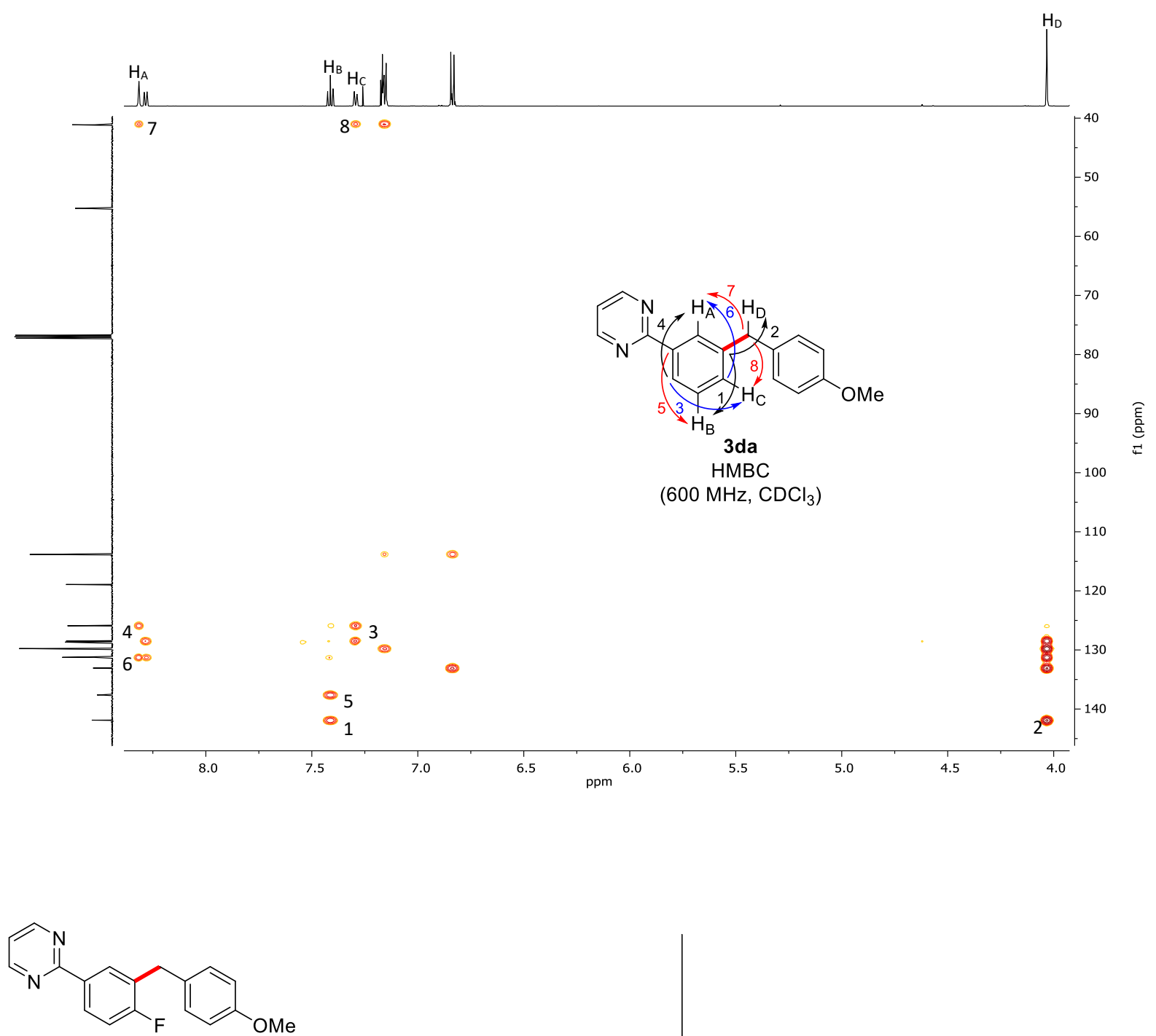

$3 e a$

$\left(300 \mathrm{MHz}, \mathrm{CDCl}_{3}\right)$

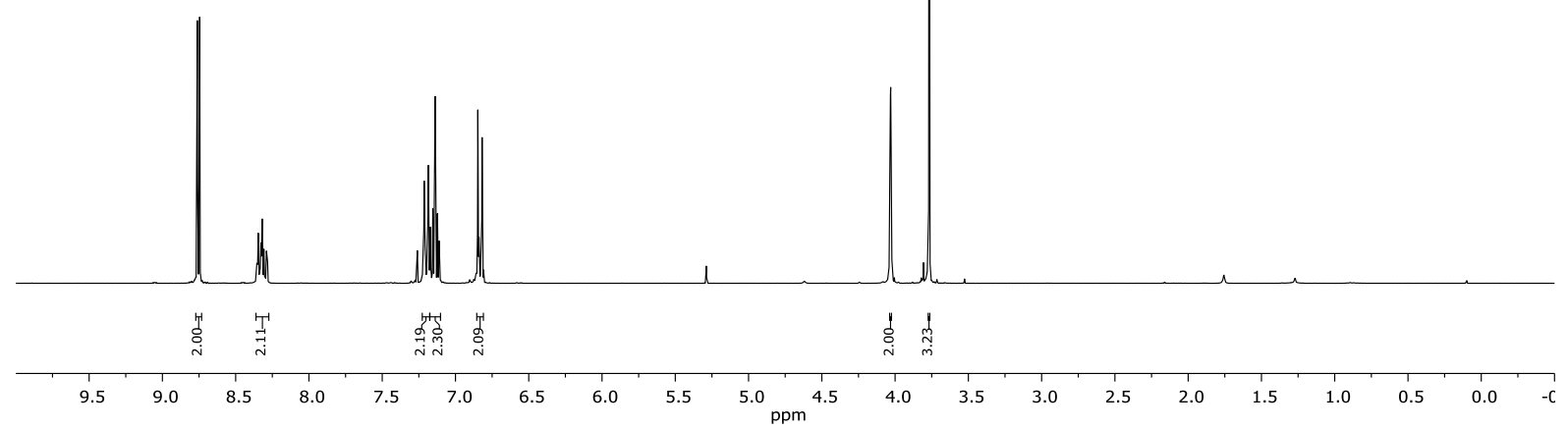



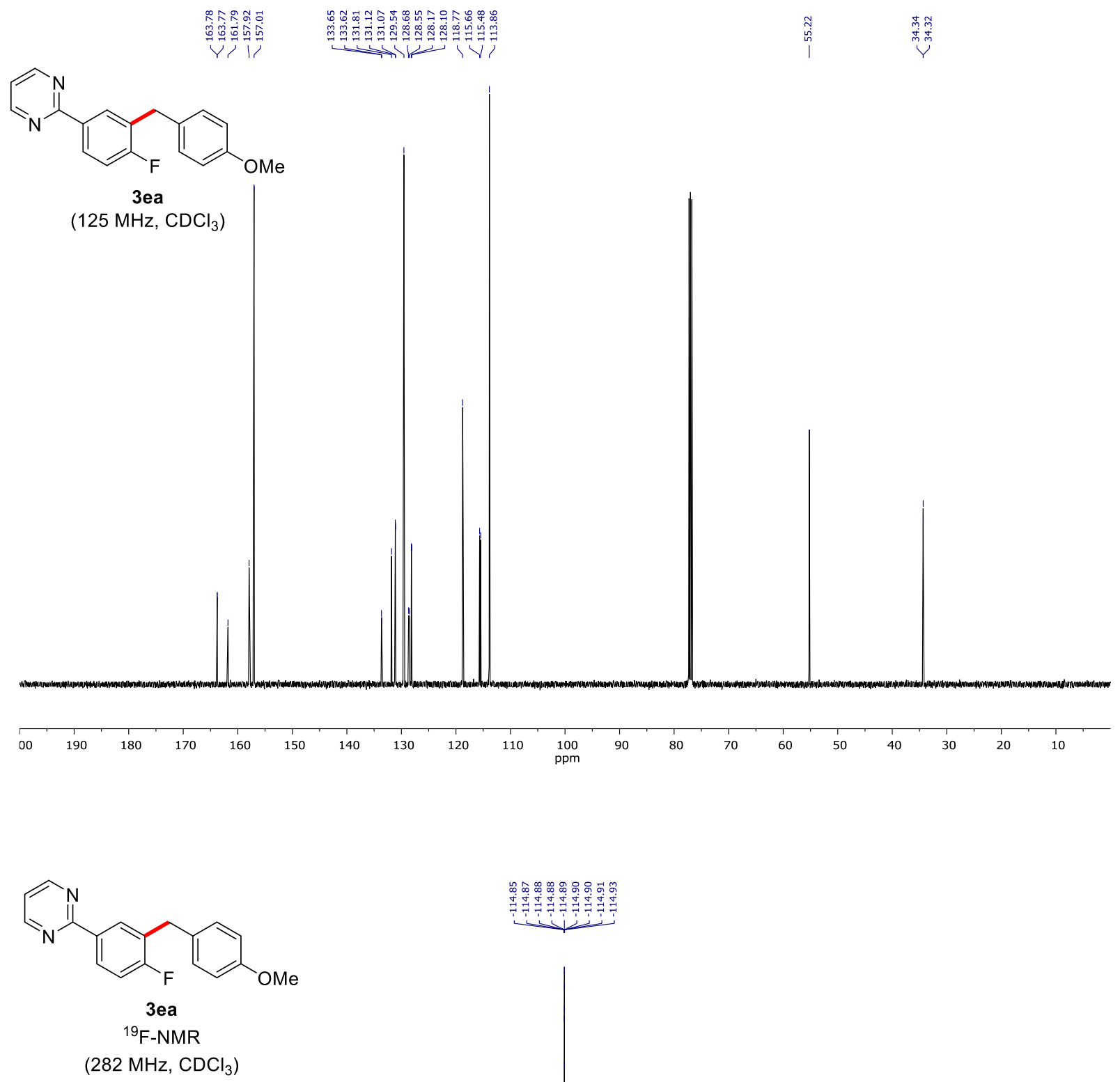

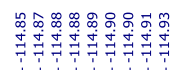

$282 \mathrm{MHz}, \mathrm{CDCl}_{3}$ 


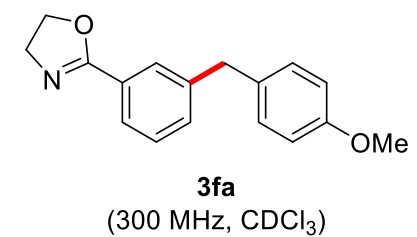

$\left(300 \mathrm{MHz}, \mathrm{CDCl}_{3}\right)$
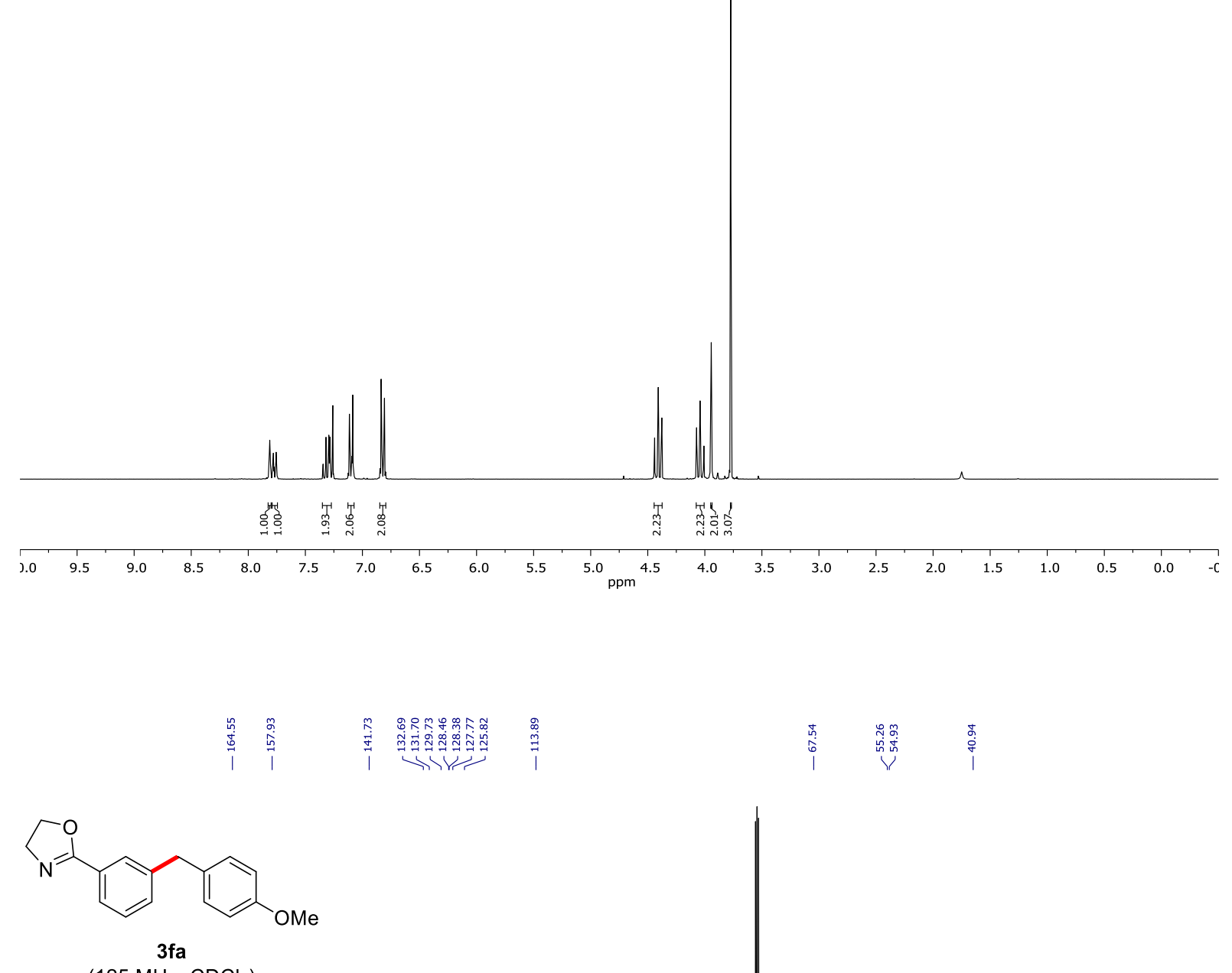

(125 MHz, $\mathrm{CDCl}_{3}$ )

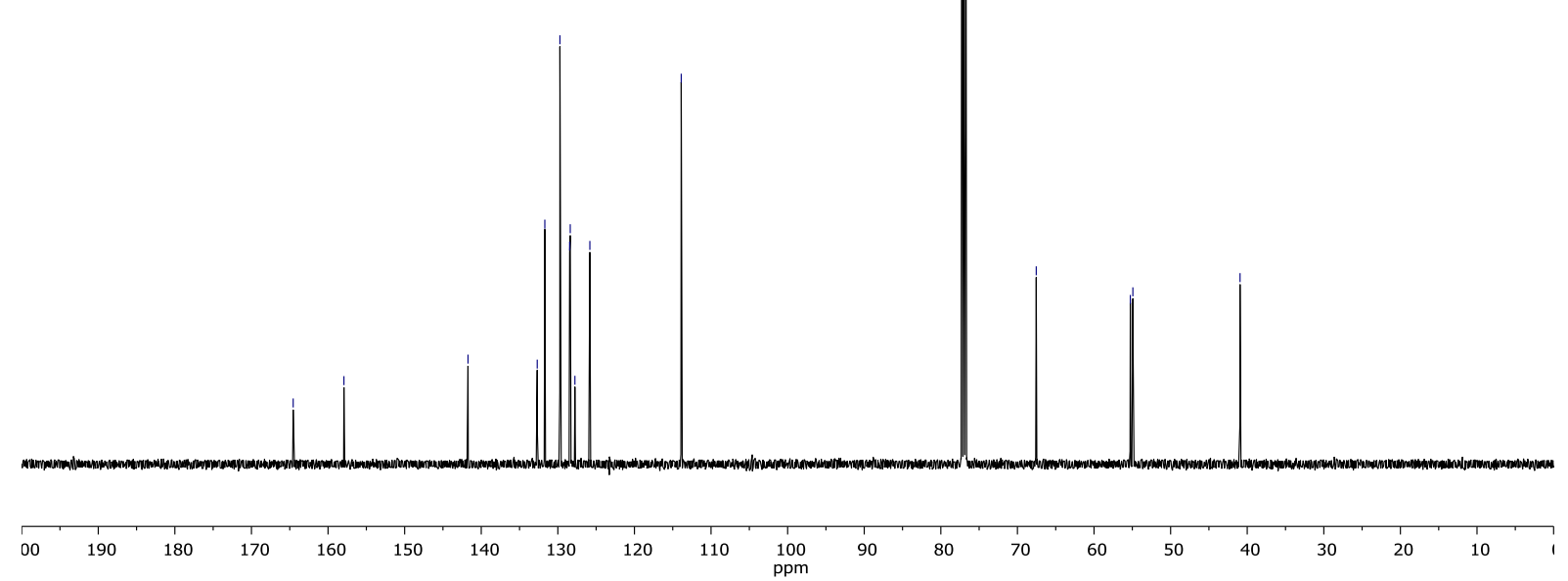




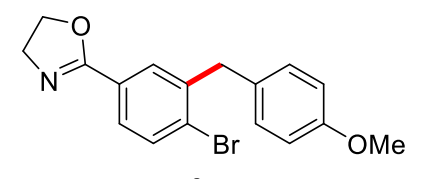

3 ga

(300 MHz, $\mathrm{CDCl}_{3}$ )

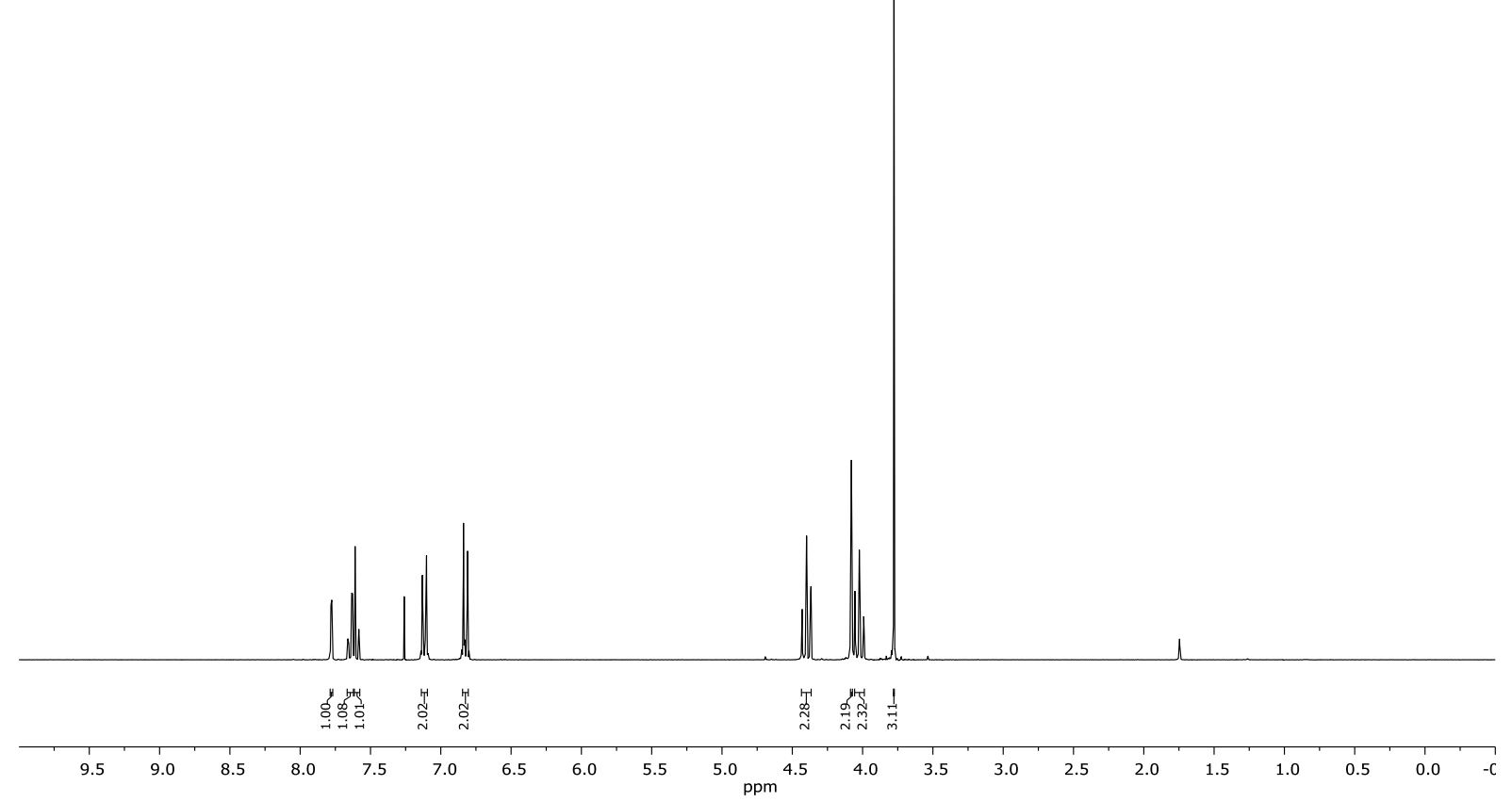

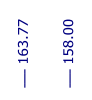

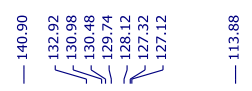

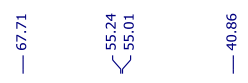

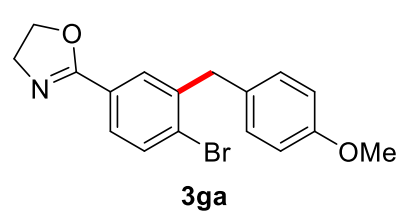

(125 MHz, $\mathrm{CDCl}_{3}$ )

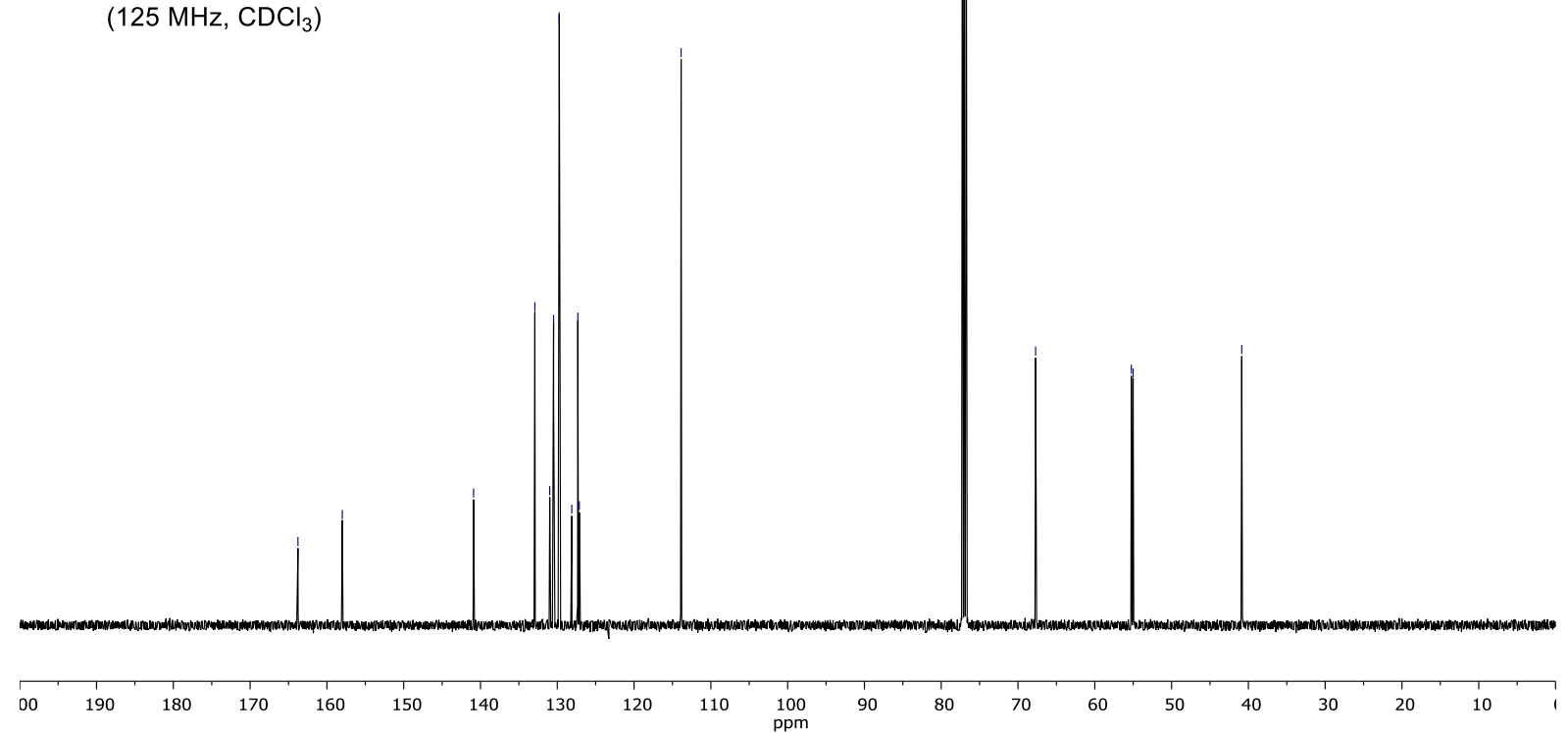



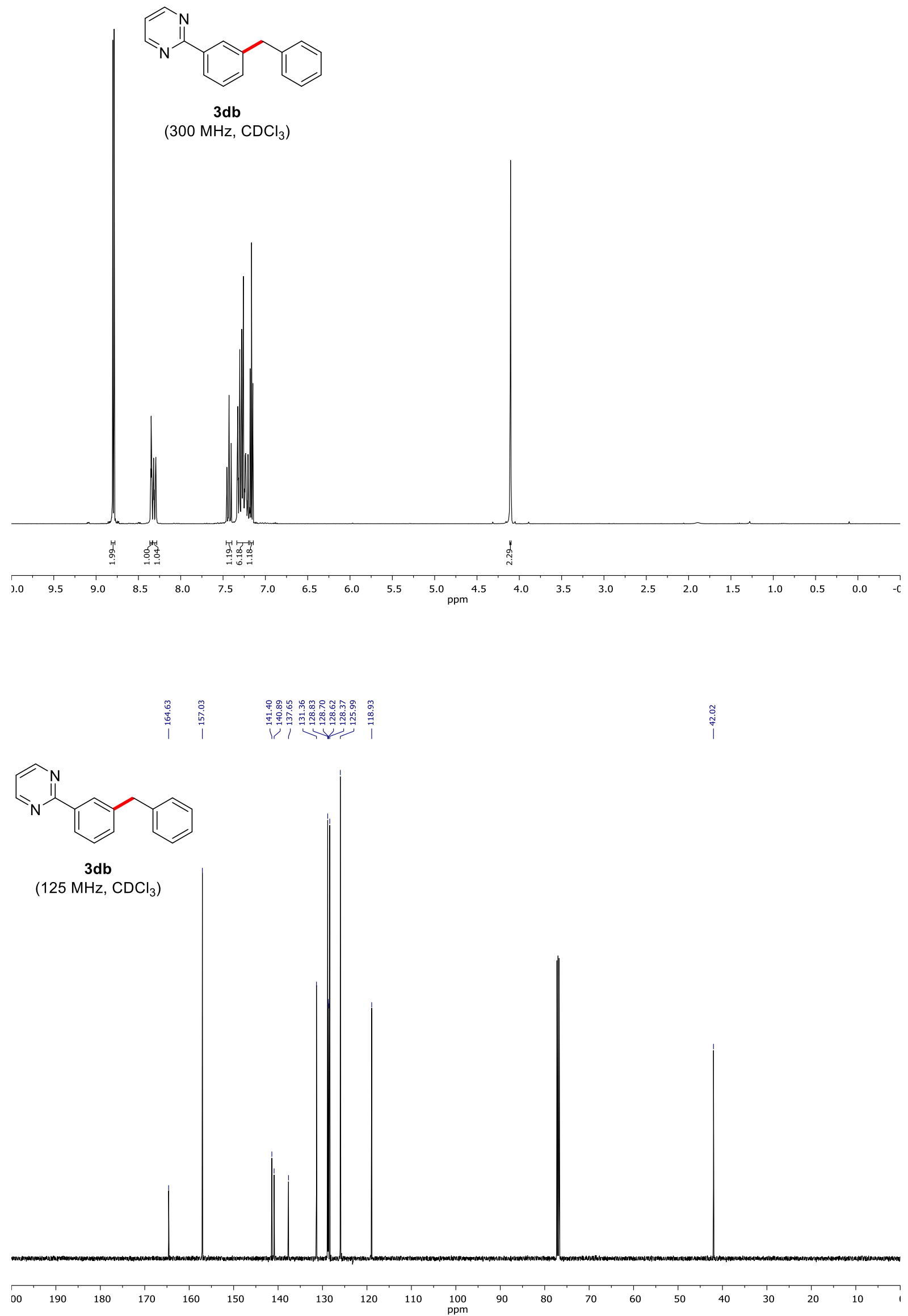


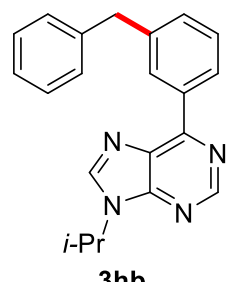

(300 MHz, $\mathrm{CDCl}_{3}$ )
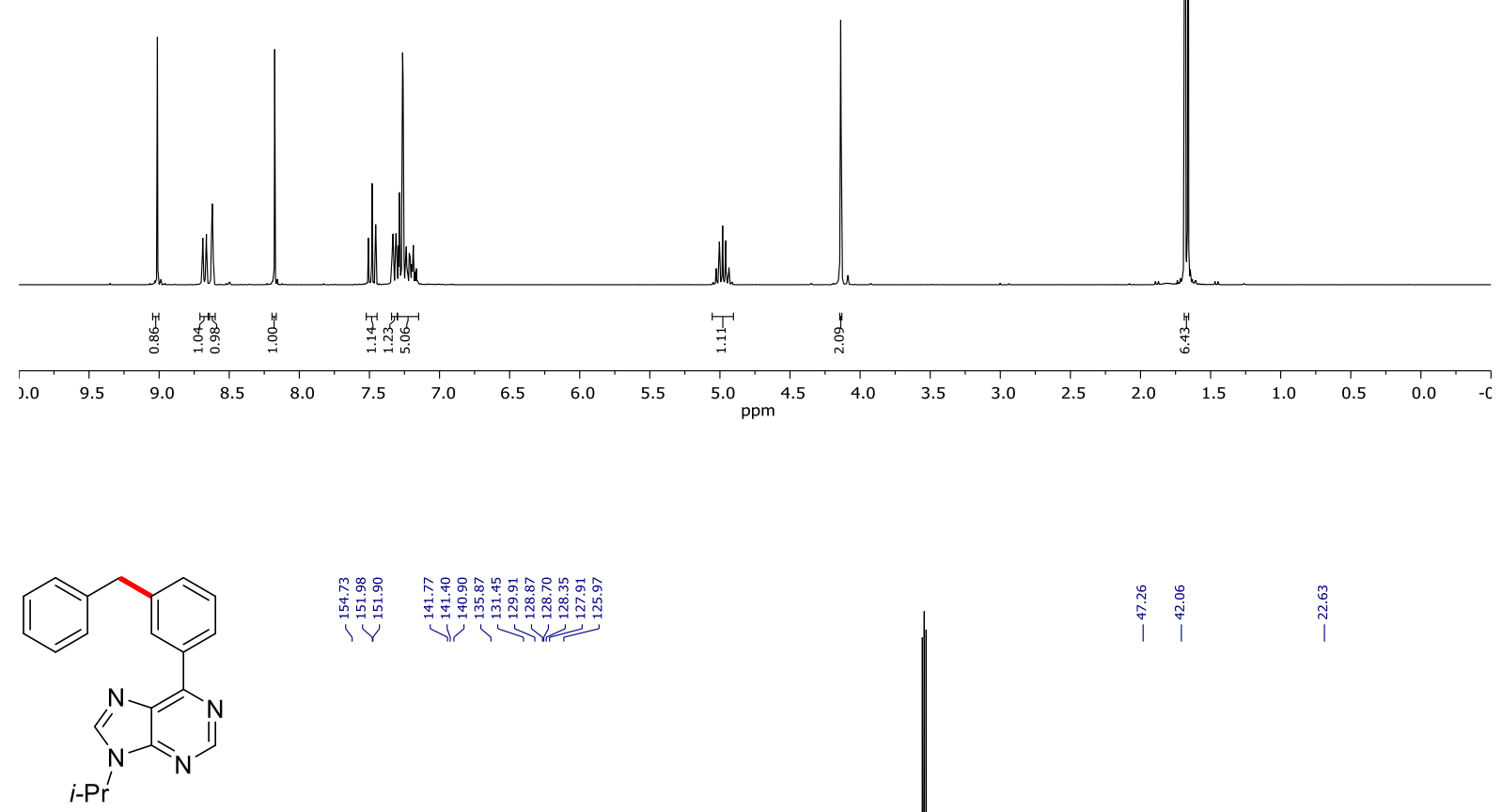

$3 \mathrm{hb}$

(125 $\mathrm{MHz} \mathrm{CDCl}_{3}$ )

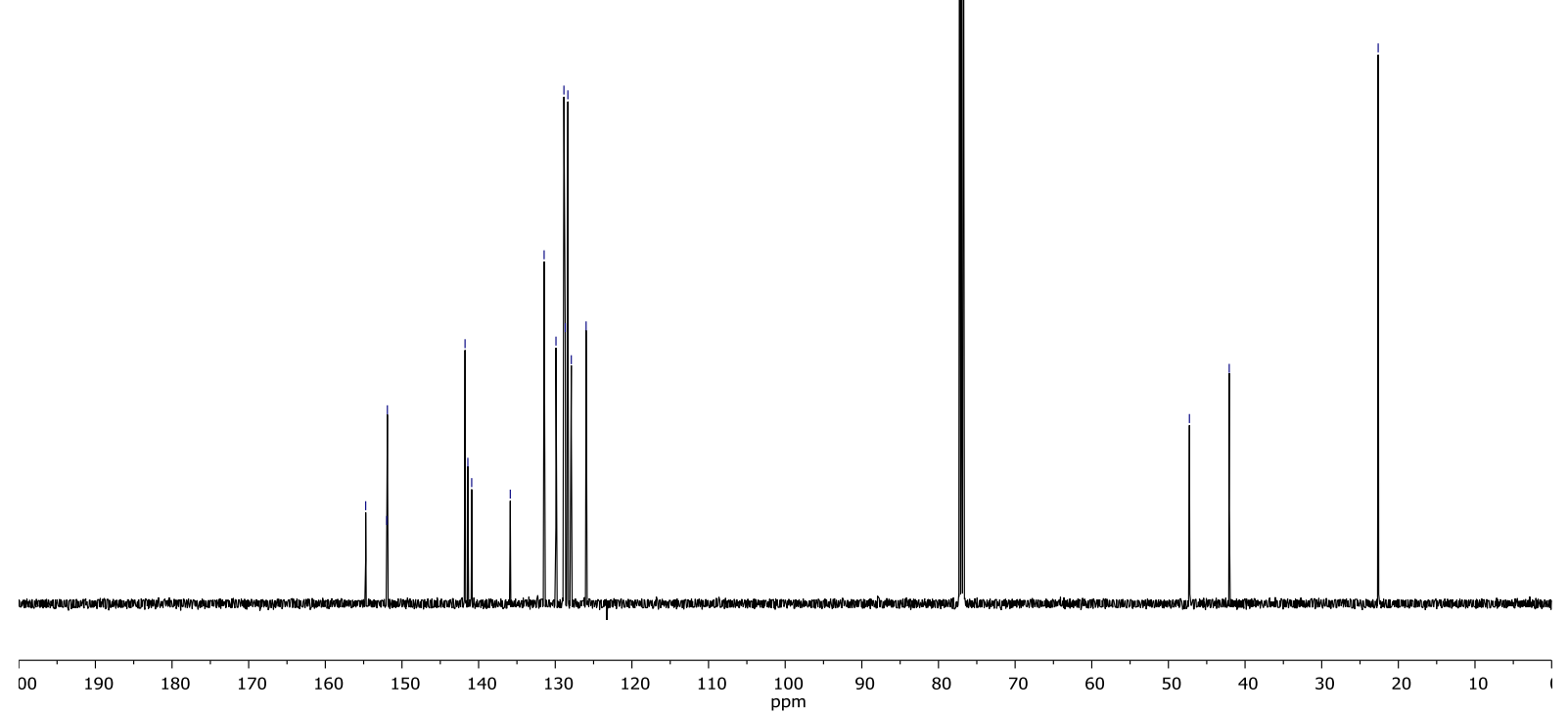




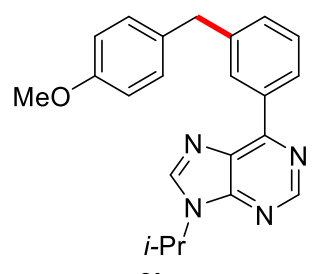

3ha

(300 MHz, $\mathrm{CDCl}_{3}$ )
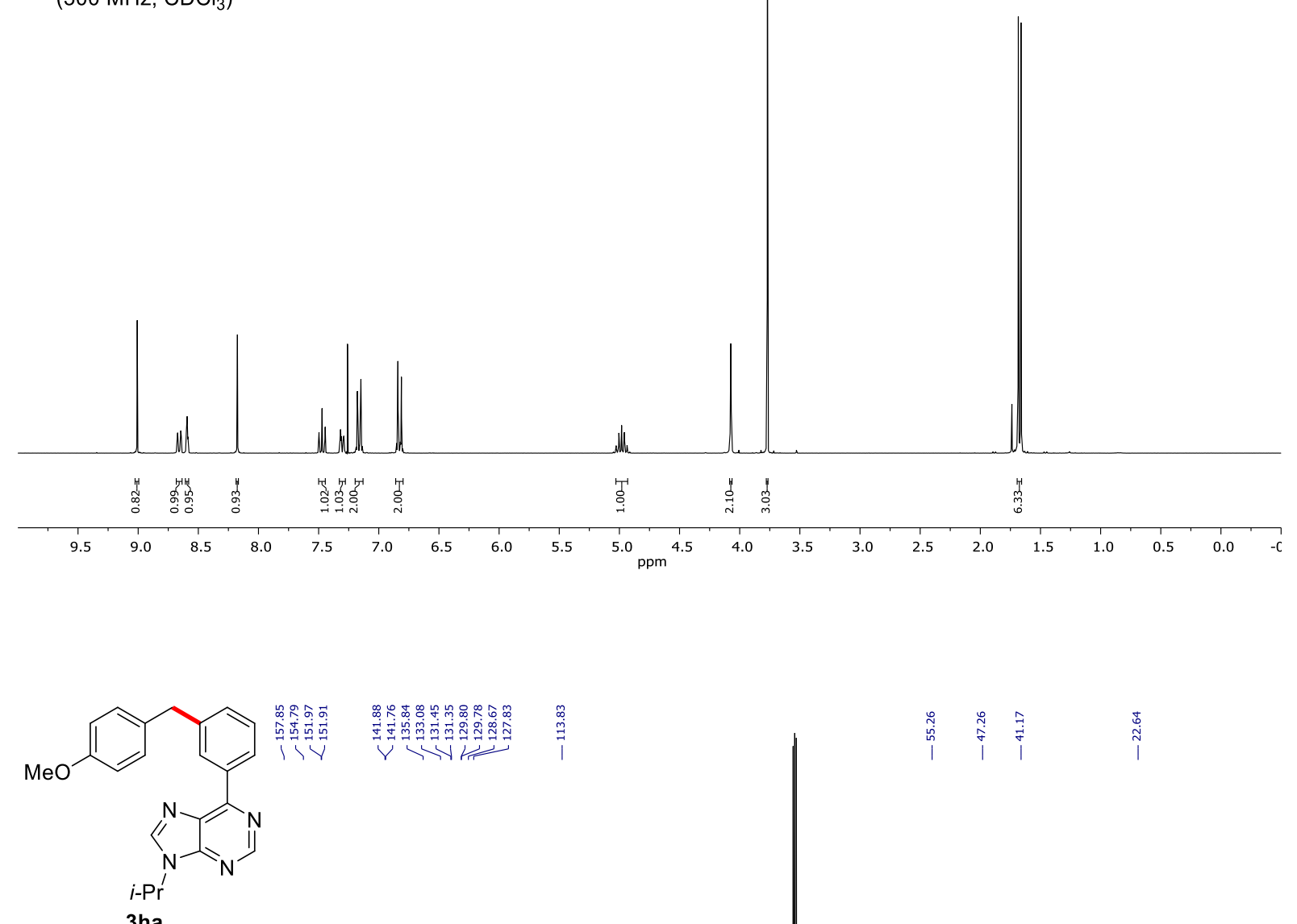

(125 MHz, $\mathrm{CDCl}_{3}$ )

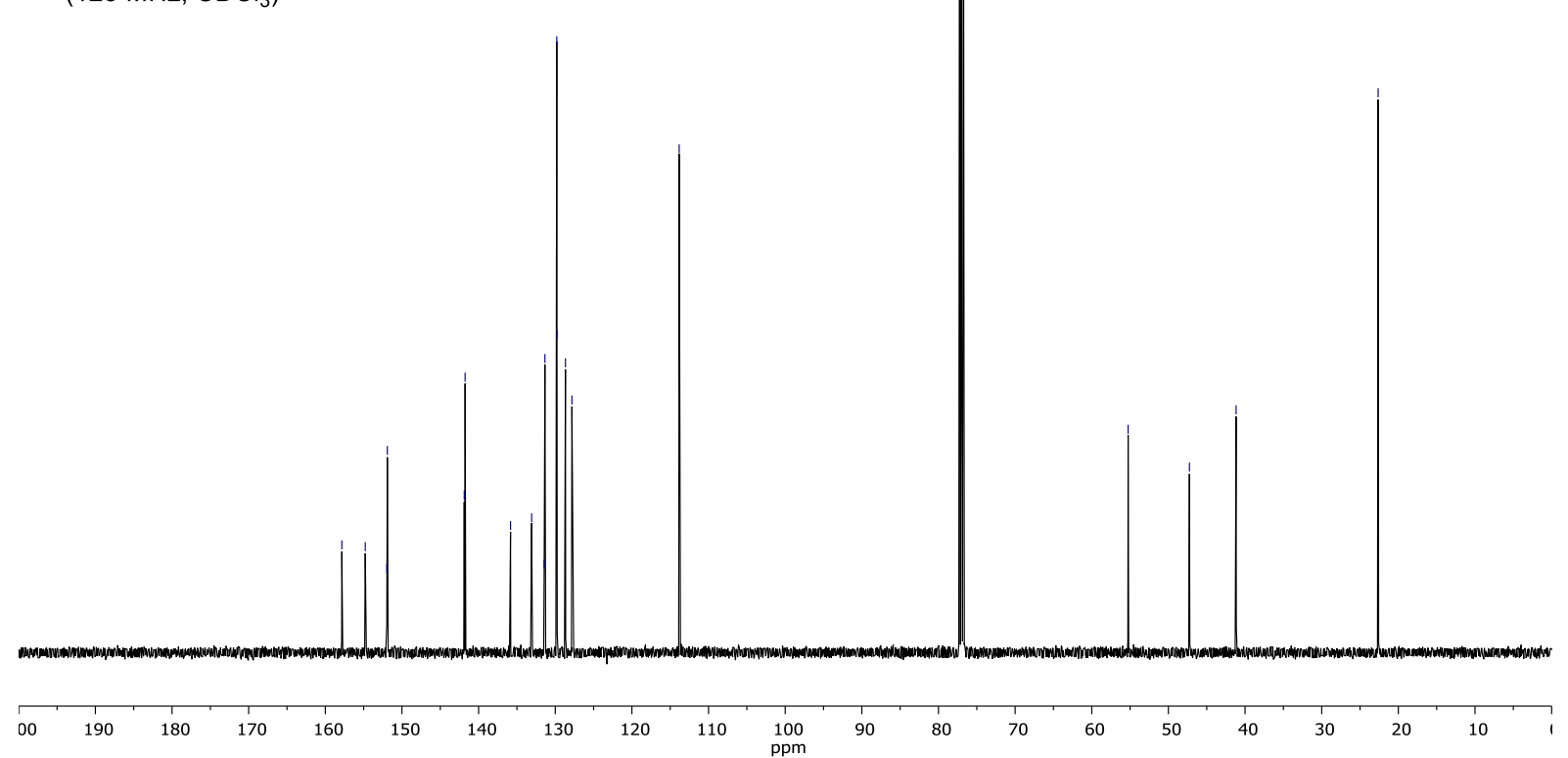



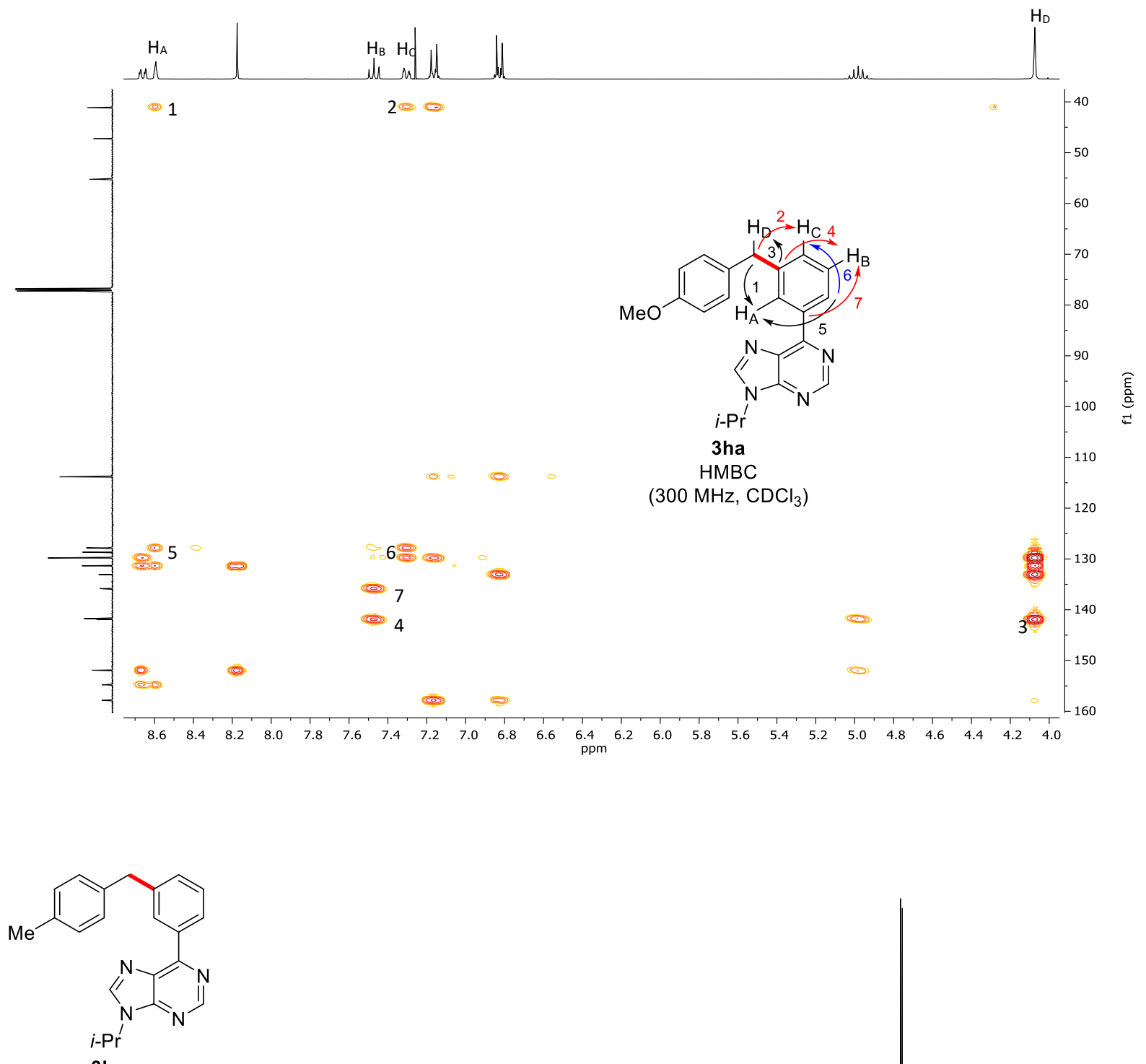

3hc

(400 $\mathrm{MHz} \mathrm{CDCl}_{3}$ )

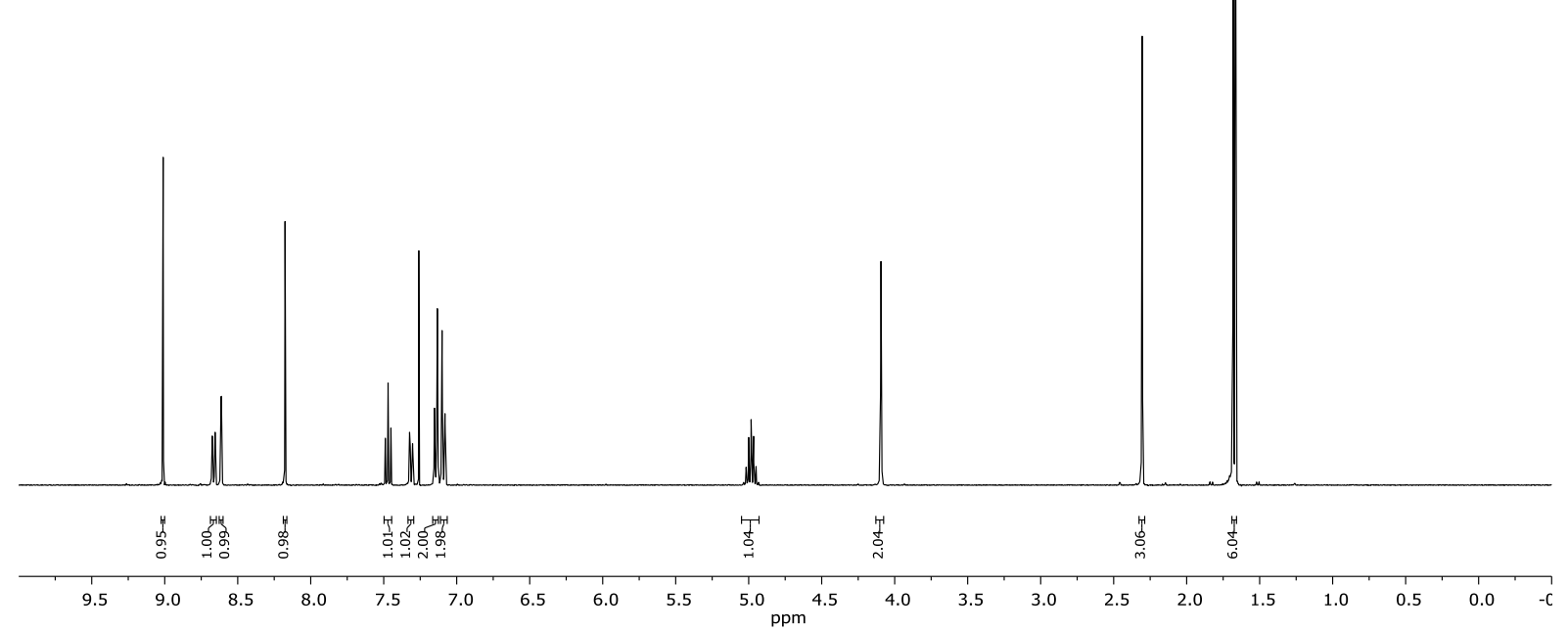



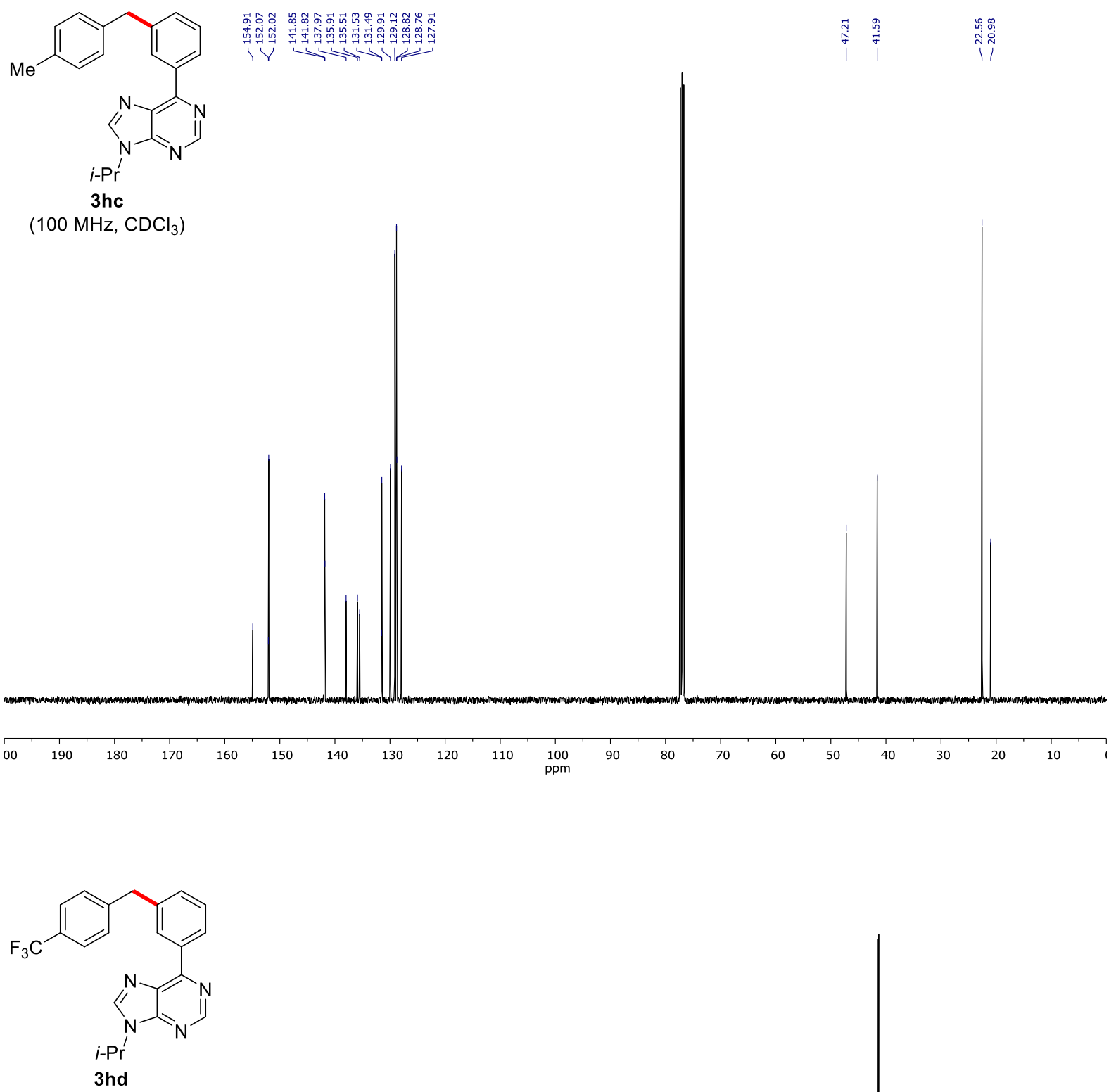

(500 MHz, $\mathrm{CDCl}_{3}$ )

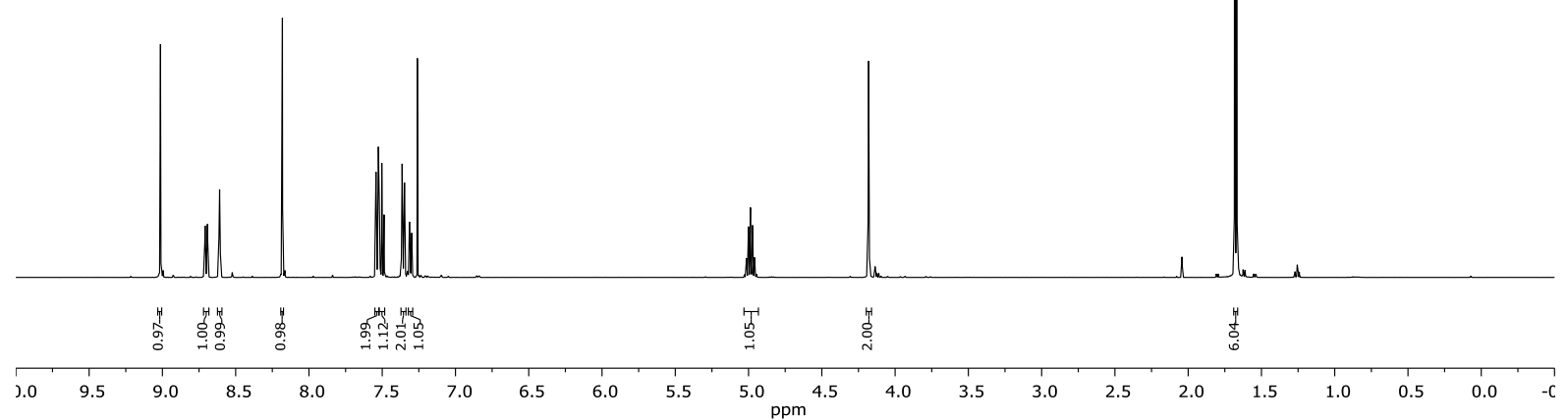



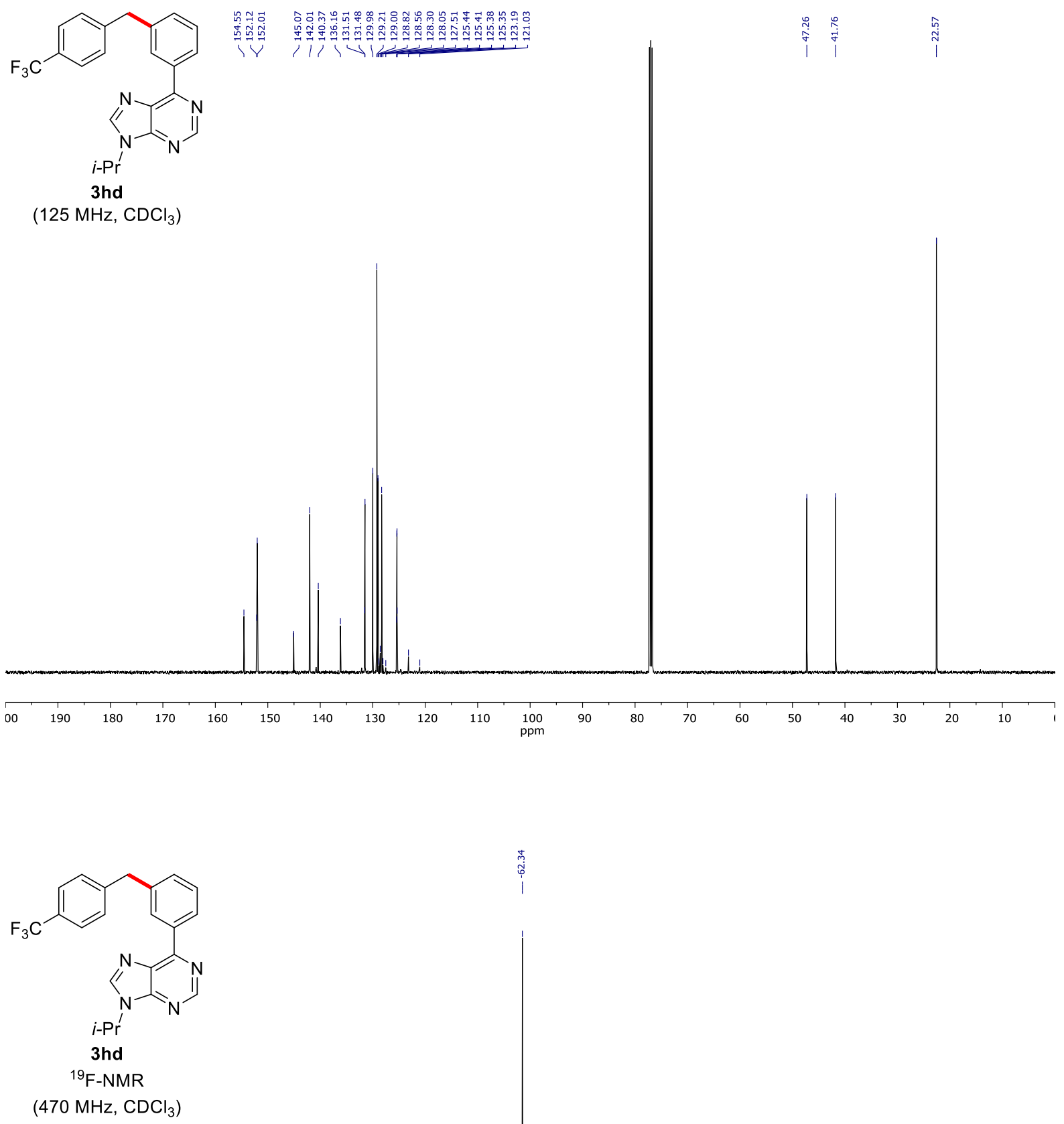

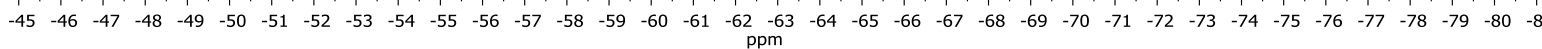




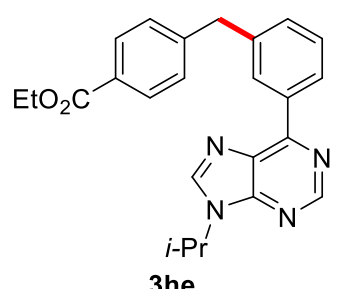

(300 $\mathrm{MHz}, \mathrm{CDCl}_{3}$ )

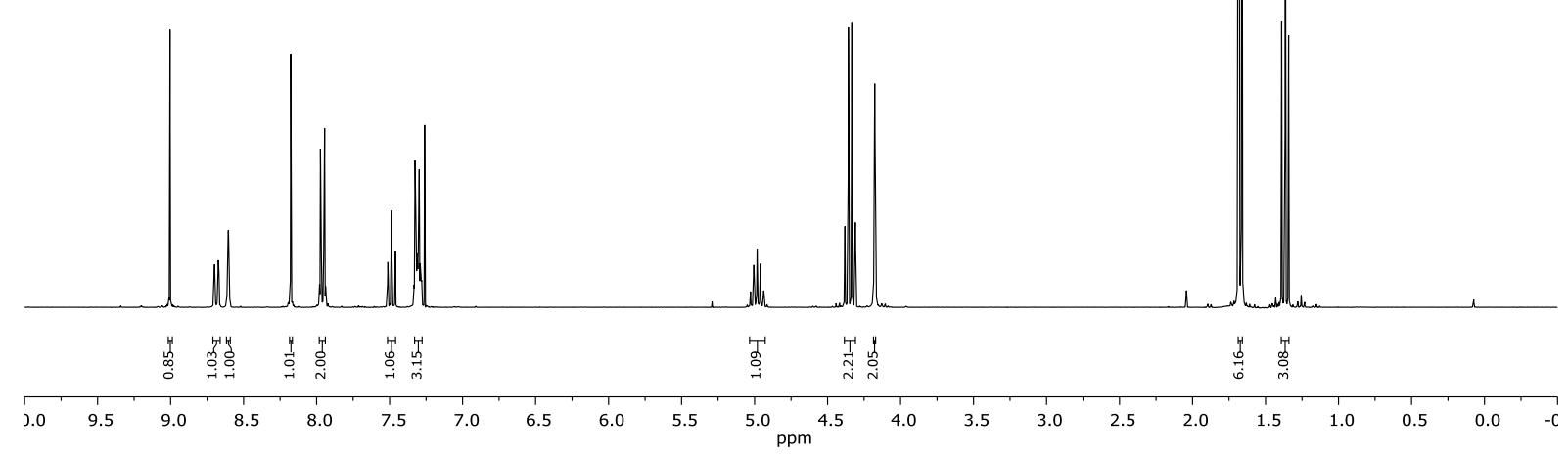

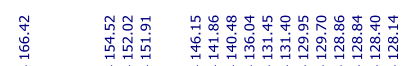

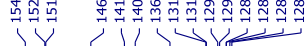

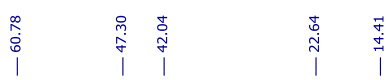

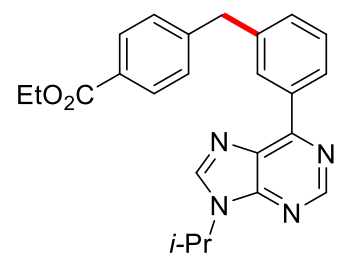

3he

(125 MHz, $\mathrm{CDCl}_{3}$ )

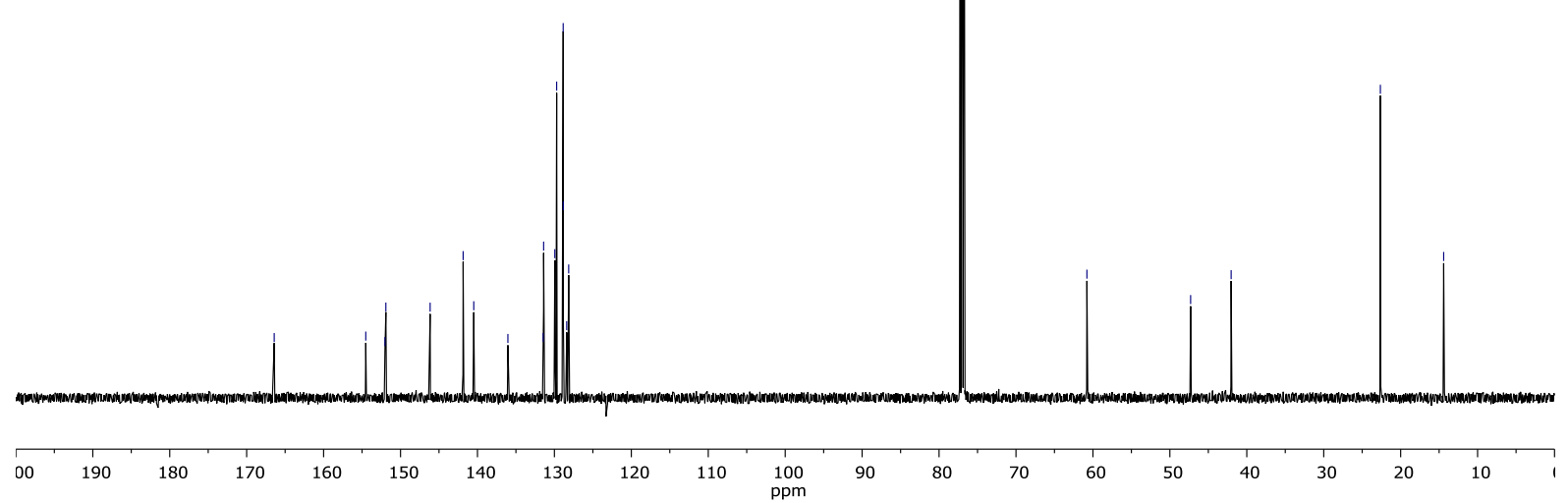




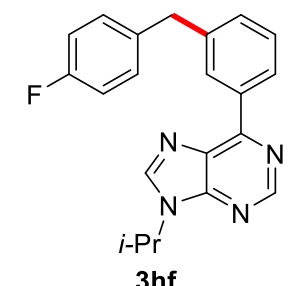

$\left(600 \mathrm{MHz}, \mathrm{CDCl}_{3}\right)$

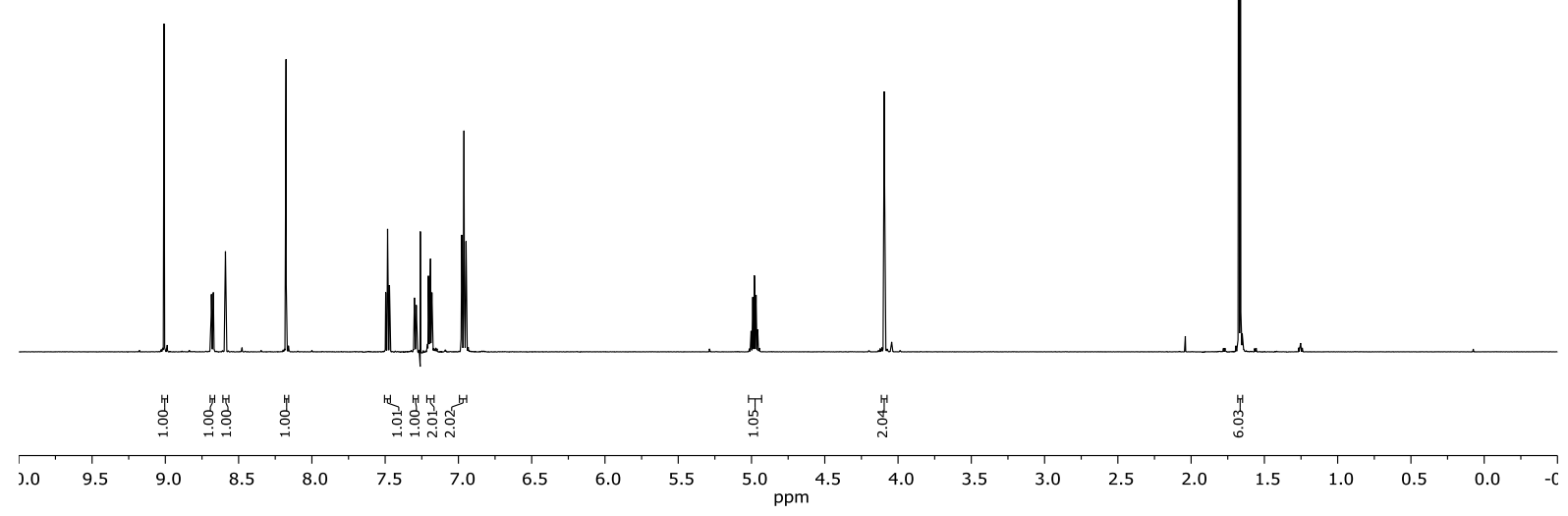

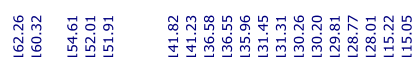

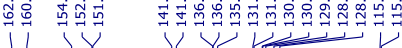

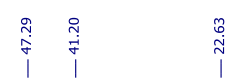<smiles>Fc1ccc(Cc2cccc(-c3ncnc4c3ncn4C(F)(F)F)c2)cc1</smiles>

$3 \mathrm{hf}$

(125 MHz, $\mathrm{CDCl}_{3}$ )

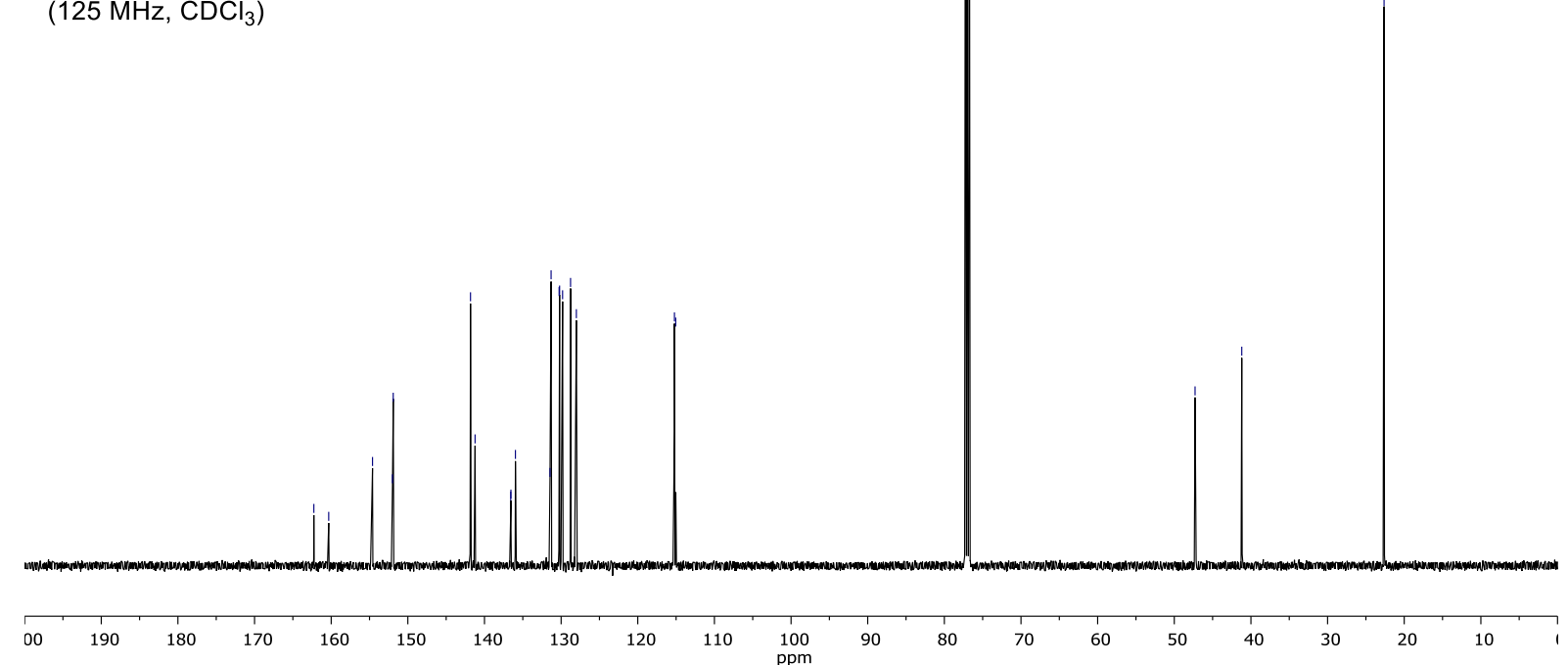




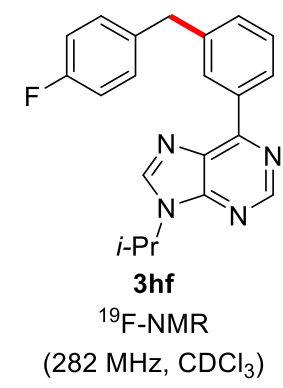

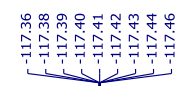

(282 MHz, $\mathrm{CDCl}_{3}$ )

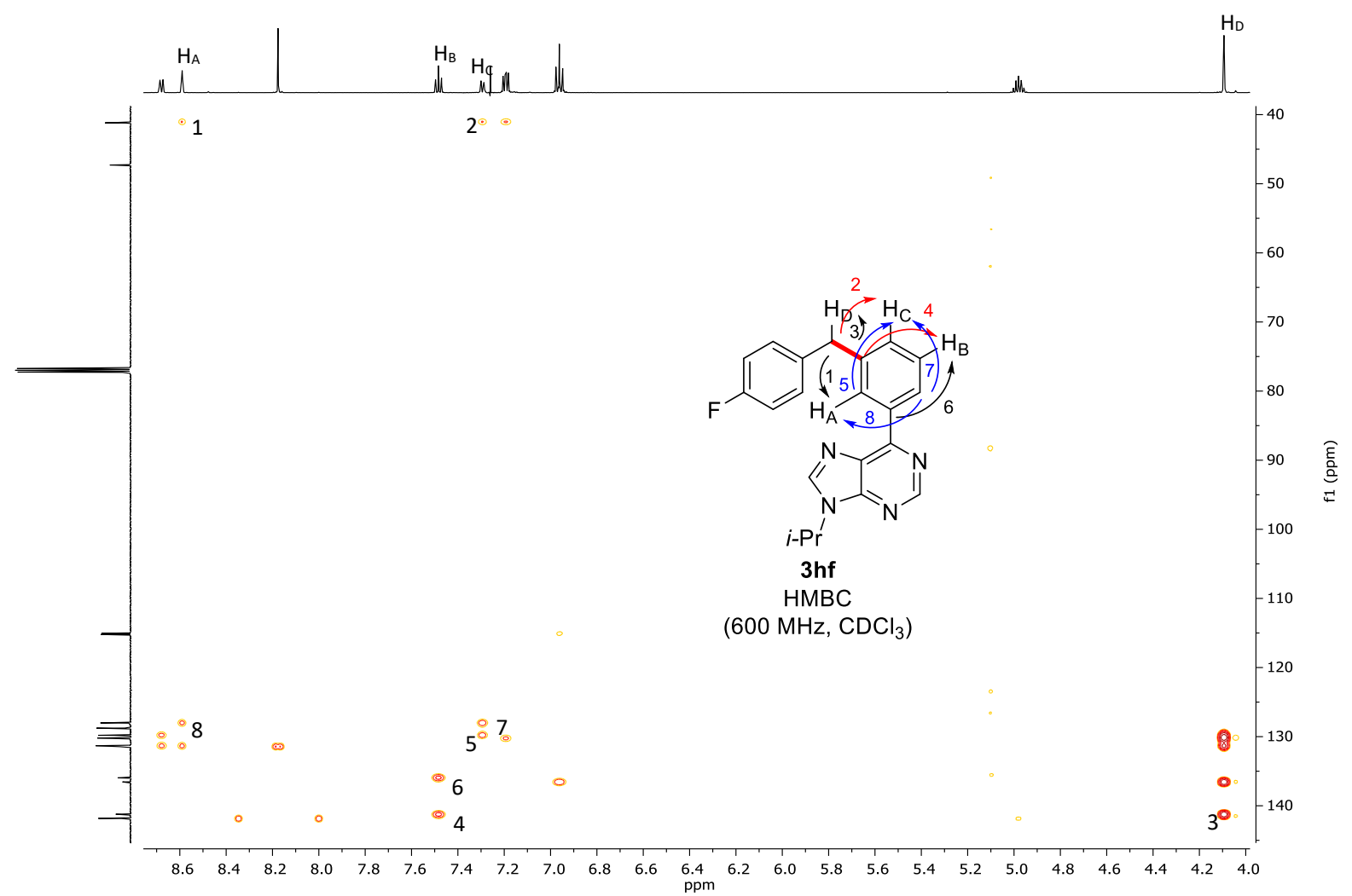




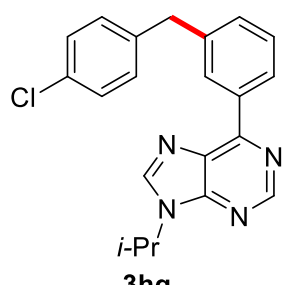

3hg

(300 MHz, $\mathrm{CDCl}_{3}$ )

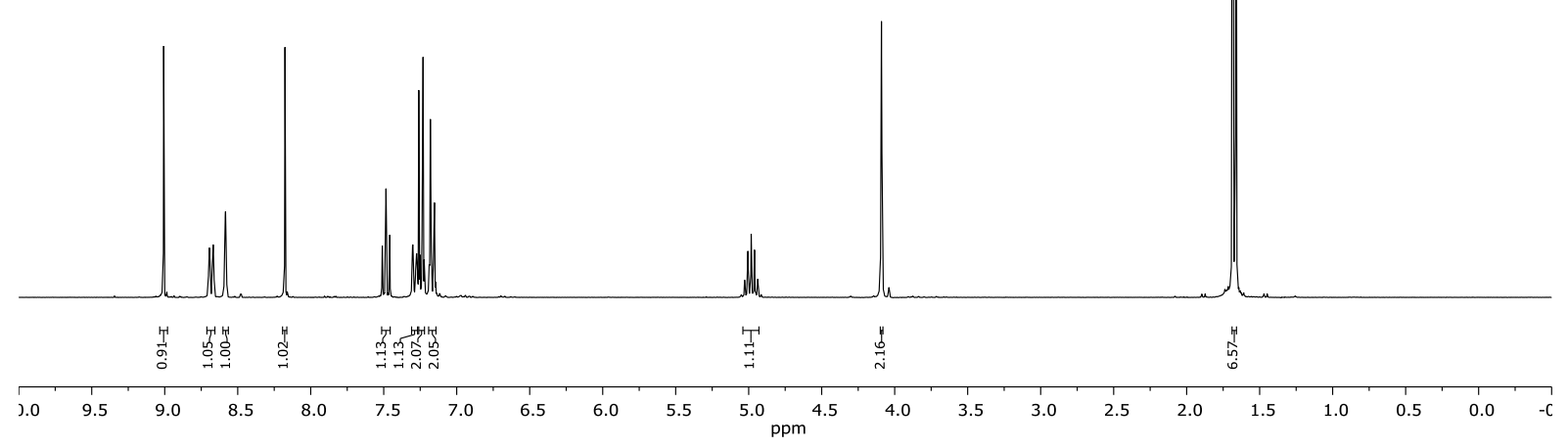

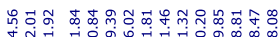

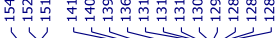

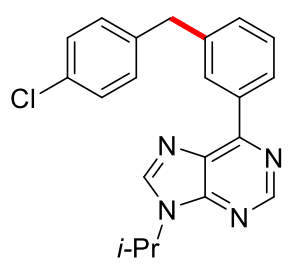

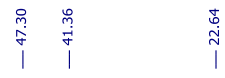

3hg

(125 MHz, $\mathrm{CDCl}_{3}$ )

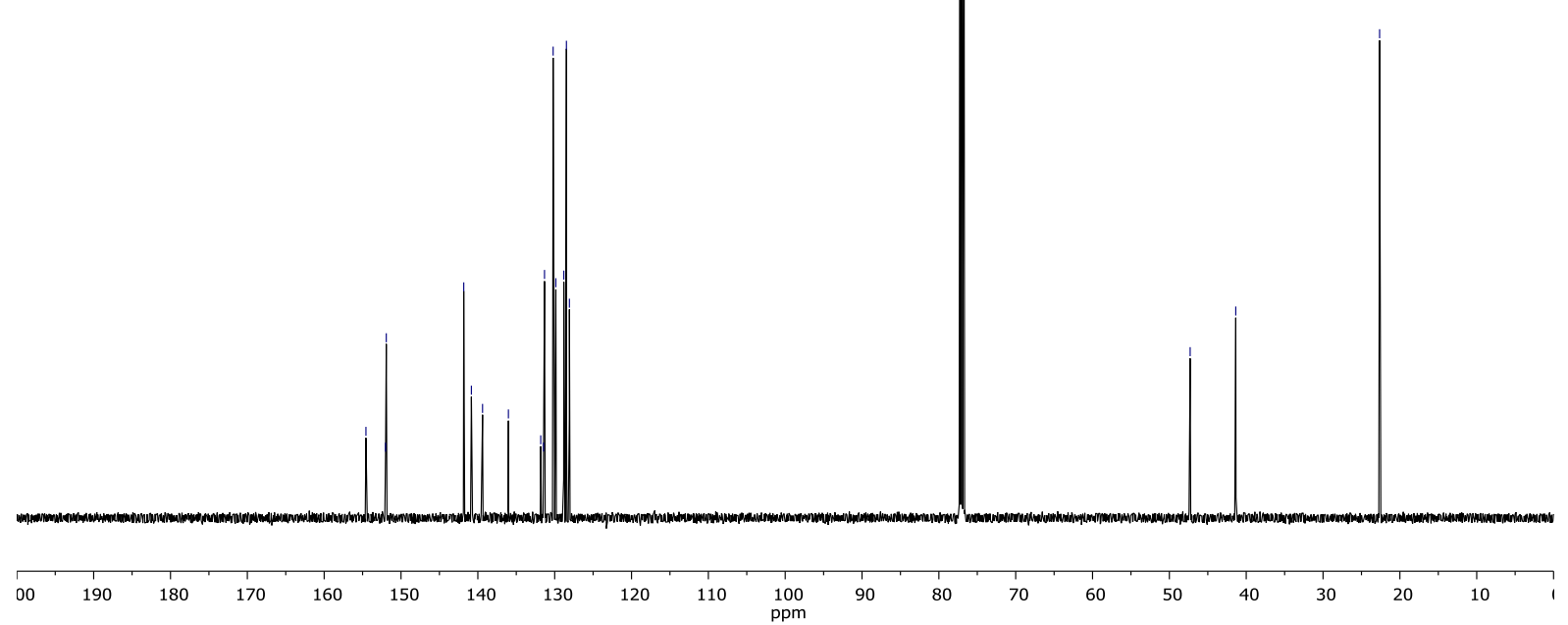




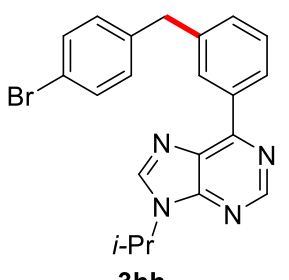

(300 MHz, $\mathrm{CDCl}_{3}$ )

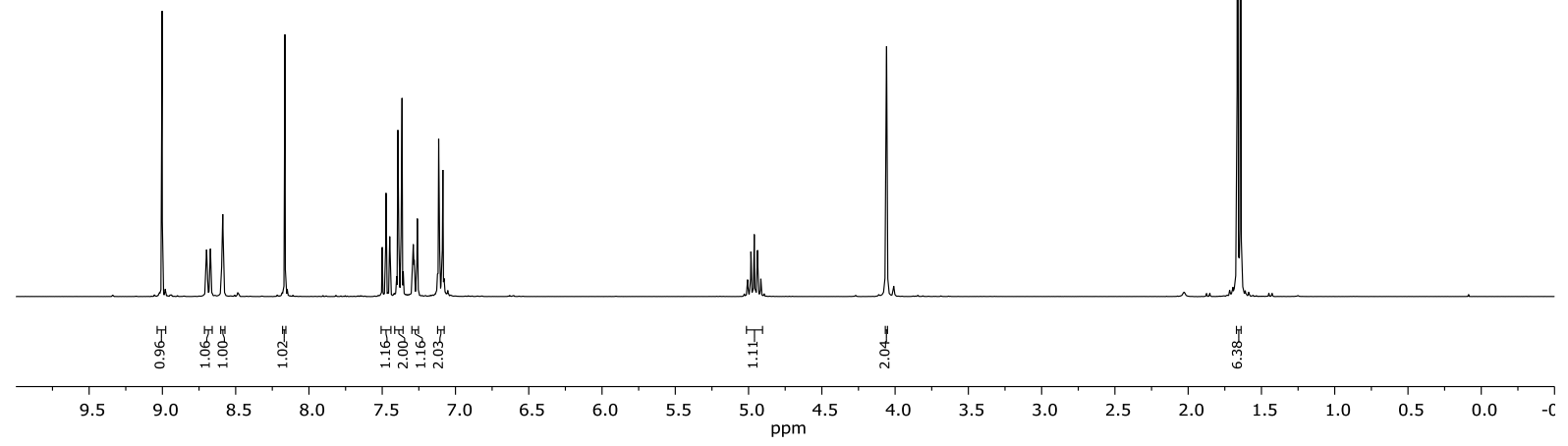

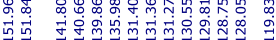

V

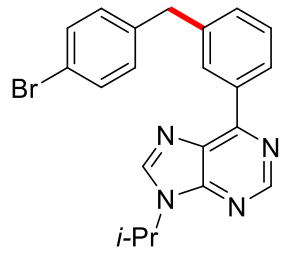

3hh

(125 $\mathrm{MHz}, \mathrm{CDCl}_{3}$ )

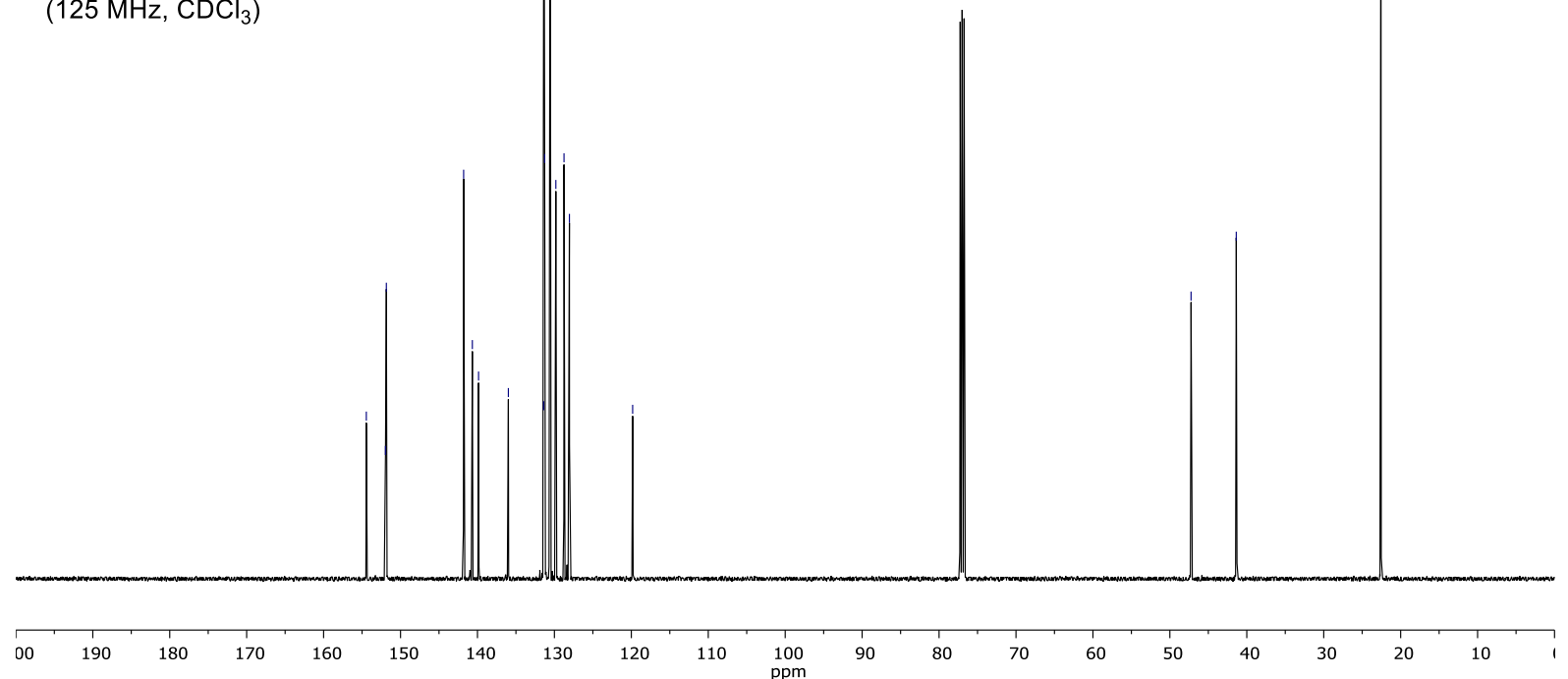



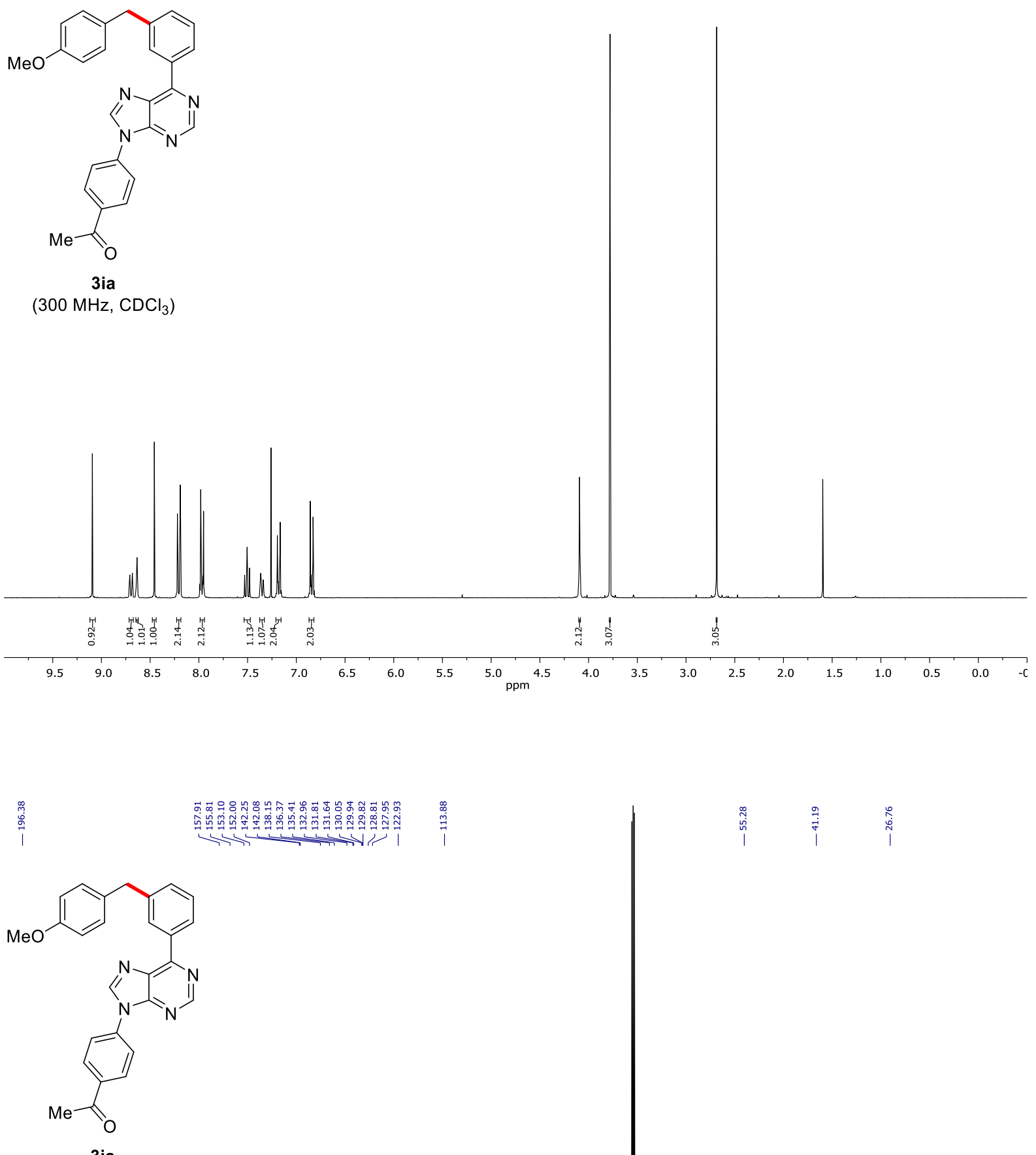

(125 MHz, $\mathrm{CDCl}_{3}$ )

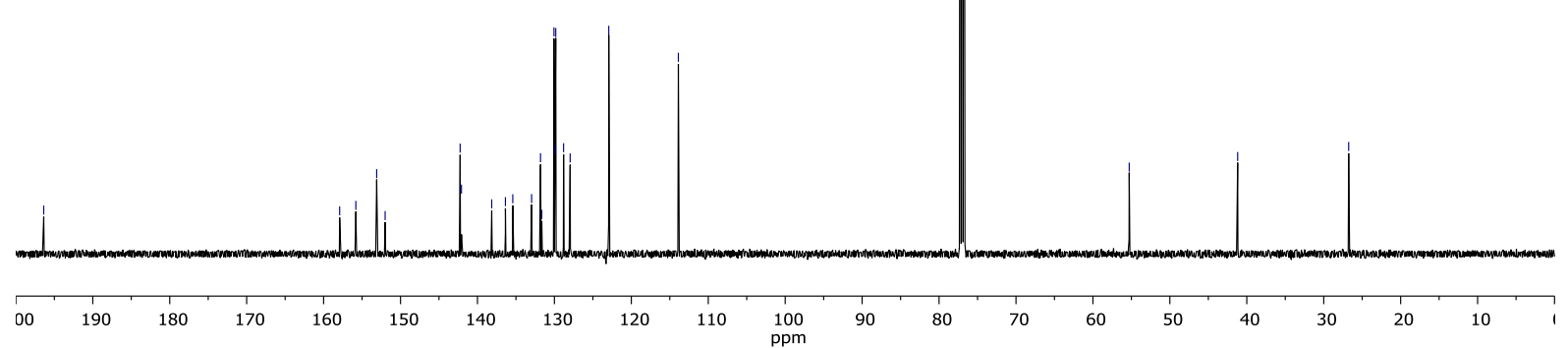



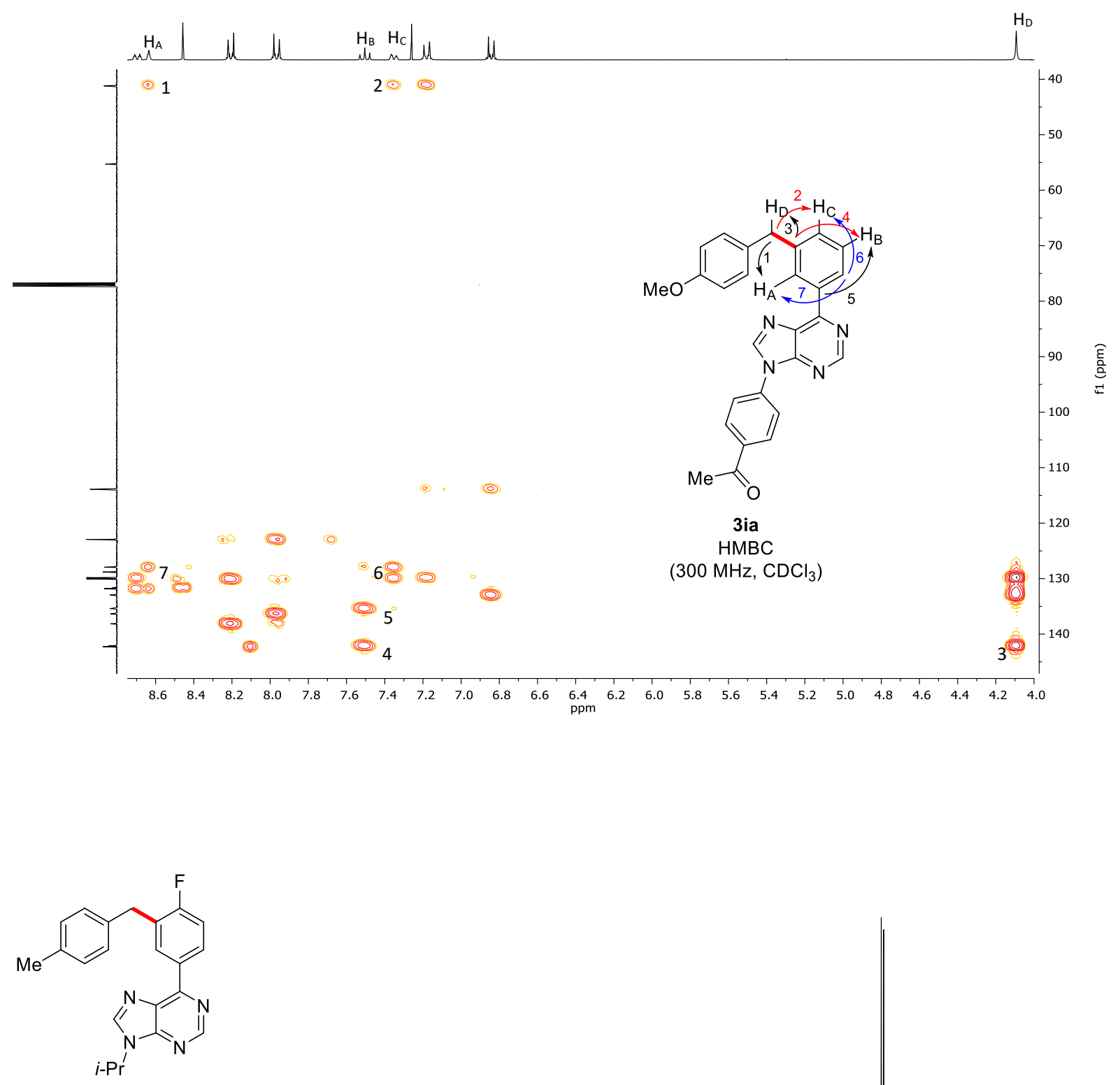

$3 j \mathrm{c}$

(300 MHz, $\mathrm{CDCl}_{3}$ )

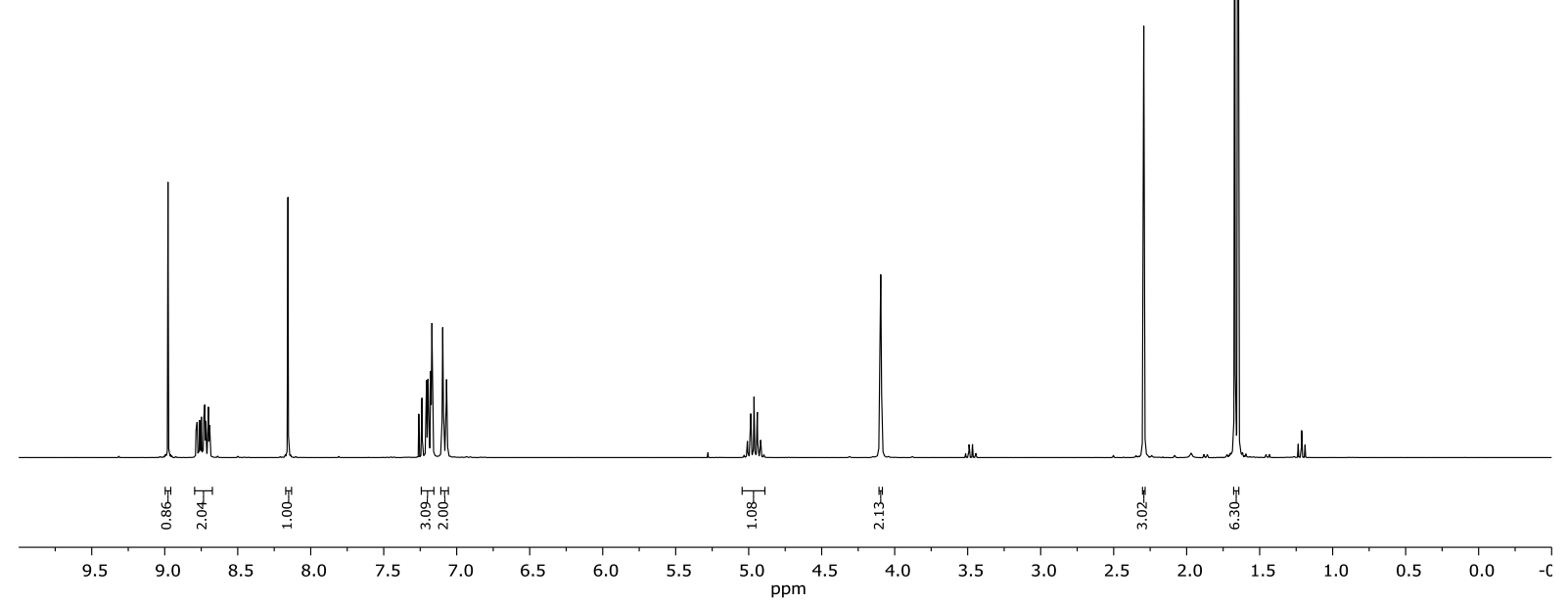



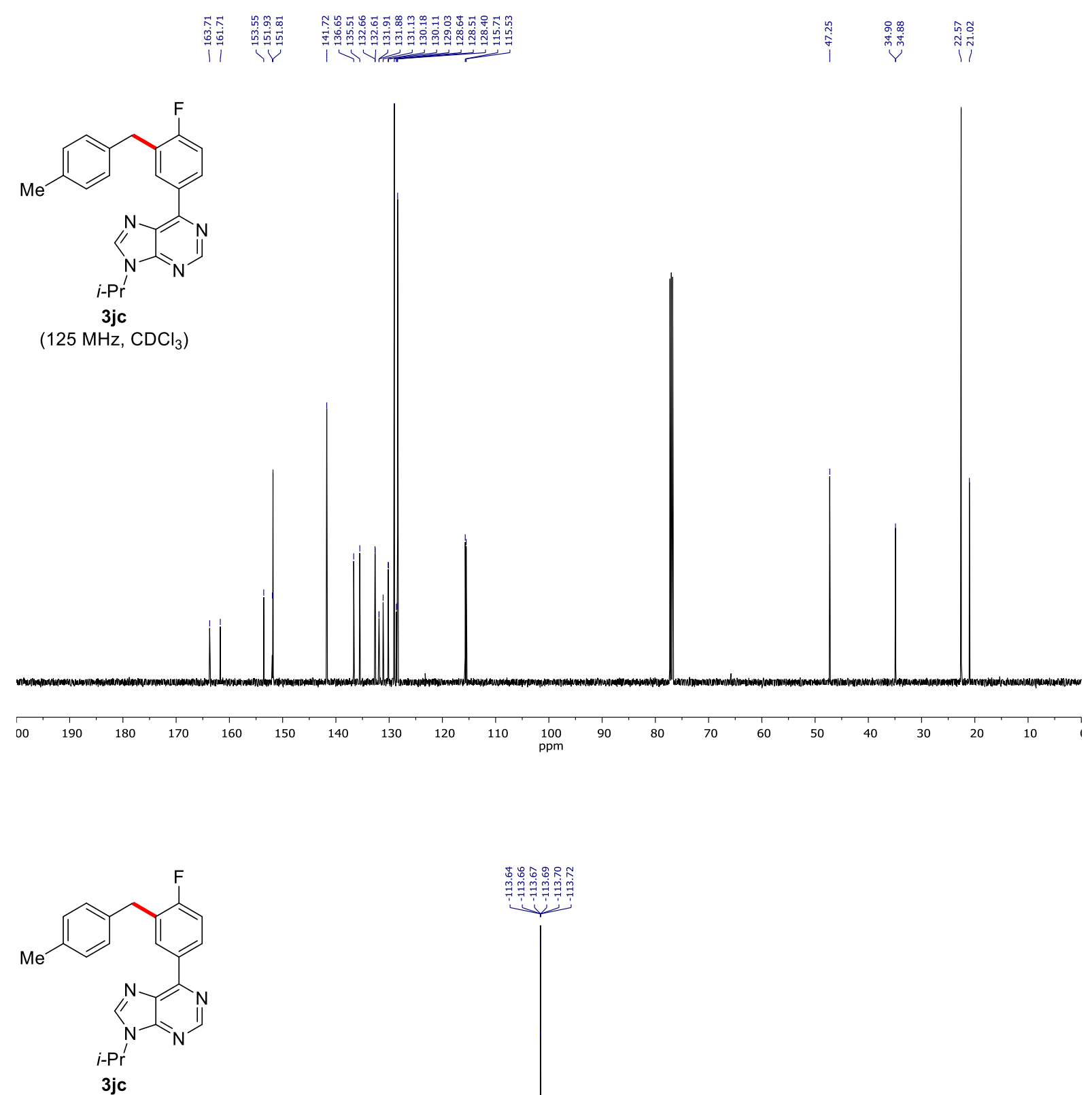

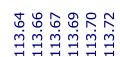

(282 MHz, $\mathrm{CDCl}_{3}$ ) 


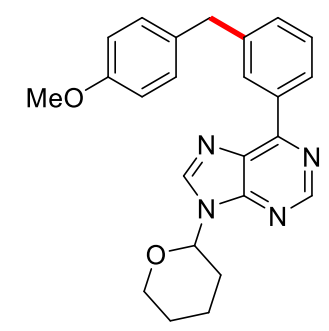

3ka

$\left(300 \mathrm{MHz}, \mathrm{CDCl}_{3}\right)$

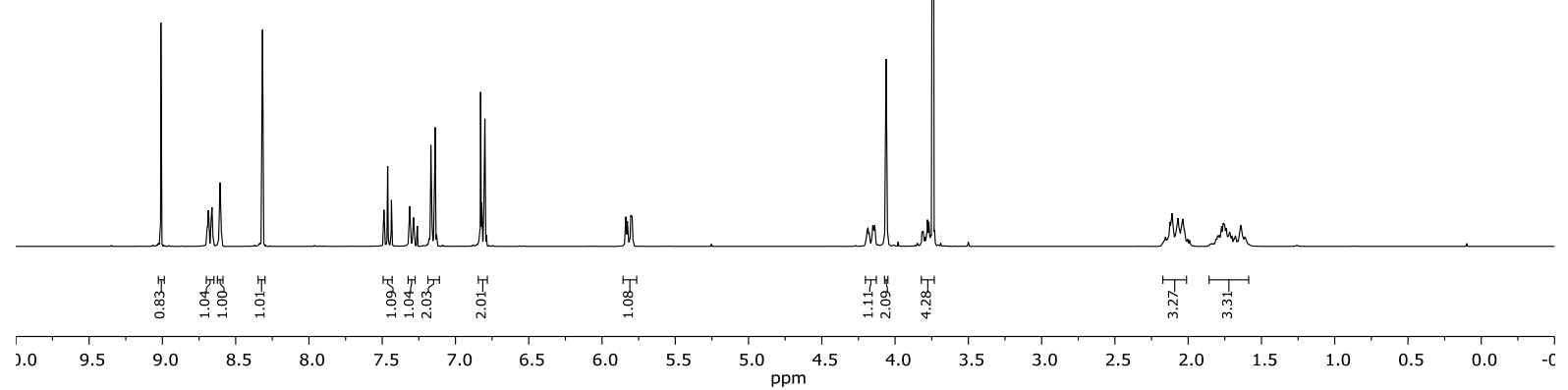

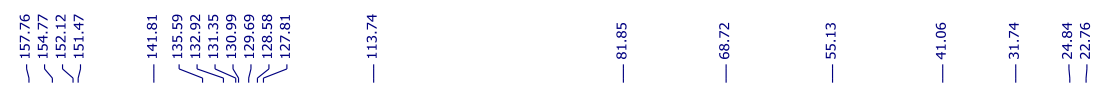

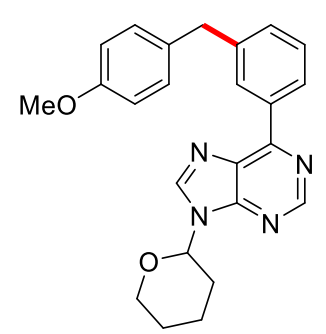

3ka

$\left(125 \mathrm{MHz}, \mathrm{CDCl}_{3}\right)$

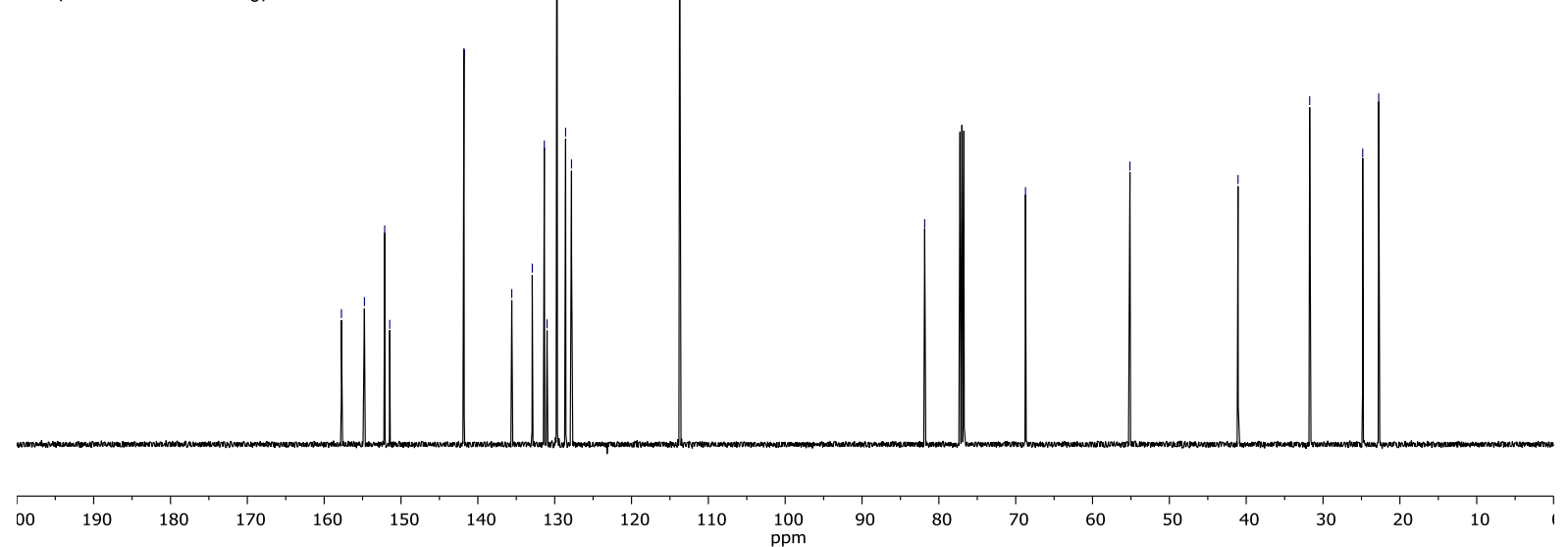



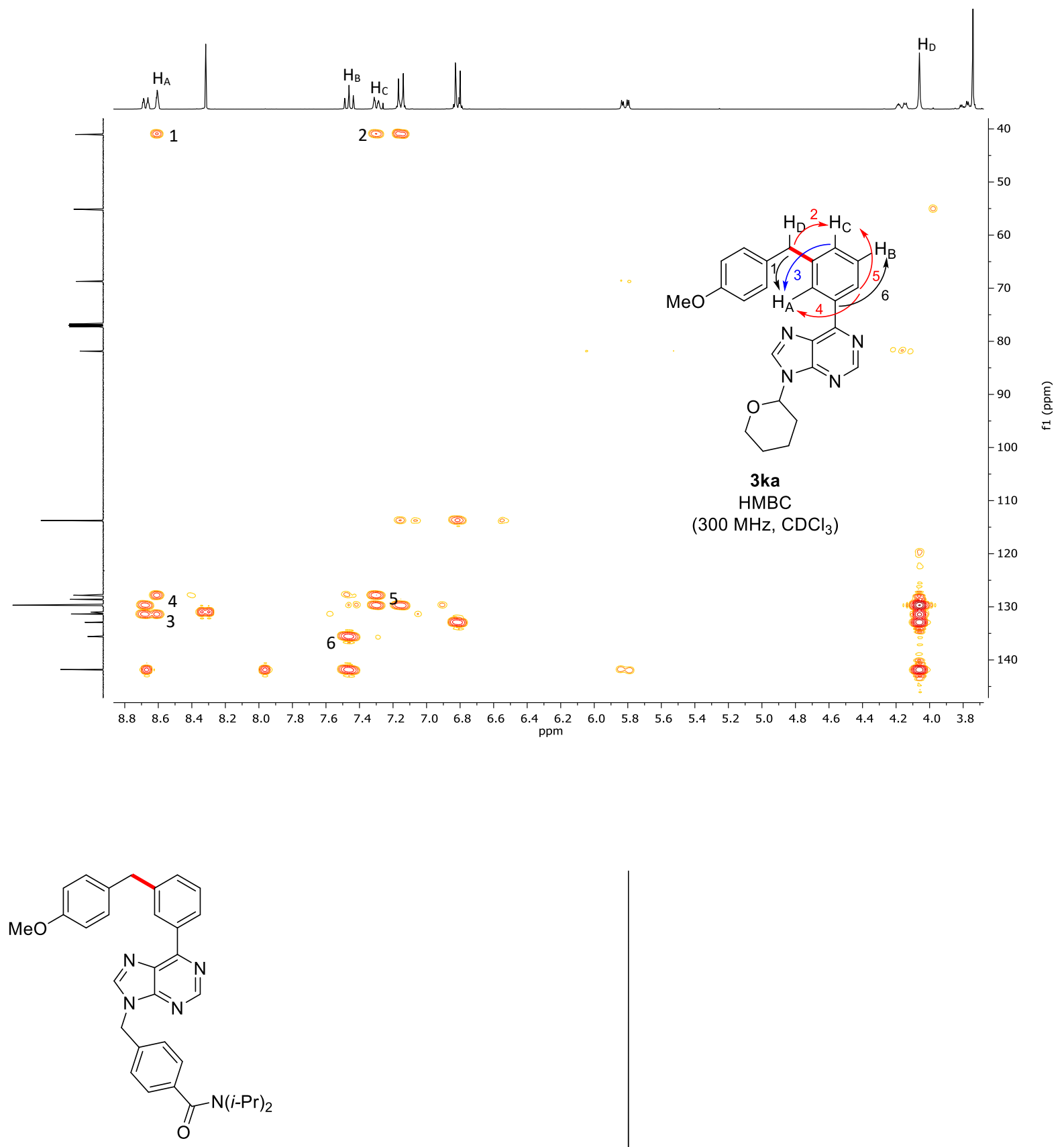

$31 a$

(400 MHz, $\mathrm{CDCl}_{3}$ )

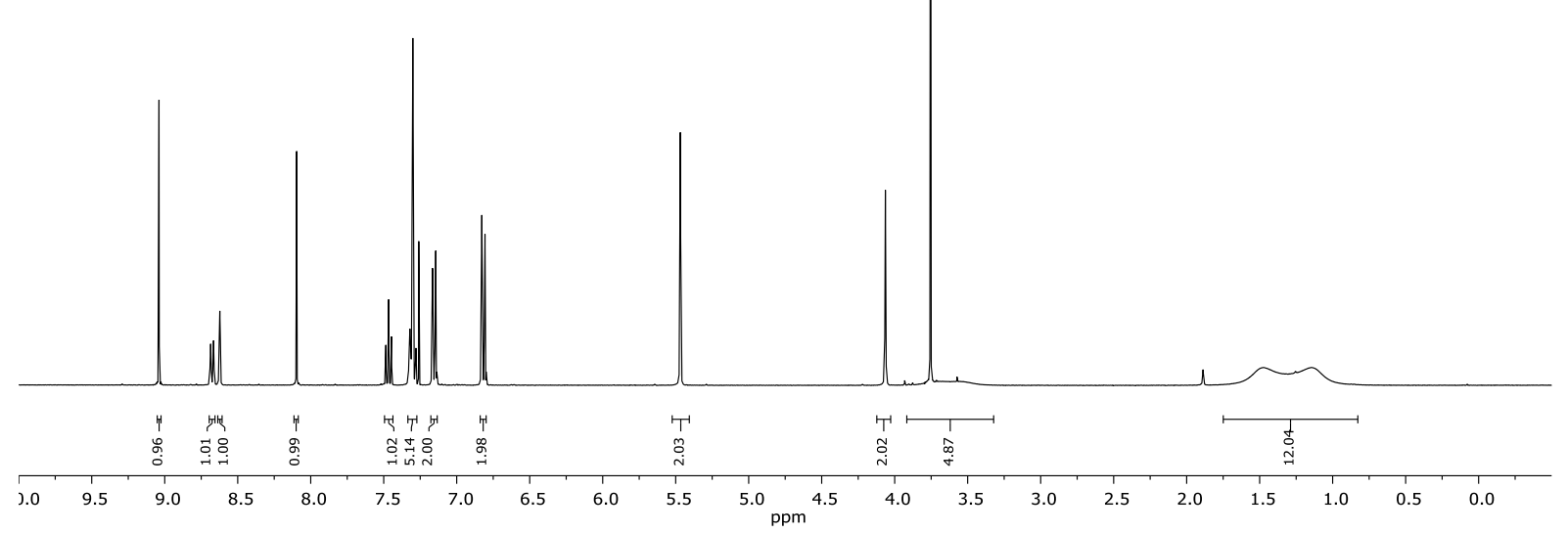



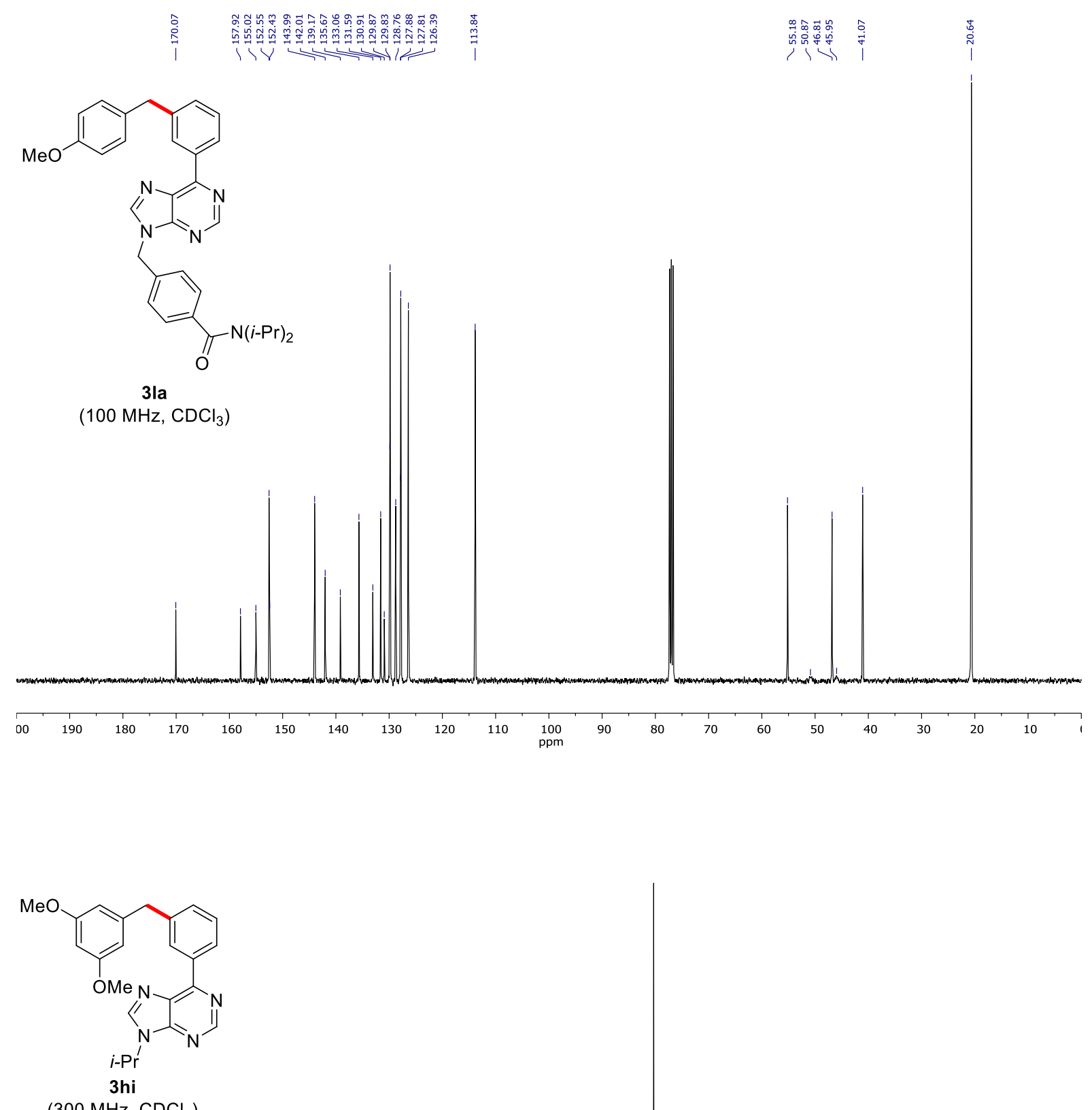

(300 MHz, $\mathrm{CDCl}_{3}$ )

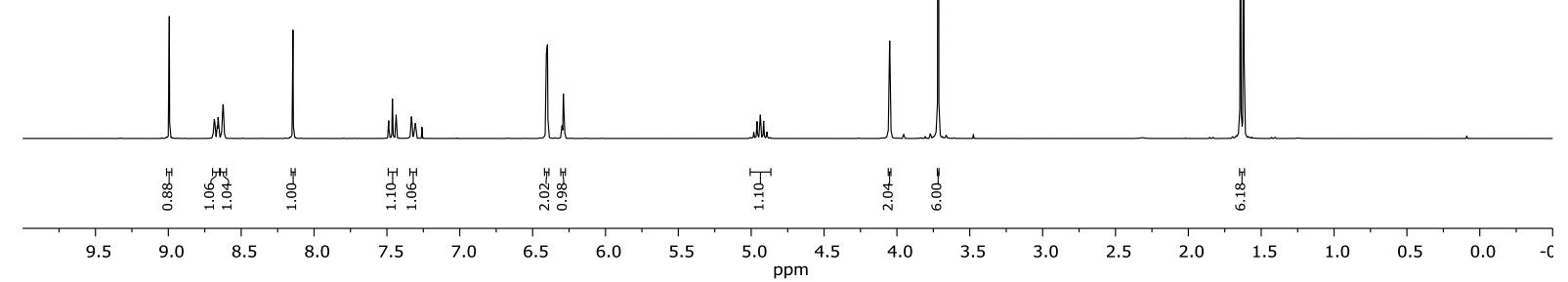




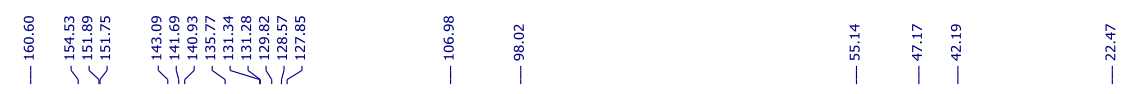

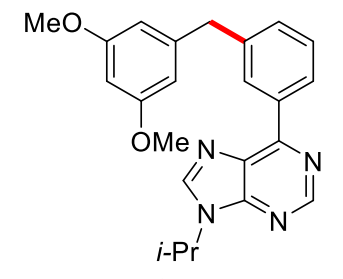

3hi

(125 $\mathrm{MHz} \mathrm{CDCl}_{3}$ )
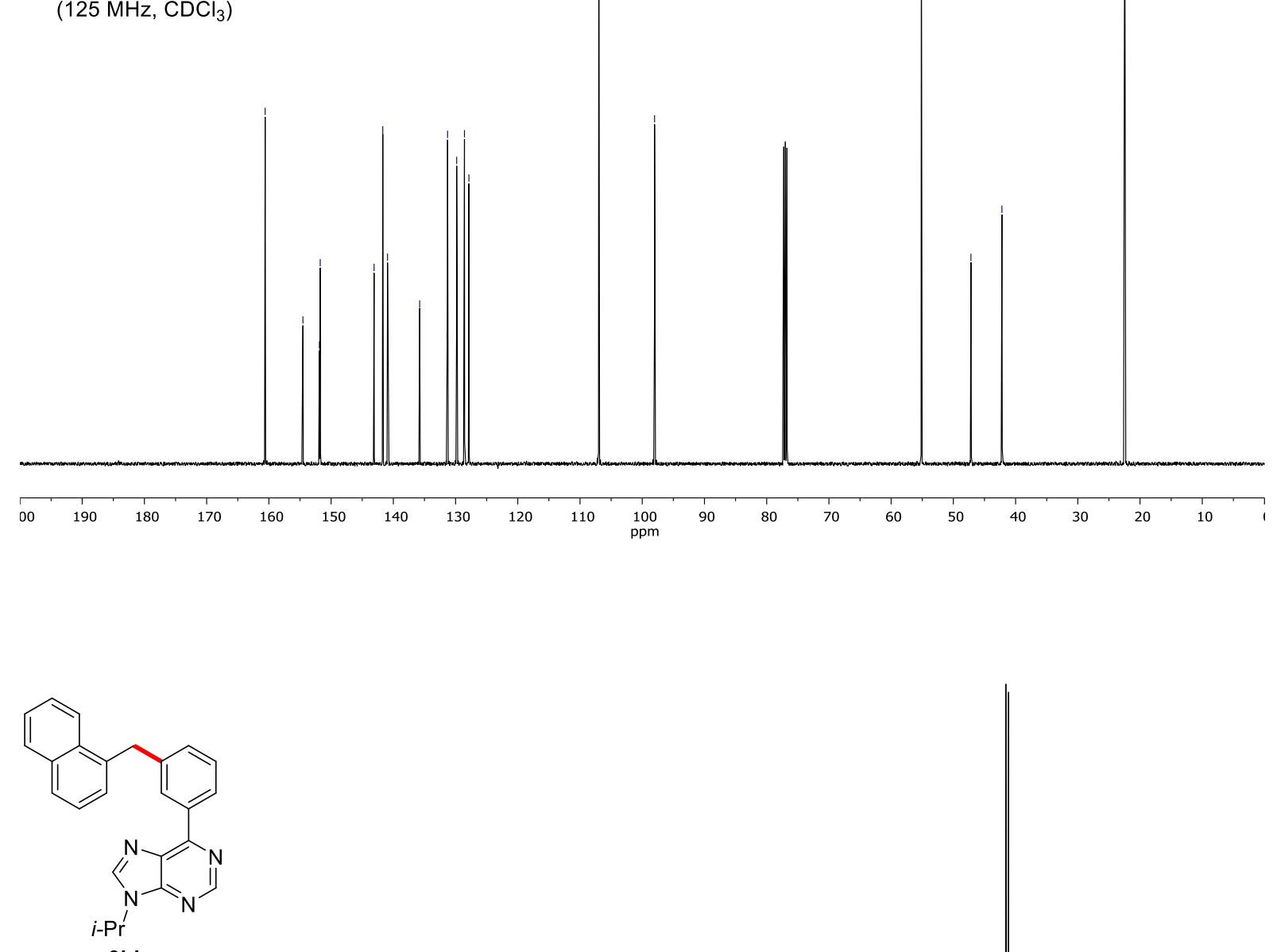

3hj

$\left(300 \mathrm{MHz}, \mathrm{CDCl}_{3}\right)$

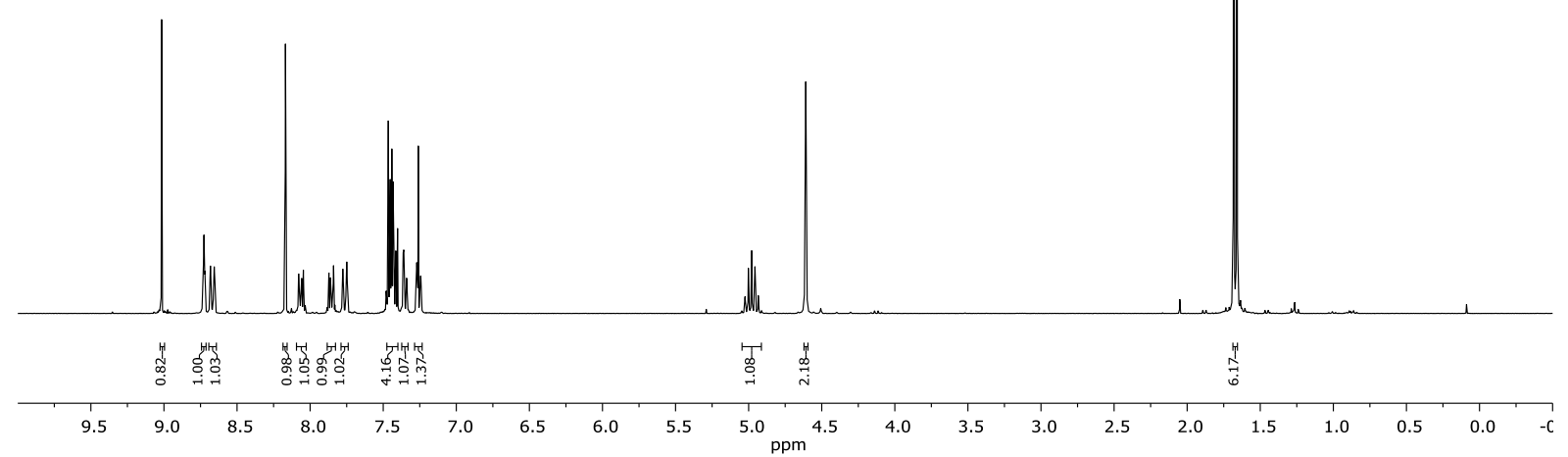



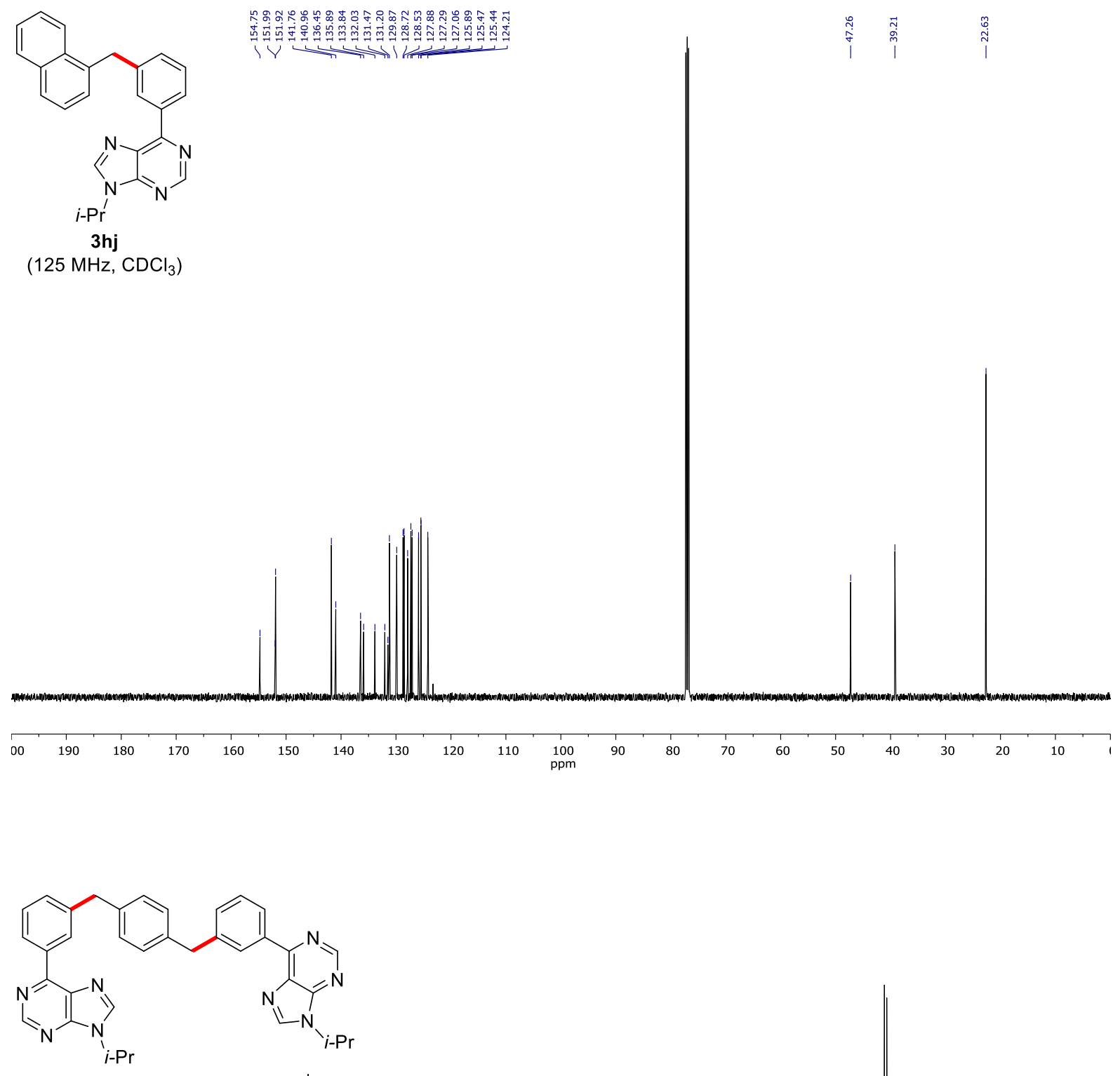

3hk

(300 MHz, $\mathrm{CDCl}_{3}$ )

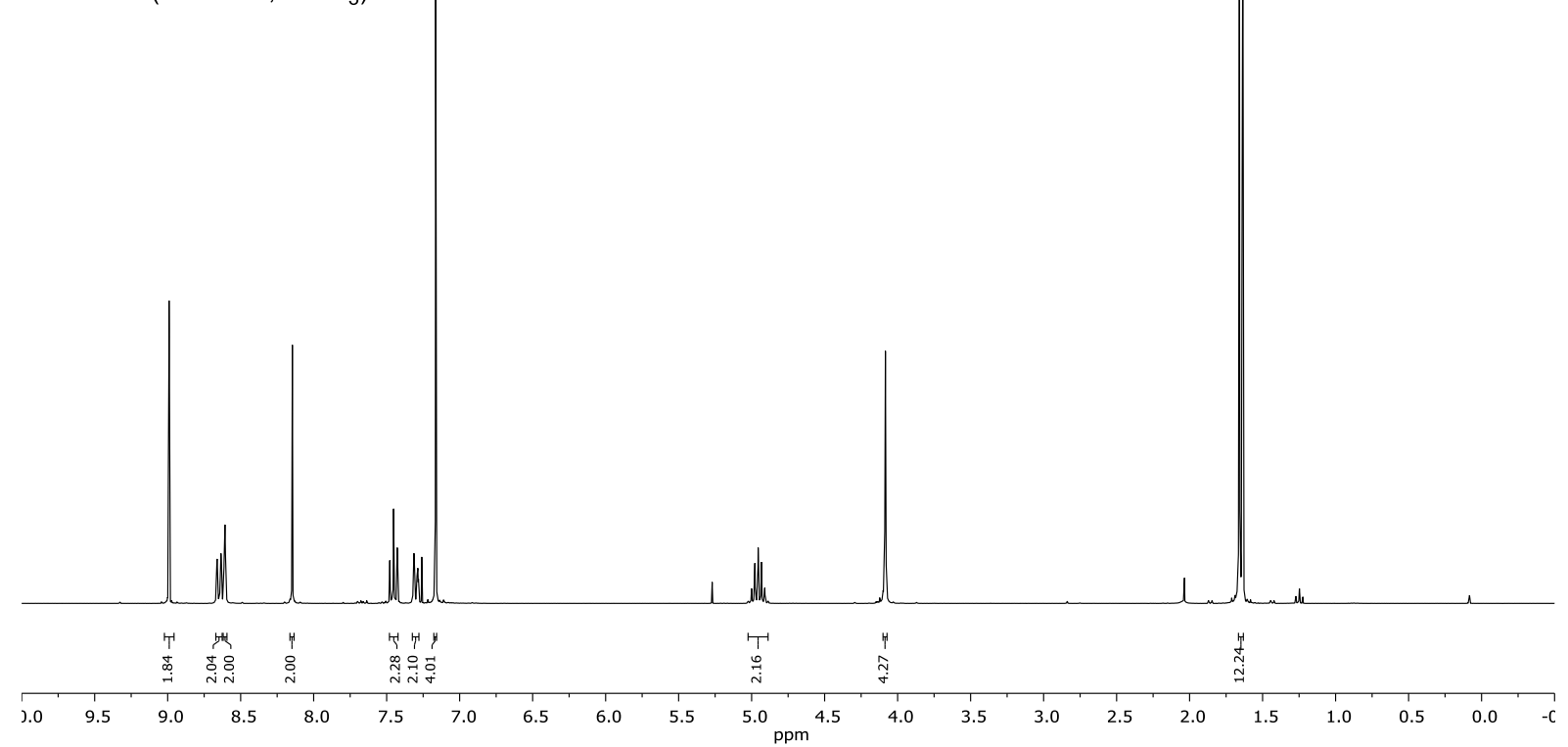


|
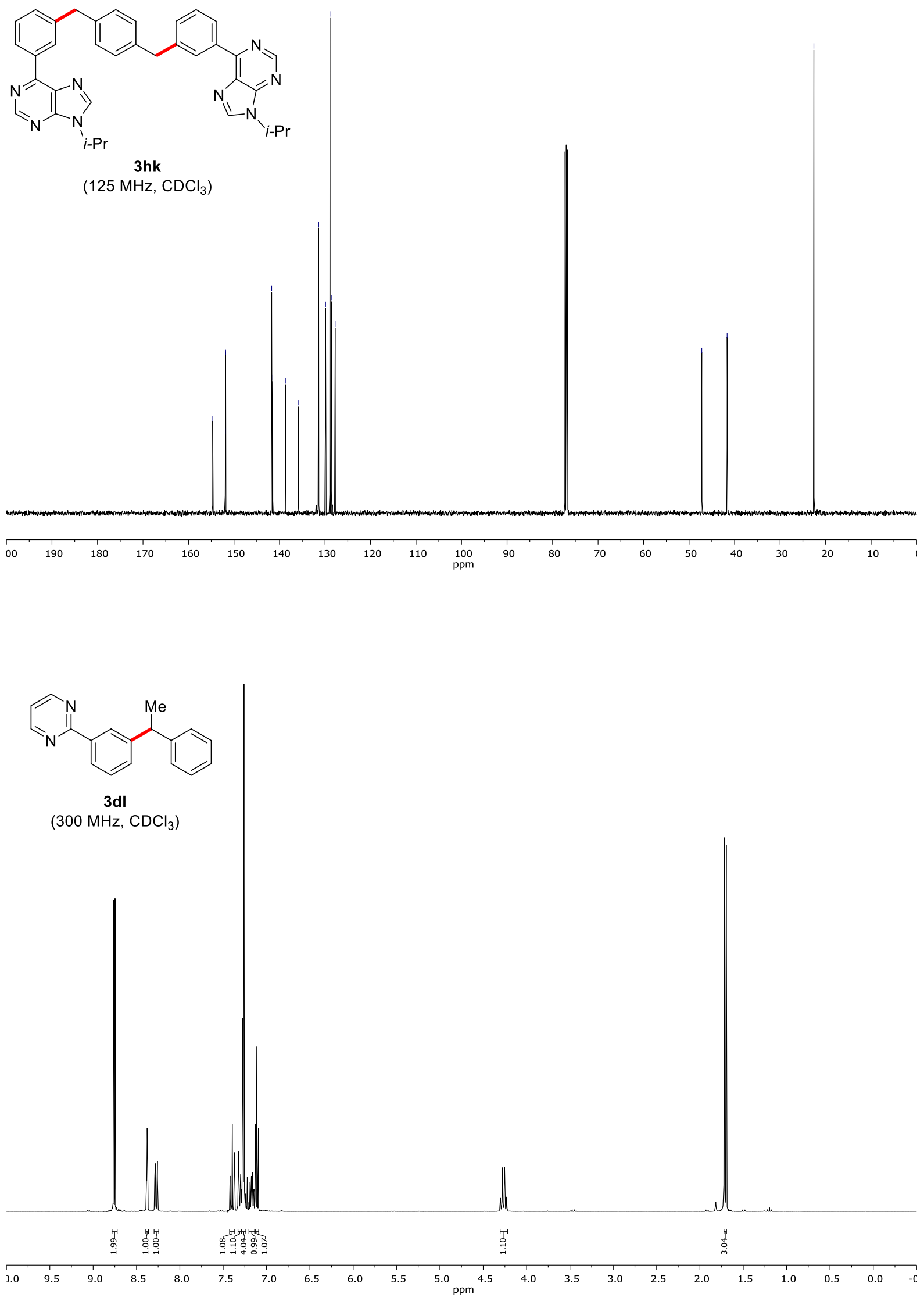

S167 


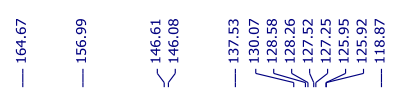

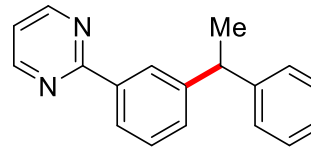

3dl

(125 MHz, $\mathrm{CDCl}_{3}$ )
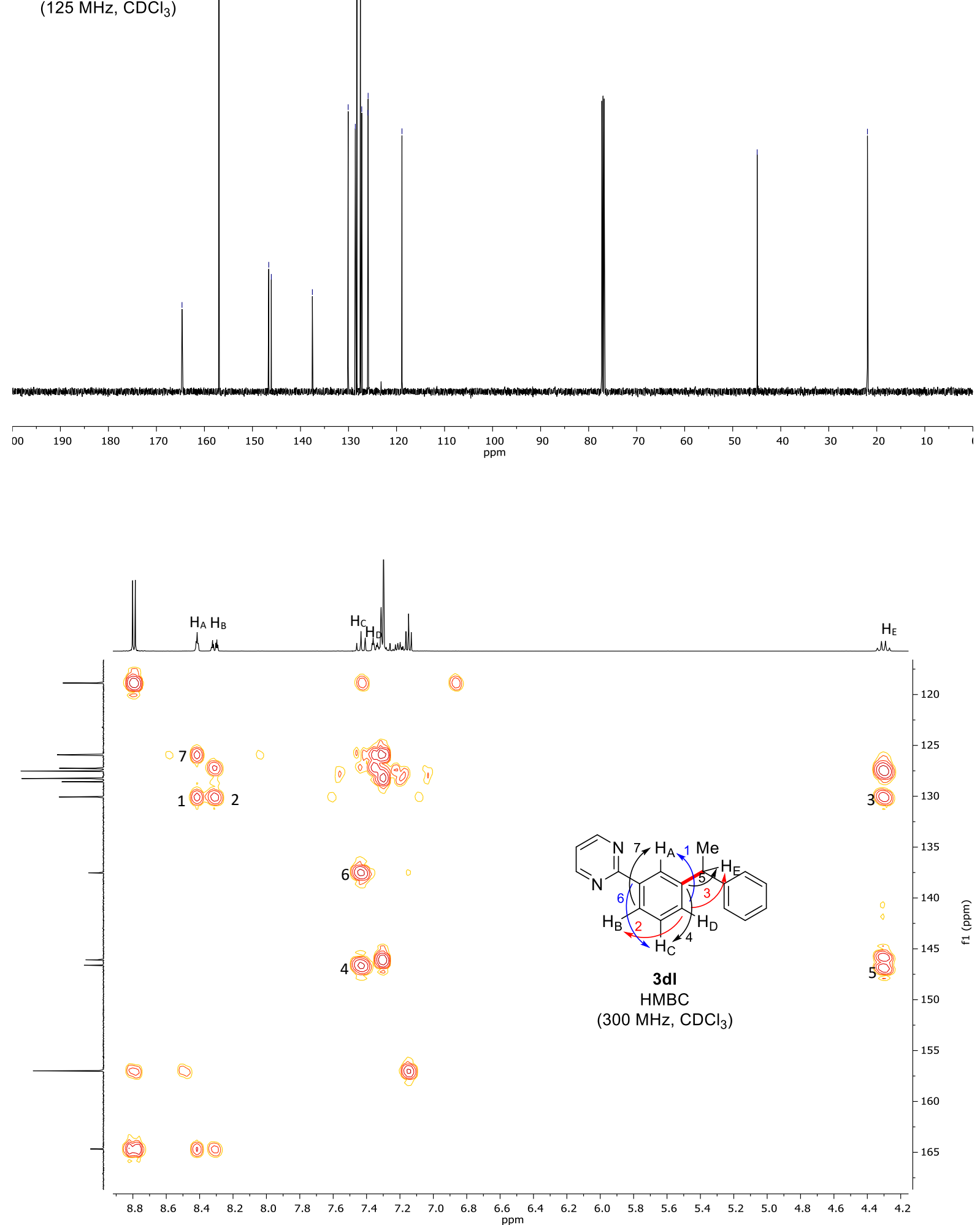


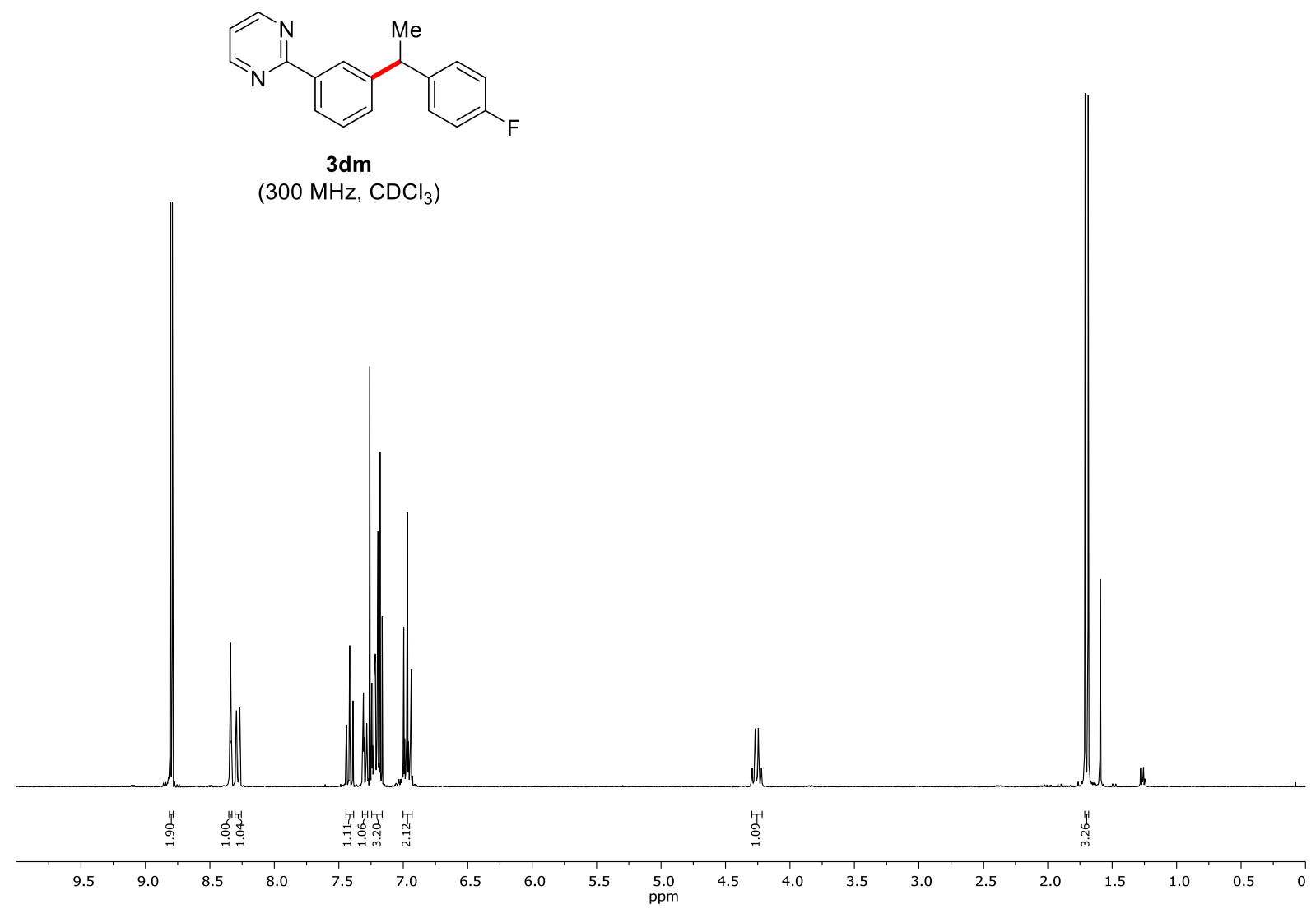

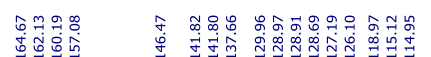

ग1। ।

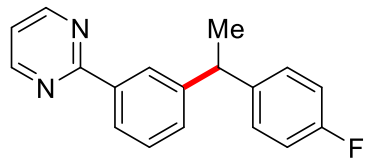

$3 \mathrm{dm}$

(125 $\mathrm{MHz} \mathrm{CDCl}_{3}$ )

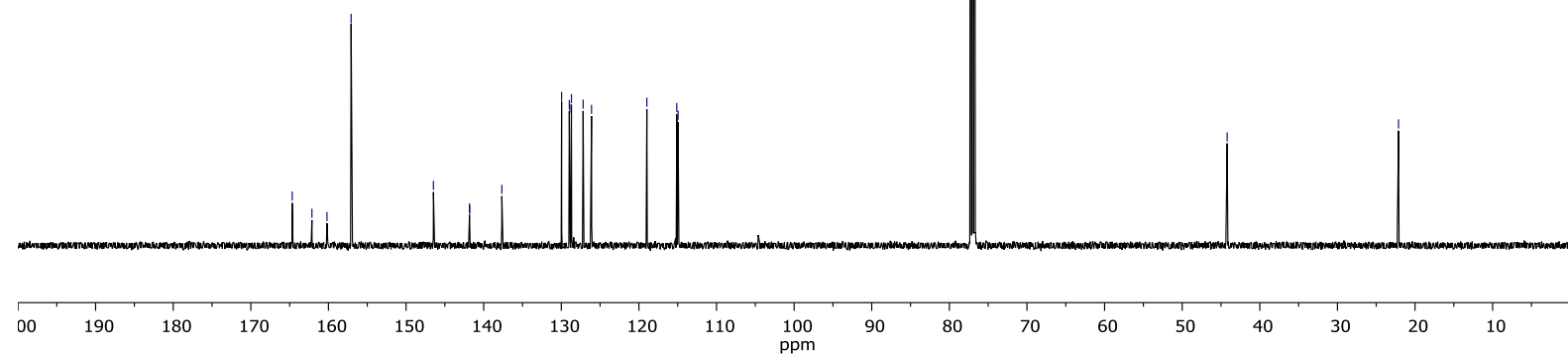



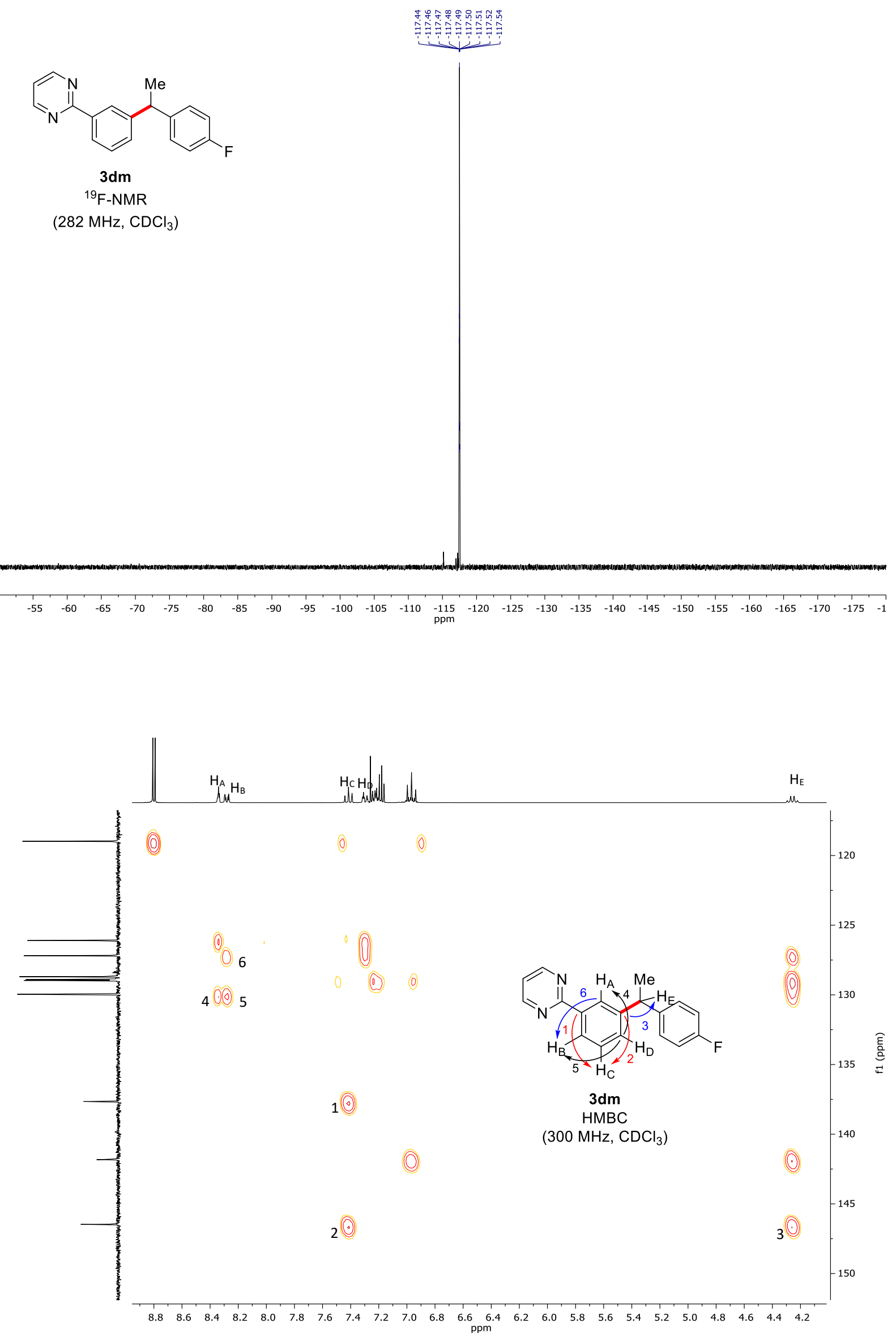


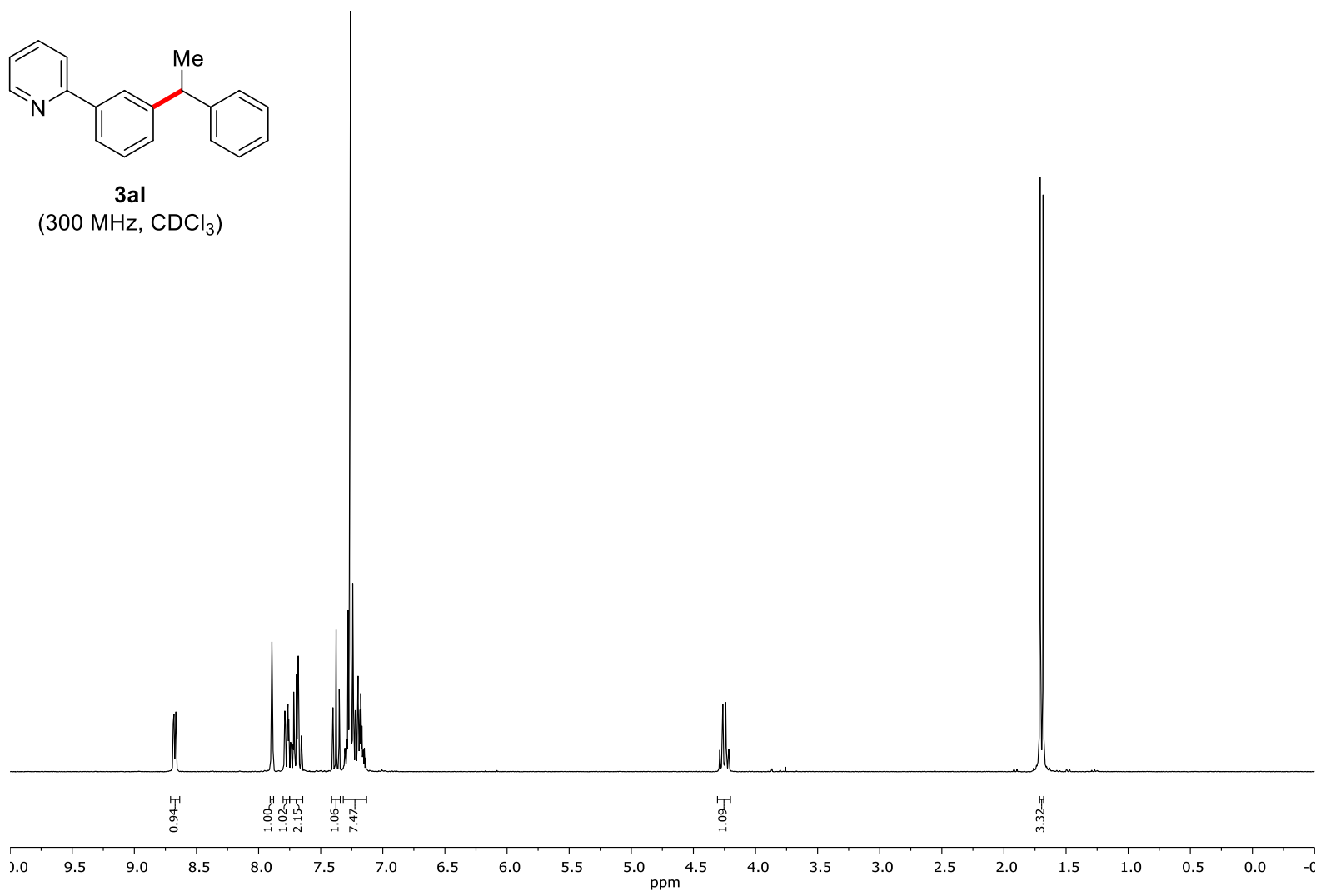

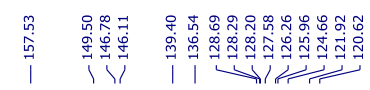

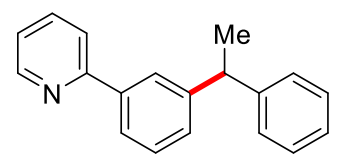

3al (125 MHz, $\mathrm{CDCl}_{3}$ )

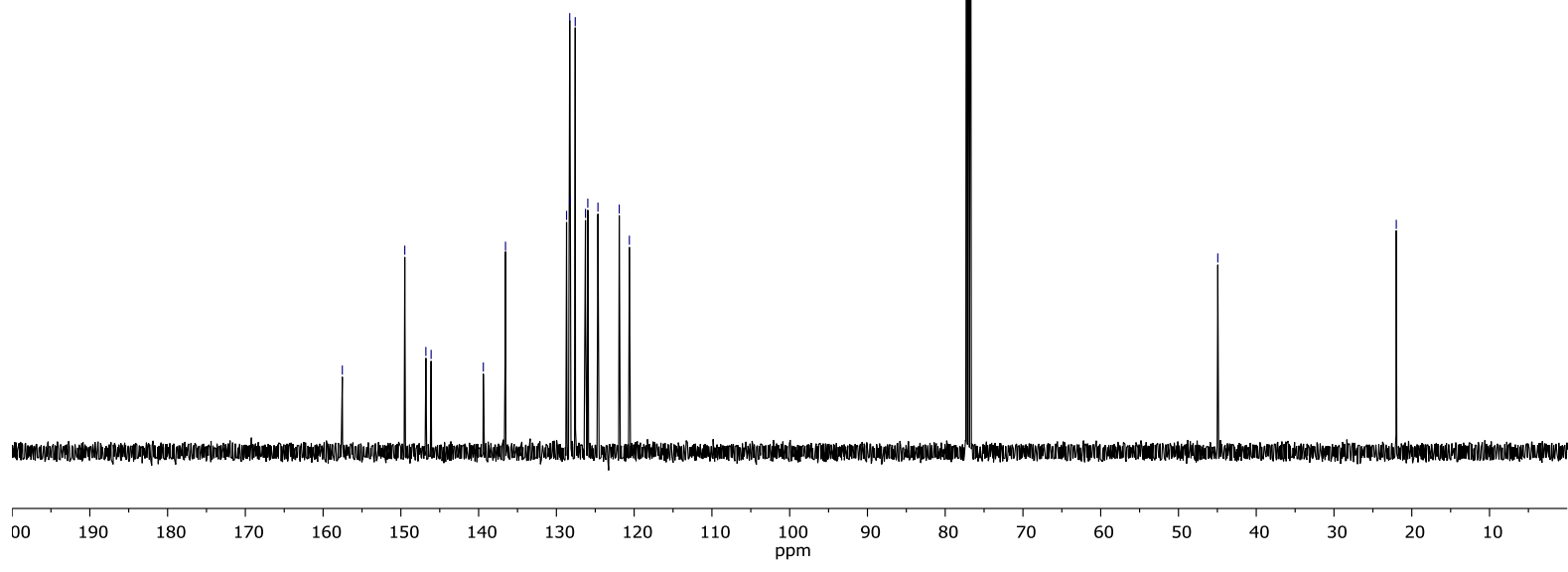

S171 

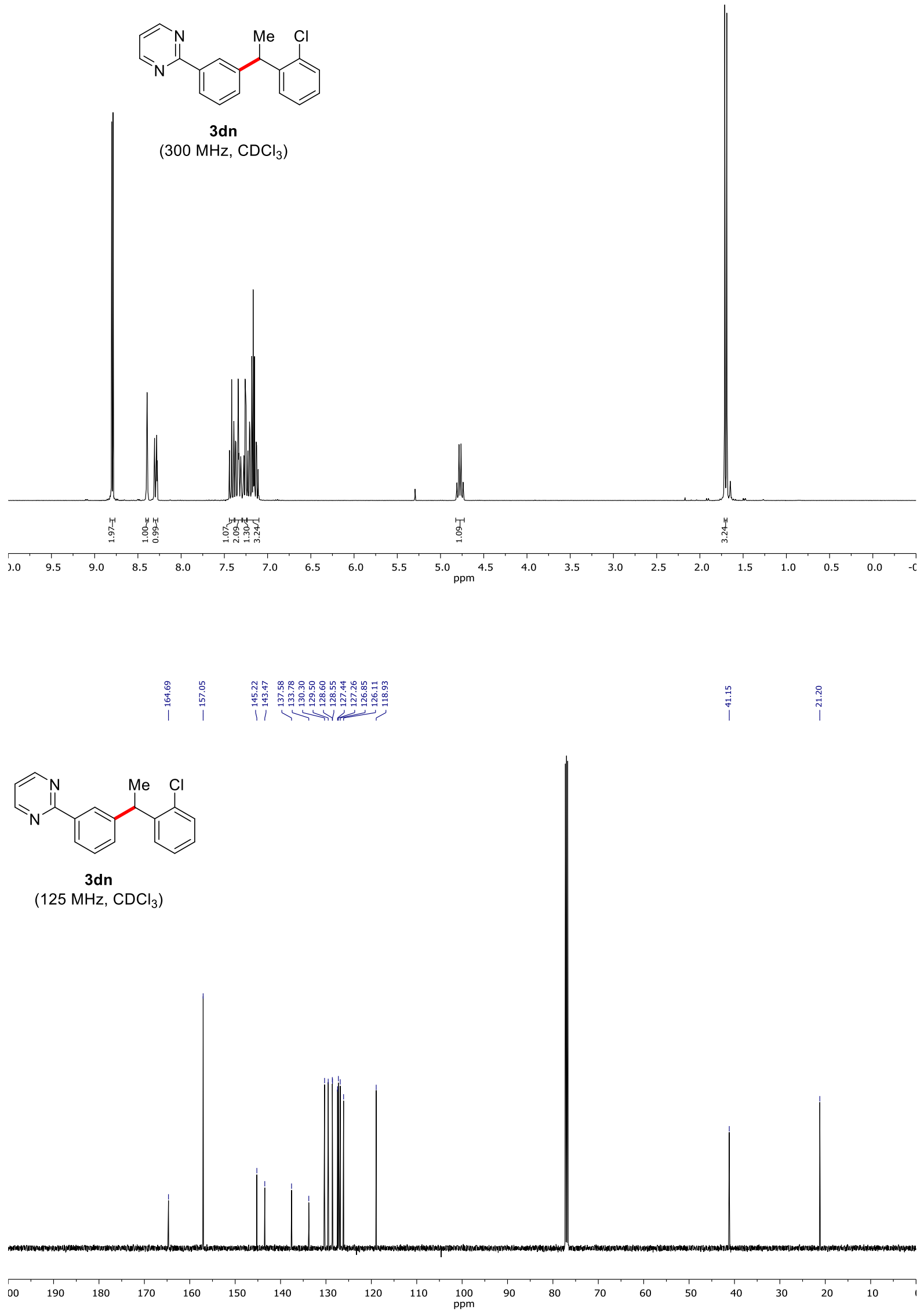

S172 

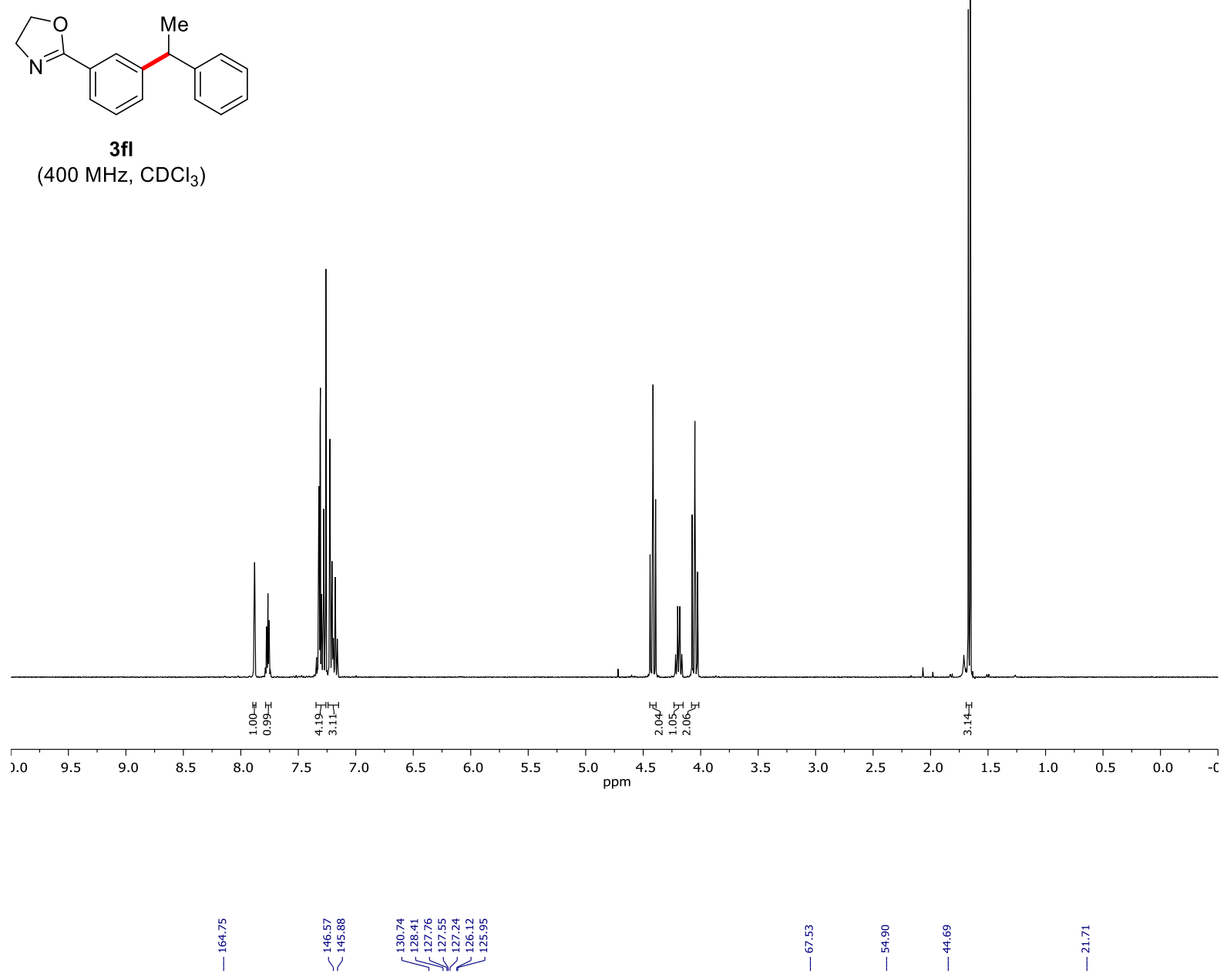

$\underset{\text { N }}{\mathbb{N}}$

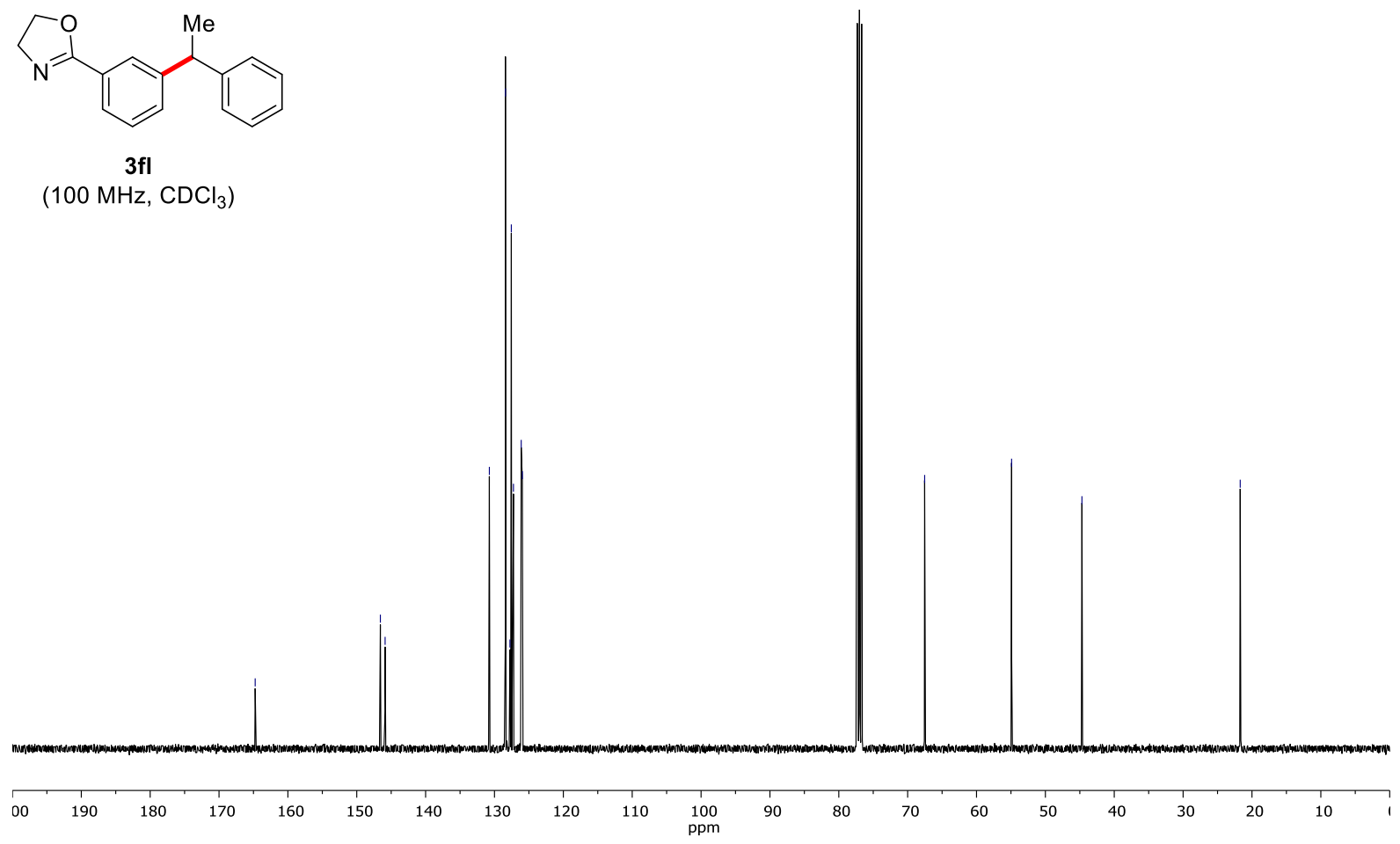

S173 


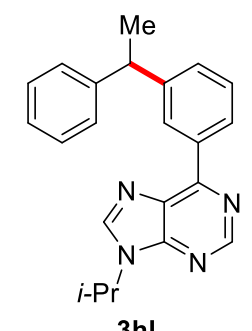

(600 $\mathrm{MHz}, \mathrm{CDCl}_{3}$ )

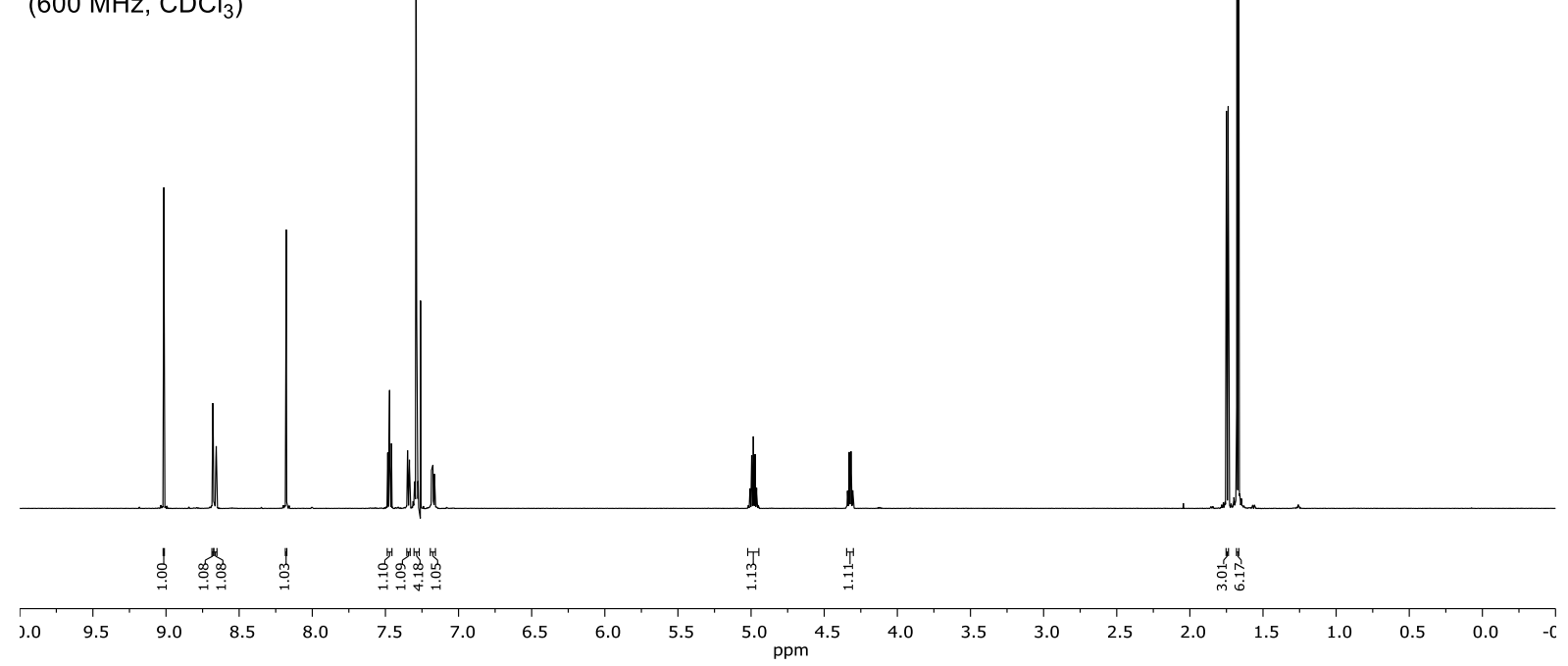

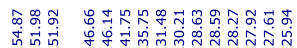

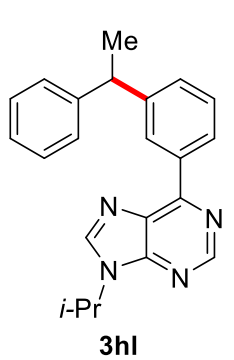

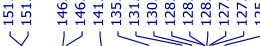

(125 $\mathrm{MHz}, \mathrm{CDCl}_{3}$ )

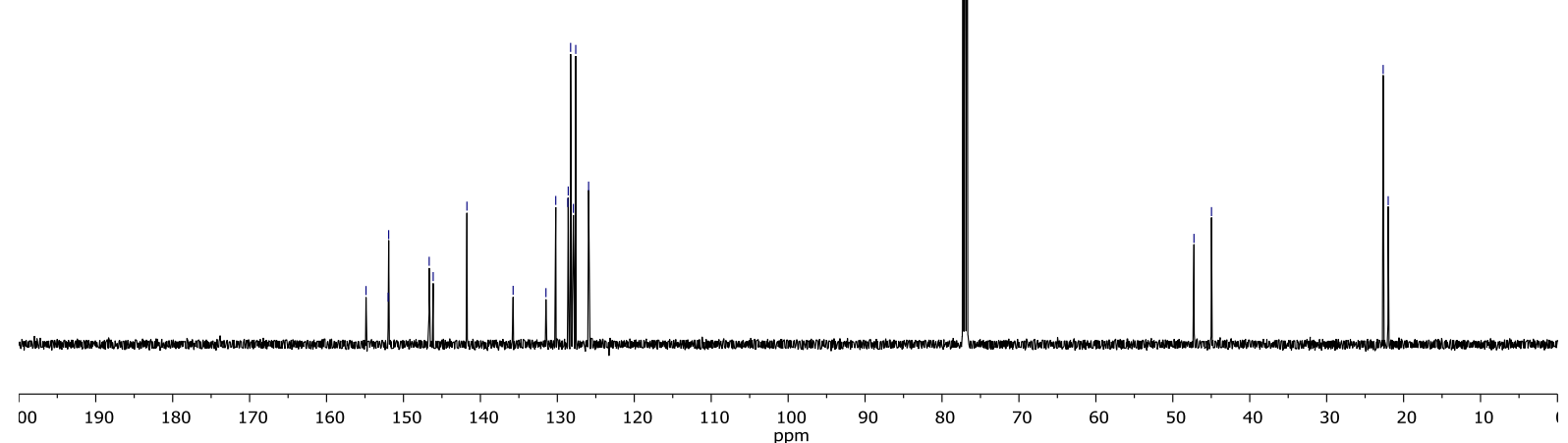




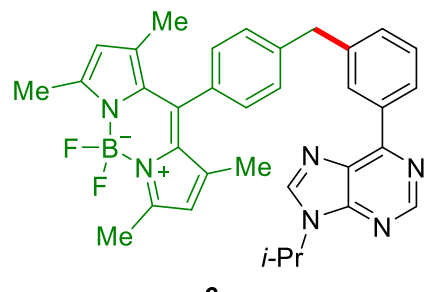

$\left(600 \mathrm{MHz}, \mathrm{CDCl}_{3}\right.$ )

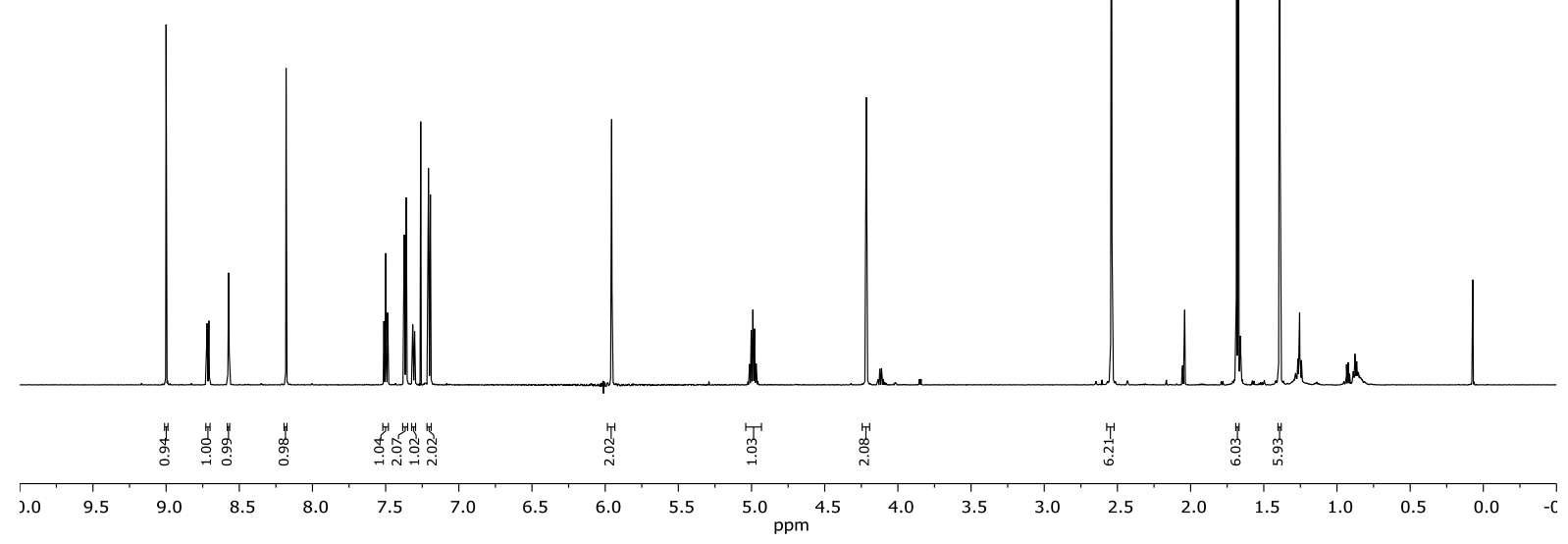

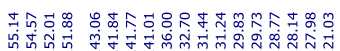

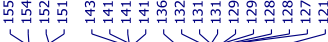

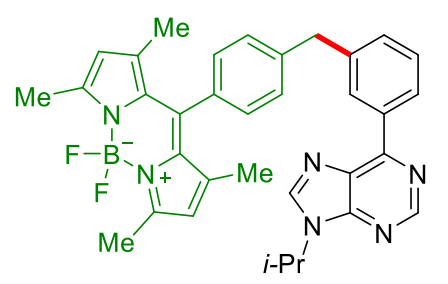

$6 a$

$\left(125 \mathrm{MHz}, \mathrm{CDCl}_{3}\right.$ )

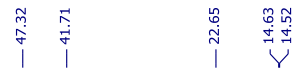

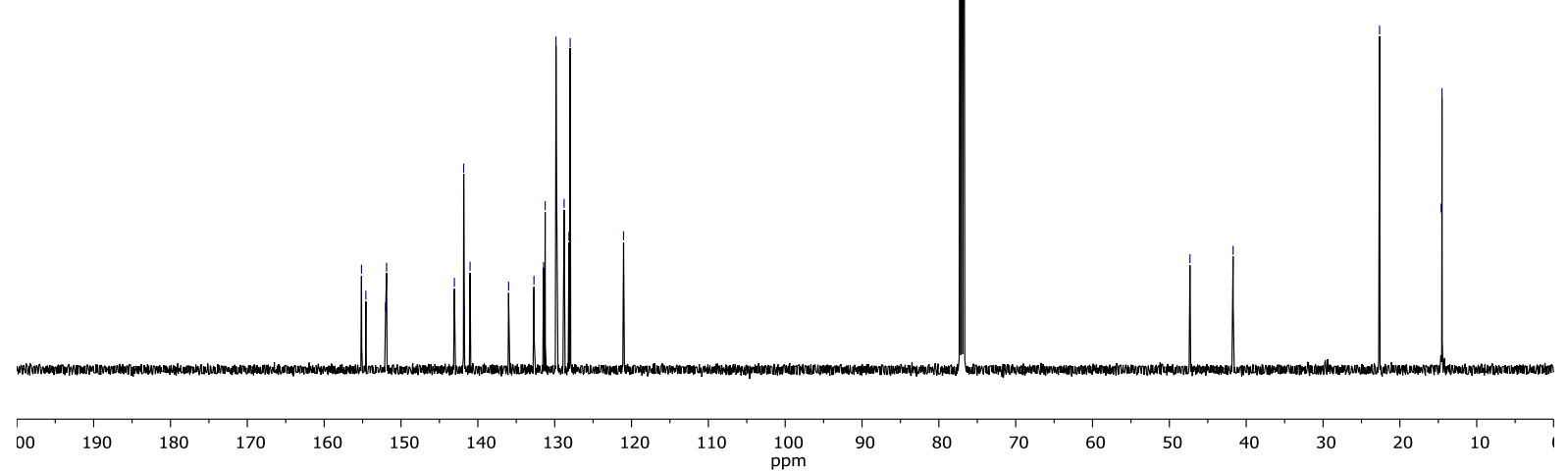




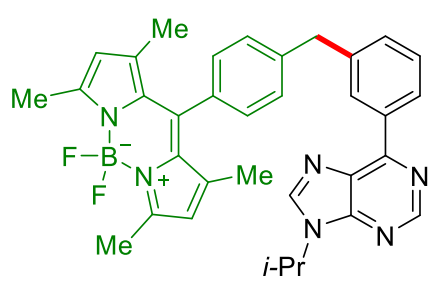

$6 a$

${ }^{11} \mathrm{~B}-\mathrm{NMR}$

$\left(128 \mathrm{MHz}, \mathrm{CDCl}_{3}\right)$

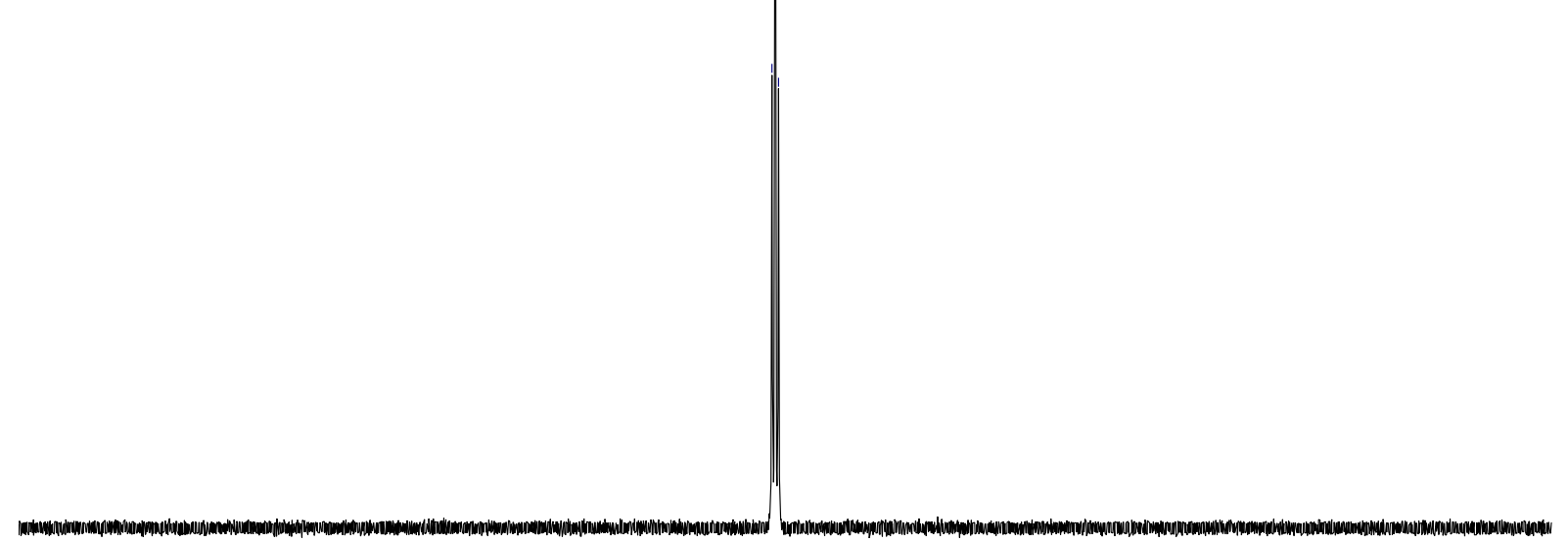

$\begin{array}{lllllllllllllllllllllllllllll}55 & 50 & 45 & 40 & 35 & 30 & 25 & 20 & 15 & 10 & 5 & \underset{p}{0} & -5 & -10 & -15 & -20 & -25 & -30 & -35 & -40 & -45 & -50 & -55 & -1\end{array}$

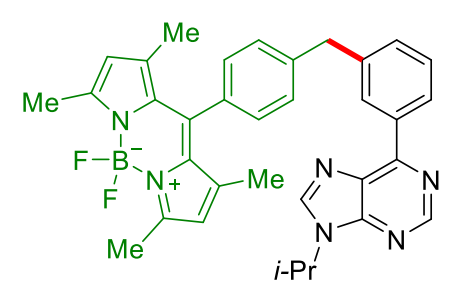

$6 a$

${ }^{19} \mathrm{~F}-\mathrm{NMR}$

(376 $\mathrm{MHz}, \mathrm{CDCl}_{3}$ )

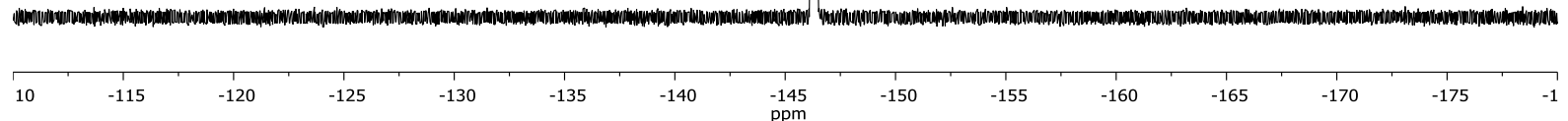



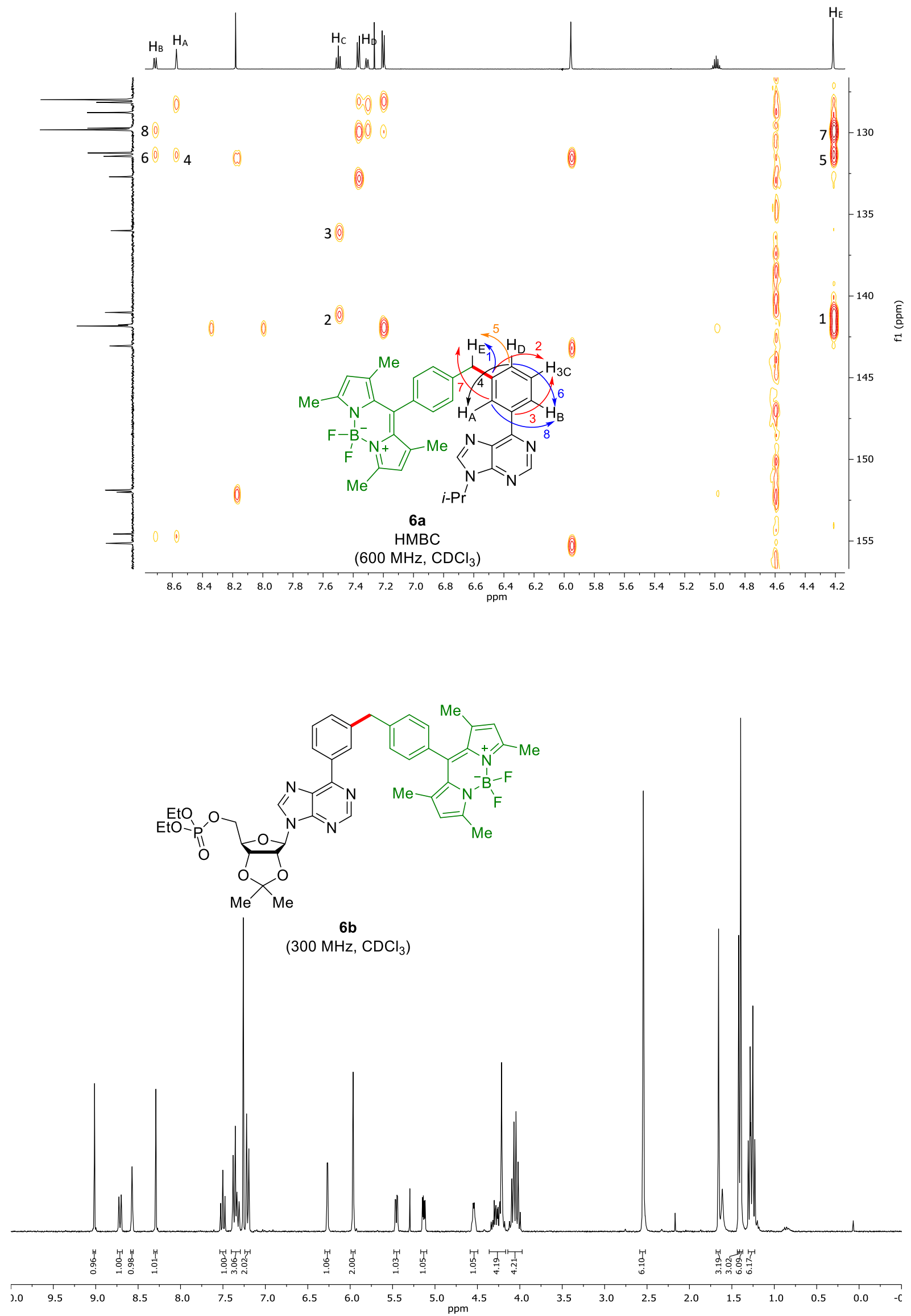

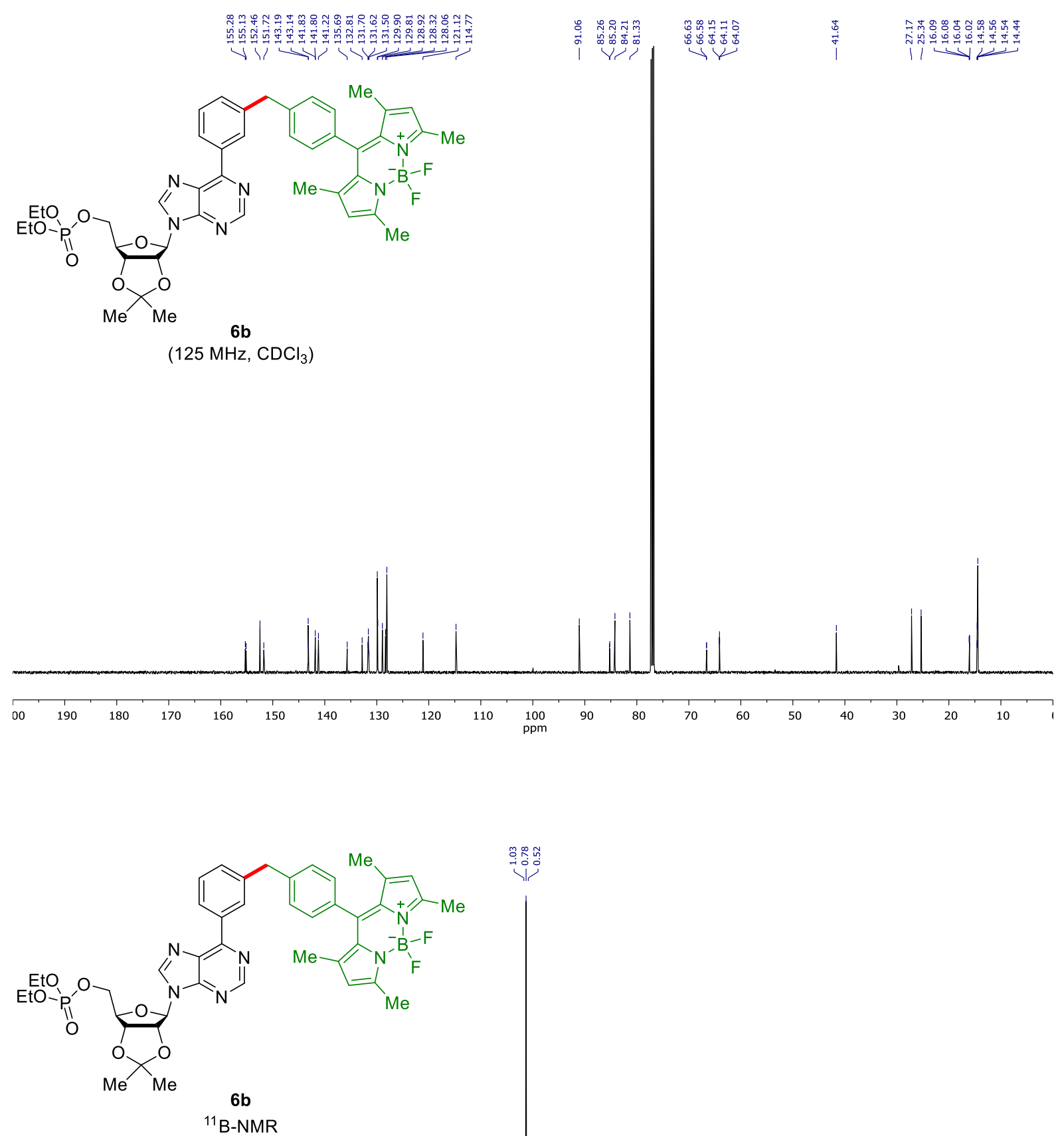

ํ.

$\left(128 \mathrm{MHz}, \mathrm{CDCl}_{3}\right.$ )

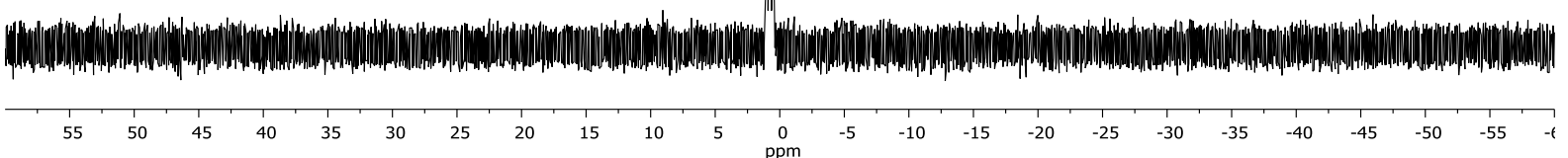




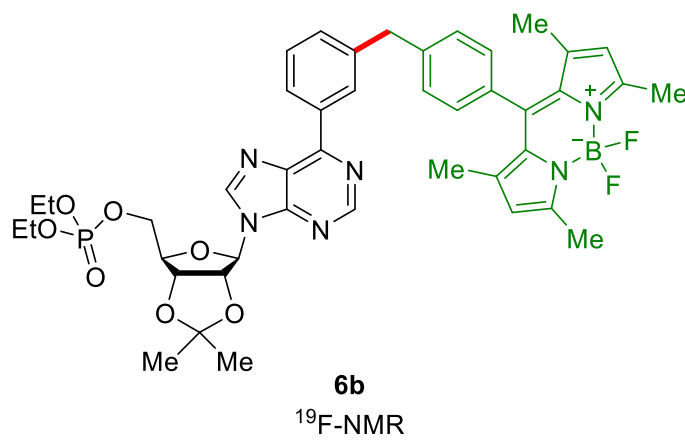

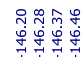

(376 MHz, $\mathrm{CDCl}_{3}$ )

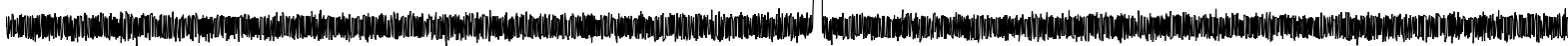

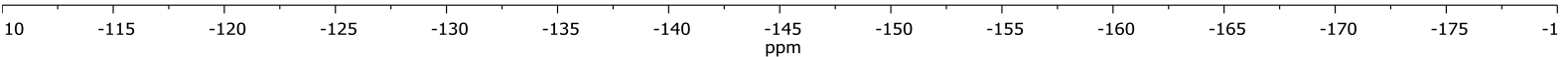

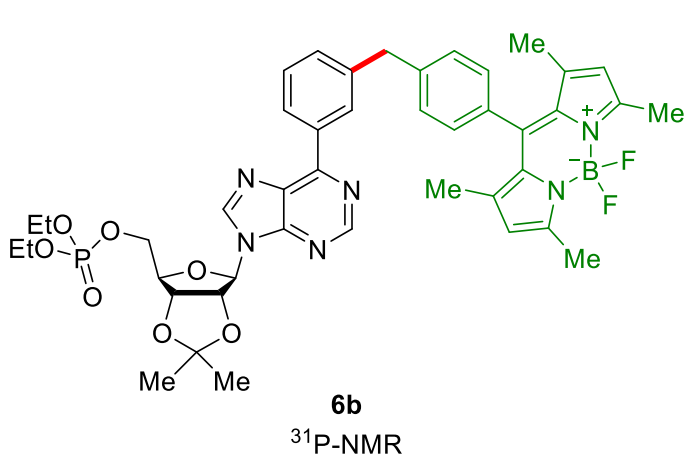

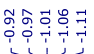

(162 MHz, $\mathrm{CDCl}_{3}$ )

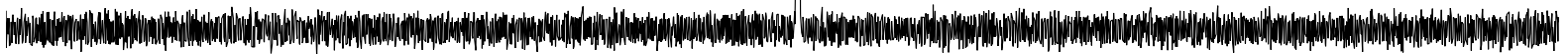

io $\quad \begin{array}{llllllllllllllllllllllll}1 & 45 & 40 & 35 & 30 & 25 & 20 & 15 & 10 & 5 & \underset{p}{0} & -5 & -10 & -15 & -20 & -25 & -30 & -35 & -40 & -45 & -!\end{array}$




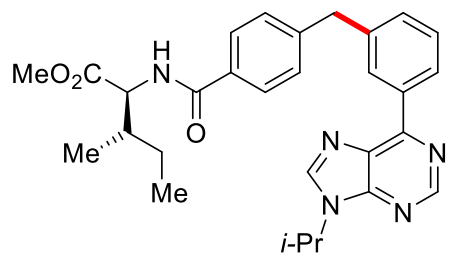

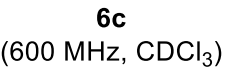

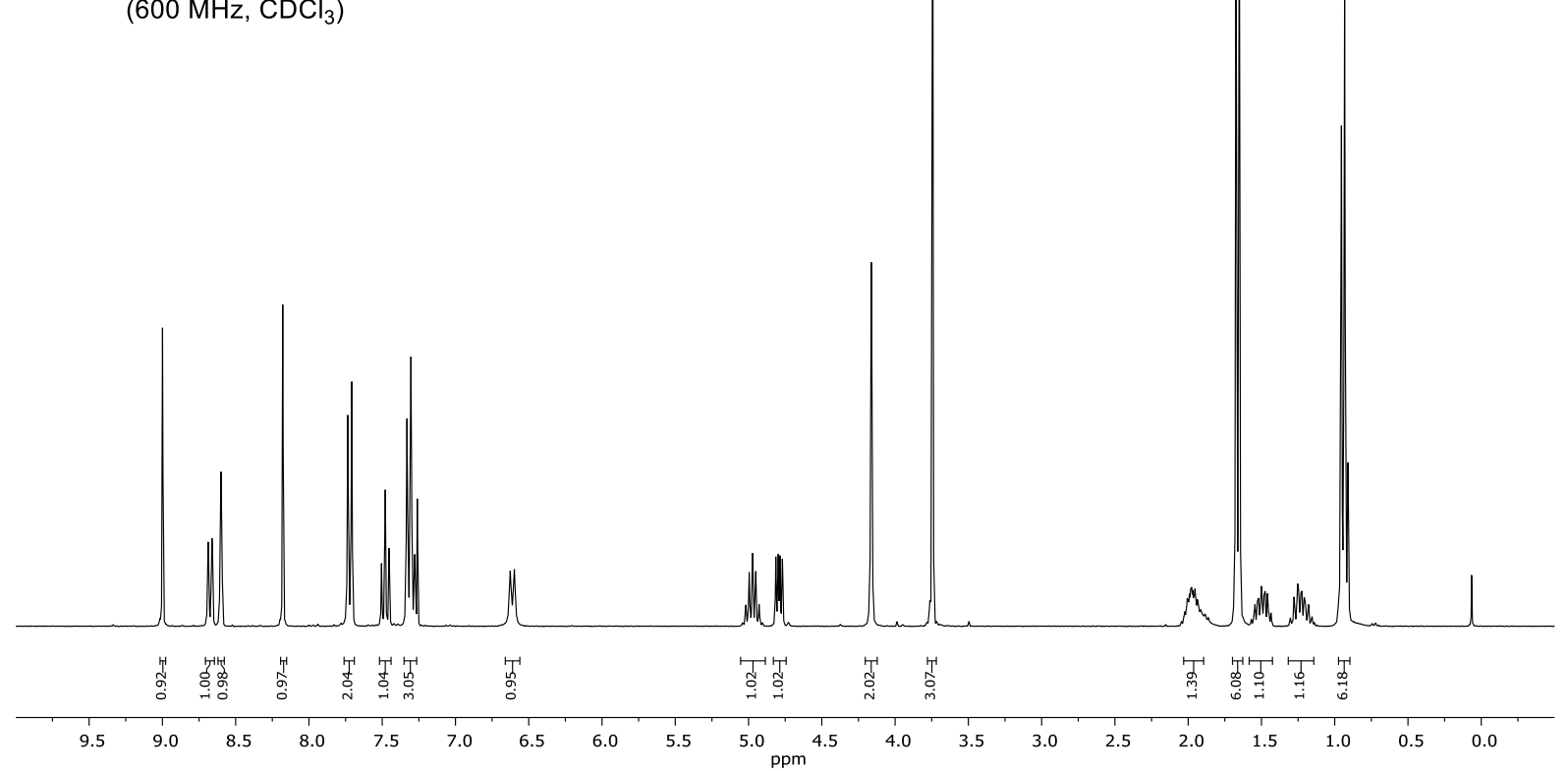

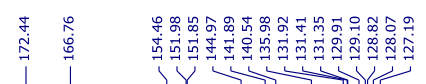

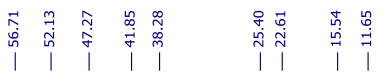

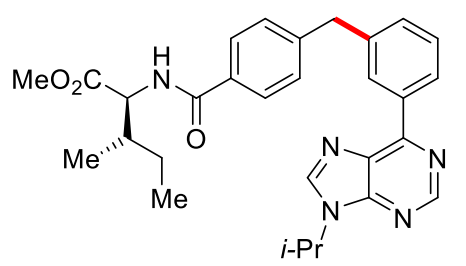

$6 \mathrm{c}$

(125 $\mathrm{MHz} \mathrm{CDCl}_{3}$ )

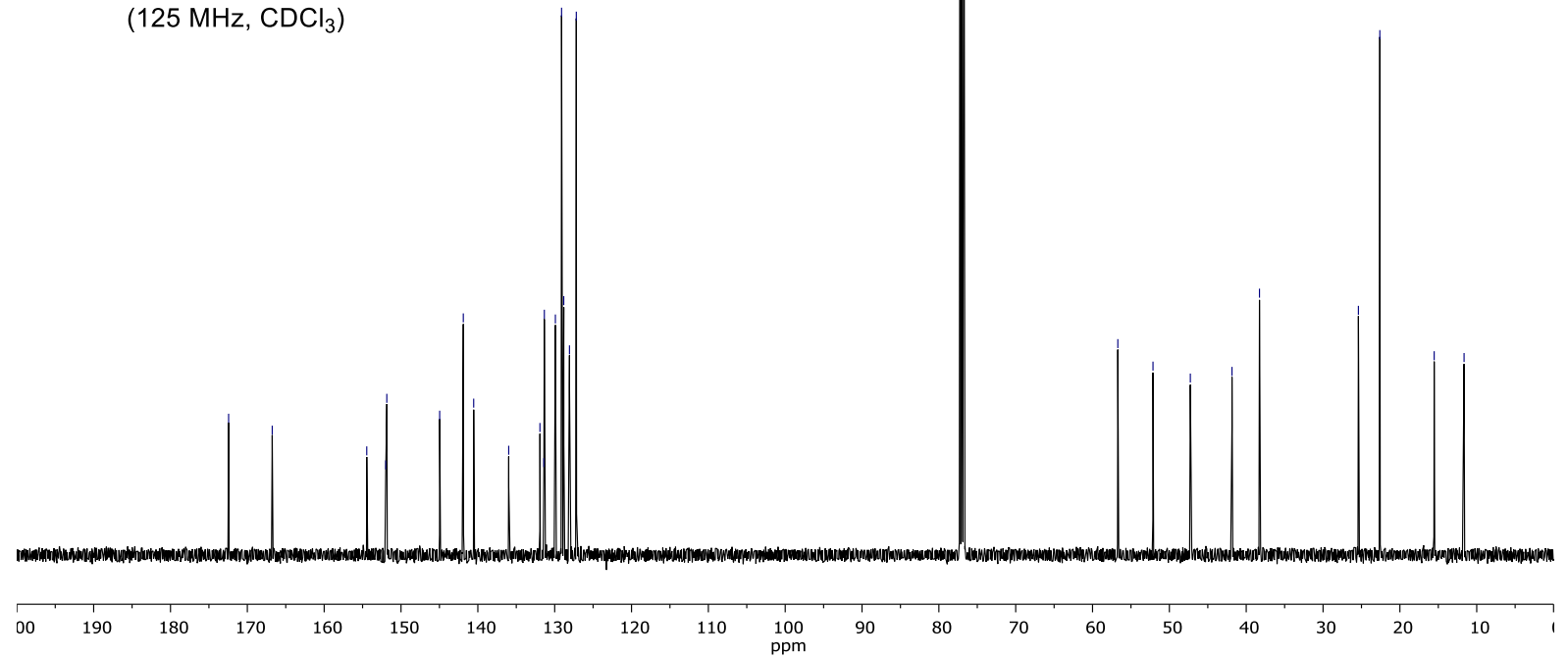



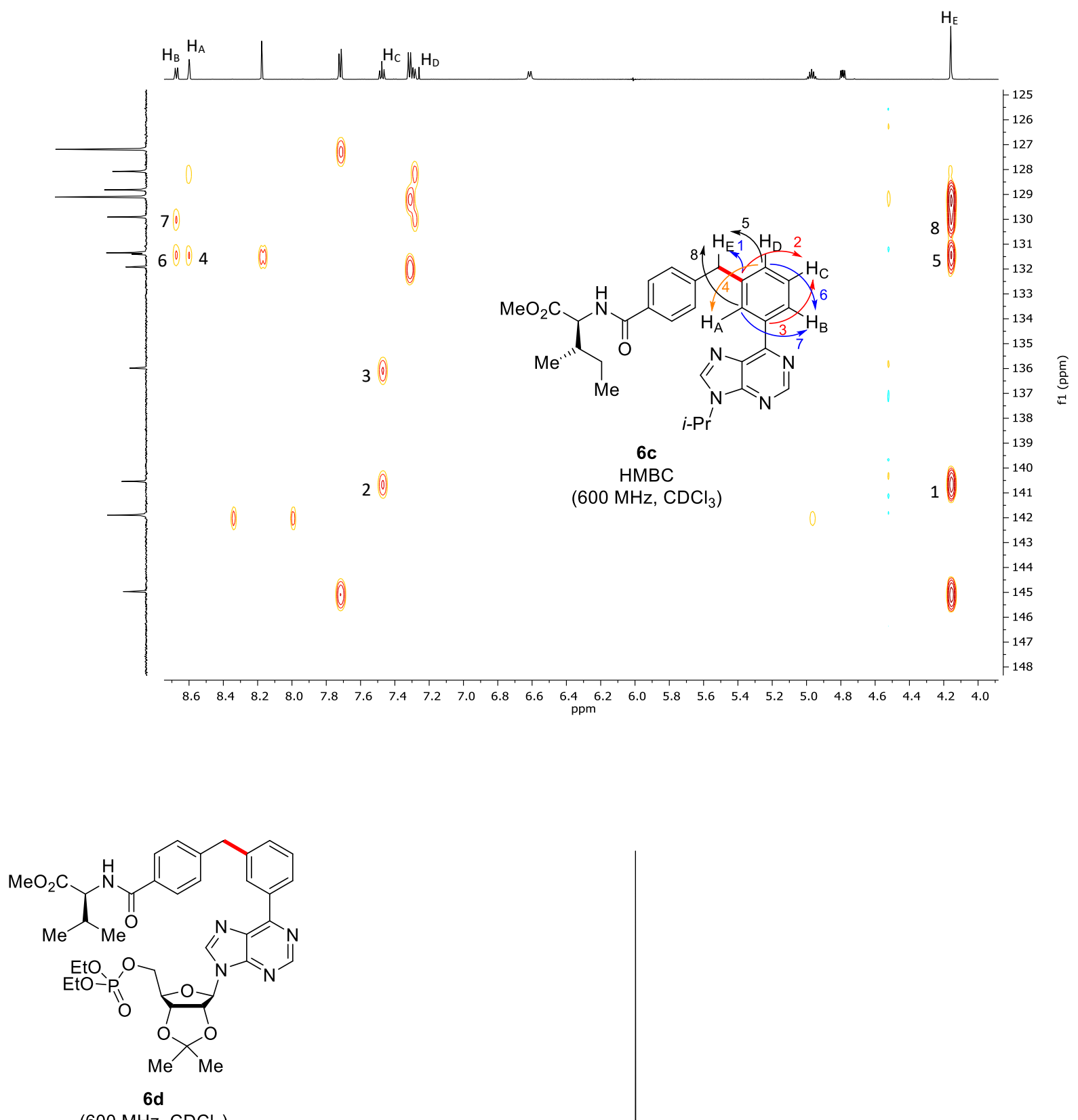

(600 $\mathrm{MHz} \mathrm{CDCl}_{3}$ )

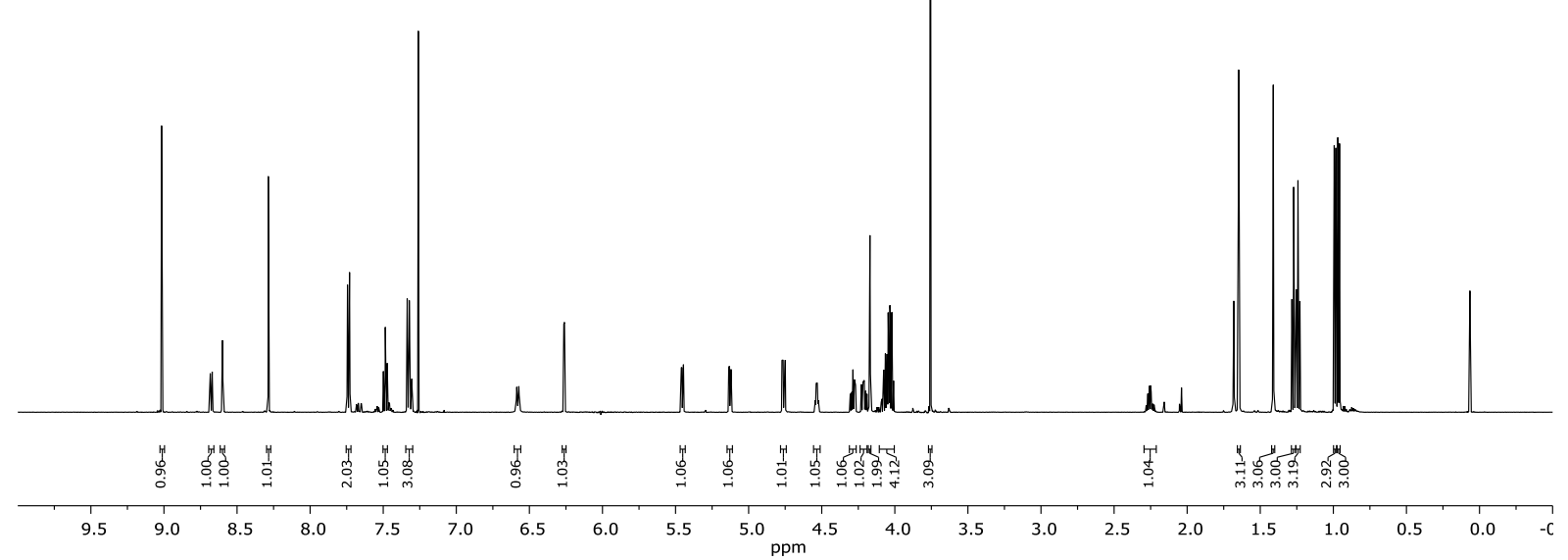




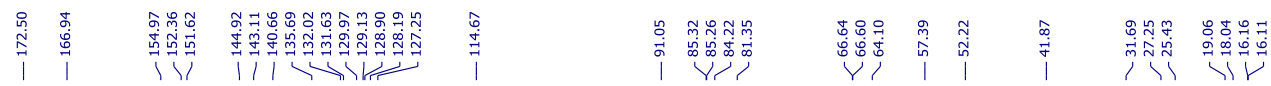

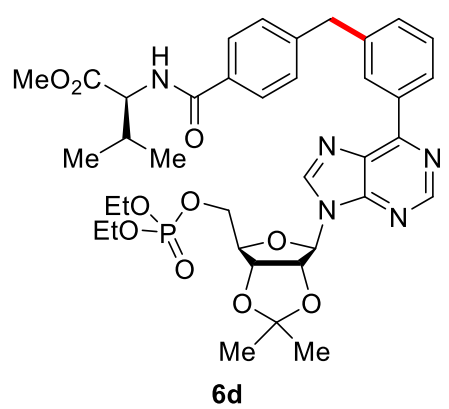

$\left(125 \mathrm{MHz} \mathrm{CDCl}_{3}\right)$
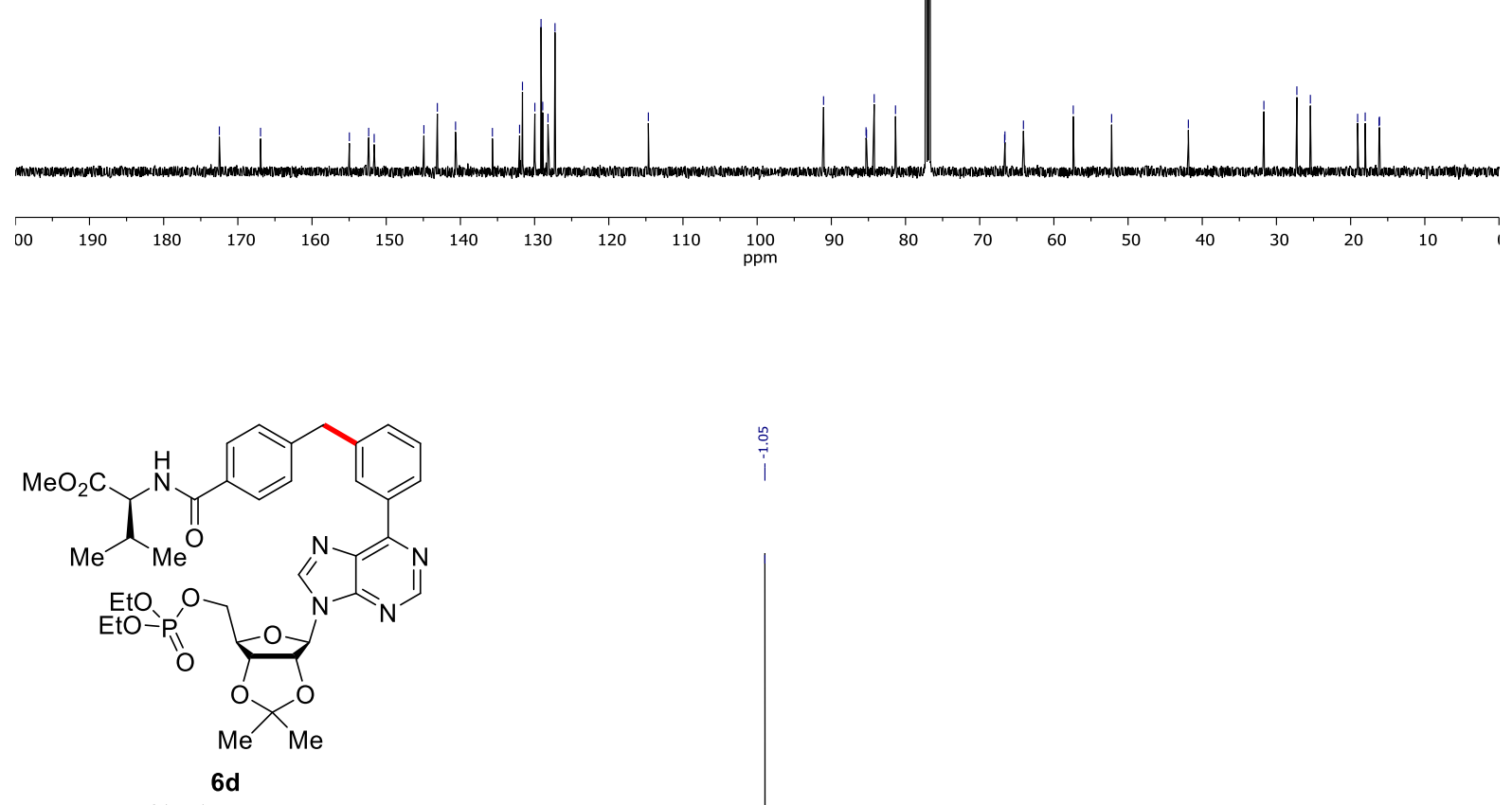

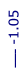

${ }^{31} \mathrm{P}\left\{{ }^{1} \mathrm{H}\right\}-\mathrm{NMR}$

(121 MHz, $\mathrm{CDCl}_{3}$ ) 
<smiles>CC(=O)C(NC(=O)c1ccc(Cc2cccc(-c3ncnc4c3ncn4C(C)C)c2)cc1)C(C)C</smiles>

$s-6 e$

$\left(600 \mathrm{MHz}, \mathrm{CDCl}_{3}\right.$ )

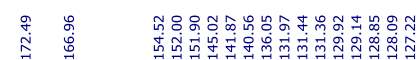

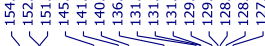

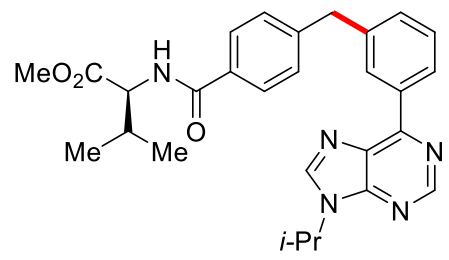

s-6e

(125 MHz, $\mathrm{CDCl}_{3}$ )

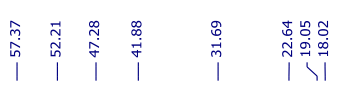

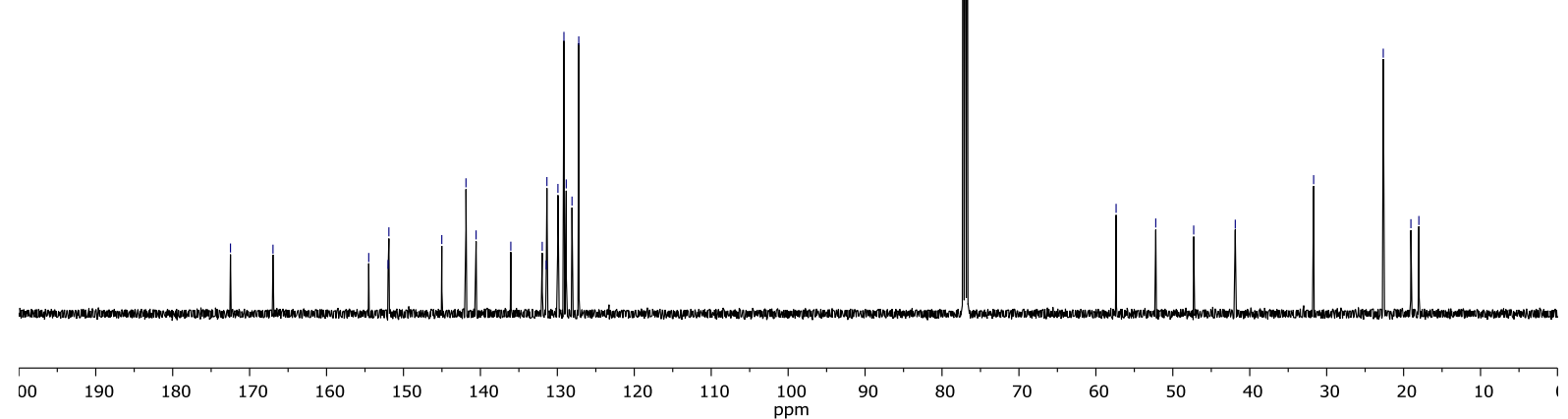




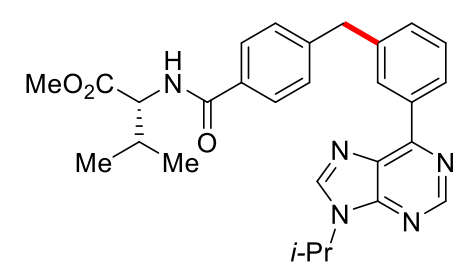

$R-6 \mathrm{e}$

(600 $\mathrm{MHz}, \mathrm{CDCl}_{3}$ )

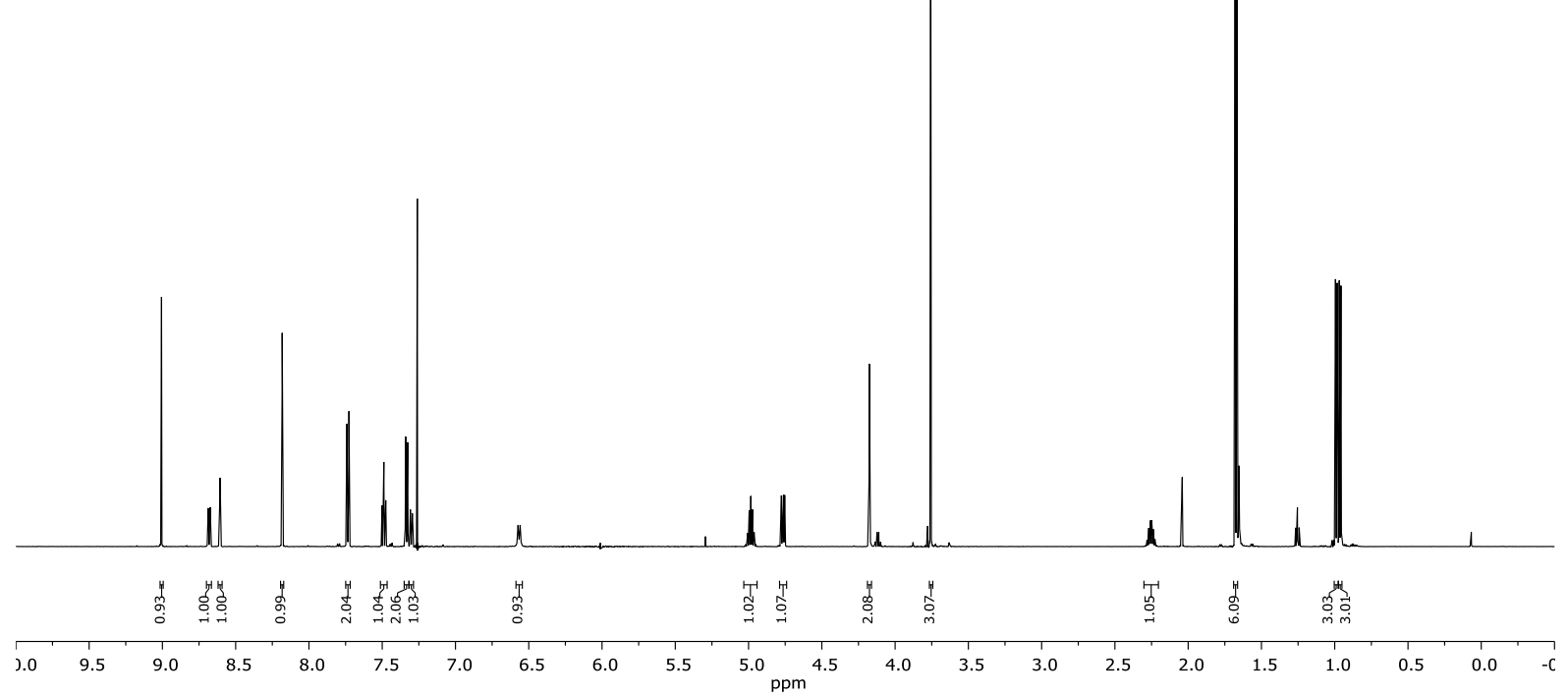

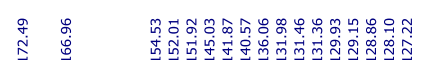

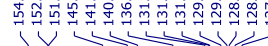

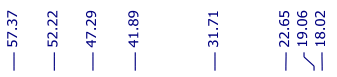<smiles>CC(=O)C(NC(=O)c1ccc(Cc2cccc(-c3ncnc4c3ncn4C(C)C)c2)cc1)C(C)C</smiles>

$R-6 \mathrm{e}$

$\left(125 \mathrm{MHz}, \mathrm{CDCl}_{3}\right)$

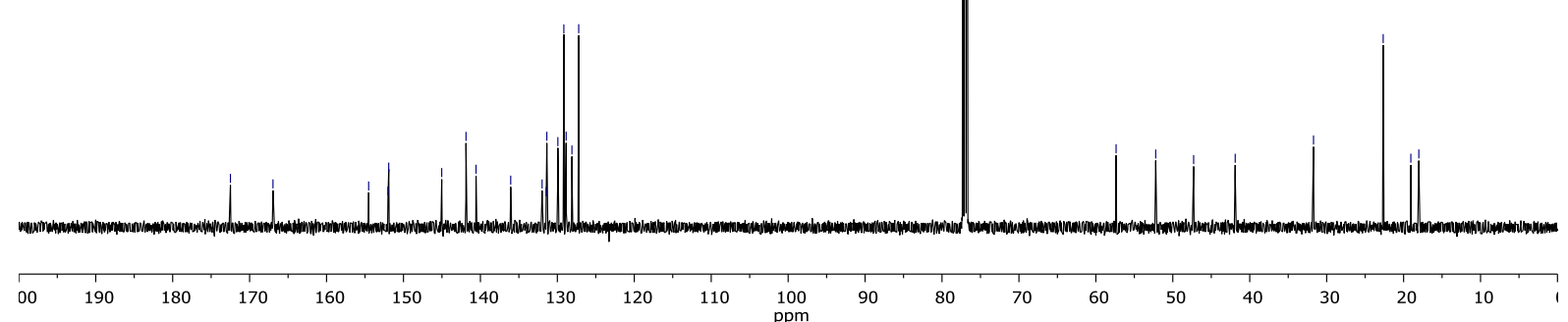




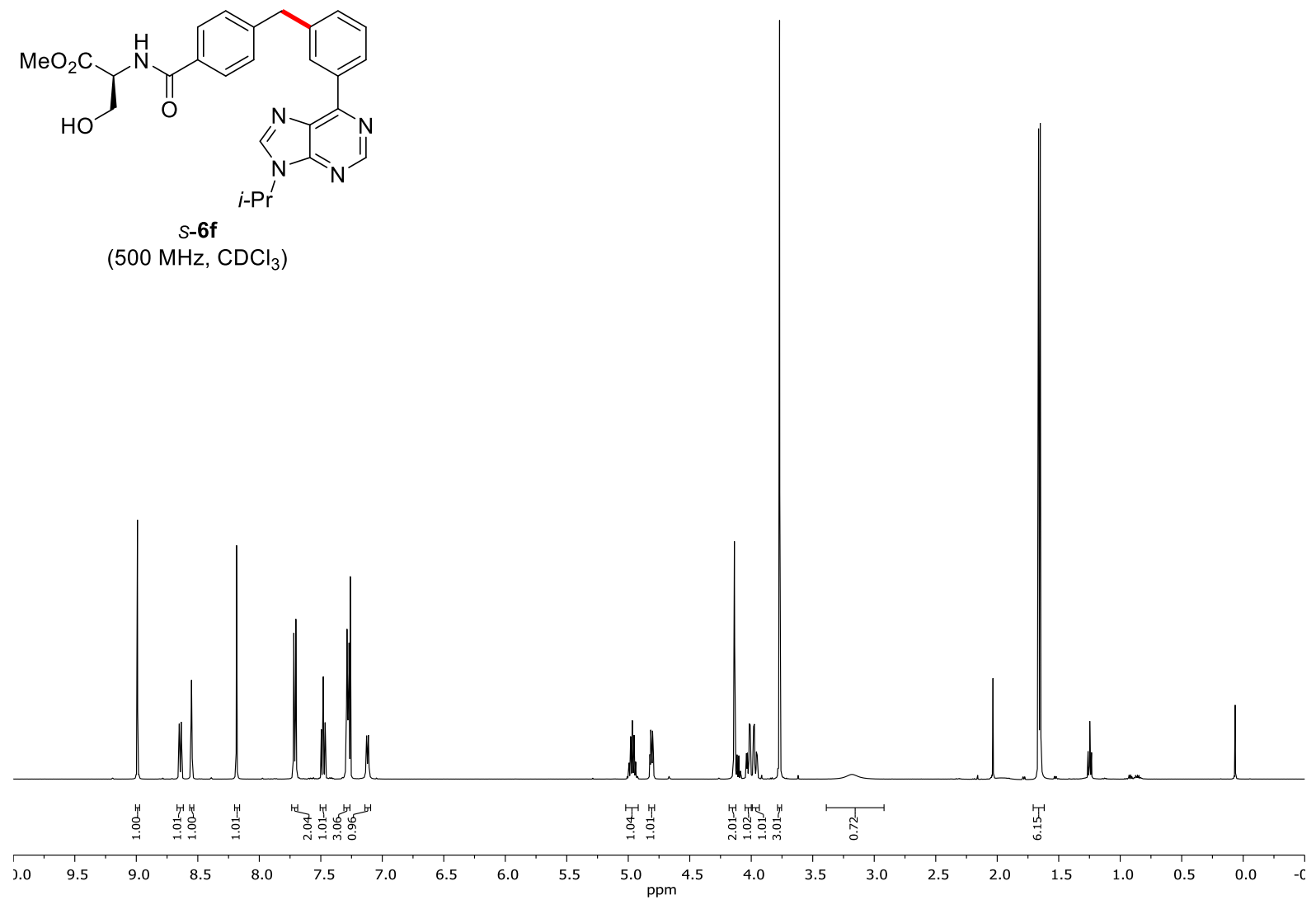

年

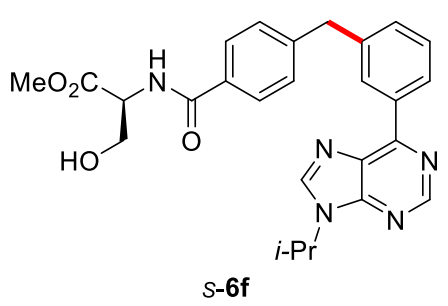

(125 $\mathrm{MHz}, \mathrm{CDCl}_{3}$ )

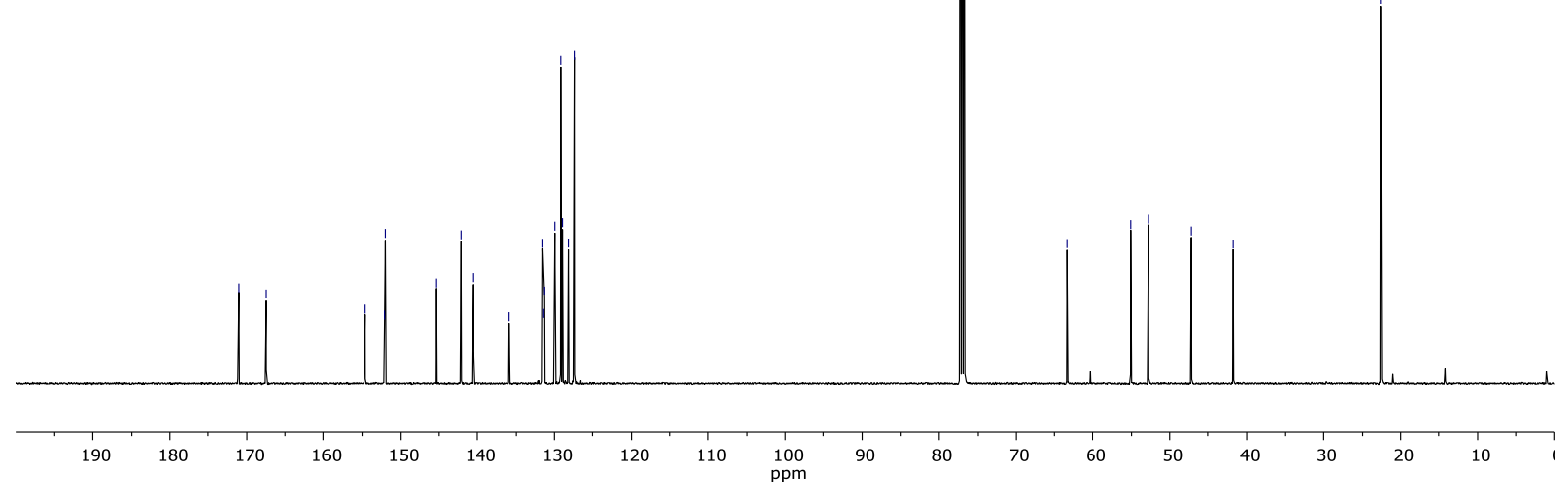




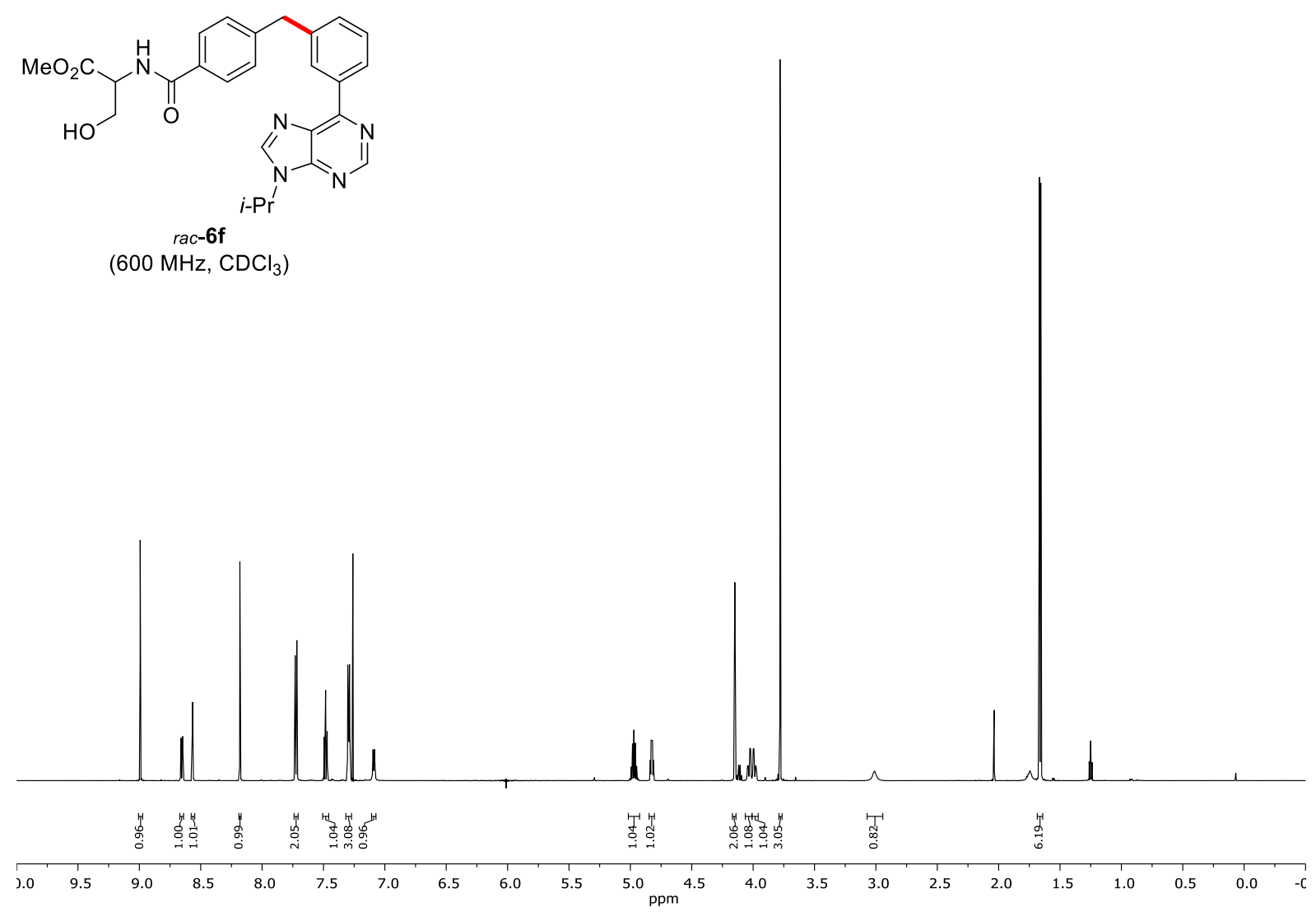

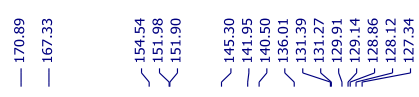<smiles>CC(=O)C(CO)NC(=O)c1ccc(Cc2cccc(-c3ncnc4c3ncn4[P+](F)(F)c3ccccc3)c2)cc1</smiles>

(125 MHz, $\mathrm{CDCl}_{3}$ )

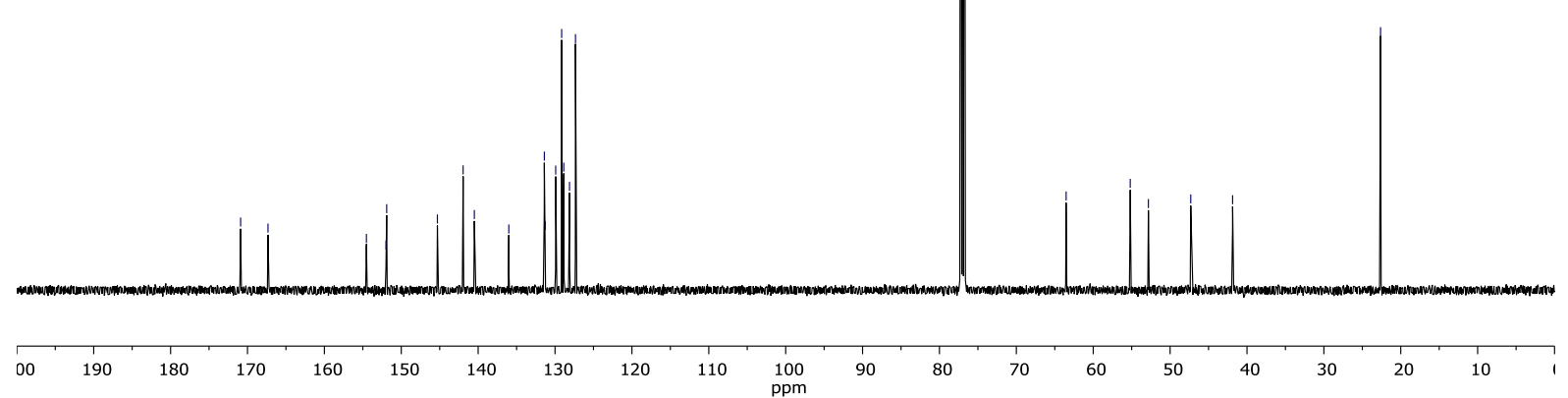




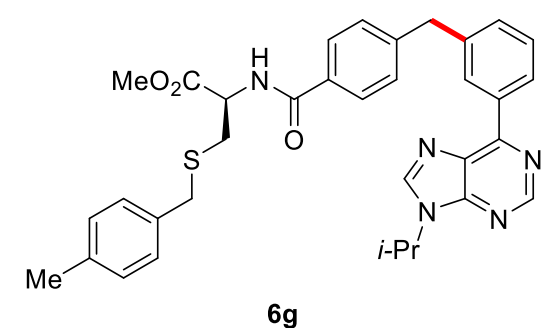

$\left(600 \mathrm{MHz} \mathrm{CDCl}_{3}\right)$

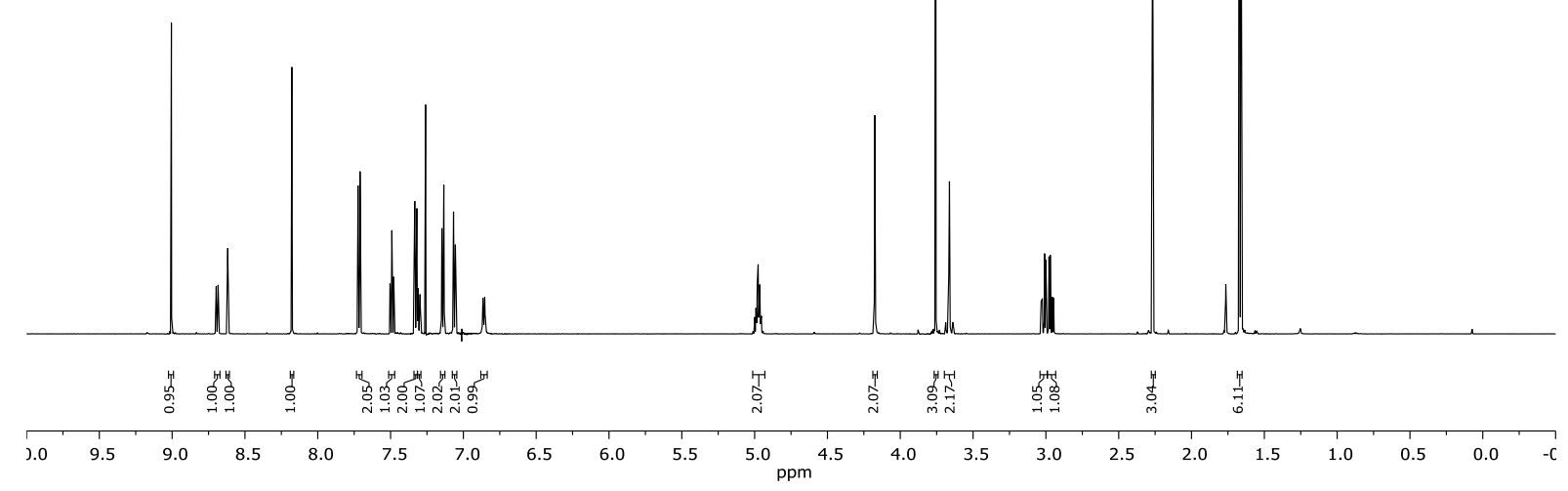

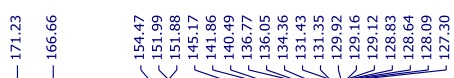

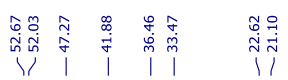

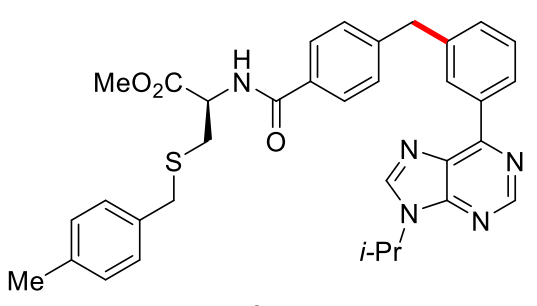

$\left(125 \mathrm{MHz} \mathrm{CDCl}_{3}\right)$

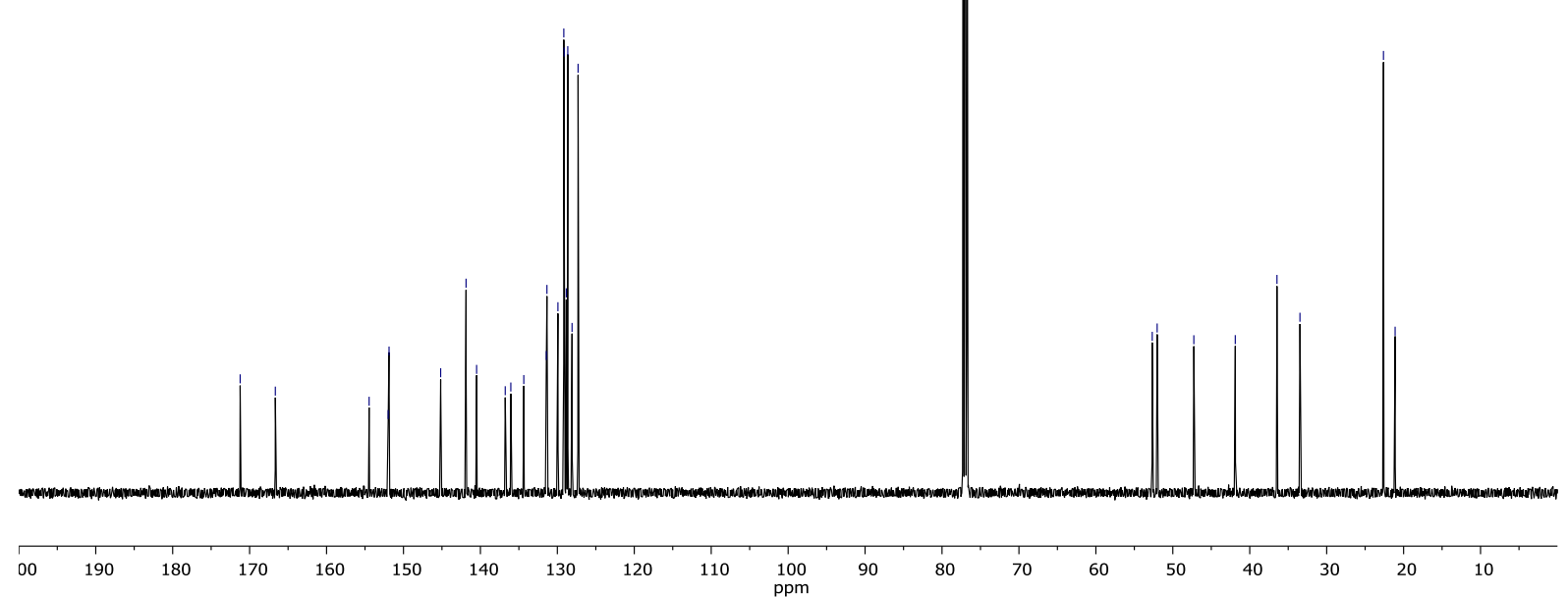



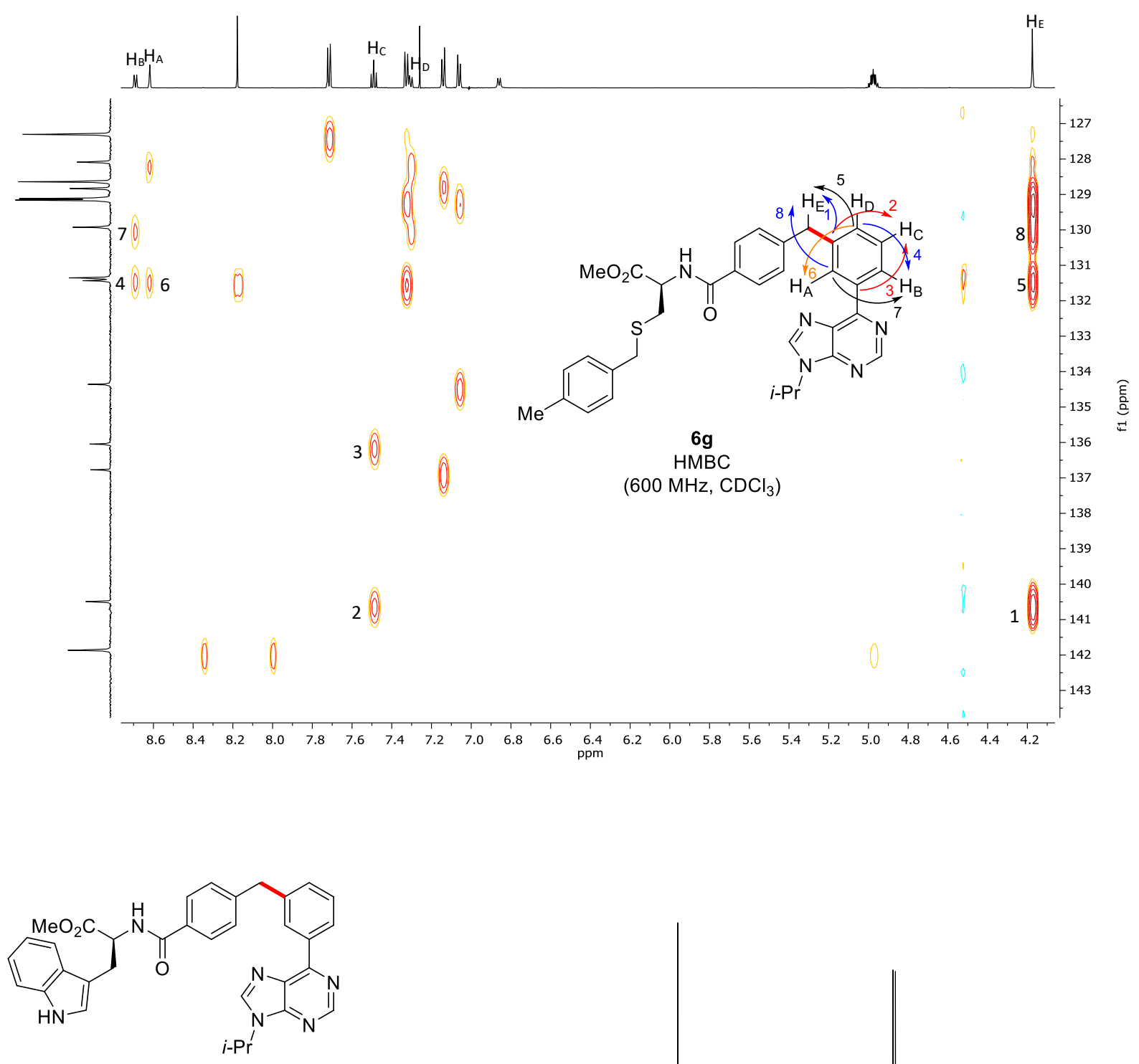

$\left(600 \mathrm{MHz}, \mathrm{CDCl}_{3}\right)$

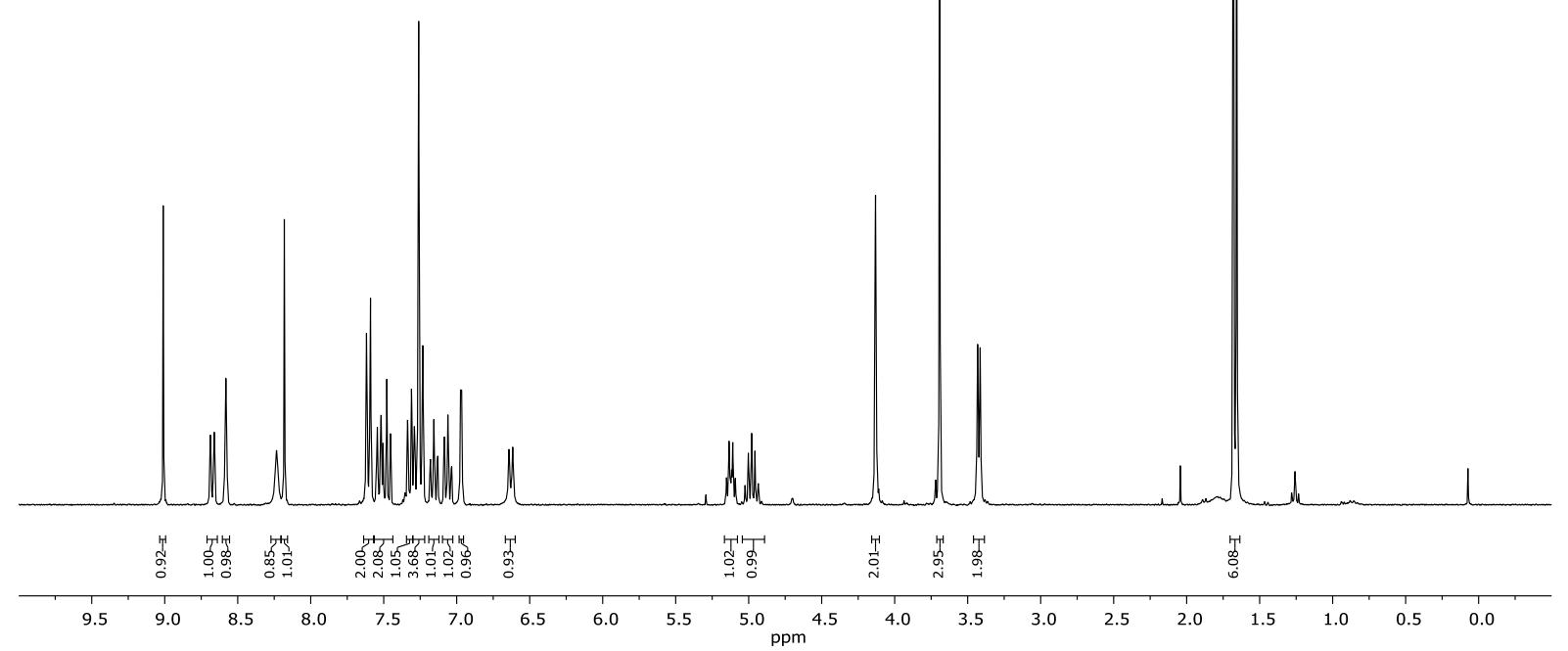




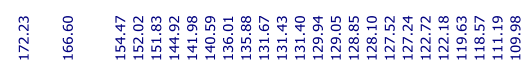

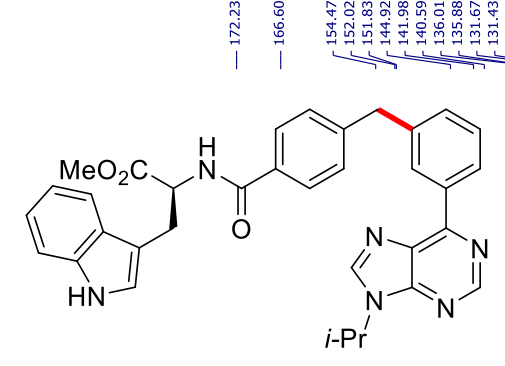

6h

$\left(125 \mathrm{MHz}, \mathrm{CDCl}_{3}\right)$

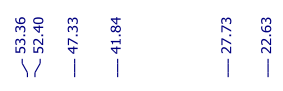
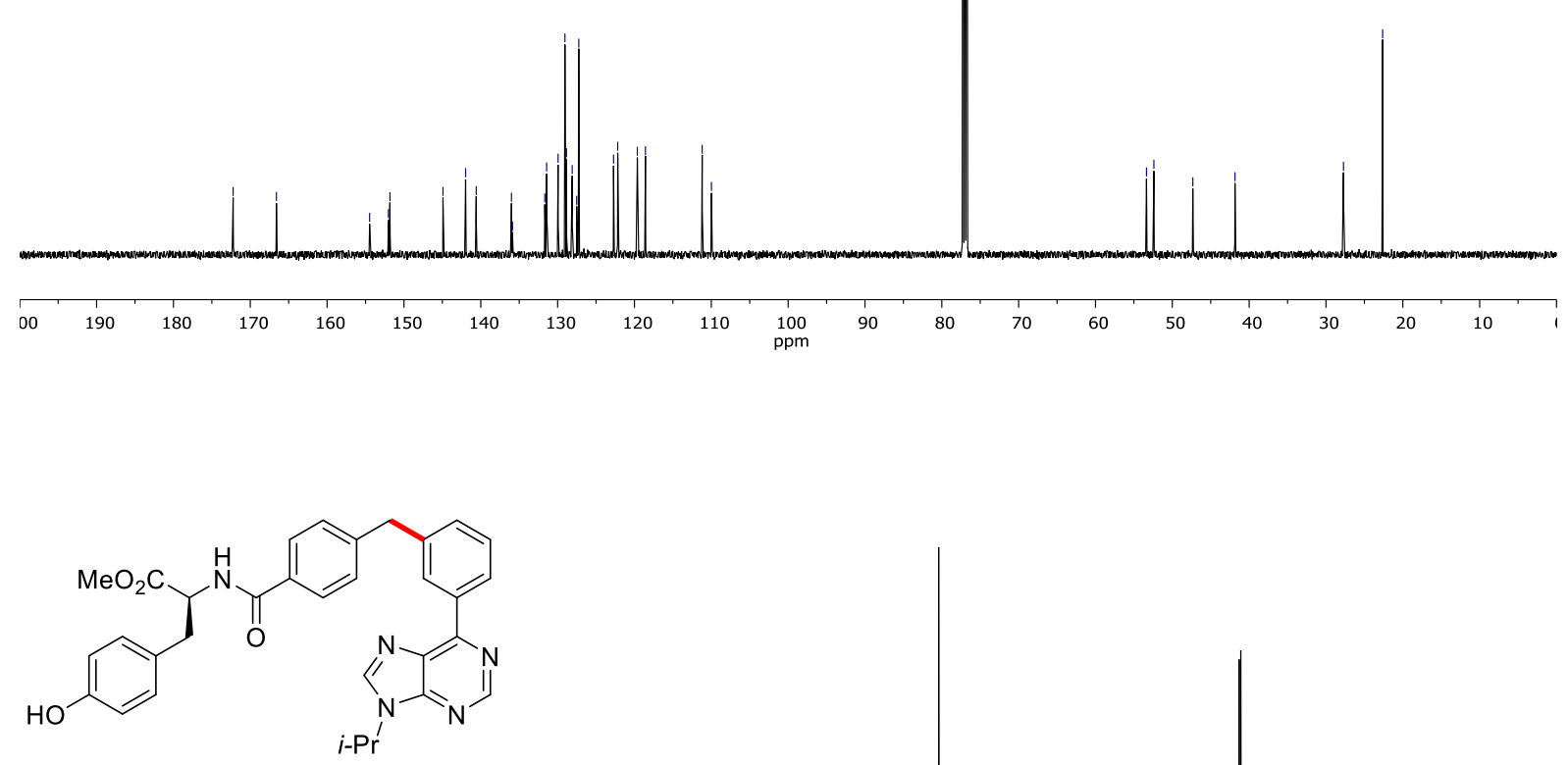

$6 i$

$\left(600 \mathrm{MHz}, \mathrm{CDCl}_{3}\right)$

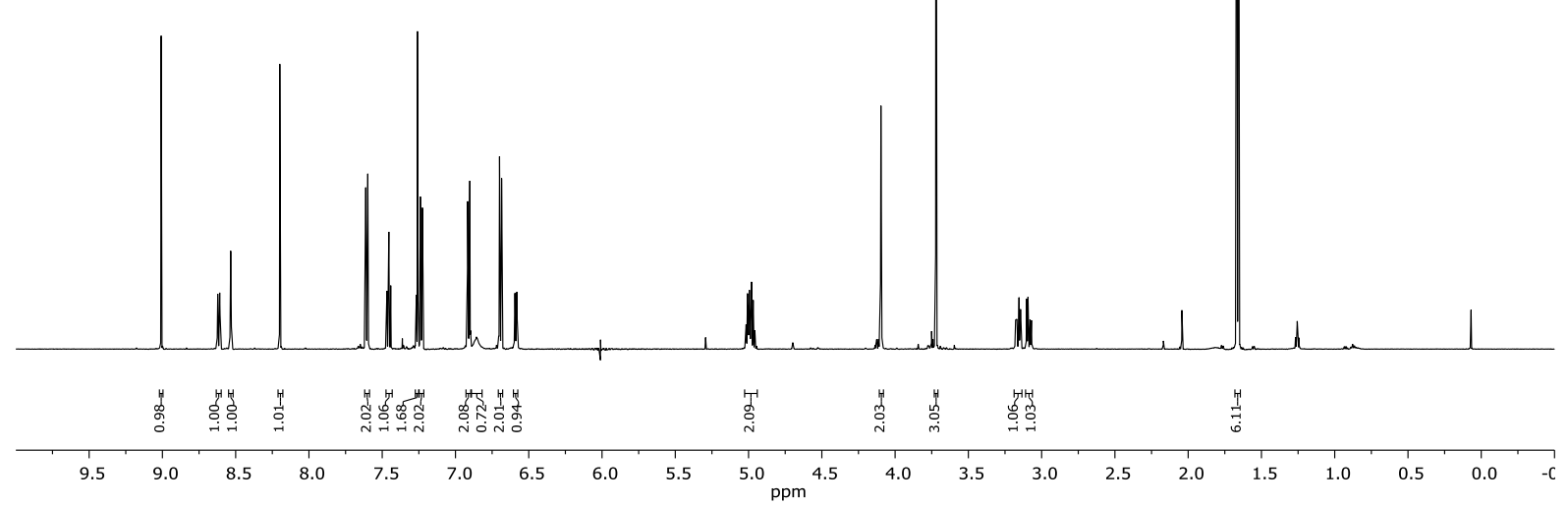



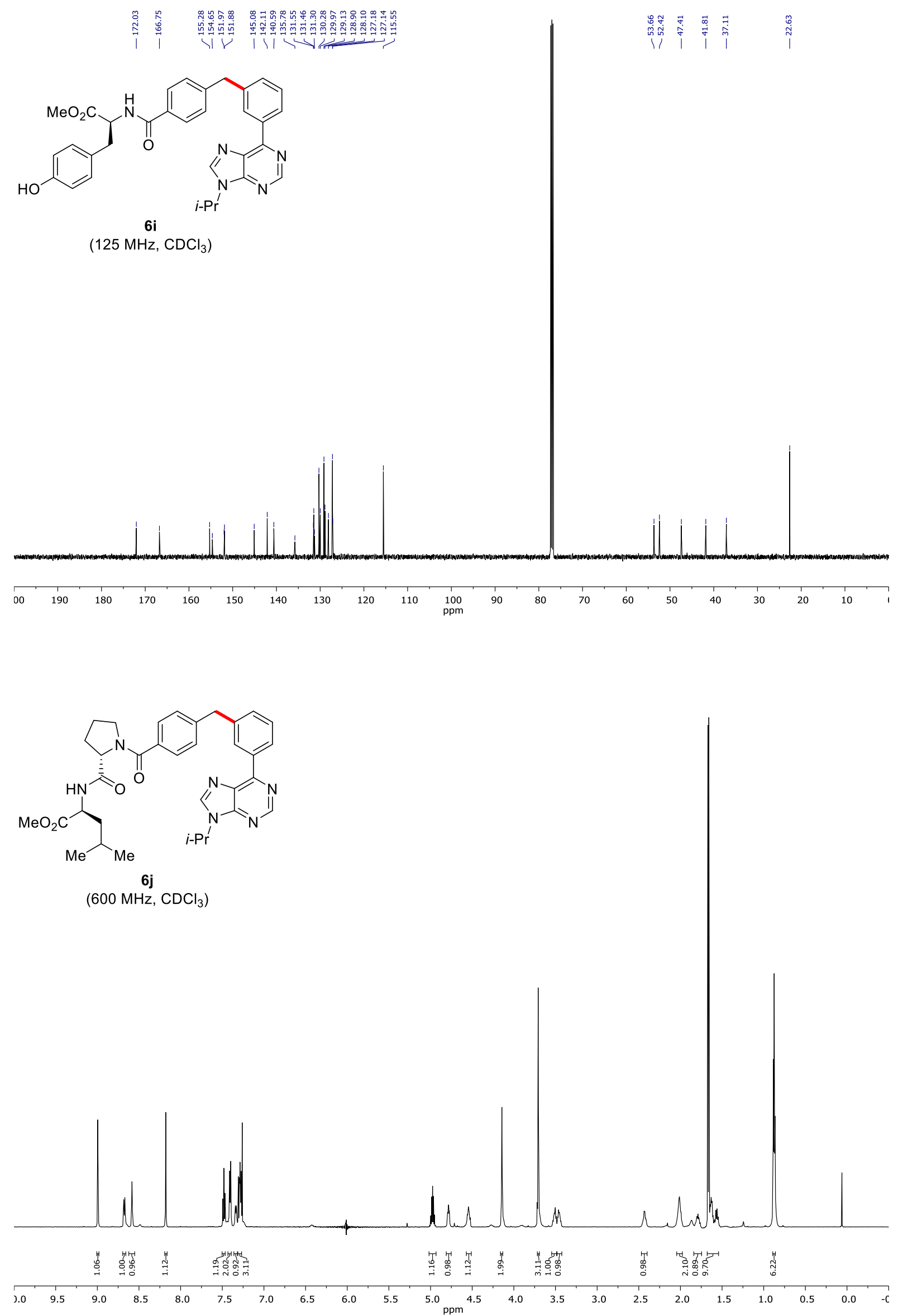

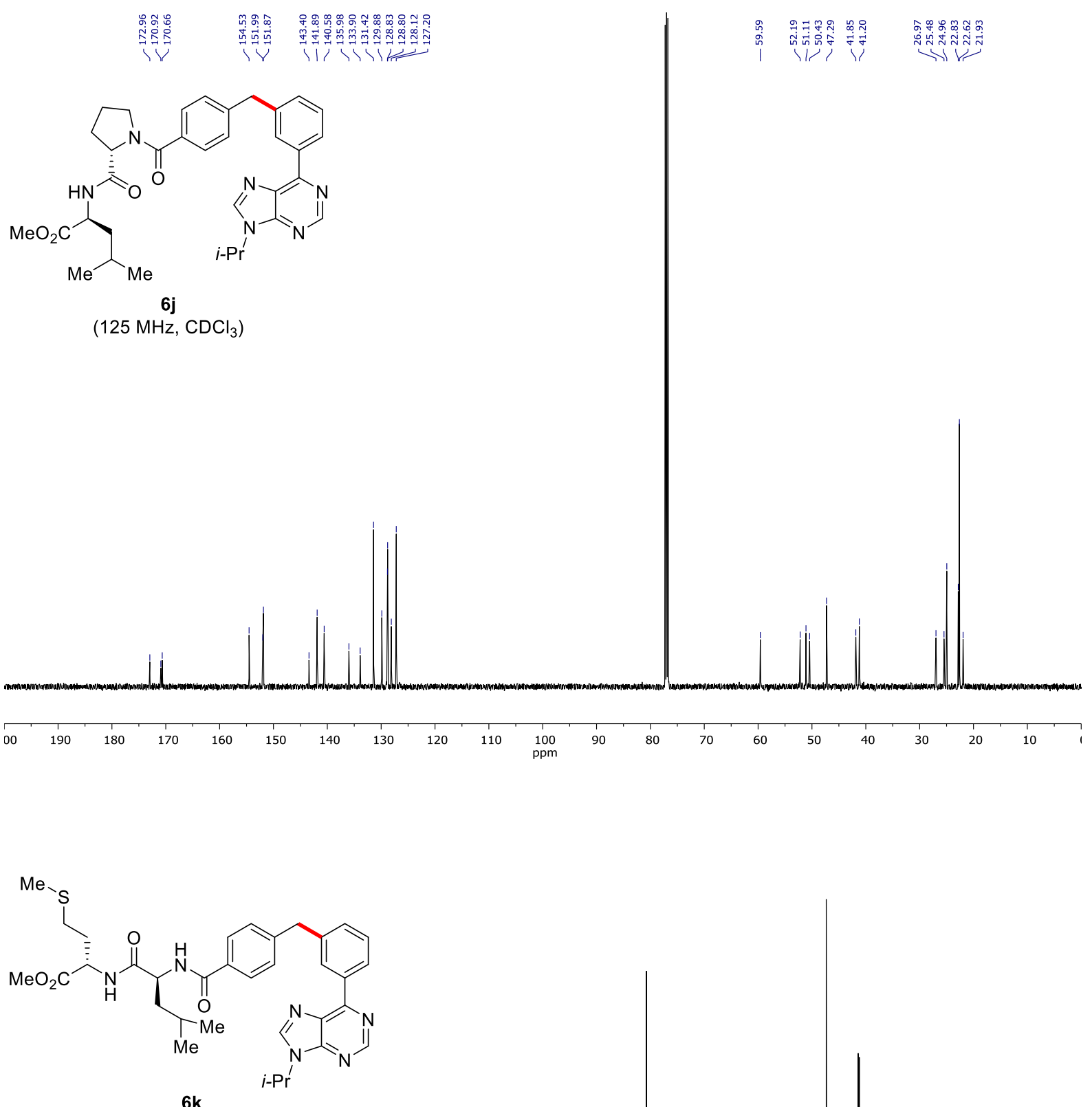

$\left(600 \mathrm{MHz}, \mathrm{CDCl}_{3}\right.$ )

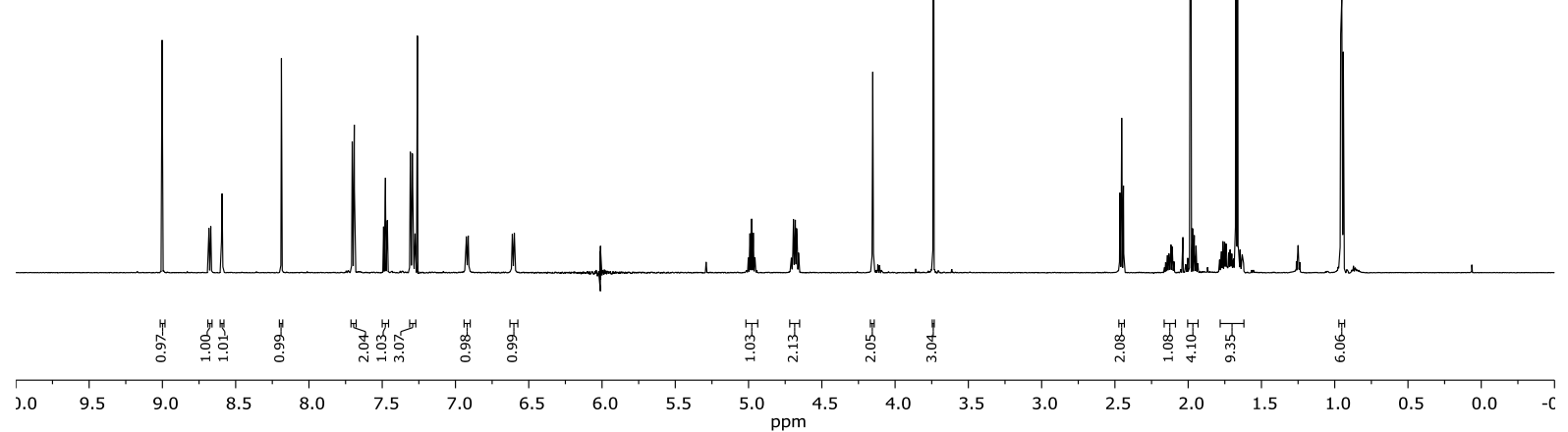




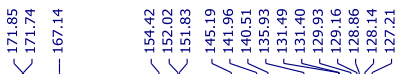

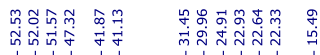

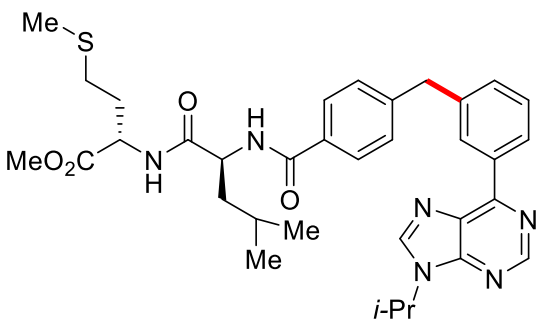

(125 MHz, $\mathrm{CDCl}_{3}$ )
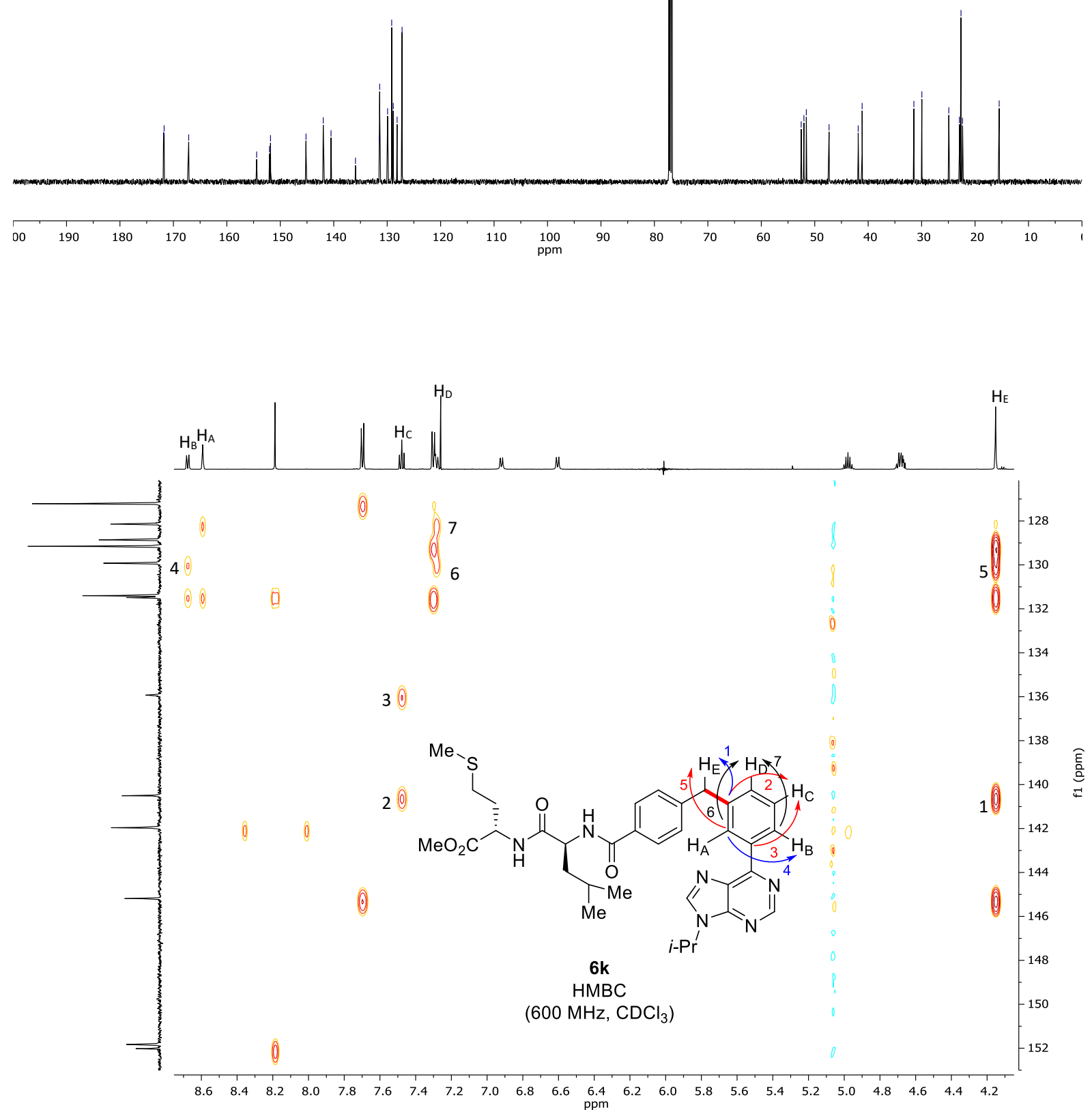


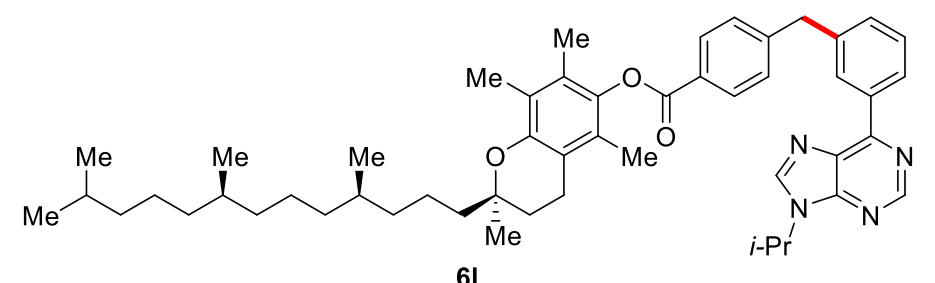

$\left(600 \mathrm{MHz} \mathrm{CDCl}_{3}\right)$

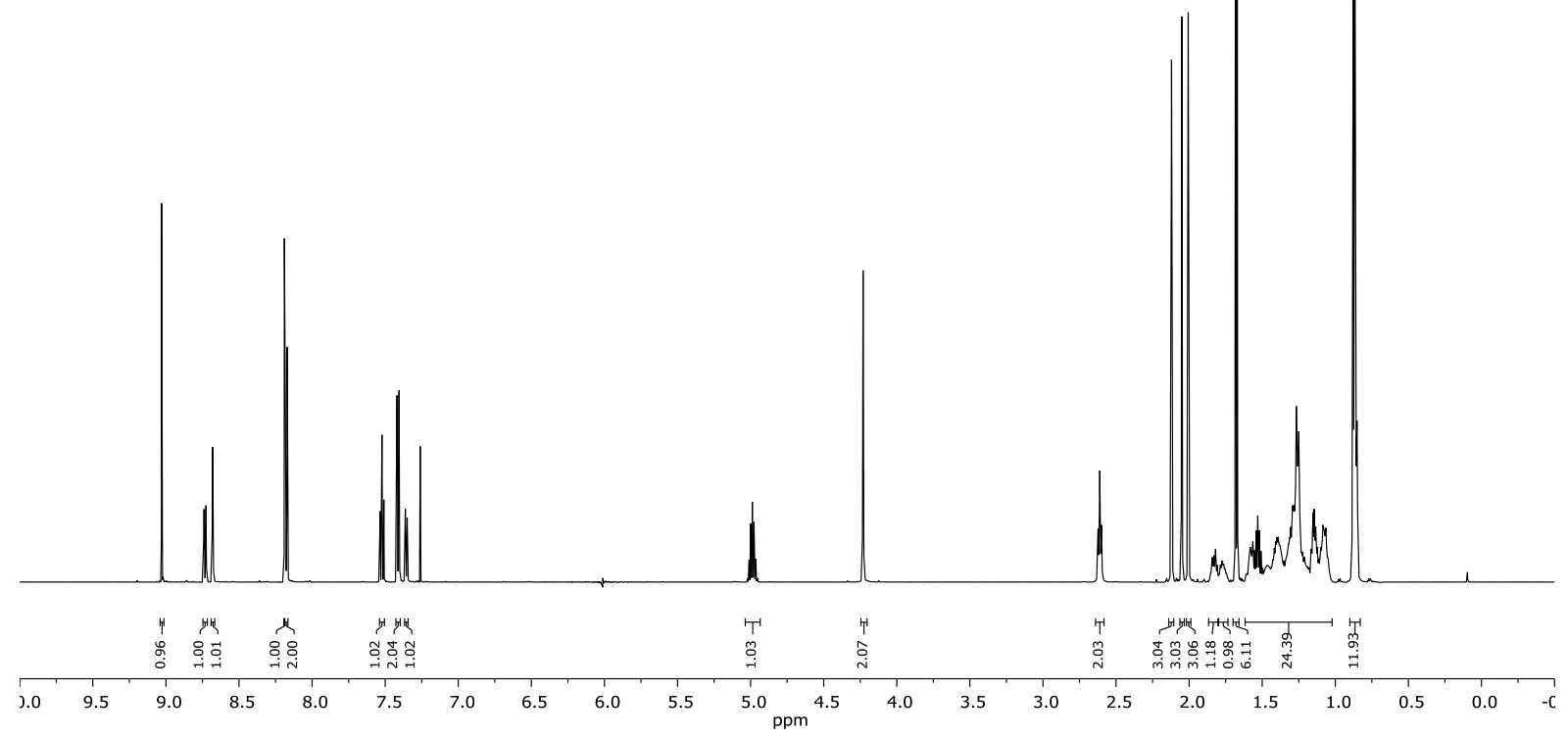

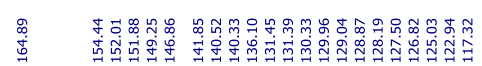

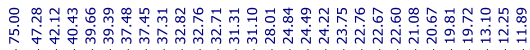

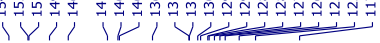

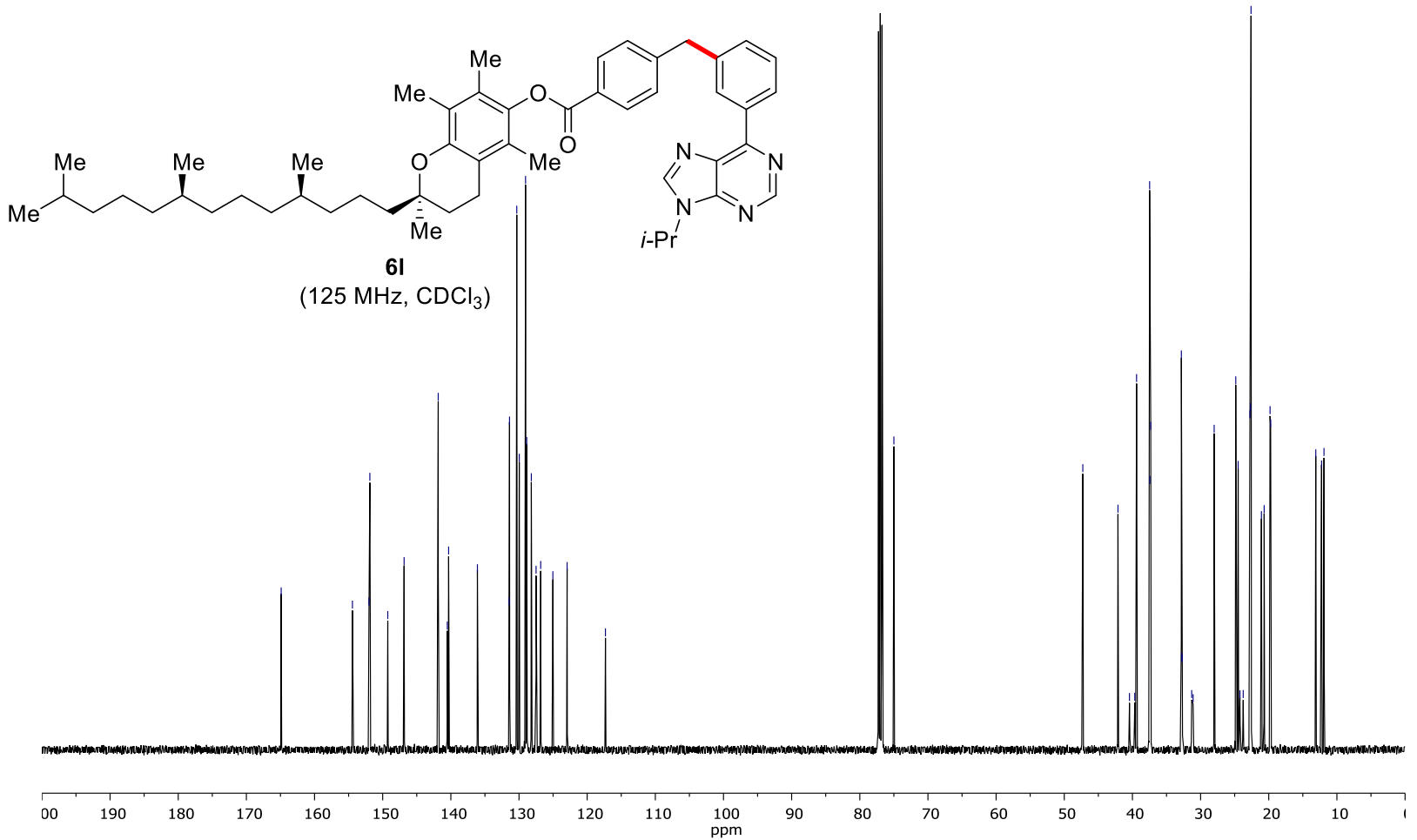




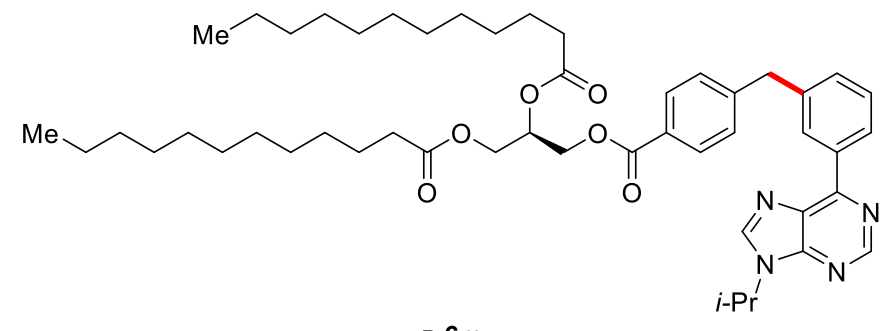

(400 $\mathrm{MHz}, \mathrm{CDCl}_{3}$ )

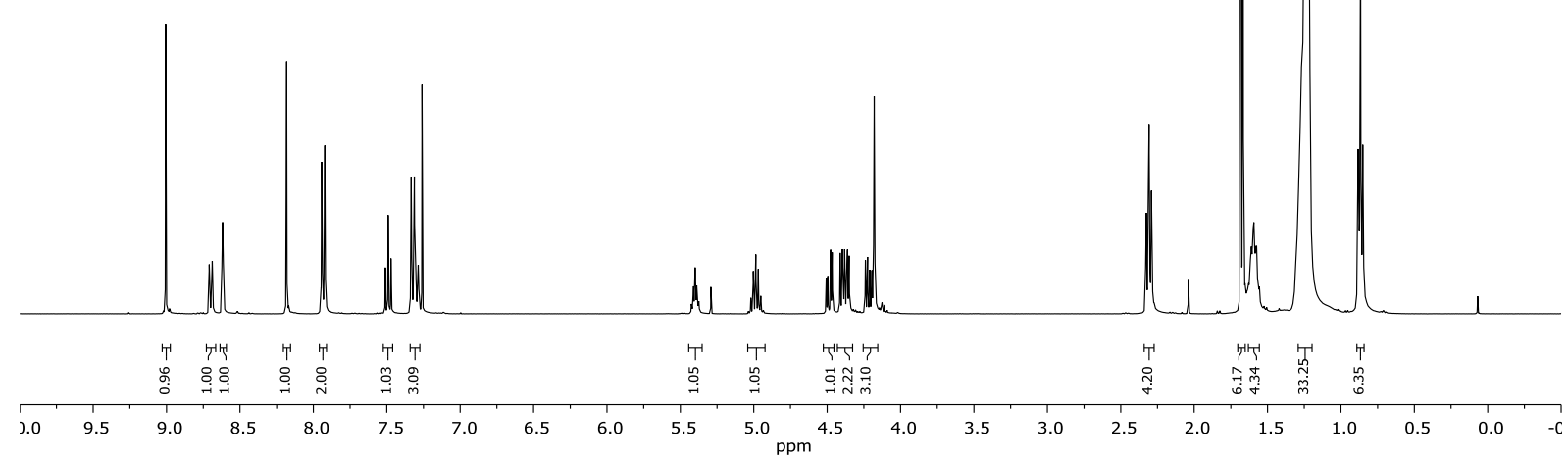

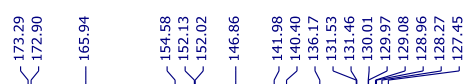

$\mathrm{Me}$

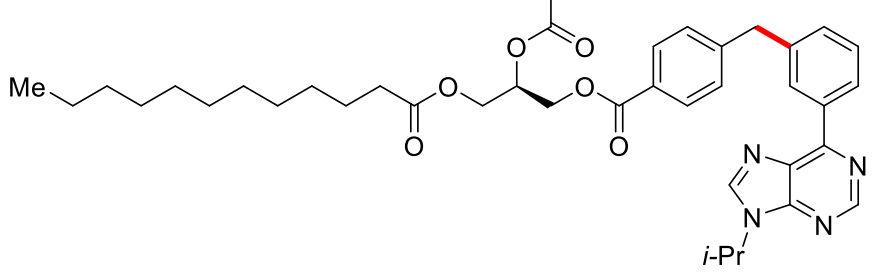

R-6m

(100 $\mathrm{MHz}, \mathrm{CDCl}_{3}$ )

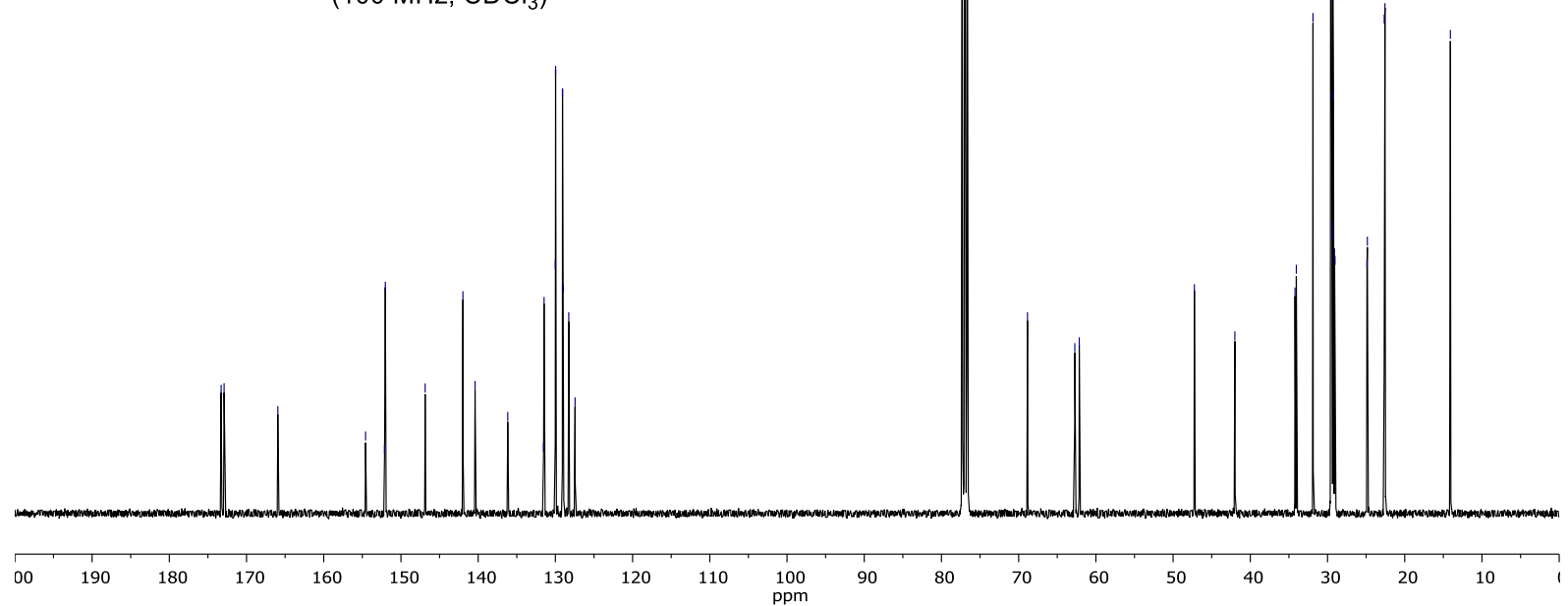




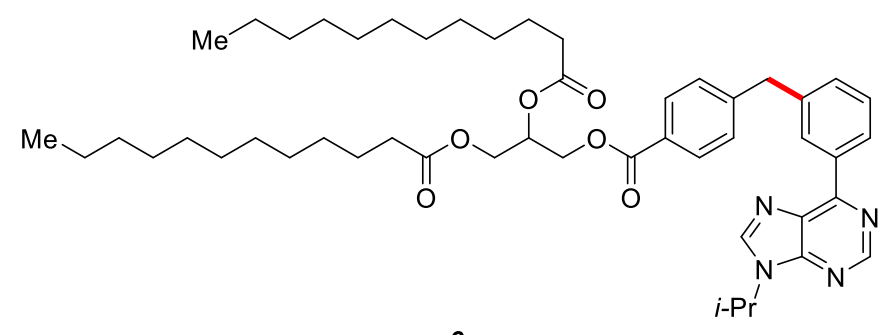

rac-6m

(600 $\mathrm{MHz}, \mathrm{CDCl}_{3}$ )

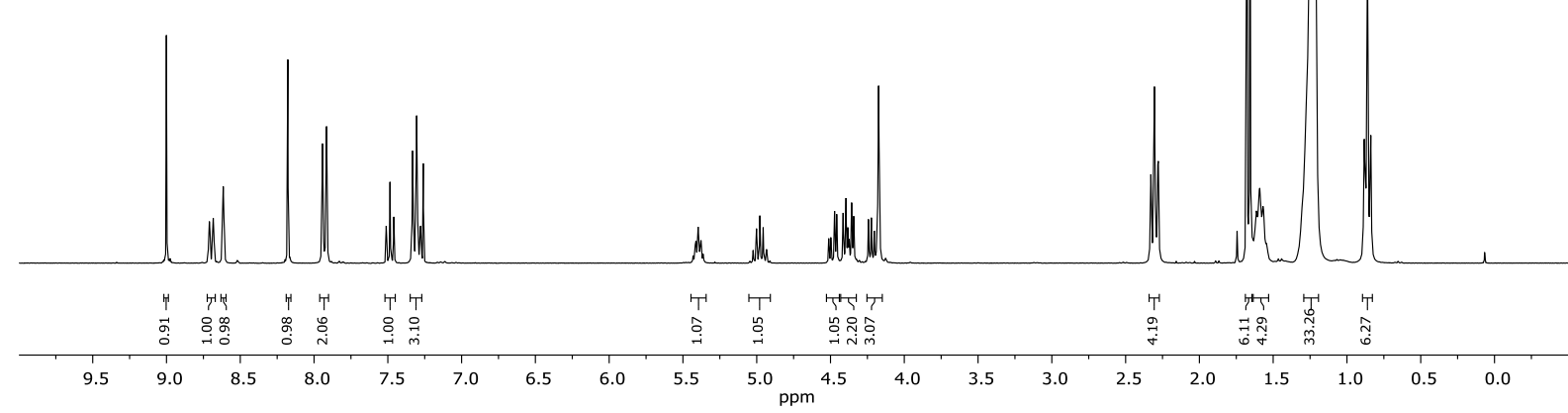

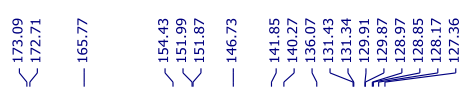<smiles>CCCCCCCCCCCC(=O)OC(COC(=O)CCCCCCCCCC)COC(=O)c1ccc(Cc2cccc(-c3ncnc4c3ncn4C(C)C)c2)cc1</smiles>

rac-6m

(125 $\mathrm{MHz}, \mathrm{CDCl}_{3}$ )

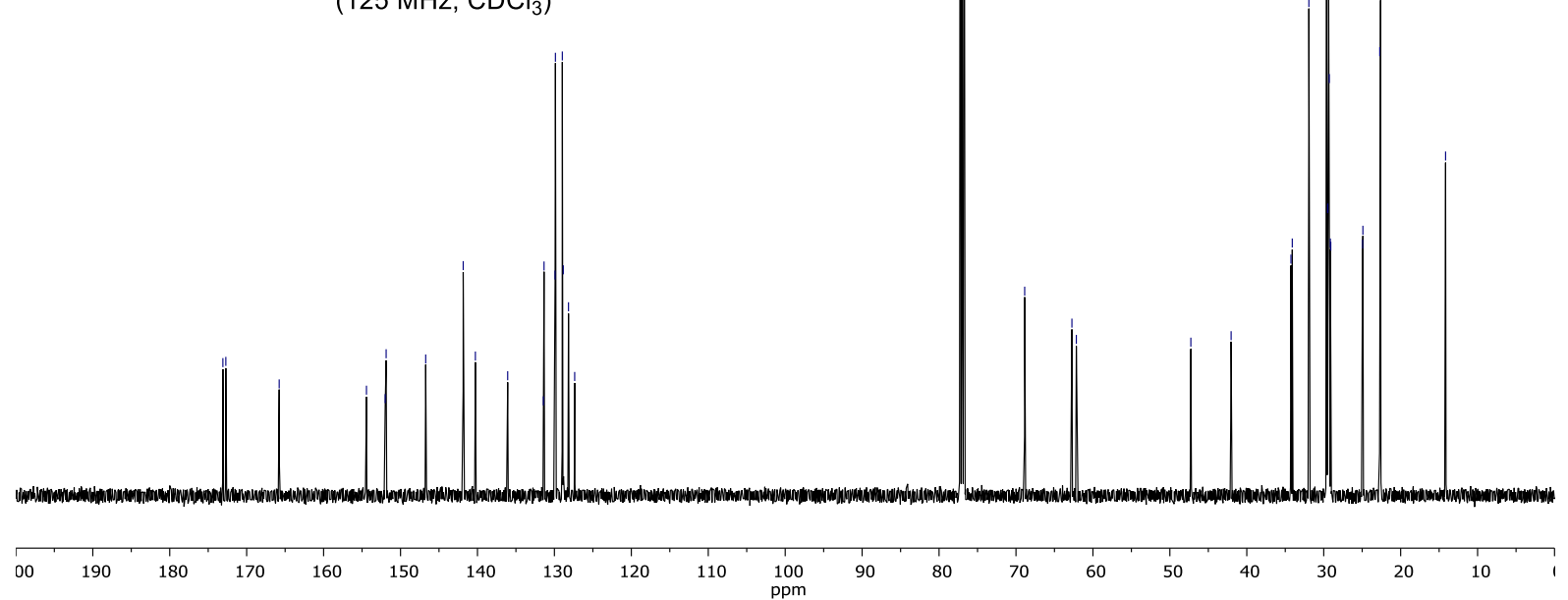




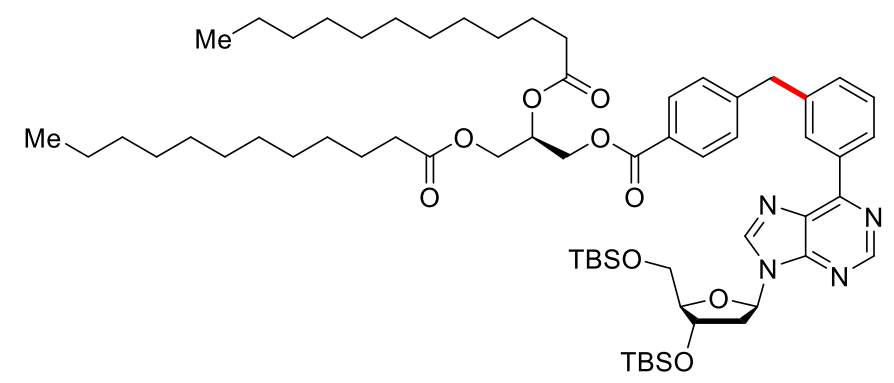

$6 n$

$\left(400 \mathrm{MHz}, \mathrm{CDCl}_{3}\right)$
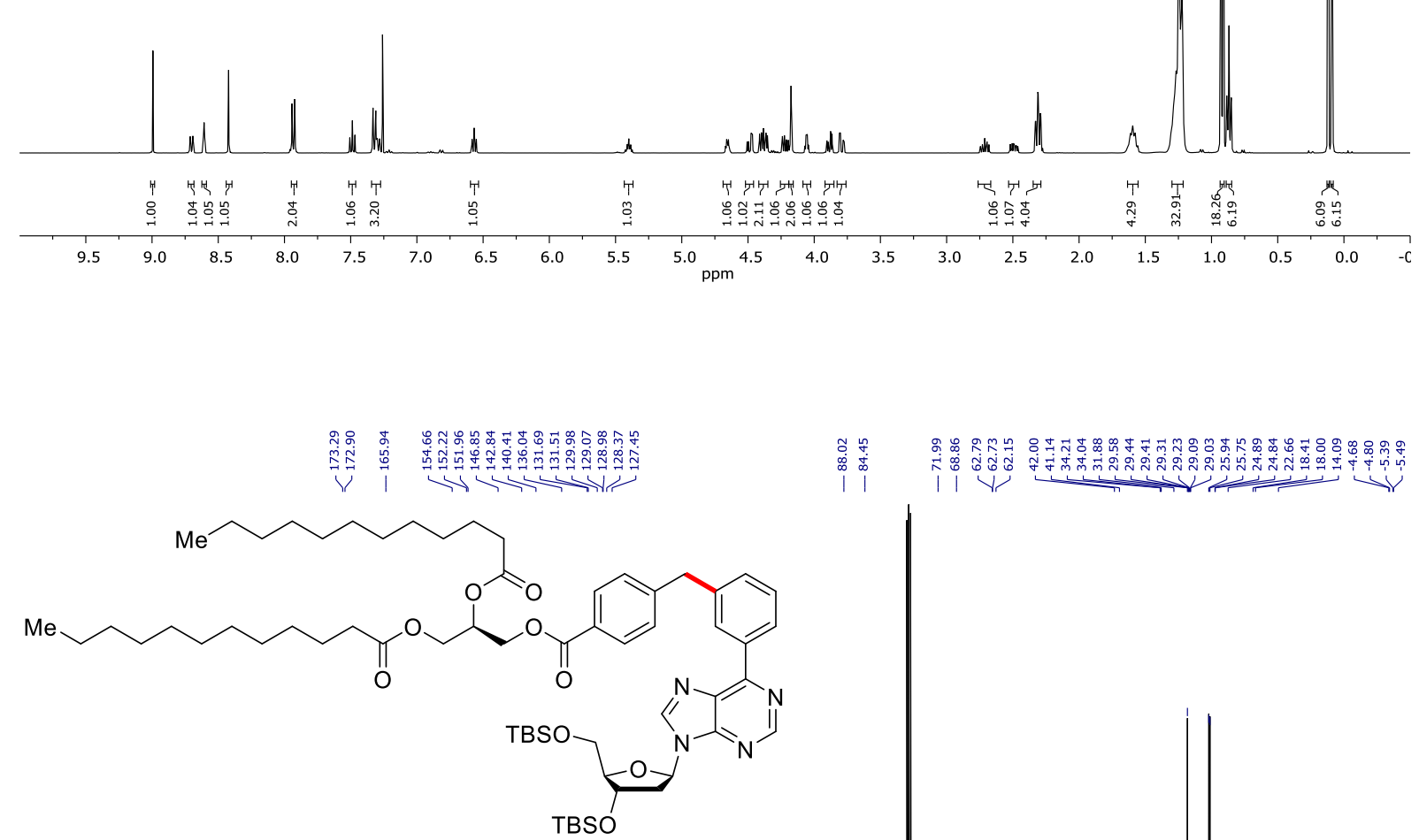

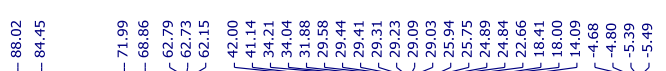

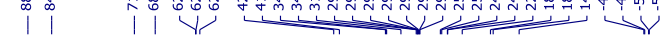

(100 $\mathrm{MHz}, \mathrm{CDCl}_{3}$ )

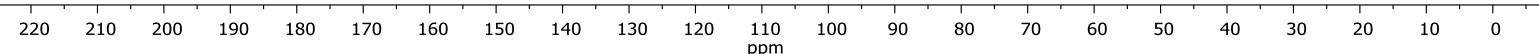




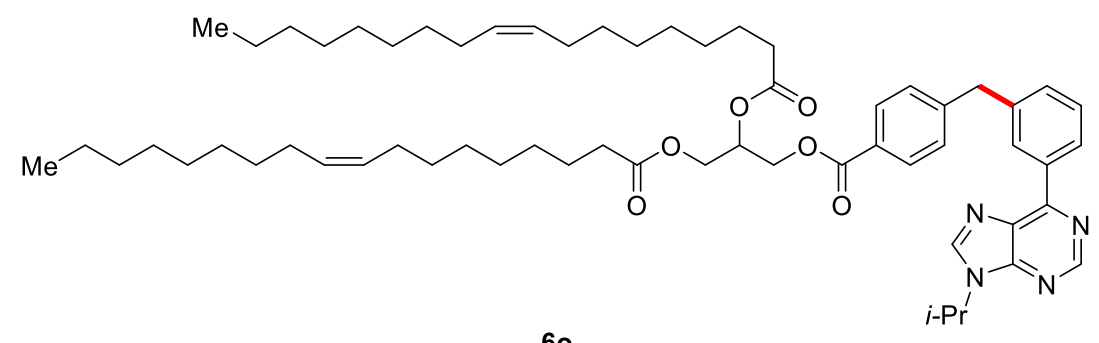

60

(600 MHz, $\mathrm{CDCl}_{3}$ )

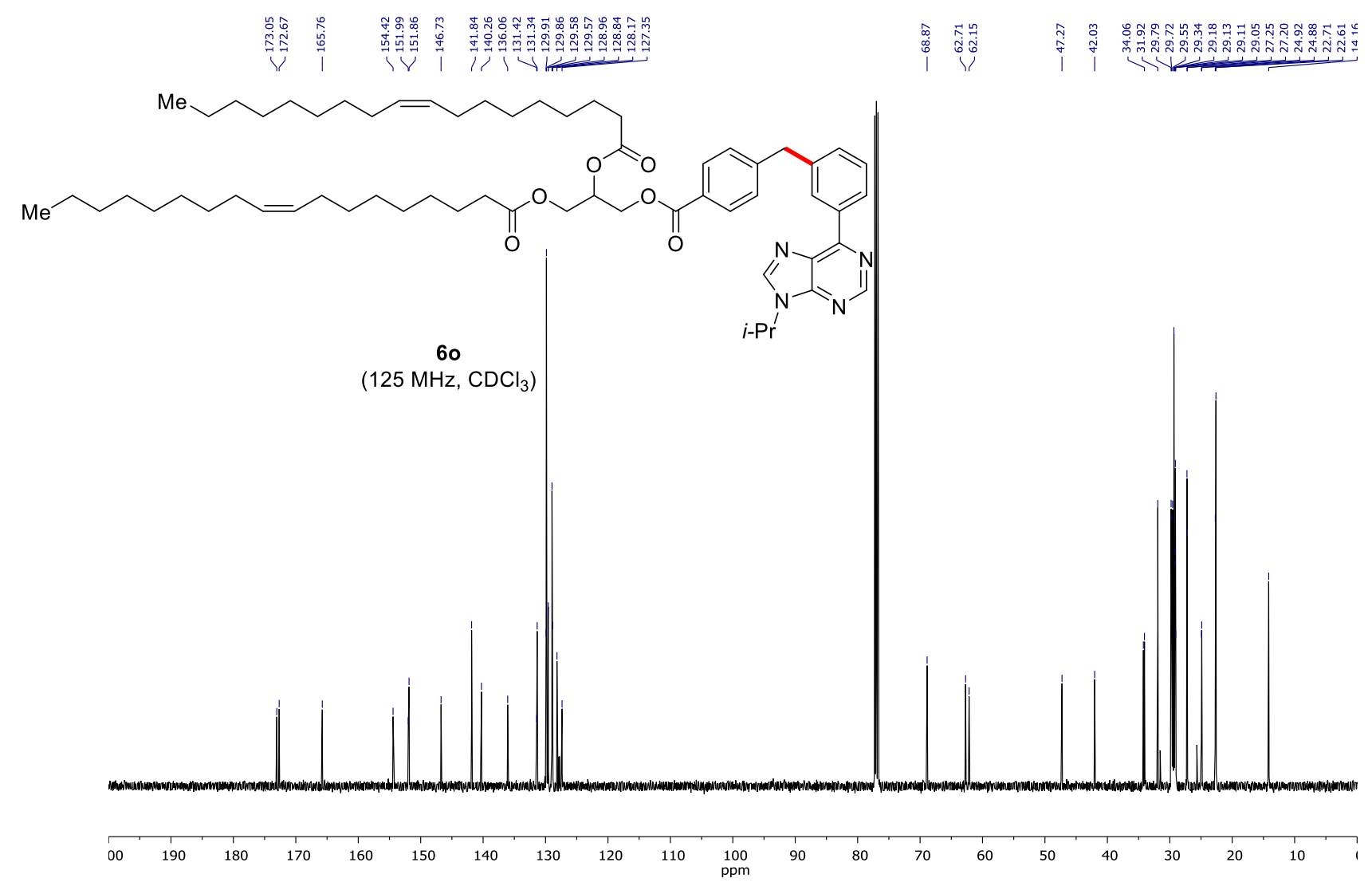




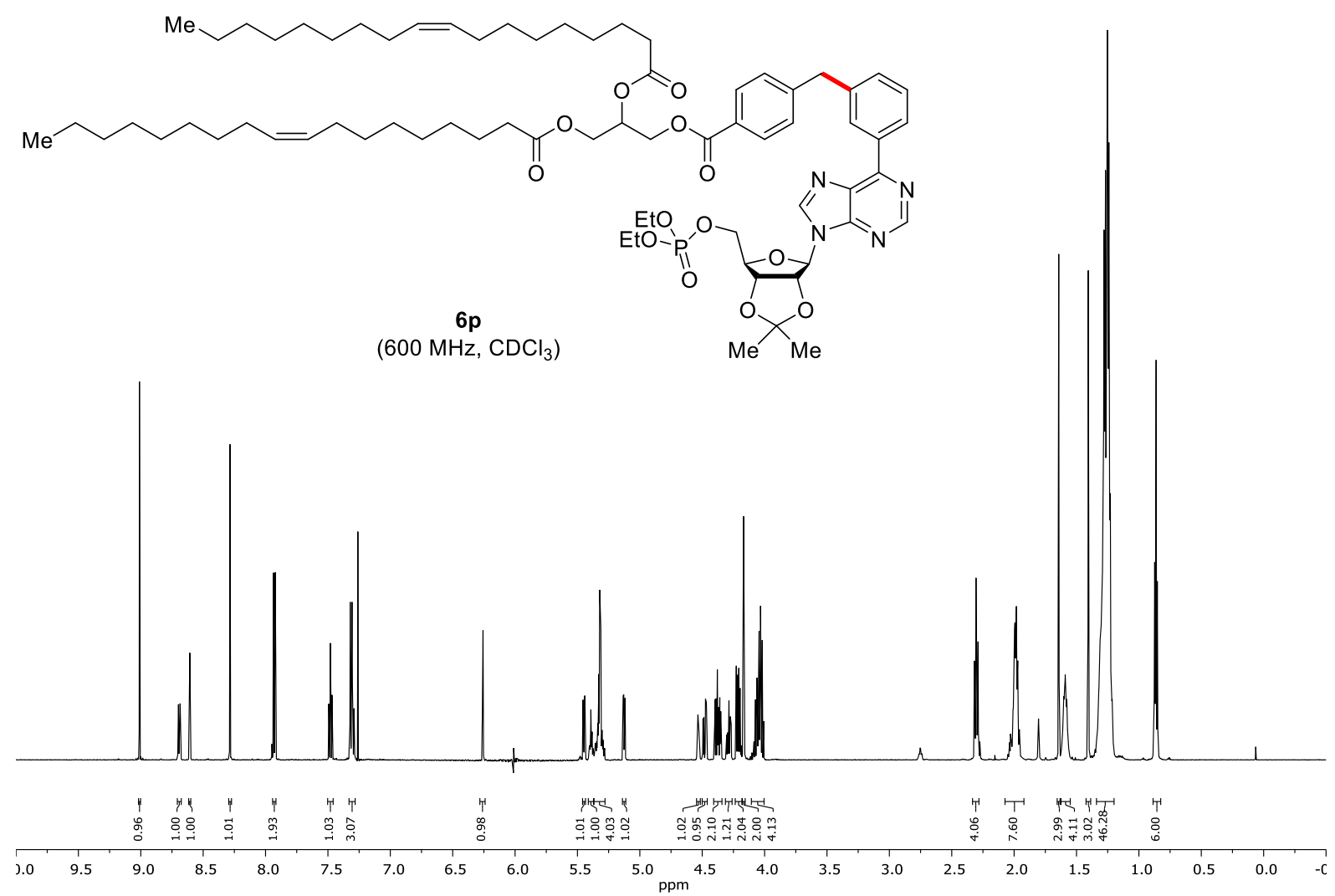

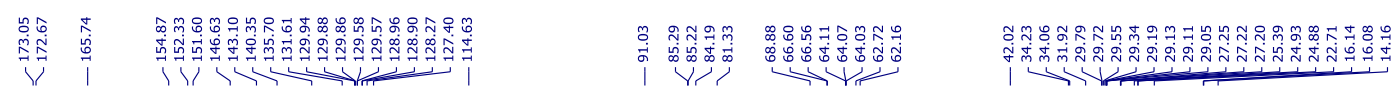

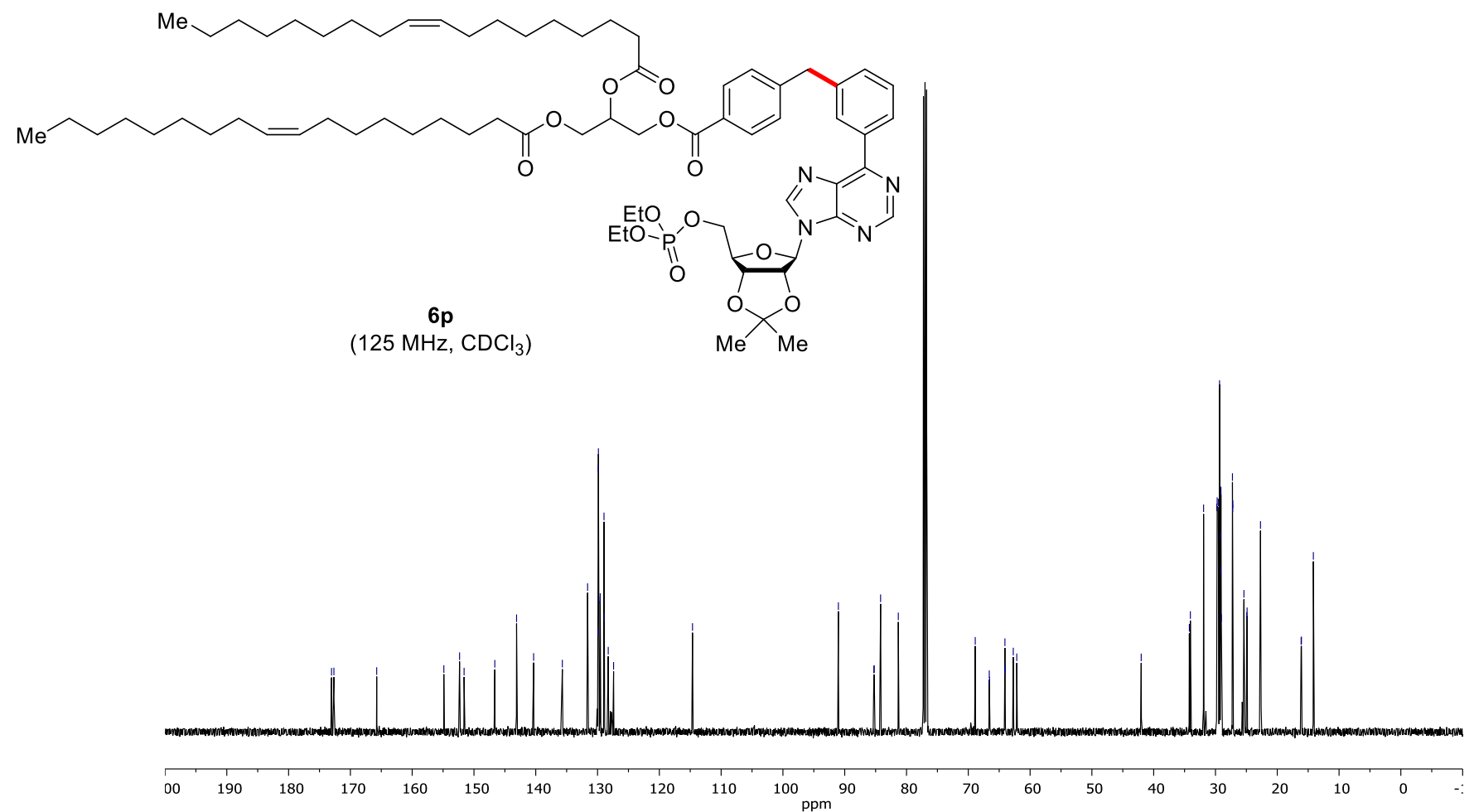



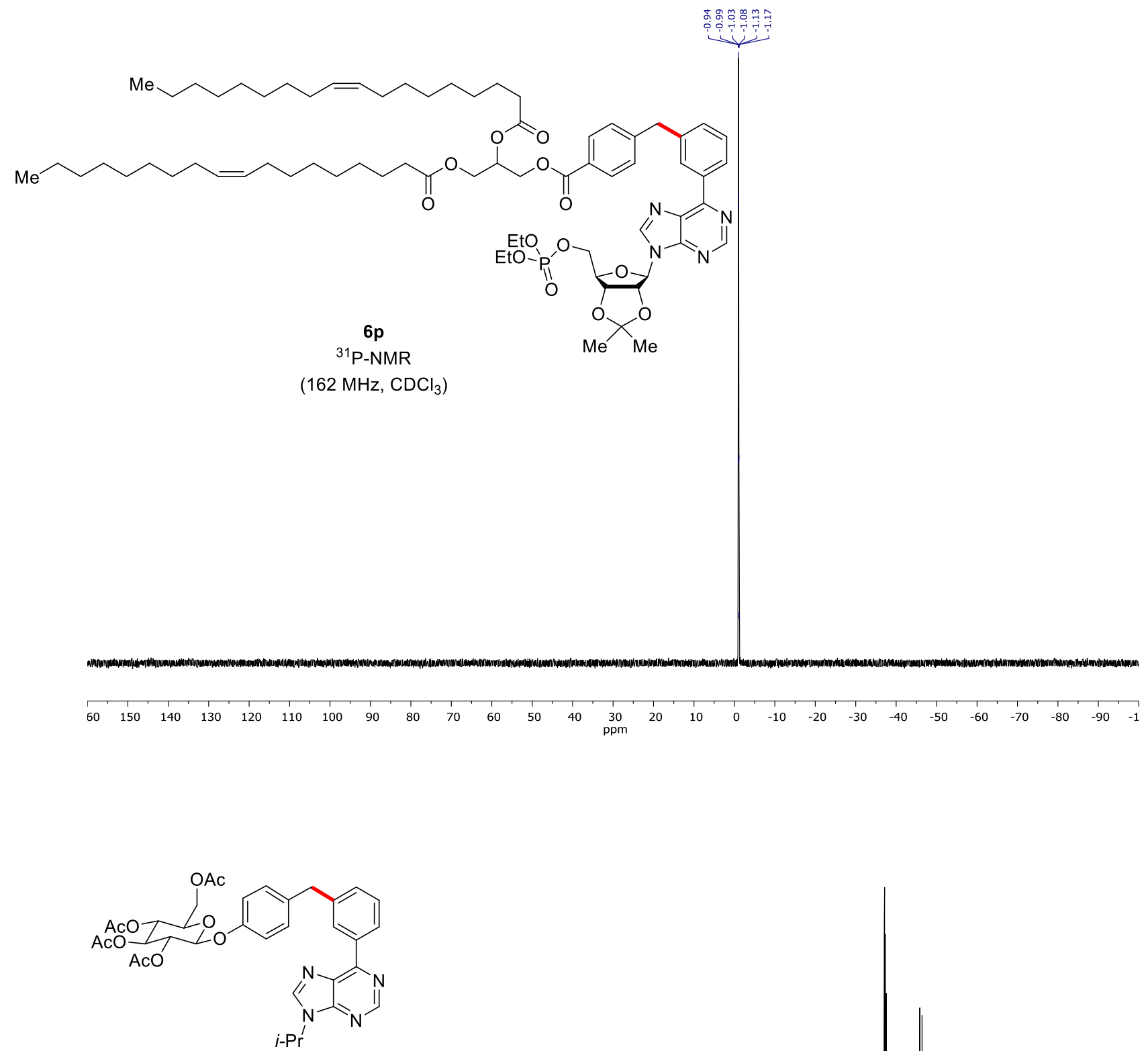

$6 q$

(300 MHz, $\mathrm{CDCl}_{3}$ )

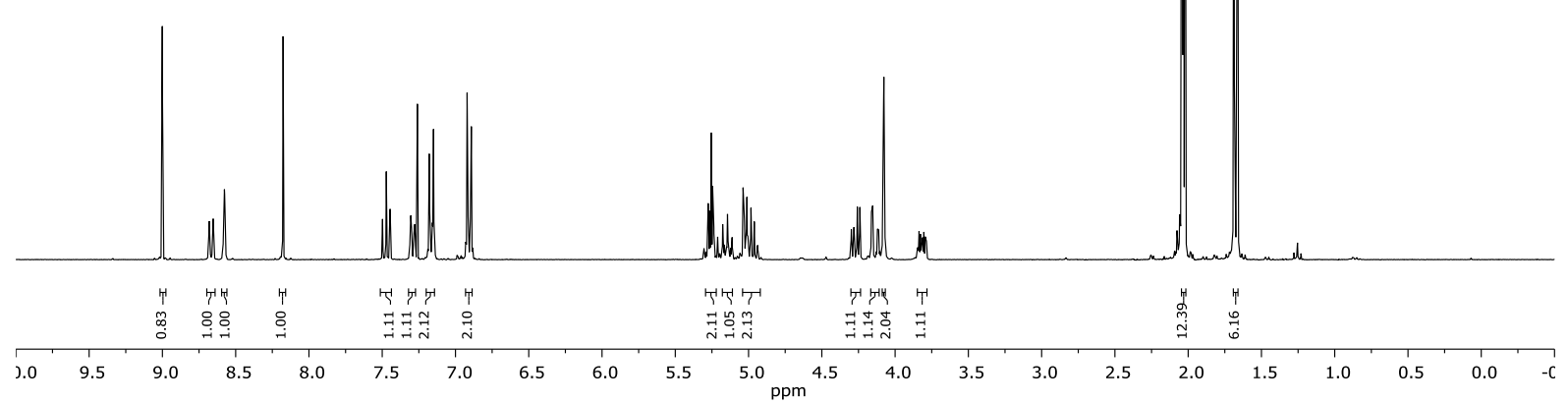




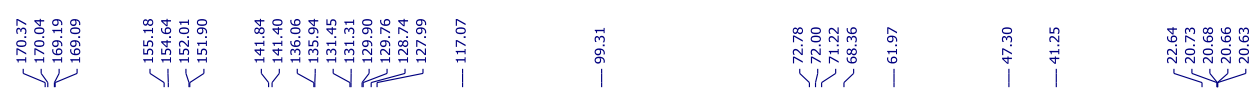

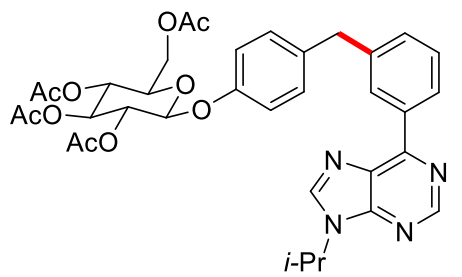

$6 q$

(125 MHz, $\mathrm{CDCl}_{3}$ )
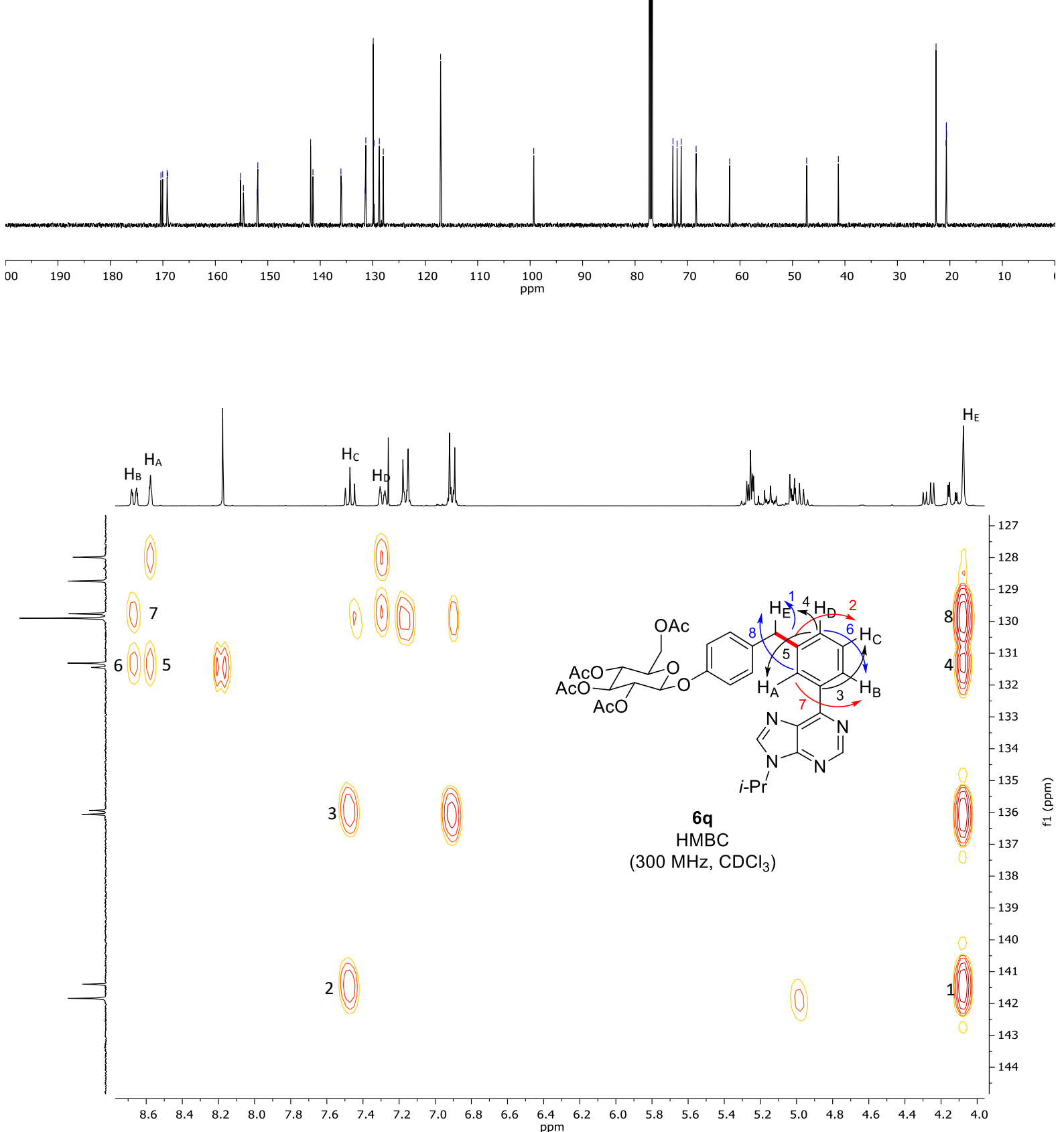


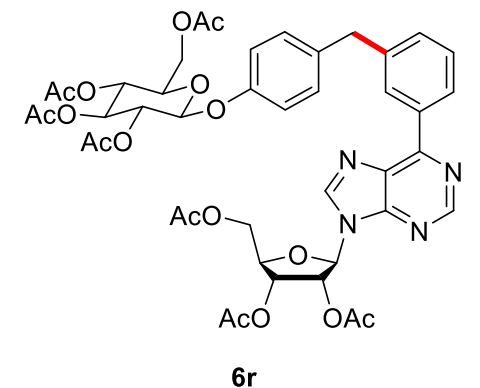

$\left(600 \mathrm{MHz} \mathrm{CDCl}_{3}\right)$

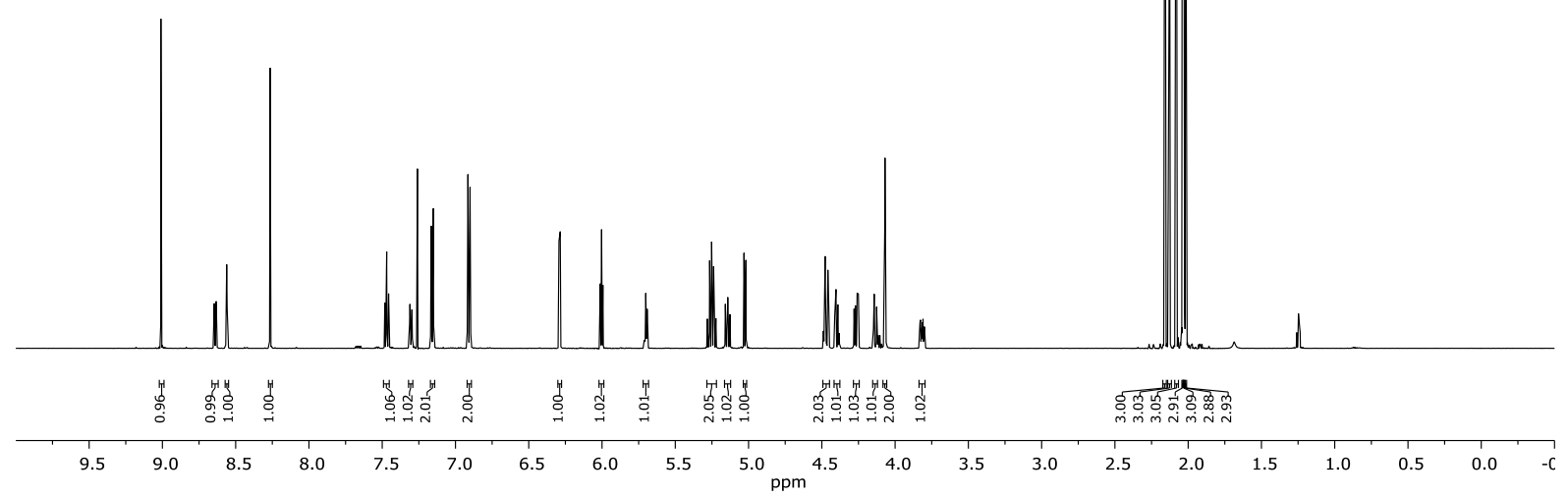

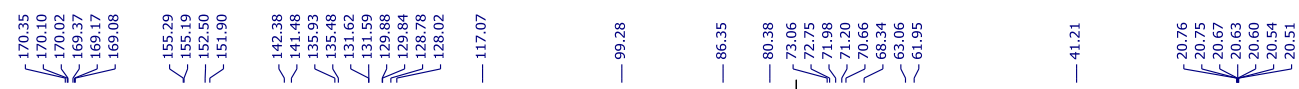

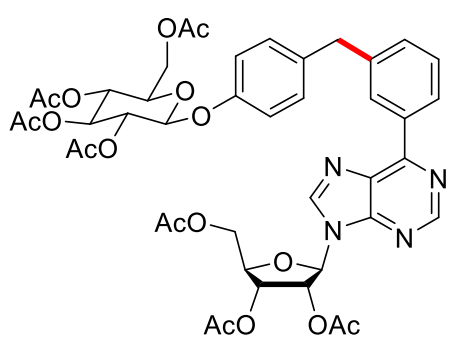

$6 r$

(125 $\mathrm{MHz} \mathrm{CDCl}_{3}$ )

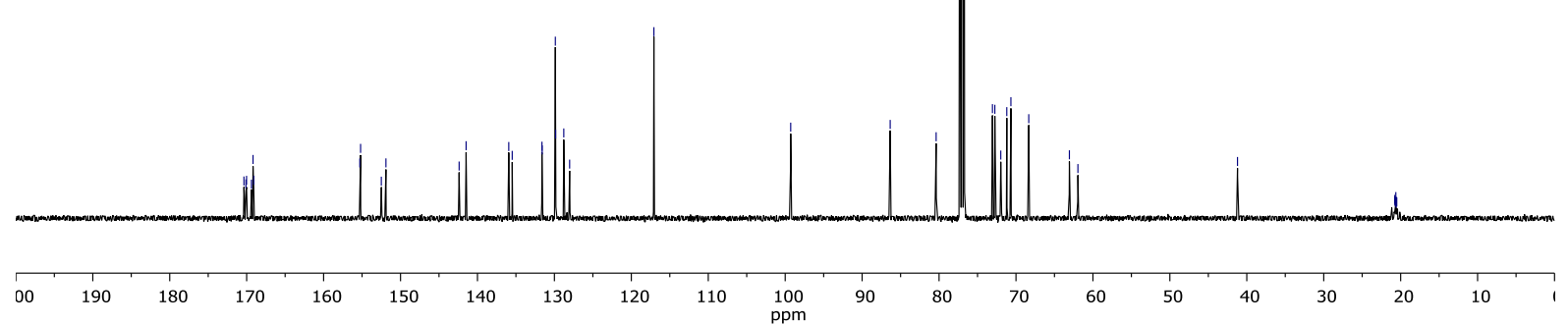



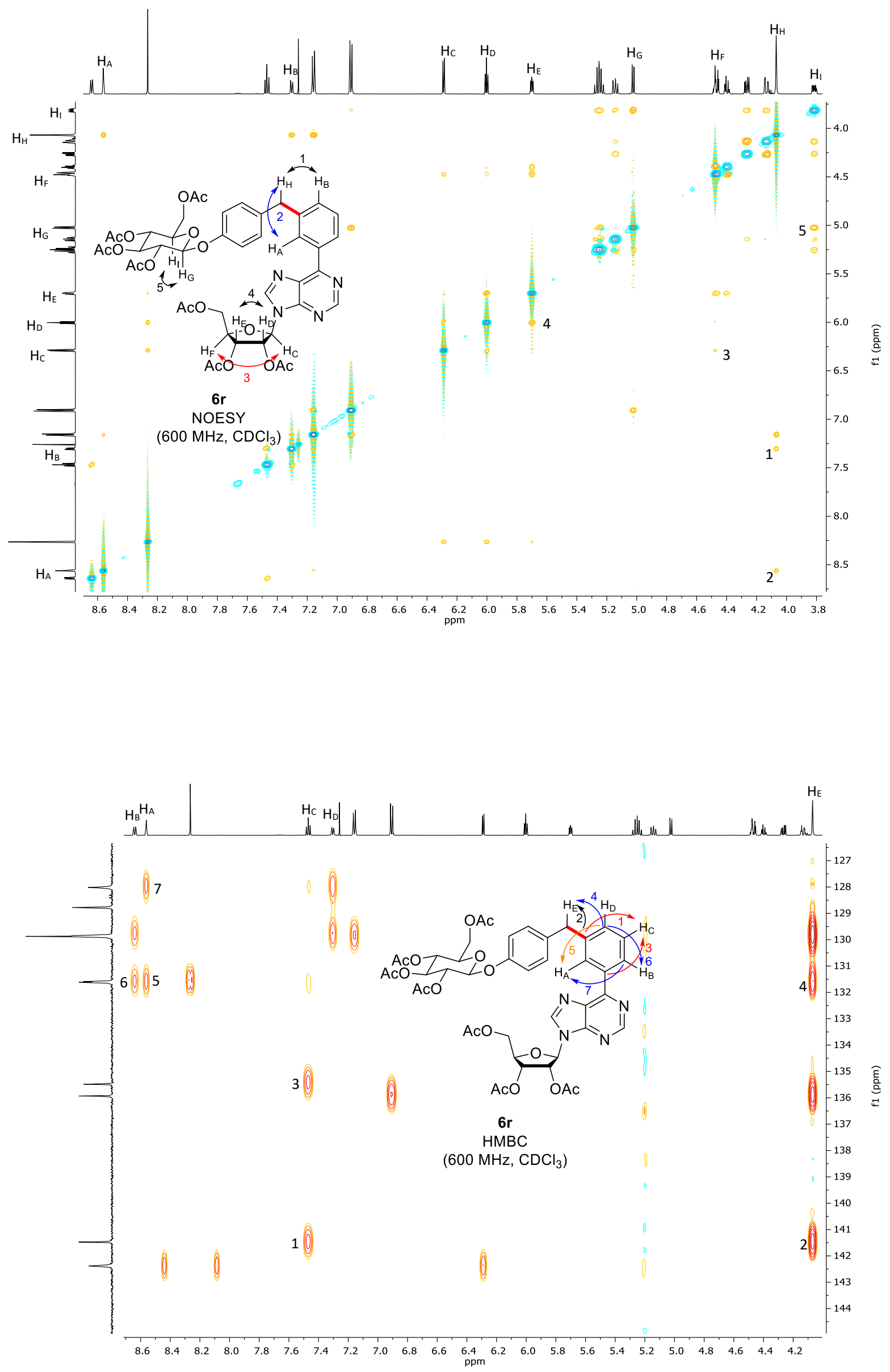


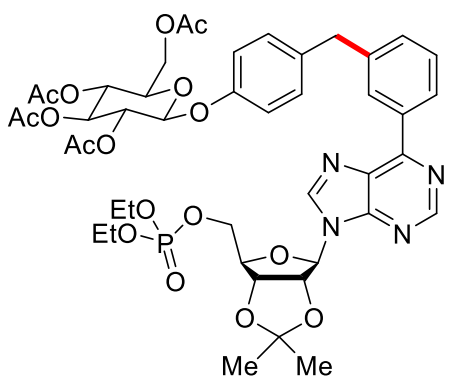

$6 s$

(600 MHz, $\mathrm{CDCl}_{3}$ )

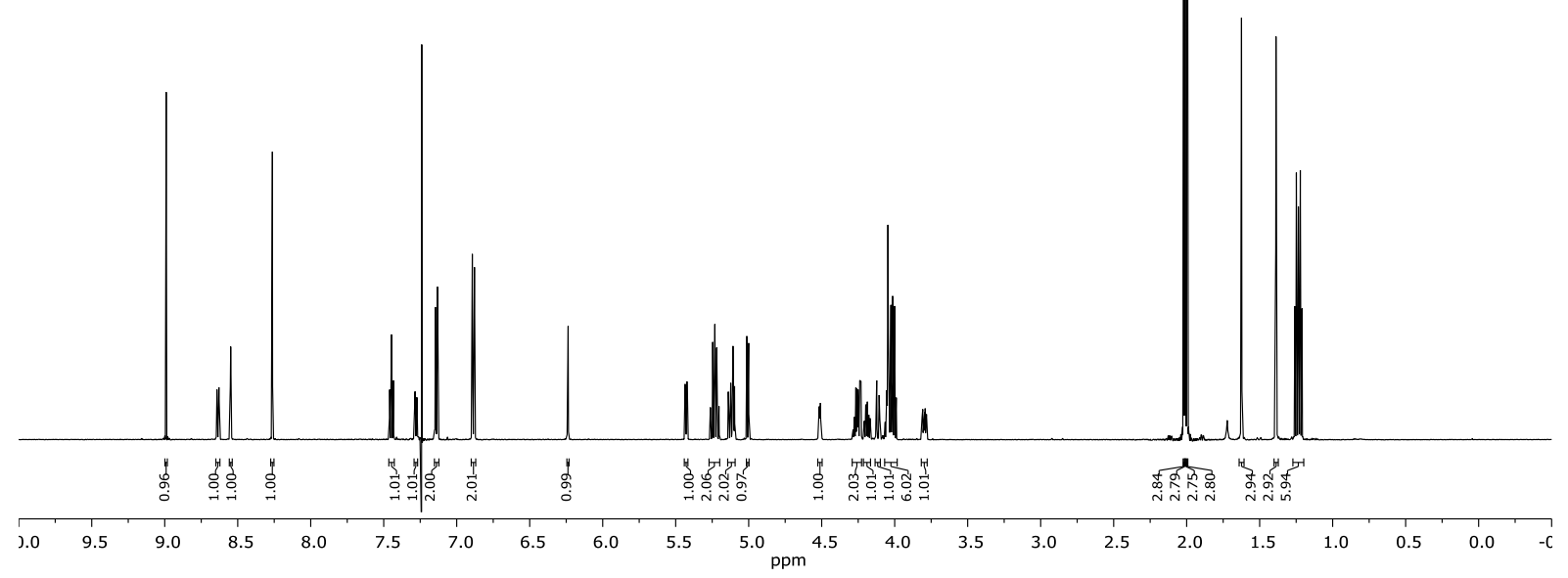

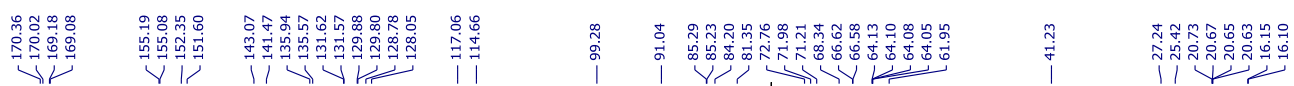

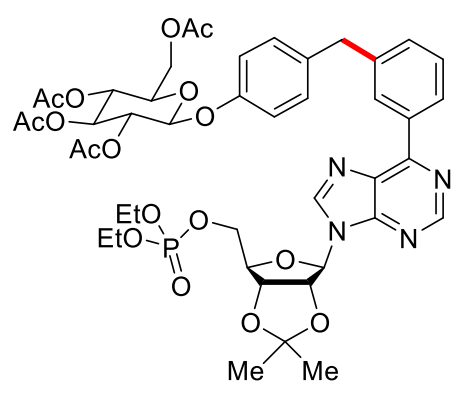

$6 s$

(125 $\mathrm{MHz}, \mathrm{CDCl}_{3}$ )

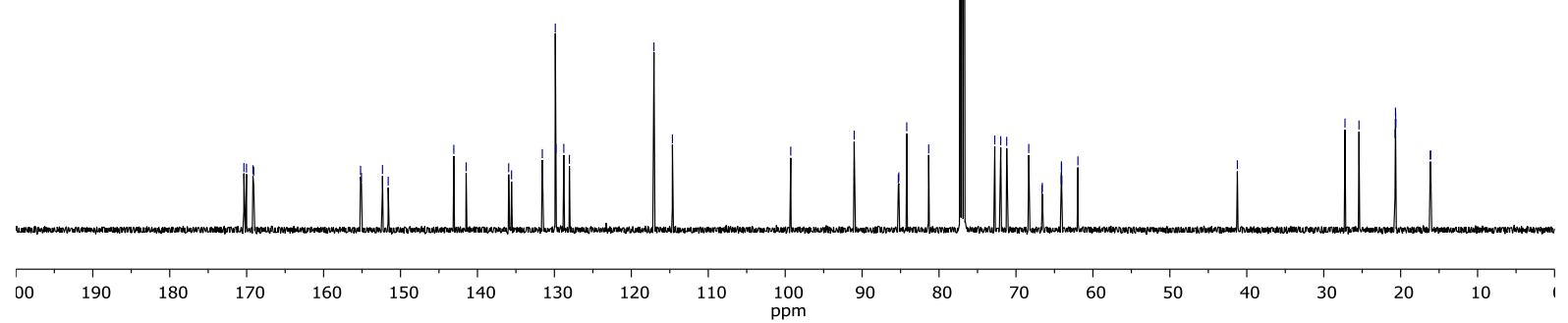




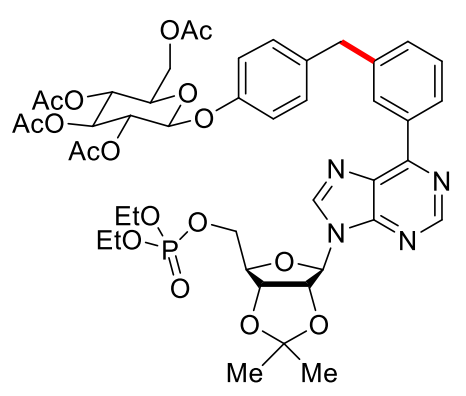

$6 s$

${ }^{31} \mathrm{P}\left\{{ }^{1} \mathrm{H}\right\}-N M R$

(121 MHz, $\mathrm{CDCl}_{3}$ )

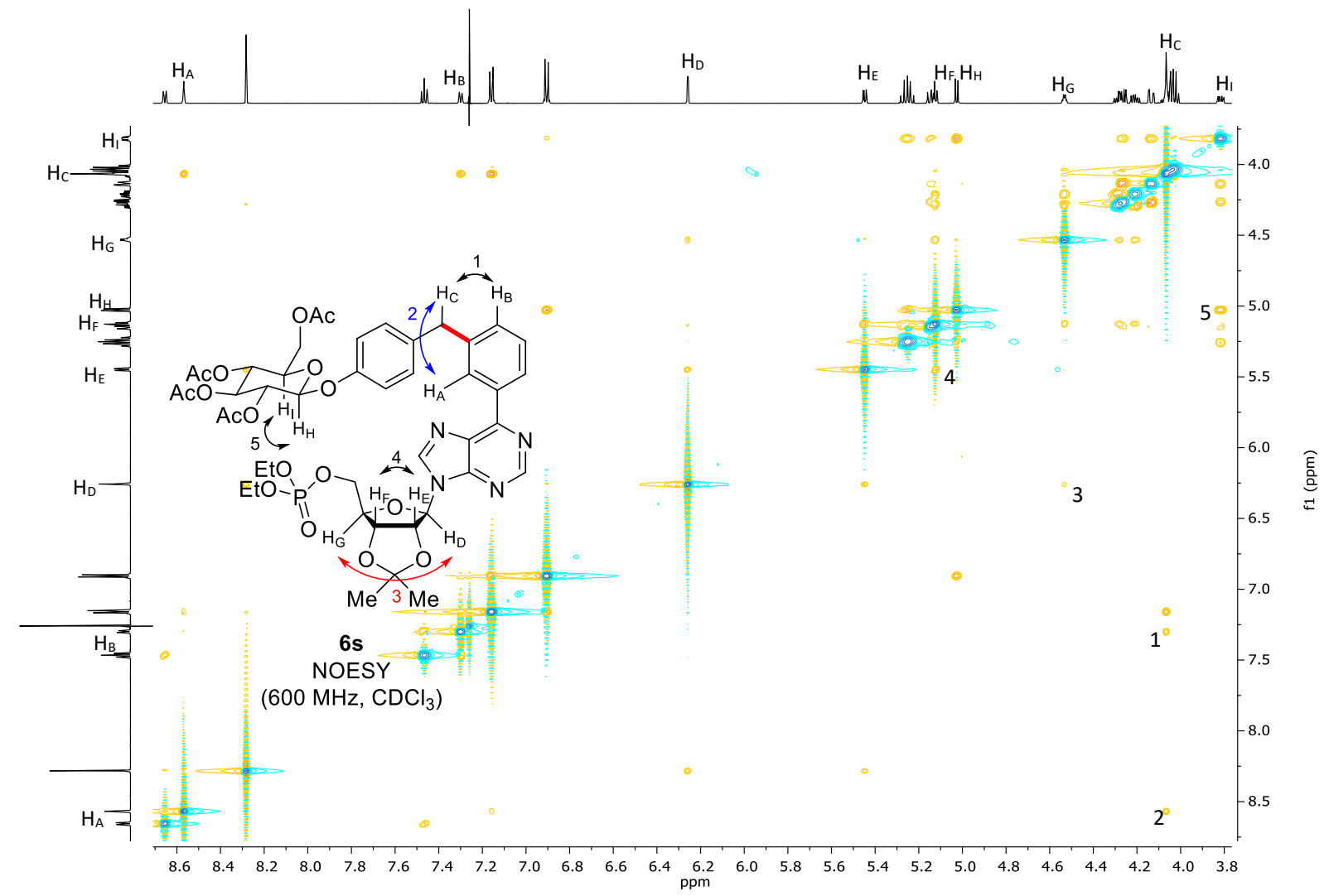



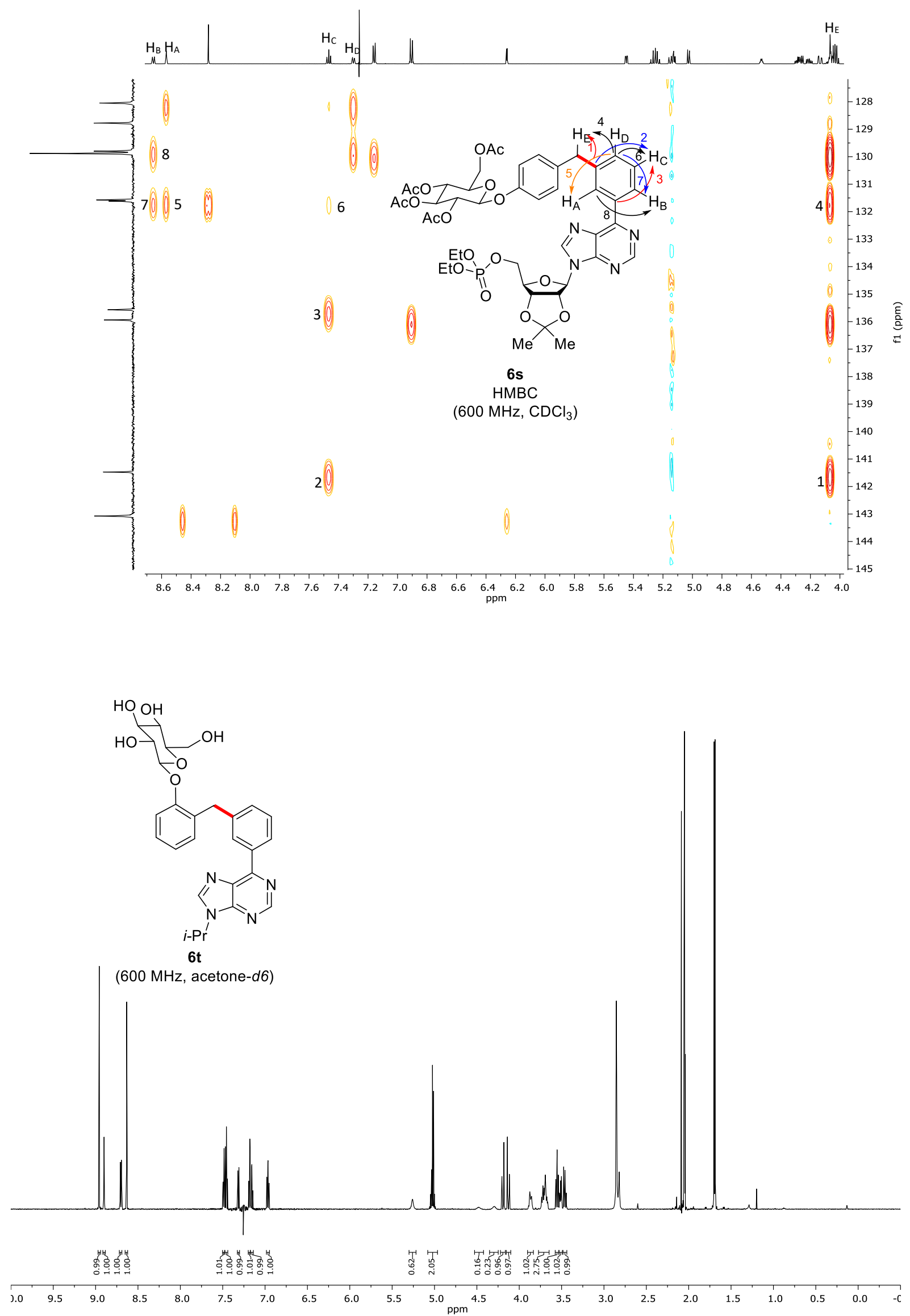

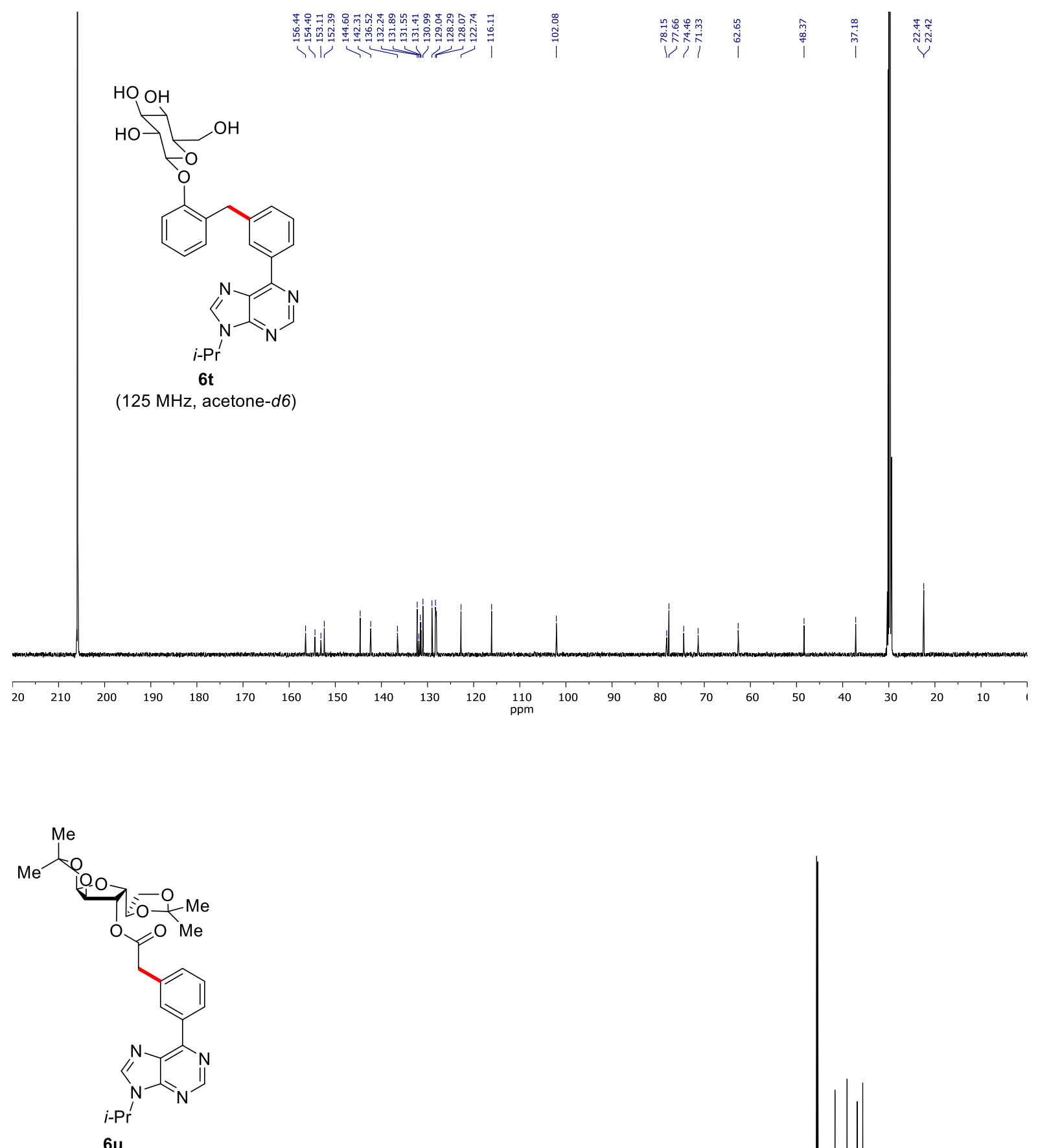

$\left(600 \mathrm{MHz}, \mathrm{CDCl}_{3}\right.$ )

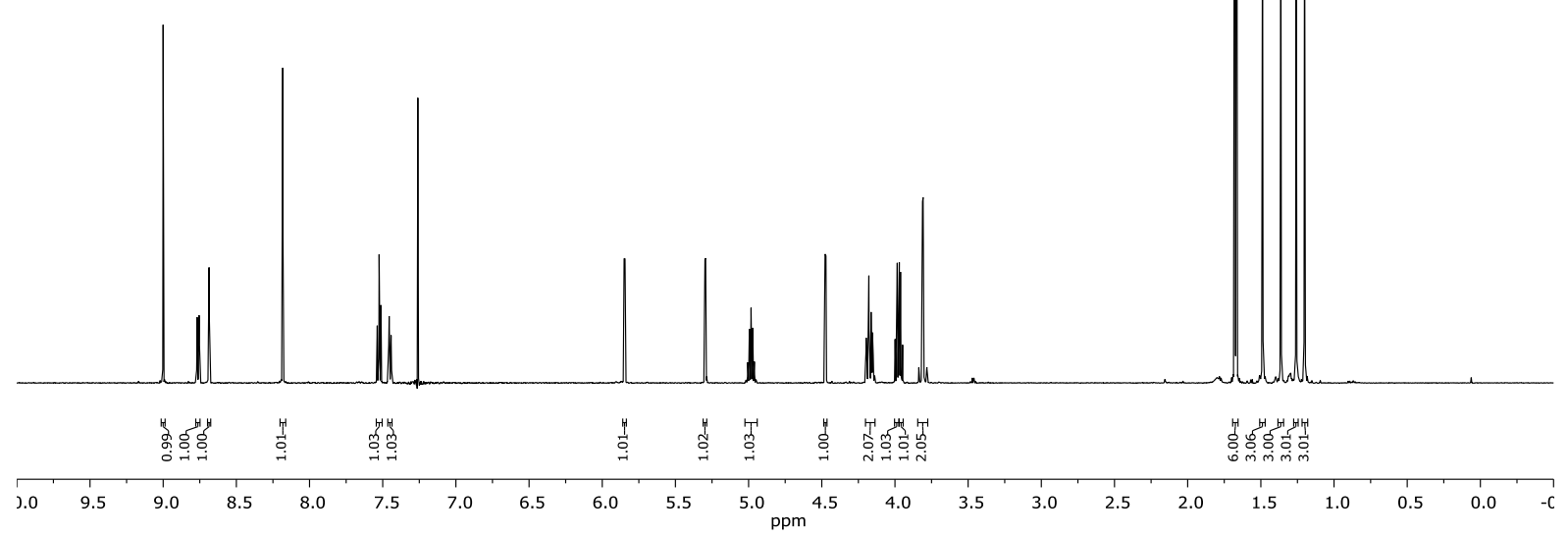




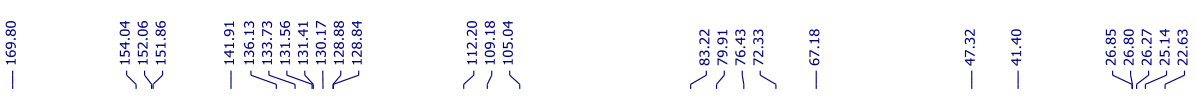

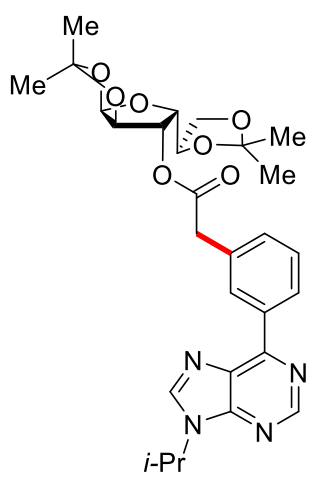

$6 \mathrm{u}$

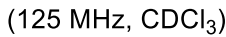
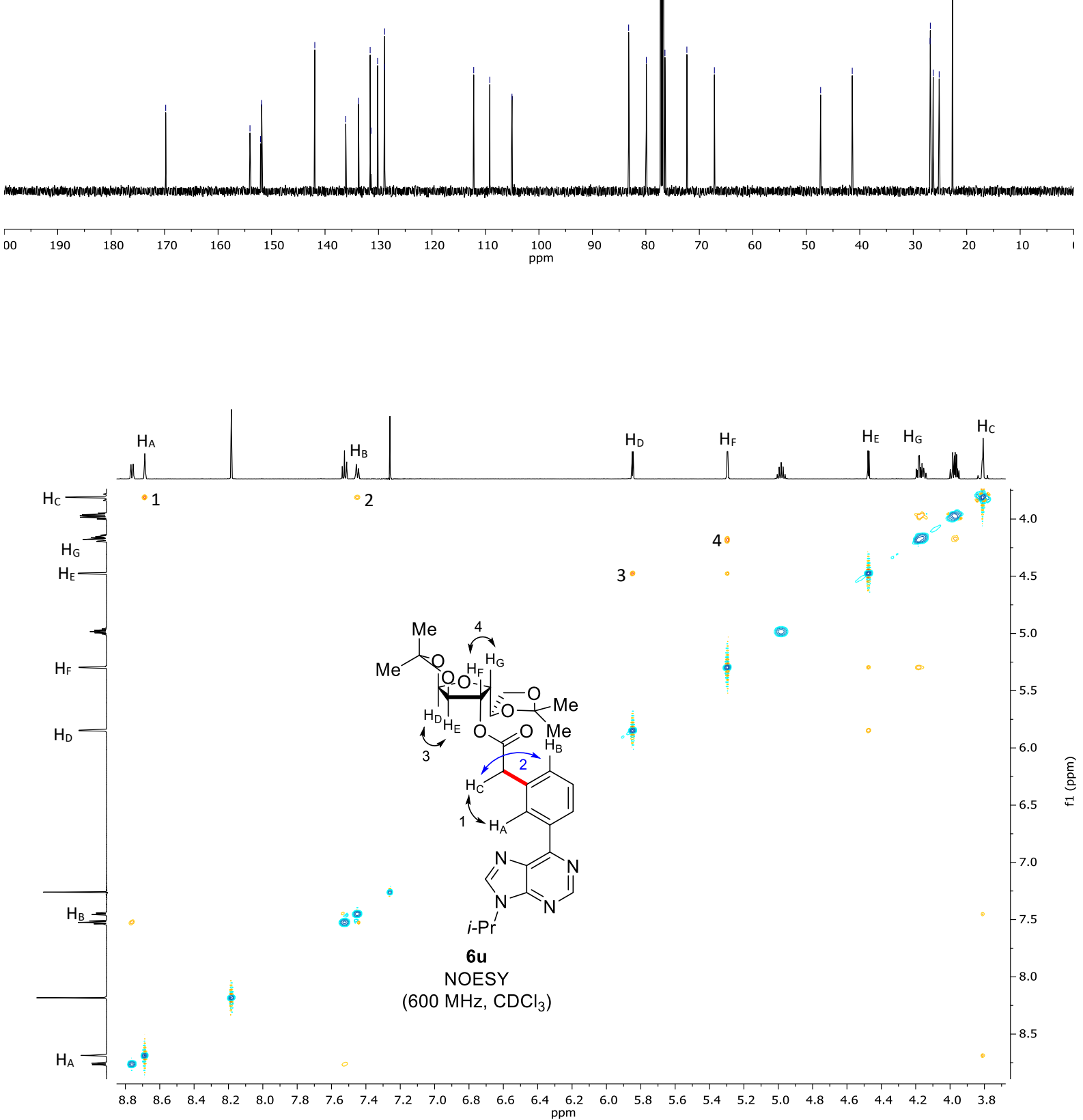


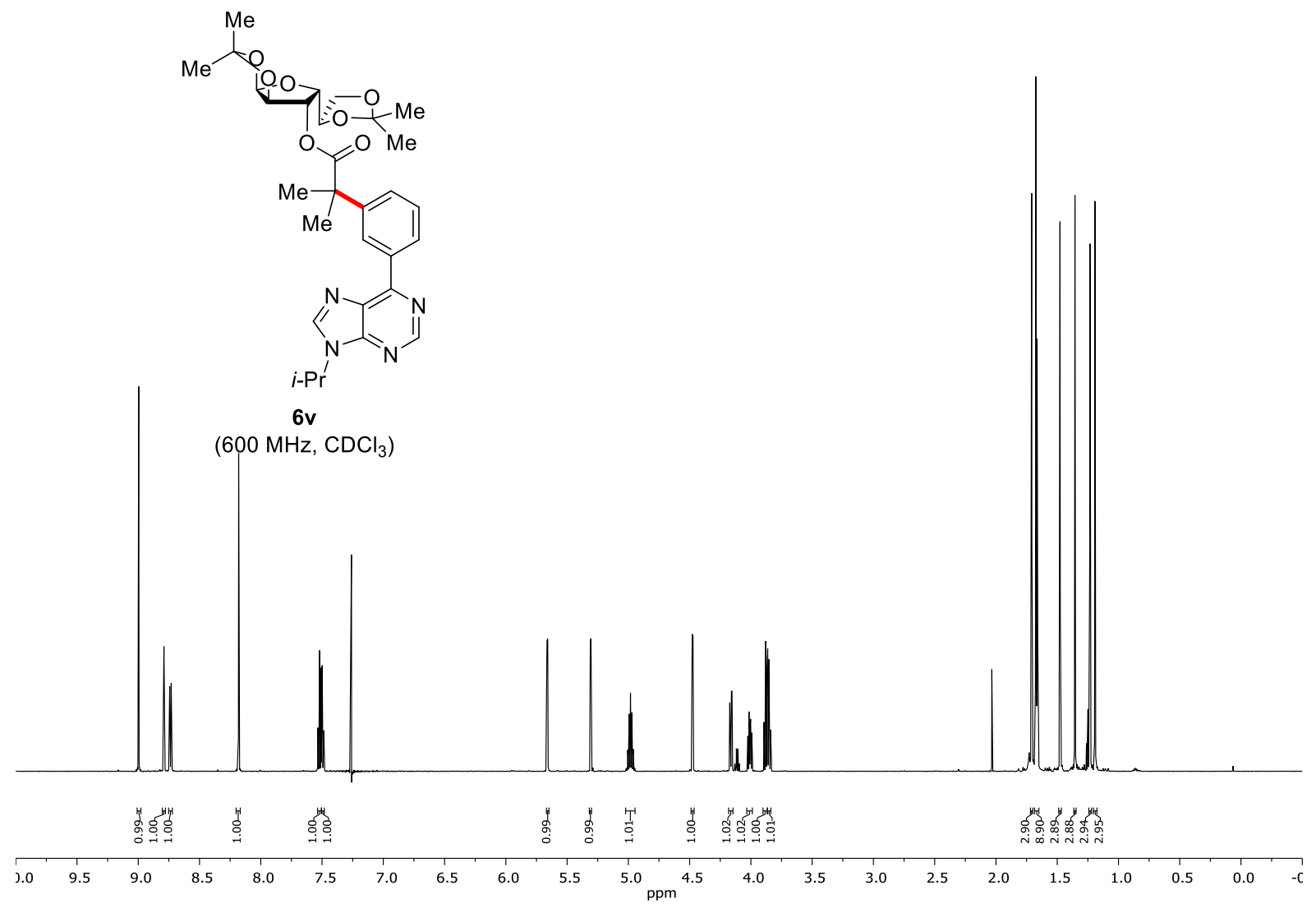

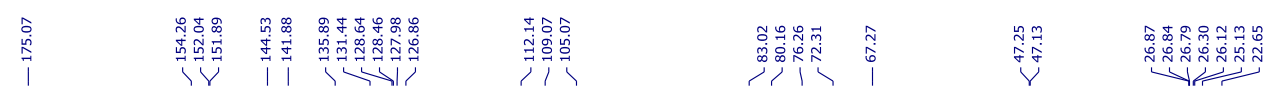

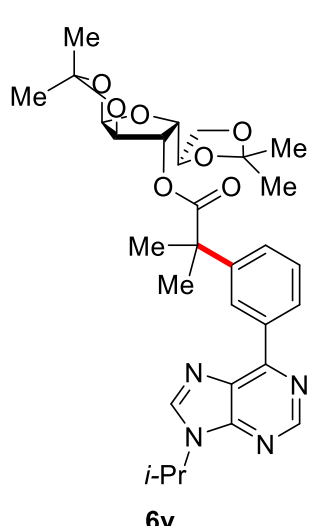

(125 $\mathrm{MHz} \mathrm{CDCl}_{3}$ )

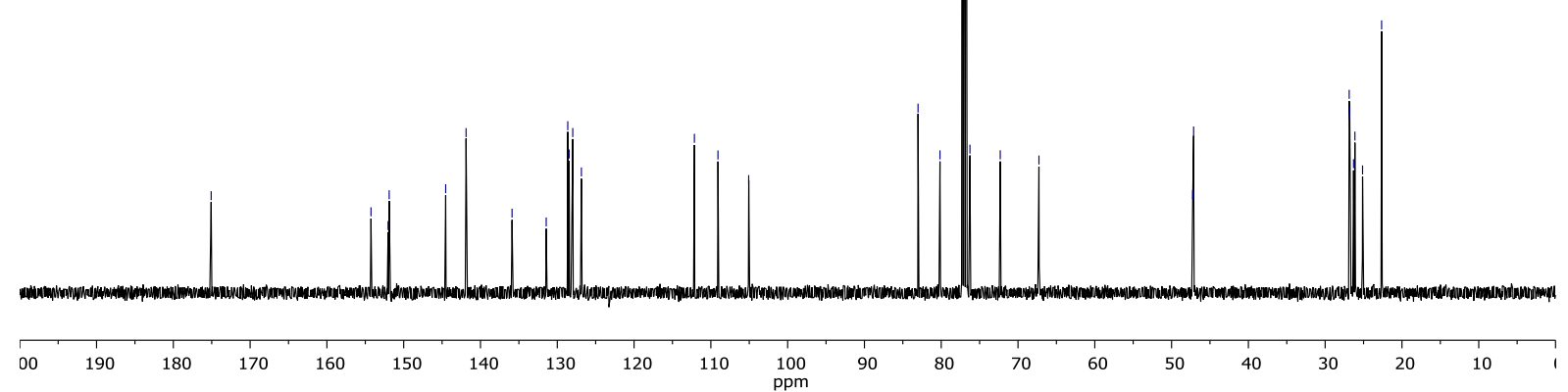



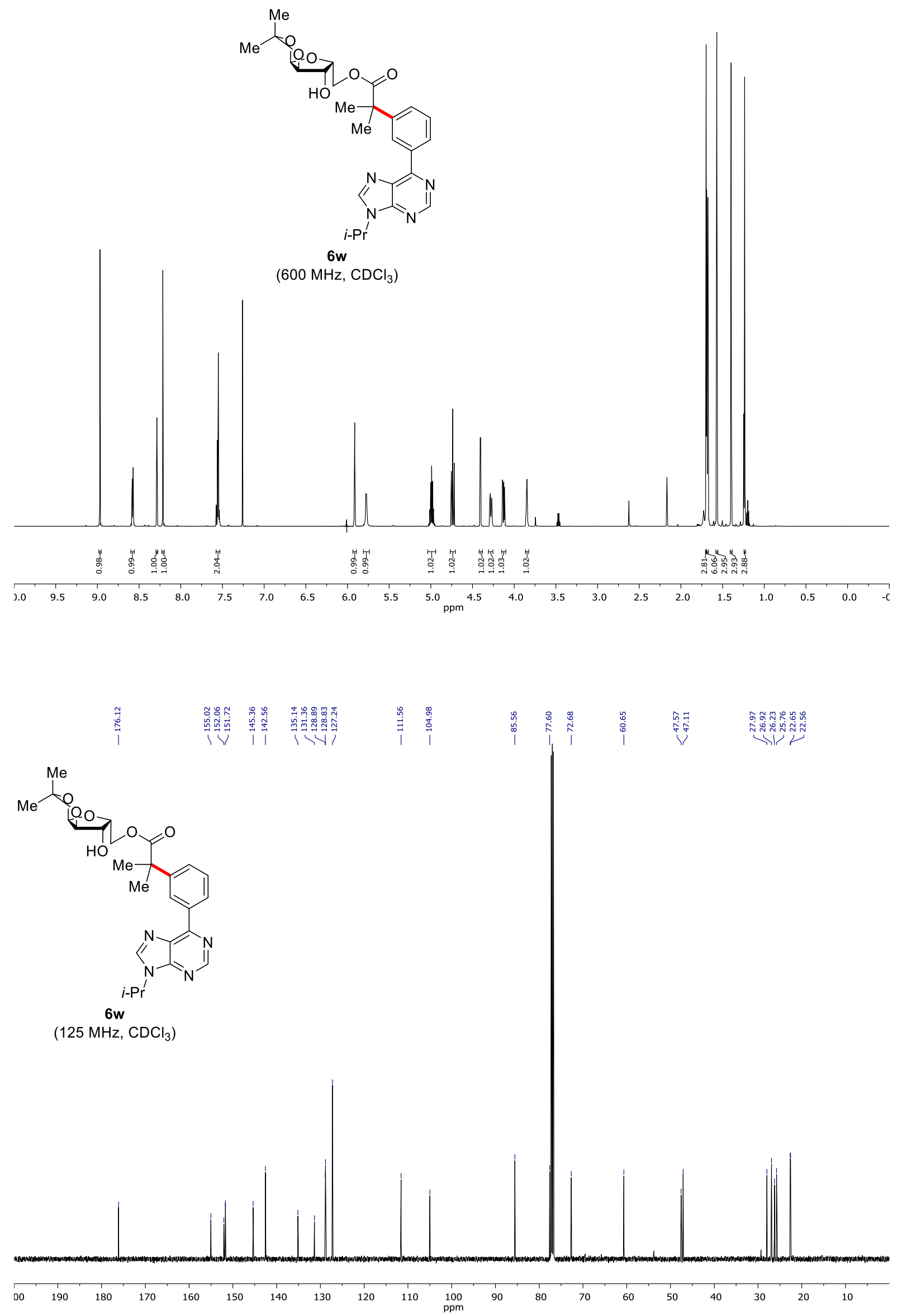


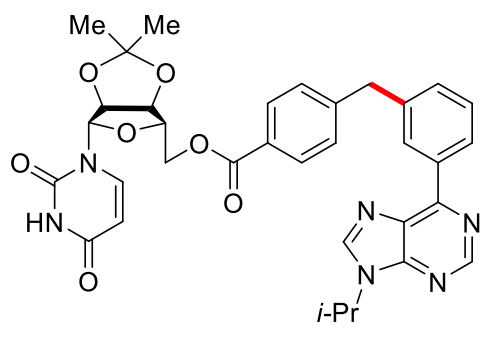

6x
$\left(600 \mathrm{MHz}, \mathrm{CDCl}_{3}\right)$

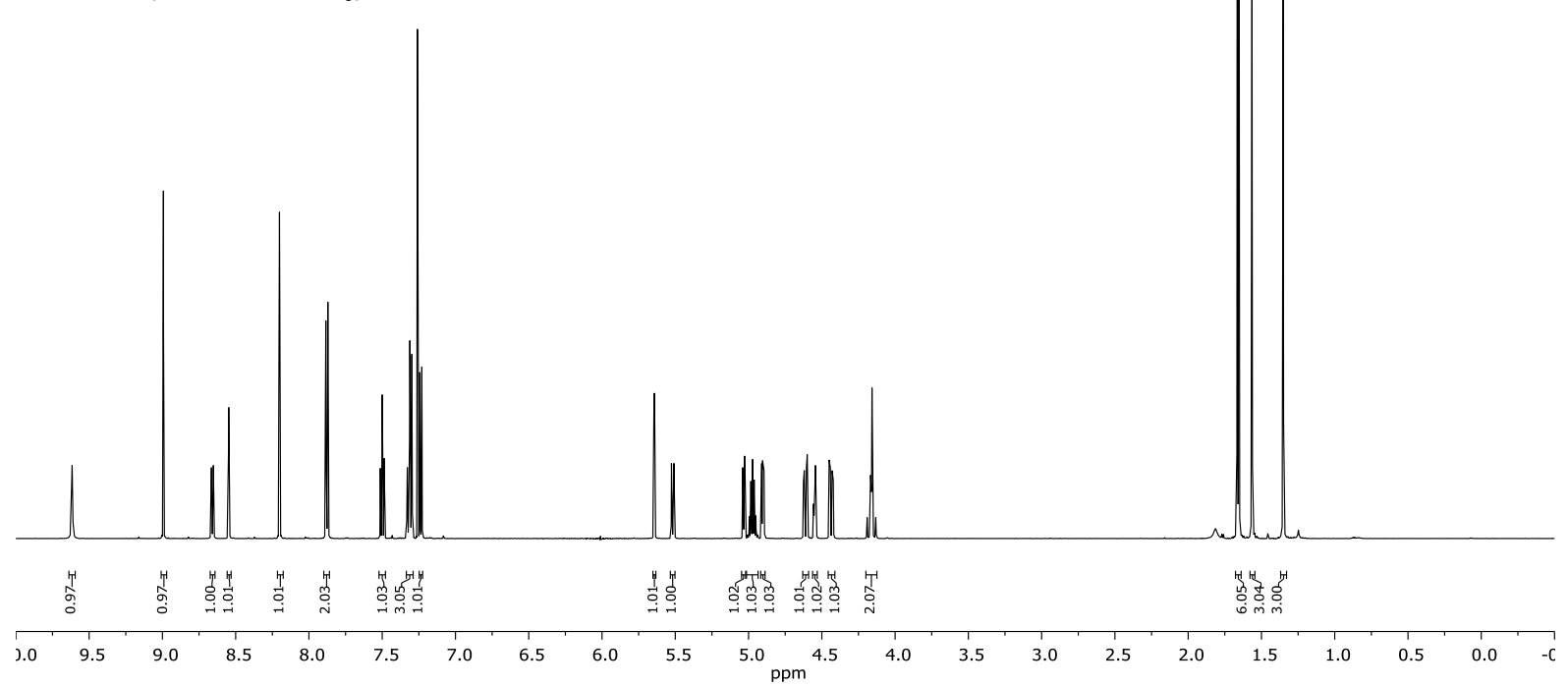

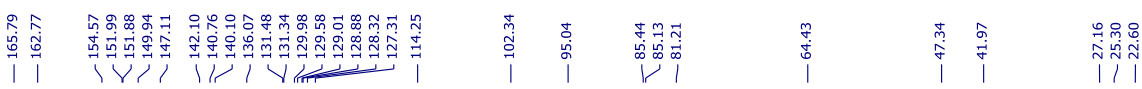

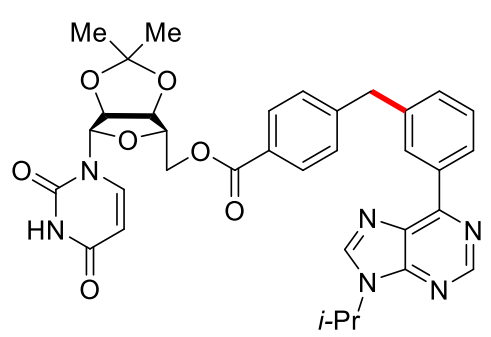

6x

(125 $\mathrm{MHz} \mathrm{CDCl}_{3}$ )

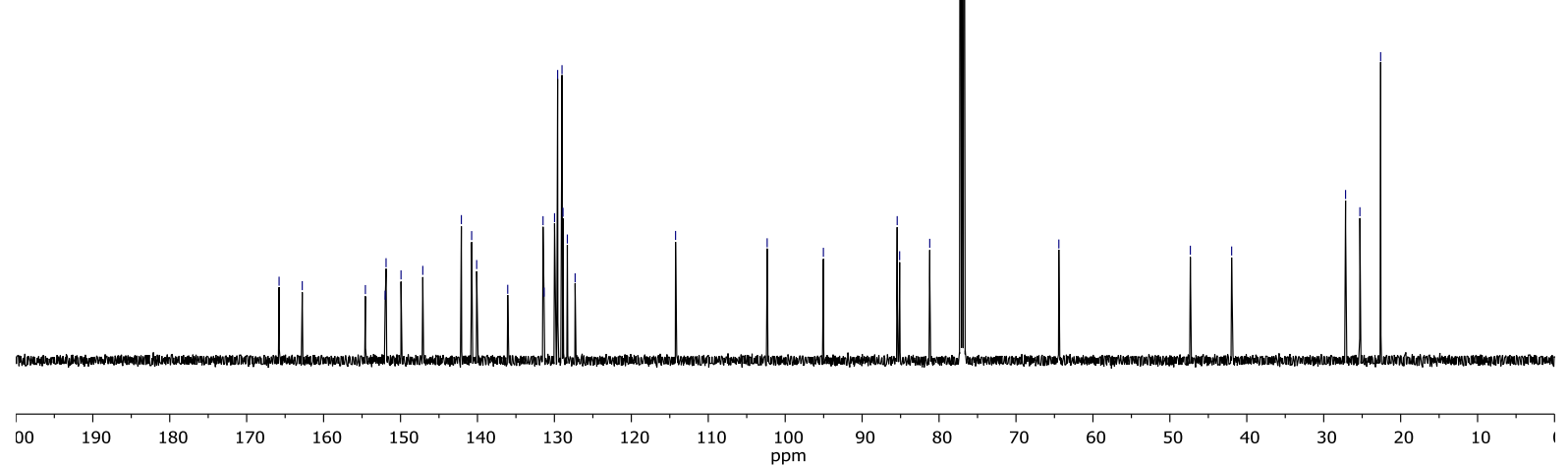



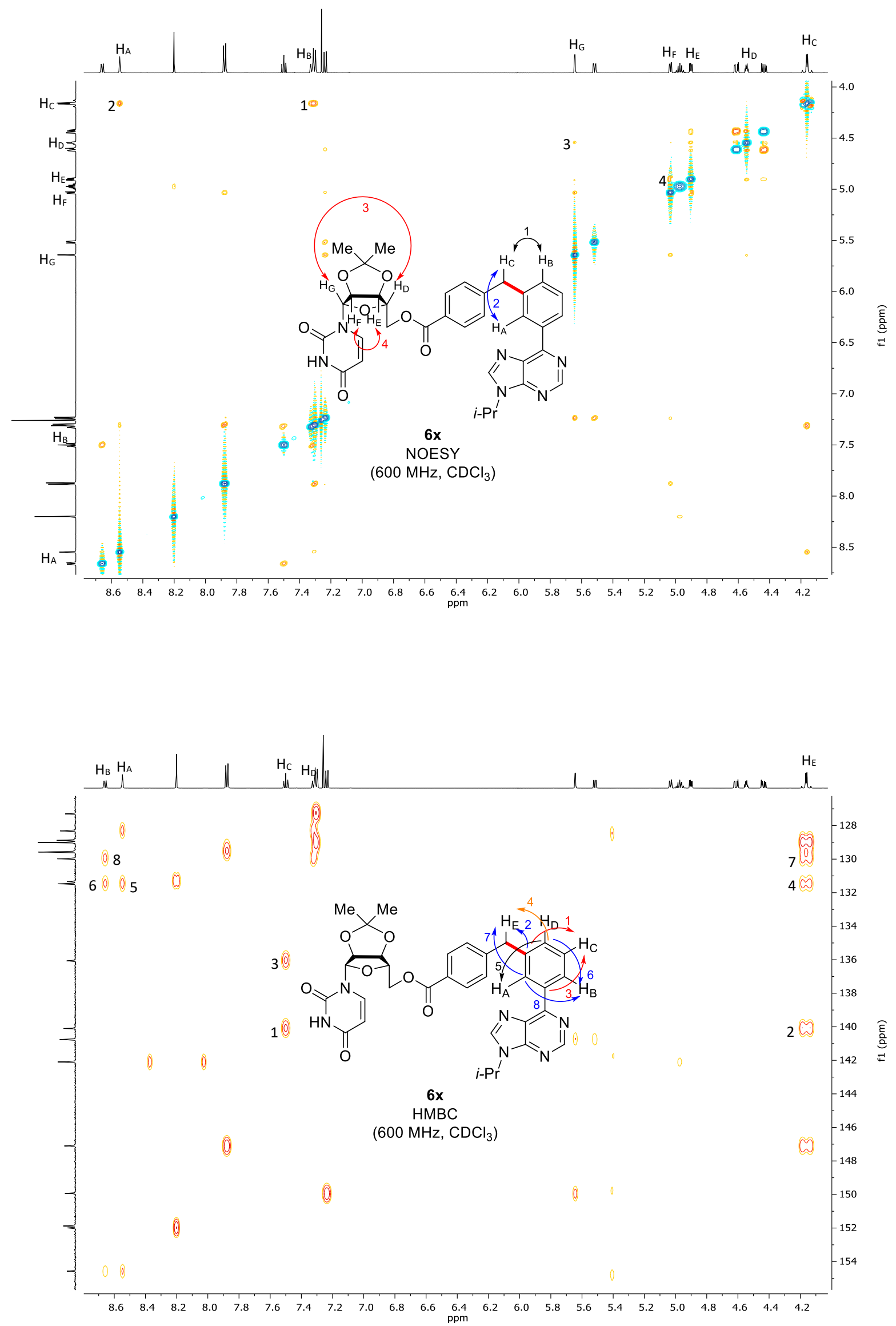


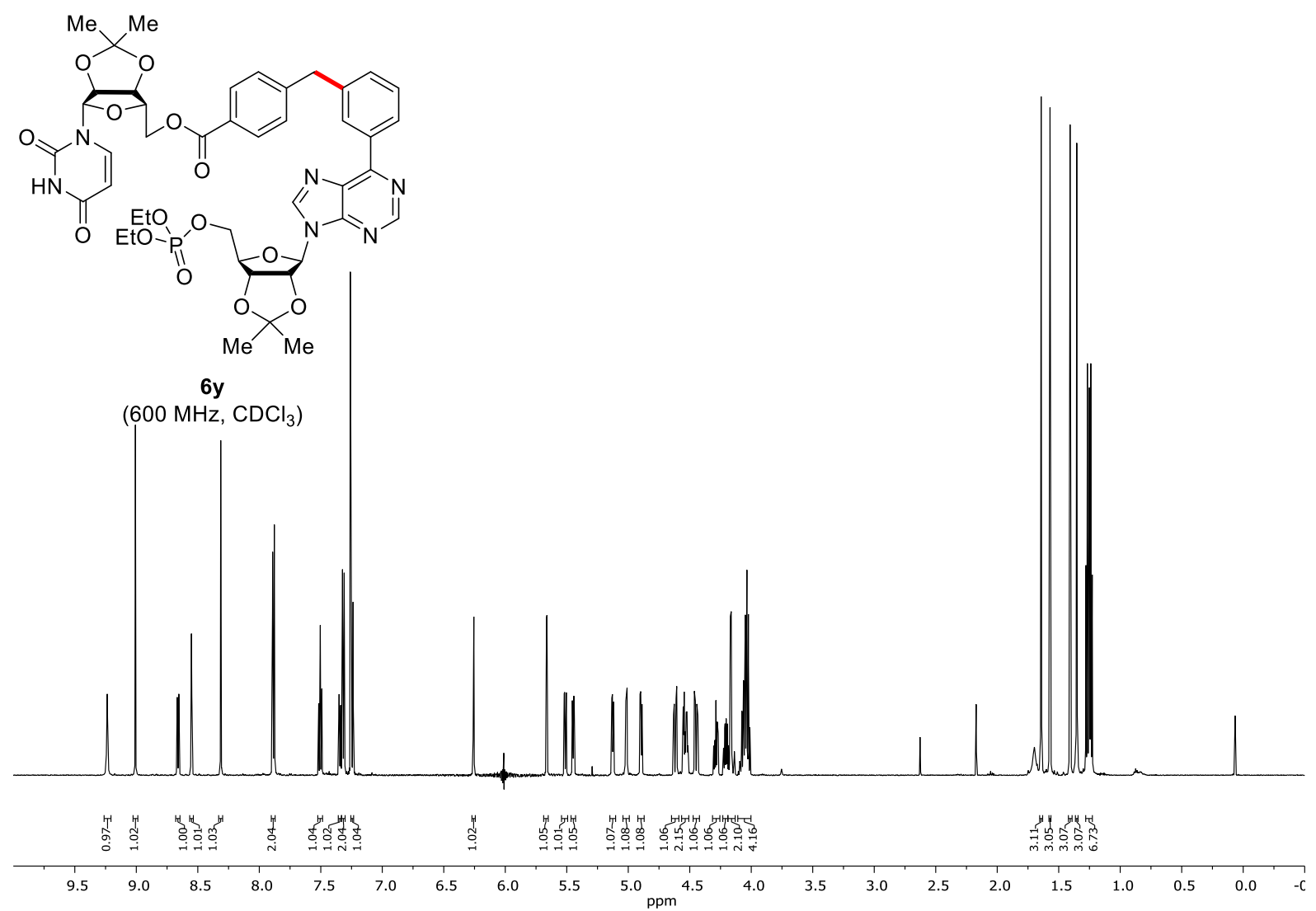

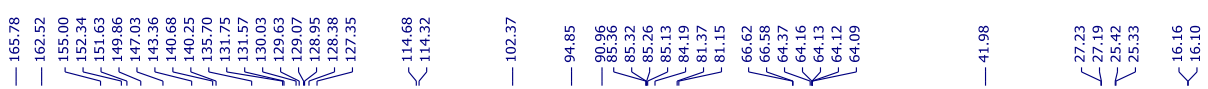<smiles></smiles>

$6 y$

(125 MHz, $\mathrm{CDCl}_{3}$ )

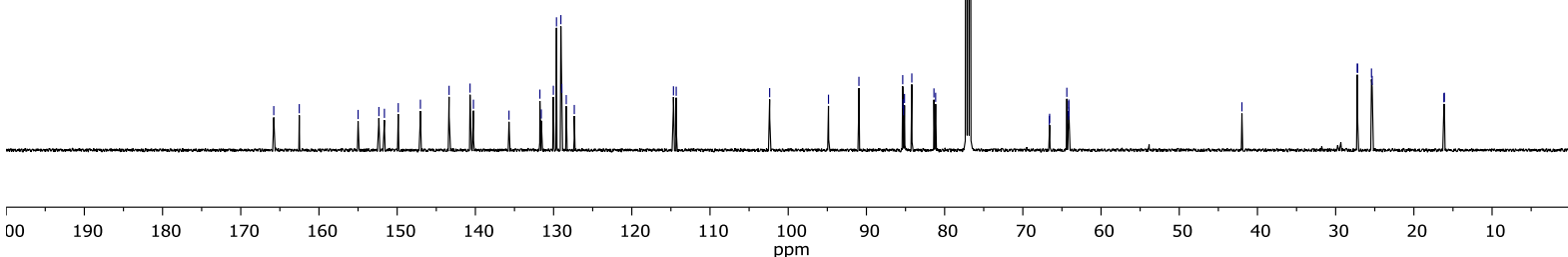




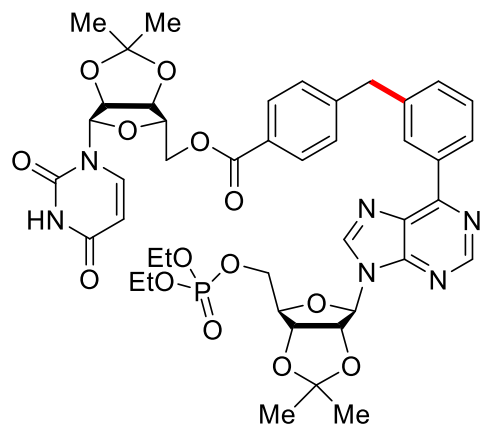

$6 y$

${ }^{31} \mathrm{P}\left\{{ }^{1} \mathrm{H}\right\}-N M R$

(121 MHz, $\mathrm{CDCl}_{3}$ )
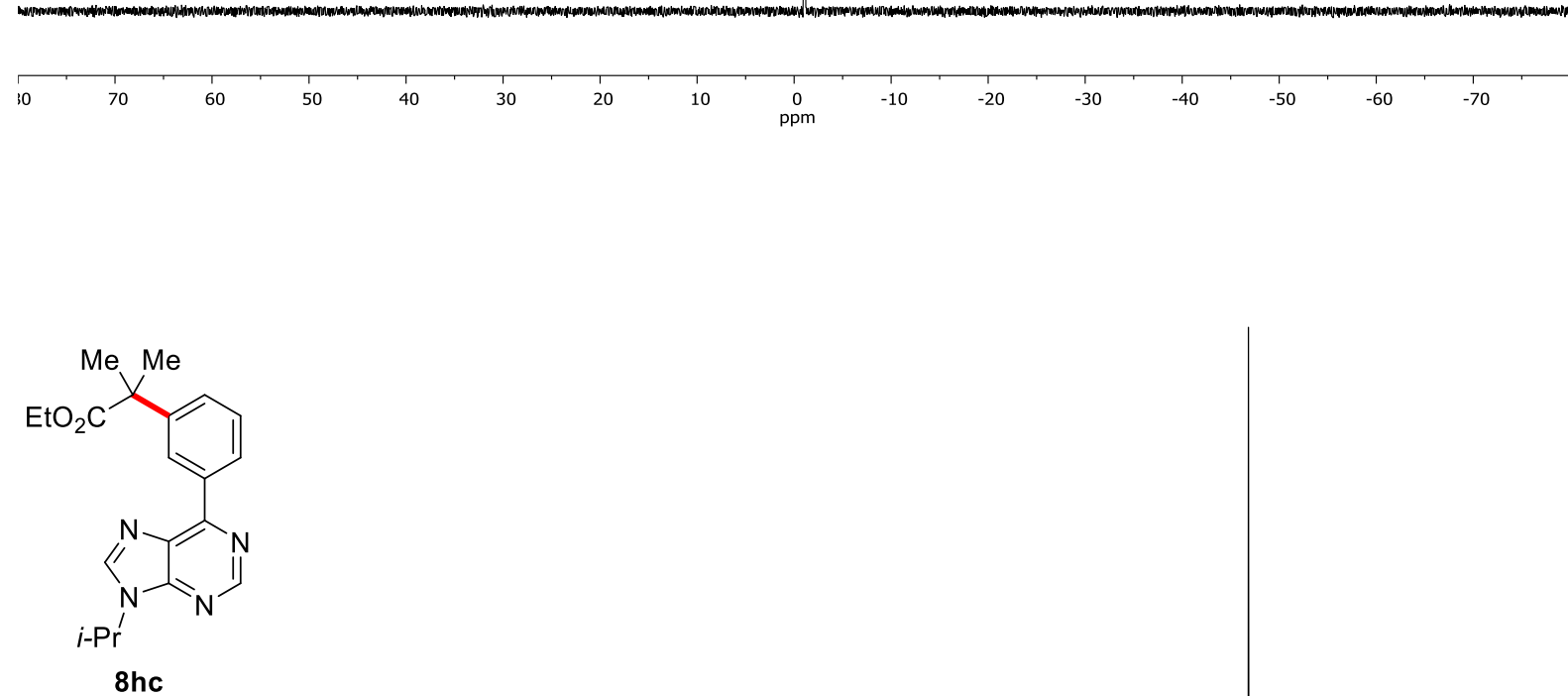

(600 MHz, $\mathrm{CDCl}_{3}$ )

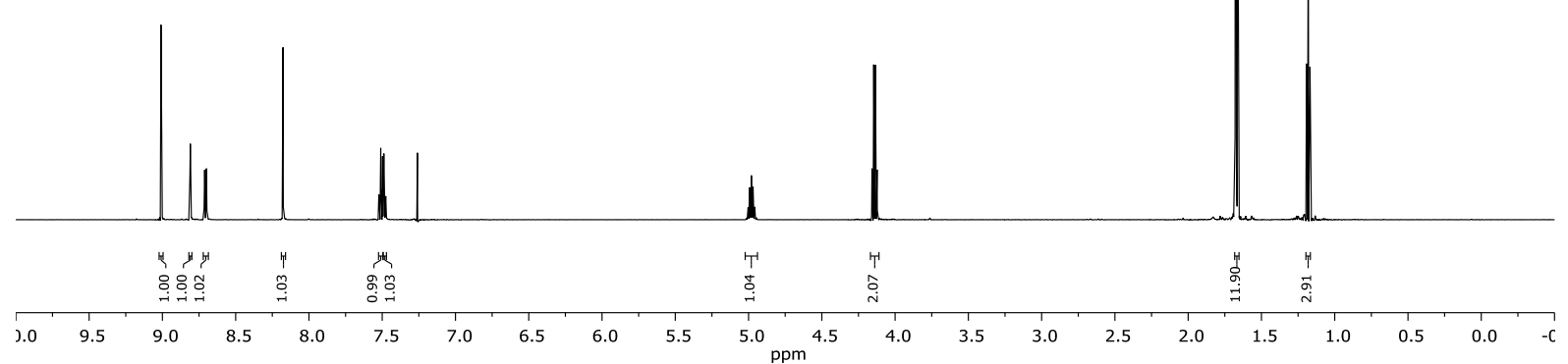




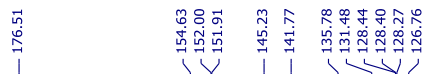

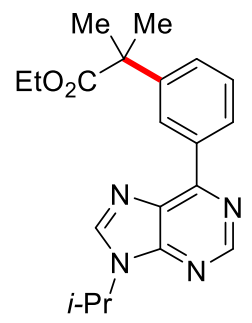

8hc

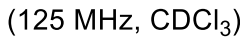
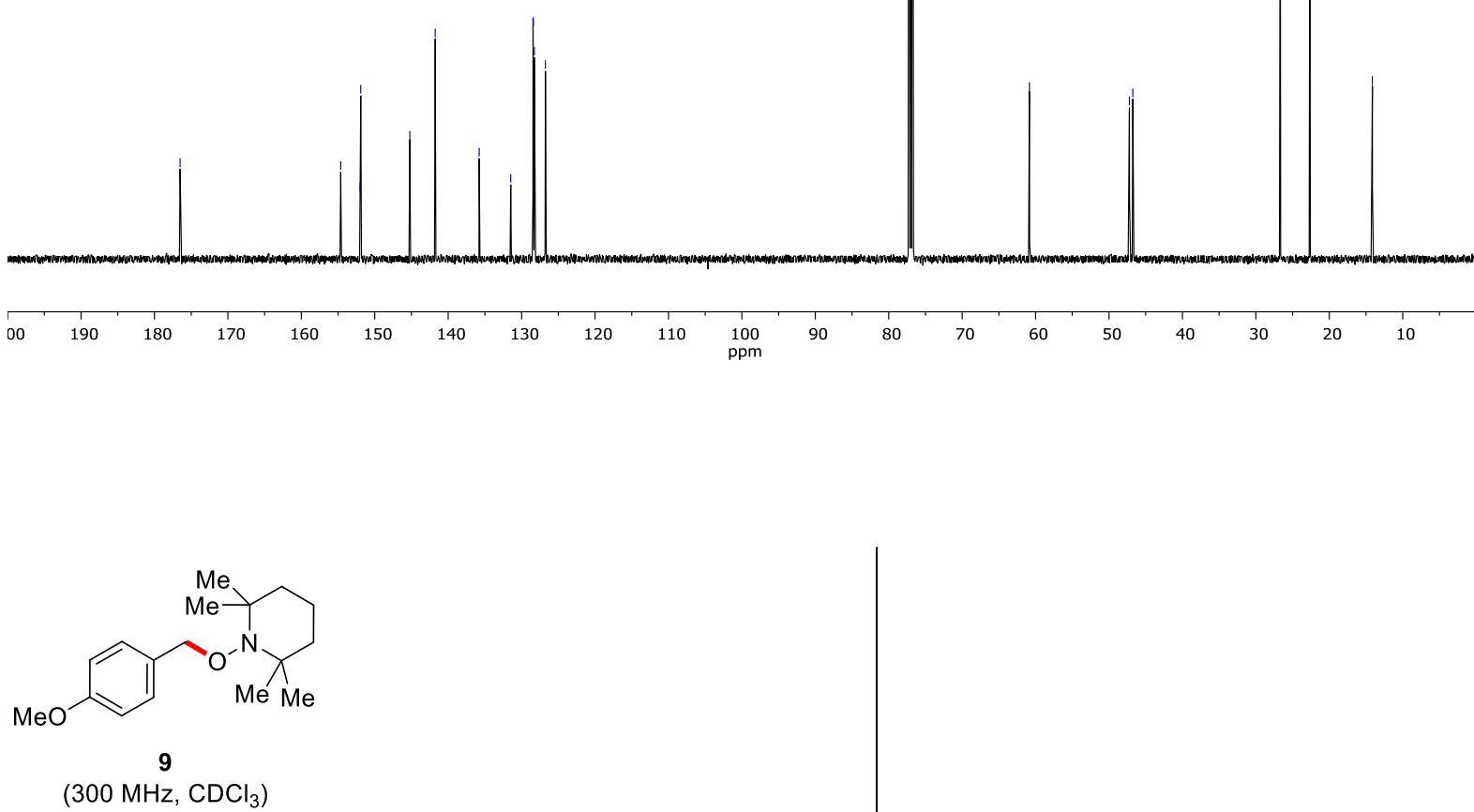

$\left(300 \mathrm{MHz}, \mathrm{CDCl}_{3}\right)$

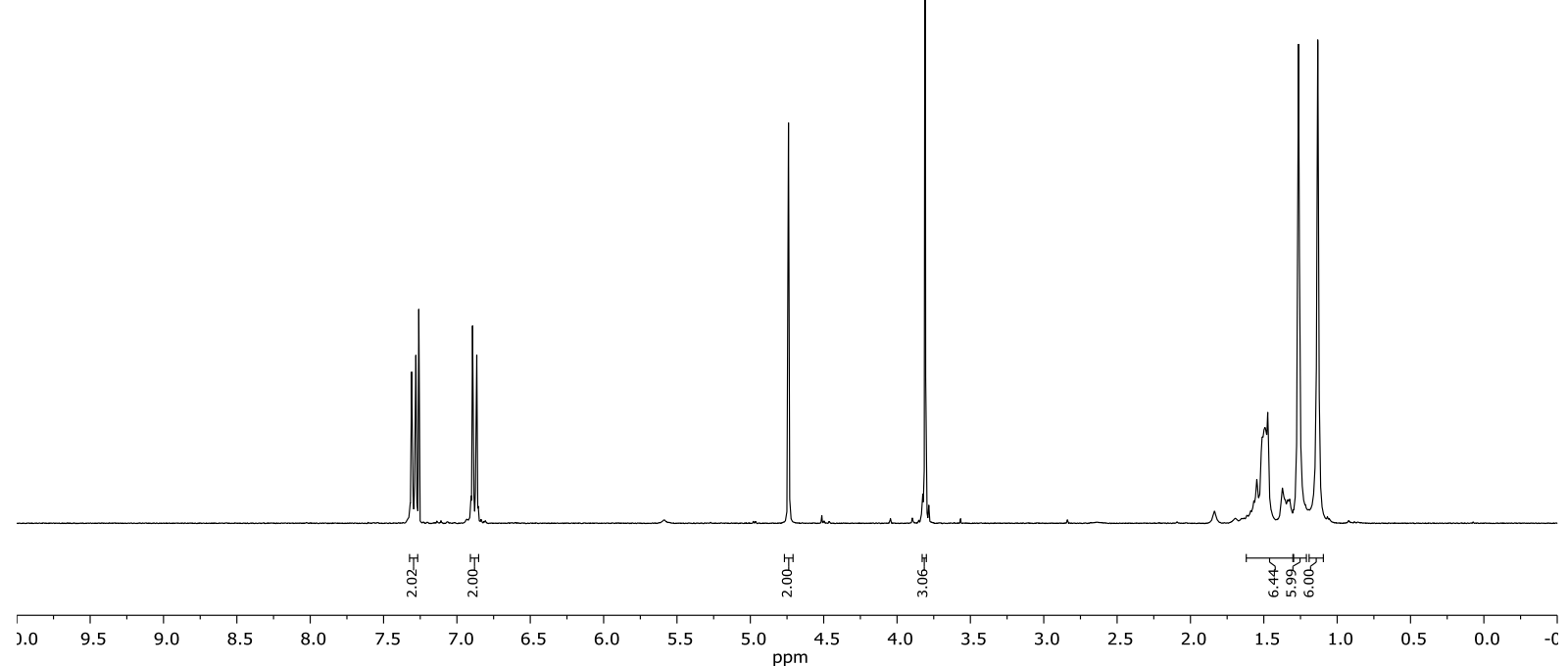



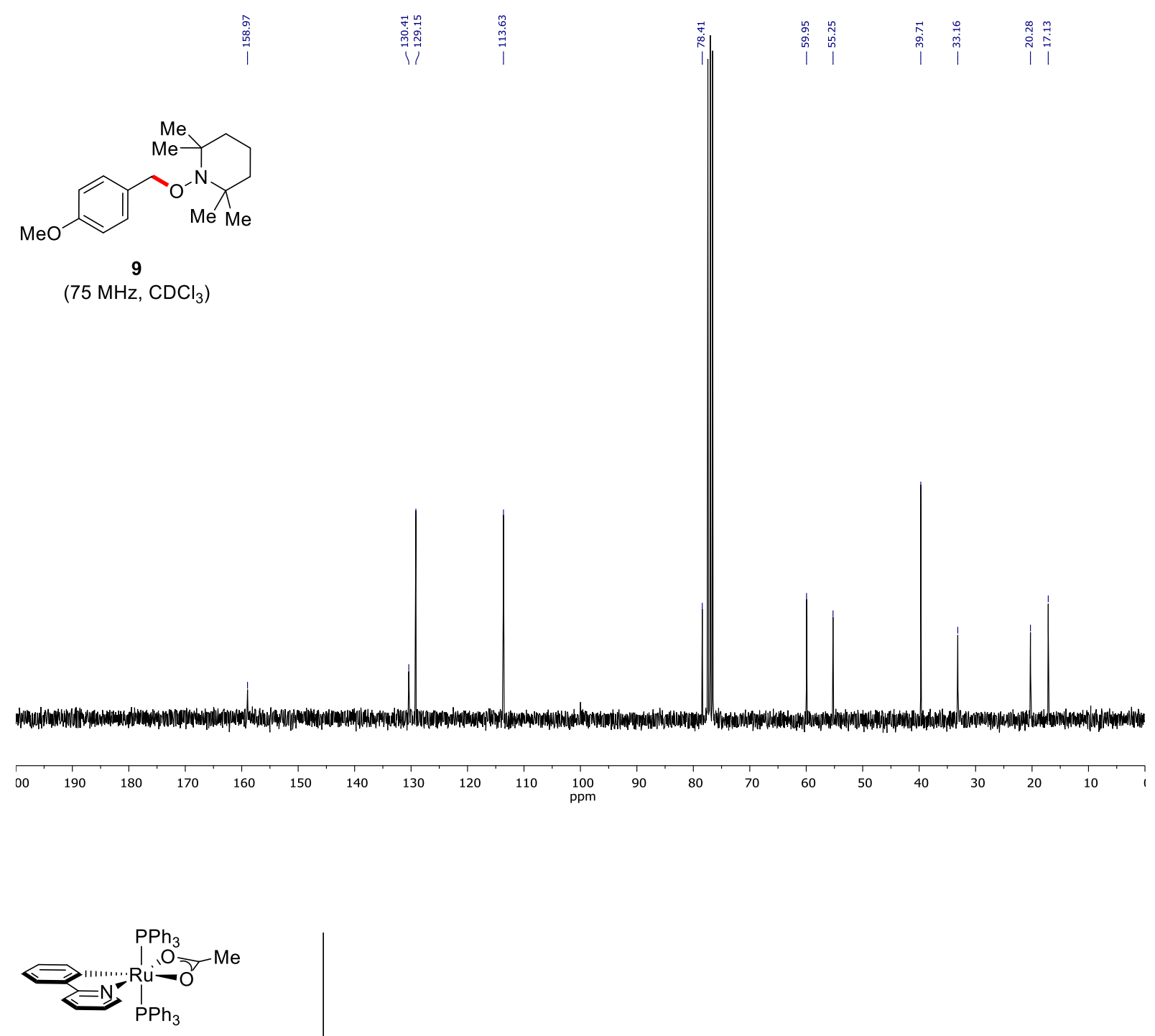

trans-Ru-II

(300 MHz, $\mathrm{CD}_{2} \mathrm{Cl}_{2}$ )

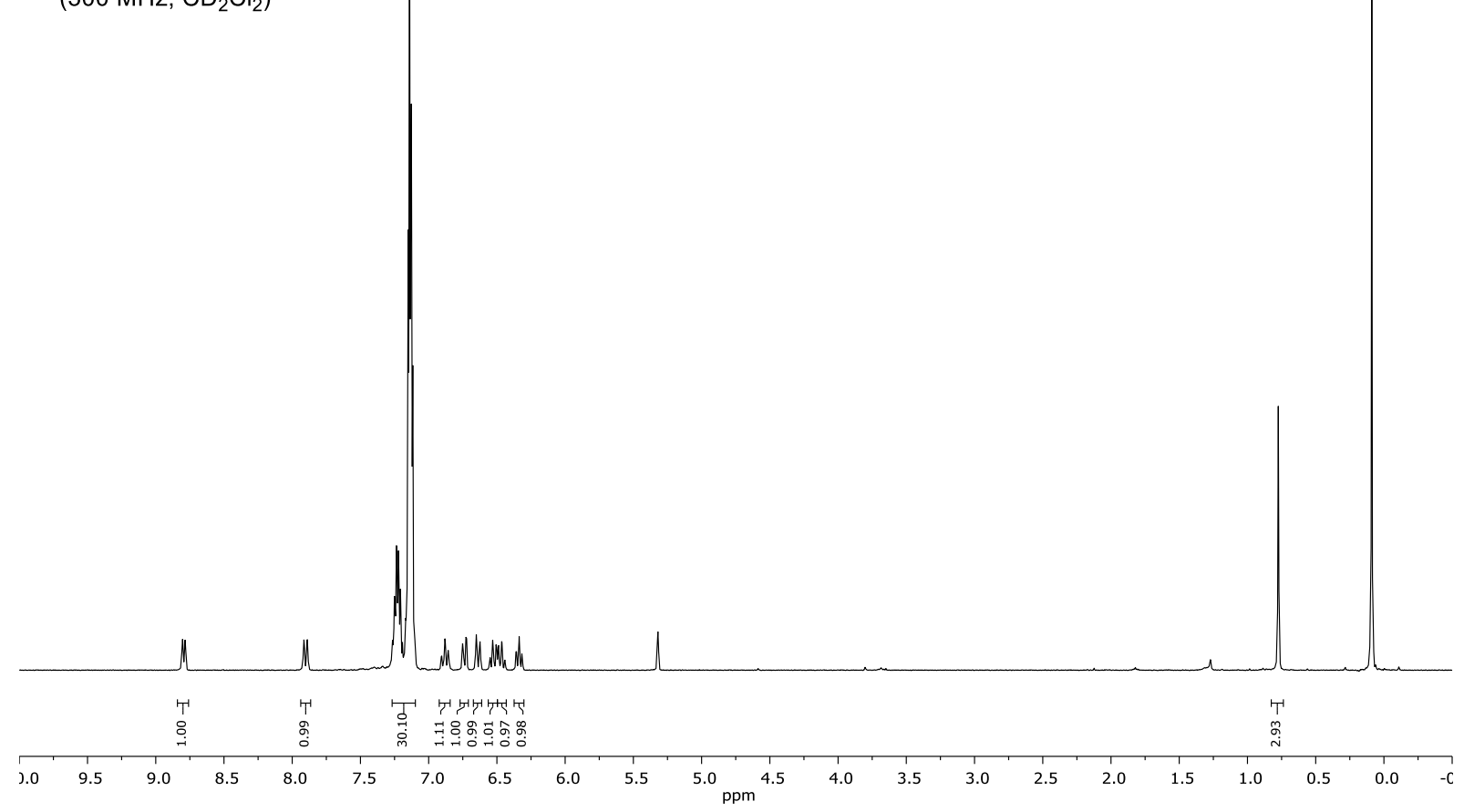



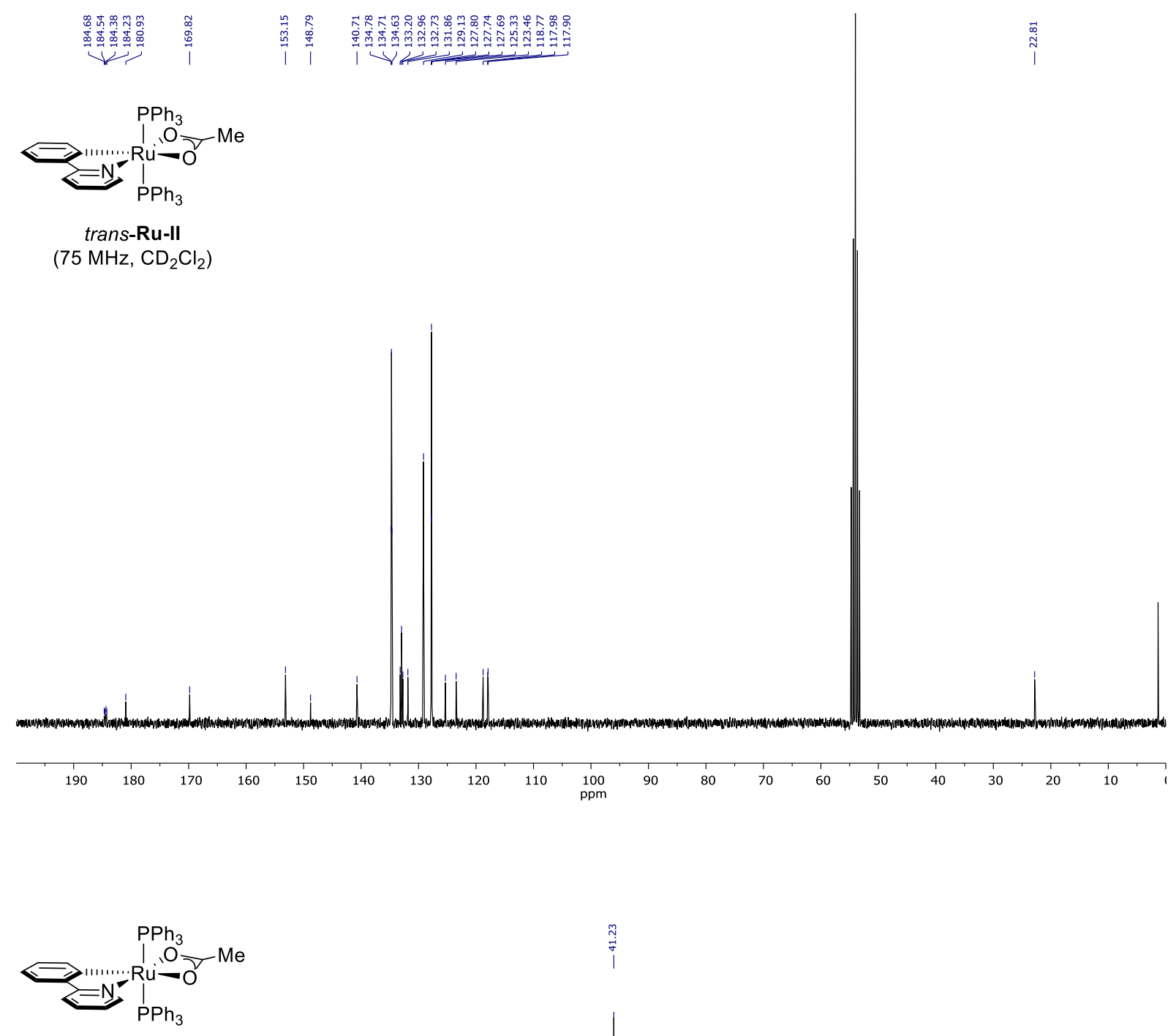

trans-Ru-II

${ }^{31} \mathrm{P}\left\{{ }^{1} \mathrm{H}\right\}-N M R$

(121 MHz, $\mathrm{CD}_{2} \mathrm{Cl}_{2}$ )

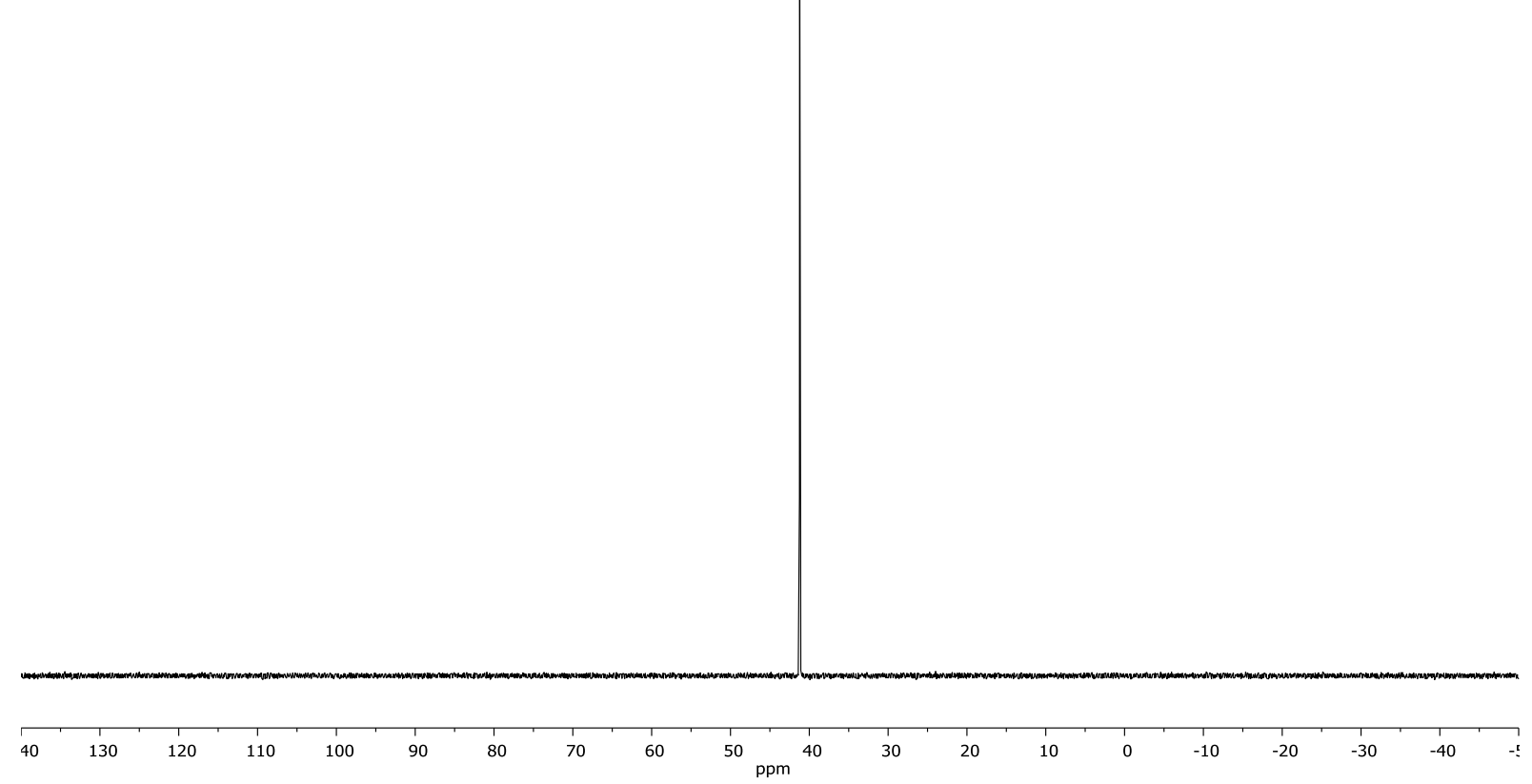




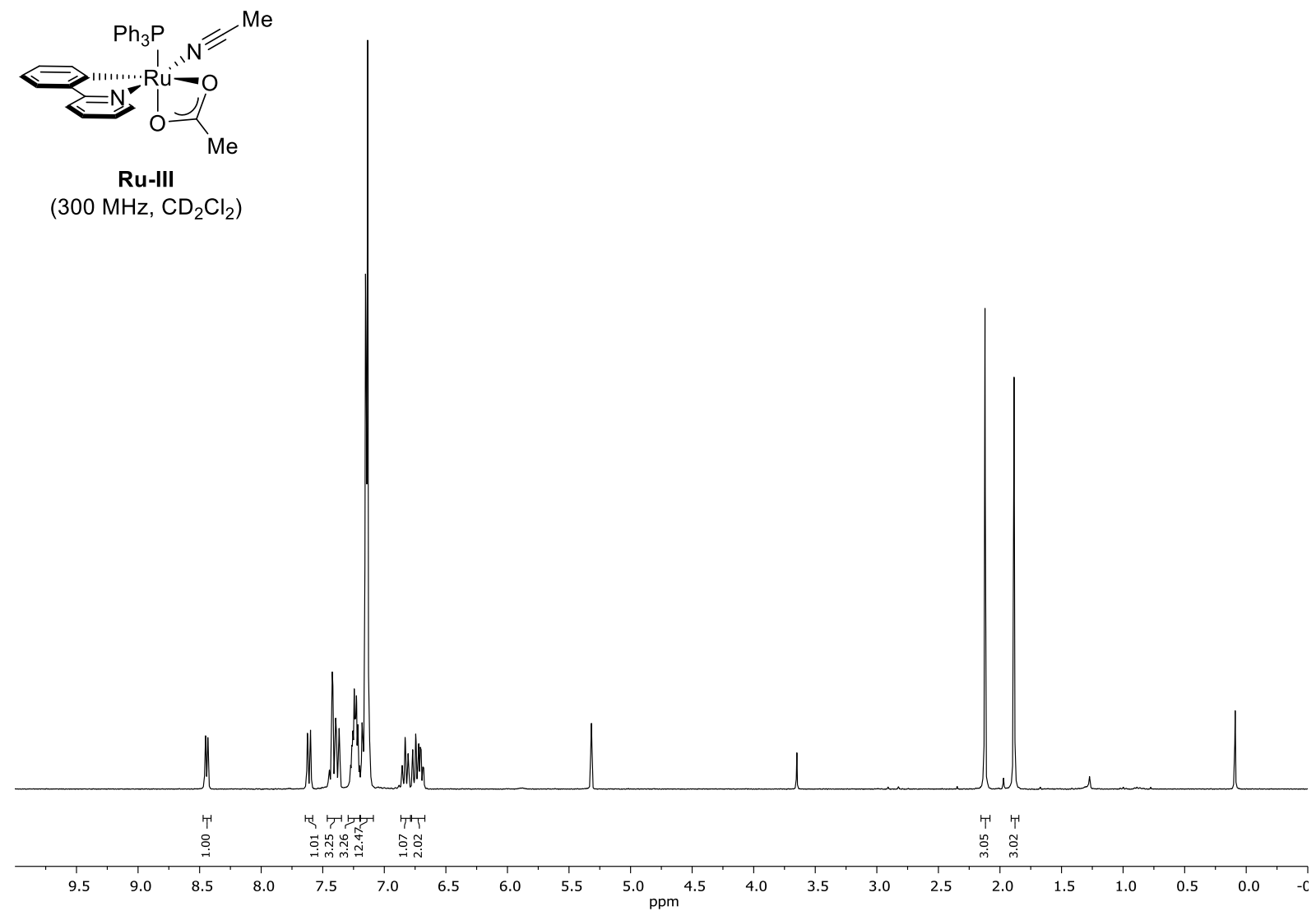

V|l |
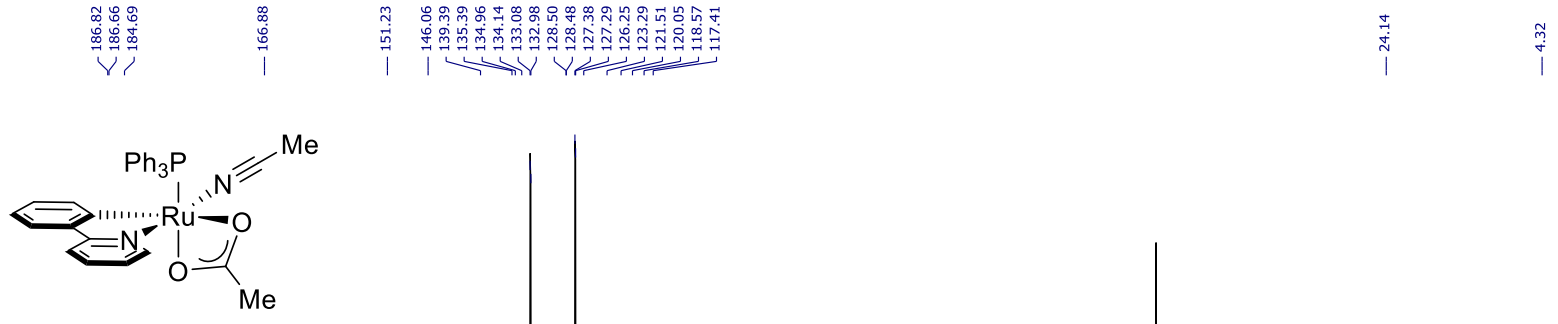

Ru-II

(100 MHz, $\mathrm{CD}_{2} \mathrm{Cl}_{2}$ )

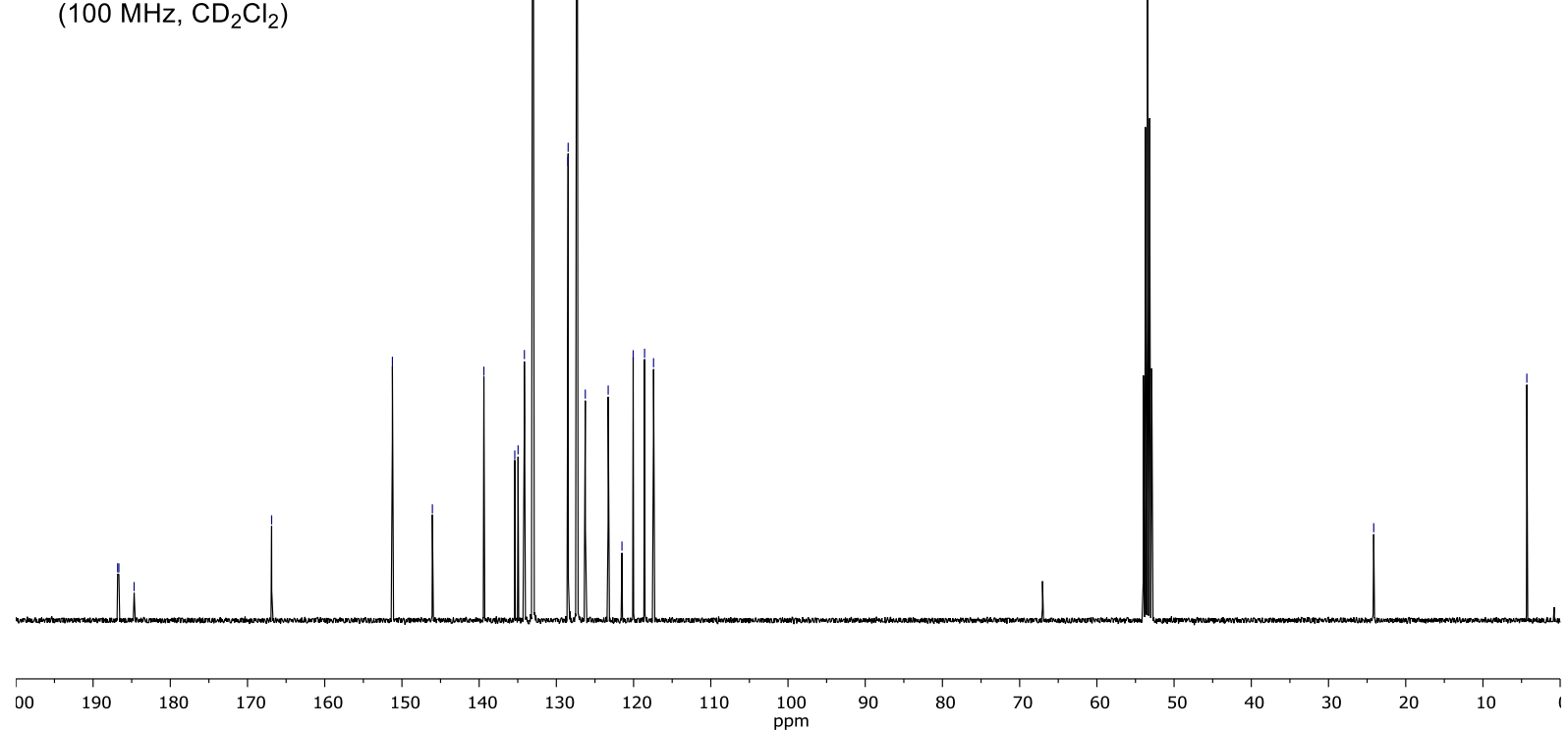




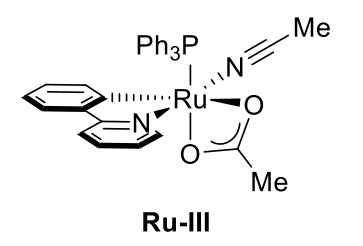

${ }^{31} \mathrm{P}\left\{{ }^{1} \mathrm{H}\right\}-\mathrm{NMR}$

(162 $\mathrm{MHz}, \mathrm{CD}_{2} \mathrm{Cl}_{2}$ )
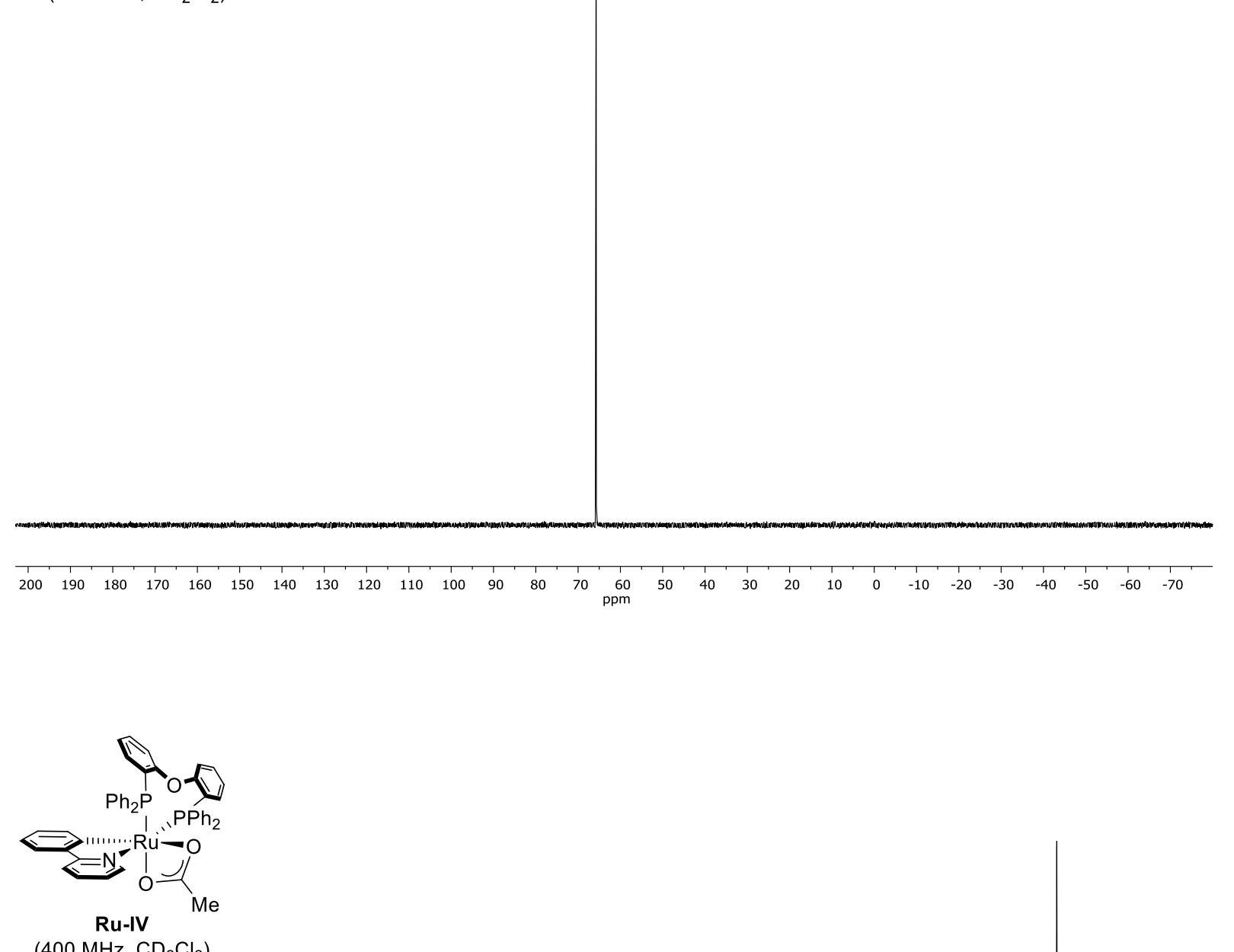

(400 MHz, $\mathrm{CD}_{2} \mathrm{Cl}_{2}$ )

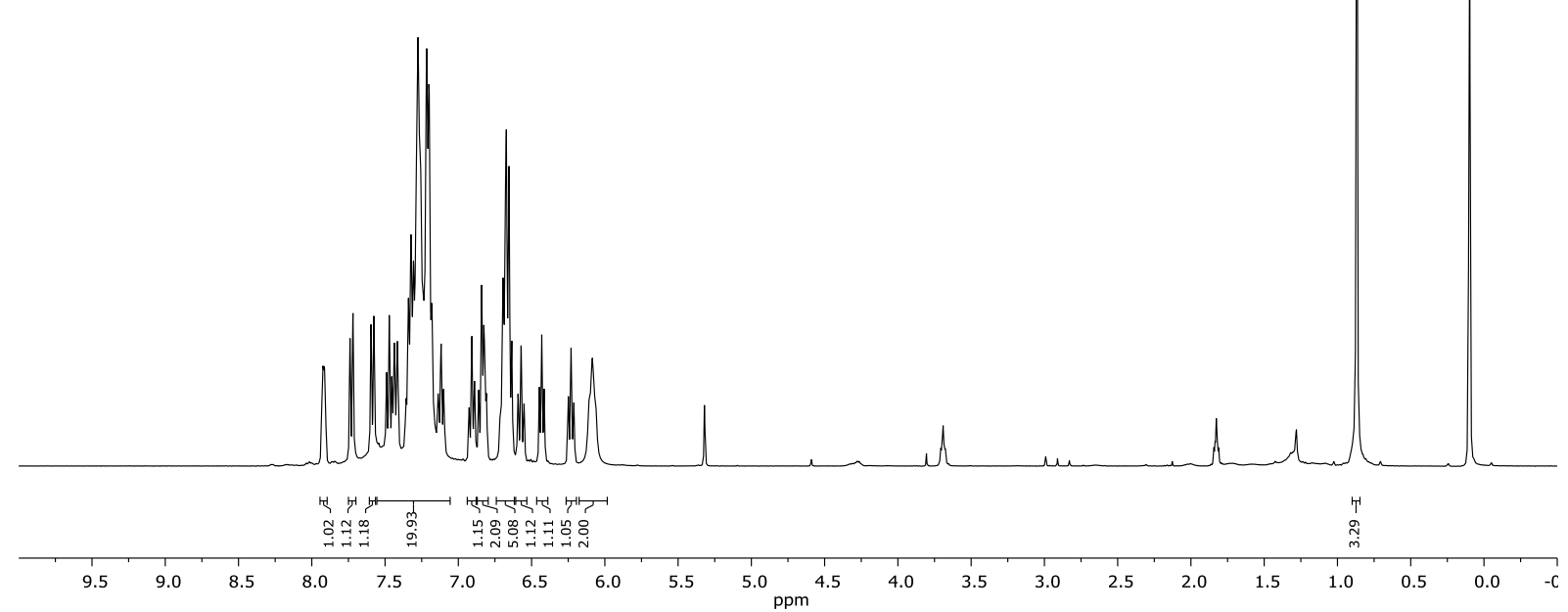




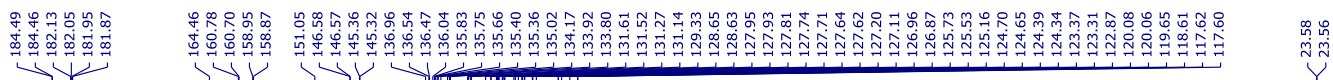
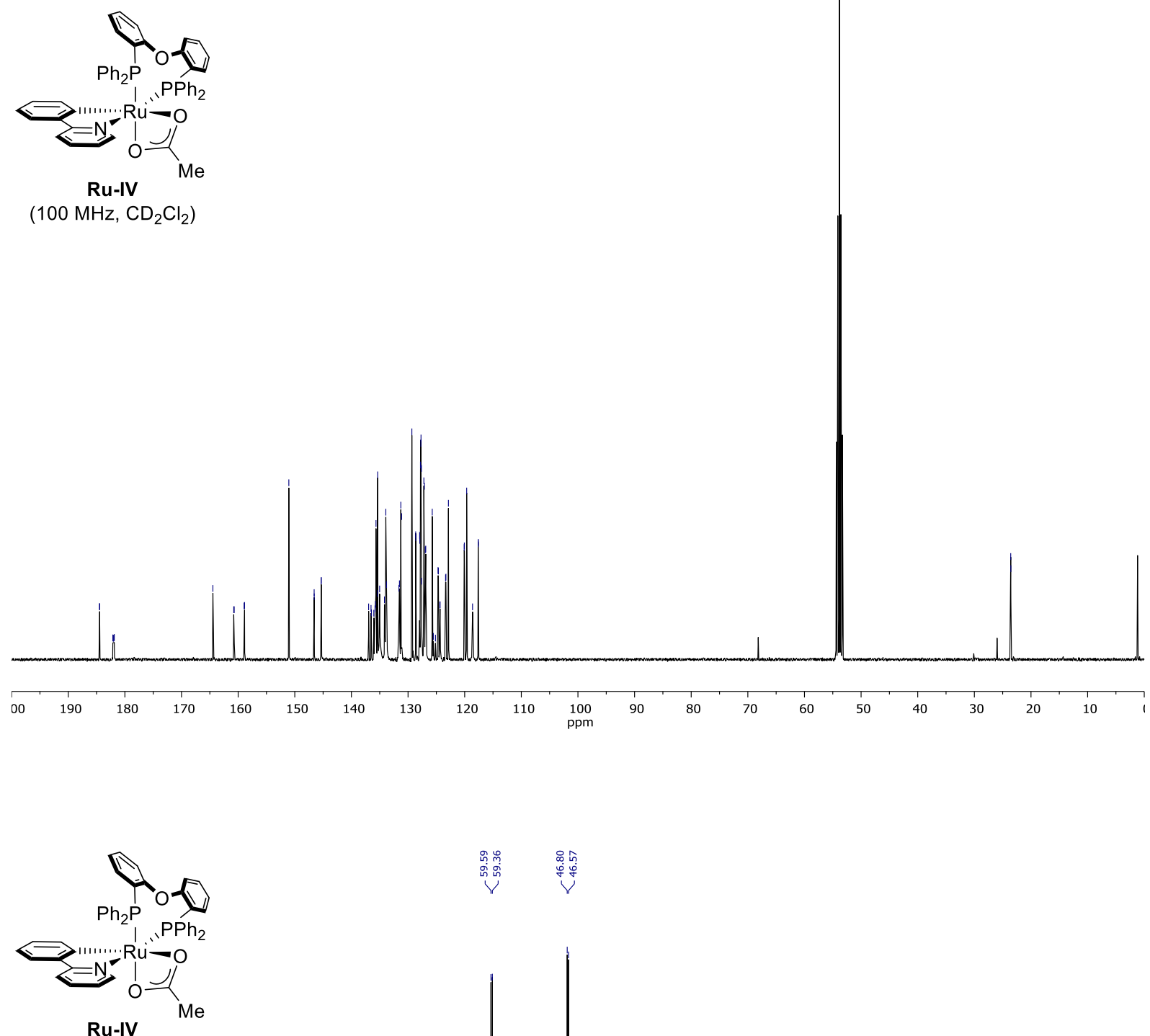

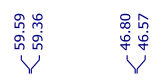

${ }^{31} \mathrm{P}\left\{{ }^{1} \mathrm{H}\right\}-\mathrm{NMR}$

(162 MHz, $\mathrm{CD}_{2} \mathrm{Cl}_{2}$ )

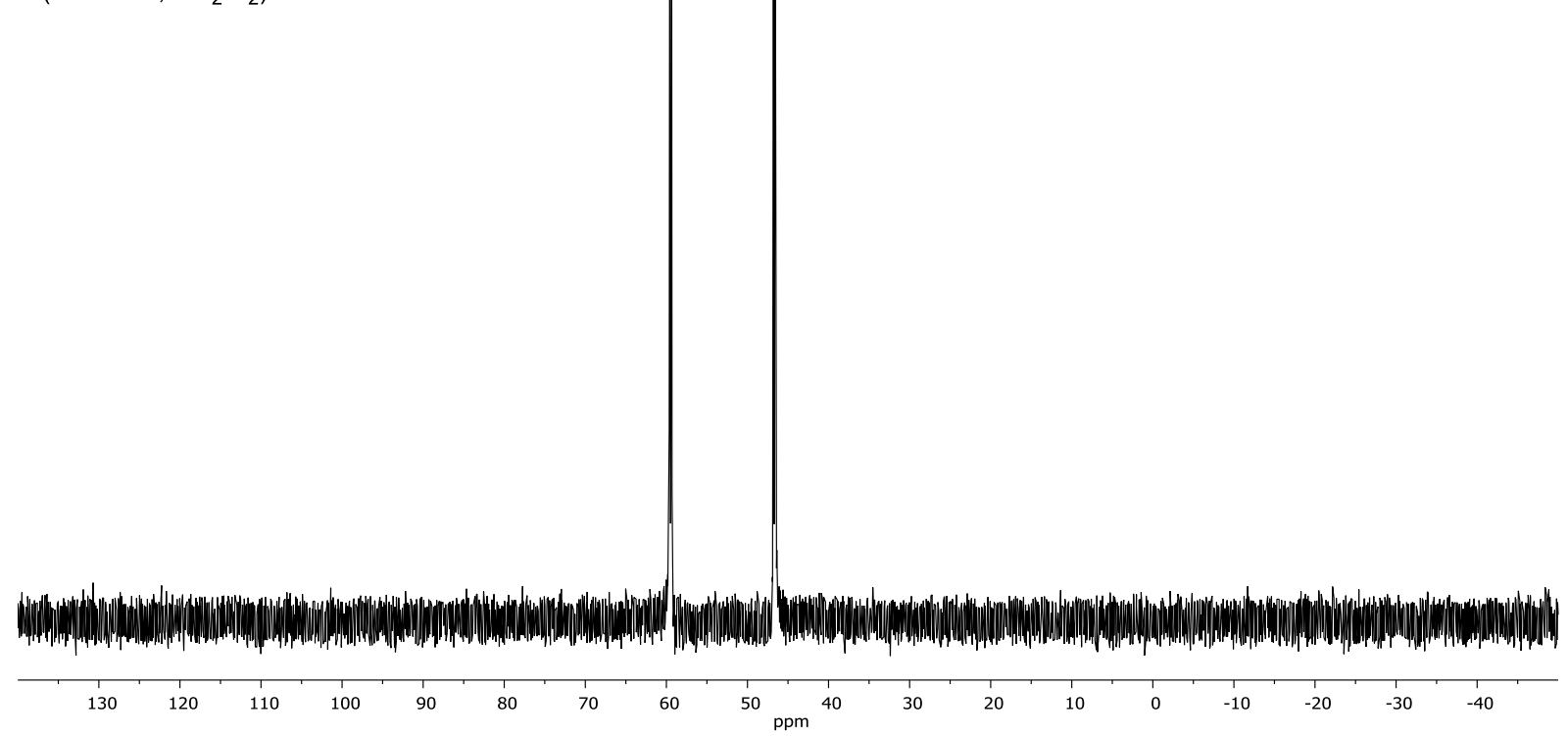

